\title{
Reliability-Based, Safety-Explicit Horizontal Curve Design Using Naturalistic Driving Study
}

by

\author{
Bashar Dhahir \\ B.Sc., Al-Mustansiriya University, Iraq \\ M.A.Sc. University of Baghdad, Iraq
}

A thesis submitted to the Faculty of Graduate and Postdoctoral Affairs in partial fulfilment of the requirements for the degree of

Doctor of Philosophy

in Civil Engineering
Department of Civil and Environmental Engineering Carleton University
Ottawa, Ontario

(C) 2018 Bashar Dhahir

The Doctor of Philosophy in Civil Engineering is a joint program with the University of Ottawa, administered by the Ottawa-Carleton Institute for Civil Engineering 


\section{Abstract}

The high rate of collisions on horizontal curves compared to other roadway elements makes them one of the most critical elements in a transportation network. In this regard, the current practice to design horizontal alignment needs to be reviewed. The current design guides adopt a deterministic approach to design horizontal curves considering only the driver comfort criterion and using a point mass model in the analysis. Therefore, the current design procedure suffers from several shortcomings. First, it ignores the importance of the probabilistic factors of highway operations and overlooks the variability effects of the factors included in the design. Second, adopting the comfort criterion and ignoring other design criteria, such as vehicle dynamic stability and sight distance, might lead to unsafe design in some cases. Third, using the point mass model in the analysis is questionable since it is insensitive to vehicle characteristics and the road profile. Also, the comfort thresholds used in the design are obtained from experiments conducted in the 1930s. This thesis aims at overcoming some of these issues through developing a new framework to design horizontal curves.

The new design framework adopts the reliability based-design approach and considers four design criteria in the design procedure to accommodate the driver behaviour in a quantitative manner. The naturalistic driving study database is used to examine driving behaviour on horizontal curves and to develop new models to predict the distributions of speed on curves and driver comfort thresholds. In addition, CarSIM software was used to develop models to estimate lateral friction demand with a higher level of accuracy. The first order reliability method is used to evaluate horizontal curve performance. The outputs 
of the reliability analysis were used as predictors in developing safety performance functions.

Reliability-based design concept is a promising concept that can be implemented in transportation engineering. This concept can provide a quantitative evaluation and can be used as a surrogate measure for safety performance. The research found that evaluation of horizontal curves performance should consider all design criteria simultaneously because changing geometric parameters to improve one of the design criterion, would affect the other design criteria. 


\section{Acknowledgements}

The author would like to express his deepest and sincerest appreciation to his supervisor, Professor Yasser Hassan, for his valuable support and guidance throughout the completion of this research. His time, efforts, and patience are highly appreciated.

The author would like to express his gratitude to Ben Dupery in the Mechanical Simulation Corporation for providing technical assistance with CarSIM software, and to Anusha Nujjetty (HSIS lab manager) and Kevin Perkins (WSDOT-Multimodal Planning Division) for providing necessary data and information to conduct the analysis of this research. The author is grateful to Miguel Perez, Christina Witcher, and Whitney Atkins (Virginia Tech Transportation Institute) for their help in providing the naturalistic driving study data.

The author is grateful to Dr. Joseph Hilbe for his assistance in modelling safety performance functions. In addition, support by the Natural Sciences and Engineering Council (NSERC) is gratefully acknowledged.

Bashar S. Dhahir 


\section{Dedication}

To my mother and father, I wish you were here,

To my beloved wife, thank you for your patience,

To my sister and brothers, thank you for your support. 


\section{TABLE OF CONTENTS}

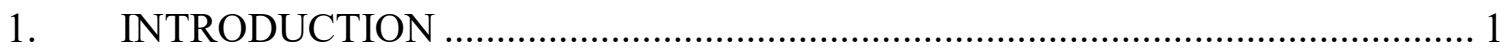

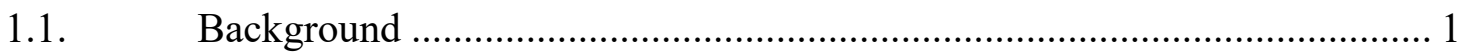

1.2. Horizontal Curve Design .................................................................... 3

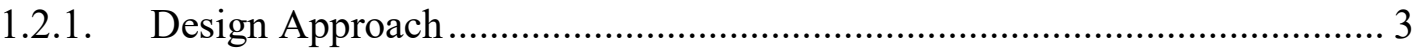

1.2.2. Design Criteria ........................................................................... 4

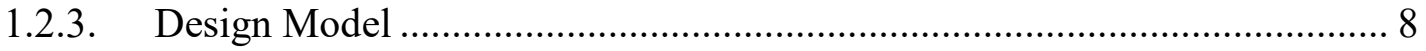

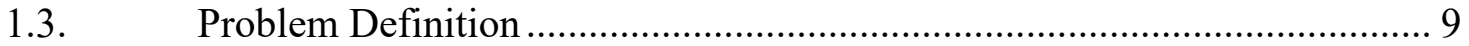

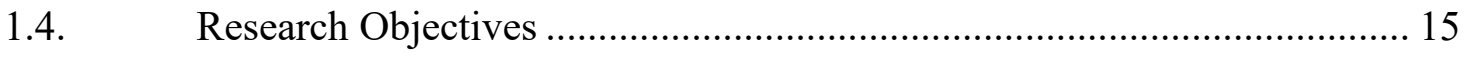

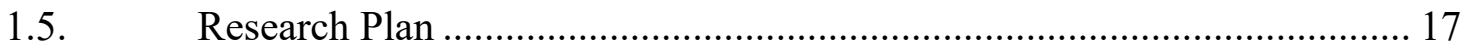

1.6. Research Scope and Organization .................................................. 18

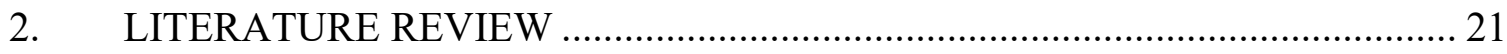

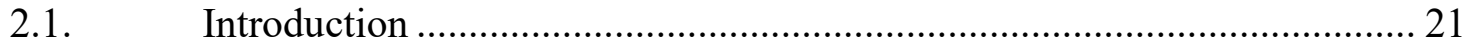

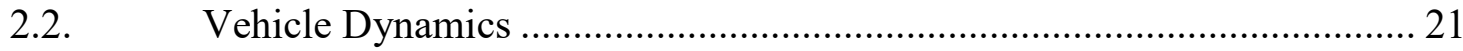

2.2.1. Vehicle Stability Models ............................................................................. 23

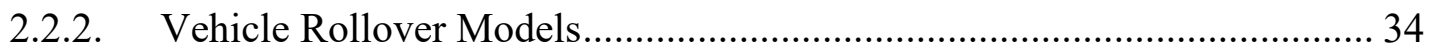

2.2.3. Driver Comfort Model........................................................................ 38

2.3. Vehicle Stability and Control ..................................................... 38

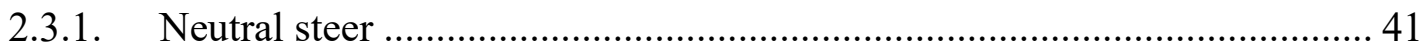

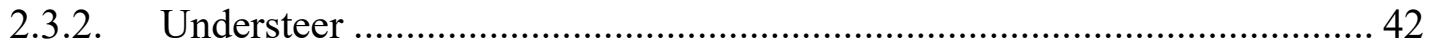

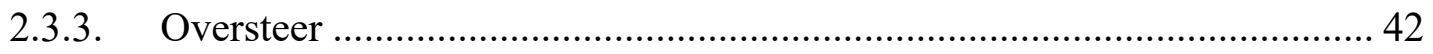

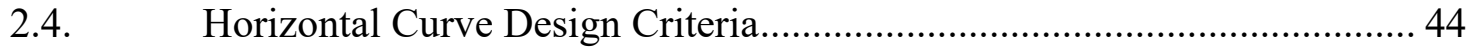

2.4.1. Driver Comfort Criterion................................................................. 45

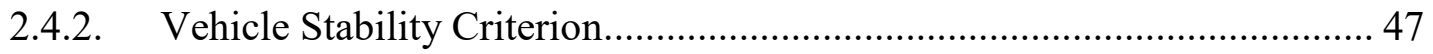




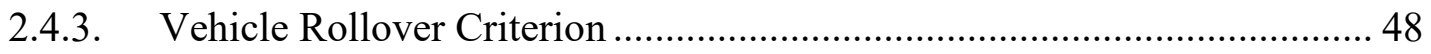

2.4.4. Sight Distance Criterion ............................................................................. 49

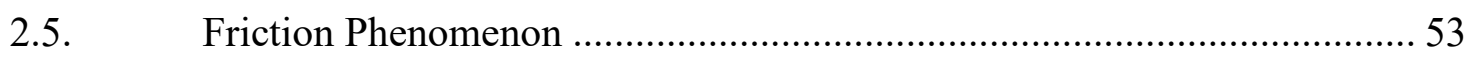

2.5.1. Pavement Texture.................................................................................. 54

2.5.2. Tire-Pavement Friction Coefficient and Force............................................ 56

2.5.3. Factors Affecting Available Friction.......................................................... 56

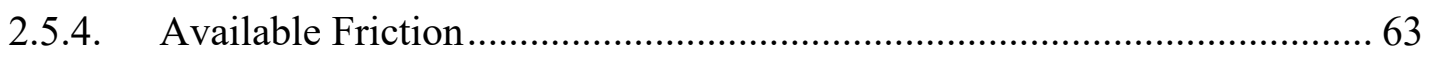

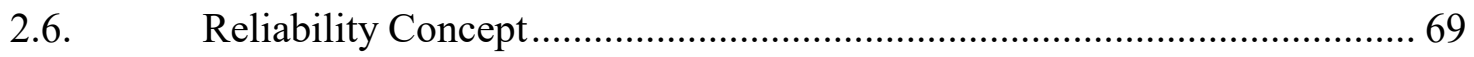

2.6.1. Reliability Performance Functions ........................................................... 70

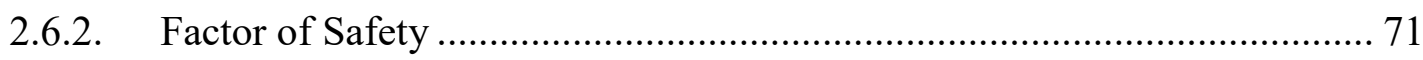

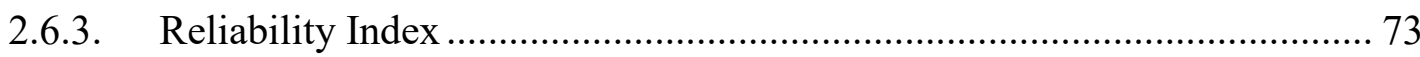

2.6.4. System Reliability ................................................................................. 74

2.6.5. Reliability-Based Design........................................................................ 77

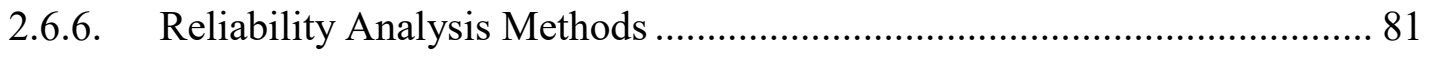

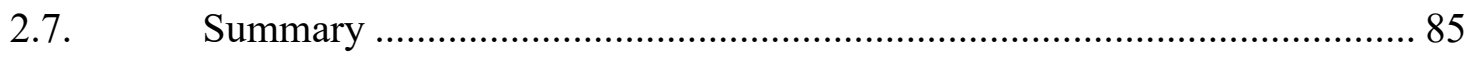

3. RESEARCH METHODOLOGY AND DATA ……………………………........ 86

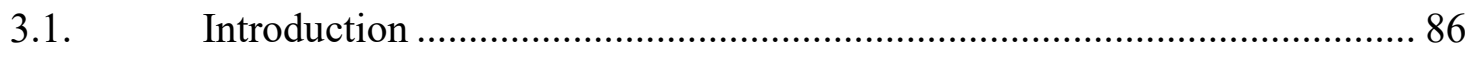

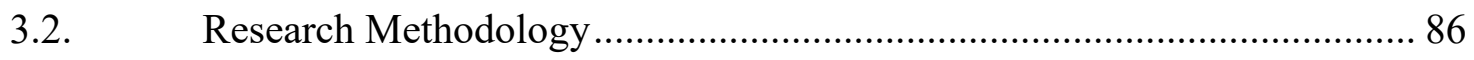

3.3. Horizontal Curve Design Framework ………………….......................... 90

3.4. Evaluation of Design Criteria .............................................................. 92

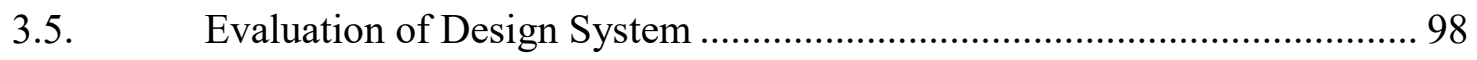

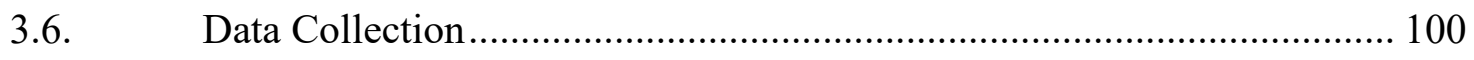

3.6.1. Naturalistic Driving Study Database ........................................................ 101

3.6.2. Pavement Friction Data .......................................................................... 113

3.6.3. Roadway Geometric Data .................................................................. 115 
3.6.4. Lateral Friction Demand Data ................................................................ 119

3.6.5. Lateral Acceleration Data...................................................................... 120

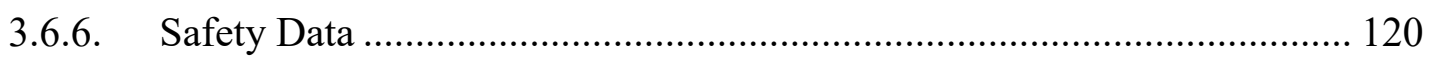

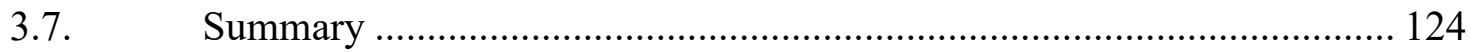

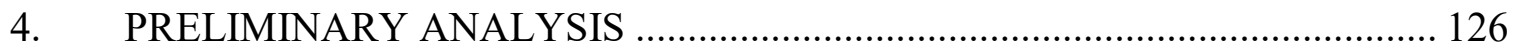

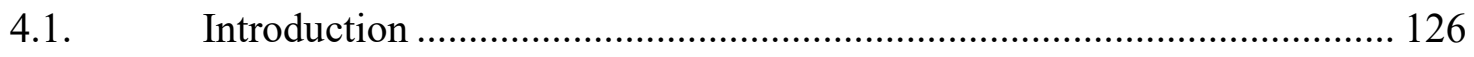

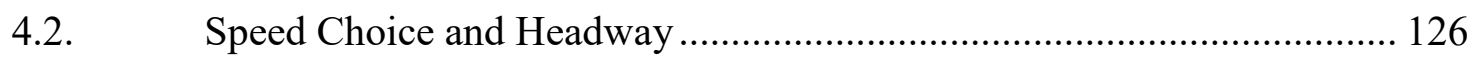

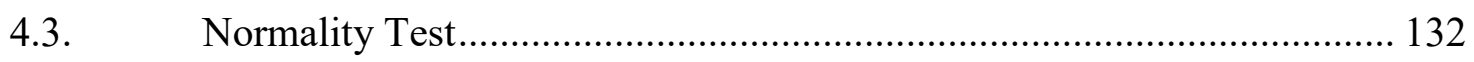

4.4. Comparing Instantaneous and Average Speeds ................................... 133

4.5. Comparing Speeds for Comfort Threshold ........................................... 133

4.6. Comparing Other Speed Variables ........................................................ 134

4.7. Comparing Variances ...................................................................... 134

4.8. Comparing Left and Right Turn Curves................................................ 135

4.8.1. Variables Correlation .......................................................................... 138

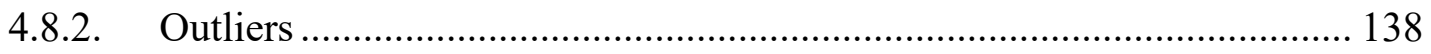

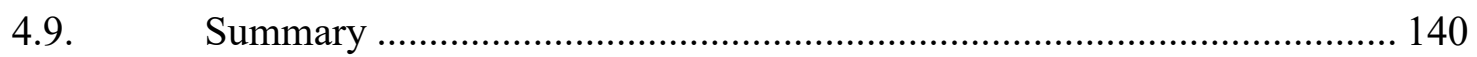

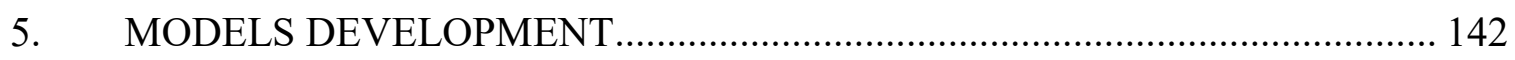

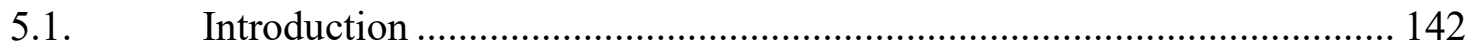

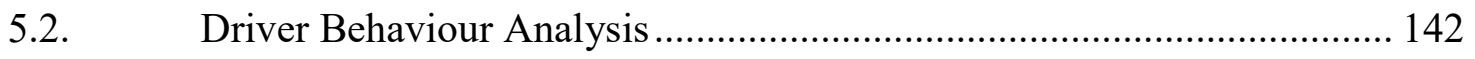

5.2.1. General Speed State........................................................................... 143

5.2.2. Curve Speed Measures ………………………………........................ 146

5.2.3. Driver Comfort Threshold ..................................................................... 147

5.2.4. Sensitivity Analysis ............................................................................. 150

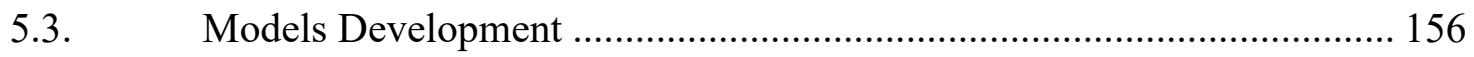

5.3.1. Speed models ........................................................................................ 157

5.3.2. Comfort Threshold Models .................................................................. 160 
5.3.3. Lateral Friction Demand Model .............................................................. 164

5.3.4. Lateral Acceleration Model ...................................................................... 167

5.3.5. Available Friction Models....................................................................... 169

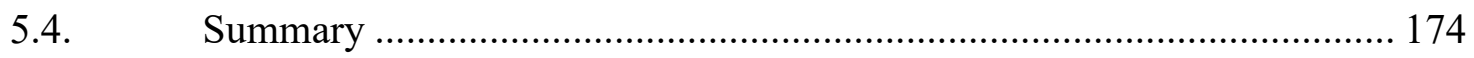

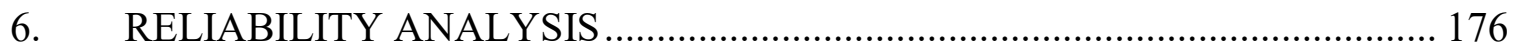

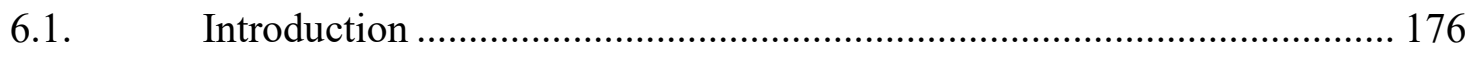

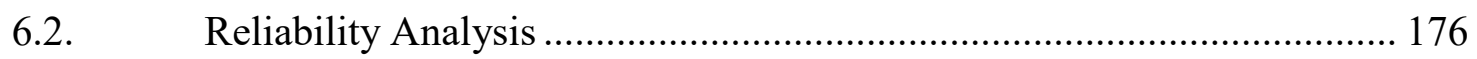

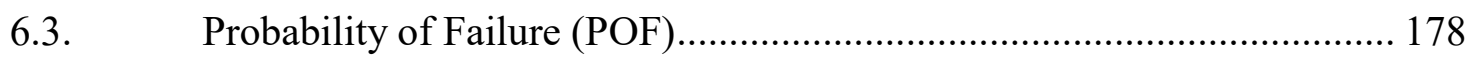

6.4. Probability of Noncompliance (PNC) ……….................................... 182

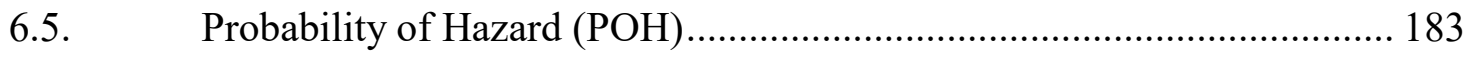

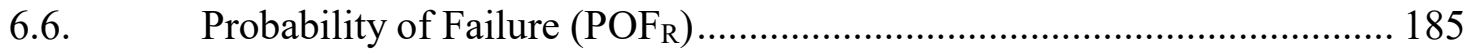

6.7. Two-Lane Highway Assessment ............................................................ 187

6.7.1. Probability of Failure Adjustment........................................................... 189

6.7.2. Vehicle Stability Assessment .................................................................. 190

6.7.3. Driver Comfort Assessment …………………….................................... 194

6.7.4. Sight Distance Assessment.................................................................. 198

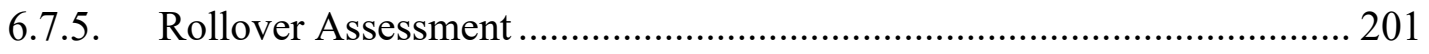

6.8. Reliability Indices Correlations ............................................................ 203

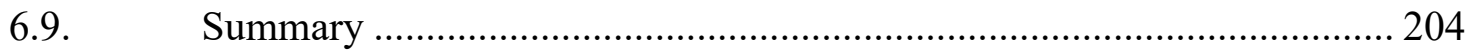

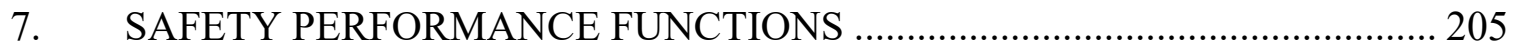

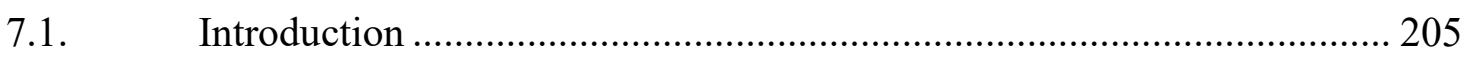

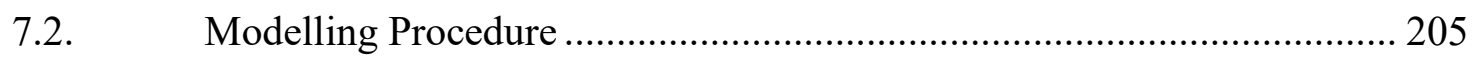

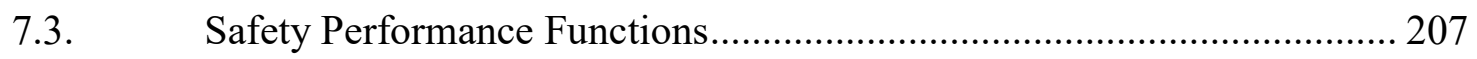

7.3.1. SPFs of Annual Collision Frequency ………………………………...... 208

7.3.2. SPFs of 5-Year Collisions .................................................................. 219

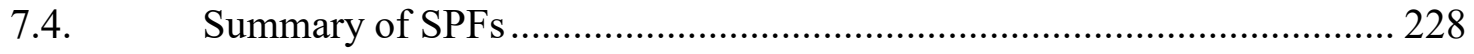


7.5. Horizontal Curve Design Procedure ........................................................ 229

7.5.1. Initial Values Selection ...................................................................... 230

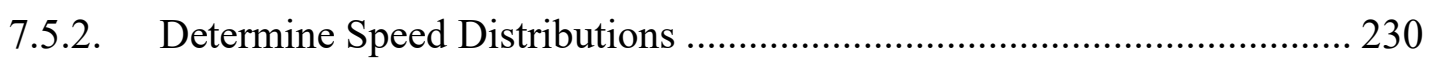

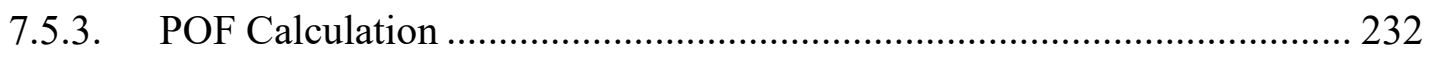

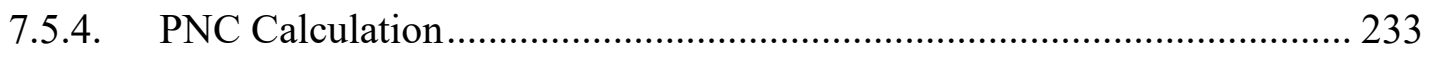

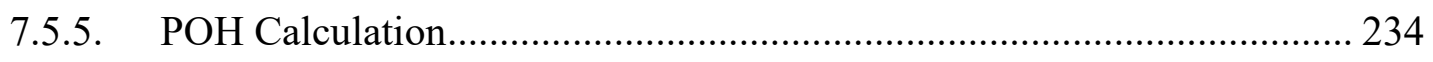

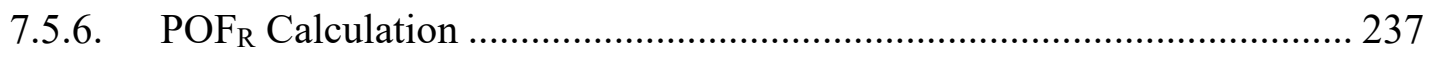

7.5.7. Overall Probability of Failure................................................................. 239

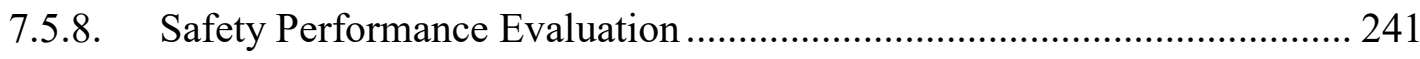

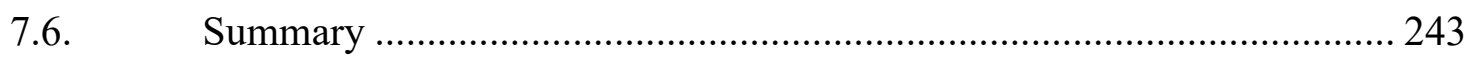

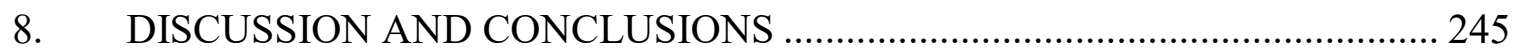

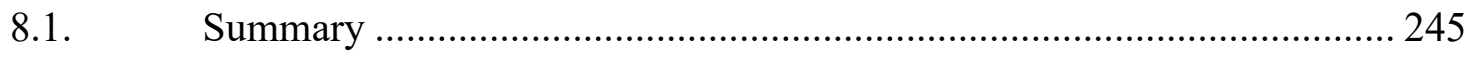

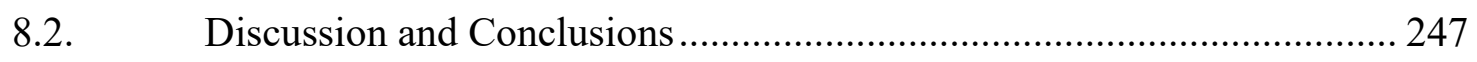

8.3. Recommendations for Future Research ………………....................... 250

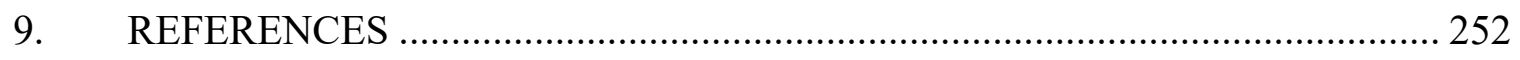

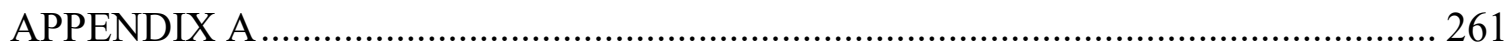

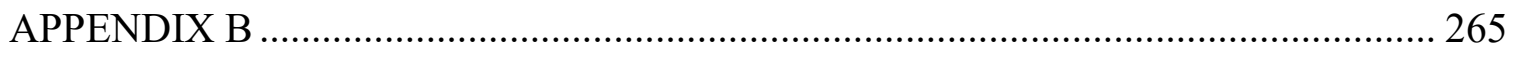

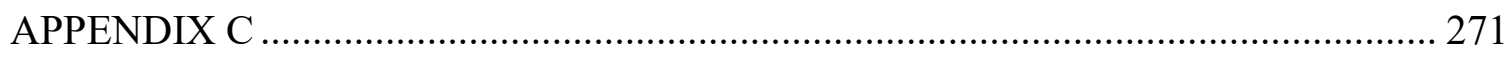

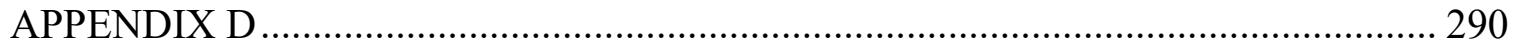

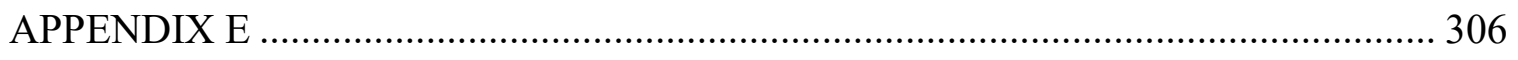

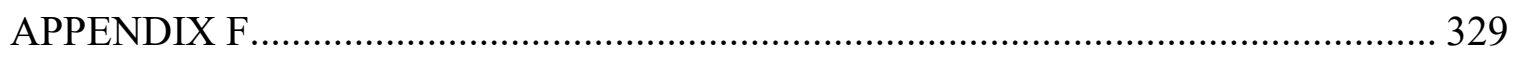

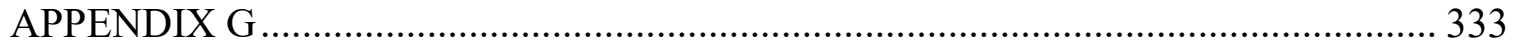




\section{LIST OF TABLES}

Table 2-1: Lateral Friction for Slide Failure (Bonneson, 2000)........................................ 64

Table 2-2: Wet and Dry Friction Factor (MacIsaac and Riley 2002),................................ 65

Table 2-3: Lateral Friction Available for Passenger Car and Truck (Harwood and Mason

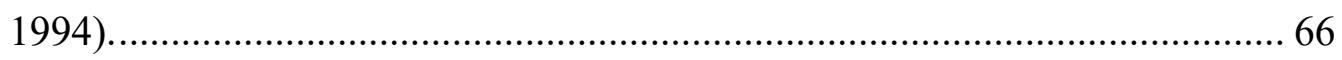

Table 2-4. Lateral Friction Factor for PC for Different Curve Speeds (Himes 2013)...... 67

Table 2-5. Lateral Friction Factor for Truck for Different Curve Speeds (Himes 2013). 67

Table 2-6: Friction Factor and Classification of Road Surface (Al-Qadi et al. 2002)..... 68

Table 2-7: Deterministic and Probabilistic Approaches Characteristics (Based on Singh et

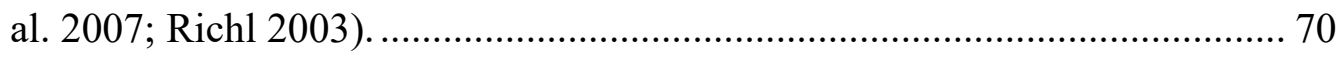

Table 3-1: Potential Factors Affecting Design Criteria................................................... 91

Table 3-2: Consequences of Violating Horizontal Curve Design Criteria. ....................... 99

Table 3-3: Geometric Data of Horizontal Curves........................................................ 110

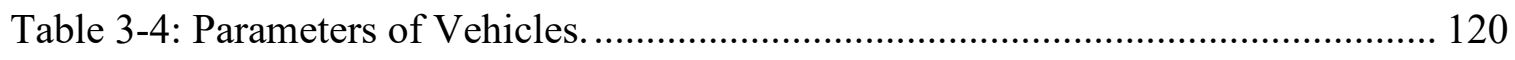

Table 4-1: Count of Readings for Each Headway Category (daytime and favourable

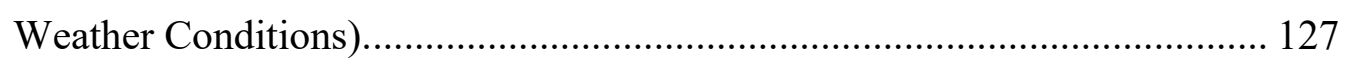

Table 4-2: One-Way-ANOVA for Speed on Curve, Approach Tangent and Headway

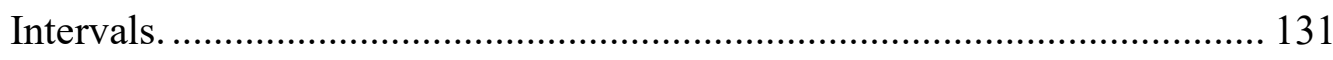

Table 4-3: One-Way-ANOVA and Test of Homogeneity in Rolling and Mountainous

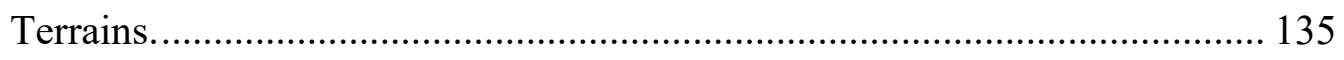

Table 4-4: ANOVA Results for Right Turn vs. Left Turn Curves................................ 136

Table 4-5: ANOVA-Two-Way Results for Right Turn vs. Left Turn Curves -

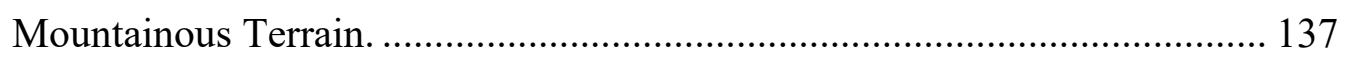

Table 4-6: Bivariate (Pearson) Correlation Matrix for Rolling and Mountainous Terrains. 
Table 5-1: Speed Models at Driver Level................................................................. 158

Table 5-2: Speed Models - Panel Models. …………………………….................. 159

Table 5-3: 85th Percentile Speed Models.................................................................... 159

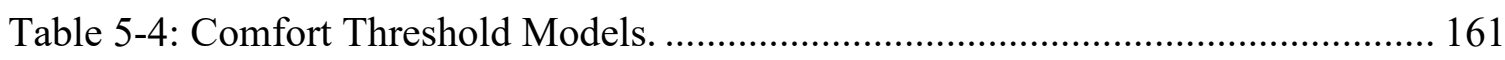

Table 5-5: Speed and Comfort Threshold Panel Models for Wet Pavement Conditions.

Table 5-6: Peak Lateral Friction Demand for Different Vehicle Types......................... 167

Table 5-7: Lateral Acceleration for Different Vehicle Types........................................ 169

Table 5-8: Friction Data Distribution for Passenger Car for Wet and Dry Pavement.... 170

Table 6-1: Descriptive Statistics for the Selected Curves............................................ 188

Table 6-2: Number of Collisions, AADT, and Reliability Index $(\beta V S) \ldots \ldots \ldots \ldots \ldots \ldots \ldots \ldots \ldots . . . .193$

Table 6-3: Number of Collisions on Curves within Specific Reliability Index $(\beta c m f)$

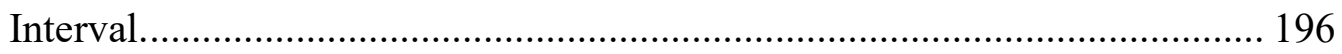

Table 6-4: Number of Collisions on Curves within Specific Reliability Index Interval $\mathrm{POH}$

Table 6-5: Bivariate (Pearson) Correlation Matrix for Reliability Indices...................... 203

Table 7-1: SPFs for Annual Collision Frequency (Base Models). ................................. 209

Table 7-2: Annual Collision Frequency Models (Considering AADT and L), ............. 210

Table 7-3: Annual Collision Frequency Models (Considering AADT, L, and R). ........ 211

Table 7-4: SPFs for Annual Collision Frequency Considering All Reliability Indices.. 212

Table 7-5: ZIP and ZINB Models to Predict Annual Collision (Base Models).............. 213

Table 7-6: ZIP Models to Predict Annual Collision Frequency. ……………………..... 214

Table 7-7: ZINB Models to Predict Annual Collision Frequency................................. 217

Table 7-8: SPFs for Five-Year Collisions Frequency (Base Models). ............................ 219

Table 7-9: NB Models to Predict Five-Year Collisions Frequency.................................. 221 
Table 7-10: SPFs for Five-Year Collisions Frequency Considering All Reliability Indices.

Table 7-11: ZIP and ZINB Base Models to Predict Five-Year Collision Frequency..... 223

Table 7-12: ZIP Models to Predict Five-Year Collision Frequency............................. 224

Table 7-13: ZINB Models to Predict Five-Year Collision Frequency. ......................... 226

Table 7-14: Adjusted POF, PNC, $\mathrm{POH}$ and $\mathrm{POF}_{\mathrm{R}}$ for Weather and Pavement Conditions. 


\section{LIST OF FIGURES}

Figure 1.1: Casualties on Curved Roadways in Canada (Transport Canada 2015)........... 2

Figure 1.2: Collisions on Curved Roadways in Different Pavement Conditions (Transport

Canada 2015). 6

Figure 1.3: Percentages of Collisions on Curved Road Sections to Straight Ones for Different Provinces. (Data Provided by Alberta Transportation, Road Safety Research Service/Quebec, Traffic Engineering and Road Safety/Nova Scotia, and Road Safety Research Office/Ontario).................................................. 7

Figure 1.4: Collision Distribution According to Weather Conditions for Province Ontario 2013- (Data Provided by Road Safety Research Office/Ontario).................... 8

Figure 1.5: Horizontal Curves Design Framework................................................. 16

Figure 2.1: Forces Affecting Vehicle Movement on Circular Path............................. 23

Figure 2.2: Forces Distribution Used to Develop PMM........................................... 25

Figure 2.3: Kinematic Bicycle Model - Reproduced from (Gillespie 1992).................. 26

Figure 2.4: Slip Angle - Reproduced from (Gillespie 1992)...................................... 26

Figure 2.5: Bicycle Model - Reproduced from (Gillespie 1992)................................. 27

Figure 2.6: Cornering Stiffness- Reproduced from (Gillespie 1992). .......................... 28

Figure 2.7: Vehicle Dynamic Sub-Models (Allen et al. 1998)...................................... 33

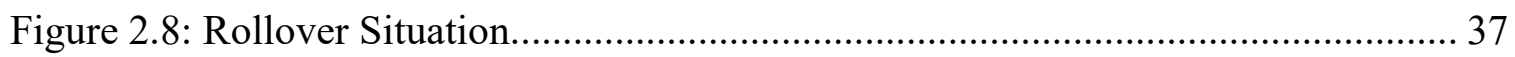

Figure 2.9: Steady State Handling Model in the Bicycle Model- Reproduced from Wong 2008 41

Figure 2.10: Relationship between Stability Factor and Derivative Control Action

(Masato 2009). 43

Figure 2.11: Typical and Electronic Ball-Bank Indicator (Misaghi 2003; Hildebrand and Lewis 2010). 46 
Figure 2.12: Minimum Clearance for Sight Distance Criterion-Reproduced from (Garber

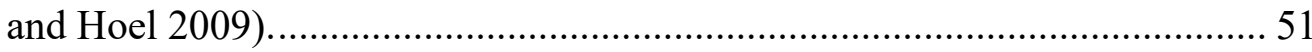

Figure 2.13: Texture wavelength influence on pavement-tire interaction (Shuo et al.

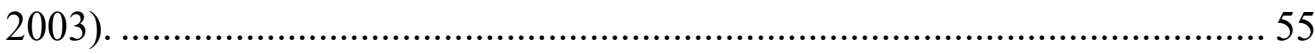

Figure 2.14: Tire-Pavement friction (Hall et al. 2009)................................................. 56

Figure 2.15: Friction Force and Slip Ratio for Traction and Braking States Based on ASE

Definition-Reproduced from (Milliken and Milliken 1995)........................ 59

Figure 2.16: Relationship between Longitudinal and Lateral Forces (Reproduced Milliken

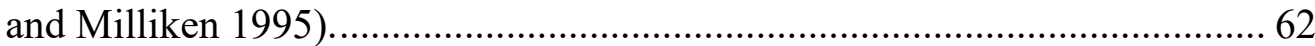

Figure 2.17: Factor of Safety- Reproduced from (Haldar and Sankaran, 2000 and Kapur

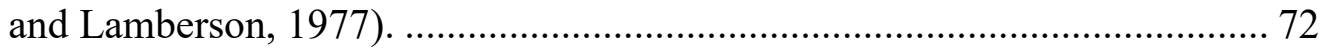

Figure 2.18: Probability Density Function of the Performance Function and Reliability Index, Reproduced from Singh et al. (2007)............................................ 73

Figure 2.19: Reliability Systems - Reproduced from (Kapur and Lamberson 1977)...... 75

Figure 2.20: Combination of Parallel and Series Subsystems (Kapur and Lamberson 1977). 76

Figure 2.21: Transformation of Non-Normal Variables to Standard Normal SpaceReproduced from (Singh et al. 2007)................................................... 84

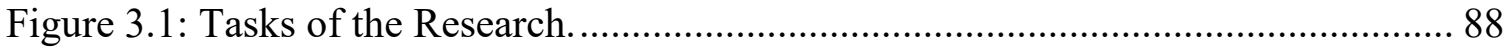

Figure 3.2: Typical Data Acquisition System Components (Dingus et al. 2015)......... 102

Figure 3.3: Number of Trips for Road Segments in Washington State (Insight Data

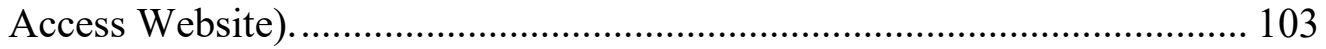

Figure 3.4: Location of Segments in Washington State (ArcMap-WSDOT database). . 105

Figure 3.5: Trips Distribution in Daytime and Night. ............................................ 106

Figure 3.6: Trip Distributions by Month of the Year and Time of the Day. ................. 106

Figure 3.7: Trips Frequency According to Weather Conditions.................................. 107 
Figure 3.8: Distributions of Driving Test Participants Age and Profession and Vehicle Type. 108

Figure 3.9: Pavement Database- Provided by Washington State Transportation

Department...... 114

Figure 3.10: Geometry and Collision Datasets (HSIS).......................................... 116

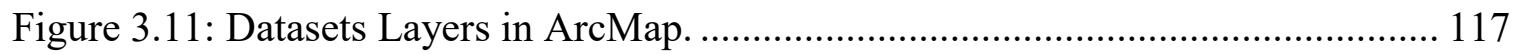

Figure 3.12: State Route Web Tool (WSDOT 2017)............................................. 118

Figure 3.13: Using AutoCAD to Check the Geometric Data of Horizontal Curves....... 119

Figure 3.14: Frequency Distribution of the Collisions. ......................................... 122

Figure 3.15: Frequency of Collisions for Weather, Light, and Pavement Conditions.... 123

Figure 3.16: Yearly and Monthly Collision Frequency Distributions. .......................... 124

Figure 4.1: Relationship between Mean and 85th percentile of Speed and Headway (Rolling Terrain). 129

Figure 4.2: Mean and 85th percentile of Speed vs. Specific Headway Intervals on Curve and at Approach Tangent (Rolling Terrain)......................................... 130

Figure 5.1: Different Speed Profiles on Curve MT 01........................................... 143

Figure 5.2: Example of Profiles of Speed and Longitudinal and Lateral Accelerations. 144

Figure 5.3: Schematic for Determining Vehicle Speed State. ................................... 146

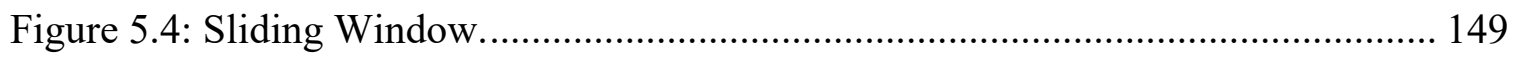

Figure 5.5: Change of Frequencies of General Speed State on All Curves with pvalThreshold.

Figure 5.6: Change of Mean Comfort Threshold with pvalThreshold. 152

Figure 5.7: Change of Frequencies of General Speed State on All Curves with accThreshold. 153

Figure 5.8: Change of Mean and Standard Deviation Comfort Threshold with minSSWin. 155 
Figure 5.9: Speed on Curve and Comfort Threshold for Different Weather Conditions.

Figure 5.10: Relationship between Lateral Friction Demand and Speed and Radius. ... 165

Figure 5.11: Lateral Friction Demand on Horizontal Curve............................................ 166

Figure 5.12: Lateral Acceleration Profile. ................................................................. 168

Figure 6.1: POF for PC with Different Available Lateral Friction Values..................... 179

Figure 6.2: Relationship between POF and Speed and Radius..................................... 180

Figure 6.3: POF for Horizontal Curves with Different Superelevation Rates $(e)$.......... 181

Figure 6.4: Relationship between PNC and Radius of Curve......................................... 183

Figure 6.5: Relationship between POH and Radius of Curve. ...................................... 184

Figure 6.6: Forces Affecting on Sprung and Unsprung Vehicle Parts. ......................... 186

Figure 6.7: Relationship between POF (Rollover) and Mean Speed of Vehicle on Curve. 186

Figure 6.8: Locations of Horizontal Curves for Reliability Analysis............................ 187

Figure 6.9: Reliability Indices for a Sample Curves.................................................. 190

Figure 6.10: Distribution of Curves According to Reliability Index $(\beta V S)$................... 191

Figure 6.11: Relationship between POF, AADT, and Collisions.................................. 192

Figure 6.12: Trend of Average Collisions per Curve with Reliability Index $(\beta V S) \ldots \ldots . .194$

Figure 6.13: Number of Curves According Reliability Index $(\beta c m f)$.......................... 195

Figure 6.14: Relationship between Number of Collisions, AADT, and PNC............... 196

Figure 6.15: Trend of Collision Frequency with Reliability Index $(\beta c m f) \ldots \ldots \ldots \ldots \ldots \ldots . . . . .197$

Figure 6.16: Number of Curves According Reliability Index $(\beta S D)$........................... 199

Figure 6.17: Relationship among Collisions, AADT, and POH of Sight Distance Criterion. 199

Figure 6.18: Relationship between Collisions per Curve and Reliability Index ( $\beta S D)$. 200 
Figure 6.19: Relationship between Collisions Rate and Reliability Index $(\beta$ Roll $) \ldots \ldots . . .201$

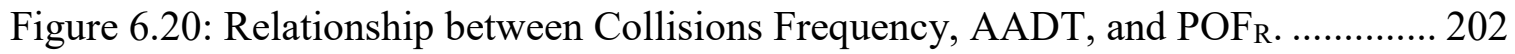

Figure 6.21: Relationship between Collisions per Curve and Reliability Index ( $\beta$ Roll). 203

Figure 7.1: Observed Collisions (per year) Compared with Poisson and NB Distributions.

Figure 7.2: Observed Collisions (five-year) Compared with Poisson and NB

Distributions 220

Figure 7.3: Predicted Mean Speed at MC and BC for Different Weather Conditions. .. 231

Figure 7.4: Radius Versus POF for Dry and Wet Pavement Conditions....................... 232

Figure 7.5: Mean Comfort Threshold for Different Radii. ....................................... 233

Figure 7.6: Radius Versus PNC for different Weather and Pavement Conditions......... 234

Figure 7.7: Determining Available Sight Distance................................................ 235

Figure 7.8: Mean Sight Distance and Stopping Sight Distance.................................. 236

Figure 7.9: Radius Versus POH for Dry and Wet Pavement...................................... 236

Figure 7.10: Radius Versus POF $_{\mathrm{R}}$ for Dry and Wet Pavement.................................. 237

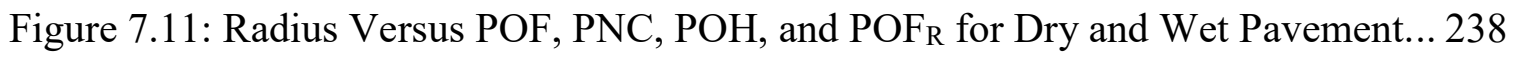

Figure 7.12: Adjusted POF, PNC, POH, and POF $\mathrm{R}_{\mathrm{R}}$ for Different Radii. ..................... 240

Figure 7.13: Radius Versus Adjusted Reliability Indices.......................................... 241

Figure 7.14: Predicted Annual and 5-Year Collisions Frequency............................. 242 


\section{LIST OF ACRONYMS}

\begin{tabular}{|c|c|}
\hline AADT & Annual Average Daily Traffic \\
\hline AASHTO & American Association of State Highway and Transportation Officials \\
\hline AT & Approach Tangent \\
\hline $\mathrm{BC}$ & Beginning of Curve \\
\hline $\mathrm{CDF}$ & Cumulative Distribution Function \\
\hline $\mathrm{COV}$ & Coefficient of Variation \\
\hline $\mathrm{EC}$ & End of Curve \\
\hline $\mathrm{ESC}$ & Electronic Stability Control \\
\hline FHWA & United States Federal Highway Administration \\
\hline FORM & First Order Reliability Method \\
\hline HSIS & Highway Safety Information System \\
\hline IHSDM & Interactive Highway Safety Design Model \\
\hline IRI & International Roughness Index \\
\hline LTR & Load Transfer Ratio \\
\hline $\mathrm{MC}$ & Middle Point of Curve \\
\hline MCS & Monte Carlo Simulation \\
\hline NDS & Naturalistic Driving Study \\
\hline NHTSA & National Highway Traffic Safety Administration \\
\hline $\mathrm{PC}$ & Passenger Car \\
\hline PDF & Probability Density Function \\
\hline $\mathrm{PNC}$ & Probability of Noncompliance \\
\hline POF & Probability of Failure \\
\hline
\end{tabular}




\begin{tabular}{|c|c|}
\hline $\mathrm{POF}_{\mathrm{R}}$ & Probability of Failure (Rollover) \\
\hline $\mathrm{POH}$ & Probability of Hazard \\
\hline PMM & Point Mass Model \\
\hline PRC & Pavement Rutting Condition \\
\hline PSC & Pavement Structural Condition \\
\hline $\mathrm{PPC}$ & Pavement Profile Condition \\
\hline SHRP 2 & The Second Strategic Highway Research Program \\
\hline SORM & Second Order Reliability Method \\
\hline SPF & Safety Performance Function \\
\hline SSD & Stopping Sight Distance \\
\hline SUV & Sport Utility Vehicle \\
\hline TAC & Transportation Association of Canada \\
\hline TCS & Traction Control System \\
\hline VDM & Vehicle Dynamic Model \\
\hline VTTI & Virginia Tech Transportation Institute \\
\hline WSDOT & Washington State Department of Transportation \\
\hline WSPMS & Washington State Pavement Management System \\
\hline
\end{tabular}




\section{CHAPTER ONE}

\section{INTRODUCTION}

\subsection{Background}

Since vehicles became available for the general public in 1920s, the number of vehicles has significantly increased. Many technical developments have happened in their design and performance characteristics (Merritt 1988). The driving behaviour has changed, and drivers nowadays tend to accept higher levels of risk (Said et al. 2009). Meanwhile, the number of collisions and fatalities are remarkable. These issues are the main concerns for automobile manufacturers and transportation designers because of their backlash on the economy, environment and even on people's life style. While vehicle manufacturers attempt to improve traffic safety by producing safer and more comfortable vehicles, road designers work to provide a suitable and safe environment for road users and for these vehicles to travel in.

In Canada, 17,322 people were injured and 432 were killed in traffic collisions on curved roadways in 2013 as shown in Figure 1.1 (Transport Canada 2015). It is reported that the collision rate on horizontal curves is $1.5-4$ times greater than those on similar straight elements (Kordani and Molan 2014). A similar finding was reported by Glennon et al. (1985) who found that the average collision rate for horizontal curves was approximately three times the average collision rate for highway tangents. The high number of collisions on horizontal curves compared to other roadway elements make them one of the most critical roadway elements in any transportation system (Glennon et al. 1985; Torbic et al. 2004). 

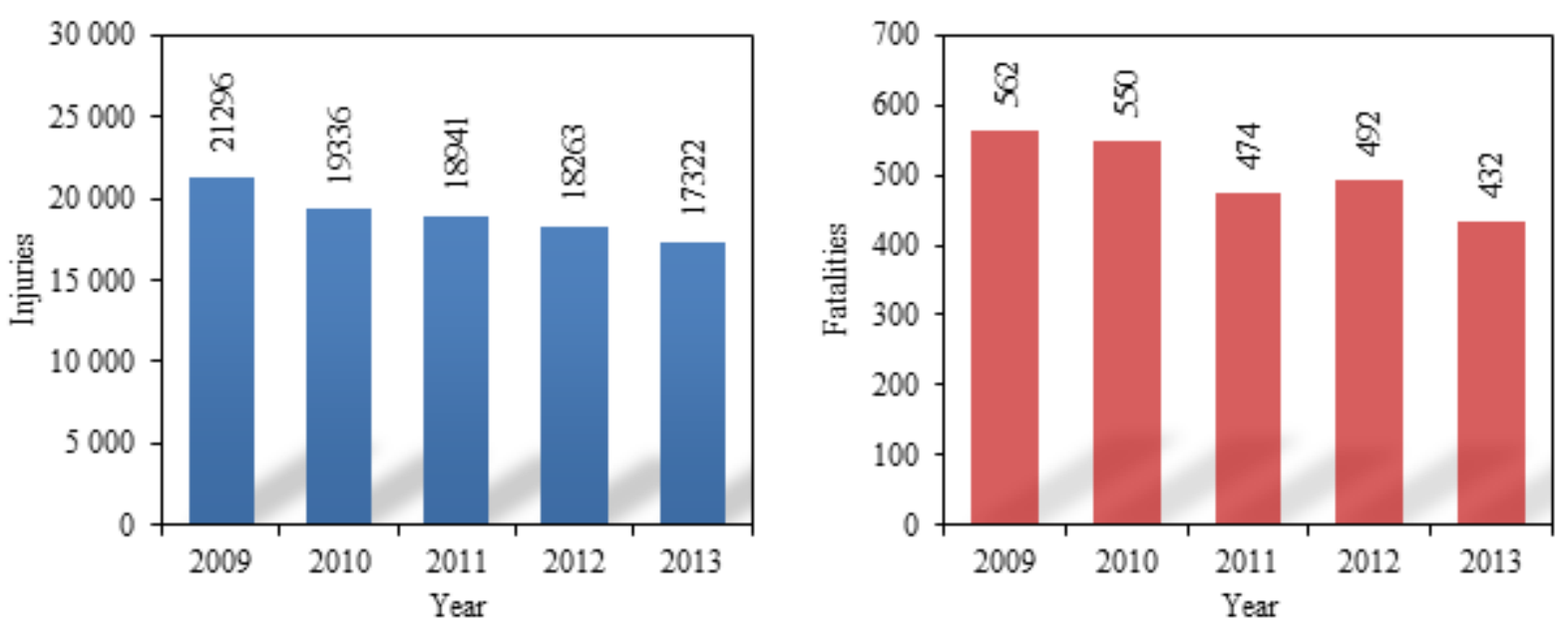

Figure 1.1: Casualties on Curved Roadways in Canada (Transport Canada 2015).

Several studies have shown that human factors, roadway's physical features, vehicle and pavement conditions, and traffic situation are potential contributing factors for collision events (Felipe 1996). The geometry of roadway elements and collision rates were found to be correlated (NRC 1987). The radius of a curve, for example, has generally an inverse relationship with collision rate (Glennon et al. 1985). Said et al. (2009) found that the roadway geometry is one of the main factors that affect driving behaviour. In other words, the roadway geometry would affect the path and speed of vehicles on curves (Said et al. 2009). Accordingly, improving the geometric design of the horizontal curves would enhance driving behaviour, reduce the probability of driver errors, and thus increase safety on roadways. Therefore, the design should take into consideration all these potential contributing factors for collision events in order to provide a safe and comfortable ride for vehicle's occupants on horizontal curves. The design also has to consider other factors that influence decision-making process such as available funds, economic benefits, and social and environmental impacts. 


\subsection{Horizontal Curve Design}

The horizontal alignment of a roadway generally consists of three components; tangent sections, horizontal curves, and in some cases, transitional spiral curves. Many factors control the design such as the safety of roadway's users, design speed, topography, climatic conditions, traffic volumes, and the existence of major utilities (TAC 2017). Considering these factors in the design, and predicting driver behaviour and vehicle response makes the design task more complicated. To design a horizontal alignment, several design criteria and requirements have been established to come up with safe and comfortable horizontal curves. Generally, driver comfort, vehicle dynamic stability, vehicle rollover, sufficient sight distance for drivers to see ahead, drainage, and roadway aesthetics are the key design criteria that need to be ensured. The following paragraphs explain the design of horizontal curves from three perspectives; the approach, criteria, and models.

\subsubsection{Design Approach}

To evaluate the safety of a design, two approaches are used to compensate for uncertainties associated with the factors involved in the design, a deterministic approach and probabilistic approach. The deterministic approach adopts near worst-case scenarios to determine a specific conservative value for each factor involved in the design model. On the other hand, the probabilistic approach involves a probabilistic assessment of the system performance considering all possible conditions and the probability of these conditions to take place (Singh et al. 2007).

The current highway design guides adopt the deterministic approach and ignore the importance of the probabilistic factors of highway operations (Hassan 2012). That is, in 
the context of horizontal curve design, their objective is to determine the radius and superelevation rate for a horizontal curve based on a certain design speed and estimated lateral friction (De Solminihac et al. 2007). In other words, the variability effects of the factors included in the design are ignored. In addition, the Margin of Safety (MOS) and the safety factor have been included implicitly in the recommended design values. Thus, the MOS is unknown particularly when using radii different from the recommended one.

\subsubsection{Design Criteria}

Horizontal curves can be designed based on several criteria as mentioned earlier. The vehicle dynamic stability criterion was developed to ensure a sufficient margin of safety for the vehicle against skidding. That is, it ensures that the design value of lateral friction is less than the available lateral friction (You and Sun 2013). The rollover criterion addresses the overturning moment to ensure that it will not exceed the counterbalance moment generated by the weight of the vehicle. Although, a vehicle tends to slide before rolling over (Himes 2013), the stability of vehicle against rolling over might need to be checked in particular when having a vehicle with high centre of gravity position. A failure in either of these criteria would lead to loss of control, run-off the road collision, or rollover accident. On the other hand, the driver comfort criterion is concerned with avoiding vehicle's occupants experiencing a feeling of excessive discomfort due to a high level of lateral acceleration. In other words, it ensures that the developed lateral acceleration while negotiating a horizontal curve does not exceed a specific limit, which is commonly known as the comfort threshold (Dhahir and Hassan 2015). Also, the sight distance criterion provides sufficient sight distance for drivers to see ahead (Sarhan and Hassan 2008). Failing to comply with these criteria, driver comfort and sight distance, would not directly 
result in a collision.

The American Association of State Highway and Transportation Officials (AASHTO) and the Transportation Association of Canada (TAC) current design guides (A Policy 2011; TAC 2017) adopt the driver comfort criterion to design horizontal curves. The comfort threshold was found to be lower than the vehicle dynamic stability and rollover thresholds. That is, these guides assume that the margin of safety based on the driver comfort threshold is sufficient against skidding and rolling over (A Policy 2011; TAC 2017). However, these latter criteria could be a critical situation for a horizontal alignment in some cases, especially when having pavement surface with available lateral friction lower than the lateral friction demand such as for icy or snow packed pavement. Figure 1.2a shows the number of collisions on curved roadways in Canada for the last ten years (Transport Canada 2015). The collisions are distributed according to the condition of pavement. The percentage of collisions on snow, slush, and icy pavement conditions for these years is approximately $21 \%$ as shown in Figure $1.2 \mathrm{~b}$. The number of collisions corresponding to these pavement conditions is 35,173 . These collisions resulted in 48,171 injuries and 800 fatalities. These casualties, which did not happen on dry or wet pavement, are not considered explicitly in the current design guides. 


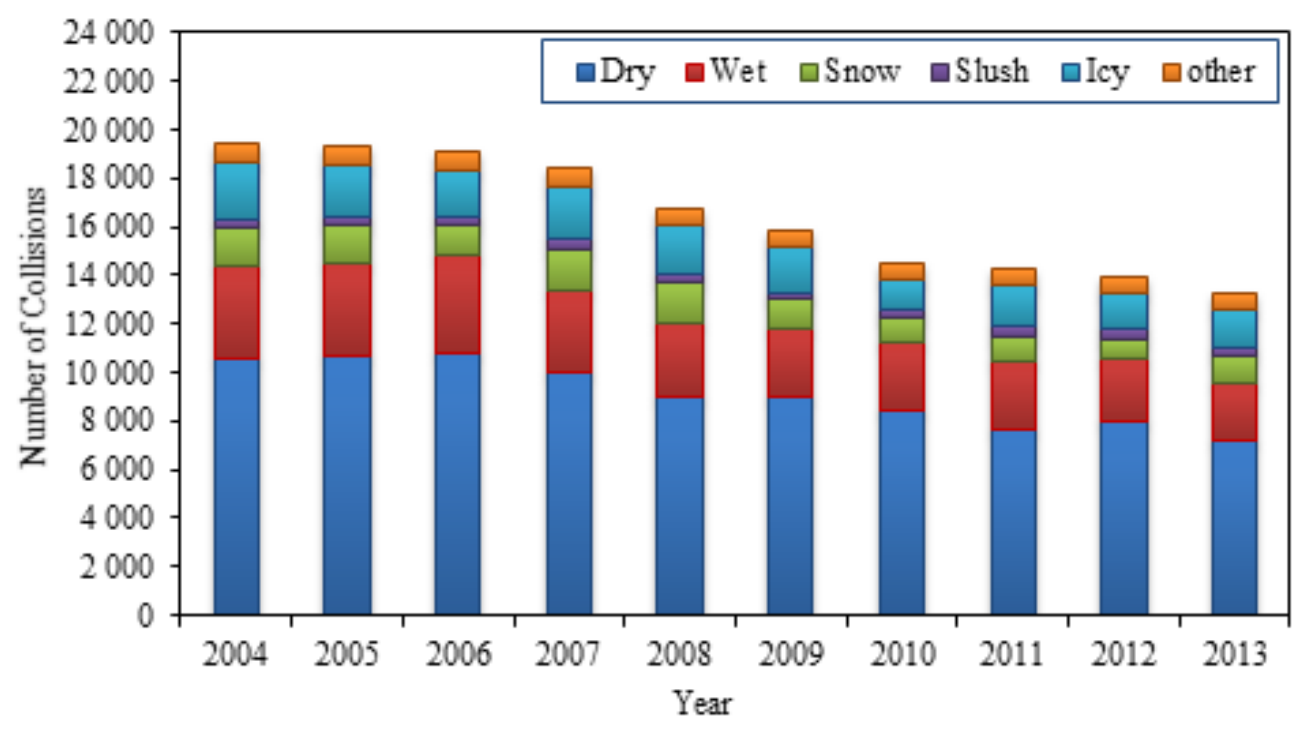

(a) Number of Collisions for Different Pavement Conditions.

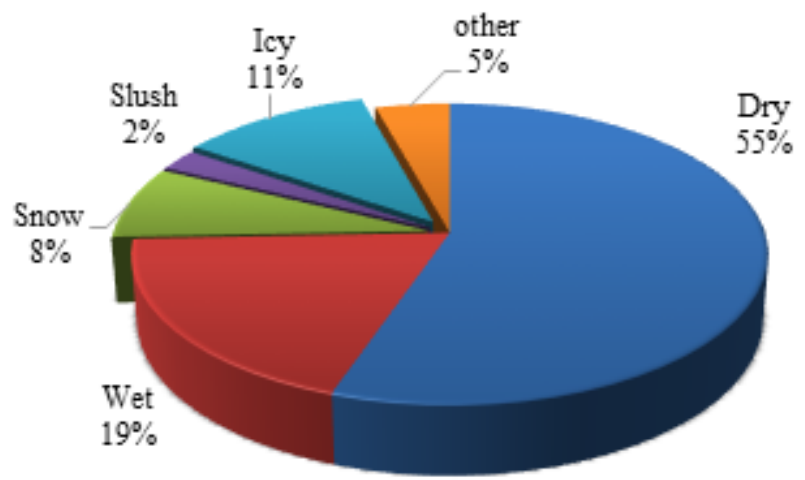

(b) Percentages of Collisions for Different Pavement Conditions.

Figure 1.2: Collisions on Curved Roadways in Different Pavement Conditions (Transport Canada 2015).

The location of the horizontal alignments would give an indication of the dominant weather and environment conditions. The data provided by the engineering traffic and road safety departments of Quebec, Alberta, Ontario, and Nova Scotia to the author show that the percentage of collisions (based on three-year collision data from 2007 to 2009) on 
curved roadway elements to the straight ones is different from one province to another (Figure 1.3). However, these percentages need to be calibrated by considering the length of roadways elements.

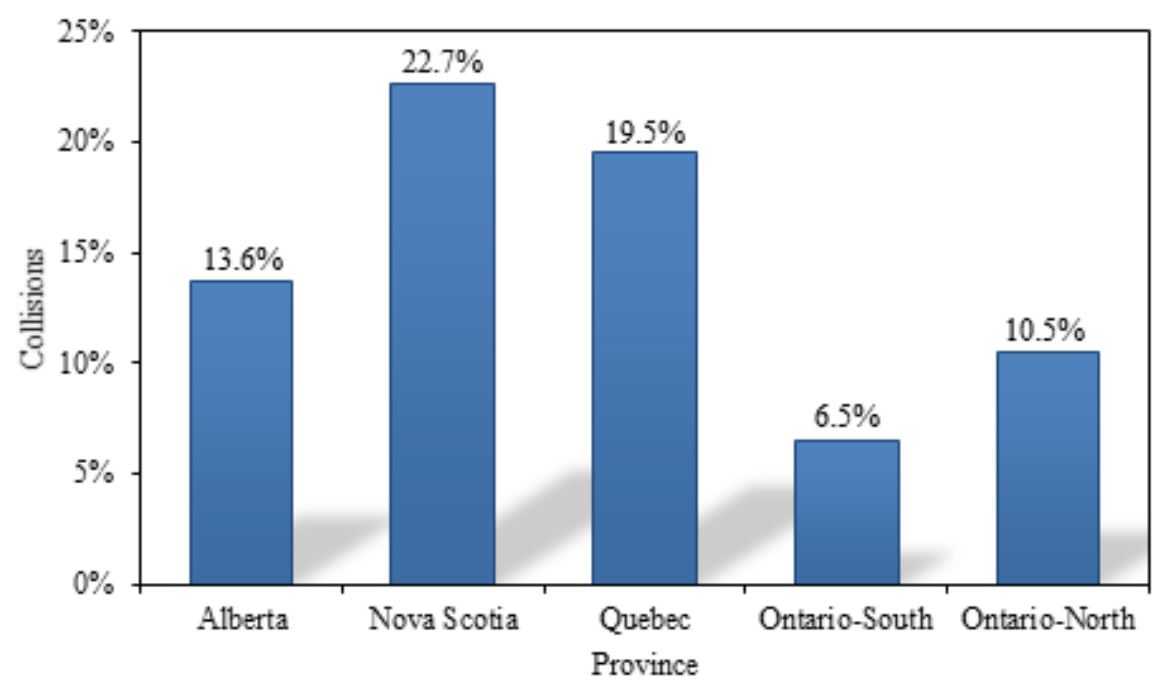

Figure 1.3: Percentages of Collisions on Curved Road Sections to Straight Ones for Different Provinces. (Data Provided by Alberta Transportation, Road Safety Research Service/Quebec, Traffic Engineering and Road Safety/Nova Scotia, and Road Safety Research Office/Ontario).

The collision percentages on curves according to weather conditions in these provinces are different as well. Figure 1.4 shows collision distributions in the south and north of province Ontario. It could be concluded that the dominant weather and environmental conditions do impact the collision rates on curved roads. That is, they should be considered in the design of horizontal curves because they affect, in one way or another, the safety performance of the curve. It should be noted that TAC mentioned that fatal run-off-road collisions are often associated with curved road segments, rural roads, high-speed-limit roadways, and adverse weather conditions (TAC 2017). Manipulating the variability of weather and pavement surface conditions in the design of horizontal curves using the 
deterministic approach would be difficult and most likely lead to considerable overdesign because it will be based on near worst-case scenario. This shortcoming would support the idea of using the probabilistic approach, which is capable of dealing with many variables considering their distributions.

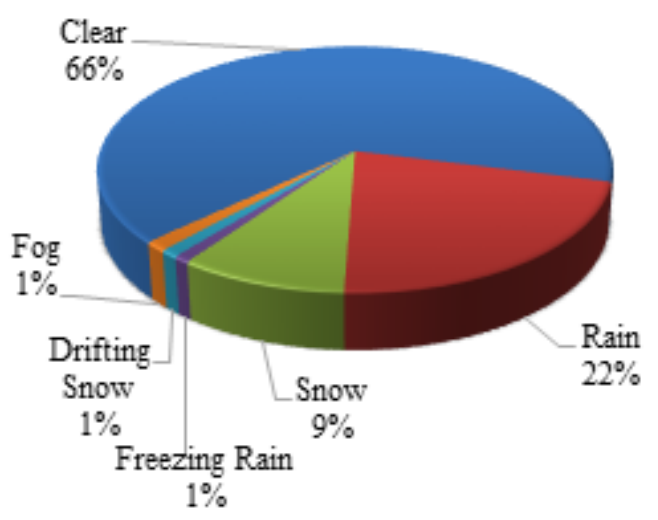

(a) South Ontario (Windsor, Hamilton).

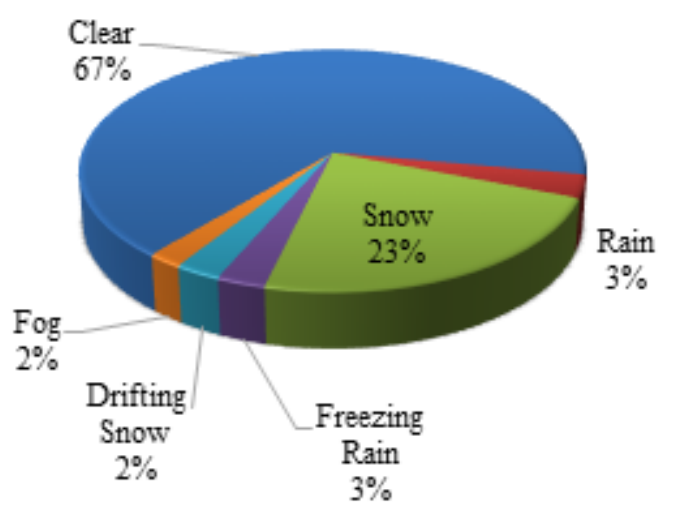

(b) North Ontario (Thunder Bay, Sudbury).

Figure 1.4: Collision Distribution According to Weather Conditions for Province Ontario 2013- (Data Provided by Road Safety Research Office/Ontario).

\subsubsection{Design Model}

Generally, when a vehicle negotiates a horizontal curve, a centripetal acceleration is developed. The direction of the centripetal acceleration is towards the centre of curvature and its magnitude depends on the speed of the vehicle and the radius of the curve (TAC 2017). Several models have been developed to simulate the motion of a vehicle on a circular curve and to determine the developed forces, moments, and accelerations. These models differ from one another in the level of complexity and accuracy. The model commonly used in the design is the Point Mass Model (PMM). The PMM is based on the equilibrium of a point mass moving on a curve through relating the radius of curve, vehicle 
speed, friction factor, and superelevation rate (Echaveguren et al. 2005). The current design guides use the PMM to design the horizontal curves through determining the minimum radius of curve required for a particular design speed, maximum superelevation rate, and maximum lateral friction factor (A Policy 2011; TAC 2017). The PMM assumes a uniform vehicle speed and that drivers follow a path of a constant radius, which is known as the steady state situation.

\subsection{Problem Definition}

The procedure used to design horizontal curves in the current design guides suffers from several major issues. These issues arise from the main components of the current design framework: adopting a deterministic approach, implementing only the driver comfort criterion, and using the PMM model. Each component has some shortcomings. The consequences of adopting the deterministic approach to design horizontal curves can be summarized as follows:

- The deterministic approach lacks a quantitative evaluation for safety.

- Aggregating data into a specific percentile and ignoring driving behaviour on curves would result in losing important information.

- The effects of variation of design factors are overlooked because of using unique and conservative values for these factors.

- The design might be overestimated because design factors' values are determined based on near worst-case scenarios.

Adopting the comfort criterion and ignoring other design criteria might lead to unsafe design in some cases as explained in Section 1.2.2. Although, the lateral friction values 
recommended by AASHTO and TAC based on the driver comfort criterion are most likely lower than the available friction factor and greater than lateral friction demand thresholds, it is important to check the dynamic stability and controllability of the vehicle and other design criteria for several reasons:

- The maximum lateral friction values used in the design are estimated based on the comfort and safety by the vast majority of drivers (TAC 2017; A Policy 2011). Driving in adverse weather conditions or on packed-snow or icy pavement is not explicitly addressed. The available lateral friction in such cases decreases significantly. For instance, the recommended lateral friction factor by AASHTO at speed $70 \mathrm{~km} / \mathrm{h}$ is 0.15 , while it could decrease to 0.1 for icy conditions (Bosch 2006). In such cases, the stability and control of a vehicle is questionable, and vehicles would skid off the track or at least drivers would experience vehiclecontrol difficulties before reaching the discomfort thresholds.

- The significant improvements in vehicle design can have a backlash on driving behaviour. Drivers tend to accept higher levels of lateral acceleration because they might not feel great discomfort when driving with speed higher than the design speed (Said et al. 2009). This means that the lateral friction demand will increase due to higher lateral acceleration. Simultaneously, the available lateral friction is expected to decrease because it has an inverse relationship with speed (Himes 2013).

- The dynamic stability and control of a vehicle does not only need to be checked within the steady state cornering situation (constant speed and path of a constant radius), but also other driving scenarios should be considered as well. In braking 
condition, for instance, the available lateral friction decreases. Furthermore, it is important to examine areas on the horizontal curve that are expected to have maximum lateral friction demand, like the transition area from straight to curve and vice versa.

- When driving in adverse weather conditions and having pavement with low available friction factor, the required stopping distance increases. Thus, the available sight distance in such a case might be insufficient for drivers to see ahead and the available stopping sight distance is not enough.

- Vehicles might be susceptible to rolling over especially for those with high centre of gravity.

The accuracy of the design depends mainly on the model used in the analysis and the level of accuracy of the data of the factors included in the model. In spite of the simplicity of implementing PMM model in the design, using this model might have the following disadvantages:

- The model is insensitive to vehicle type since it considers the vehicle as a rigid body and approximates it to a point mass.

- The model overlooks some characteristics of the vehicle such as the suspension system and centre of gravity. As a result, the design does not incorporate the sprung part of the vehicle since it ignores the effect of body roll which induces load transfer from the inner wheels to the outer ones (Chang 2001).

- It overestimates the margin of safety against skidding and rollover for all vehicle types (Torbic et al. 2014).

- The model does not consider the effect of road profile since it is developed to design 
horizontal curves on flat grades only (Kontaratos et al. 1994).

- The values of lateral friction used in this model, which are obtained from experiments conducted between 1920 and 1952, might be outdated and do not reflect the current characteristics of vehicles, pavement surface, and driver behaviour (Bonneson 2001).

To overcome problems associated with the current design procedure of horizontal curves, several measures can be considered. First, a probabilistic approach should be adopted. Thus, the effect of variability of the factors would be considered in conducting the analysis. The reliability analysis could be employed to determine the performance of horizontal curve in terms of Probability of Failure. This approach would help as well in clarifying the feasibility between the cost and safety of the design because of its capability to provide a quantitative evaluation for safety. For instance, it can be used to evaluate the effect of flattening the curve (increasing the radius of the curve) on the safety performance of the curve. Accordingly, the decision-making process to select the most efficient design among alternatives would be more objective. However, there are some limitations associated with implementing the probabilistic approach such as using approximation methods in conducting the analysis. Also, a considerable amount of data should be collected, which means, in turns, more time and cost. Note that three technical terms are used in this research to describe the probability of failure of the design. The use of these terms is based on the design criterion itself and the consequences of violating this criterion. The Probability of Failure (POF and $\mathrm{POF}_{\mathrm{R}}$ ) is used for evaluating the safety of the design according to vehicle dynamic stability and roll over criteria. Any violation for these design criteria would end up with losing control over the vehicle. The Probability of Non- 
Compliance (PNC) and Probability of Hazard ( $\mathrm{POH})$ will be used to describe the probability of failure of driver comfort and sight distance design criteria. Violating these design criteria would affect vehicle's driver workload, comfort, and stress.

Second, a new design framework should be developed to consider all design criteria mentioned earlier in Section 1.2.2. Accordingly, the design of horizontal curve would ensure that vehicle's occupants are not subjected to uncomfortable sensation of the forces pushing them to the outside of the curve. Also, the lateral friction demand or overturning moment would not exceed the safe limits. The available stopping distance would be ensured to be greater than the required as well. Implementing the probabilistic approach in the analysis, as mentioned earlier, would be a powerful tool to quantify the level of safety for each design criterion. The crucial task in achieving this goal is the selection of suitable thresholds for the POF, $\mathrm{PNC}, \mathrm{POH}$, and $\mathrm{POF}_{\mathrm{R}}$, while noting the different consequences of failing to comply to each criterion. In addition, these design criteria should be examined simultaneously because they would work as a double-edged sword in some cases. For instance, a design with a low value of PNC based on driver comfort may encourage drivers to speed up on the horizontal curve because they will not be exposed to uncomfortable sensation of the lateral acceleration (Bashar and Hassan 2015). As a result, the vehicle stability, rollover, and sight distance criteria would be affected. Also, selecting a low value for POF based on dynamic stability might end up with inefficient cost-safety trade-off design.

Third, the accuracy of the design can be improved by using more accurate models such as bicycle model or Vehicle Dynamic Model (VDM). The bicycle model can include the effect of the centre of the mass and the frictional factors at the front and rear wheels 
(Gillespie 1992). The VDM on the other hand, accounts for the vehicle characteristics with different degrees of freedom through including several features of the vehicle such as suspension force mechanism, steering suspension geometry, and lateral force deflection of tire, etc. (Allen et al. 1998). These models can provide better descriptions of the actual movement of a vehicle on a horizontal curve and thus provide results with higher levels of accuracy. However, they are more complicated than the PMM. The significant development in computer applications would assist in using models that are more complicated. Currently, several software products have the capability to include vehicle characteristics, driving behaviour, and road conditions and simulate the movement of a vehicle on various curvilinear paths. CarSIM, for instance, has the ability to deal with more than 800 variables in the VDM (Mechanical Simulation 2014). These variables include forces (longitudinal, lateral, and normal), angles (slip, steering, yaw, and roll), location of the vehicle (radius and offset from the path, and road profile), accelerations (longitudinal and lateral), understeer gradient, and stability factor.

Finally, it is an important issue to re-visit and update, if needed, the design comfort threshold used in the current design guides. This would help in developing safer and more cost-effective design for horizontal curves. In addition, it will provide helpful information to vehicles' manufactures in developing autonomous vehicles. In other words, developing autonomous vehicles equipped with sensors, artificial intelligence applications, and advanced control systems should consider the comfortable sensation of vehicle's occupants as well. In addition, reliability analysis could be implemented to evaluate the safety performance of new generation of vehicles (connected and autonomous vehicles) considering several scenarios. 


\subsection{Research Objectives}

This research aims at rectifying the current procedure to design horizontal curves and overcoming some problems associated with its applications. The main objective of the research is to develop a new framework to design horizontal curves capable of providing a quantitative evaluation for performance and safety considering the interaction among design criteria and influencing factors such as environment and traffic characteristics. The discussions in previous sections described the current design procedure and some associated issues. According to the current design procedure, the required data to design a horizontal curve are the design speed, superelevation rate, and lateral friction, which is based on comfort criterion. The minimum radius is determined using the PMM formula. The designer might then select the most appropriate radius after checking the available and minimum required sight distance, the grade, the drainage and aesthetics requirements, and consulting the previous experiences to finalize the design.

The proposed design framework comprises, in addition to the driver comfort criterion, the vehicle dynamic stability, rollover, and sight distance criteria. These criteria will be satisfied through four stages as shown in Figure 1.5; Stage I (Inputs) is concerned with determining the distributions of design parameters and thresholds. Stage II (Reliability Analysis) focuses on determining the probability of failure of the design for each design criterion. In this stage, a preliminary check for probabilities of failure is conducted to determine whether the outputs are within acceptable limits. The influencing factors that would affect the performance of the curve such as weather conditions and traffic characteristics are considered in Stage III. The evaluation of the curve performance would be adjusted to reflect the effect of these factors. Finally, Stage IV (Safety Analysis) is 


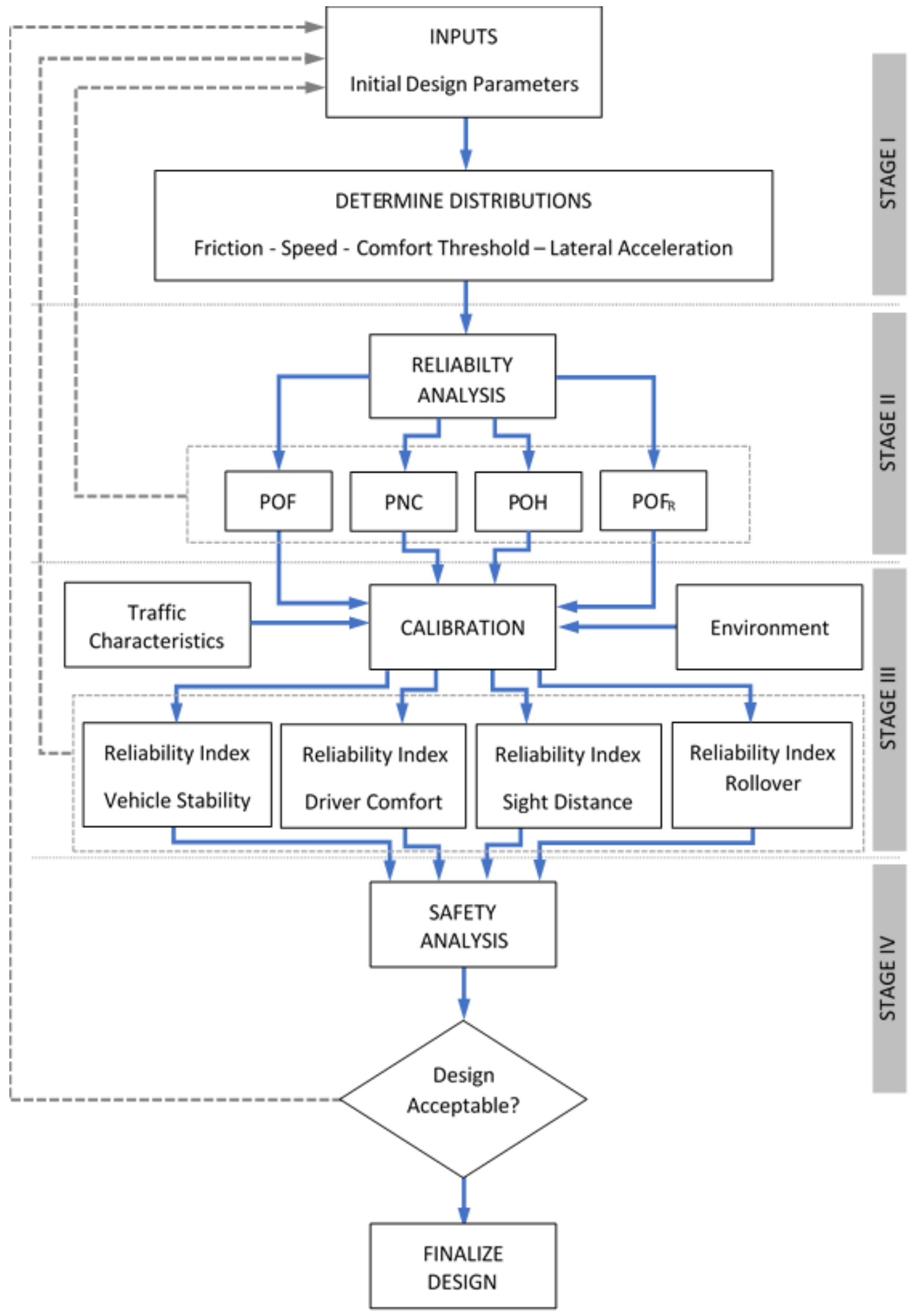

Figure 1.5: Horizontal Curves Design Framework. 
fulfilled. The main task to initiate stage I is determining the distributions of the variables included in the analysis. Therefore, considerable data should be collected and analysed of driving behaviour and available friction. In stage II, reliability analysis will be conducted to determine the POF, PNC, POH, and $\mathrm{POF}_{\mathrm{R}}$. Stage III calibrates the reliability indices considering the influential factors such as environment and traffic characteristics. Stage IV requires sufficient historical safety data to develop proper models to predict safety performance of the curve.

\subsection{Research Plan}

Based on the previous discussion, several tasks have to be accomplished in order to achieve the objective of the research. These tasks have to fill in the four stages of horizontal curve design and evaluation. The following tasks were conducted for each stage.

1. Stage I: selecting dependent and independent variables and determine their distributions using data extracted from the naturalistic driving study database (NDS). Using this database would overcome several issues associated with current procedure in data collection. Thus, the human and technical problems would be mitigated. In addition, the geometry parameters of horizontal curves are collected to be used with the NDS to develop the required models for predicting speed, comfort threshold, and friction demand. These models should consider driving behaviour on horizontal curves. Also, CarSIM is used in developing model to estimate lateral friction demand and lateral acceleration.

2. Stage II: reliability methods are used to evaluate the design of horizontal curves in terms of POF, PNC, POH, and $\mathrm{POF}_{\mathrm{R}}$. Because the analyses are conducted 
for relatively high number of curves, a Matlab program was developed to conduct the analysis. Note that the uploading the input data in the current software that handle reliability analysis can only be done manually.

3. Stage III: weather and pavement conditions data are collected from the closest weather stations and transportation agencies to adjust the outputs of the previous stage in order to reflect the influence of some factors on the performance of the curve. A procedure is developed to adjust the outputs of reliability analysis for each design criterion.

4. Stage IV: historical safety data are collected for five years. The safety database is screened to remove collisions caused by improper driving practice such as driving under the influence of alcohol or drugs. The safety data (dependent variable) associated with the outputs of Stage III, horizontal curve geometry data, and traffic characteristics are used to develop safety performance functions. Thus, the developed safety performance functions would mitigate the effect of unrelated collisions to horizontal curve geometry, and the effect of weather and pavement conditions on collision frequency. The negative binomial regression models are used in developing these models.

\subsection{Research Scope and Organization}

The scope of the research is limited to horizontal alignments located on arterial twolane highways in rural areas. Two types of terrain are investigated; rolling and mountainous. Washington State in the USA is selected to be the study area because of the presence of different terrain types, varying weather conditions, and availability of the 
naturalistic driving study. In addition, other pertinent data such as traffic characteristics, historical safety data, and horizontal curves parameters in this state could be collected from several transportation agencies. The CarSIM software is used to simulate various driving scenarios to develop models to estimate lateral friction demand and lateral acceleration. Typical passenger cars (Sedan) and sport utility vehicle (SUV) are considered in these simulations. Four design criteria are considered in the reliability analysis.

The structure of the thesis for the remaining chapters is organized in the following manner:

1. Chapter Two, literature review, is divided into five parts. First part is devoted to understanding the movement of vehicle on a curved path. The common models used to explain this movement will be addressed in this part. The second part focuses on the stability of vehicles considering vehicle response and current control improvements. The third part is dedicated to review the previous literature of the current methodology to design horizontal curves and design criteria. The fourth part is dedicated to explaining the frictional forces between the tire and pavement, factors influencing the frictional forces, and the relationship between the longitudinal and lateral friction forces. The fifth part mainly focuses on explaining the reliability concept and the analysis methods.

2. Chapter Three, research methodology and data, is devoted to represent the methodology used to achieve research objectives and the data used in the analyses. This chapter is divided into two sections. The first section illustrates the procedure should be followed and tasks that should be accomplished to achieve the objectives the research. The second section describes the data that should be collected, 
extracted, and used in the analysis.

3. Chapter Four, preliminary analysis, focuses on screening and examining the collected data through performing several statistical tests and conducting preliminary analysis. Thus, this chapter is devoted to prepare the data for models' development.

4. Chapter Five, models' development, focuses on two areas; comprehending driving behaviour on curves, and developing models. A procedure is produced to determine driving behaviour in terms of speed, acceleration, and comfort threshold. Also, several models are developed to predict speeds at different locations on curves and determine driver comfort threshold. In addition, models to estimate available friction factors and lateral acceleration were developed as well.

5. Chapter Six, reliability analysis, illustrates the concept of reliability analysis and provides assessment for horizontal curve design criteria.

6. Chapter Seven, safety analysis, focuses on developing safety performance functions and provides an example to illustrate the new framework to design horizontal curves.

7. Chapter Eight, discussions and conclusions, presents a summary showing the main conclusions and findings of this research. In addition, recommendations for future research work were provided. 


\section{CHAPTER TWO}

\section{LITERATURE REVIEW}

\subsection{Introduction}

The main objective of this research is to develop a new framework to design horizontal curves. This would require highlighting problems and shortcomings associated with the current practice to design horizontal curves, if any. The purpose of this chapter is to review published literature and studies that are pertinent to the scope of the research. the design of horizontal curves is reviewed considering three different areas; the design approach, the design criteria, and models used in the analysis. In order to cover these areas properly, the chapter is arranged in the following order; the movement of a vehicle on a horizontal curves and models used to describe this movement are explained in Section 2.2, vehicle dynamic stability and control are discussed in Section 2.3, horizontal curve design criteria are explained in Section 2.4, friction phenomenon between vehicle's tires and pavement is covered in Section 2.5, and the concept of reliability analysis is presented in Section 2.6.

\subsection{Vehicle Dynamics}

Generally, when a vehicle negotiates a horizontal curve, a centripetal acceleration is developed. The magnitude of this acceleration depends on the vehicle speed and curve radius. The centripetal force is the force that keeps the vehicle moving at a uniform speed along a circular path. The centripetal force acts towards the centre of curvature and it is counterbalanced by an equal in magnitude and opposite in direction force known as the centrifugal force. The friction between vehicle tires and pavement surface and a weight 
component, if the road is superelevated, sustain the centripetal acceleration (Bonneson 2001). Since the friction force acts at the surface of the roadway and the centrifugal force acts at the centre of gravity of the vehicle, a moment is generated that tends to overturn the vehicle as shown in Figure 2.1. Therefore, the design of horizontal curves needs to address these forces, moments, and accelerations which influence the vehicle stability and the comfort of its occupants.

As shown in Figure 2.1, a vehicle consists of two parts; the vehicle body (sprung mass) and the wheels (unsprung mass). The vehicle body is connected to the wheels by a suspension system to provide comfortable ride for vehicle's occupants. The main function for the suspension system is to provide an up-down relative displacement between the vehicle body and the wheels. The vehicle body rolls when the centrifugal force acts at the centre of gravity of the vehicle (Masato 2009). Due to this rolling movement, loads transfer from the inner wheels of the vehicle to the outer ones when cornering and thus the loads on the wheels are redistributed. As a result, the applied load and lateral frictional force (the force between the tire and pavement) on each tire will change. The transferred load from one wheel to another depends on vehicle characteristics such as the ratio of the centre of gravity height to the width of the vehicle. In braking case, the load transfers from rear axle to the front axle. The ratio of centre of gravity height to the wheelbase has the main influence on the load transfer. These two cases (cornering and braking) influence the dynamic stability of the vehicle especially when they occur simultaneously because of the variation in friction demand at each tire. Taking into account the closed-loop system of driver-vehicle interaction (driver action, vehicle response, and driver reaction) has to be considered in vehicle stability and control as well. In this closed-loop system, the motion 
of a vehicle on a circular path would be influenced by driver's acts (throttling, braking, and steering) and vehicle response, which is affected by the characteristics of the vehicle and roadway (Gillespie 1992).

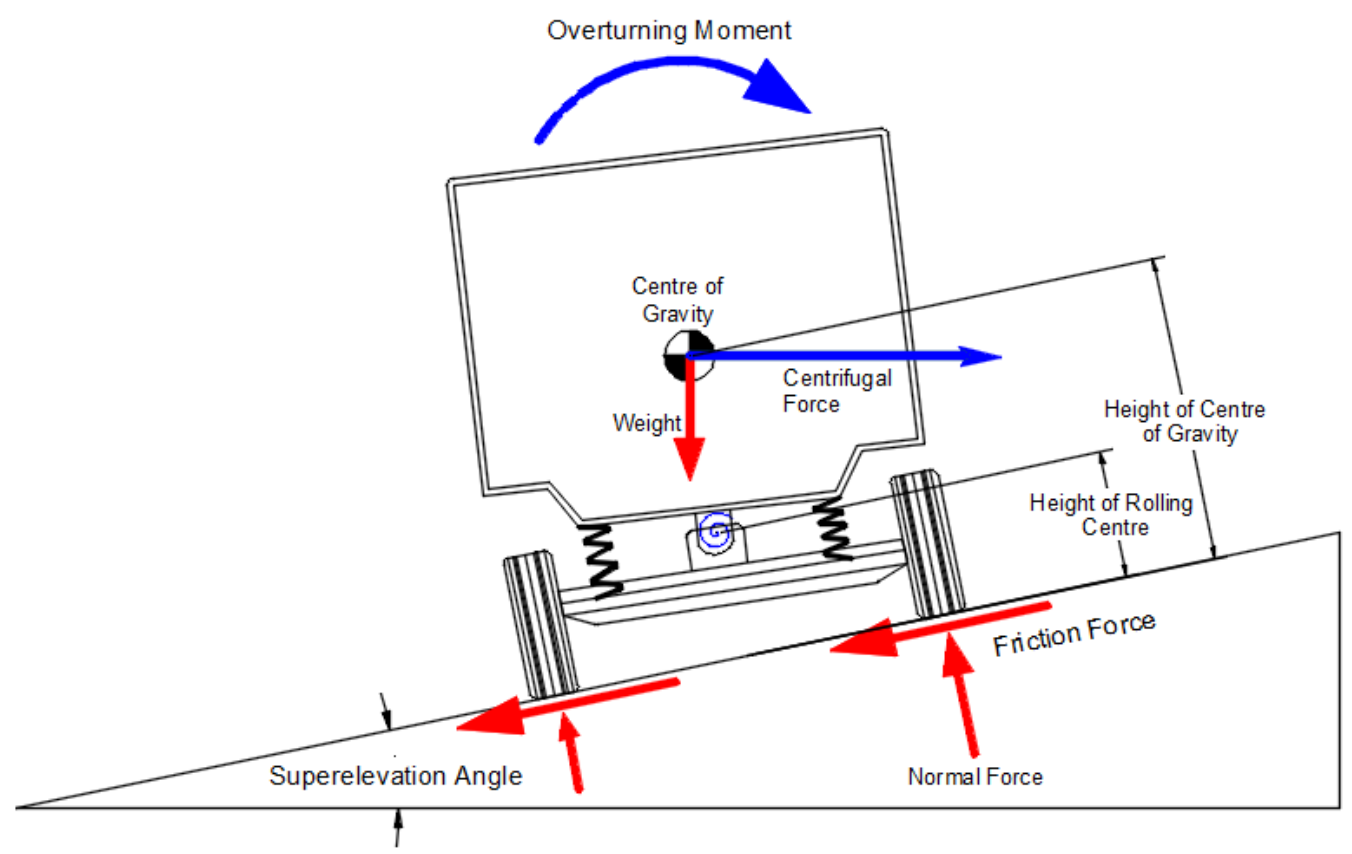

Figure 2.1: Forces Affecting Vehicle Movement on Circular Path.

\subsubsection{Vehicle Stability Models}

Generally, when curve design parameters (radius, superelevation rate, and speed) are combined in such a way that the pavement and tires cannot supply enough lateral friction, the vehicle will lose stability and skid-off the roadway. Vehicle stability models are used to determine whether the vehicle will skid off the road or will keep moving within the lane. These models analyse vehicle dynamics through determining the forces that push the vehicle to the outwards of the curve and those work to counterbalance these forces and sustain the vehicle on the curved track. Several models have been developed to simulate vehicle movement on a circular path and to estimate the forces that affect the stability of 
the vehicle. These models differ from one another in the level of accuracy and complexity. Following are the main models that can be used to estimate the friction demand, or to determine the minimum radius required.

\subsection{1.a. Point Mass Model}

The Point Mass Model (PMM) is the most popular model used to simulate the movement of a vehicle on a horizontal curve and widely used in the design guides. This model approximates the vehicle into a point mass and uses dynamics laws to determine the relationship among speed of vehicle, radius of the curve, lateral friction factor, and superelevation rate to ensure the dynamic equilibrium of a point mass (Echaveguren et al. 2005). For example, Lamm et al (1999) presented Figure 2.2 where all forces acting on the vehicle are assumed to act at a single point.

The PMM formula is shown in Equation (2.1) (A Policy 2011; TAC 2017).

$$
f=\frac{V^{2}}{127 R}-0.01 e
$$

Where $f=$ lateral friction factor (unitless), $V=$ speed of vehicle $(\mathrm{km} / \mathrm{h}), R=$ radius of curve (m), and $e=$ superelevation rate (percent).

In spite of the simplicity of the PMM, this model suffers from several shortcomings. First, the model ignores vehicle characteristics by assuming uniform distribution of frictional forces on all tires of the vehicle. Second, it assumes a uniform speed along the curve, and that vehicles follow a curve of a constant radius (Glen 2002). Third, the effect of overlapping vertical alignment is overlooked because the model assumes vehicles move on a plane curved surface (Kontaratos et al 1994). Fourth, the effect of lateral friction spikes at the beginning of the horizontal curves is not considered (Hassan 2004). Finally, the 
model cannot distinguish the differences among vehicle types (Torbic et al. 2014).

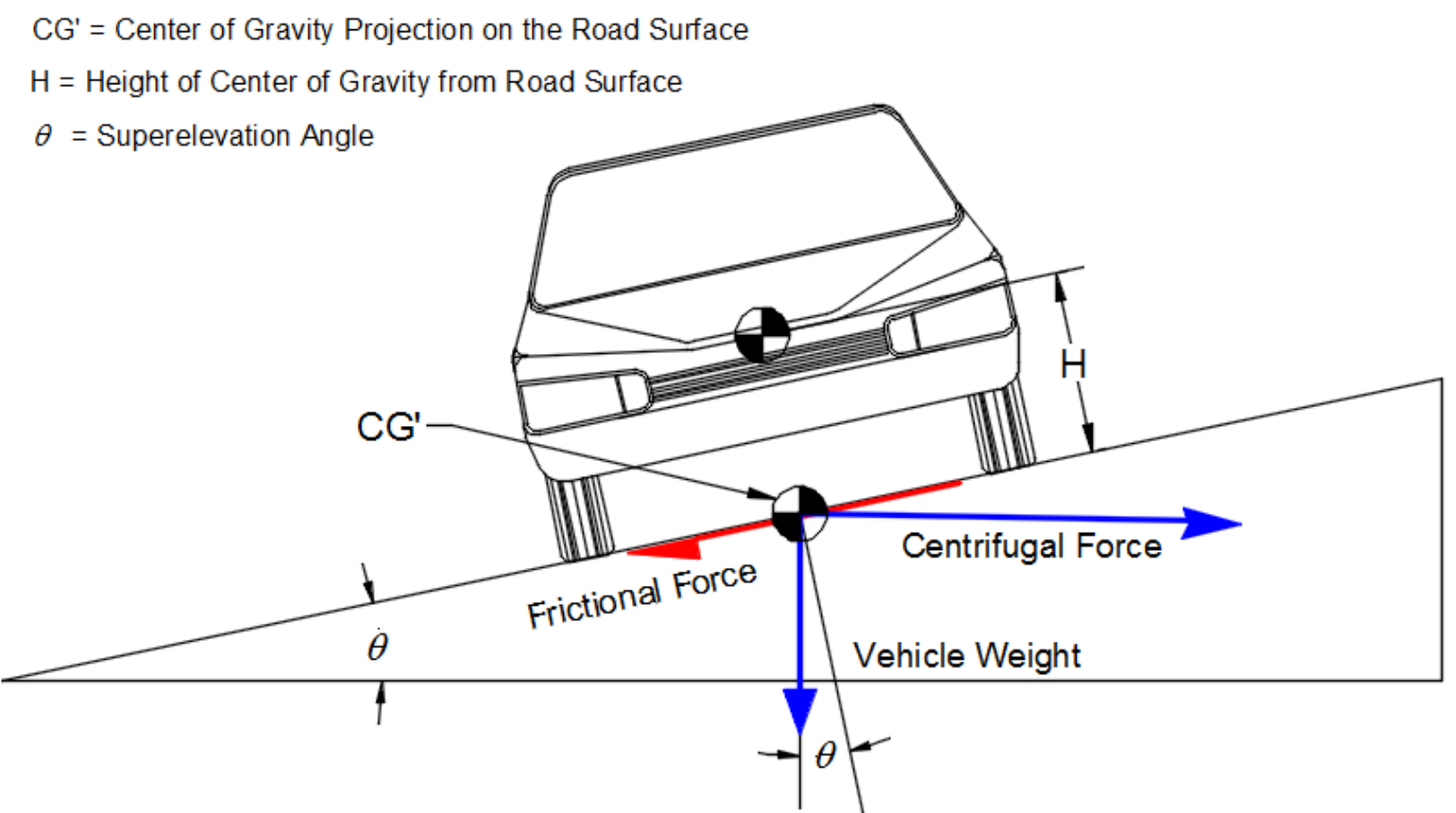

Figure 2.2: Forces Distribution Used to Develop PMM.

\subsection{1.b. Bicycle Model}

The bicycle model can be considered an extension for the kinematic bicycle model which was developed to describe the lateral motion of a vehicle. The kinematic bicycle model represents the front wheels of the vehicle by a one wheel and same thing with the rear wheels as shown in Figure 2.3. It uses geometric relationships to describe the motion without considering the forces affecting the motion. However, the model provides reasonable descriptions for only low speed motion since the velocity vectors at front and rear wheels are in the direction of the orientation. As shown in Figure 2.4, direction of velocity vector (direction of travel) may differ from the direction of wheel heading (direction of orientation), with the difference referred to as slip angle (Rajamani 2006). In 
the kinematic model, the slip angles $(\alpha)$ for the front and rear wheels are equal to zero. Though, using this model for higher speeds (greater than $5 \mathrm{~m} / \mathrm{s}$ ) has some concerns because slip angles for vehicle's tires are presented and the geometric relationship is no longer capable of describing the motion (Gillespie 1992).

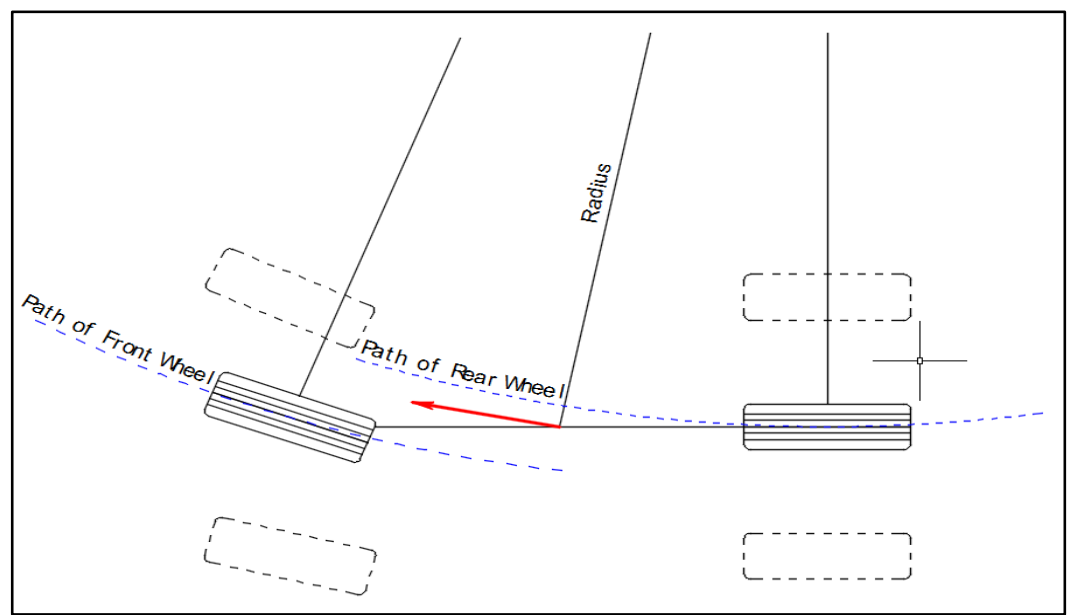

Figure 2.3: Kinematic Bicycle Model - Reproduced from (Gillespie 1992).

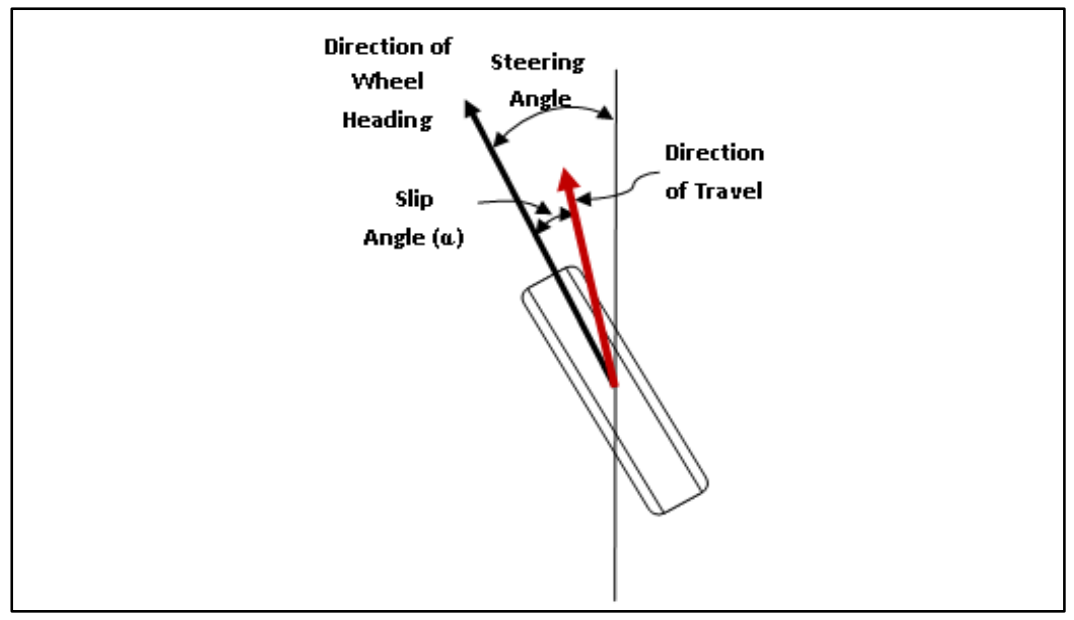

Figure 2.4: Slip Angle - Reproduced from (Gillespie 1992). 
Similar to the kinematic model, the classic bicycle model represents the front and rear wheels of the vehicle by a single wheel for each axle as shown in Figure 2.5. The summation of forces in the lateral direction of the tires is equal to the centripetal acceleration times the mass according to Newton's second law:

$$
\sum F_{y}=\sum F_{y f}+\sum F_{y r}=m V^{2} / R
$$

The forces in the lateral direction on the front and rear wheels are determined as follows (Gillespie 1992).

$$
\begin{aligned}
& F_{y f}=F_{y r} \frac{c}{b} \\
& F_{y r}=m \frac{b}{L}\left(\frac{V^{2}}{R}\right)
\end{aligned}
$$

Where $F_{y f}$ and $F_{y r}=$ the lateral force on the front and rear wheels respectively, $m=$ mass of the vehicle, $b$ and $c=$ distances from the centre of gravity of the vehicle to the front and rear wheel, respectively, $L=$ wheelbase distance, $V=$ speed of the vehicle, and $R=$ radius of the curve.

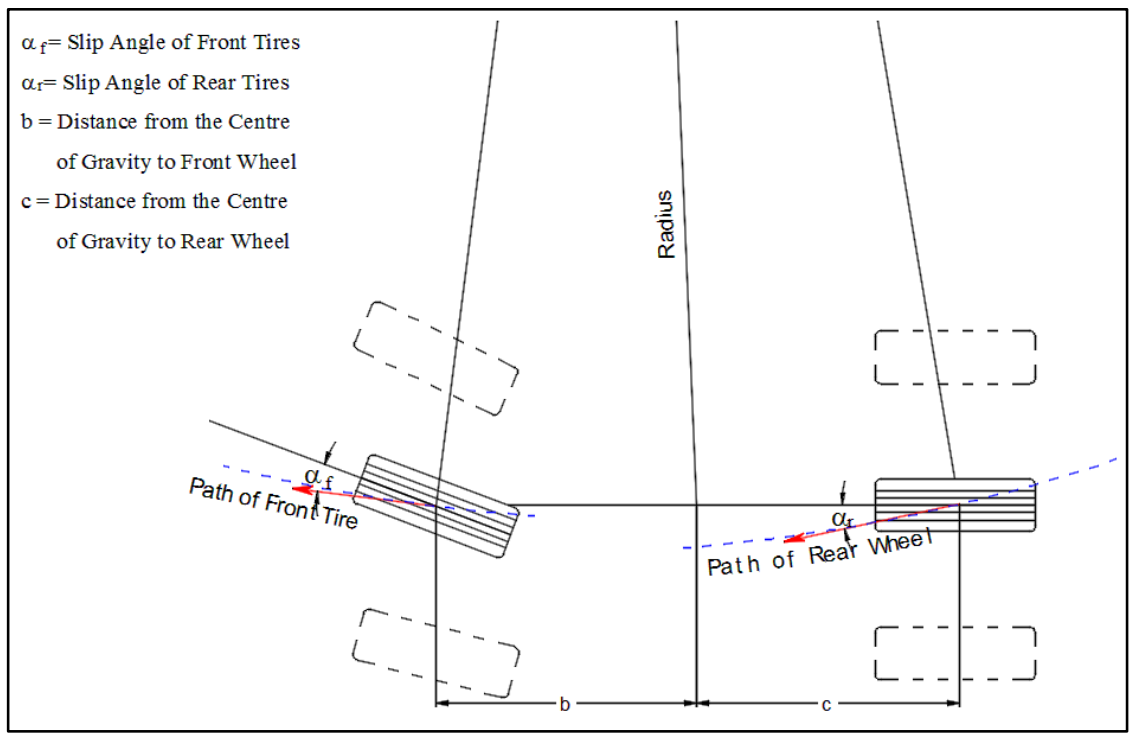

Figure 2.5: Bicycle Model - Reproduced from (Gillespie 1992). 
The lateral force for the front and rear tire can be found by using the cornering stiffness (Figure 2.6). The cornering stiffness $\left(C_{\alpha}\right)$ can be estimated for small slip angles, less than 5 degrees, since the relationship between slip angle $(\alpha)$ and lateral force is linear (Gillespie 1992).

$$
F_{y}=C_{\alpha} \alpha
$$

The general equation to represent the relationship among steering angle $(\delta)$, radius of curve $(R)$, loads on front and rear wheels $(W)$, wheelbase $(L)$, speed of vehicle $(V)$, and cornering stiffness is (Gillespie 1992):

$$
\delta=57.3 \frac{L}{R}+\left(\frac{W_{f}}{C_{\alpha f}}-\frac{W_{r}}{C_{\alpha r}}\right) \frac{V^{2}}{g R}
$$

Thus, the radius of the curve is:

$$
R=\frac{5.73 L C_{\alpha f} C_{\alpha r}+V^{2} W_{f} C_{\alpha r}-V^{2} W_{f} C_{\alpha f}}{\delta C_{\alpha f} C_{\alpha r} g}
$$

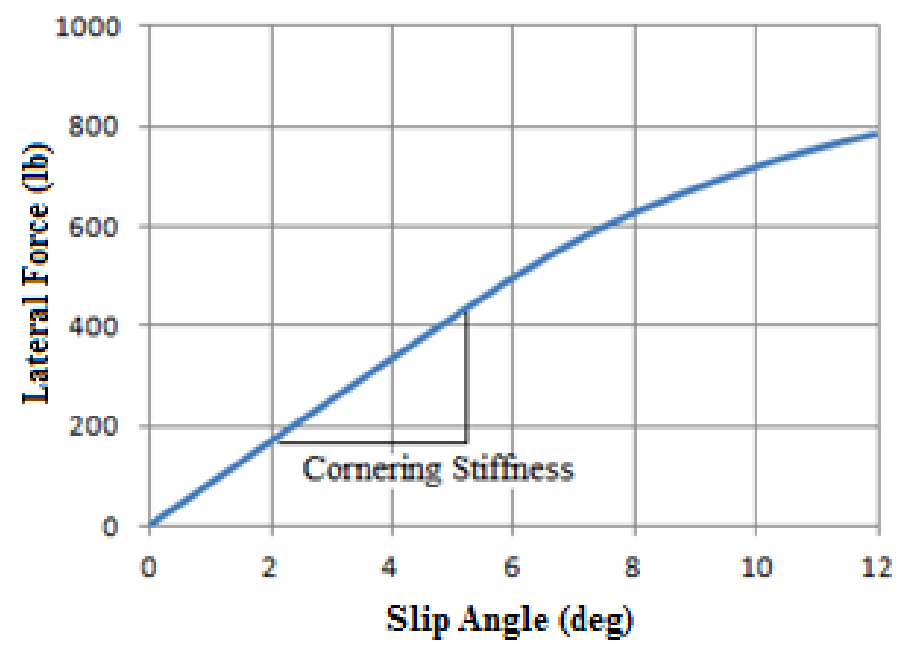

Figure 2.6: Cornering Stiffness- Reproduced from (Gillespie 1992). 
Kontaratos et al (1994) used the bicycle model to develop vehicle-road model to determine the minimum horizontal curve radius for a passenger car. The vehicle was defined as a rigid body with a specific height. The interior and exterior wheels of the vehicle were assumed to be subjected to equal forces whereas the forces on the front and rear wheels depend on the type of vehicle driving mode (Kontaratos et al. 1994). The minimum radius for a specific design speed can be calculated using Equation (2.8):

$$
R_{\text {min }}=\frac{V^{2}}{g\left(n f_{y \text { Max }}+e\right)}
$$

The value $n$ for front and rear drive vehicle is obtained from Equations (2.9) and (2.10), respectively.

$$
\begin{aligned}
& n=\sqrt{1-\frac{f_{X f}^{2}}{f_{X M a x}^{2}}}\left[1-\frac{h}{I_{R}} \cdot G-\frac{A_{z}}{m \cdot g}-\frac{A_{z}}{m \cdot g} \cdot \frac{h}{I_{R}}\right] \\
& n=\sqrt{1-\frac{f_{X f}^{2}}{f_{X M a x}^{2}}}\left[1+\frac{h}{I_{f}} \cdot G-\frac{A_{z}}{m \cdot g}-\frac{A_{z}}{m \cdot g} \cdot \frac{h}{I_{f}}\right]
\end{aligned}
$$

Where $A_{z}=$ air resistance force component, $f_{X M a x}$ and $f_{y \operatorname{Max}}=$ maximum available tangential and lateral coefficient of frictions, respectively, $f_{X f}=$ coefficient of friction to respective direction and wheels, $h=$ height of centre of gravity, $g=$ gravity acceleration, $I_{f}$ and $I_{R}=$ distance between Centre of gravity and front and rear wheel respectively, $m=$ vehicle mass, $G=$ grade, $e=$ superelevation rate, and $V=$ vehicle speed.

Tejas (2011) implemented the bicycle model on 3D road to estimate the friction demand at the front and rear wheels for transient and steady states. The vehicle is in a "steady state" when all the states of a vehicle are in equilibrium, such as uniform speed and moving on curve of constant radius. The vehicle is in a transient response before it reaches 
the steady. The main assumptions of the steady state model were (Tejas 2011):

- Lateral load transfer, roll and pitch dynamics, rolling resistance, and suspension compliance effects are ignored.

- Vehicle longitudinal acceleration and deceleration are zero.

- The magnitude of vehicle velocity vector is approximated to be equal to the longitudinal speed.

The lateral friction demand for steady state at front and rear axles are shown in Equation (2.11) and (2.12) respectively.

$$
\begin{aligned}
& f_{y f}=\frac{F_{c f}}{W_{f}}=\frac{\frac{b}{L}\left(m \frac{V^{2}}{R}-W \frac{e}{100}\right)}{W \frac{b}{L}-\left(m\left(-g \frac{G}{100}+a_{x}\right)\right) \frac{h}{L}} \\
& f_{y r}=\frac{F_{c r}}{W_{r}}=\frac{\frac{a}{L}\left(m \frac{V^{2}}{R}-W \frac{e}{100}\right)}{W \frac{a}{L}-\left(m\left(-g \frac{G}{100}+a_{x}\right)\right) \frac{h}{L}}
\end{aligned}
$$

Where $F_{c f}$ and $F_{c r}=$ cornering force at front and rear axle, $W_{f}$ and $W_{r}=$ normal force at front and rear axle, $f_{y f}$ and $f_{y r}=$ lateral friction demand at front and rear axles, $a$ and $b=$ centre of gravity location to front and rear axle distance, $e=$ superelevation rate, $V=$ longitudinal velocity, and $G=$ grade.

These equations provided by Tejas (2011) could be rearranged to determine the minimum radius of curve by entering the maximum allowable lateral friction demand and vehicle parameters. Thus, equation (2.11) can be rewritten as follows:

$$
R=\frac{b m V^{2}}{f_{y f} W b+f_{y f} m h g \frac{G}{100}-f_{y f} m h a_{x}+b W \frac{e}{100}}
$$


The main difference between Tejas bicycle model and Kontaratos model is that the latter considers the available lateral friction in determining the minimum radius of the horizontal curve.

Although the bicycle model considers the vehicle as a rigid body with two wheels and can consider the effect of the roadway grade, the following shortcomings can be highlighted:

- The lateral load transfer between left and right wheels is not considered.

- The suspension effects, rolling resistance, and aerodynamics are ignored.

- The camber angle (angle between the wheel plane and the vertical axes) and roll angle are ignored.

- The longitudinal acceleration/deceleration is considered constant.

\subsection{1.c. Two-Axle Model}

This model can be considered as an extension to the bicycle model and a further step toward realism. In this model, the mass of vehicle is elevated some distance above the ground and the width of vehicle is considered (Glen 2002). The normal load is permitted to transfer from one side another. As a result, the load transfer due to body roll is considered in determining friction demand at each tire. That is, the friction demand at the two axles of the vehicle will not be equal to the friction demand of PMM and the friction demand at each tire would be different.

\subsection{1.d. Vehicle Dynamics Models}

The vehicle dynamic model (VDM) is more sophisticated than the previous models; however, it provides better understanding for the relationship between the vehicle and roadway (Glen 2002). The model is virtually developed to cover all types of lateral, 
longitudinal, and angular motions and it takes into consideration vehicle's characteristics such as body roll, pitch, yaw, and lateral weight distribution. Therefore, several sub-models have been imbedded in the VDM such as tire model, suspension model, steering model, power and drive train model, and brakes model (Figure 2.7). You and Sun (2013), for example, developed a three degree of freedom vehicle model including angular, yaw, and lateral velocity to study vehicle stability when moving on combined horizontal and vertical alignments. Based on fundamental laws of Newtonian force and moment balance, several equations have been derived for lateral movement, yaw motion, and roll motion of vehicle body. Considering the profile and geometry of the curve and several vehicle characteristics such as suspension system, equations to determine the normal and lateral force at each tire have been derived. A Matlab/Simulink to establish three models (vehicle model, driver model, and road model) to simulate a closed-loop vehicle driving (You and Sun 2013).

Several software packages, based on vehicle dynamic analysis, have been developed to simulate the motion of various types of vehicles such as CarSIM and Interactive Highway Safety Design Model (IHSDM). CarSIM, first introduced in 1990, is a software developed by Mechanical Simulation Corporation to simulate the dynamic performance of cars, trucks, motorcycles, and specialty vehicles accurately and efficiently (Mechanical Simulation 2014). CarSIM is equipped with several kinds of virtual sensors that detect various types of vehicle motion, including acceleration, speed, and previews of the road ahead, and uses many ordinary differential equations for each sub-model. For instance, the math model for a four-wheeled vehicle has over 110 ordinary differential equations and the solver programs generate over 800 built-in output variables (Mechanical Simulation 2014). 


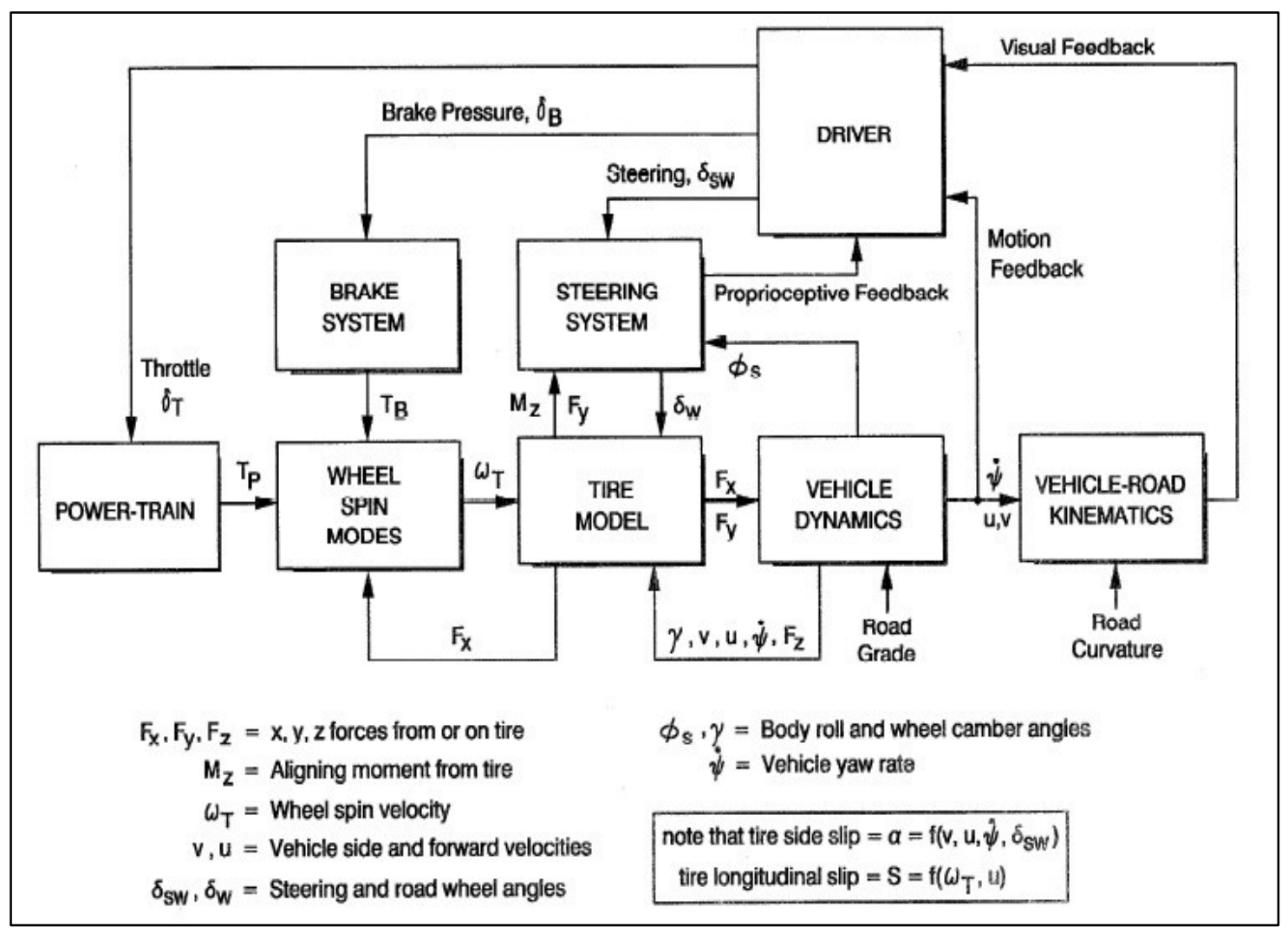

Figure 2.7: Vehicle Dynamic Sub-Models (Allen et al. 1998).

On the other hand, the IHSDM, developed by the United States Federal Highway Administration (FHWA), is "a suite of software analysis tools for evaluating safety and operational effects of geometric design in the highway project development process" (FHWA 2015). It includes six modules (Crash Prediction, Design Consistency, Intersection Review, Policy Review, Traffic Analysis, and Driver/Vehicle) used to evaluate an existing or proposed geometric design from different perspectives and estimates measures to describe expected safety and operational performance of the design. The Driver-Vehicle Module simulates driving behaviour and vehicle dynamics along a highway. Thus, this module is a combination of two models: the driver performance model and the non-linear vehicle dynamic analysis model (FHWA 2015). Therefore, the IHSDM is geared towards 
assisting geometric design decision makers in the highway design process while CarSIM is geared towards vehicles' manufacturers.

\subsubsection{Vehicle Rollover Models}

When a vehicle negotiates a horizontal curve, an overturning moment is developed due to two equal and opposite forces; the frictional force that acts at the roadway surface and a centrifugal force that acts at the centre of gravity of the vehicle (TAC 2017). Note that rollover requires sufficient tire lateral force or a tripping mechanism to turn over the vehicle before it would skid off the road (Allen et al. 1992).

The vehicle starts to rollover when the vertical load on the inside wheels reaches zero and the wheels lift off the ground. For unsprung vehicle case, the rollover threshold can be determined by taking moment at the outside wheels as shown in Equation (2.14) (Chang 2001).

$$
\frac{v^{2}}{g R}=e+\frac{t}{2 h}
$$

Where $R=$ radius, $v=$ speed of vehicle, $t=$ average $(\mathrm{c} / \mathrm{c})$ width between wheels, $h=$ height of the centre of gravity, $e=$ superelevation rate, and $g=$ acceleration of gravity.

Chang (2011) incorporated the body roll approach, which considers the sprung part of the vehicle in the analysis, in defining the relationship among minimum radius, speed of vehicle, lateral friction, and superelevation rate. That is, it considers load transfer from the inner wheels to the outer ones as explained earlier.

When considering the effect of the suspension system of the vehicle, the rollover threshold will be: 


$$
\frac{v^{2}}{g R}=\left[\left(1-\frac{h_{o}}{h}\right)(e-\phi)\right]+\frac{t}{2 h}
$$

Where $h_{o}=$ height of the rolling pivot, which is the distance from the ground to the rolling centre of sprung part of vehicle body, and $\phi=$ roll angle.

By considering that the roll angle of the sprung vehicle equal to the roll rate $\left(r_{\phi}\right)$ of the sprung mass times the lateral acceleration, the radius will be:

$$
R=\frac{v^{2}}{g\left[\left(1-\frac{h_{o}}{h}\right) e+f\right]}\left[1+r_{\phi}\left(1-\frac{h_{o}}{h}\right)\right]
$$

Chang (2011) found that the radius calculated using Equation 2.16 is always greater than radius calculated using Equation $2.1\left(R=\frac{V^{2}}{127(e+f)}\right)$.

Based on (Gillespie 1992), the approximate typical passenger car and truck height ratios $\left(\frac{h_{o}}{h}\right)$ are 0.5 and 0.25 respectively, and the roll rates are 0.1 and 0.05 radians $/ g$. Thus, the radius of the horizontal curve for passenger car considering the sprung part of the vehicle is (Chang 2001):

$$
R=\frac{V^{2}}{121(0.5 e+f)}
$$

For truck is:

$$
R=\frac{V^{2}}{122.5(0.75 e+f)}
$$

The models show that the passenger cars need radii greater than trucks. Chang (2001) explained that a truck should be more stable in body rolling if it is loaded within a confined range and without exceeding the height ratio because the suspension stiffness is harder than those of passenger car. It should be noted that the model was utilizing common passenger 
car's parameters for improving horizontal design criteria. In addition, the models do not consider the deformation on outside tires and ignore load distributions at the front and rear tires. However, these models contradict several studies that stated that the rollover threshold for passenger cars (higher than $1.2 \mathrm{~g}$ ) is higher than those for trucks (within a range of 0.27 to $0.4 \mathrm{~g}$ ) (Lamm et al. 1999).

Similarly, Torbic et al. (2014) used the quasi static model on superelevated roads to predict wheel lift. The model assumed that the rolling centre of the sprung mass does not change, aligned with the centre of the vehicle, and a fixed height above the road surface. The equilibrium equation between the applied and sustained forces for a rigid vehicle with a simple suspension model moving on superelevated road is:

$$
\frac{v^{2}}{g R}=\frac{\frac{T}{2 h}+e}{1+\left(1-\frac{h_{o}}{h}\right) r_{\phi}}
$$

Where $v=$ speed, $T=$ truck width, $h=$ height of sprung-mass centre of gravity, $h_{o}=$ height of roll centre, and $r_{\phi}=$ roll rate.

For the suspension model and considering worst case-scenario $\left(r_{\phi}=0.17 \mathrm{rad} / \mathrm{g}, \frac{h_{o}}{h}=\right.$ zero, and $e=$ zero), Equation (2.30) will be:

$$
\frac{v^{2}}{g R}=0.85\left(\frac{T}{2 h}\right)
$$

Considering transient manoeuvres, Torbic et al. (2014) used high-order multibody models to predict wheel lift. CarSIM and TruckSIM were used in the analysis. The Load Transfer Ratio (LTR) was developed to predict wheel lift and examine relative severity of a manoeuvre as shown in Equation (2.35). 


$$
L T R=\frac{N_{i}-N_{o}}{N_{i}+N_{o}}
$$

Where $N_{i}$ and $N_{o}$ are the normal loads on the inner and outer axels. The LTR ranges from -1 (the inner wheels lift) and +1 (Torbic et al. 2014).

Allen et al. (1992) showed that the lateral acceleration divided by gravity acceleration (g) must be greater than one half the vehicle track width divided by the centre of gravity height in order to generate sufficient overturning moment to rollover a vehicle (Figure 2.8). Note that this relation does not consider the effect of grade or superelevation rate.

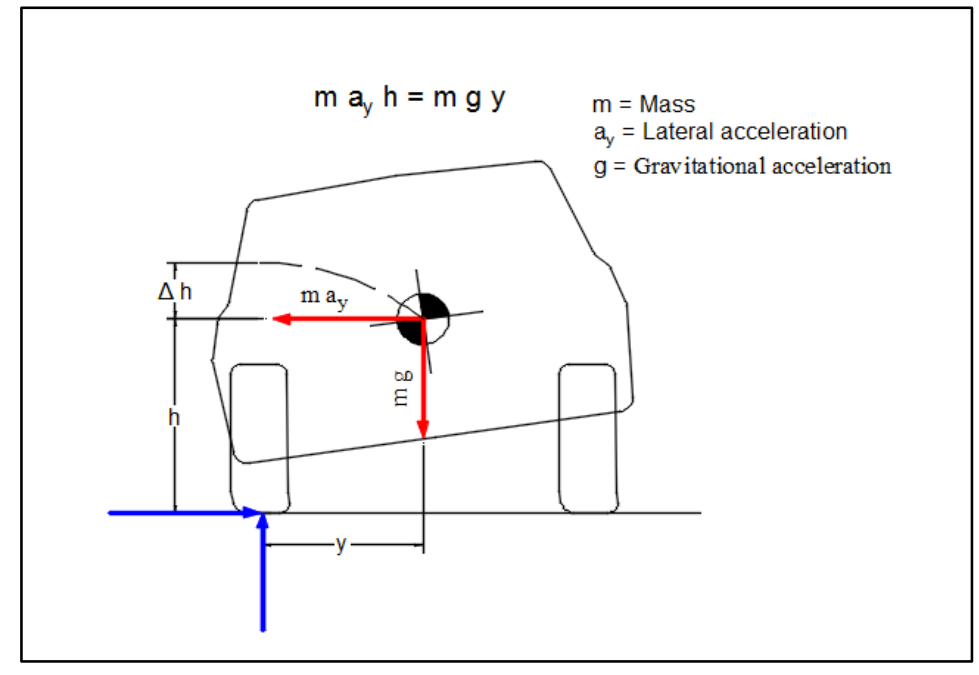

Figure 2.8: Rollover Situation.

Lamm et al. (1999), based on the PMM and considering the height of the centre of gravity, the width of the vehicle, and superelevation rate in the analysis, stated that rollover due to centrifugal force cannot occur as long as the tractive moment is smaller than the resistant moment. Thus, the radius of curve for impeding rollover is:

$$
R \geq \frac{v^{2}(h-b e)}{g b+h e}
$$


Where $v=$ speed of vehicle $(\mathrm{m} / \mathrm{s}), b=$ half the width of vehicle, $e=$ superelevation rate, and $h=$ height of the centre of gravity.

\subsubsection{Driver Comfort Model}

The driver comfort model is the same as PMM, which is used to determine the radius of a horizontal curve based on a design speed, superelevation rate, and friction factor. The friction factors have been determined based on the sensation of vehicle's occupants to forces pushing them to the outside of the curve. That is, the friction factor used in the design is the level of lateral acceleration that is sufficient to cause discomfort feelings to the majority of drivers (TAC 2017; A Policy 2011). The values of friction factors in the design guides are based on experiments conducted in 1930s using the ball-bank indicator. These values were confirmed for high speed design and modified for low speed design starting in the 2001 edition of the Green Book (A Policy 2011) based on the work by (Bonneson 2000).

\subsection{Vehicle Stability and Control}

Although a driver controls the motion of a vehicle, the response of the vehicle depends on the driver's steering and throttle/braking inputs, surface conditions, geometry of the road, and the dynamic characteristics of the vehicle. The steering action of the driver depends on information collected from the surroundings such as the current position of the vehicle with reference to the target path, the current vehicle motion, and predicting vehicle behaviour (Masato 2009). Therefore, it is important to study the inherent dynamic characteristics of a vehicle; the response of a vehicle for driver's actions; and the applied and generated forces that affect the motion of the vehicle relative to the ground. Also, 
understanding vehicle stability situation would give an indication of driver workload, which is defined as "the time rate at which drivers must perform the driving task which changes continuously until it is completed" (Messer 1989).

Control characteristics of a vehicle refer to its response to steering commands and to environmental inputs. The directional stability of a vehicle can be defined as "its ability to stabilize its direction of motion against disturbances. A vehicle is considered to be directionally stable if, following a disturbance, it returns to a steady state regime within a finite time" (Wong 2008). The main factors affecting the steady state controlling characteristics are the weight distribution of the vehicle and the cornering stiffness of the tires. The operational parameters that affect the cornering stiffness and thus the controlling characteristics are (Masato 2009; Wong 2008):

- tire material, construction, and shape such as radial-ply or bias-ply tires,

- tire inflation pressure,

- tire vertical load (lateral load transfers from the inner to the outer tires will increase the slip angle required to generate a given cornering force), and

- roadway conditions.

Studying the stability and control mechanism of the vehicle is out of the scope of this research. However, it is important to understand stability and understeer factors because they describe the driving situation of the vehicle. These two factors will be observed when running simulations to develop the models.

When a vehicle moves on a horizontal curve at moderate or higher speeds, vehicle's tires develop appropriate lateral forces to balance the centrifugal force. These lateral forces 
develop in turn lateral slip angles. Using the bicycle model to simulate the movement of the vehicle as shown in Figure 2.9, the steering angle for front tire $\left(\delta_{f}\right)$ will be a function of wheelbase $(L)$, radius $(R)$, and slip angles of the front tires $\left(\alpha_{f}\right)$ and rear tires $\left(\alpha_{r}\right)$ as shown in the Equation (2.23) (Wong 2008).

$$
\delta_{f}=\frac{L}{R}+\alpha_{f}-\alpha_{r}
$$

Considering normal load distribution on the front and rear wheels, the steering angle will be (Wong 2008):

$$
\delta_{f}=\frac{L}{R}+\left(\frac{W_{f}}{C_{\alpha f}}-\frac{W_{r}}{C_{\alpha r}}\right) \frac{V^{2}}{g R}
$$

Where $C_{\alpha f}$ and $C_{\alpha r}=$ the cornering stiffness (the slope of the lateral force vs. slip angle as shown in Figure 2.6 for the front and rear tires, respectively), and $W_{f}$ and $W_{r}=$ the normal load at front and rear tires, respectively. The background calculations to derive this equation can be found in Wong (2008).

The $\left(\frac{W_{f}}{C_{\alpha f}}-\frac{W_{r}}{C_{\alpha r}}\right)$ is known as the understeer coefficient $\left(K_{u s}\right)$. Depending on the value of this coefficient, the handling characteristics may be classified as neutral, oversteer, and understeer. Thus, the previous equation could be rewritten as follows:

$$
\delta_{f}=\frac{L}{R}+K_{u s} \frac{V^{2}}{g R}=\frac{L}{R}+K_{u s} \frac{a_{y}}{g}
$$

Where $a_{y}=$ lateral acceleration. 


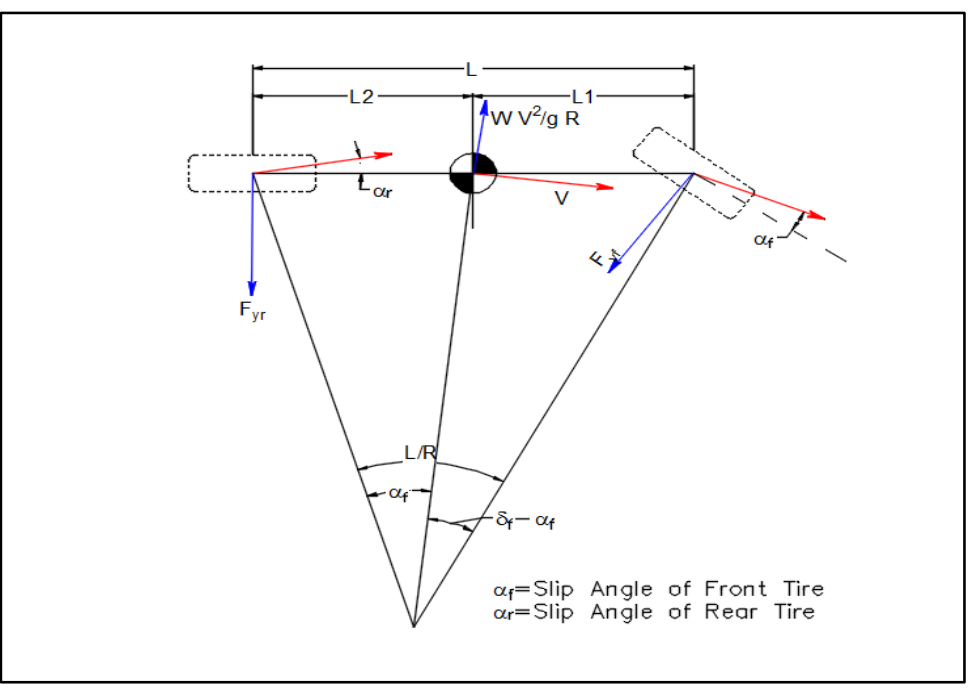

Figure 2.9: Steady State Handling Model in the Bicycle Model- Reproduced from Wong 2008.

Within this context, vehicle handling characteristics may be determined using the stability factor $(A)$, which represents the relationship between vehicle mass, position of the vehicle's centre of gravity, wheelbase, and cornering stiffness as shown in Equation (2.28) (Jazar 2014).

$$
A=\frac{m}{L^{2}}\left(\frac{L_{2}}{C_{\alpha f}}-\frac{L_{1}}{C_{\alpha r}}\right)
$$

Where $m=$ mass $(\mathrm{kg})$, and $L_{1}$ and $L_{2}=$ the distance between the center of gravity of the vehicle and the front and rear wheels respectively.

The value of the understeer coefficient or the stability factor is used to indicate the control characteristics of the vehicle. For the steady state, it is classified into three categories; neutral steer, understeer, and oversteer (Jazar 2014; Wong 2008).

\subsubsection{Neutral steer}

The values of stability factor and understeer coefficient in this case are both equal to 
zero because the slip angles are equal for the rear and front tires. The control characteristics will be independent of the speed of the vehicle. The driver needs to maintain the steering angle when accelerating on a horizontal curve of constant radius (Wong 2008).

\subsubsection{Understeer}

The understeer coefficient and stability factor for vehicles of understeer control characteristics are positive. The slip angle at the front wheels is greater than that at rear wheels. When a vehicle accelerates on a horizontal curve of a constant radius, the driver should increase the steering angle. Otherwise, the vehicle will follow a path of increasing radius if the steering angle is constant.

\subsubsection{Oversteer}

Vehicles with oversteer control characteristics have negative understeer coefficient and stability factor. The slip angle for the front wheels is less than that of the rear wheels (Wong 2008). When an oversteering vehicle accelerates on a horizontal curve, the driver needs to decrease the steering angle in order to maintain constant radius. Otherwise, the path radius would decrease if the steering angle remained fixed. As the driver reduces the steering angle to maintain a constant radius, the steering angle may end up to be equal to zero and the driver would then lose control. The speed at which the steering angle equals zero is known as the critical speed. Vehicles will exhibits directional instability when speeding up over the critical speed (Wong 2008).

It is important to ensure that the vehicle in neutral or understeer conditions for the following reasons:

- The motion of the vehicle does not have a critical speed above which the vehicle 
would be unstable and the stability would be questionable (Karnopp 2004).

- Masato (2009) reported that driver derivative time decreases with the stability factor (Figure 2.10). The driver derivative time is considered to reflect vehicle driving control since it is one of the causes of the workload of the driver. In other words, vehicles with less control actions are considered easier to handle.

- It is found that drivers of understeer vehicles have lower heart beat than those of oversteer characteristics vehicles. This might give an indication of reduced driver workload (Masato 2009).

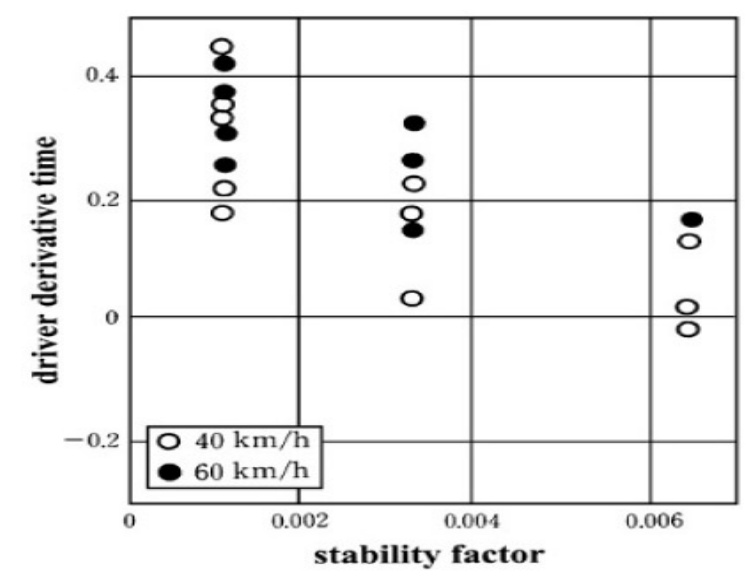

Figure 2.10: Relationship between Stability Factor and Derivative Control Action (Masato 2009).

Although most of the vehicles are designed to be in an understeer situation, the change in load distribution on front and rear wheels may change the steady state controlling behaviour of the vehicle. For example, vehicles might exhibit oversteer behaviour when decelerating on a curve due to load transfer. In this case, the slip angle decreases at front wheels and increases at the rear wheels (Wong 2008). 
It is worth to note that many developments and enhancements have taken place to the design of vehicles to increase their directional stability, safety, and ride comfort. Some of these developments are based on the estimation of the friction coefficient between the pavement surface and the tire under dynamic and chaotic situations such as Traction Control System (TCS), and Electronic Stability Control (ESC) (Vieira et al. 2012). Although these developments have shown effectiveness in reducing the likelihood of collision occurrence, they have, in turn, the potential of increasing unsafe driving. Changing driver behaviour, which is commonly known as behavioural adaptation, is the main negative effect of such developments on safety. Drivers with vehicles equipped with ESC, for example, tend to drive faster and more aggressively in adverse weather conditions (Rudin-Brown et al. 2009).

\subsection{Horizontal Curve Design Criteria}

Generally, the design of roads has to provide road's visible features and dimensions based on acceptable balance between level of service, cost, environmental impact, and level of safety. Therefore, the main challenge for the designer during the design process is to select the best solution among various alternatives. The geometric design of the horizontal alignment should provide specifications and dimensions for the circular curves, spiral transition curves, and the development of superelevation. Many factors control the design such as driver expectation, design speed, topography, climatic conditions, traffic volumes, and the existence of major utilities (TAC 2017). Wolshon (2004) stated that horizontal curve design should allow drivers to maintain vehicle control within the lane of travel, at a desired speed, and to provide an adequate distance to see ahead. The design should also consider the rollover situation for vehicles with high centre of gravity. In addition, the 
design should correspond with avoiding vehicle's occupants experiencing excessive discomfort due to a high level of lateral acceleration (TAC 2017; A Policy 2011). These requirements are known as the design criteria. These design criteria (driver comfort, vehicle dynamic stability, rollover, and sight distance) are discussed in the coming sections.

\subsubsection{Driver Comfort Criterion}

Driver comfort is a human factor issues related to "kinaesthesia", which is defined as "the sensation by which bodily position, weight, muscle tension, and movement are perceived" (Thambirajah 2011). When a vehicle moves on a curvilinear path, vehicle's occupants will be subjected to a lateral acceleration that affects their comfort. The driver's comfort threshold of lateral acceleration depends on the driver's sensation of the forces pushing them to the outside of the curve, which is the combined effect of the lateral acceleration and roll angle, and it is basically the unbalanced lateral force (frictional force), which is commonly explained in lateral acceleration form (Glennon et al. 1985). Thus, the unbalanced lateral force (side pitch) is affected principally by vehicle suspension system and weight distribution. This side pitch has been noticed to be felt by vehicle's occupants at speed much lower than those at which skidding impends. The difference between the two speeds (speed at which vehicle starts skidding and speed at which side pitch be felt) provides an ample margin of safety against skidding (Barnett 1936). Based on that, the maximum safe speed was defined as "the minimum speed at which the centrifugal force created by the movement of a vehicle around the curve causes the driver or passenger to feel a side pitch outward" (Merritt 1988). This was the criterion used to identify driver discomfort as correlated with the centrifugal force sensation. Note that the comfort threshold for the same curve differs from one driver another depending on several factors 
such as the gender and age of driver.

Ball-Bank Indicator has been used to determine driver comfort threshold or what was known as "driver's judgment of incipient instability" (Merritt 1988). The classical ballbank indicator consists of a steel ball moving freely in a sealed glass tube. Figure 2.11 shows the typical and electronic ball-bank indicators. The developed centrifugal force causes the ball to roll out to a fixed angle position. The ball-bank reading (in degrees) at any time measures the combined effect of vehicle body roll angle, superelevation angle, and lateral acceleration angle (TAC 2017; A Policy 2011). It is reported that the ball-bank indicator readings of $14^{\circ}$ for speeds $30 \mathrm{~km} / \mathrm{h}$ or less, $12^{\circ}$ for speeds of 40 and $50 \mathrm{~km} / \mathrm{h}$, and $10^{\circ}$ for speeds of 55 to $80 \mathrm{~km} / \mathrm{h}$ would provide sufficient margin of safety against vehicle skidding or rollover. However, recent studies showed that these readings should be increased to reflect current improvements in vehicle dynamics (TAC 2017). Also, an electronic accelerometer, a gravity-sensitive electronic device, can be used to determine advisory speed for horizontal curves because of its capability to measure the lateral forces and accelerations that vehicle's occupants experience (A Policy 2011).
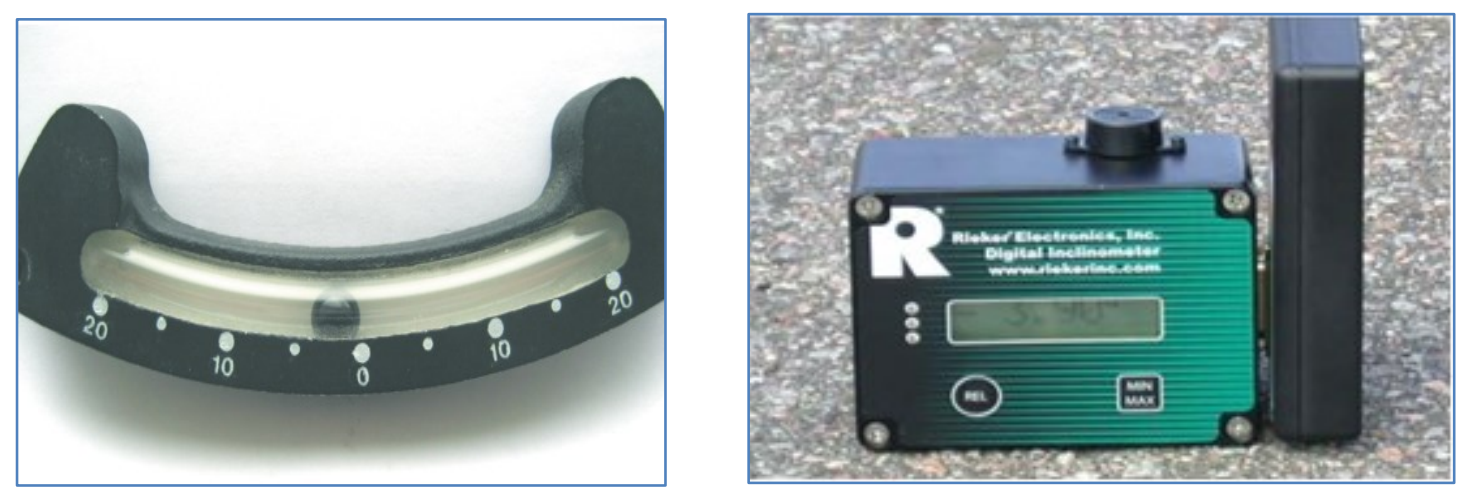

Figure 2.11: Typical and Electronic Ball-Bank Indicator (Misaghi 2003; Hildebrand and Lewis 2010). 
To determine the comfort threshold, Equation (2.1) can be adjusted to and consider the body roll of the vehicle and rewritten as:

$$
\frac{v^{2}}{R}=g(f+e)-\rho g
$$

Where $v=\operatorname{speed}(\mathrm{m} / \mathrm{s}), f=$ lateral friction factor, $e=$ superelevation rate, $\rho=$ body roll angle, and $g=$ gravity acceleration $\left(\mathrm{m} / \mathrm{s}^{2}\right)$.

As explained earlier, vehicle's occupants feel the unbalanced lateral acceleration, which will be (Said 2008):

$$
a_{L}=\frac{v^{2}}{R}-e g+\rho g=g f_{d}+\rho g
$$

Where $a_{L}=$ lateral acceleration perceived by vehicle's occupants $\left(\mathrm{m} / \mathrm{s}^{2}\right)$, and $f_{d}=$ lateral friction demand.

Driver comfort criterion is concerned with avoiding vehicle's occupants experiencing excessive discomfort due to a high level of lateral acceleration. In other words, it ensures that the lateral acceleration developed while negotiating a horizontal curve does not exceed a specific limit (the comfort threshold). Note that failing to comply with the driver comfort criterion would not directly result in a collision. However, a location where a significant number of drivers' experience discomfort can lead to improper manoeuvres and would likely experience a relatively high number of collisions.

\subsubsection{Vehicle Stability Criterion}

This criterion is important because the consequences of failing to comply with it likely ends up with a collision, if the driver unable to take over vehicle control. The stability of the vehicle depends on the frictional interaction between tires and pavement, and the 
horizontal component of the vehicle weight in case the roadway is superelevated. The design of horizontal curves according to this criterion ensures sufficient margin of safety against vehicle skidding off the roadway. The vehicle is considered stable as long as the applied force does not exceed the total balancing force. In other words, the vehicle is stable as long as the lateral friction demand is less than friction available. However, to ensure vehicle stability, the following issues have to be considered:

- Vehicle lateral Slip (offset): When a vehicle negotiates a horizontal curve, it starts to deviate from the path due to lateral forces. As the speed of vehicle increases, the direction of movement will not be the same as the direction of tires, and slip angles will present. That is, the vehicle starts to deviate from the planned path. Drivers adjust steering and vehicle speed in order to keep the vehicle moving on the path. For the vehicle still under driver control in this situation, the lateral slippage or offset from the path has to be kept small enough to ensure that the vehicle still travels within the lane width.

- Stability Factor: Vehicle stability has to be concerned with predicting whether a small perturbation from a basic motion will tend to die out or will tend to grow (Karnopp 2004). Within this context, the stability factor (understeer coefficient) can be used to evaluate vehicle steering situation as mentioned in Section 2.3.

\subsubsection{Vehicle Rollover Criterion}

The vehicle rollover criterion should ensure sufficient margin of safety against vehicle rollover. The Green Book does not adequately consider the rollover criterion (Awadallah 2005). It assumes that the design of horizontal curves based on driver comfort criterion provides sufficient margin of safety for vehicle stability and rollover criteria (A Policy 
2011). Also, vehicles (passenger cars) would likely skid off the roadway before rolling over (Lamm et al. 1999). However, Harwood and Mason (1994) have evaluated the margin of safety for vehicle skidding and rollover for horizontal curves designed according to AASHTO policy. The main findings for high speed horizontal curves are:

- Passenger cars with poor tires travelling on a poor wet pavement will skid off the road before rolling over.

- Most trucks with high centres of gravity (unstable trucks) moving on dry pavement will rollover before skidding off the road.

- Trucks travelling on a poor wet pavement will skid off the roadway at lower speed than rollover for horizontal curve with design speed up to $64 \mathrm{~km} / \mathrm{h}(40 \mathrm{mi} / \mathrm{h})$, while it will rollover at lower speed than skidding off the roadway for horizontal curves with design speed greater than $64 \mathrm{~km} / \mathrm{h}(40 \mathrm{mi} / \mathrm{h})$.

- The margin of safety against rollover provided in design Green Book appears to be adequate for trucks travelling lower than the design speed. However, most unstable trucks would rollover when travelling on freeway ramps at speed 8 to $16 \mathrm{~km} / \mathrm{h}$ higher than the design speed.

Note that the PMM was used in conducting the analysis to provide these findings. Thus, vehicle characteristics were ignored in the analysis. Also, the effect of grade was not considered. For transient situation, the LTR mentioned in Section 2.2.2 can be used to ensure that there is sufficient margin of safety against vehicle rollover.

\subsubsection{Sight Distance Criterion}

The sight distance is "the distance along a roadway throughout which an object of 
specified height is continuously visible to the driver" (A Policy 2011). The criterion used to measure sight distance on horizontal curves define that the driver's eye and the object to be seen are in the centre of the inside lane (TAC 2017). According to the TAC (2017) and Green Book (A Policy 2011), the height of driver's eye (for passenger car) of $1.08 \mathrm{~m}$ and the height of an object on the travel way of $0.6 \mathrm{~m}$ have been considered in determining the sight distance. The available sight distance for horizontal curves could be limited in some cases by lateral topographical or manmade obstructions such as cut slopes, trees, or buildings. Additional considerations should be taken when having a horizontal curve superimposed with vertical curve. In such case, the crest of the vertical curve would limit driver's sight line. Therefore, the obstruction for the sight distance should be checked considering the combined vertical and horizontal alignments (Sarhan and Hassan 2008).

The design of horizontal curves should provide an adequate sight distance for a driver to see ahead in order to be able to take evasive action in case of having a hazard in the path (Wolshon 2004). In other words, the sight distance criterion ensures that the available sight distance is greater than the required stopping sight distance. To attain this criterion when having an obstruction along the horizontal curve, a minimum clearance from the inside lane to the obstruction, such as road surface or physical features outside the travel way, as shown in Figure 2.12, should be ensured (TAC 2017).

Equation (2.29) is used to calculate the required lateral clearance in case of continuous or single obstruction (TAC 2017).

$$
\mathrm{LC}=R\left[1-\cos \frac{(90 S)}{\pi R}\right]
$$

Where $\mathrm{LC}=$ lateral clearance distance between the restrictive element and the centreline 
of the inside lane (m), $R=$ radius (m), and $S=$ stopping sight distance (m). Thus, the available sight distance $\left(S_{a v i}\right)$ for a specific curve radius and lateral clearance is:

$$
S_{a v i}=\frac{R}{28.65} \cos ^{-1}\left(1-\frac{\mathrm{LC}}{R}\right)
$$

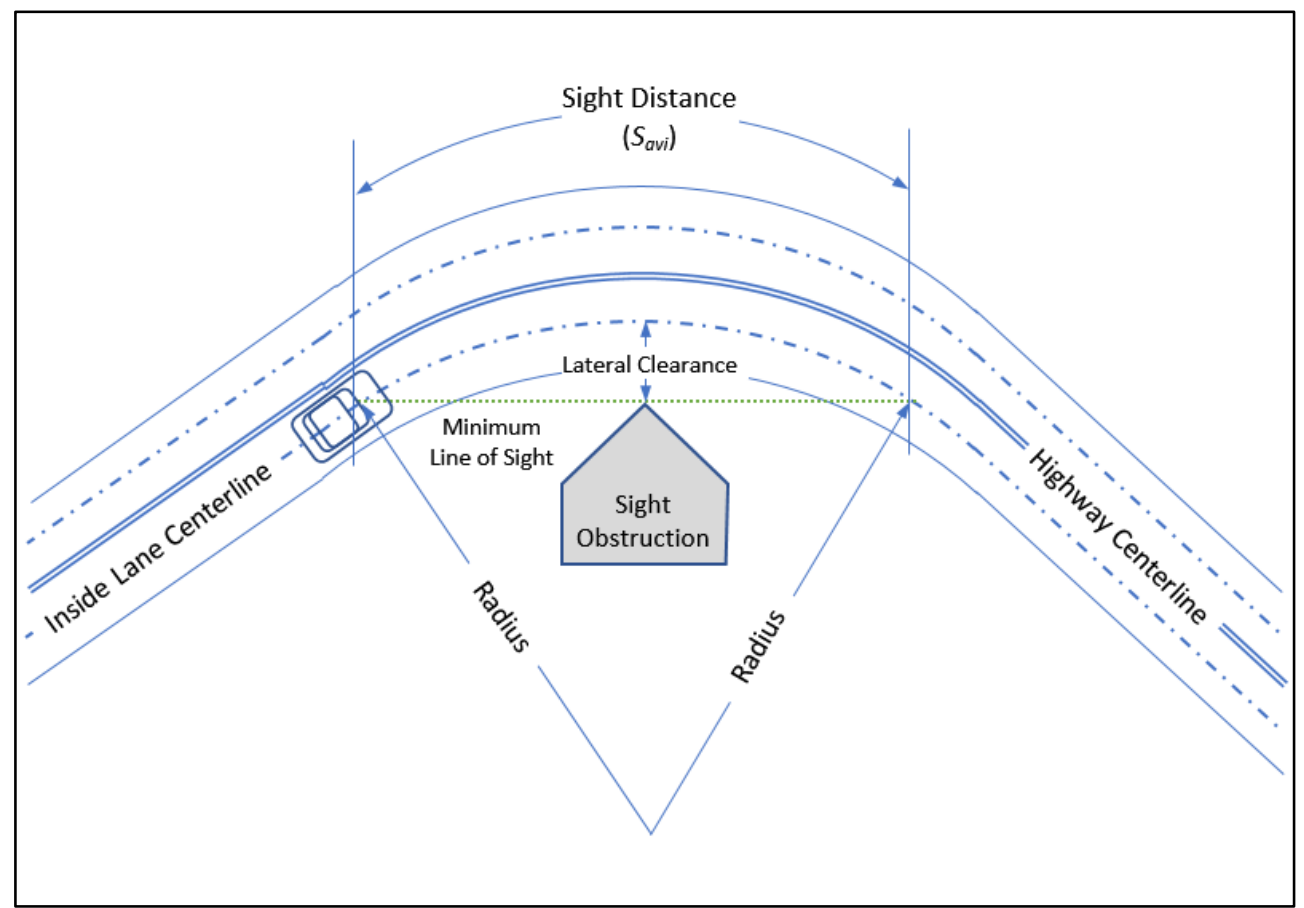

Figure 2.12: Minimum Clearance for Sight Distance Criterion-Reproduced from (Garber and Hoel 2009).

It should be noted that this method is precise only when having the vehicle and the sideway obstruction located on the same simple horizontal curve. In other words, the sight distance (length of curve) is greater than the sight line. The available sight distance would be limited by the roadway itself when the horizontal curve superimposed with a crest vertical curve or limited by an overpass when having the horizontal curve on a sag vertical curve. Equations (2.31) and (2.32) can be used to calculate the available sight distance when the sight distance is greater or less than the length of the crest vertical curve, 
respectively.

$$
\begin{aligned}
& L=\frac{A S_{a v i}^{2}}{100\left(\sqrt{2 h_{1}}+\sqrt{2 h_{2}}\right)^{2}} \\
& L=2 S_{a v i}-\frac{200\left(\sqrt{h_{1}}+\sqrt{h_{2}}\right)^{2}}{A}
\end{aligned}
$$

Where $L=$ length of crest vertical curve (m), $h_{1}=$ height of driver's eye above roadway surface (m), $h_{2}=$ height of object above roadway surface (m), and $A=$ algebraic difference in grades $(\%)$.

On the other hand, the sight distance is restricted at dark when having sag vertical curve. In this case, the available sight distance depends on the height of the headlights and its divergence degree that enables drivers to see ahead. Thus, the minimum length of sag curves is:

$$
\begin{aligned}
& L=\frac{A S_{a v i}^{2}}{200\left(h_{3}+S_{a v i} \tan \alpha\right)} \\
& L=\frac{200\left(h_{3}+S_{a v i} \tan \alpha\right)}{A}
\end{aligned}
$$

Where $h_{3}=$ headlight height (m), and $\alpha=$ upward divergence angle of the light beam $\left({ }^{\circ}\right)$

The stopping sight distance (SSD) according to AASHTO and TAC (1999) is calculated using Equation (2.35) and Equation (2.36), respectively.

$$
S S D=0.278 V t+\frac{V^{2}}{254\left(\frac{a}{9.81} \mp G\right)}
$$

Or, 


$$
S S D=0.278 V t+\frac{V^{2}}{254\left(f_{L} \mp G\right)}
$$

Where $t=$ perception reaction time $(\mathrm{s}), G=\operatorname{grade}($ decimal $), a=$ deceleration rate $\left(\mathrm{m} / \mathrm{s}^{2}\right)$, and $f_{L}=$ longitudinal friction factor. Therefore, for a simple horizontal curve, the available sight distance should always be greater than the stopping sight distance.

It is important to mention that the relationship between the deceleration rate and available longitudinal friction. When a driver decelerates at or below comfortable deceleration threshold $\left(3.4 \mathrm{~m} / \mathrm{s}^{2}\right)$ on a horizontal curve of low available longitudinal friction, the available friction would be the threshold that governed the relationship, and vice versa. For this reason, Equation 2.36 are used in the performance function of reliability analysis. Note that the comfortable deceleration threshold is the rate at which most drivers can stay within their lane and maintain steering control during the braking manoeuvre on wet surfaces (TAC 2017).

\subsection{Friction Phenomenon}

Examining the interrelationship between pavement surface and vehicle's tires is important to understand the dynamic stability of vehicles while negotiating a horizontal curve considering different driving scenarios such as accelerating and decelerating. In addition, the required sight distance and even the driving behaviour would be impacted by the condition of the pavement surface and the available friction factor.

Roadway pavements are designed to provide safe skid resistance, structural integrity, and satisfactory ride quality. In other words, pavement has to provide a good combination of adequate friction, low level of roughness and noise (Flintsch et al. 2003). Pavement surface characteristics such as frictional properties have a great influence on the frictional 
forces developed due to tire-pavement interface. These forces enable drivers to control their vehicles during negotiating curves or performing acceleration or deceleration actions. The following paragraphs focus on the tire-pavement frictional properties and the relationship between the longitudinal and lateral friction factors.

\subsubsection{Pavement Texture}

Surface texture can be defined as the "deviation of the pavement surface from a true planar surface" (ASTM 2012). Depending on the wavelength $(\lambda)$ and its amplitude $(A)$, pavement texture is divided into three levels of texture as follows (Hall et al. 2009) :

1. Micro-texture: the wavelength in this level is less than $0.5 \mathrm{~mm}$ and $A$ from 1 to $500 \mu \mathrm{m}$ in which surface roughness quality is influenced by the surface properties of the aggregate particles of the concrete paving or asphalt paving materials.

2. Macro-texture: the wavelength in this level ranges between $0.5 \mathrm{~mm}$ and $50 \mathrm{~mm}$ and $A$ between 0.1 and $20 \mathrm{~mm}$ in which the surface roughness quality is influenced by the mixture properties of the asphalt paving materials and the finishing method of the concrete paving materials.

3. Mega-texture: the wavelength in this level ranges between $50 \mathrm{~mm}$ and $500 \mathrm{~mm}$ and $A$ between 0.1 and $50 \mathrm{~mm}$ and usually defined by the distress and defects on the pavement surface.

The difference in wavelength has an impact on the interface between pavement surface and the tire. For instance, the frictional forces due to the interaction between the tire and road pavement surface occur within the micro and macro texture levels while the tire noise takes place in the macro and mega-texture levels as shown in Figure 2.13. 


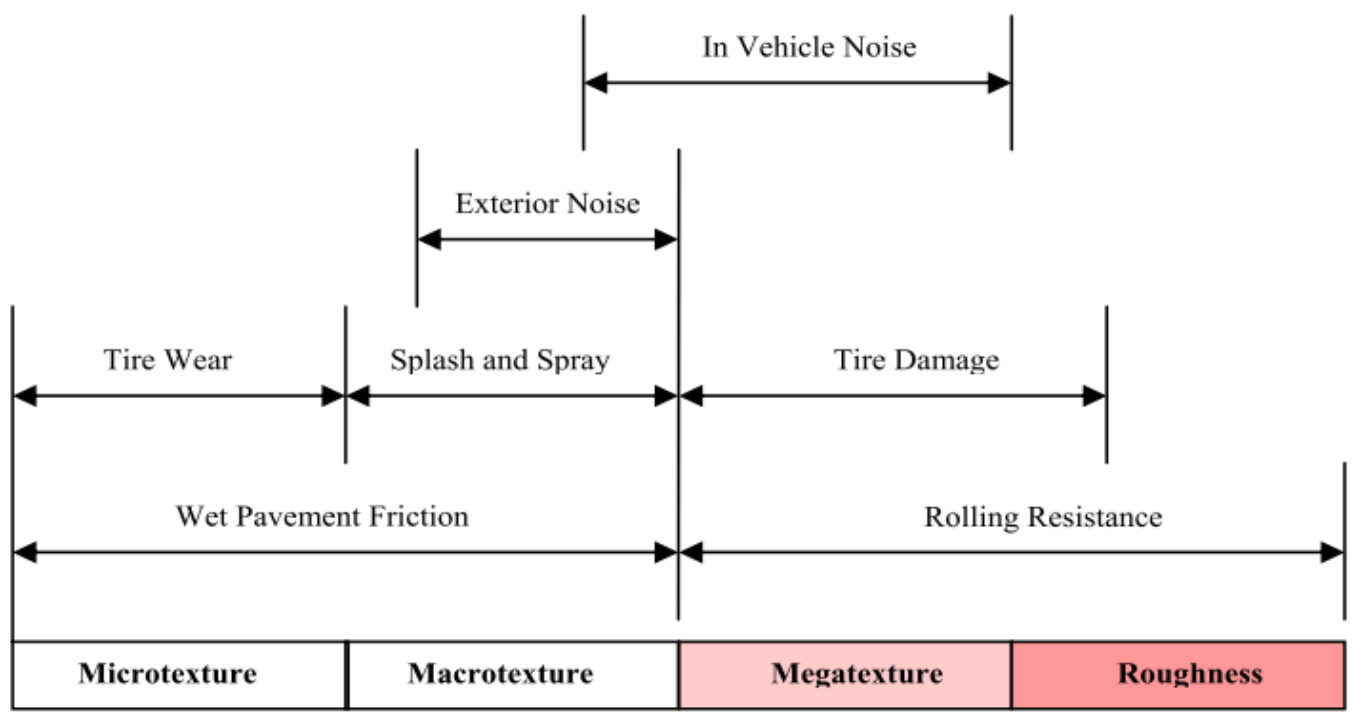

Figure 2.13: Texture wavelength influence on pavement-tire interaction (Shuo et al. 2003).

The micro-texture affects pavement friction at low speed because it has a great influence on the adhesion area between aggregate and tire rubber (Figure 2.14). On the other hand, macro-texture affects pavement friction at higher speed since it has a great effect on the hysteresis friction (Lu et al. 2008). It has been found that increasing macrotexture reduces total collisions on both dry and wet pavements (Henry 2000). However, attention has to be paid for other pavement characteristics when increasing surface asperity. For instance, increasing the texture of pavement surface (increasing amplitudes and wavelengths) may increase the noise, vibration, and level of discomfort, fuel consumption, and tire wear due to increasing interaction between tire and pavement surface (Henry 2000; Flintsch et al. 2003). 


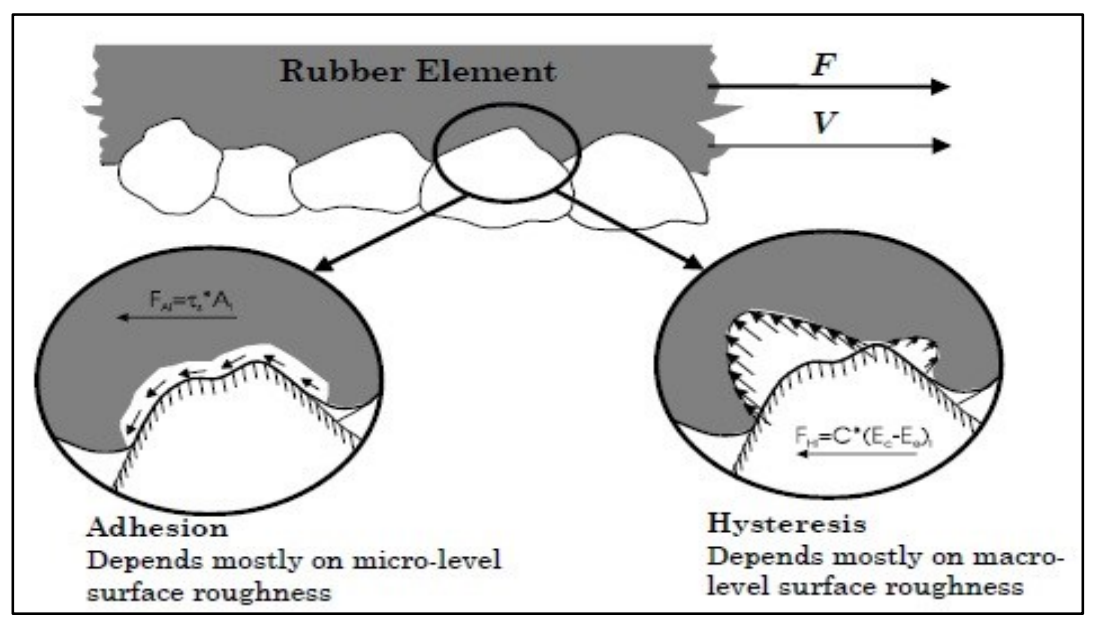

Figure 2.14: Tire-Pavement friction (Hall et al. 2009).

\subsubsection{Tire-Pavement Friction Coefficient and Force}

Pavement friction is "the force that resists the relative motion between vehicle's tire and the pavement surface" (Hall et al. 2009). As explained in previous section, the developed pavement frictional force due to the interaction between pavement and tire is the result of two main frictional forces; adhesion and hysteresis. The adhesion is the force that is developed due interface between tire rubber and pavement surface within the microtexture level and it is a function of the interface shear strength and contact area. The hysteresis force is a result of losing energy due to the total tire deformation which is caused by enveloping of the tire around pavement surface within the macro-texture level.

\subsubsection{Factors Affecting Available Friction}

Many factors are known in the literature to affect the available friction between vehicle's tires and road surface. These factors can be categorized into the following: 


\subsection{3.a. Pavement Characteristics}

Most of the tire-pavement interaction outputs such as friction, noise, splash and spray, rolling resistance, and tire wear are determined by pavement texture (Flintsch et al. 2003). As explained earlier, pavement surface texture levels, micro-texture and macro-texture, affect the frictional force developed at tire-pavement interaction. Their effect depends mainly on the relative speed between tire and pavement surface. While micro-texture predominates at lower speed, macro-texture has a significant influence at higher speeds (Hall et al. 2009).

\subsection{3.b. Tire Characteristics}

Besides supporting the weight of the vehicle, tires should provide sufficient traction for driving and braking, and provide adequate steering control (Wong 2008). The influence of rubber material properties of the pneumatic tires on friction is complex. The polymers and other components affect the frictional properties through influencing the physical characteristics such as hardness, damping loss, surface roughness and adhesion (Clark 1971). The friction and rolling resistance are affected by other tire conditions such as tire tread (type, pattern, and depth), tire condition, velocity, and tire design (thickness of sidewalls and treads). In addition, tire inflation pressure has a significant impact on pavement frictional force especially when having wet pavement condition and high speed (Gillespie 1992; Hall et al., 2009). Yet, there is no single tire that can produce high friction with all pavement surfaces under all conditions.

\subsection{3.c. Vehicle Operating Parameters}

Although speed of vehicles is the most crucial factor in designing horizontal curves, the effect of speed on the performance of the tire is not consistent enough to be generalized 
(Milliken and Milliken 1995). Generally, the friction coefficient has an inverse relation to tire speed. However, understanding the relationship between friction coefficient and speed needs to consider other properties such as the slip ratio, the slip angle, and the relationship between lateral and longitudinal friction.

- Slip Ratio: There are several definitions for slip ratio used worldwide. The slip ratio or longitudinal slip is defined by the Society of Automotive Engineers (SAE) as "the ratio of the longitudinal slip velocity to the spin velocity of the straight freerolling tire", which is expressed by Equation (2.37) (Milliken and Milliken 1995).

$$
S=\frac{r \omega}{V \cos \alpha}-1
$$

Where $S=$ Slip ratio, $r=$ effective rolling radius of the free-rolling tire, $\omega=$ the angular speed of the tire, $V=$ the linear speed of the tire centre, and $\alpha=$ slip angle. Therefore, the slip ratio equals zero when having free rolling tire, -1 when having locked wheel, and greater than +1 when spinning.

The longitudinal friction force equals zero when having a free-rolling wheel then it escalates quickly as the slip ratio increases until the peak friction value is reached as shown in Figure 2.15. Then, for braking state, the friction force starts to decrease with further increases in the slip ratio until the condition of a "locked wheel" is reached when the slip ratio is -1 . The peak value of the longitudinal friction force generally occurs when the slip ratio is approximately $20 \%$ (Bosch 2006). 

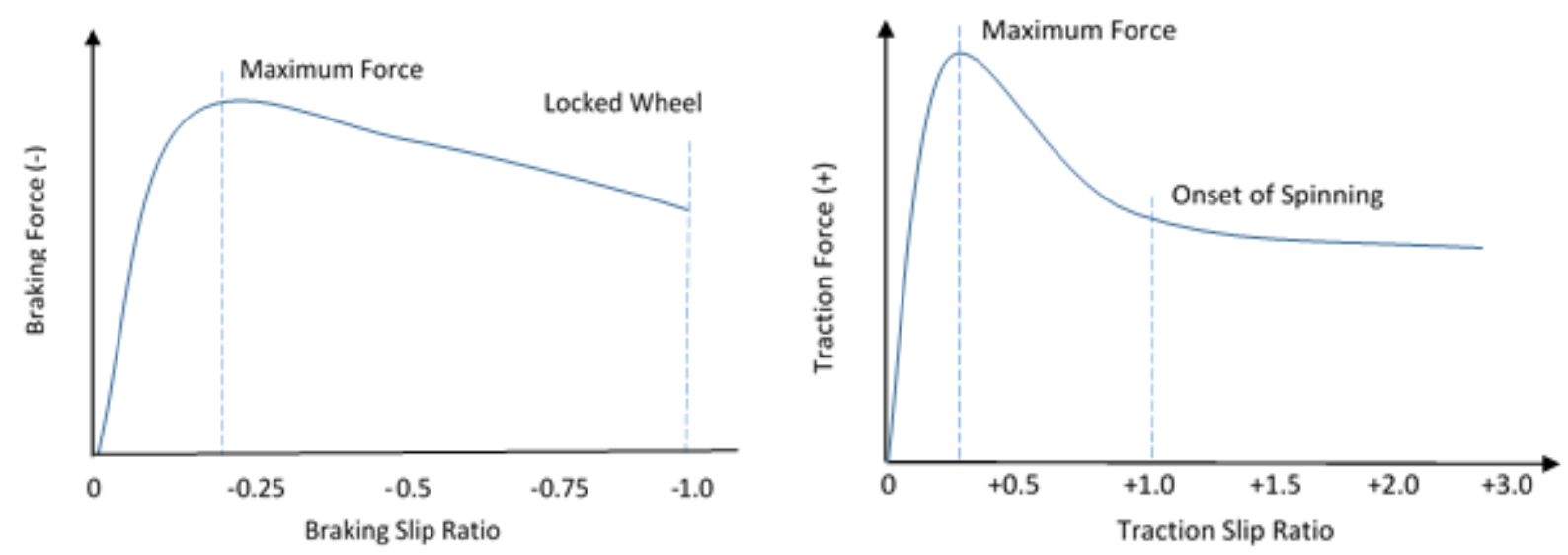

Figure 2.15: Friction Force and Slip Ratio for Traction and Braking States Based on ASE Definition-Reproduced from (Milliken and Milliken 1995).

- Slip Angle: as defined in Section 2.2.1.b, the slip angle exists when a lateral force is applied to the wheel causing a lateral reaction force to develop at the contact patch between the tire and pavement. The developed force causes the wheel to deviate from its pointed direction. Thus, the wheel acquires lateral velocity in addition to the forward velocity and the total velocity vector of the wheel will travel in direction other than the direction of the wheel (Karnopp 2004).

The relationship between the lateral friction and slip angle is almost linear for small values of lateral force and slip angle as shown in Figure 2.6. The relationship becomes nonlinear for relatively large slip angles. The stability analysis of vehicle's pneumatic tires typically considers the linear relationship between the slip angle and the lateral force (slip angles up to $10^{\circ}$ ) (Karnopp 2004; Masato 2009).

- Interaction between Longitudinal and Lateral Friction: In addition to the factors mentioned earlier, the available lateral friction depends on the interaction between lateral and longitudinal friction. The interaction is more significant when 
a vehicle accelerates or decelerates on a curvilinear path. In this case, the maximum available lateral friction depends on the utilized friction in the longitudinal direction. The approximate relationship between these two perpendicular forces can be represented mathematically as a circle or ellipse. The circular relationship is used to represent the relationship between longitudinal and lateral friction when there is no direction preference assumed (Taborek 1957). In this case, the relationship can be represented as follows (Lamm et al. 1999):

$f_{\text {Total }}^{2}=f_{T}^{2}+f_{R}^{2}$

Where $f_{\text {Total }}=$ total available friction, $f_{T}=$ available longitudinal friction, and $f_{R}=$ available lateral friction.

The vehicle will be in an unstable condition if the total friction available is less than the total friction demand (Lamm et al. 1999). This approach was followed by Olson et al. (1984) who used the force equilibrium relationship between the available total lateral and longitudinal friction to calculate the braking distance on a curvature. The utilized friction in the lateral direction reduces the available longitudinal friction for stopping (Olson et al. 1984).

$$
f_{T}^{2}=f_{\text {Total }}^{2}-\left(\frac{V^{2}}{127 R}-e\right)^{2}
$$

Where $f_{T}=$ available longitudinal friction, $f_{\text {Total }}=$ available total friction, $V=$ speed of vehicle $(\mathrm{km} / \mathrm{h}), R=$ radius $(\mathrm{m})$, and $e=$ superelevation rate.

Similarly, if the vehicle movement requires a specific longitudinal friction for braking or acceleration, the maximum available lateral friction can be obtained by subtracting the longitudinal friction consumed from the total available friction. 
The interaction between lateral and longitudinal friction was the basis of the recommendation by Lamm et al. (1999) to set a maximum value of the permissible lateral friction as shown in the following equation (Lamm et al. 1999).

$$
f_{R, p e r m}=n 0.925 f_{T, p e r m}
$$

Where $f_{T, \text { perm }}$ and $f_{R, \text { perm }}=$ maximum permissible longitudinal and lateral friction coefficients, respectively, 0.925 = reduction factor for an assumed elliptical shape for the relationship between longitudinal and lateral friction, and $n=$ utilization ratio of lateral friction ranging from 0.4 to 0.6 .

Based on data obtained by Japan Automobile Research Institute (JARI) from experiments on a small passenger car tire at a load of $400 \mathrm{~kg}$ and speed of $20 \mathrm{~km} / \mathrm{h}$, Milliken and Milliken (1995) developed a single diagram to illustrate the relationship between longitudinal and lateral friction demand considering the effect of the slip angle and slip ratio as shown in Figure 2.16. The relationship between longitudinal and lateral friction was found to take an elliptic shape, whose shape depends on the magnitude of the slip angle. In other words, the figure illustrates two points; the maximum available friction factor (supply), which is represented by the outer semi-circle or ellipse shape, and the demand friction factor for various slip ratios and slip angles for the speed and normal force mentioned in the figure.

The figure shows the significant effect of the slip angle on the lateral force. In braking status for instance, at speed of $20 \mathrm{~km} / \mathrm{h}$, normal load of $3900 \mathrm{~N}$, and longitudinal force of $2,000 \mathrm{~N}$, the lateral force is $900 \mathrm{~N}$ at a slip angle of $1^{\circ}$. For the same conditions, the lateral force would increase to $3,500 \mathrm{~N}$ at a slip angle of $4^{\circ}$. The big difference between the two 
values of the lateral force demand indicates that the lateral force (lateral friction demand) increases with steering/slip angle with all other conditions unchanged. In other words, designing horizontal curves with smaller radius would result in larger steering and slip angles. Accordingly, the lateral friction demand increases and, in turn, reduces the available longitudinal friction.

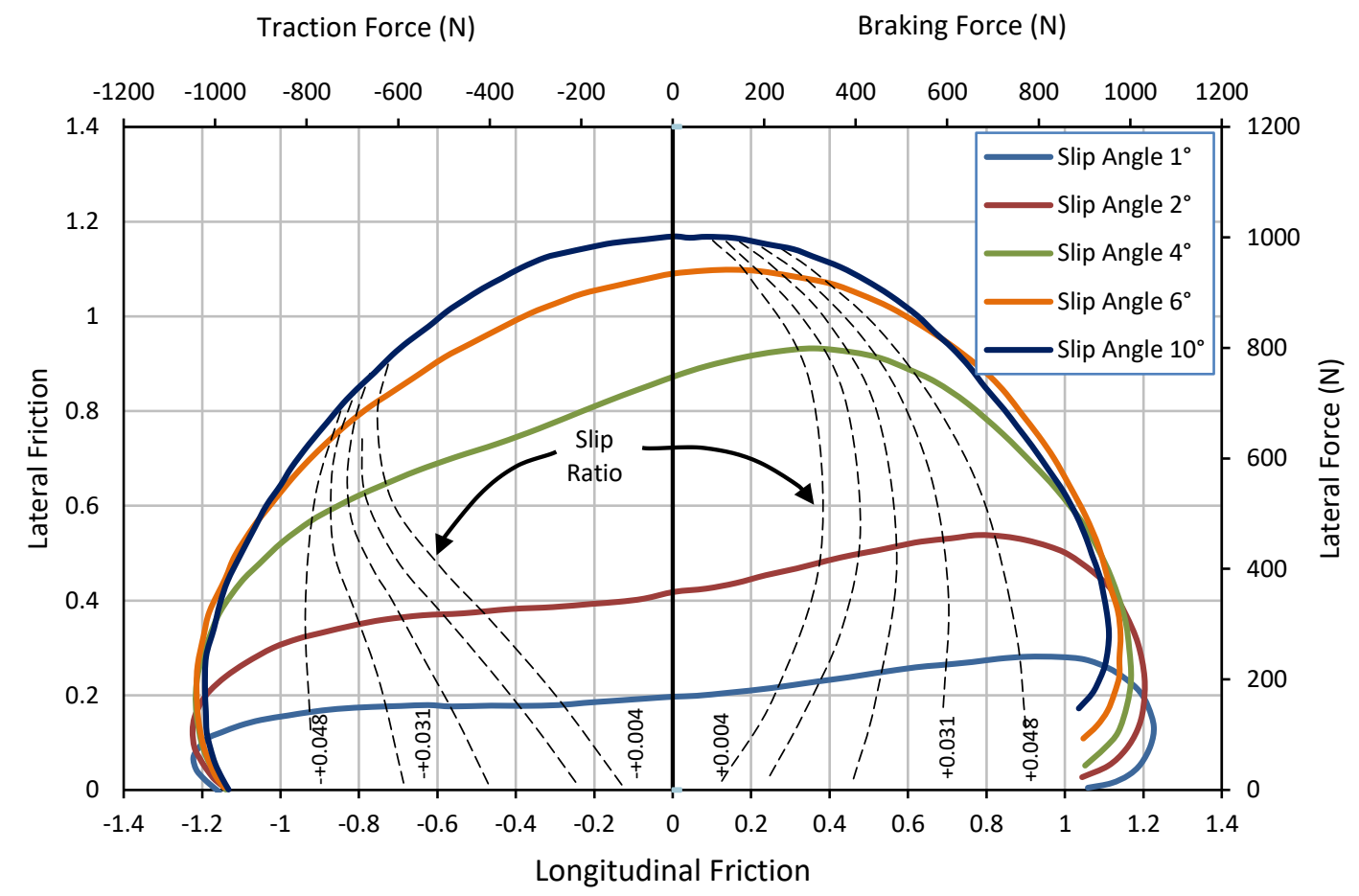

Figure 2.16: Relationship between Longitudinal and Lateral Forces (Reproduced Milliken and Milliken 1995).

\subsection{3.d. Environment}

The weather conditions would impact pavement surface conditions and, in turn, affect the available friction factor. The temperature has a significant impact on skid resistance since the tire is made of visco-elastic materials. The existence of water, snow, ice, and other contaminants (such as oil dripping, layer of dust, or organic materials) may reduce friction to very hazardous levels (Hall et al. 2009; A Policy 2011). In wet pavement conditions, for 
instance, water may be present in the contact area between the tire and the pavement and would work as a lubricant. As a result, the friction force would decrease because the adhesion force component would diminish. Furthermore, the hydroplaning phenomenon could occur if the tire and pavement surface textures are unable to provide an adequate capacity to channel and squeeze the water (Shuo et al. 2003). The highest values observed of friction are on a clean and dry surface and the lowest when having icy conditions.

\subsubsection{Available Friction}

Considerable research has been conducted to study pavement friction. However, studies that focused on determining available lateral friction were relatively scarce compared to longitudinal friction. In most of these studies, pavement friction has been assumed to be random and normally distributed. However, Himes (2013) confirmed this assumption.

Bonneson (2000) reported that the dynamic friction factors provided in the Green Book (A Policy 2011) are applicable to a locked-wheel skid on a worst-case of poor, wet pavement and worn tires. The dynamic friction for a locked-wheel skid represents a maximum slip (or "dynamic") condition as the tire is moving relative to the pavement surface. Table 2-1 shows the maximum longitudinal and lateral friction supply for skid failure for each speed category for passenger car and truck. As shown in the table, the maximum design longitudinal friction factor was taken from the Green Book version 1994. Equation 2.41 provided by Olson et al. (1984) was used to calculate the maximum (peak) friction supply for skid failure for passenger car (PC) vehicles.

$$
f_{x, \max -P C}=0.2+1.12 \times f_{x, \text { skidding }}
$$


Where $f_{x, \max -P C}=$ maximum (peak) longitudinal available friction for $\mathrm{PC}$, and $f_{x, \text { skidding }}=$ skidding friction factor.

Equation 2.42 shows the peak friction supply for heavy trucks $\left(f_{x, \max -T}\right)$.

$$
f_{x, \max -T}=1.45 \times f_{x, \text { skidding }}
$$

Table 2-1: Lateral Friction for Slide Failure (Bonneson, 2000).

\begin{tabular}{|c|c|c|c|c|}
\hline \hline \multirow{2}{*}{$\begin{array}{c}\text { Design } \\
\text { Speed } \\
(\mathrm{km} / \mathrm{h})\end{array}$} & \multirow{2}{*}{$\begin{array}{c}\text { Max. Design } \\
\text { Longitudinal }\end{array}$} & \multirow{2}{*}{$\begin{array}{c}\text { Max. } \\
\text { Friction Factor }\end{array}$} & $\begin{array}{c}\text { Fondinal } \\
\text { Friction Supply }\end{array}$ & \multicolumn{2}{|c|}{$\begin{array}{c}\text { Maximum Lateral Friction Supply } \\
\text { for Slide Failure }\end{array}$} \\
\cline { 4 - 5 } & & 0.53 Said Failure & Passenger Car & Heavy Truck \\
\hline 30 & 0.40 & 0.53 & 0.79 & 0.54 \\
40 & 0.38 & 0.48 & 0.74 & 0.49 \\
50 & 0.35 & 0.44 & 0.69 & 0.45 \\
60 & 0.33 & 0.4 & 0.65 & 0.41 \\
70 & 0.31 & 0.36 & 0.60 & 0.37 \\
80 & 0.30 & 0.34 & 0.58 & 0.35 \\
90 & 0.30 & 0.33 & 0.57 & 0.33 \\
100 & 0.29 & 0.31 & 0.55 & 0.31 \\
110 & 0.28 & 0.30 & 0.54 & 0.30 \\
120 & 0.28 & 0.29 & 0.52 & 0.29 \\
\hline \hline
\end{tabular}

It should be noted that Olson et al. also provided equations to estimate sliding friction for PC and heavy trucks using skid number as shown in the following equations.

$$
\begin{aligned}
& f_{S-P C}=1.2 \times S N_{V} \\
& f_{S-T}=0.84 \times S N_{V}
\end{aligned}
$$

Where $f_{S-P C}$ and $f_{S-T}=$ sliding friction factor for PC and heavy truck tires, respectively, and $S N_{V}=$ ASTM skid number at speed $V(\mathrm{mi} / \mathrm{h})$.

The National Highway Traffic Safety Administration's (NHTSA) Vehicle Research and Test Centre conducted a preliminary investigation into the effects of tire inflation 
pressure on the peak and slide coefficients in wet and dry conditions (MacIsaac and Riley 2002). The tests were conducted on a section of rural state route that experiences moderate to heavy traffic volume on pavement of 7-year-old. The friction factor values for different speed and pavement conditions are shown in Table 2-2.

Table 2-2: Wet and Dry Friction Factor (MacIsaac and Riley 2002).

\begin{tabular}{|c|c|c|c|c|c|c|c|}
\hline \multicolumn{2}{|c|}{$\begin{array}{c}\text { Tire / Inflation } \\
\text { Pressure Tire }\end{array}$} & \multirow{2}{*}{$\begin{array}{c}\text { SRTT* } \\
35 \text { psi } \\
(241 \\
\mathrm{kPa})\end{array}$} & \multirow{2}{*}{$\begin{array}{l}\text { SRTT } \\
17 \mathrm{psi} \\
(117 \\
\mathrm{kPa})\end{array}$} & \multirow{2}{*}{$\begin{array}{c}\text { EAGLE } \\
35 \text { psi } \\
(241 \\
\mathrm{kPa})\end{array}$} & \multirow{2}{*}{$\begin{array}{c}\text { EAGLE } \\
24 \mathrm{psi} \\
(165 \mathrm{kPa})\end{array}$} & \multirow{2}{*}{$\begin{array}{c}\text { EAGLE } \\
17 \mathrm{psi} \\
(117 \mathrm{kPa})\end{array}$} & \multirow[b]{2}{*}{ Average } \\
\hline $\begin{array}{l}\text { Speed } \\
(\mathrm{mi} / \mathrm{h})\end{array}$ & $\begin{array}{c}\text { Speed } \\
(\mathrm{km} / \mathrm{h})\end{array}$ & & & & & & \\
\hline \multicolumn{8}{|c|}{ Wet Slide Coefficients of Friction } \\
\hline 10 & 16 & 0.72 & 0.73 & 0.68 & 0.69 & 0.70 & 0.704 \\
\hline 20 & 32 & 0.65 & 0.67 & 0.58 & 0.65 & 0.62 & 0.634 \\
\hline 30 & 48 & 0.60 & 0.61 & 0.56 & 0.57 & 0.55 & 0.578 \\
\hline 40 & 64 & 0.57 & 0.55 & 0.51 & 0.53 & 0.50 & 0.532 \\
\hline 50 & 80 & 0.51 & 0.50 & 0.46 & 0.48 & 0.48 & 0.486 \\
\hline 60 & 97 & 0.49 & 0.44 & 0.46 & 0.41 & 0.41 & 0.442 \\
\hline 70 & 113 & 0.45 & 0.45 & 0.40 & 0.36 & 0.37 & 0.406 \\
\hline \multicolumn{8}{|c|}{ Dry Slide Coefficients of Friction } \\
\hline 10 & 16 & 0.83 & - & - & 0.79 & 0.77 & 0.797 \\
\hline 20 & 32 & 0.80 & - & - & 0.73 & 0.73 & 0.753 \\
\hline 30 & 48 & 0.78 & - & - & 0.76 & 0.75 & 0.763 \\
\hline 40 & 64 & 0.78 & - & - & 0.81 & 0.80 & 0.797 \\
\hline 50 & 80 & 0.79 & - & - & - & 0.87 & 0.830 \\
\hline 60 & 97 & 0.80 & - & - & - & 0.85 & 0.825 \\
\hline
\end{tabular}

* = Standard Reference Test Tires

Harwood and Mason (1994) evaluated margin of safety against skidding and rolling over by extracting the available lateral friction data for passenger car and truck in wet and dry from tire-pavement friction provided in the Green Book (version 1994). The available tire-pavement friction under wet pavement conditions for PC was calculated by multiplying the locked-wheel braking coefficient by 1.45 . The available friction for truck was assumed to be $70 \%$ of the PC. The extracted data are shown in Table $2-3$. 
Table 2-3: Lateral Friction Available for Passenger Car and Truck (Harwood and Mason 1994).

\begin{tabular}{|c|c|c|c|c|c|}
\hline \hline \multirow{2}{*}{ Speed } & \multicolumn{4}{|c|}{ Available Lateral Friction } \\
\cline { 3 - 6 } \multicolumn{2}{|c|}{} & \multicolumn{2}{c|}{ Passenger Car } & \multicolumn{2}{c|}{ Truck } \\
\hline $\mathrm{Mi} / \mathrm{h}$ & $\mathrm{km} / \mathrm{h}$ & Wet & Dry & Wet & Dry \\
\hline 20 & 32 & 0.58 & 0.94 & 0.41 & 0.66 \\
30 & 48 & 0.51 & 0.94 & 0.36 & 0.66 \\
40 & 64 & 0.46 & 0.94 & 0.32 & 0.66 \\
50 & 80 & 0.44 & 0.94 & 0.3 & 0.66 \\
60 & 97 & 0.42 & 0.94 & 0.29 & 0.66 \\
70 & 113 & 0.41 & 0.94 & 0.28 & 0.66 \\
\hline \hline
\end{tabular}

Himes (2013) conducted analysis to determine pavement-tire friction for PC and heavy truck tires. Friction data were collected from two projects that used dynamic friction tester and circular texture meter. The skid number for wet pavement was determined using the following equations:

$$
\begin{aligned}
& S_{p}=14.2+89.7 \mathrm{MPD} \\
& F_{60}=0.081+0.732 \times D F T_{20} \times e^{20-60 / S_{p}} \\
& S N_{v}=\frac{F_{60}+0.023-0.098 \times M P D}{0.607 \times e^{v-60 / S_{p}}}
\end{aligned}
$$

Where $S_{p}=$ the slip speed $(\mathrm{km} / \mathrm{h}), \mathrm{MPD}=$ mean profile depth $(\mathrm{mm}), F_{60}=$ the adjusted friction measurement at $60 \mathrm{~km} / \mathrm{h}, D F T_{20}=$ dynamic friction measurement at speed $20 \mathrm{~km} / \mathrm{h}$, and $S N_{v}=$ the speed for which the skid number is calculated $(\mathrm{km} / \mathrm{h})$.

Considering equation (2.41) to estimate longitudinal peak friction and the reduction factor $(0.925)$ presented by Lamm et al. (1999) to estimate the lateral friction values, Himes (2013) provided friction data distribution shown in Table 2-4. The friction data for dry pavement were extracted from study conducted by MacIsaac and Riley (2002). 
Table 2-4. Lateral Friction Factor for PC for Different Curve Speeds (Himes 2013).

\begin{tabular}{|c|c|c|c|c|c|c|}
\hline \hline \multirow{2}{*}{$\begin{array}{c}\text { Speed } \\
(\mathrm{mi} / \mathrm{h})\end{array}$} & \multicolumn{4}{|c|}{ Wet Pavement } & \multicolumn{2}{c|}{ Dry Pavement } \\
\cline { 2 - 6 } & $\begin{array}{c}\text { Longitudinal } \\
\text { Friction }\end{array}$ & $\begin{array}{c}\text { Standard } \\
\text { Deviation }\end{array}$ & $\begin{array}{c}\text { Mean } \\
\text { Lateral } \\
\text { Friction }\end{array}$ & $\begin{array}{c}\text { Standard } \\
\text { Deviation }\end{array}$ & $\begin{array}{c}\text { Peak } \\
\text { Friction }\end{array}$ & $\begin{array}{c}\text { Lateral } \\
\text { Friction }\end{array}$ \\
\hline 10 & 0.694 & 0.114 & 0.663 & 0.105 & 1.09 & 0.768 \\
15 & 0.648 & 0.117 & 0.597 & 0.108 & 1.088 & 0.764 \\
20 & 0.584 & 0.097 & 0.54 & 0.09 & 1.085 & 0.759 \\
25 & 0.526 & 0.084 & 0.487 & 0.077 & 1.073 & 0.754 \\
30 & 0.475 & 0.071 & 0.439 & 0.066 & 1.06 & 0.749 \\
35 & 0.429 & 0.063 & 0.397 & 0.058 & 1.048 & 0.745 \\
40 & 0.388 & 0.056 & 0.359 & 0.052 & 1.035 & 0.74 \\
45 & 0.351 & 0.052 & 0.324 & 0.048 & 1.028 & 0.736 \\
50 & 0.318 & 0.05 & 0.294 & 0.046 & 1.02 & 0.731 \\
55 & 0.289 & 0.048 & 0.268 & 0.044 & 1.003 & 0.727 \\
60 & 0.261 & 0.047 & 0.241 & 0.043 & 0.985 & 0.722 \\
65 & 0.237 & 0.047 & 0.219 & 0.043 & 0.968 & 0.717 \\
70 & 0.215 & 0.046 & 0.199 & 0.043 & 0.95 & 0.712 \\
\hline \hline
\end{tabular}

Friction factors provided by Himes (2013) for heavy trucks are shown in Table 2-5.

Table 2-5. Lateral Friction Factor for Truck for Different Curve Speeds (Himes 2013).

\begin{tabular}{|c|c|c|c|c|}
\hline \hline $\begin{array}{c}\text { Speed } \\
(\mathrm{mi} / \mathrm{h})\end{array}$ & $\begin{array}{c}\text { Mean } \\
\text { Longitudinal } \\
\text { Friction }\end{array}$ & $\begin{array}{c}\text { Standard } \\
\text { Deviation }\end{array}$ & $\begin{array}{c}\text { Mean Lateral } \\
\text { Friction }\end{array}$ & $\begin{array}{c}\text { Standard } \\
\text { Deviation }\end{array}$ \\
\hline 10 & 0.486 & 0.107 & 0.464 & 0.094 \\
15 & 0.454 & 0.110 & 0.418 & 0.097 \\
20 & 0.409 & 0.090 & 0.378 & 0.081 \\
25 & 0.368 & 0.078 & 0.341 & 0.069 \\
30 & 0.333 & 0.066 & 0.307 & 0.059 \\
35 & 0.300 & 0.059 & 0.278 & 0.052 \\
40 & 0.272 & 0.052 & 0.251 & 0.047 \\
45 & 0.246 & 0.049 & 0.227 & 0.043 \\
50 & 0.223 & 0.047 & 0.206 & 0.041 \\
55 & 0.202 & 0.045 & 0.188 & 0.040 \\
60 & 0.183 & 0.044 & 0.169 & 0.039 \\
65 & 0.166 & 0.044 & 0.153 & 0.038 \\
70 & 0.151 & 0.043 & 0.139 & 0.038 \\
\hline \hline
\end{tabular}


Due to lack of distribution data for friction for dry pavement, Himes (2013) assumed that the coefficient of variation (COV) values of dry-pavement friction are the same of those of wet-pavement. Thus, the mean values of friction for dry pavement mentioned in Table 2-4 were used to calculate the standard deviation of the dry pavement for different mean speed.

The lateral friction supply data for other than dry and wet pavement surfaces is extremely rare and sparse (Morrall and Talarico 1994). Most of the studies reported that available friction for icy pavement conditions is between 0.00 and 0.15 . The range for available friction for different pavement conditions are shown in Table 2-6 (Al-Qadi et al. 2002; Fu et al 2016). This table provides classification for pavement surface in three countries (Sweden, Finland, and Japan) based on the available friction factor. These values usually used by transportation agency for comprehensive winter maintenance program.

Table 2-6: Friction Factor and Classification of Road Surface (Al-Qadi et al. 2002).

\begin{tabular}{|l|c|l|l|l|c|}
\hline \multicolumn{2}{|c|}{ Sweden } & \multicolumn{2}{c|}{ Finland } & \multicolumn{2}{c|}{ Japan } \\
\hline $\begin{array}{c}\text { Road } \\
\text { Surface }\end{array}$ & $\begin{array}{c}\text { Friction } \\
\text { Factor }\end{array}$ & Road Surface & $\begin{array}{c}\text { Friction } \\
\text { Factor }\end{array}$ & \multicolumn{1}{c|}{ Road Surface } & $\begin{array}{c}\text { Friction } \\
\text { Factor }\end{array}$ \\
\hline Good & $\geq 0.40$ & Bare and dry & $0.45-1.00$ & Dry, wet & $0.45 \sim$ \\
\hline $\begin{array}{l}\text { Medium } \\
\text { to good }\end{array}$ & $0.36-0.39$ & Bare and wet & $0.3-0.44$ & $\begin{array}{l}\text { Slush, Granular snow on } \\
\text { ice crust, Powder snow }\end{array}$ & $0.25-0.35$ \\
\hline Medium & $0.30-0.35$ & $\begin{array}{l}\text { Packed ice } \\
\text { and snow }\end{array}$ & $0.25-0.29$ & $\begin{array}{l}\text { Compacted snow, } \\
\text { Granular snow on ice crust }\end{array}$ & $0.20-0.30$ \\
\hline $\begin{array}{l}\text { Medium } \\
\text { to poor }\end{array}$ & $0.26-0.29$ & $\begin{array}{l}\text { Tightly } \\
\text { packed snow }\end{array}$ & $0.20-0.24$ & Ice film & $0.15-0.30$ \\
\hline Poor & $\leq 0.25$ & icy & $0.15-0.19$ & $\begin{array}{l}\text { Powder snow on ice crust, } \\
\text { ice crust }\end{array}$ & $0.15-0.20$ \\
\cline { 5 - 6 } & & Wet ice & $0.00-0.14$ & $\begin{array}{l}\text { Very slippery compacted } \\
\text { snow }\end{array}$ & $\sim 0.20$ \\
\cline { 5 - 7 } & & $\begin{array}{l}\text { Very slippery ice crust, ice } \\
\text { film }\end{array}$ & $\sim 0.15$ \\
\hline \hline
\end{tabular}




\subsection{Reliability Concept}

All activities involve many variables and have, in one way or another, a degree of risk. Dealing with uncertainties and reducing the probability of failure can be considered as the main concern for both, the user and the producer. This situation exposes the need for a system, technique, or a method capable of dealing with uncertainties, quantifying risks, and predicting future performance for a product or a system. From this point, reliability concept has emerged and grown rapidly during the last century. The International Standard Organization (ISO 8402) defined reliability as "the ability of an item to perform a required function, under given environmental and operational conditions and for a stated period of time" (Marvin and Arnljot 2004). The definition presents a probabilistic approach to deal with uncertainty rather than using the traditional deterministic approach, which relies on the near worst-case scenario of a problem. In other words, relying only on a factor of safety might lead to overdesign in some cases and not necessarily result in higher level of safety or reliability (Singh et al. 2007). In addition, the reliability concept affects both safety and productivity with many possible applications of this concept. Therefore, it has been employed in many engineering disciplines. However, this concept in transportation has not got much attention compared to other engineering fields. The geometric design guides, for instance, still adopt the deterministic approach in the design procedure. Table 2-7 shows the main characteristics of the deterministic and probabilistic approaches.

In transportation engineering literature, the terms probability of failure (POF), probability of non-compliance (PNC), or probability of hazard ( $\mathrm{POH})$ are used to represent the probability of demand (apply) exceeding supply or capacity (available) of the system. Note that the consequence of failing to comply to some geometric design criteria is not 
necessarily end up with a collision.

\subsubsection{Reliability Performance Functions}

Table 2-7: Deterministic and Probabilistic Approaches Characteristics (Based on Singh et al. 2007; Richl 2003).

\begin{tabular}{|l|l|}
\hline \multicolumn{1}{|c|}{ Deterministic approach } & \multicolumn{1}{|c|}{ Probabilistic approach } \\
\hline $\begin{array}{l}\text { Looks for the near worst scenario and } \\
\text { extreme values and build the design to cope } \\
\text { with them. }\end{array}$ & $\begin{array}{l}\text { Uses statistical methods to build up a } \\
\text { reliable design. }\end{array}$ \\
\hline $\begin{array}{l}\text { Provides a factor of safety to cope with the } \\
\text { effects of unknown. }\end{array}$ & $\begin{array}{l}\text { Uses statistical methods to determine the } \\
\text { probability and magnitude of related } \\
\text { variables. }\end{array}$ \\
\hline $\begin{array}{l}\text { The weakness of this approach is that the } \\
\text { factor of safety could be too large or in some } \\
\text { cases too small. }\end{array}$ & $\begin{array}{l}\text { Would result in cost saving and efficient use } \\
\text { of resources. }\end{array}$ \\
\hline $\begin{array}{l}\text { Would be insensitive to some operational } \\
\text { factors such as volume and collision rate. }\end{array}$ & $\begin{array}{l}\text { Safety would be presented in terms of } \\
\text { probability of failure or reliability index. }\end{array}$ \\
\hline Safety is provided in terms of safety factor. & Each variable is presented in a distribution \\
\hline Each variable has only one value.
\end{tabular}

Reliability can be described as a complement of failure as shown in Equation (2.48). The term probability of failure (POF) will be used in the following paragraphs to represent all types of failure modes unless there is a need to use another technical term.

$$
R=1-P_{f}
$$

Where $R=$ Reliability, $P_{f}=$ Probability of failure/noncompliance/hazards. 
The performance function $(Z)$ of any system can be formulated based on the difference between demand $(D)$ and supply $(S)$ as follows (Felipe and Navin 1998):

$$
Z=S-D
$$

Based on this equation, the system is unable to provide the demand when $(Z=S-D<$ 0 ). The safe and reliable performance of the system when the supply exceeds the demand $(S-D>0)$. The limit state of the function when the demand equals the supply $(S-D=$ $0)$.

Considering Equation (2.49), the probability of failure is:

$$
P_{f}=P(S<D)=\int_{-\infty}^{\infty} F_{S}(x) f_{D}(x) d x
$$

Where $P_{f}=$ probability of system failure, $F_{S}=$ cumulative probability distribution function for the supply, and $f_{D}=$ probability density function for the demand.

\subsubsection{Factor of Safety}

In the deterministic approach, the safety of the design is ensured by requiring the supply $(S)$ be greater than the demand $(D)$ with a specific margin of safety (Haldar and Sankaran 2000). Where, the margin of safety $(M)$ is the difference between the expected value of the supply and that of demand as shown in Equation (2.51).

$$
M=E(S)-E(D)
$$

The Factor of Safety (FOS) is a nominal supply divided by a nominal demand.

$$
F O S=\frac{S_{N}}{D_{N}}
$$

Where $M=$ margin of safety, $E(S)=$ expected value of supply, $E(D)=$ expected value of demand, $S_{N}, D_{N}=$ nominal supply and demand, respectively. 
The value of the nominal supply $\left(S_{N}\right)$ is usually conservative and representing one, two, or three standard deviations below the mean value as shown in Figure 2.17. On the other hand, the nominal value for the demand $\left(D_{N}\right)$ is some standard deviations above the mean value. The shaded region in the figure (the overlap area between the PDF curves of the Supply and Demand) provides a "qualitative measure" of the probability of failure (Haldar and Sankaran 2000). The POF decreases when overlapped area decreases.
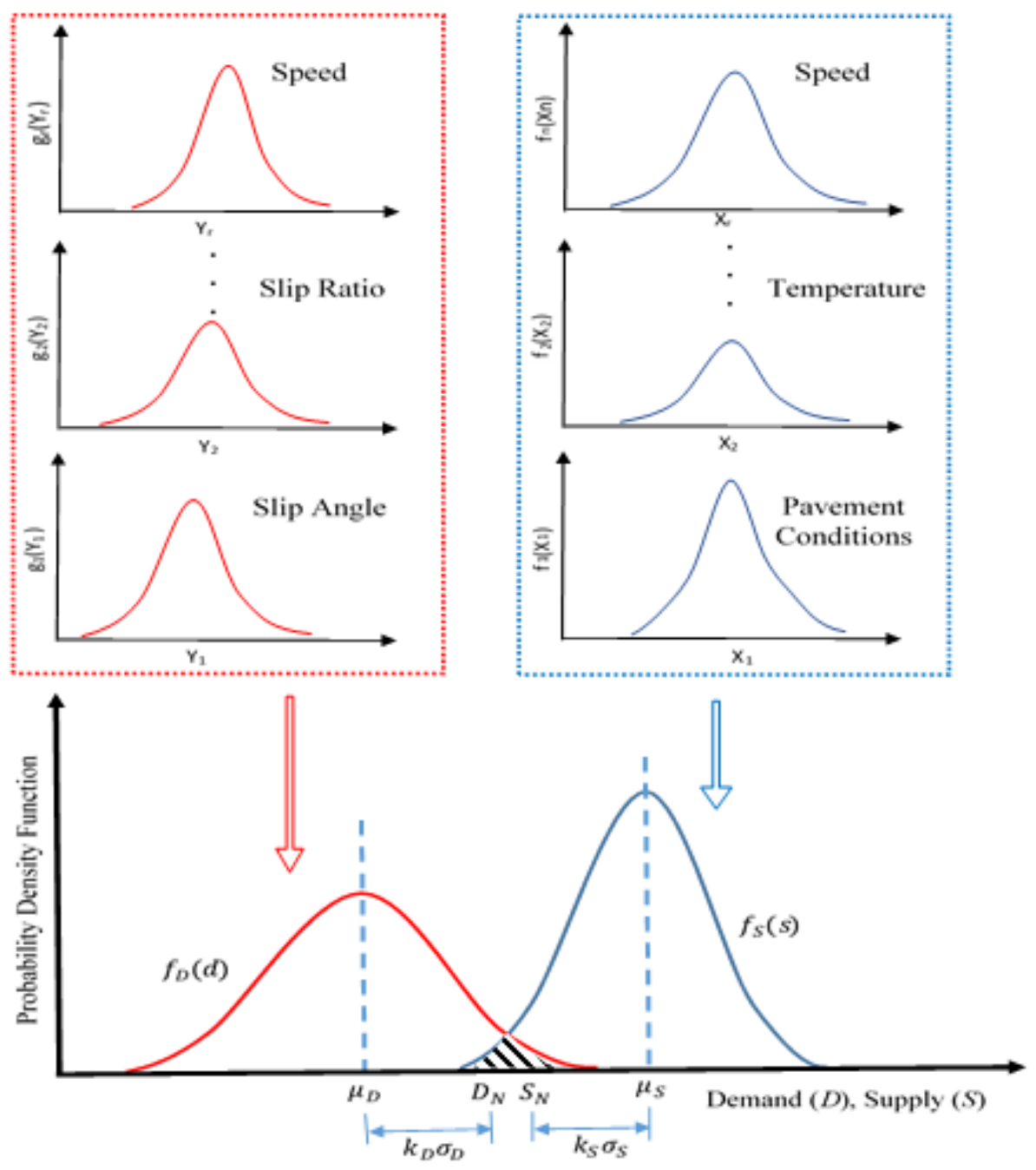

Figure 2.17: Factor of Safety- Reproduced from (Haldar and Sankaran, 2000 and Kapur and Lamberson, 1977). 


\subsubsection{Reliability Index}

In the probabilistic approach, the performance of a system is evaluated in terms of POF, PNC, or POH. However, dealing with very small values of these terms might not be the best way to evaluate the system. Therefore, reliability index can be a more appropriate way to describe the reliability of a system or product. Reliability index is defined as the ratio of the margin of safety and the combined variance (Zheng 1997).

$$
\beta=\frac{M}{\sqrt{\operatorname{VAR}(S)+\operatorname{VAR}(D)}}
$$

Where $\beta=$ reliability index, $\operatorname{VAR}(S)=$ variance of supply, and $\operatorname{VAR}(D)=$ variance of demand.

The unsafe region or the probability of failure is the shaded area of the probability distribution function of the performance function $(Z<0)$ shown in Figure 2.18.

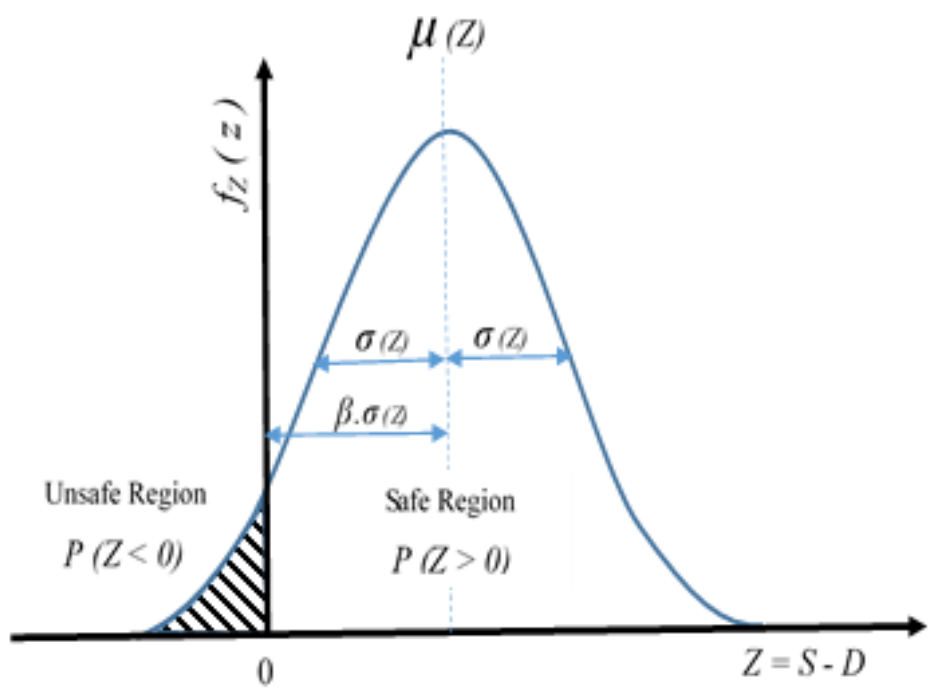

Figure 2.18: Probability Density Function of the Performance Function and Reliability Index, Reproduced from Singh et al. (2007). 
The probability of failure $\left(P_{f}\right)$ for a normally distributed performance function can be calculated as follows:

$$
P_{f}=P(Z<0)=\int_{-\infty}^{0} f_{Z}(z) d z=\int_{-\infty}^{0} \frac{1}{\sigma(Z) \sqrt{2 \pi}} \exp \left[-\frac{1}{2}\left(\frac{z-\mu(Z)}{\sigma(Z)}\right)^{2}\right]
$$

Since $\beta=\frac{\mu(Z)}{\sigma(Z)}$ and $Z=0$, then:

$$
\begin{aligned}
& P_{f}=\int_{-\infty}^{0} \frac{1}{\sigma(Z) \sqrt{2 \pi}} \exp \left[-\frac{1}{2}(-\beta)^{2}\right] \\
& P_{f}=\phi(-\beta)=1-\phi(\beta) \\
& \beta=-\phi^{-1}\left(P_{f}\right)
\end{aligned}
$$

Where $\phi^{-1}\left(P_{f}\right)=$ the inverse of the standard normal probability distribution function.

\subsubsection{System Reliability}

Whether the system is static (time independent) or dynamic (time dependent), it may contain more than one component or performance function. System reliability analysis is used when the failure is defined in terms of more than one performance function. The system reliability might be series system, parallel system, or series and parallel combination system as shown in Figure 2.19 (Kapur and Lamberson 1977).

In series reliability system, the system is characterized by operating successfully if all performance functions of the system are functioning appropriately (Kapur and Lamberson 1977). In other words, assuming that failures of the subsystems are independent events, the entire system will fail if any component of the system fails (at least one performance function is negative). 


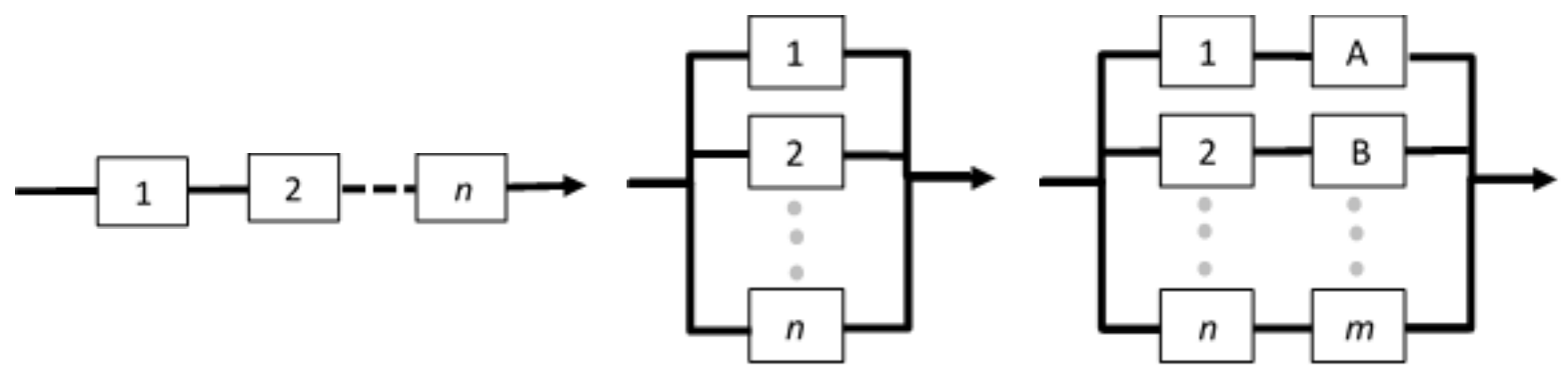
(a) Series
(b) Paralle
(c) Combined

Figure 2.19: Reliability Systems - Reproduced from (Kapur and Lamberson 1977).

$$
\begin{aligned}
& R_{s}=P\left[E_{1} \cap E_{2} \cap \ldots \cap E_{n}\right] \\
& R_{s}=P\left(E_{1}\right) P\left(E_{2}\right) \ldots P\left(E_{n}\right) \\
& R_{s}=\prod_{i=1}^{n} R_{i}
\end{aligned}
$$

Where $R_{S}=$ system reliability, $R_{i}=$ reliability of subsystem $i, E_{i}=$ event that the subsystem $i$ operates successfully. Note that the reliability of the series system will be always equal to or less than the least reliable component (subsystem).

In contrast to the series reliability system, the parallel system will fail if all components of the system fail. That is, all the performance functions are negative.

$$
\begin{aligned}
& Q_{s}=P\left[\bar{E}_{1} \cap \bar{E}_{2} \cap \ldots \cap \bar{E}_{n}\right] \\
& Q_{s}=P\left(\bar{E}_{1}\right) P\left(\bar{E}_{2}\right) \ldots P\left(\bar{E}_{n}\right) \\
& Q_{s}=\prod_{i=1}^{n}\left(1-R_{i}\right) \\
& R_{s}=1-\prod_{i=1}^{n}\left(1-R_{i}\right)
\end{aligned}
$$


Where $Q_{s}=$ unreliability of the system, $\bar{E}_{i}=$ complementary event, and $R_{i}=$ reliability of subsystem.

The analysis for the combination of parallel and series subsystems can be conducted by collapsing subsystems into equivalent parallel or series components as shown in Figure 2.20 (Kapur and Lamberson 1977).

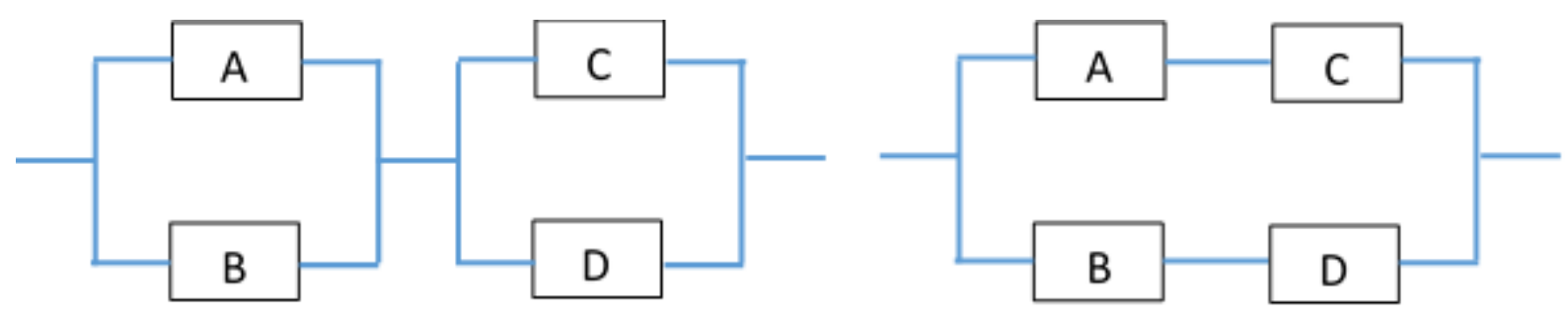

Figure 2.20: Combination of Parallel and Series Subsystems (Kapur and Lamberson 1977).

Essa et al. (2015) determined the PNC for a system considering two limit state functions, the vehicle dynamic stability and sight distance criteria, using the following expression:

$$
P N C_{\text {system }}=P_{N C 1}+P_{N C 2}-P_{N C 1 N C 2}
$$

Where $P_{N C 1}=$ probability of noncompliance for the first limit-state function (insufficient sight distance), $P_{N C 2}=$ probability of noncompliance for the second limit-state function (skidding), and $P_{N C 1 N C 2}=$ joint failure probability.

Although this approach could help in estimating the probability of failure of the system, associating two design criteria of different failure consequences is questionable. You and Sun (2013) calculated the total reliability of a system considering two design criteria, skid 
off the road and rollover criteria, as shown in Equation (2.66). The accidents are assumed to be independent events.

$$
P_{\text {system }}=1-\left[1-P\left(Z_{1}<0\right)\right]\left[1-P\left(Z_{2}<0\right)\right]
$$

Where $P\left(Z_{1}<0\right)=$ probability of skidding, and $P\left(Z_{2}<0\right)=$ probability of rollover.

\subsubsection{Reliability-Based Design}

Several research studies have been conducted to implement the reliability concept in geometric design the highway elements. Adopting the probabilistic approach, Ben-Akiva et al. (1985) proposed a process of three steps to select an optimal design value for a highway element. These steps are: defining input value distributions, developing physical and behavioural relationships, and applying the design criterion. The optimal design values were derived by minimizing cost function over different alternatives. They applied this process on a climbing lane example and found that the deterministic approach provides an overestimated road user's cost. Also, they found that the expected cost is sensitive to the construction cost, traffic volume, local conditions, and collision rates. To implement reliability analysis in designing horizontal curves, Echaveguren et al. (2005) studied the dynamic stability of a vehicle negotiating a horizontal curve using the relationship between speed limit $\left(S_{\text {lim }}\right)$ and vehicle operating speed $\left(S_{o p}\right)$ as a performance function. The speed limit was defined as "the maximum speed that ensures the dynamic stability of a vehicle for a given superelevation, radius of curvature, and friction of pavement" (Echaveguren et al. 2005). The methodology used in the study included the following steps:

- analyse probability distributions of friction supply to determine the speed limit,

- characterize probability distribution of operating speed, 
- define the limit state function as the difference between the speed limit and operating speed $\left(Z=S_{\text {lim }}-S_{o p}\right)$ and calculate the reliability index and probability of failure.

Richl and Sayed (2006) used reliability analysis to estimate the probability of being unable to stop within the available sight distance. Two performance functions were used in the analysis as shown in Equations (2.67) and (2.68) (Richl and Sayed 2006).

$$
\begin{aligned}
& Z=0.925 f_{\text {supply }}-\left(\frac{V_{o}^{2}}{g R}-e\right) \\
& Z=S S D_{\text {supply }}-\left(V_{o} P R T+\frac{V^{2}}{2 g f_{\text {rem }}}-e\right)
\end{aligned}
$$

Where $f_{\text {supply }}=$ random variable representing coefficient of friction supply, $V_{o}=$ random variable representing initial vehicle speed $(\mathrm{m} / \mathrm{s}), g=$ gravitational constant $\left(\mathrm{m} / \mathrm{s}^{2}\right)$, $R=$ radius $(\mathrm{m}), e=$ superelevation rate $(\mathrm{m} / \mathrm{m}), S S D_{\text {supply }}=$ stopping sight distance supplied $(\mathrm{m}), P R T=$ perception reaction time (s), and $f_{\text {rem }}=$ random variable calculated using Equation 2.69.

$$
1 \leq\left(\frac{f_{R}}{f_{\text {Rmax }}}\right)^{2}+\left(\frac{f_{\text {rem }}}{f_{\text {Tmax }}}\right)^{2}
$$

Where $f_{R}=$ available lateral friction, $f_{\text {Tmax }}=$ random variable representing friction supplied by the road, and $f_{R \max }=0.925 f_{T \max }$.

Himes (2013 and 2014) studied the design of horizontal curves through investigating the dynamic stability (skid off the road and vehicle rollover) of passenger cars and trucks. The performance functions used in the skid-off-the-road failure analysis were based on the classical and modified PMM as shown in Equations (2.70) and (2.71), respectively. 


$$
\begin{aligned}
& Z=f_{y, \text { supply }}-\frac{V^{2}}{15 R}+\frac{e}{100} \\
& Z=f_{y, \max } \sqrt{1-\left(\frac{\frac{a_{x}}{g} \mp \frac{G}{100}}{f_{x, \max }}\right)^{2}-\frac{V^{2}}{15 R}+\frac{e}{100}}
\end{aligned}
$$

Where $f_{y, \text { supply }}=$ available lateral friction, $V=$ operating speed $(\mathrm{mi} / \mathrm{h}), R=$ Radius of horizontal curve $(\mathrm{ft}), a_{x}=$ deceleration $\left(\mathrm{ft} / \mathrm{s}^{2}\right), G=$ grade (positive for uphill grade), $f_{y, \max }$ and $f_{x, \max }=$ maximum available lateral and longitudinal friction, respectively, and $e=$ superelevation rate.

The skid-off-the-road probability of failure for heavy trucks was found to be much larger than for passenger cars for the same mean speed value. Also, the design radius for passenger car according to skid-off-the-road failure criterion was found to be larger than for rollover failure. First and Second Order Reliability Methods were used in the analysis. However, the difference in the results of both methods were insignificant. Note that Himes (2013) has not considered several factors in the analysis such as weather conditions.

Himes (2013) also conducted reliability analysis for rollover. The performance function was the difference between the lateral acceleration threshold for rolling over and the lateral acceleration demand as shown in Equation (2.72). Note that the lateral acceleration threshold was determined based on static rollover using PMM.

$$
Z=\left(\frac{\frac{t}{2 h}+e}{1+\left(1-\frac{h_{o}}{h}\right) R_{\phi}}\right)-\frac{V^{2}}{15 R}
$$

Where $t=$ vehicle width (ft), $h=$ centre of gravity height (ft), $h_{o}=$ Roll centre height (ft), and $R_{\phi}=$ Roll rate ( $\left.\mathrm{rad} / \mathrm{g}\right)$. 
You and Sun (2013), used reliability analysis to determine vehicle stability on combined horizontal and vertical alignments. One of the performance functions presented in this study was built based on the difference between the available and demanded radii. Note that the PMM was used to determine the radius demand.

$$
Z=R_{S}-\frac{V^{2}}{g\left(e+f_{y}\right)}
$$

Where $R_{S}=$ radius of circular curve supply, $f_{y}=$ lateral friction coefficient, and $V=$ operating speed of vehicle.

Hussein et al. (2014) used risk-based reliability analysis to provide calibrated design charts for the middle ordinate (lateral clearance) of horizontal curves. A pre-specified value for the PNC was targeted in the calibration to determine the middle ordinate of the horizontal curve. The performance function was the difference between the available and demanded sight distance. Braking deceleration, perception and reaction time, and operating speed distributions were the input variables in the analysis.

$$
Z=\left[2 R \cos ^{-1}\left(1-\frac{M}{R}\right)\right]-\left[V P R T+\frac{V^{2}}{2(a+g G)}\right]
$$

Where $M=$ middle ordinate (m), $P R T=$ perception and brake reaction time (s), and $a=$ deceleration $\left(\mathrm{m} / \mathrm{s}^{2}\right)$.

They found that the current design guides are conservative especially at high speeds and sharp curves. Note that their analysis ignored grade effects and have not considered the factors mentioned earlier in Himes (2013) research. 


\subsubsection{Reliability Analysis Methods}

Solving reliability equations using direct or exact methods would be difficult when having more than two variables in the performance functions. Therefore, simulation and approximation approaches are used in such cases (Singh et al. 2007). Several methods have been developed to calculate the reliability of a system or product such as First and Second Order Reliability Methods, and Monte Carlo Simulation. Each of these methods has its characteristics and requirements. For the purpose of this research, the following methods are discussed.

\subsection{6.a. First Order Approximation Method}

The first-order approximation (FOA) method can be used to estimate the amount of uncertainty, or scatter, of a dependent variable owing to uncertainty about the independent variables included in a functional relationship (Singh et al. 2007). It is an approximation method for means and variances and its estimation is influenced by the degree of nonlinearity in the model equation and in the extent of parameter uncertainty. FOA would provide good results when having near linearity in functional relations, small coefficients of variation of the most sensitive uncertain variables, and near normal distribution (Tyagi and Haan 2001).

To illustrate the FOA mathematically, variable $Y$ can be expressed as a function of $n$ random variables.

$$
Y=g(\underline{X})
$$

The first order term is truncated with a Taylor series expansion of the model outputs. The expected value of the model can be approximated as (Singh et al. 2007): 


$$
Y=g\left(\underline{X}_{e}\right)+\sum_{i=1}^{n}\left(X_{i}-X_{i e}\right)\left(\frac{\partial g}{\partial X_{i}}\right)_{X e}
$$

Since the expansion point is commonly the mean value of the basic variables, the expected value of $Y$ will be:

$$
E[Y] \approx g(\underline{X})
$$

And the variance of $(Y)$ will be:

$$
\operatorname{var}(Y)=\sigma_{Y}^{2} \approx \sum_{i=1}^{n}\left[\left(\frac{\partial g(\underline{X})}{\partial X_{i}}\right)_{\bar{X}_{i}} \sigma_{X_{i}}\right]^{2}
$$

Where $\underline{X}=\left(X_{1}, X_{2}, \ldots, X_{n}\right)=$ a vector containing $n$ random variables, $\underline{X}_{e}=$ $\left(X_{1 e}, X_{2 e}, \ldots, X_{n e}\right)=$ a vector representing the expansion points, $\sigma_{Y}=$ standard deviation of $Y$, and $\bar{X}=\left(\bar{X}_{1}, \bar{X}_{2}, \ldots, \bar{X}_{n}\right)=$ a vector of mean values of the statically independent input basic variables.

\subsection{6.b. First Order Reliability Method}

The First Order Reliability Method (FORM) is considered as an extension or an advancement to the First Order Approximation (FOA) because it was developed to overcome some technical difficulties associated with FOA. The FORM manipulates the lack of invariance of the solution relative to the formulation performance function. Thus, the assessment of the uncertainity propagation would not be affected significantly when having simple algebric changes in the formulation of the problem (Singh et al. 2007). The expected value and the variance of joint performance function in this method can be obtained by determining the failure point $\left(X^{*}\right)$, at the limit state where $Z=0$, then expanding the performance function using a Taylor series expansion as follows: 


$$
\begin{aligned}
& E(Z) \approx \sum_{i=1}^{n}\left(\frac{\partial Z}{\partial X_{i}}\right)\left(\bar{X}_{i}-X_{i}^{*}\right) \\
& V A R(Z)=\sum_{i=1}^{n}\left(\frac{\partial Z}{\partial X_{i}}\right)_{X_{i}^{*}}^{2} \sigma_{X_{i}}^{2}
\end{aligned}
$$

Where $\quad \bar{X}_{i}=$ mean value, and $\sigma=$ standard deviation.

When the values of Cumulative Distribution Function (CDF) and Probability Density Function (PDF) are non-normal distributions, a transformation technique is used to convert these distributions to a standard normal space to determine the failure point or what is commonly known as most propbable point (MPP) as shown in Figure 2.21. The values of the mean and standard deviation of the equivalent normal distribution can be found by equating the cumulative probabilities at the failure point. The transformation procedure is out of the scope of the research and the detailed analysis can be found in Singh et al. (2007).

It is shown that the reliability index for the FORM represents the shortest distance in the standardized normal space between the system mean state (origin) and the failure surface (limit state surface). In other words, it represents the most likely combination of input variable values that produce the critical target level. Therefore, the reliability index in the standard normal space will be (Singh et al. 2007):

$$
\text { Minimize } \beta=\sqrt{U_{1}^{2}+U_{2}^{2}}
$$

The equation in the above is subjected to limit state condition. 

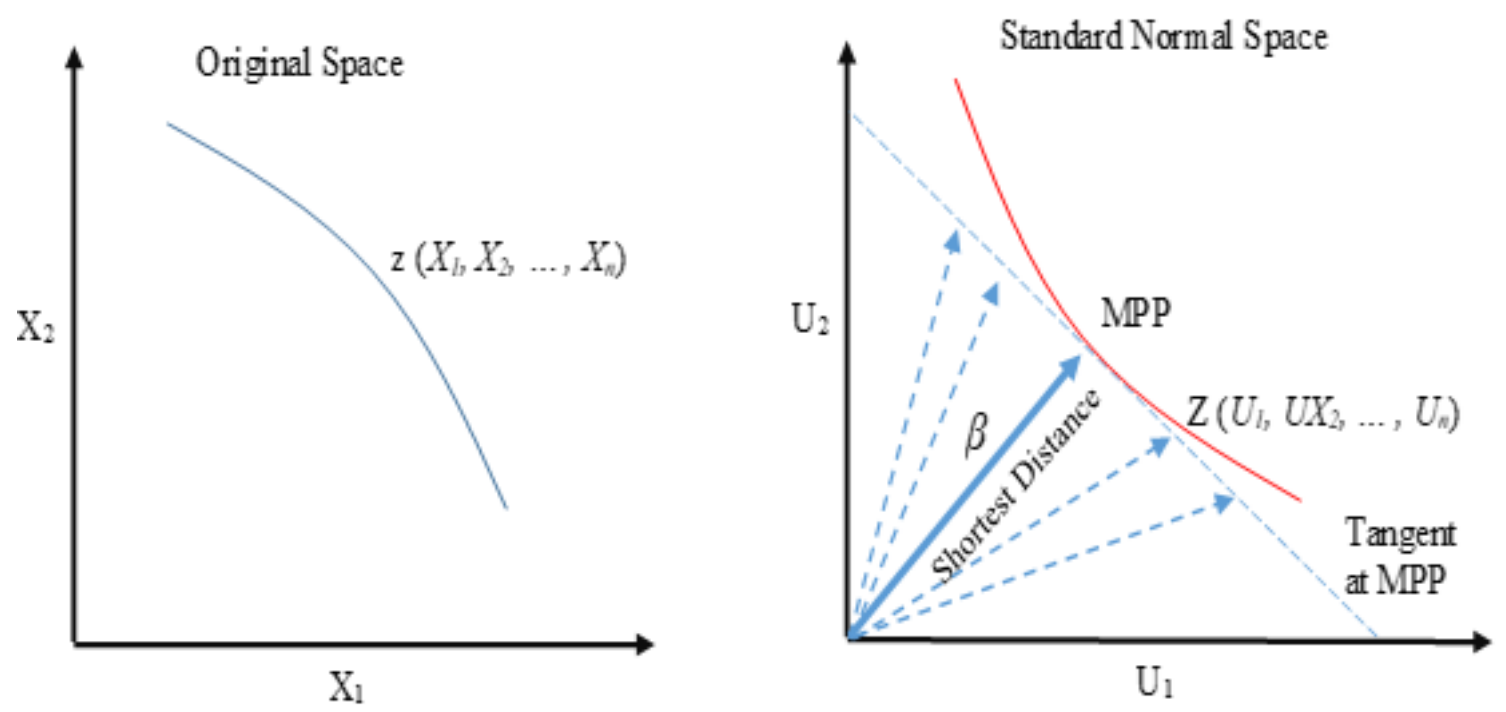

Figure 2.21: Transformation of Non-Normal Variables to Standard Normal Space-Reproduced from (Singh et al. 2007).

\subsection{6.c. Second Order Reliability Method}

When the limit state is nonlinear because the relationship between the random variables in the limit state equation or some variables being non-normally distributed, or a linear limit state in the original space becomes nonlinear when transformed to a standard space, it is better to use higher order approximation for the failure probability computation (Haldar and Sankaran 2000). Thus, the Second Order Reliability Method (SORM) improves the results of FORM by including additional information regarding the curvature of the limit state function, adding another term of Taylor series. The Taylor series expansion of a general nonlinear function at the failure point is:

$$
\begin{aligned}
g\left(X_{1}, X_{2}, \ldots, X_{n}\right) & \\
& =g\left(x_{1}^{*}, x_{2}^{*}, \ldots, x_{n}^{*}\right) \\
& +\sum_{i=1}^{n}\left(x_{i}-x_{i}^{*}\right) \frac{\partial g}{\partial X_{i}}+\frac{1}{2} \sum_{i=1}^{n} \sum_{j=1}^{n}\left(x_{i}-x_{i}^{*}\right)\left(x_{j}-x_{j}^{*}\right) \frac{\partial^{2} g}{\partial X_{i} \partial X_{j}}
\end{aligned}
$$




\subsection{6.d. Monte Carlo Simulation}

Monte Carlo Simulation (MCS) is another mathematical technique used to quantify risk. This method generates random values of each uncertain variable based on their respective probability distributions and the model describing the system. Statistics and an empirical probability distribution of the model are determined by repeating the random generation of the variable values and model execution steps many times (Singh et al. 2007). The MCS can be used to overcome some weaknesses associated with the FORM and SORM methods such as the inability of estimating the error bounds or confidence intervals (Padmanabhan 2003).

\subsection{Summary}

This chapter reviewed four research areas. First, vehicle dynamics and control along with main models that depict vehicle's movement on horizontal curves was addressed. This would help in determining critical points on curves and thus developing more accurate models. In addition, the chapter covered the design criteria that have been used in design guides to ensure the safety and comfort to roadway users. Four design criteria were covered; vehicle stability, comfort threshold, rollover, and sight distance. In addition, pavement friction phenomenon and interrelationship between vehicle's tires and pavement surface were covered. Knowing the factors that affect pavement friction would help in understanding and determining vehicle performance and safety while negotiating horizontal curves. Two design criteria would be affected directly by the available friction; vehicle stability and sight distance. Finally, reliability concept was provided. Also, the main methods that are used to evaluate system performance, in terms of probability of failure, were explained as well. 


\section{CHAPTER THREE}

\section{RESEARCH METHODOLOGY AND DATA}

\subsection{Introduction}

In order to develop the new framework to design horizontal curves mentioned in Chapter 1 to provide safe and comfortable trip for vehicles' occupants, all design criteria have to be satisfied. This chapter is the kernel of the research and aims at providing the methodology used to connect framework components (data, design criteria, reliability analysis, and safety analysis) in one skeleton to achieve the objective of this research. Detailed descriptions for the collected data used in the analysis are explained as well. In addition, this chapter focuses on the first task of the methodology (data collection) through describing the data used in the analysis.

\subsection{Research Methodology}

The methodology used to achieve the objectives of this research includes five major tasks. The first task concerns collecting data and building the main database. Since the data are obtained and extracted from several data sets, the main database should be organized properly to serve several purposes such as studying driving behaviour, developing models, and conducting reliability and safety analysis. The main key in this task is that the collected data should overcome the issues associated with other data collection techniques. This task was addressed in Section 3.6.

Second task focuses on analysing the data through extracting several variables from the database and conducting preliminary analyses and statistical tests. This task aims at 
preparing the data to serve three purposes; $(i)$ determine variables' distributions that are used in the reliability analysis (ii). study driving behaviour on horizontal curves. (iii) remove the outliers and prepare the data to develop several models. This task is addressed in Chapter 4.

Third task is devoted to developing several models that will be used in the reliability analysis. These models include predicting speed on curves, determine comfort threshold, available and demanded friction factors, and lateral acceleration. It should be noted that studying driving behaviour on curve was conducted before developing the models. The task is addressed in Chapter 5.

Fourth task concerns conducting reliability analysis. Four design criteria are addressed in the reliability analysis: vehicle stability, driver comfort, sight distance, and rollover. The outputs of the analysis (POF, $\mathrm{PNC}, \mathrm{POH}$, and $\mathrm{POF}_{\mathrm{R}}$ ) are adjusted considering weather and pavement conditions. The analysis is explained in Chapter 6.

The last task focuses on the developing safety performance functions. The outputs of reliability analysis, horizontal curves design parameters, traffic parameters, and historical safety data were included in the analysis. The task is addressed in Chapter 7. To accomplish these tasks, the following steps were conducted (Figure 3.1):

- Selecting study area was the first step in this research. Washington State was selected to be the study area for several reasons as explained in Section 3.6.1. Five types of data were collected from several resources. First, the data related to vehicle movement on curves. Second, horizontal curves geometric parameters. Third, historical data for safety and traffic volumes. Fourth, pavement conditions and frictions. Finally, weather conditions. 


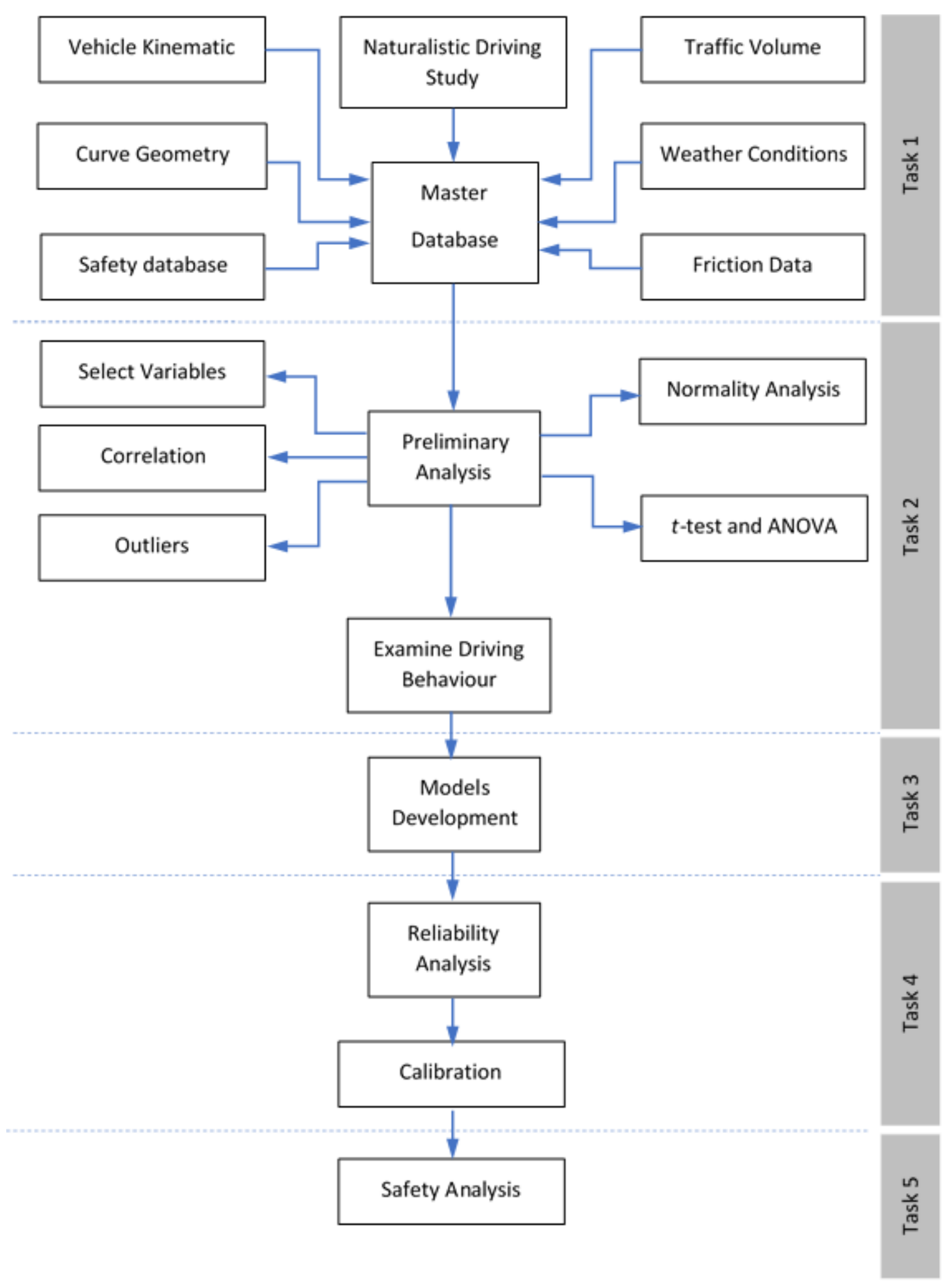

Figure 3.1: Tasks of the Research. 
- A master database was built by combining and compiling datasets in such a way to help reaching the data easily and serve research objectives. This includes matching vehicle movements (using naturalistic driving study database) with the geometric parameters of horizontal curves, weather conditions, pavement conditions, and safety dataset. Matlab scripts were developed to achieve this task.

- Several dependant and independent variables were extracted from the datasets.

- Preliminary analysis and statistical tests were conducted prior to model development such as determining the distribution of the variables, correlation, and determine outliers. This step was necessary to select the variables to be used in models' development.

- The driving behaviour throughout horizontal curves was conducted by developing a procedure to determine vehicle speed state. A Matlab script was developed to achieve this step.

- Different types of models were developed to predict speed on curve, comfort threshold, lateral acceleration, and friction available and demand.

- Reliability analyses were conducted to determine probability of failure for four design criteria.

- The probabilities of failure and reliability indices were adjusted considering weather and pavement conditions.

- Safety analysis was conducted to develop several safety performance functions considering the developed reliability indices for each design criterion, horizontal curve geometry, and traffic characteristics.

- A design example was provided to illustrate the applicability of the new framework 
to design horizontal curves.

\subsection{Horizontal Curve Design Framework}

The philosophy used in designing horizontal curves in the current design guides depends only on driver comfort criterion. The design speed concept is used to establish the range of other design parameters of the curve, such as the radius and superelevation rate. The design procedure considers only driving in favourable weather conditions and it ignores adverse weather conditions, which represents approximately $23 \%$ of the collisions on curved roadways as mentioned in Chapter 1. On the other hand, the suggested philosophy to design horizontal curves would provide more realistic evaluation for horizontal curve design through considering the effect of other influencing factors on the safety and comfort of vehicle's occupants.

To adopt this philosophy and achieve the objectives of the new design framework, the methodology used in the research include all design criteria and consider the factors that affect the performance of horizontal curves. Therefore, the effect of different driving behaviour, environment, time of day, traffic characteristics, roadway classification, and pavement surface conditions should be considered when conducting reliability analysis for each design criterion. Table 3-1 shows the potential factors that would affect the design criteria of horizontal curves. The vehicle dynamic stability criterion, for instance, would be affected by pavement surface conditions because the available lateral friction changes according to these conditions as explained in Section 2.5.3. Also, the available lateral friction has an inverse relationship with speed of vehicle. The weather conditions would affect the dynamic stability of the vehicle indirectly because their influences are reflected 
Table 3-1: Potential Factors Affecting Design Criteria.

\begin{tabular}{|c|c|c|c|c|c|c|c|c|c|c|c|c|c|c|c|c|}
\hline \multirow[b]{2}{*}{ Criterion } & \multicolumn{5}{|c|}{ Pavement } & \multicolumn{2}{|c|}{ Time } & \multicolumn{3}{|c|}{ Weather } & \multicolumn{2}{|c|}{ Traffic } & \multicolumn{4}{|c|}{$\begin{array}{c}\text { Road } \\
\text { Classification } \\
\text { and Terrain } \\
\end{array}$} \\
\hline & $\vec{\Delta}$ & $\sum_{3}^{\overline{0}}$ & क् & $\stackrel{0}{0}$ & $\begin{array}{l}\bar{ज} \\
\frac{5}{\omega}\end{array}$ & 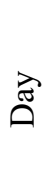 & 营 & $\frac{\vec{\Xi}}{U}$ & 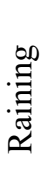 & की & $\frac{\Xi}{\Xi}$ & $\begin{array}{l}\bar{d} \\
\mathbb{2} \\
\text { की }\end{array}$ & $\underset{\pi}{\stackrel{\pi}{*}}$ & 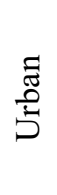 & $\begin{array}{l}\bar{d} \\
\stackrel{d}{=}\end{array}$ & 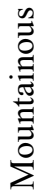 \\
\hline $\begin{array}{l}\text { Vehicle Dynamic } \\
\text { Stability }\end{array}$ & $\sqrt{ }$ & $\sqrt{ }$ & $\sqrt{ }$ & $\sqrt{ }$ & $\sqrt{ }$ & $x$ & $x$ & $\nabla$ & $\nabla$ & $\nabla$ & $\nabla$ & $\sqrt{ }$ & $x$ & $x$ & $\boldsymbol{\nabla}$ & $\sqrt{ }$ \\
\hline Vehicle Rollover & $\sqrt{ }$ & $\sqrt{ }$ & $\sqrt{ }$ & $\sqrt{ }$ & $\sqrt{ }$ & $x$ & $x$ & $\boldsymbol{\nabla}$ & $\boldsymbol{\nabla}$ & $\nabla$ & $\nabla$ & $\sqrt{ }$ & $\sqrt{ }$ & $\sqrt{ }$ & $\sqrt{ }$ & $\sqrt{ }$ \\
\hline Driver Comfort & $\sqrt{ }$ & $\sqrt{ }$ & $\sqrt{ }$ & $\sqrt{ }$ & $\sqrt{ }$ & $\sqrt{ }$ & $\sqrt{ }$ & $\sqrt{ }$ & $\sqrt{ }$ & $\sqrt{ }$ & $\nabla$ & $\sqrt{ }$ & $\nabla$ & $\nabla$ & $\nabla$ & $\boldsymbol{\nabla}$ \\
\hline Sight Distance & $\sqrt{ }$ & $\sqrt{ }$ & $\sqrt{ }$ & $\sqrt{ }$ & $\sqrt{ }$ & $\sqrt{ }$ & $\sqrt{ }$ & $\sqrt{ }$ & $\sqrt{ }$ & $\sqrt{ }$ & $x$ & $\sqrt{ }$ & $x$ & $x$ & $\nabla$ & $\nabla$ \\
\hline
\end{tabular}

$\sqrt{ }=$ direct effect, $\boldsymbol{\nabla}=$ indirect effect, and $\times=$ no effect.

in pavement surface conditions and speed. On the other hand, this criterion would not be affected directly by traffic volume. However, the speed of vehicle would change with traffic volume. The terrain type would affect the lateral friction demand because of the presence or absence of roadway grade. Almost all these factors would affect rollover criterion as well. However, the impact of pavement conditions is different. The vehicle would be more prone to rollover than skidding when having high lateral friction supply, if the turning moment is high enough. Vehicles are more likely to skid-off the road when having snow or icy pavement. Weather conditions would affect indirectly since they affect available friction of the pavement. This criterion would not be affected by time of day. Also, road classification would influence this criterion because the urban roadways most likely have low superelevation rate than rural ones. All these factors would affect the driver comfort criterion. Thus, the driving behaviour would change according to pavement and weather conditions, day time, traffic characteristics, and road classification and terrain. For 
example, drivers would tend to drive faster when driving in favourable weather conditions and daytime than adverse weather conditions and night. Accordingly, the comfort threshold would be different. Road classification and terrain have indirect effect in speed. Pavement conditions would impact directly the sight distance criterion because the demanded stopping sight distance increases when having pavement surface with low friction factor values. The time of day and adverse weather conditions would affect the available sight distance. The other factor would have no or indirect effect on this criterion. It should be noted that some of these factors are discussed in reliability analysis chapter.

Accordingly, the performance of the horizontal curves should be evaluated considering these factors. Also, the performance should be examined as a function of time especially for driver dynamic stability. This could be used to determine the time to reach critical threshold for probability of failure for the design and hence manage and schedule the required maintenance for road segment.

\subsection{Evaluation of Design Criteria}

Based on the previous section, considering all these factors would provide more realistic evaluation for safety and design performance. The reliability-based design concept used in some studies provided evaluation for safety for specific situations such as driving during favourable weather conditions or considering one or two design criteria as provided in El-Bassioni and Sayed (2011), Hussein et al (2014) and Himes and Donnel (2014). When estimating the probability of failure against skidding, for instance, the available lateral friction has been considered for only wet pavement condition and for single selected design speed. However, the real situation is that the pavement is not always wet, and the operating 
speed varies based on several factors such as weather, daytime, and traffic conditions. Therefore, the available lateral friction should be addressed as a function of the influencing potential factors mentioned in Table 3-1. Thus, the performance function for vehicle dynamic stability $\left(Z_{S}\right)$ should be:

$$
Z_{S}=f_{S}-f_{D}
$$

The available lateral friction $\left(f_{S}\right)$ is influenced by several factors as mentioned in Chapter 2. Thus, the available friction can be taken as a function of the following factors:

$$
f_{S}=f\left(V, P_{C}, T\right)
$$

Where $V=$ speed of vehicle, $P_{C}=$ pavement surface conditions, and $T=$ temperature.

On the other hand, the lateral friction demand $\left(f_{D}\right)$ will be influenced by other factors. Traffic characteristics, for example, would change with time of the day, which would affect the speed of vehicle (free flow speed conditions or slow speed at peak hours). Also, the type of vehicle (PC, SUV, truck, ... etc) and suspension system characteristics would affect load transfer and load distribution on wheels. Accordingly, the lateral friction demand should be presented as a function of the influencing factors.

$$
f_{D}=f(V, A)
$$

Where $A=$ vehicle type.

Although the traffic volume is not included explicitly in the equations above, the effect of traffic volume should be considered from two points of view. First, the effect of traffic volume on the available friction factor because it has a direct effect on the deterioration of the pavement. Therefore, it is important to measure the available friction on regular bases, or use generalized models to determine pavement conditions, before estimating the POF. Thus, the evaluation for horizontal curve in terms of POF should be based on pavement 
deterioration and traffic volume growth. That is, the POF is a time dependent function and should be estimated, at least, for each year. Secondly, the effect of traffic volume on the collision frequency. The collision frequency is expected to increase up to a point as the traffic volume increases. Several studies used traffic volume as an exposure variable in the safety performance functions (Sawalha and Sayed 2006). Therefore, the traffic volume can be used to determine the weighted average probability of failure $\left(P O F_{\text {avg }}\right)$. Thus, the calibrated probability of failure equation can be written as:

$$
P O F_{\text {avg }}=\frac{\sum_{i=1}^{n} P O F_{i} \times T V_{i}}{\sum_{i=1}^{n} T V_{i}}
$$

Where $T V_{i}=$ traffic volume per hour.

It should be noted that the POF is expected to change every year due to the change in available friction of the pavement (deterioration, and minor or major rehabilitation). Thus, the average POF for one year, considering only pavement conditions, would be:

$$
P O F_{\text {avg }}=\frac{\sum_{i=1}^{n 1} P O F_{i}+\sum_{i=1}^{n 2} P O F_{i}+\sum_{i=1}^{n 3} P O F_{i}+\cdots+\sum_{i=1}^{n j} P O F_{i}}{365}
$$

Where $n_{i}=$ number of days for dominant pavement conditions, subscript $(i)$ represent pavement conditions (dry, wet, packed-snow, icy, etc.). Note that the speed distribution for each pavement conditions is different. Thus, the equation assumes average speed along the day in calculating the available and demanded lateral friction.

For the driver comfort criterion, the performance function $Z_{C}$ is based on the difference between comfort threshold $a_{L}$ and the actual lateral acceleration experienced by vehicle's occupants $a_{a c t}$. Thus, the performance function is: 


$$
Z_{C}=a_{L}-a_{a c t}
$$

As explained in previous section, the driver comfort threshold would be influenced, in addition to the horizontal curve geometry, by several factors such as time of day, roadway classification, weather and pavement conditions, and traffic characteristics. Thus,

$$
a_{L}=f\left(t, R_{C}, W_{c}, P_{C}, V, T V, G_{H C}\right)
$$

Where $t=$ time of the day (day or night), $R_{c}=$ road classification, $W_{c}=$ weather conditions, $V=$ traffic speed, $T V=$ traffic volume, and $G_{H C}=$ horizontal curve geometry.

That means that the PNC would vary depending on the prevailing conditions. Therefore, the total PNC should be the cumulative of the entire PNC's along different periods of the day (i.e. Daytime and night) and year (dry and wet seasons).

That is, the calibrated PNC for driving is:

$$
P N C_{j}=\frac{\sum_{i=1}^{n} P N C_{i}}{n}
$$

Where $n=$ hour or day.

It should be noted that the PNC, unlike the POF, is almost constant every year because the prevailing weather conditions, in terms of number of hours or days around the year, are almost constant. Thus, Equation 3.7 could be rewritten considering weather conditions only as shown below:

$$
P N C_{\text {avg }}=\frac{\sum_{i=1}^{n 1} P N C_{i}+\sum_{i=1}^{n 2} P N C_{i}+\cdots+\sum_{i=1}^{n j} P N C_{i}}{365}
$$

Where $n_{i}=$ number of days for dominant weather conditions, subscript $(i)$ represent weather conditions (clear, raining, snowing, etc.). 
The same principle can be used to modify the performance function of rollover and sight distance criteria. For vehicle rollover criterion, the performance function is the difference between the counterbalance moment $\left(M_{c}\right)$ and the applied turning moment $\left(M_{a}\right)$ as shown in Equation (3.10).

$$
Z_{R}=M_{c}-M_{a}
$$

The turning moment depends on the speed of vehicle, height of centre of gravity of the vehicle and rolling centre, and the characteristic of the suspension system of the vehicle (Equation 3.11). Note that a vehicle would rollover when the turning moment is greater than the counterbalance moment and there is a sufficient available friction factor or any other tripping mechanism in pavement such as curbs. Otherwise the vehicle would normally skid-off-the-road before rolling over.

$$
M_{a}=f\left(V, h, h_{o}, S\right)
$$

Where $V=$ speed of vehicle, $h=$ height of centre of gravity, $h_{o}=$ height of rolling centre, $S=$ suspension system characteristics.

The counterbalance moment depends mainly on the weight of the vehicle, the width of the vehicle (distance between wheels), and the superelevation rate of the horizontal curve. Therefore, the counterbalance moment is:

$$
M_{c}=f\left(W, T_{w}, e\right)
$$

Where $W=$ weight of the vehicle, $T_{w}=$ vehicle width, and $e=$ superelevation rate.

Since the speed is included in the performance function, the traffic characteristics should be included in determining the probability of failure. Thus, POF will be:

$$
P O F_{\text {Roll }}=\frac{\sum_{i=1}^{n} P O F_{R o l l, i}}{n}
$$


The discussion provided in vehicle stability criterion can be applied for rolling over criterion.

For sight distance criterion, the performance function is the difference between the available sight distance $S_{a v i}$ and demanded stopping sight distance $S_{D}$.

$$
Z_{S D}=S_{a v i}-S_{D}
$$

The available sight distance would be limited by several issues as explained in Section 2.4.4. In addition, weather conditions and time of day (daytime or night) might affect the available sight distance. Also, the factors that control sight distance at night are conceptually different from daytime.

$$
S_{a v i}=f\left(\mathrm{LC}, t, W_{c}, h_{1}, h_{2}\right)
$$

Where LC= lateral clearance (2-D analysis is assumed), and $h_{1}$ and $h_{2}=$ height of driver's eye and object, respectively.

The stopping sight distance would be a function of available longitudinal friction and driver perception reaction time.

$$
S_{D}=f\left(f_{L}, P I E V, G, a\right)
$$

Where $f_{L}=$ available longitudinal friction, $P I E V=$ perception reaction time, $G=$ grade, and $a=$ longitudinal acceleration.

The same factors affecting vehicle stability, besides perception reaction time, would affect stopping sight distance criterion.

$$
\mathrm{POH}=\frac{\sum_{i=1}^{n} \mathrm{POH}_{i}}{n}
$$

Similar to the vehicle stability criterion, the POH should be calculated every year because of the change in pavement friction with time. Considering only the effect of 
pavement friction, Equation 3.16 can be rewritten as:

$$
P O H_{\text {avg }}=\frac{\sum_{i=1}^{n 1} \mathrm{POH}_{i}+\sum_{i=1}^{n 2} \mathrm{POH}_{i}+\sum_{i=1}^{n 3} \mathrm{POH}_{i}+\cdots+\sum_{i=1}^{n j} P O H_{i}}{365}
$$

Where $n_{i}=$ number of days for dominant pavement conditions, subscript $(i)$ represent pavement conditions (dry, wet, packed-snow, icy, etc.).

\subsection{Evaluation of Design System}

The previous section provided explanations and evaluation of the design criteria and performance functions for each criterion. However, it is important to estimate the overall design reliability or system reliability. This can be done through understanding the relationships among design criteria whether they are working as a parallel or series system, or a combination of both. It should be noted that failure of a system can take two shapes; performance failure and structural failure. While the performance failure takes place when the system is unable to perform as per expectations while the structure of the system has not been altered, the structural failure involves damage or change of the structure (Singh et al. 2007).

Before going forward, it is worth to note that the outputs of evaluating a horizontal curve might be different from other engineering discipline evaluation, such as structural engineering. For example, probability of noncompliance (comfort criterion) in the former means, in fact, the probability of violating specific design requirements. Thus, failure to comply to these requirements might not end up with a collision; however, it would reduce the level of driver comfort and putting more stress on road users, and hence should increase the probability of having a collision (Sarhan and Hassan 2008). Similarly, violating the sight distance criterion might not end up with collision unless other events occur 
simultaneously such as presence of a stationary object ahead. Thus, failure to comply with these two criteria will not necessarily fail the entire system. However, collision frequency is expected to increase. On the other hand, failure in the latter (structural failure) definitely ends up with a system failure such as a building collapse. Note that failure in vehicle stability criterion might result in losing control over the vehicle, which is a very serious issue. Thus, this failure might end up with a collision unless the driver is able to take control over the vehicle again. On the other hand, failure to comply with rollover criterion likely fail the entire system. Thus, there are different consequences when violating design criteria as shown in Table 3-2.

Table 3-2: Consequences of Violating Horizontal Curve Design Criteria.

\begin{tabular}{|l|l|l|}
\hline \multicolumn{1}{|c|}{ Criterion } & Consequence of Violation & \multicolumn{1}{|c|}{ Notes } \\
\hline Vehicle Stability & $\begin{array}{l}\text { Driver loses control over the } \\
\text { vehicle }\end{array}$ & $\begin{array}{l}\text { Likely ends up with a collision. } \\
\text { Driver action is required to take } \\
\text { over vehicle control again. }\end{array}$ \\
\hline Driver Comfort & $\begin{array}{l}\text { Vehicle's occupants traverse } \\
\text { the curve uncomfortably }\end{array}$ & $\begin{array}{l}\text { Increase the probability of having a } \\
\text { collision. }\end{array}$ \\
\hline Sight Distance & $\begin{array}{l}\text { Increase driver workload and } \\
\text { stress }\end{array}$ & $\begin{array}{l}\text { Drivers likely travel safely unless a } \\
\text { stationary object exists at the } \\
\text { moment. }\end{array}$ \\
\hline Rollover & $\begin{array}{l}\text { Vehicle rolls over and driver } \\
\text { loses control over the vehicle }\end{array}$ & Most likely ends up with a collision. \\
\hline
\end{tabular}

As explained in Section 2.6.4 and based on the previous discussion, estimating the probability of failure for the horizontal curve design considering all design criteria as a reliability system (series, parallel, or combined) would not be feasible because the consequences of violating design criteria are different. Furthermore, conducting reliability analysis considering more than two design criteria (series system) is very difficult to 
achieve. This part of reliability analysis is still an open research area to develop an accurate approximation (Haukaas 2014).

\subsection{Data Collection}

The first task in achieving the objectives of the research is collecting the required data. The data collection task was planned to overcome problems associated with other traditional data collection techniques. The data were obtained from several resources such as transportation agencies, and research institutes. These datasets were collected to serve several areas such as developing models that describe vehicles dynamics, examine driving behaviour, conduct reliability analysis, and perform safety analysis. Thus, the following datasets were collected:

1. Naturalistic Driving Study (NDS): it was used in studying different patterns of driving behaviour on horizontal curves. In addition, the dataset is used to develop several models that predict speed on curve and determine driver comfort threshold.

2. Road Information Database (RID): this database was used to match all other datasets and provide information about roadway segments.

3. Pavement Friction Data: it was used in reliability analysis to evaluate vehicle stability and sight distance criteria.

4. Outputs of Software Simulations: these datasets were obtained from the CarSIM simulation runs and were used to develop several models to determine the lateral friction demand and applied lateral acceleration for two types of vehicle; sedan passenger car and SUV.

5. Weather Conditions: weather database was used in the analysis to examine the 
effect of location and environment on horizontal curve safety performance.

6. Safety Data: historical collision database was used to develop safety performance functions for horizontal curves.

\subsubsection{Naturalistic Driving Study Database}

Data collection task might be associated with some issues that could affect the accuracy of the analysis and models' development. For example, speed data might have human errors if data are collected manually; technical errors such as the cosine error need to be accounted for if data are collected using radar/laser guns; unrepresentative sample might be used in simulator experiments; driving behaviour and speed selection of drivers might be altered due the presence of observers and/or measuring devices or during short-term field experiments using instrumented vehicles (Hassan 2004). On the other hand, data of lateral acceleration affecting vehicle occupants are mostly calculated and are rarely collected (Said et al. 2009). In addition, the data collection usually takes place within a short time period under specific, normally favourable weather conditions. Therefore, recording many variables for a long period of time without human interference should overcome most of these issues and provide data that reflect the naturalistic driving behaviour. The second Strategic Highway Research Program (SHRP 2) has been designed to collect data about driving behaviour for a large sample of participants and for a long period without any human interference (Campbell 2012). Therefore, it has the potential of providing more realistic data over other approaches.

The SHRP 2 was authorized in 2005, and is managed by the Transportation Research Board (TRB) on behalf of the National Research Council, to conduct the largest and most comprehensive research to study the naturalistic driving behaviour (Dingus et al. 2015). 
The study has recorded up to three years of all trips for more than 3,100 volunteer drivers of ages 16-80 in six states in USA: Florida, Indiana, North Carolina, New York, Pennsylvania, and Washington. The data acquisition system (DAS) installed in vehicles included six primary components as shown in Figure 3.2; NextGen main unit (computing engine for the system), head unit, network box, radar, radar interface box, and solid-state data drive. The recorded data include vehicle speed, acceleration, braking, vehicle controls, lane position, forward radar, and video views to the front and rear of the vehicle and on the driver's face and hands. The Naturalistic Driving Study (NDS) dataset contains excess of $33,000,000$ travel miles from some 3,800 vehicle-years of driving - more than 4 petabytes of data (Dingus et al. 2015).

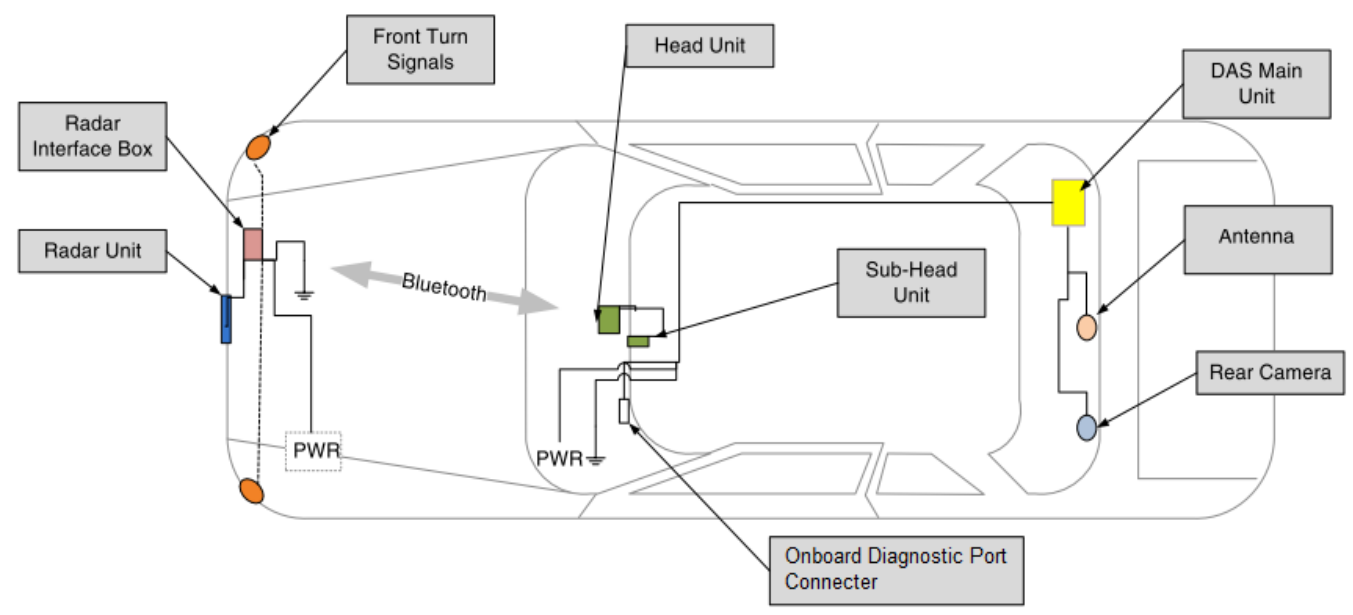

Figure 3.2: Typical Data Acquisition System Components (Dingus et al. 2015).

\section{a. Site and Curves Selection}

Considering the presence of different terrain types, varying weather conditions, and availability of the naturalistic driving study data through Virginia Tech Transportation 
Institute (VTTI), road geometric data and collision data through the Washington State Department of Transportation (WSDOT) and Highway Safety Information System (HSIS), the selected curve sites for this research were all located in Washington State. Based on the number of participants and trips provided in Insight Data Access Website (Figure 3.3), which is managed by VTTI, several two-lane highway segments in rolling and mountainous terrains in rural area were primarily selected. The line colours in the figure represents the frequency intensity of trips and number of participants on each road segment.

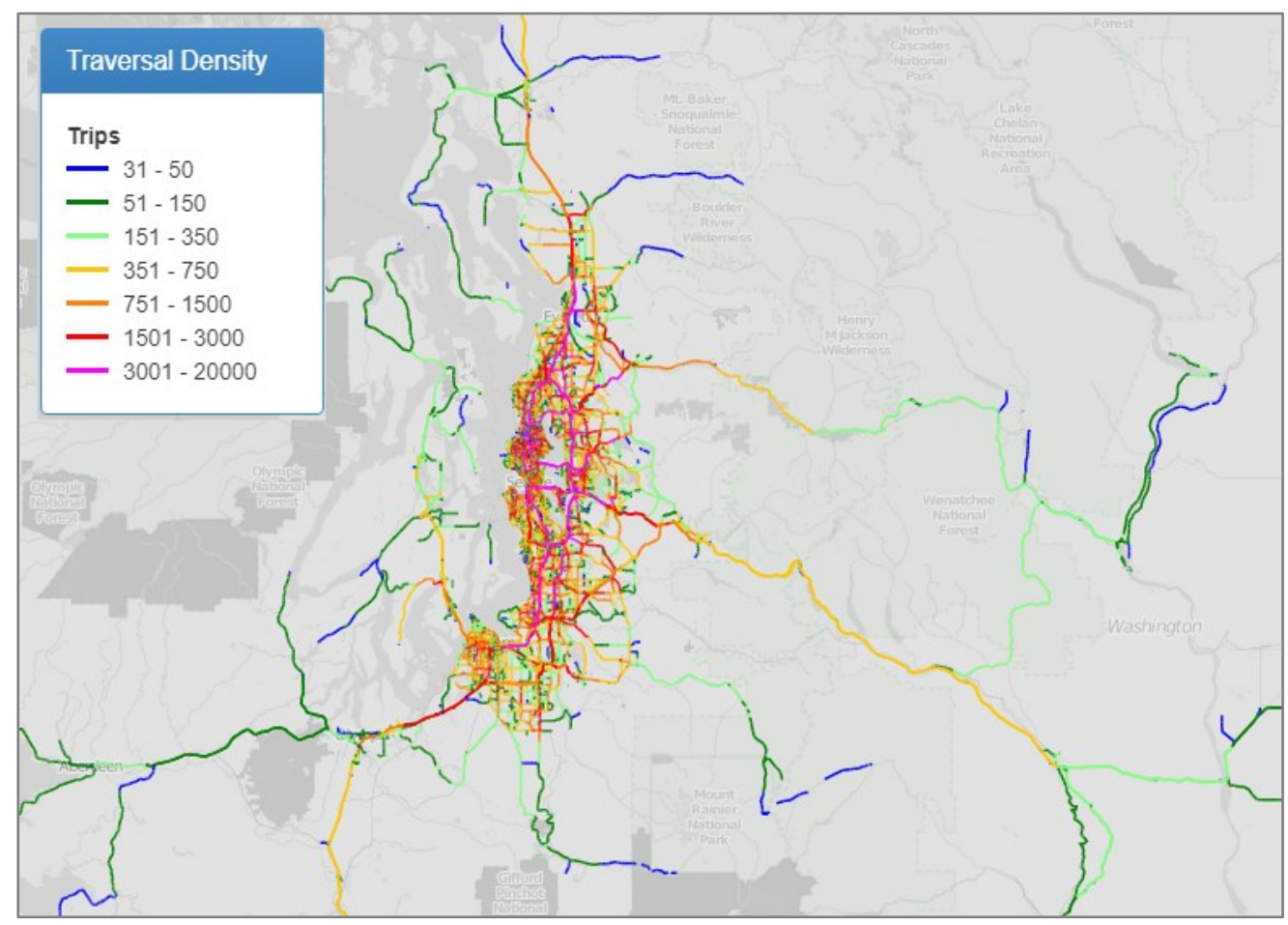

Figure 3.3: Number of Trips for Road Segments in Washington State (Insight Data Access Website).

Data were further screened to identify appropriate horizontal curves on two-lane arterial highways in rolling and mountainous terrains and rural areas and ultimately select 
24 curves in rolling terrain and 25 curves in mountainous terrain. The location of these curves on highway segments are shown in Figure 3.4. Fitzpatrick and Carlson (2002) showed that the terrain might not affect speed on curve. However, considering the effect of grade on the driving behaviour was the reason behind selecting the curves on rolling and mountainous terrains. Each selected curve is preceded by an independent tangent, which should be long enough for vehicles to reach their desired speeds independently from the curvature on either side of the tangent. Fitzpatrick et al (1999) suggested that drivers would be travelling at the tangent's desired speed if they are at least $200 \mathrm{~m}$ away from the curve. Therefore, the threshold for an independent tangent between two successive curves in this study was set at $400 \mathrm{~m}$. In rolling terrain, 12 curves were preceded by independent tangents in both directions and were therefore considered in the two directions. The remaining 12 curves were preceded by an independent curve in one direction and were considered in that direction only. On the other hand, only 4 curves were considered in the two directions in the mountainous terrain. Thus, the total number of curves in rolling and mountainous terrains, considering each direction as a unique curve, were 36 and 29 , respectively. It should be noted that there are five posted speed limits (ranging from $40 \mathrm{mi} / \mathrm{h}(64.37 \mathrm{~km} / \mathrm{h}$ ) to $60 \mathrm{mi} / \mathrm{h}(114.36 \mathrm{~km} / \mathrm{h}))$ in rolling terrain and only one posted speed limit on mountainous terrain $(60 \mathrm{mi} / \mathrm{h}(96.56 \mathrm{~km} / \mathrm{h}))$.

The total number of files provided by VTTI was 4,598. Each file provides data for one trip on one segment. Each segment (file) contains at least one curve. The total number of trips extracted from the NDS was 3,503 and 2,731 for rolling and mountainous terrains, respectively. The trip data were screened to remove trips that were missing essential information such as speed or GPS coordinates or speed. Approximately, $18 \%$ of the trips 
were discarded ending up with a total number of trips of 2,874 and 2,316 on curves in rolling and mountainous terrains, respectively.

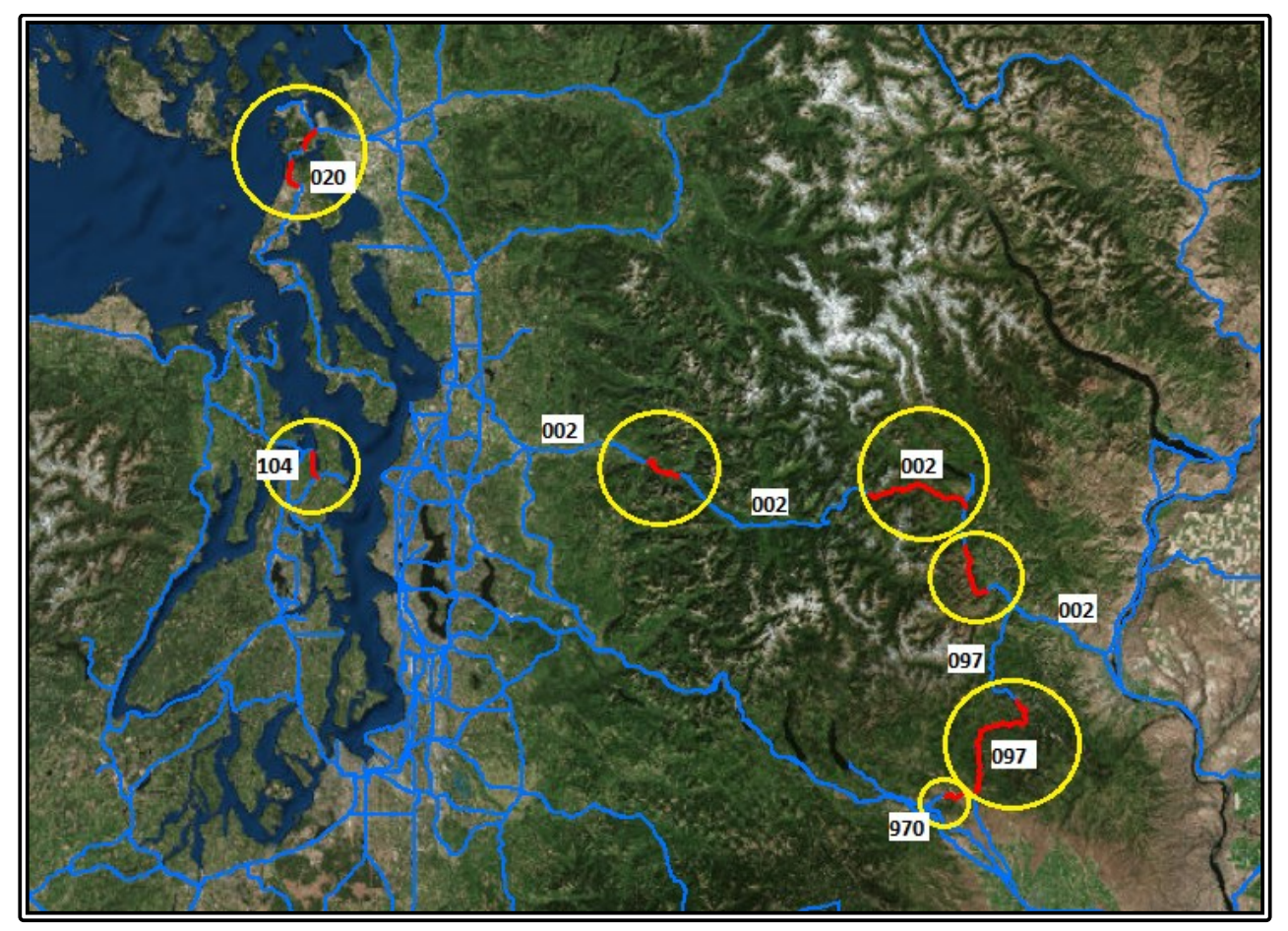

Figure 3.4: Location of Segments in Washington State (ArcMap-WSDOT database).

For uniformity, the trips were divided into daytime and night. Daytime trips are those occurring after the civil twilight in the morning and before the civil twilight in the evening, where civil twilight is defined as the instant in the morning or evening when the centre of the sun is at a depression angle of $6^{\circ}$ below an ideal horizon (Geoscience 2017). The distribution of trips based on the day time is shown in Figure 3.5. Approximately $91 \%$ of the trips had taken place during the daytime. 


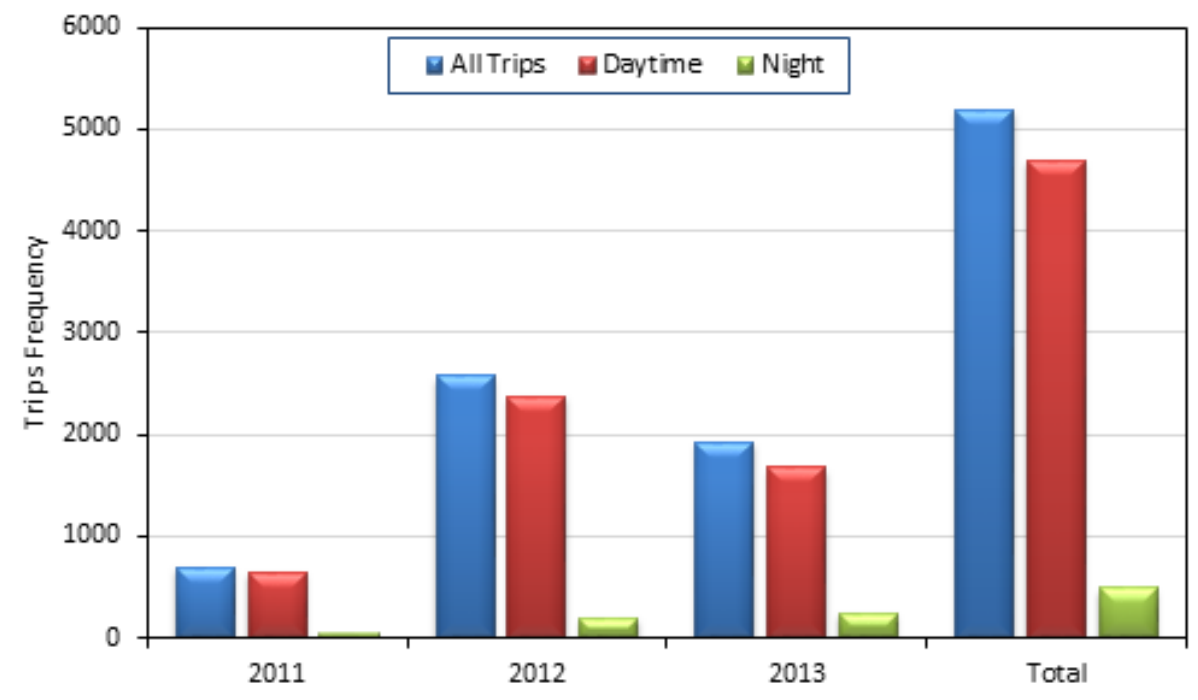

Figure 3.5: Trips Distribution in Daytime and Night.

Figure 3.6 shows the distribution of trips classified as daytime and night by month of the year and time of the day. As shown in the figure, most trips occurred in the daytime, and the night time sample may not be big enough for statistical analysis and modelling.

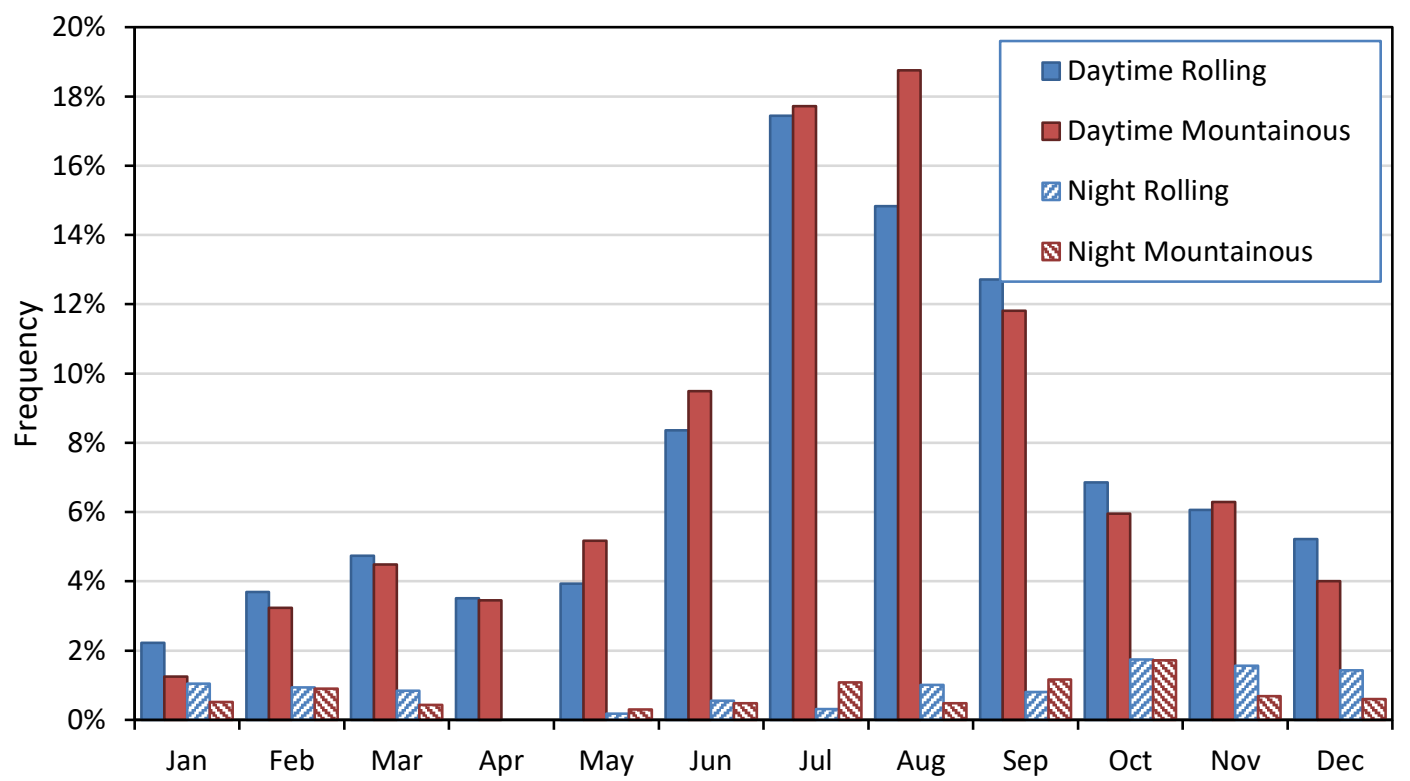

Figure 3.6: Trip Distributions by Month of the Year and Time of the Day. 
In addition, the trip data were matched with weather conditions to identify the prevalent weather conditions during each trip. Daily weather conditions data for three years (2011-2013) of seven selected weather stations were obtained from the Office of Washington State Climatologist, where each curve was approximately 2 to $9 \mathrm{~km}$ away from at least one station. Trips distribution according to weather conditions (clear, raining, and snowing) is shown in Figure 3.7. The percentages of trips that took place in favourable weather conditions and in rain were approximately 78 and $21 \%$, respectively. The number of trips that occurred in snowy weather conditions represents about $1 \%$ of the trips, thus the sample is not statistically big enough to be modelled for snow weather conditions.

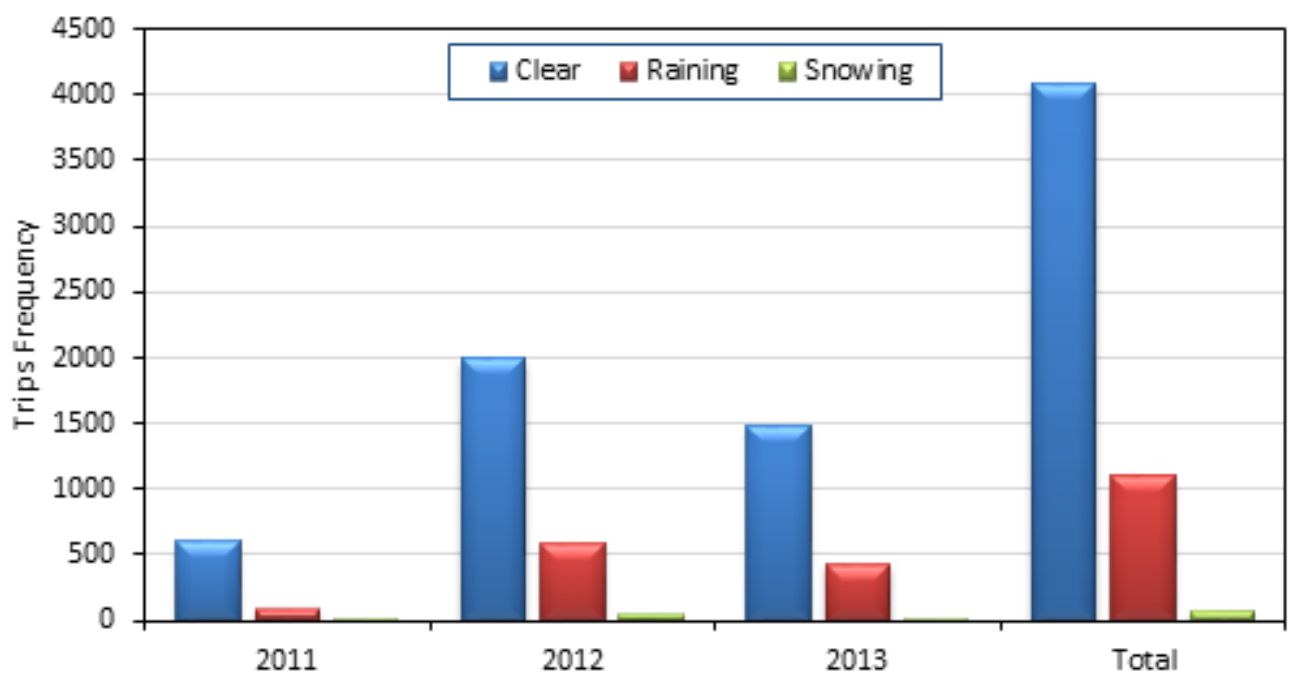

Figure 3.7: Trips Frequency According to Weather Conditions.

The total number of trips during daytime and favourable weather conditions in rolling and mountainous terrain were 1,952 and 1,816, respectively. The lowest number of unique drivers and total trips on any curve was 30 and 34, respectively. It should be noted that these trips were driven by 178 driving test participants ( 98 males and 80 females). Figure 3.8 presents the distributions of participants age and profession and the distribution of 
vehicle types used in these trips. As shown in the figure, four types of light vehicles were used: passenger car (PC), sport and utility vehicle (SUV), van and minivan, and pickup/light truck vehicles.

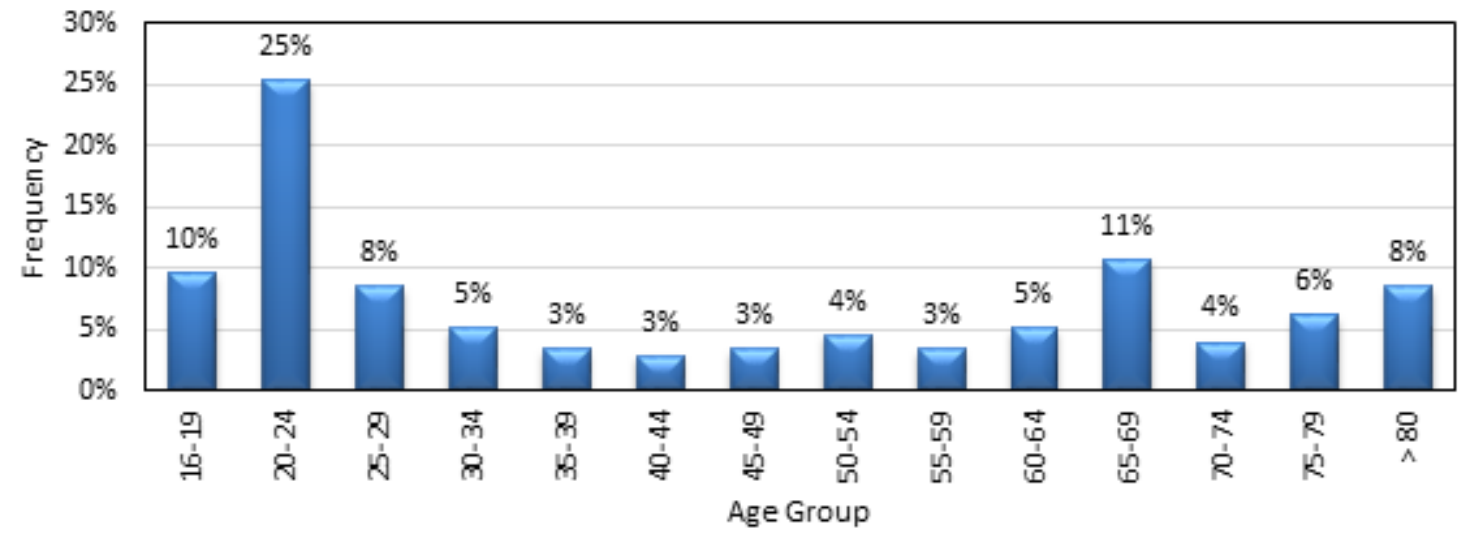

(a) Drivers' Age Groups.

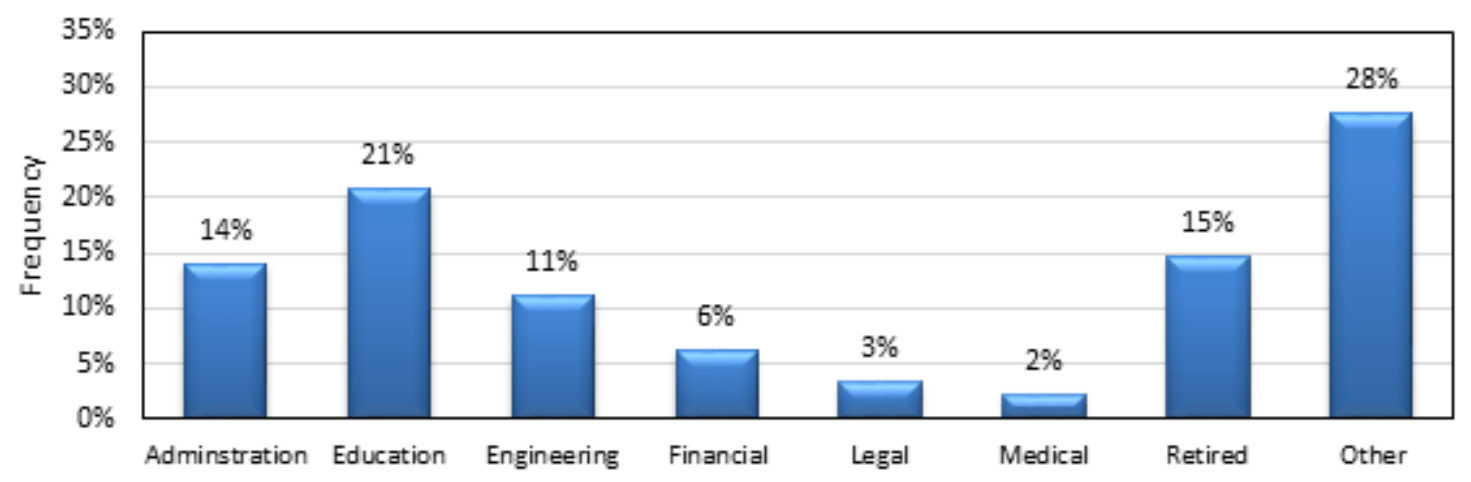

(b) Drivers' Professions.

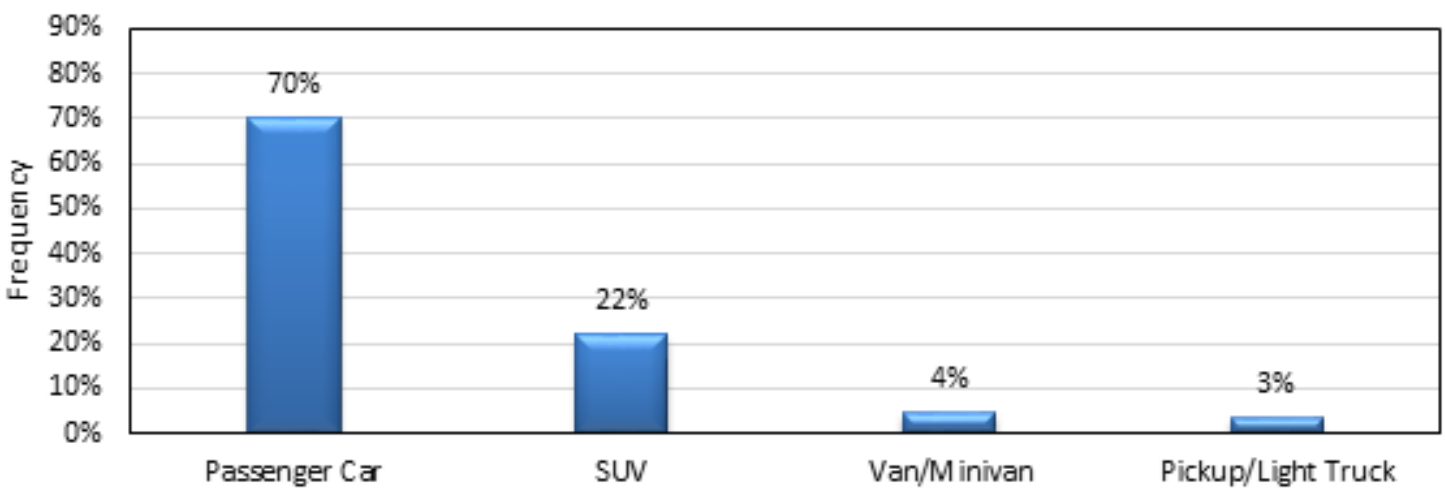

(c) Vehicle Types.

Figure 3.8: Distributions of Driving Test Participants Age and Profession and Vehicle Type. 
The final trip data were matched with the roadway information database to determine the points in the trip file that correspond to beginning and end of curve (BC and EC). The GPS data and $\mathrm{BC} / \mathrm{EC}$ coordinates were used to determine the points where the vehicle is closest to $\mathrm{BC} / \mathrm{EC}$. Because speed data were available every $0.1 \mathrm{~s}$ while the GPS data were available every $1.0 \mathrm{~s}$, the vehicle locations at $\mathrm{BC} / \mathrm{EC}$ were refined by interpolating the location based on vehicle speed and time stamp.

Another challenge in extracting the data from the NDS was to interpret the vehicle headway data. According to Virginia Tech Transportation Institute (VTTI), the trip data would not include headway information if no objects were detected ahead of the host vehicle at the maximum detection range of $200 \mathrm{~m}$ or if detected objects were not classified as a lead vehicle. Therefore, points where data were available except for headway information were classified as free-flow conditions, if sensors were sampling at same rate.

\section{b. Variables Selection}

The parameters recorded in the NDS include; speed, lateral acceleration, longitudinal acceleration/deceleration, date and time of trip, GPS coordinates, light intensity, and headway distance. The speed of a vehicle in the data was a GPS speed at a frequency of 1 $\mathrm{Hz}$ (a measurement every second) with a resolution of $0.02 \mathrm{~km} / \mathrm{h}$ and accuracy of $\pm 1 \mathrm{~km} / \mathrm{h}$. In addition, vehicle speed and lateral and longitudinal accelerations were measured by equipment installed on each vehicle at a rate of $10 \mathrm{~Hz}$ (a measurement every 0.1 seconds) (InSight 2016). The latter measurements were used in the analysis. The geometric data of the horizontal curves were obtained from two agencies. The first set was obtained from the Highway Safety Information System (HSIS) database. The second set of geometric data was obtained from Washington State Department of Transportation (WSDOT), which 
included additional information as explained in Section 3.6.3. Based on these two datasets, the geometric characteristics of the selected curves are shown in Table 3-3.

Table 3-3: Geometric Data of Horizontal Curves.

\begin{tabular}{|c|c|c|c|c|c|c|c|c|c|}
\hline $\begin{array}{c}\text { Curve } \\
\text { ID }^{\mathrm{a}}\end{array}$ & $R(\mathrm{~m})$ & $L(\mathrm{~m})$ & $e$ & $\mathrm{TD}^{\mathrm{b}}$ & $\begin{array}{c}\text { Curve } \\
\text { ID }^{\mathrm{a}}\end{array}$ & $R(\mathrm{~m})$ & $L(\mathrm{~m})$ & $e$ & $\mathrm{TD}^{\mathrm{b}}$ \\
\hline RT 01 & 218 & 257 & 0.080 & $\mathrm{~L}$ & MT 01 & 348 & 511 & 0.060 & $\mathrm{~L}$ \\
\hline RT 02 & 437 & 186 & 0.070 & $\mathrm{~L}$ & MT 02 & 289 & 319 & 0.076 & $\mathrm{~L}$ \\
\hline RT 03 & 218 & 158 & 0.080 & $\mathrm{~L}$ & MT 03 & 349 & 252 & 0.060 & $\mathrm{R}$ \\
\hline RT 04 & 218 & 158 & 0.080 & $\mathrm{R}$ & MT 04 & 582 & 496 & 0.032 & $\mathrm{R}$ \\
\hline RT 05 & 291 & 421 & 0.068 & $\mathrm{~L}$ & MT 05 & 635 & 763 & 0.045 & $\mathrm{~L}$ \\
\hline RT 06 & 388 & 276 & 0.068 & $\mathrm{R}$ & MT 06 & 291 & 320 & 0.072 & $\mathrm{~L}$ \\
\hline RT 07 & 388 & 276 & 0.068 & $\mathrm{~L}$ & MT 07 & 582 & 424 & 0.053 & $\mathrm{~L}$ \\
\hline RT 08 & 291 & 297 & 0.068 & $\mathrm{~L}$ & MT 08 & 698 & 507 & 0.060 & $\mathrm{R}$ \\
\hline RT 09 & 291 & 297 & 0.068 & $\mathrm{R}$ & MT 09 & 698 & 507 & 0.060 & $\mathrm{~L}$ \\
\hline RT 10 & 318 & 261 & 0.060 & $\mathrm{R}$ & MT 10 & 279 & 201 & 0.096 & $\mathrm{~L}$ \\
\hline RT 11 & 318 & 261 & 0.060 & $\mathrm{~L}$ & MT 11 & 303 & 381 & 0.070 & $\mathrm{R}$ \\
\hline RT 12 & 274 & 254 & 0.080 & $\mathrm{R}$ & MT 12 & 283 & 308 & 0.060 & $\mathrm{R}$ \\
\hline RT 13 & 274 & 254 & 0.080 & $\mathrm{~L}$ & MT 13 & 349 & 277 & 0.067 & $\mathrm{R}$ \\
\hline RT 14 & 274 & 164 & 0.080 & $\mathrm{~L}$ & MT 14 & 349 & 277 & 0.067 & $\mathrm{~L}$ \\
\hline RT 15 & 274 & 164 & 0.080 & $\mathrm{R}$ & MT 15 & 332 & 124 & 0.060 & $\mathrm{~L}$ \\
\hline RT 16 & 146 & 120 & 0.080 & $\mathrm{~L}$ & MT 16 & 279 & 116 & 0.096 & $\mathrm{~L}$ \\
\hline RT 17 & 174 & 299 & 0.100 & $\mathrm{~L}$ & MT 17 & 410 & 587 & 0.068 & $\mathrm{~L}$ \\
\hline RT 18 & 297 & 313 & 0.080 & $\mathrm{R}$ & MT 18 & 436 & 270 & 0.062 & $\mathrm{~L}$ \\
\hline RT 19 & 250 & 326 & 0.073 & $\mathrm{R}$ & MT 19 & 332 & 225 & 0.060 & $\mathrm{~L}$ \\
\hline RT 20 & 349 & 271 & 0.060 & $\mathrm{~L}$ & MT 20 & 436 & 158 & 0.061 & $\mathrm{~L}$ \\
\hline RT 21 & 349 & 262 & 0.092 & $\mathrm{~L}$ & MT 21 & 436 & 158 & 0.060 & $\mathrm{R}$ \\
\hline RT 22 & 349 & 262 & 0.092 & $\mathrm{R}$ & MT 22 & 698 & 442 & 0.055 & $\mathrm{~L}$ \\
\hline RT 23 & 291 & 174 & 0.068 & $\mathrm{~L}$ & MT 23 & 698 & 442 & 0.037 & $\mathrm{R}$ \\
\hline RT 24 & 291 & 174 & 0.068 & $\mathrm{R}$ & MT 24 & 434 & 258 & 0.073 & $\mathrm{~L}$ \\
\hline RT 25 & 318 & 298 & 0.050 & $\mathrm{R}$ & MT 25 & 582 & 244 & 0.058 & $\mathrm{~L}$ \\
\hline RT 26 & 318 & 298 & 0.050 & $\mathrm{~L}$ & MT 26 & 696 & 371 & 0.053 & $\mathrm{R}$ \\
\hline RT 27 & 250 & 177 & 0.073 & $\mathrm{R}$ & MT 27 & 388 & 488 & 0.086 & $\mathrm{R}$ \\
\hline RT 28 & 250 & 177 & 0.073 & $\mathrm{~L}$ & MT 28 & 498 & 303 & 0.060 & $\mathrm{~L}$ \\
\hline RT 29 & 349 & 445 & 0.090 & $\mathrm{~L}$ & MT 29 & 873 & 161 & 0.039 & $\mathrm{R}$ \\
\hline RT 30 & 349 & 445 & 0.090 & $\mathrm{R}$ & & & & & \\
\hline RT 31 & 218 & 196 & 0.080 & $\mathrm{R}$ & & & & & \\
\hline RT 32 & 291 & 165 & 0.068 & $\mathrm{~L}$ & & & & & \\
\hline RT 33 & 291 & 165 & 0.068 & $\mathrm{R}$ & & & & & \\
\hline RT 34 & 146 & 120 & 0.080 & $\mathrm{R}$ & & & & & \\
\hline RT 35 & 174 & 299 & 0.100 & $\mathrm{R}$ & & & & & \\
\hline RT 36 & 297 & 313 & 0.080 & $\mathrm{R}$ & & & & & \\
\hline
\end{tabular}


Several variables were extracted from the NDS database to study driving behaviour and comfort threshold on horizontal curves. A Matlab script was developed to extract the instantaneous speed at different points on the curve and approach tangent in addition to average value of observations within a specific distance or time period of these points (Dhahir and Hassan 2018). Details for the Matlab script to extract speed parameters were provided in Section 5.2.1. Consequently, several speed variables along each curve and the approach tangent were extracted for each trip in the NDS database as follows:

1. $V_{B C}, V_{M C}$, and $V_{E C}$ : instantaneous speed measurements at the beginning, middle, and end of curve (BC, $\mathrm{MC}$, and $\mathrm{EC})$, respectively.

2. $V_{T B C}, V_{T M C}$, and $V_{T E C}$ : speeds at $\mathrm{BC}, \mathrm{MC}$, and $\mathrm{EC}$, respectively, estimated by averaging speed observations within a short period of time of $1.0 \mathrm{~s}$ around the relevant point. Specifically, the ranges used to average speed observations are BC to $(\mathrm{BC}+1.0 \mathrm{~s}),(\mathrm{MC}-0.5 \mathrm{~s})$ to $(\mathrm{MC}+0.5 \mathrm{~s})$, and $(\mathrm{EC}-1.0 \mathrm{~s})$ to $\mathrm{EC}$.

3. $V_{D B C}, V_{D M C}$, and $V_{D E C}$ : speeds at $\mathrm{BC}, \mathrm{MC}$, and $\mathrm{EC}$, respectively, estimated by averaging speed observations within a short distance of $50 \mathrm{~m}$ around the relevant point. Specifically, the ranges used to average speed observations are $\mathrm{BC}$ to $(\mathrm{BC}+$ $50 \mathrm{~m}),(\mathrm{MC}-25) \mathrm{m}$ to $(\mathrm{MC}+25 \mathrm{~m})$, and $(\mathrm{EC}-50) \mathrm{m}$ to $\mathrm{EC}$.

4. $V_{c r v-a v g}$ : mean curve speed, estimated as the average of all speed observations along the curve.

5. $V_{c r v-\min }$ : minimum curve speed, estimated as the minimum speed observation recorded along the curve.

6. $V_{c r v-\max }$ : maximum curve speed, estimated as the maximum speed observation recorded along the curve. 
7. $V_{a p p-\max }$ : maximum approach tangent speed, estimated as the maximum speed observation along $200 \mathrm{~m}$ before $\mathrm{BC}$.

8. $V_{a p p}$ : instantaneous approach tangent speed observed at $200 \mathrm{~m}$ before BC.

9. $V_{a p p-a v g}$ : approach tangent speed, estimated as the average of all speed observations along 50-m distance from $(B C-250 \mathrm{~m})$ to $(\mathrm{BC}-200 \mathrm{~m})$.

For studying driving behaviour on horizontal curve and developing models to determine driver comfort threshold, the following variables were extracted for each trip:

1. $a_{L}$ : driver Comfort threshold, estimated as the minimum lateral acceleration experienced by a driver when vehicle is traveling at approximately constant speed.

2. $V_{c m f-a v g}$ : mean speed corresponding to driver comfort threshold, estimated as the average of all speed observations along the segment corresponding to driver comfort threshold.

3. $V_{c m f-\min }$ : minimum speed corresponding to driver comfort threshold, estimated as the minimum of all speed observations along the segment corresponding to driver comfort threshold.

It should be noted that the objective behind selecting more than one parameter to determine the speed and comfort threshold for approximately the same location or situation on the curve is to examine whether there are differences between them. Thus, it will provide the opportunity to use them interchangeably in developing models and to select the parameters that would suite the model better. 


\subsubsection{Pavement Friction Data}

The frictional data were provided by Washington State Pavement Management System (WSPMS) (Pavement Office). Five datasets have been provided as shown in Figure 3.9. The Survey Units dataset includes main data and it is used to connect with other datasets. The Contract History dataset includes construction, and minor and major rehabilitation activities for the duration from 1981 to 2014. The database includes the following information; historical data for maintenance (date and type of maintenance, location, ESAL, pavement type and thickness, and cost), distress details, and survey details (rutting, International Roughness Index (IRI), Pavement Structural Condition (PSC), Pavement Profile Condition (PPC), and Pavement Rutting Condition (PRC)). The scores of the latter three variables range from 0 (very poor) to 100 (very good) and calculated using the following equations:

$$
\begin{aligned}
& P S C=100-15.8 \sqrt{D D} \\
& P P C=100-0.05355(I R I)^{1.268} \\
& P R C=100-113.288(R u t)^{1.18}
\end{aligned}
$$

Where $D D=$ total distress deducted, $I R I=$ International Roughness Index, and $R u t=$ rutting depth (inch). 


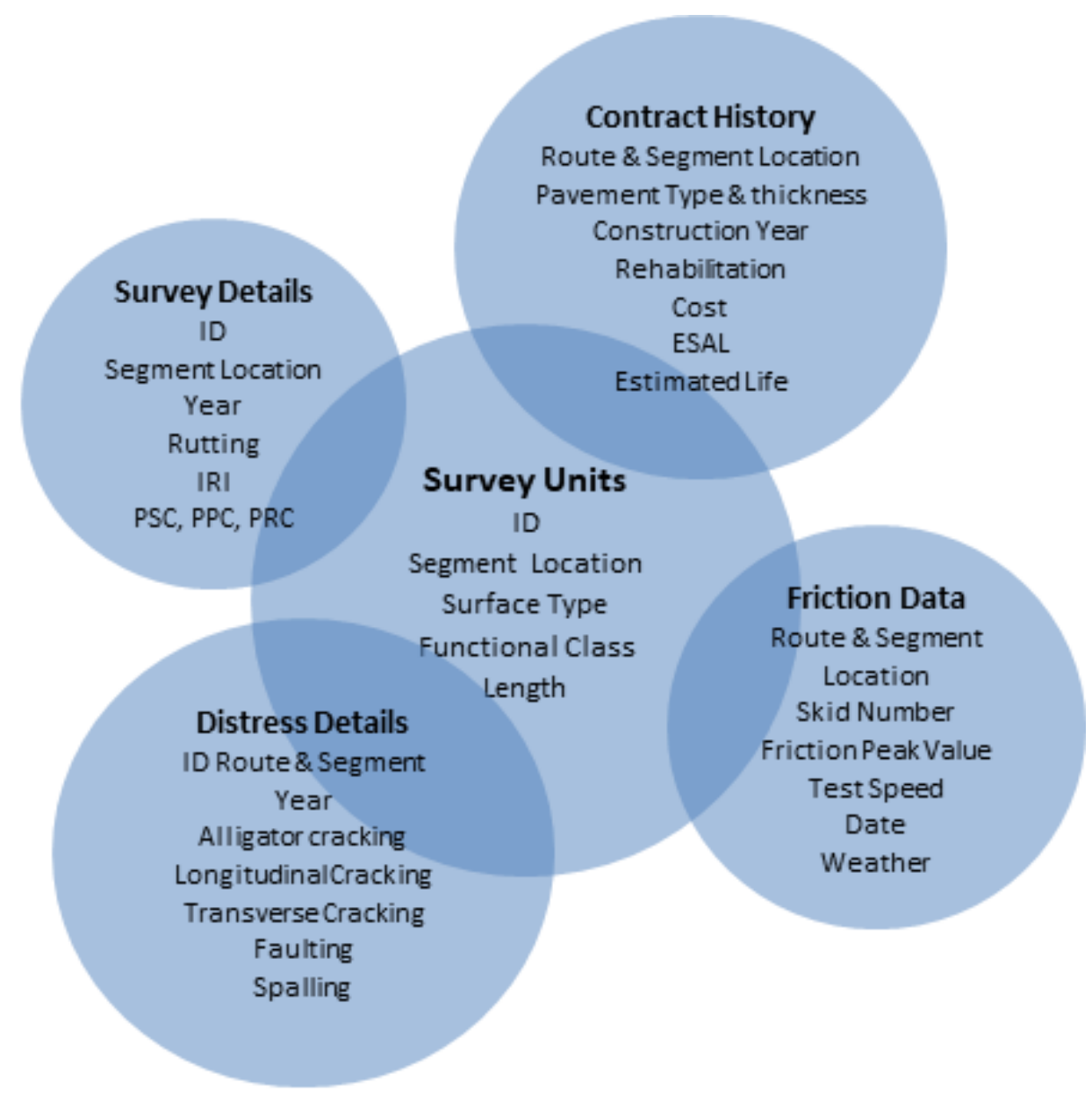

Figure 3.9: Pavement Database- Provided by Washington State Transportation Department.

The Friction Data dataset includes measured friction of pavement for the period from 2007 to 2014 for each roadway segment. The variables included in the dataset were the location and date of the test, skid number, peak value of the skid number, test speed, and weather conditions. It should be noted that WSDOT uses the locked wheel skid trailer to measure pavement friction. The friction is reported in the database in terms of the skid number at a standard measuring speed of $40 \mathrm{mph}$. Since speed limits on most local roads are slower than $40 \mathrm{mph}$, and highways are normally around $60 \mathrm{mph}$ and due to various 
factors, such as the traffic congestion, intersections, the driving speed on highways may run as low as $20 \mathrm{mph}$. Therefore, the collected skid numbers is adjusted to speed of $40 \mathrm{mph}$ according to ASTM E1960-07 requirements (ASTM 2011). The equation developed by Wisconsin Department of Transportation in 2012 is used to adjust skid number based on measurement speed (Gokhale and Bischoff 2012).

$$
F R_{40}=F R S-0.57(40-S)
$$

Where $F R_{40}=$ calculated friction at speed of $40 \mathrm{mph}, F R S=$ measured friction at a slip speed of $S$ (mph), and $S=$ driving speed when collecting the pavement friction (mph). For the purpose of the research, the reduction factor (0.925) suggested by Lamm et al. (1999) mentioned earlier was used to calculate the available lateral friction. It should be noted that WSDOT measures surface friction for the same segment every two years.

\subsubsection{Roadway Geometric Data}

As mentioned earlier, one set of the geometric data of the horizontal curves was obtained from Highway Safety Information System (HSIS). The database was arranged into three datasets (Figure 3.10); road, grade, and curve. The datasets provide information regarding the radius of the curve, length, central angle, direction of the curve, number and width of lanes, shoulder type and width, and the grade. The location of the curve is referenced by providing the station (in mile post) of the beginning of the curve for every route. 


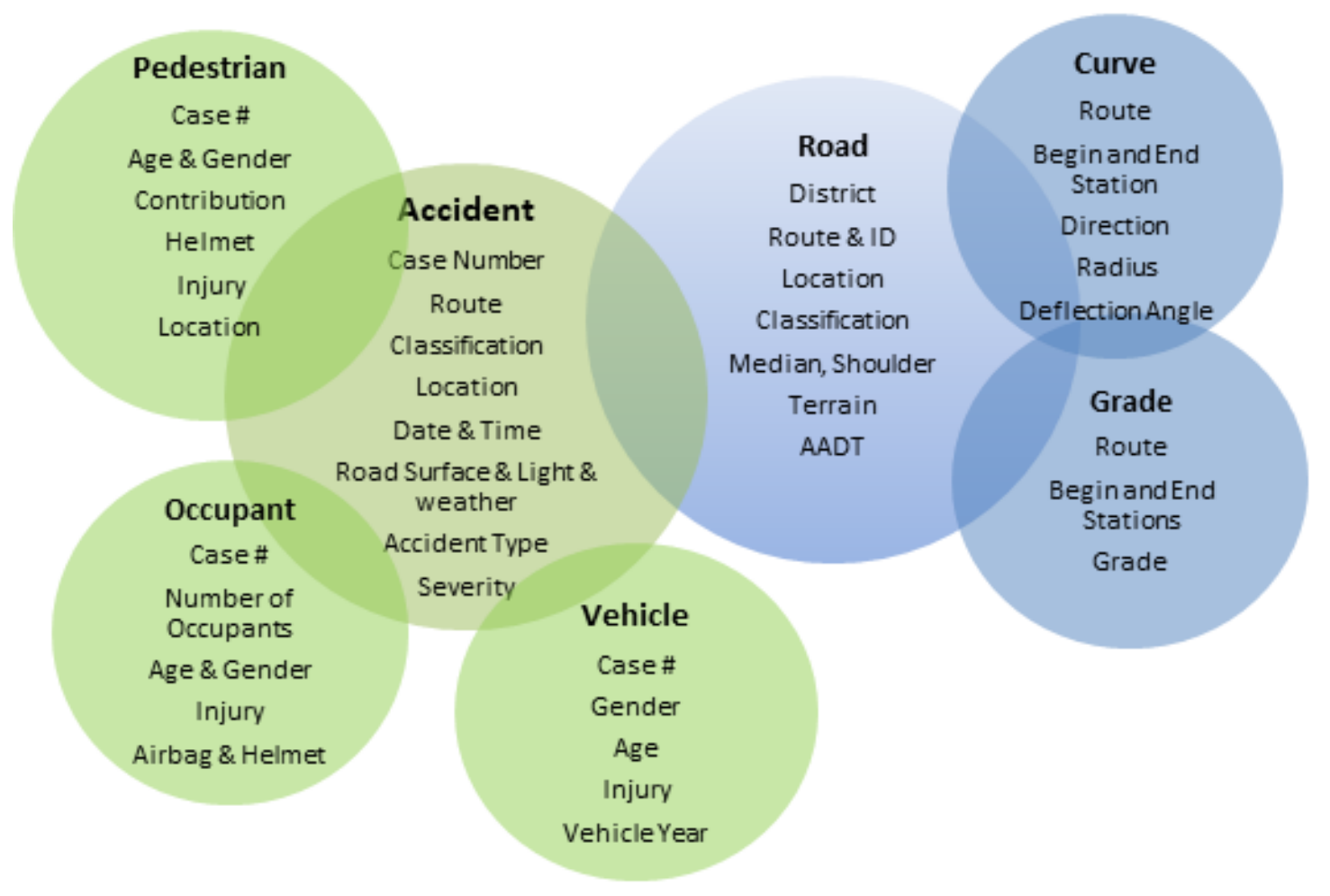

Figure 3.10: Geometry and Collision Datasets (HSIS).

In addition, another set of geometric data were obtained from the Washington State Department of Transportation (WSDOT) as well. The provided data were compared with those of HSIS, and both datasets were found to be identical. However, the datasets provided by WSDOT include additional information such as roadway level of service, legal speed limit, design speed (for some curves), terrain, and historical Annual Average Daily Traffic (AADT). These datasets were arranged and then uploaded into ArcMap software as shown in Figure 3.11. 


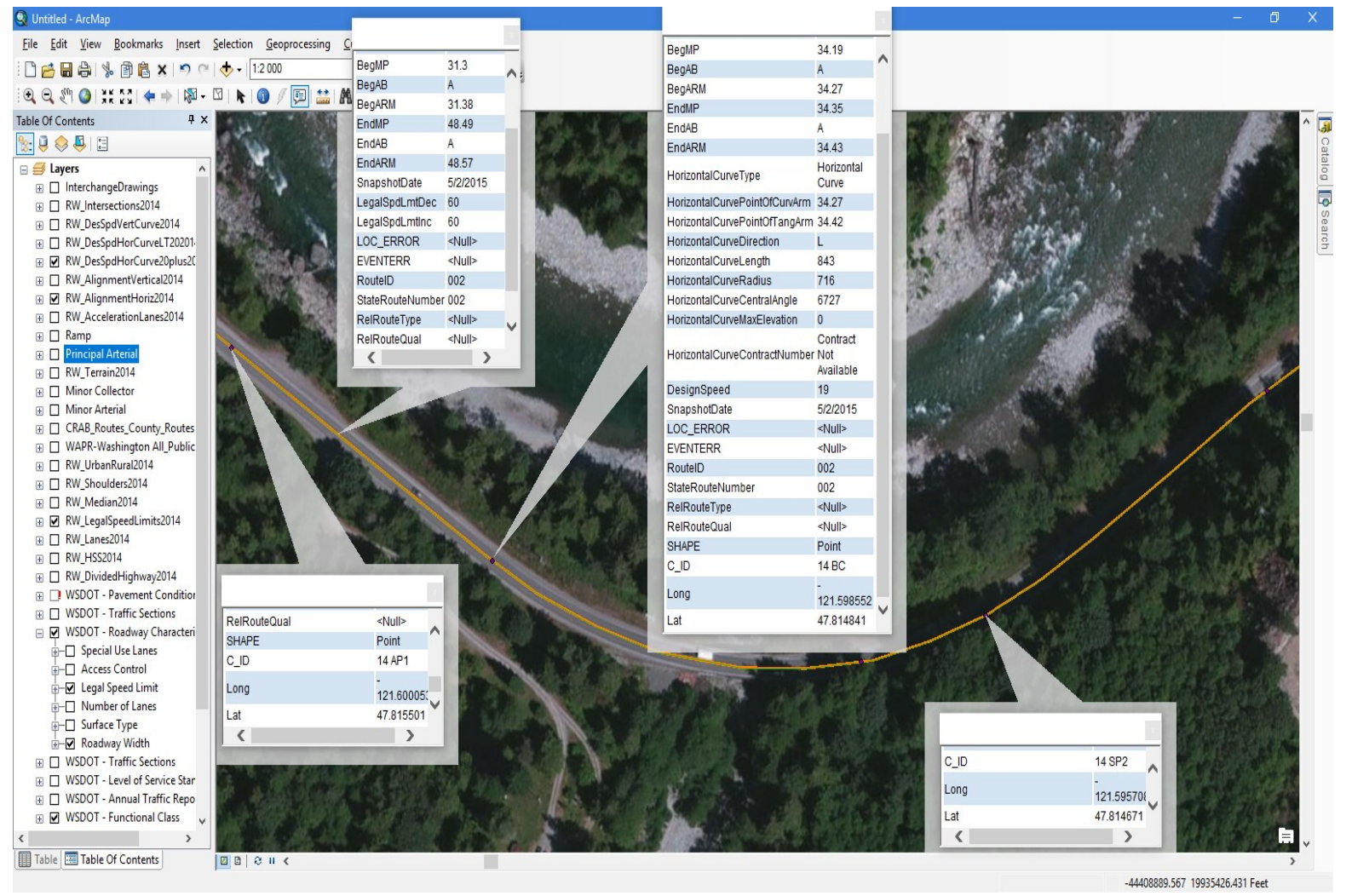

Figure 3.11: Datasets Layers in ArcMap.

The website of WSDOT provides the State Route Tool (SRweb), which is an application that allows users to view digital images of the Washington State Highway System using web browser as shown in Figure 3.12. The application is a powerful tool and can provide portions of the Washington State Highway Log with images at each of the milepost locations for both directions and enabling the user to "drive" the highway at a distance interval of $1 / 100^{\text {th }}$ of a mile. The SRweb can also provide pictures of $360^{\circ}$ at any location. This application was used to check the accuracy of the data, such as the posted speed limit, and to give more information about the horizontal curves and surroundings. It should be noted that the database of SRweb is updated every other year (WSDOT 2017). 


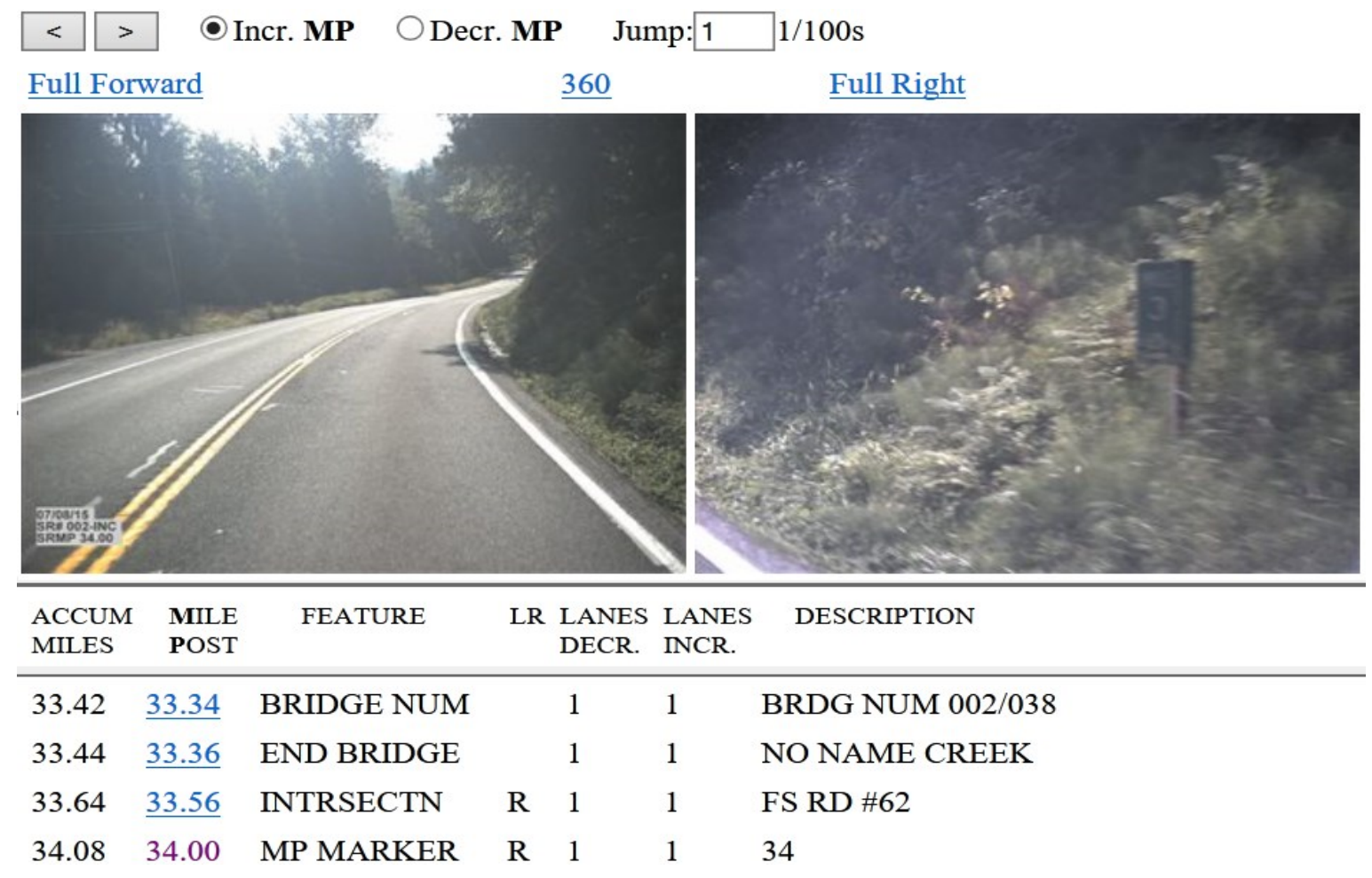

Figure 3.12: State Route Web Tool (WSDOT 2017).

Although, the beginnings of the circular curves were provided by WSDOT in dataset format (numbers in spread sheet files) or in ArcMap layouts (shape files), data of spiral transition curves have not been provided. Therefore, these layouts were exported to AutoCAD software for two reasons:

- To conduct an extra check for the data provided (check the curve with its attributes). This task included checking the radius, deflection angle, and length of curve.

- To determine whether or not the horizontal alignment has spiral transition curves.

As shown in Figure 3.13, the approaches of each curve are extended to establish the deflection angle of the curve. Then, a circle was drawn tangentially to the approaches. 
Lines were drawn from the centre of the circle perpendicularly on the tangents (approaches). Then, measurements are taken for the central angle, deflection angle, radius, and length of the curve and compared with those provided in the datasets. If the measured length of the curve and the length provided in the dataset were equal and the location of the beginning of the curve provided by WSDOT is corresponding with the tangency point, that meant that there is no spiral transition attached to the curve. Otherwise, the horizontal curve would have spiral transition curves.

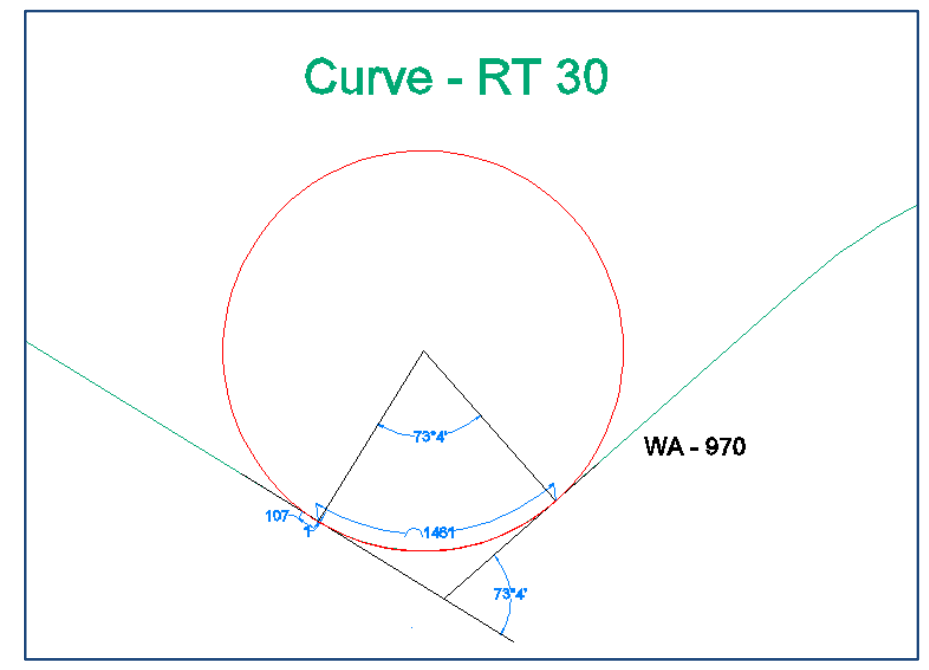

Figure 3.13: Using AutoCAD to Check the Geometric Data of Horizontal Curves.

\subsubsection{Lateral Friction Demand Data}

The CarSIM software was used to determine peak and average lateral friction demand.

Two typical types of vehicles were used in the analysis; sedan passenger car D-class and SUV D-class. The parameters of these vehicles are shown in Table 3-4 (more information are provided in Appendix A). 
Table 3-4: Parameters of Vehicles.

\begin{tabular}{|l|c|c|}
\hline \hline \multicolumn{1}{|c|}{ Item } & Sedan & SUV \\
\hline Sprung mass $(\mathrm{kg})$ & 1,370 & 1,430 \\
\hline Unsprung mass $(\mathrm{kg})$ & 80 (at each axle) & $\begin{array}{c}80 \text { (front axle) and } 100 \\
\text { (rear axle) }\end{array}$ \\
\hline Width of the vehicle $(\mathrm{mm})$ & 1,550 & 1,565 \\
\hline Height of centre of gravity $(\mathrm{mm})$ & 520 & 650 \\
\hline Tire size & $215 / 55 \mathrm{R} 17$ & $255 / 75 \mathrm{R} 16$ \\
\hline \hline
\end{tabular}

These vehicles were simulated to traverse horizontal curves designed for speeds of 50 , 70 , and $90 \mathrm{~km} / \mathrm{h}$. The superelevation rates used for these curves were $6 \%, 8 \%$, and $10 \%$, and the minimum radius for each combination of design speed and superelevation rate was calculated according to Green Book (A Policy 2011) requirements. These simulation runs were performed for vehicles travelling with approximately constant speeds ranging between 40 and $120 \mathrm{~km} / \mathrm{h}$.

\subsubsection{Lateral Acceleration Data}

Although the difference in the magnitude of applied lateral acceleration is not significant among different vehicle type, models were developed to illustrate the effect of grade and vehicle suspension systems on vehicle's occupants. The database used in determining lateral friction demand was also used to calculate the lateral acceleration applied on vehicle's occupants.

\subsubsection{Safety Data}

The datasets mentioned earlier along with safety data were compiled to examine the relationship among roadway geometry, traffic characteristics, reliability indices, and safety 
and ultimately to develop safety performance models. For this dataset, referred to here as safety dataset, three subsets of data were required: geometric parameters, traffic characteristics, and historical safety data. Collision data on curves were obtained from the HSIS database for five years (2009 - 2013). The database provided information for the location of each collision using Linear Referencing System, date of collision, pavement surface conditions (dry, wet, snow, etc.), light condition (day light, dawn, dusk, etc.), collision severity, number of vehicles involved in collision, driver information (gender and age), and weather conditions (clear, raining, snowing, etc.). The location of the collision is reported to the nearest 0.10 mile for over $95 \%$ of the collisions in rural areas (Nujjetty et al. 2014). Collisions corresponding to each curve were considered as "curve related" if they occurred on the curve or within a $50 \mathrm{~m}$ distance before the beginning or after the end point of the curve. The total collisions on the study curves for five years was 546. It should be noted that collisions caused by improper driving such as "driving under the influence of alcohol or drugs" or "apparently asleep" were removed from the dataset.

The frequency distribution of the collisions for five years on the study curves is shown in Figure 3.14. The percentage of collisions on curves (for each collision category) seems to be relatively high compared to curves with zero-collision frequency. When removing collisions that occurred on packed-snow or icy pavement, the percentage of curves with zero-collisions increased to approximately $10 \%$. This means that some of the curves had collisions only in adverse weather conditions (packed-snow or icy pavement). Therefore, more information about the circumstances of these collisions would be helpful in explaining the high number of collision frequency. 


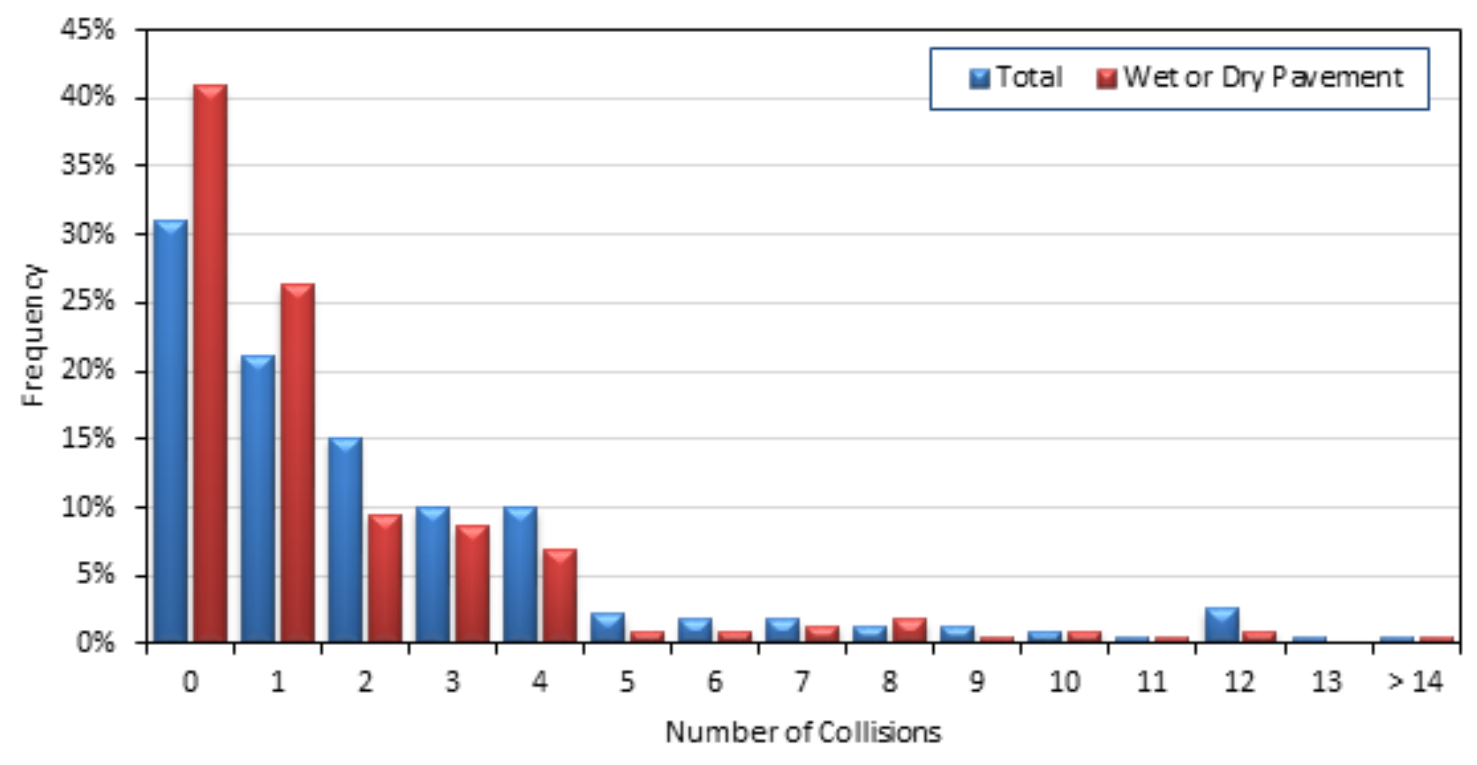

Figure 3.14: Frequency Distribution of the Collisions.

As shown in Figure 3.15, approximately $30 \%$ of collisions occurred in adverse weather conditions (plot a) and about $40 \%$ of them occurred in insufficient light conditions (plot b). Thus, the available sight distance would be most likely limited in these circumstances. Furthermore, the pavement surface conditions were not dry in approximately $45 \%$ of the total collisions and about $28 \%$ of them were in packed-snow and icy conditions. Thus, the available longitudinal and lateral friction might be insufficient for traversing the curves safely. Also, drivers would need more stopping sight distance. Accordingly, the probability of having a collision would increase. 


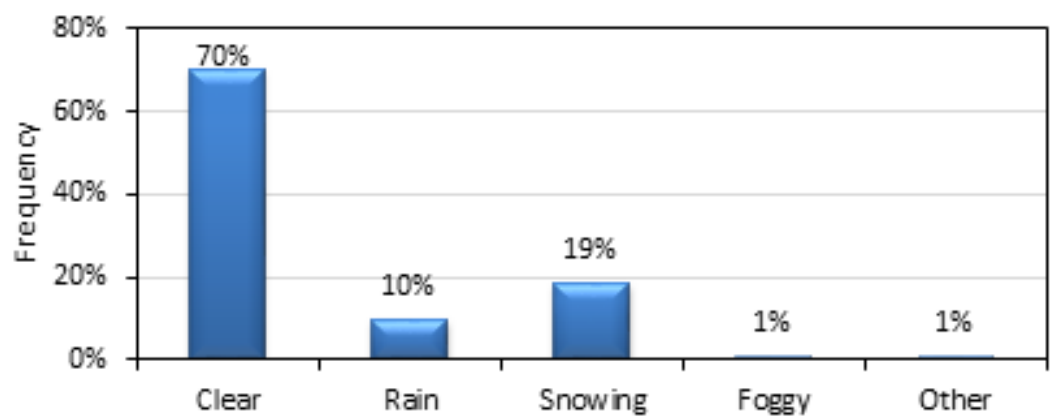

(a) Weather Conditions.

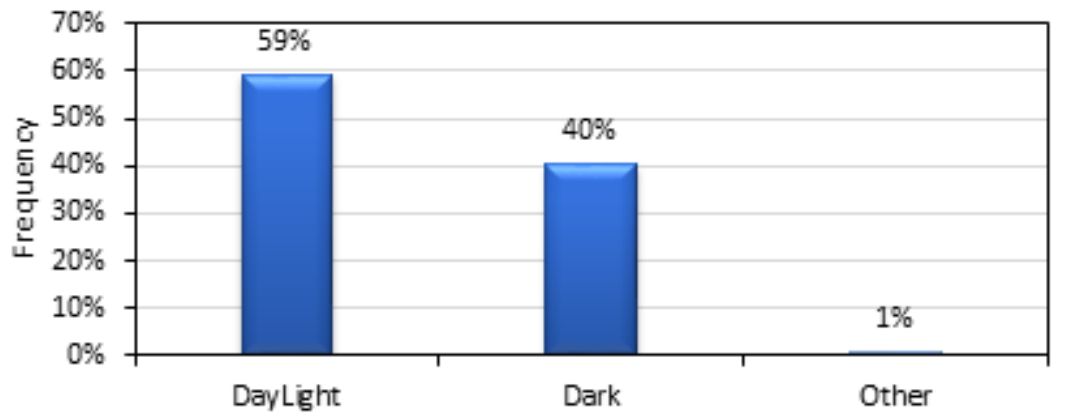

(b) Light Conditions.

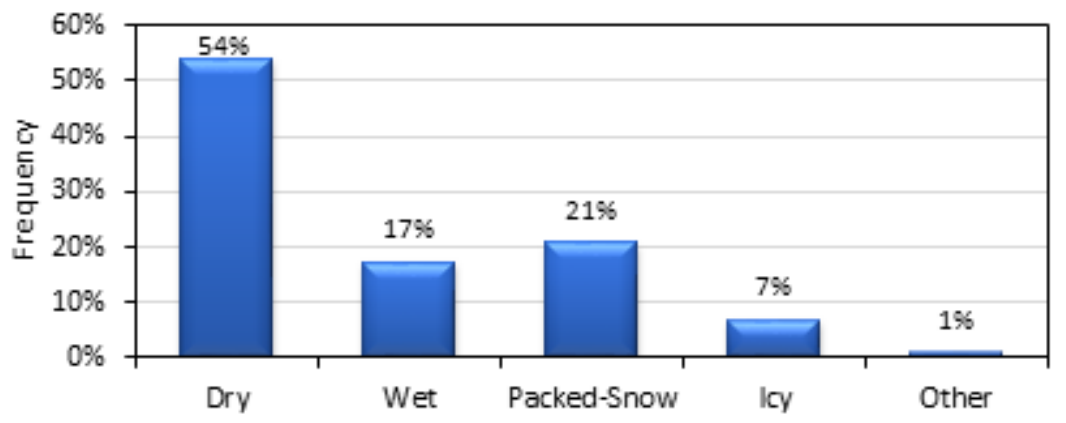

(c) Pavement Surface Conditions.

Figure 3.15: Frequency of Collisions for Weather, Light, and Pavement Conditions.

The total collisions frequency for each of the five years was almost constant as shown in Figure 3.16 a. The highest collision frequencies were recorded for winter months (October-January) as shown in plot b. The safety database shows that Friday recorded the highest weekday collision frequency. This would give an indication about the effect of weather on the safety performance of these curves. 


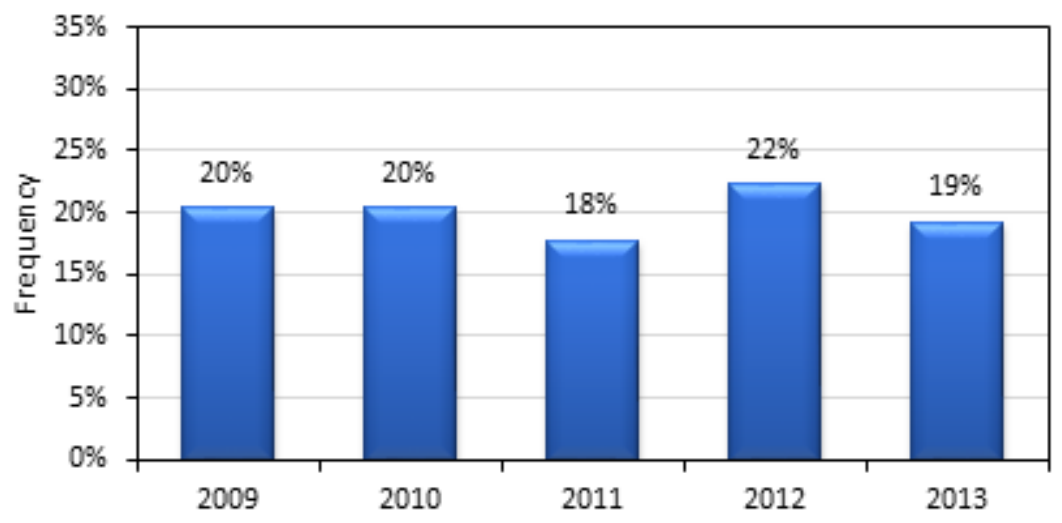

(a) Yearly Collision Frequency.

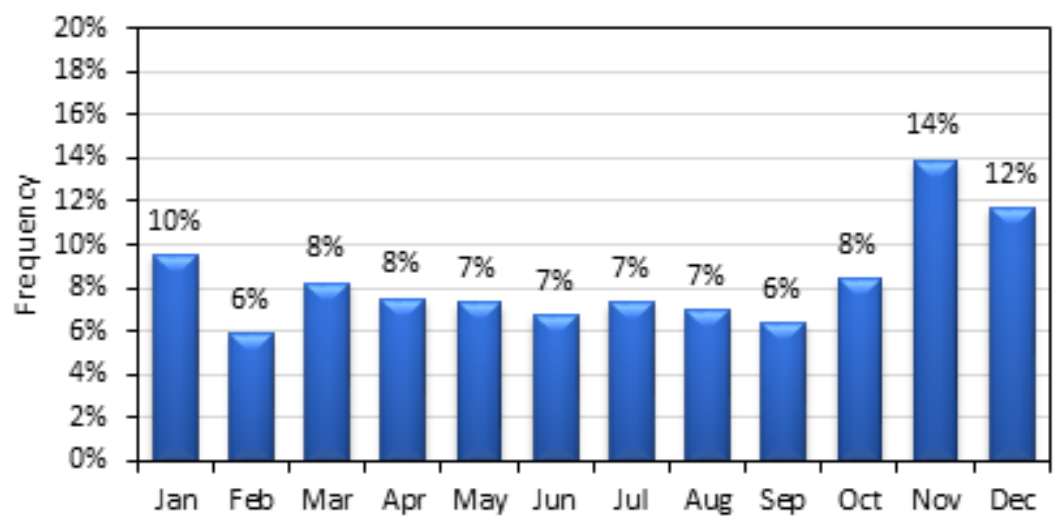

(b) Monthly Collision Frequency.

Figure 3.16: Yearly and Monthly Collision Frequency Distributions.

\subsection{Summary}

Chapter 1 presented the new framework to design horizontal curves. This chapter explained the methodology that was used to accomplish the objectives of this research and provided the guidelines to deliver the new framework to design horizontal curves. The methodology included five major tasks; building master database, conducting preliminary analysis and studying driving behaviour on curves, modelling driver behaviour on curves, conducting reliability analysis, and developing safety performance functions. Several steps 
need to be conducted at every major task.

This chapter also provided the performance function for each design criteria and the influential factors that would affect performance of horizontal curves. Based on these factors, the outputs of the reliability analysis in terms of POF, PNC, $\mathrm{POH}$, and $\mathrm{POF}_{\mathrm{R}}$ need to be adjusted in order to provide more realistic evaluation. In addition, the chapter provided detailed information regarding collecting data and building the master database. The master database comprises six datasets; naturalistic driving study database, curve geometry, traffic characteristics, friction, weather conditions, and safety. A Matlab script was developed to build up the master database. 


\section{CHAPTER FOUR}

\section{PRELIMINARY ANALYSIS}

\subsection{Introduction}

Prior to model development for speed on curve and driver comfort threshold, preliminary analyses were performed to evaluate the statistical characteristics of the speed and driver comfort variables described in the previous chapter and identify and remove the outliers from the data. These statistical tests performed in these preliminary analyses included identifying the headway threshold for free-flow speeds, normality test, analysis of variance (ANOVA), homogeneity of variance, and equality of means. All statistical tests in this chapter were performed using SPSS software.

\subsection{Speed Choice and Headway}

Knowing the relationship between speed choice and headway distance would help in understanding vehicle speed state (i.e. free-flow conditions) and also in modelling speed and comfort threshold. Typically, vehicles are considered in free-flow condition when having a headway $(h)$ of 5 seconds or more (Dimaiuta et al. 2011). However, the relationship between speed choice and headway distance (in seconds) was investigated to identify the headway threshold for free-flow conditions in rolling and mountainous terrains. Based on the headway data collected in the NDS, the speed data, considering posted speed limits, were categorized into six categories as shown in Table 4-1. As shown in the table, the number of readings in the headway category $h \geq 5 \mathrm{~s}$ is relatively high for each speed limit in rolling and mountainous terrains. 
Table 4-1: Count of Readings for Each Headway Category (daytime and favourable Weather Conditions).

\begin{tabular}{|c|c|c|c|c|c|c|}
\hline \multirow{2}{*}{$\begin{array}{l}\text { Headway } \\
\text { Category }\end{array}$} & \multicolumn{6}{|c|}{$\begin{array}{l}\text { Posted Speed Limit km/h } \\
\qquad(\mathrm{mi} / \mathrm{h})\end{array}$} \\
\hline & $\begin{array}{c}64.37 \\
(40)\end{array}$ & $\begin{array}{c}72.42 \\
(45)\end{array}$ & $\begin{array}{c}80.47 \\
(50)\end{array}$ & $\begin{array}{c}88.51 \\
(55)\end{array}$ & $\begin{array}{c}96.56 \\
(60)\end{array}$ & All \\
\hline \multicolumn{7}{|l|}{ Rolling Terrain } \\
\hline & \multicolumn{6}{|c|}{ Curve } \\
\hline $0 \leq h<1$ & 19 & 5 & 28 & 0 & 3 & 55 \\
\hline $1 \leq h<2$ & 71 & 26 & 177 & 16 & 21 & 311 \\
\hline $2 \leq h<3$ & 44 & 11 & 91 & 16 & 9 & 171 \\
\hline $3 \leq h<4$ & 22 & 13 & 58 & 9 & 13 & 115 \\
\hline $4 \leq h<5$ & 8 & 3 & 34 & 5 & 2 & 52 \\
\hline \multirow{2}{*}{$5 \leq h$} & 171 & 66 & 734 & 90 & 136 & 1197 \\
\hline & \multicolumn{6}{|c|}{ Approach } \\
\hline $0 \leq h<1$ & 19 & 10 & 31 & 1 & 5 & 66 \\
\hline $1 \leq h<2$ & 74 & 23 & 182 & 18 & 27 & 324 \\
\hline $2 \leq h<3$ & 47 & 14 & 98 & 15 & 14 & 188 \\
\hline $3 \leq h<4$ & 23 & 6 & 58 & 8 & 9 & 104 \\
\hline $4 \leq h<5$ & 8 & 2 & 18 & 1 & 2 & 31 \\
\hline $5 \leq h$ & 164 & 69 & 735 & 93 & 127 & 1188 \\
\hline \multicolumn{7}{|c|}{ Mountainous Terrain - Upgrade } \\
\hline & \multicolumn{6}{|c|}{ Posted Speed Limit km/h (mi/h) } \\
\hline & \multicolumn{3}{|c|}{$96.56(60)$} & \multicolumn{3}{|c|}{$96.56(60)$} \\
\hline & \multicolumn{3}{|c|}{ Curve } & \multicolumn{3}{|c|}{ Approach } \\
\hline $0 \leq h<1$ & \multicolumn{3}{|c|}{7} & \multicolumn{3}{|c|}{11} \\
\hline $1 \leq h<2$ & \multicolumn{3}{|c|}{52} & \multicolumn{3}{|c|}{53} \\
\hline $2 \leq h<3$ & \multicolumn{3}{|c|}{39} & \multicolumn{3}{|c|}{45} \\
\hline $3 \leq h<4$ & \multicolumn{3}{|c|}{27} & \multicolumn{3}{|c|}{20} \\
\hline $4 \leq h<5$ & \multicolumn{3}{|c|}{17} & \multicolumn{3}{|c|}{9} \\
\hline $5 \leq h$ & \multicolumn{3}{|c|}{417} & \multicolumn{3}{|c|}{421} \\
\hline \multicolumn{7}{|c|}{ Mountainous Terrain - Downgrade } \\
\hline & \multicolumn{3}{|c|}{ Curve } & \multicolumn{3}{|c|}{ Approach } \\
\hline $0 \leq h<1$ & \multicolumn{3}{|c|}{10} & & 19 & \\
\hline $1 \leq h<2$ & & 93 & & & 111 & \\
\hline $2 \leq h<3$ & & 69 & & & 84 & \\
\hline $3 \leq h<4$ & & 53 & & & 46 & \\
\hline $4 \leq h<5$ & & 34 & & & 27 & \\
\hline $5 \leq h$ & & 706 & & & 678 & \\
\hline
\end{tabular}


It should be noted that the headway category $h \geq 5$ s included trips with no headway readings, which should correspond to no object ahead within the maximum detection range of the radar in the equipped vehicles of $200 \mathrm{~m}$ distance as explained in previous section. This distance would produce a headway of $5 \mathrm{~s}$ or more for speeds up to $144 \mathrm{~km} / \mathrm{h}(89.9$ $\mathrm{mi} / \mathrm{h}$ ), which is considerably higher than the speeds observed in the data. On the other hand, the number of readings in $0 \leq h<1$ and $4 \leq h<5$ categories are relatively low.

The effect of headway threshold on speed choice on curve and approach tangent was investigated considering two situations; investigating the trend of mean and $85^{\text {th }}$ percentile speed against headway threshold and against specific headway interval. The effect of headway threshold was investigated by plotting the trends of mean and $85^{\text {th }}$ percentile speeds on curve and tangent against headway threshold $(h \geq 1, h \geq 2, \ldots, h \geq 5 \mathrm{~s})$. Figure 4.1 shows examples of these plots for $V_{D M C}$ and at $V_{a p p}$ in rolling terrain (plots for mountainous terrain are shown in Appendix B). Note that using other speed variables, such as $V_{c r v-a v g}, V_{T M C}$, and $V_{M C}$, yielded similar results (Appendix B). All parts of the figure do not show any specific trend between speed and headway. In addition, the standard deviation for each speed is almost constant for each category (Appendix B). This means that the main speed distribution parameters do not depend on the headway threshold whether it is speed on curve or approach tangent. 


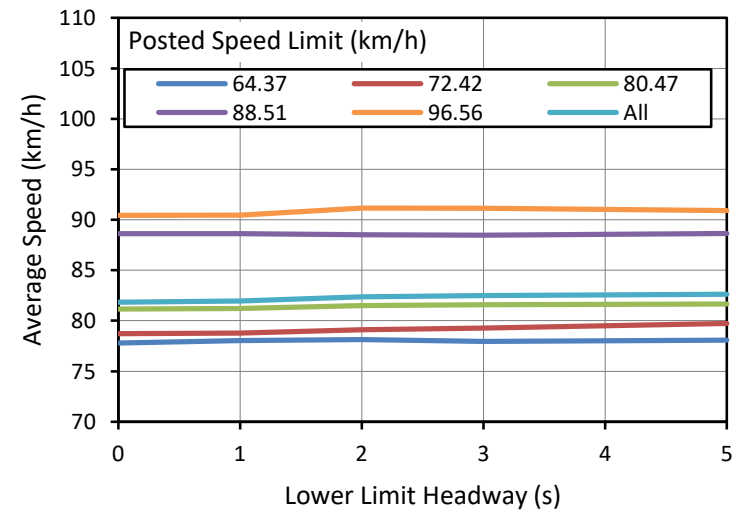

(a) Mean $V_{D M C}$.

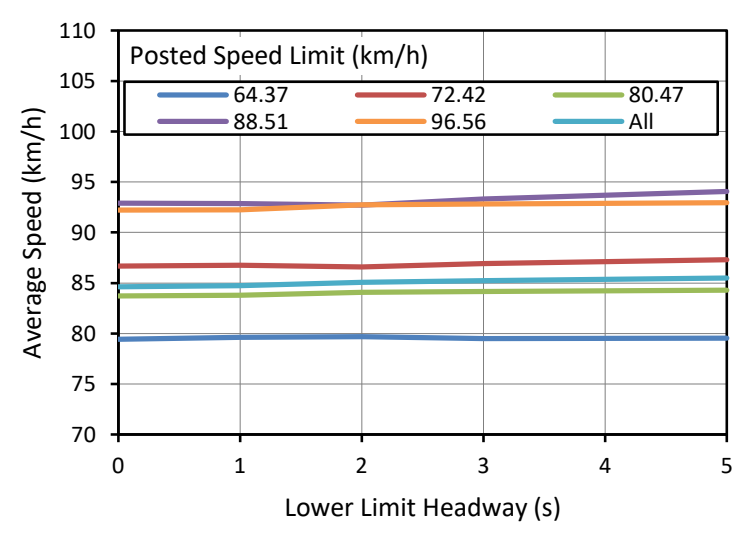

(c) Mean $V_{\text {app }}$.

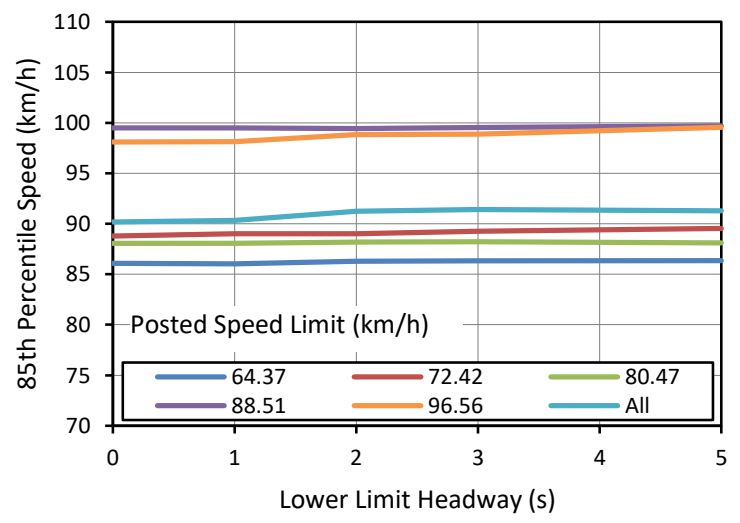

(b) $85^{\text {th }}$ Percentile $V_{D M C}$.

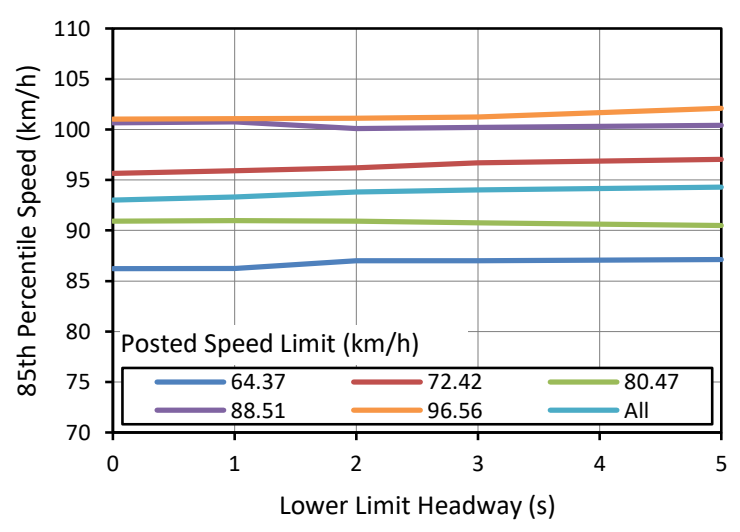

(d) 85 th Percentile $V_{a p p}$.

Figure 4.1: Relationship between Mean and 85th percentile of Speed and Headway (Rolling Terrain).

As shown in the figure, using trips in the category $h \geq 5 \mathrm{~s}$ would not yield considerably different estimates of the mean or $85^{\text {th }}$ percentile speed compared to using all trips regardless of headway threshold. However, this conclusion might have resulted from the relatively high number of readings in the category $h \geq 5 \mathrm{~s}$. Therefore, the $85^{\text {th }}$ percentile of speed and mean speed were plotted for specific headway intervals $(0 \leq h<1,1 \leq h<2, \ldots$, $h \geq 5 \mathrm{~s}$ ) as shown in Figure 4.2. The figure shows a trend of slight increase of speed with the increase of headway for both $V_{D M C}$ and $V_{a p p}$. The trend is more obvious for headway 
intervals less than $3 \mathrm{~s}$. This would indicate that headway can be a factor affecting speed choice on curve and approach tangent up to headway of $3 \mathrm{~s}$ (plots for speed parameters $V_{c r v-a v g}, V_{T M C}$, and $V_{M C}$ are shown in Appendix B).

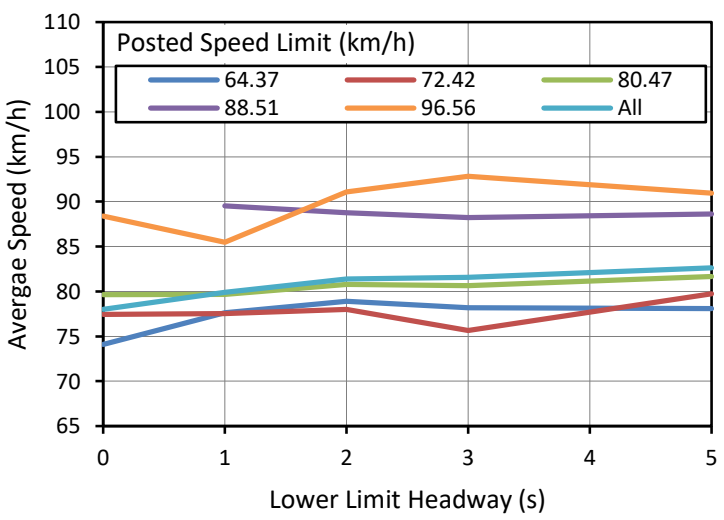

(a) Mean $V_{D M C}$.

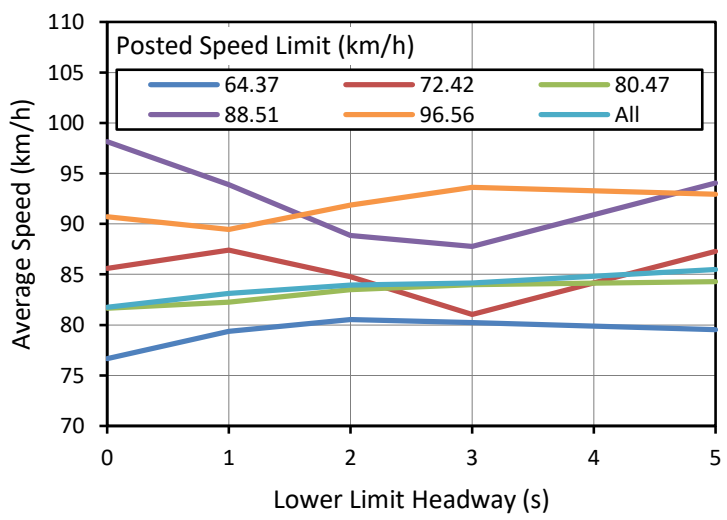

(c) Mean $V_{a p p}$.

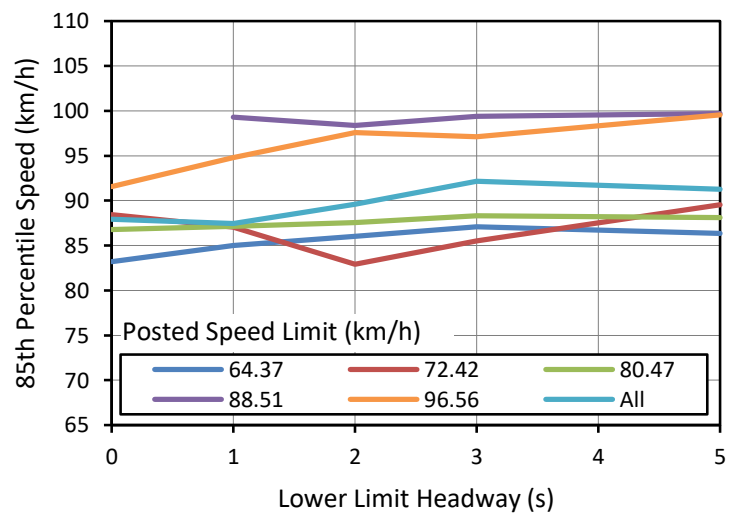

(b) 85th Percentile $V_{D M C}$.

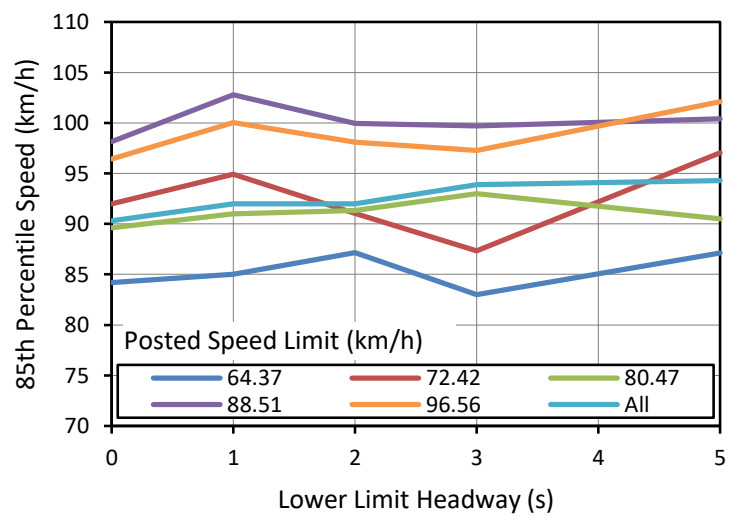

(d) 85th Percentile $V_{a p p}$.

Figure 4.2: Mean and 85th percentile of Speed vs. Specific Headway Intervals on Curve and at Approach Tangent (Rolling Terrain).

This conclusion was tested statistically using One-Way-ANOVA. Table 4-2 presents the results for the null hypothesis $\left(H_{0}\right)$ that, for each speed limit, the mean speeds were equal for the different headway intervals. The $F$-statistics and $p$-values in the table show that there is no enough evidence to reject the null hypothesis for all speed variables (i.e. 
there is no significant difference between the mean of speed for the different headway intervals) for all speed limits except the $80.47 \mathrm{~km} / \mathrm{h}(50 \mathrm{mi} / \mathrm{h})(p$-values are 0.039 and 0.007 for $V_{D M C}$ and $V_{c r v-a v g}$, respectively). Likewise, $F$-statistics and $p$-values for $V_{a p p}$ have not provided enough evidence to reject the null hypothesis for all posted speed limits except for $80.47 \mathrm{~km} / \mathrm{h}(50 \mathrm{mi} / \mathrm{h})$ ( $p$-value is 0.004$)$. For mountainous terrain, the One-WayANOVA results show that there is a significant effect for headway interval on all speed variables at $5 \%$ level of significance. It should be noted that the grade of curve (upgrade and downgrade) may have affected the analysis. Therefore, Two-Way-ANOVA was conducted considering the grade as a factor. The results shown in Table 4-2 show that $F$ statistics and $p$-values have not provided enough evidence to reject the null hypothesis for all speed parameters except for $V_{D M C}$ and $V_{a p p}$.

Table 4-2: One-Way-ANOVA for Speed on Curve, Approach Tangent and Headway Intervals.

\begin{tabular}{|c|c|c|c|c|c|c|c|c|c|c|}
\hline \multirow{2}{*}{$\begin{array}{l}\text { Speed Limit } \\
\mathrm{km} / \mathrm{h}(\mathrm{mi} / \mathrm{h})\end{array}$} & \multicolumn{2}{|c|}{$V_{M C}$} & \multicolumn{2}{|c|}{$V_{D M C}$} & \multicolumn{2}{|c|}{$V_{T M C}$} & \multicolumn{2}{|c|}{$V_{c r v-a v g}$} & \multicolumn{2}{|c|}{$V_{a p p}$} \\
\hline & $\begin{array}{c}F- \\
\text { statistic }\end{array}$ & $p$-value & $\begin{array}{c}F- \\
\text { statistic }\end{array}$ & $p$-value & $\begin{array}{c}F- \\
\text { statistic }\end{array}$ & $p$-value & $\begin{array}{c}F- \\
\text { statistic }\end{array}$ & $p$-value & $\begin{array}{c}F- \\
\text { statistic }\end{array}$ & $p$-value \\
\hline \multicolumn{11}{|c|}{ Rolling Terrain } \\
\hline $64.37(40)$ & 1.342 & 0.247 & 1.314 & 0.258 & 1.313 & 0.259 & 1.478 & 0.197 & \begin{tabular}{|l|}
0.590 \\
\end{tabular} & 0.707 \\
\hline $72.42(45)$ & 1.359 & 0.247 & 0.822 & 0.537 & 1.353 & 0.249 & 0.722 & 0.608 & 0.679 & 0.640 \\
\hline $80.47(50)$ & 1.812 & 0.108 & 2.356 & $0.039 *$ & 1.843 & 0.102 & 3.228 & $0.007^{*}$ & 3.517 & 0.004* \\
\hline $88.51(55)$ & 0.347 & 0.847 & 0.076 & 0.990 & 0.344 & 0.847 & 0.147 & 0.964 & 1.942 & 0.093 \\
\hline $96.54(60)$ & 1.063 & 0.384 & 1.921 & 0.093 & 1.064 & 0.383 & 1.828 & 0.110 & 1.178 & 0.322 \\
\hline \multicolumn{11}{|c|}{ Mountainous Terrain One-Way-ANOVA } \\
\hline $96.54(60)$ & 4.639 & $0.010^{*}$ & 4.369 & $0.013 *$ & 4.683 & $0.009 *$ & 3.956 & $0.019 *$ & 4.865 & $<0.001^{*}$ \\
\hline \multicolumn{11}{|c|}{ Mountainous Terrain Two-Way-ANOVA } \\
\hline $96.54(60)$ & 2.600 & 0.075 & 3.211 & $0.041^{*}$ & 2.607 & 0.074 & 2.554 & 0.078 & 2.534 & $0.027^{*}$ \\
\hline
\end{tabular}


Based on previous discussion, trips with headway $h \geq 3$ s were considered to correspond to traveling under free-flow conditions in this research. It is expected that the speed, and subsequently lateral acceleration and lateral friction demand, at free-flow conditions will be higher than those of non-free-flow conditions. A highway design based on free-flow conditions only will ensure safety at conditions where the geometric design is the main factor controlling driver behaviour. On the other hand, considering only free-flow conditions may lead to very high margin of safety in the design for a significant portion of the traffic that travels during peak conditions.

\subsection{Normality Test}

Kolmogorov-Smirnov and Shapiro-Wilk tests were used to check the normality of variables distributions. Only vehicles at free-flow conditions were considered in the analysis because operating speed and comfort threshold models in the literature have been traditionally developed for vehicles traveling under these conditions (Torbic et al. 2014). Based on the analysis of headway threshold for free-flow speed, the normality tests were conducted for a headway threshold of $3 \mathrm{~s}$. The results showed that at least 73\% (rolling terrain) and $72 \%$ (mountainous terrain) of the variables came from normally distributed populations. Similar results were obtained when considering a $5 \mathrm{~s}$ headway threshold, which is recommended in many studies (Hassan et al 2011; Bassani et al. 2015), where the hypothesis of a normal distribution was accepted on at least $76 \%$ and $72 \%$ of the rolling and mountainous terrain curves, respectively. The detailed results are presented in Appendix (C). 


\subsection{Comparing Instantaneous and Average Speeds}

As mentioned earlier, several variables were extracted from the trip data to describe speed of vehicle at different points (BC, MC, EC, and AT) using instantaneous speed measurements or average of speed observations over a short time period or distance. OneWay-ANOVA was used to compare the means of each set of speed variables corresponding to each point on each curve. The results showed that the differences are not significant for all speed variables on all curves at 5\% level of significance. The results also show that the assumption of homogeneity of variance was not violated at $5 \%$ level of significance for all curves. That is, the instantaneous and average speed variables at the same point $(\mathrm{BC}, \mathrm{MC}$, EC, and AT) have approximately the same mean and same variance. The results of OneWay ANOVA and homogeneity of variance tests are shown in Appendix (D).

\subsection{Comparing Speeds for Comfort Threshold}

One-Way-ANOVA was also used in comparing the average and minimum speeds corresponding to the comfort threshold $\left(V_{c m f-a v g}\right.$ and $\left.V_{c m f-m i n}\right)$. The results showed that there is no enough evidence to reject the null hypothesis for $98 \%$ of all curves. This result was generally expected given that the comfort threshold was determined for a segment with a statistically constant speed. One-way ANOVA was also used to compare $V_{c m f-a v g}$ to the average speed over the whole curve $\left(V_{c r v-a v g}\right)$. The results showed no significant difference between the two speed variables for all curves. Similarly, ANOVA results showed no statistically significant difference between $V_{c m f-\min }$ and $V_{c r v-\min }$ on $87 \%$ of the curves. This would be helpful in determining mean speed corresponding to comfort threshold based on any of the curve speed parameters without having to use the speed 
corresponding to the specific segment over which the driver comfort threshold was estimated. Finally, $V_{c m f-a v g}$ was compared with $V_{M C}$ and found no difference between variables for all curves in rolling and mountainous terrains.

\subsection{Comparing Other Speed Variables}

In addition to the tests mentioned in the previous sections, additional tests were performed to compare several speed parameters. The minimum and maximum speeds on curve $\left(V_{c r v-\min }\right.$ and $\left.V_{c r v-\max }\right)$ were compared, and a statistically significant difference was found for at least $81 \%$ of the curves. This finding indicates that the change in instantaneous speed of a typical trip along a horizontal is not insignificant. $V_{c r v-\max }$ was also compared to $V_{B C}$ and no significant difference was found for approximately $91 \%$ of the curves. Thus, $V_{B C}$ can be used to predict maximum speed on curve. $V_{c r v-a v g}$ was compared to each of $V_{c r v-\min }$ and $V_{M C}$, and no significant difference was found in either comparison for approximately $88 \%$ and $94 \%$ of the curves, respectively. Thus, $V_{M C}$ can be

used as an estimate of both $V_{c r v-m i n}$ and $V_{c r v-a v g}$. Finally, the maximum speed on the approach tangent $\left(V_{a p p-\max }\right)$ was compared to the instantaneous speed $V_{a p p}$ using ANOVA. The results showed no significant difference between $V_{a p p-\max }$, and $V_{a p p}$ on approximately $94 \%$ of the curves. Thus, the instantaneous tangent speed at $200 \mathrm{~m}$ upstream a horizontal curve can be used as a surrogate of the maximum speed on the approach tangent.

\subsection{Comparing Variances}

One-Way-ANOVA and homogeneity tests were used to examine the differences of 
variances of speed and comfort threshold of the curves in rolling and mountainous terrains (Table 4-3). The outputs of the Levene test for most variables indicated insignificant differences between the variance on all curves at $5 \%$ level of significance. However, in some cases, a significant difference existed but can be attributed to one or few curves. When these curves were removed from the dataset, the results showed no statistically significant difference between the variances, except for comfort threshold where the number of curves needed to be removed was 4 (out of 33) and 7 (out of 29) for rolling and mountainous terrain, respectively.

Table 4-3: One-Way-ANOVA and Test of Homogeneity in Rolling and Mountainous Terrains.

\begin{tabular}{|c|c|c|c|c|c|c|}
\hline \multirow{3}{*}{ Parameter } & \multicolumn{3}{|c|}{ Rolling Terrain } & \multicolumn{3}{|c|}{ Mountainous Terrain } \\
\hline & \multicolumn{2}{|c|}{ Levene Test (Quadratic) } & \multirow{2}{*}{$\begin{array}{l}\text { Number of } \\
\text { Curves } \\
\text { Removed }\end{array}$} & \multicolumn{2}{|c|}{ Levene Test (Quadratic) } & \multirow{2}{*}{$\begin{array}{c}\text { Number of } \\
\text { Curves } \\
\text { Removed }\end{array}$} \\
\hline & $F$ - Statistic & $p$-value & & $F$ - Statistic & $p$-value & \\
\hline$V_{c r v-a v g}$ & 1.183 & 0.223 & --- & 1.228 & 0.192 & --- \\
\hline$V_{c r v-\min }$ & 1.075 & 0.357 & --- & 1.304 & 0.133 & --- \\
\hline$V_{c r v-\max }$ & 1.014 & 0.446 & 1 & 1.049 & 0.396 & --- \\
\hline$V_{B C}$ & 1.325 & 0.110 & 1 & 1.114 & 0.312 & --- \\
\hline$V_{M C}$ & 1.039 & 0.408 & --- & 1.298 & 0.139 & --- \\
\hline$V_{E C}$ & 1.202 & 0.204 & --- & 1.063 & 0.376 & --- \\
\hline$a_{L}$ & 1.386 & 0.088 & 4 & 1.600 & 0.069 & 7 \\
\hline$V_{c m f-a v g}$ & 0.992 & 0.481 & --- & 0.856 & 0.678 & 1 \\
\hline$V_{\min -c m f}$ & 1.165 & 0.243 & --- & 0.789 & 0.769 & 1 \\
\hline$V_{a p p}$ & 1.296 & 0.132 & 2 & 0.914 & 0.595 & --- \\
\hline$V_{a p p-\max }$ & 1.327 & 0.116 & 3 & 0.917 & 0.591 & --- \\
\hline
\end{tabular}

\subsection{Comparing Left and Right Turn Curves}

It is reported that collisions are more common on right curves than on left curves (TAC 2017). Therefore, the effect of curve turning direction on driver comfort threshold and 
speed on curve was examined using twelve curves in rolling terrain and four curves in mountainous terrain, where both directions are included in the analysis. One-Way ANOVA is used to test the null hypothesis that the mean comfort threshold $\left(a_{L}\right)$ and speed on curve $\left(V_{c m f-a v g}\right.$ and $\left.V_{M C}\right)$ are equal for left and right turning curves. As shown in Table 4-4, for a $5 \%$ level of significance, the null hypothesis should be accepted for $V_{c m f-a v g}$ and $V_{M C}$ for approximately $92 \%$ and $100 \%$ of the curves in rolling terrain and for $50 \%$ (two out of four) and $75 \%$ of the curves (three out of four) in mountainous terrain. On the other hand, the null hypothesis should be rejected for $a_{L}$ for $50 \%$ (six of twelve) and $100 \%$ of the curves in the rolling and mountainous terrain, respectively.

Table 4-4: ANOVA Results for Right Turn vs. Left Turn Curves.

\begin{tabular}{|c|c|c|c|c|c|c|}
\hline \multirow{2}{*}{$\begin{array}{c}\text { Curve } \\
\text { ID }\end{array}$} & \multicolumn{2}{|c|}{$V_{M C}$} & \multicolumn{2}{c|}{$V_{\text {cmf-avg }}$} & \multicolumn{2}{c|}{$a_{L}$} \\
\cline { 2 - 7 } & $F$ & $p$-value & $F$ & $p$-value & $F$ & $p$-value \\
\hline R 03 & 1.566 & 0.214 & 1.463 & 0.230 & 0.850 & 0.359 \\
\hline R 06 & 0.012 & 0.911 & 1.390 & 0.242 & 7.929 & $0.006^{*}$ \\
\hline R 08 & 1.670 & 0.199 & 1.936 & 0.167 & 0.303 & 0.583 \\
\hline R 10 & 1.139 & 0.288 & 1.647 & 0.202 & 1.742 & 0.190 \\
\hline R 12 & 0.353 & 0.557 & 0.061 & 0.806 & 2.136 & 0.151 \\
\hline R 14 & 1.893 & 0.176 & 1.819 & 0.184 & 1.288 & 0.263 \\
\hline R 21 & 0.256 & 0.616 & 0.247 & 0.622 & 25.035 & $<0.001^{*}$ \\
\hline R 23 & 1.799 & 0.187 & 0.826 & 0.369 & 6.815 & $0.013^{*}$ \\
\hline R 25 & 0.409 & 0.527 & 1.178 & 0.284 & 9.295 & $0.004^{*}$ \\
\hline R 27 & 3.356 & 0.070 & 4.434 & $0.038^{*}$ & 2.316 & 0.131 \\
\hline R 29 & 0.055 & 0.815 & 0.000 & 0.990 & 22.854 & $<0.001^{*}$ \\
\hline R 32 & 0.167 & 0.684 & 0.014 & 0.905 & 4.016 & $0.048^{*}$ \\
\hline MT 08 & 3.653 & 0.060 & 3.191 & 0.078 & 89.622 & $<0.001^{*}$ \\
\hline MT 13 & 3.105 & 0.085 & 1.799 & 0.185 & 17.994 & $<0.001^{*}$ \\
\hline MT 20 & 4.525 & $0.038^{*}$ & 4.520 & $0.038^{*}$ & 25.785 & $<0.001^{*}$ \\
\hline MT 22 & 3.711 & 0.059 & 5.041 & $0.028^{*}$ & 11.780 & $0.001^{*}$ \\
\hline * Statistically significant at 5\% level of significance. & & \\
\hline \hline
\end{tabular}


It was noted that the results of the mountainous terrain could have been affected by the grade corresponding to each direction, as one direction will be on a general upgrade and the other will be on a downgrade. Therefore, a two-way ANOVA was conducted to examine the effect of turning direction and grade on $a_{L}, V_{c m f-a v g}$, and $V_{M C}$. Table 4-5 showed a significant effect for both turning direction and grade on $a_{L}$. The interaction between two parameters, grade and turning direction, had a significant effect on $a_{L}$, which means that the effect of each parameter depends on the other. $V_{M C}$ had the same results except for the interaction parameter, which was not significant. Therefore, the effect of each parameter does not depend on the other one. The turning direction and the interaction parameter between grade and turning direction were not significant for $V_{c m f-a v g}$. However, the grade was significant. It should be noted however that these results are based only on four curves.

Table 4-5: ANOVA-Two-Way Results for Right Turn vs. Left Turn Curves - Mountainous Terrain.

\begin{tabular}{|l|c|c|c|}
\hline \hline Variable & Parameter & $F$ & $p$-value \\
\hline \multirow{3}{*}{$a_{L}$} & Direction & 42.122 & $<\mathbf{0 . 0 0 1}$ \\
\cline { 2 - 4 } & Grade & 6.283 & $\mathbf{0 . 0 1 3}$ \\
\cline { 2 - 4 } & Direction $\times$ Grade & 50.320 & $<\mathbf{0 . 0 0 1}$ \\
\hline \multirow{3}{*}{$V_{c m f-\text { avg }}$} & Direction & 2.768 & 0.097 \\
\cline { 2 - 4 } & Grade & 7.335 & $\mathbf{0 . 0 0 7}$ \\
\cline { 2 - 4 }$V_{M C}$ & Direction $\times$ Grade & 3.014 & 0.084 \\
\hline & Direction & 4.892 & $\mathbf{0 . 0 2 8}$ \\
\cline { 2 - 4 } & Grade & 5.529 & $\mathbf{0 . 0 2 0}$ \\
\cline { 2 - 4 } & Direction $\times$ Grade & 2.335 & 0.128 \\
\hline \hline
\end{tabular}

Thus, although the data show that $V_{M C}$ on right-turn curves is up to $4.5 \mathrm{~km} / \mathrm{h}$ higher than on left-turn curves, there is no enough evidence to conclude that this difference is 
statistically significant and is likely the result of random sampling. However, a right-turn curve will likely have higher $a_{L}$ than the same curve when turning left with the difference of average $a_{L}$ reaching up to $0.048 \mathrm{~g}$, a finding that is consistent with Misaghi and Hassan Said et al. (2009). However, the statistical analysis does not provide a strong evidence whether this difference is statistically significant or insignificant.

\subsubsection{Variables Correlation}

Prior to model development, it is important to assess the correlation among the different variables. The bivariate (Pearson) correlation matrix among the selected variables in Table 4-6 was developed. The correlations of most variables are significant at $1 \%$ level of significance as shown in the table. As expected, the speed on curve (mean, maximum, and minimum speed) has a strong direct relationship with the speed at approach tangent and curve radius. The comfort threshold has a direct relationship with speed on curve and approach tangent and an inverse relationship with radius.

\subsubsection{Outliers}

As explained earlier, the NDS data are collected with no observer interference, which could be considered as one of the most important features over other data collection methods. However, the database could contain some extreme values that could skew measures of central tendency such as the mean. SPSS Analytic Catalyst uses the interquartile range (IQR) to identify outliers. A value is considered as outlier if it lies outside the range $Q_{1}-1.5\left(Q_{3}-Q_{1}\right)$ and $Q_{3}+1.5\left(Q_{3}-Q_{1}\right)$, where $Q_{1}$ is the first quartile (25th percentile), and $Q_{3}$ is the third quartile (75th percentile) (IBM 2017). The extreme outliers represent values more than three times $\left(Q_{3}-Q_{1}\right)$. It is noticed that these outliers 
would affect developing models at the driver level models but would only have a limited effect on aggregated models for the $85^{\text {th }}$ percentile value. The extreme outliers detected and removed from the database were less than $0.2 \%$ of the available observations.

Table 4-6: Bivariate (Pearson) Correlation Matrix for Rolling and Mountainous Terrains.

\begin{tabular}{|c|c|c|c|c|c|c|c|c|c|}
\hline & $\begin{array}{c}V_{a p p} \\
(\mathrm{~km} / \mathrm{h})\end{array}$ & $\begin{array}{c}V_{M C} \\
(\mathrm{~km} / \mathrm{h})\end{array}$ & $\begin{array}{c}V_{c r v-\max } \\
(\mathrm{km} / \mathrm{h})\end{array}$ & $\begin{array}{c}V_{c r v-m i n} \\
(\mathrm{~km} / \mathrm{h})\end{array}$ & $\begin{array}{l}a_{L} \\
(\mathrm{~g})\end{array}$ & $\begin{array}{c}R \\
(\mathrm{~m})\end{array}$ & $e$ & $\begin{array}{l}\Delta \\
\left({ }^{\circ}\right)\end{array}$ & $\begin{array}{l}V_{\text {red }}{ }^{\mathrm{a}} \\
(\mathrm{km} / \mathrm{h})\end{array}$ \\
\hline \multicolumn{10}{|c|}{ Rolling Terrain } \\
\hline$V_{a p p}$ & 1 & & & & & & & & \\
\hline$V_{M C}$ & $0.716^{* *}$ & 1 & & & & & & & \\
\hline$V_{c r v-\max }$ & $0.768 * *$ & $0.959 * *$ & 1 & & & & & & \\
\hline$V_{c r v-\min }$ & $0.721 * *$ & $0.953 * *$ & $0.906^{* *}$ & 1 & & & & & \\
\hline$a_{L}$ & $0.426 * *$ & $0.424 * *$ & $0.449 * *$ & $0.426^{* *}$ & 1 & & & & \\
\hline$R$ & $0.246^{* *}$ & $0.450 * *$ & $0.428 * *$ & $0.439^{* *}$ & $-0.329^{* *}$ & 1 & & & \\
\hline$e$ & 0.025 & $-0.180 * *$ & $-0.090^{* *}$ & $-0.194 * *$ & $0.109^{* *}$ & $-0.348^{* *}$ & 1 & & \\
\hline$\Delta$ & $0.058^{*}$ & -0.031 & 0.046 & $-0.099 * *$ & $0.225^{* *}$ & $-0.336^{* *}$ & $0.324 * *$ & 1 & \\
\hline$V_{\text {red }}{ }^{\mathrm{a}}$ & $0.154 * *$ & -0.423 & -0.216 & -0.403 & -0.001 & -0.296 & $0.280^{* *}$ & $0.119 * *$ & 1 \\
\hline \multicolumn{10}{|c|}{ Mountainous Terrain } \\
\hline$V_{a p p}$ & 1 & & & & & & & & \\
\hline$V_{M C}$ & $0.786^{* *}$ & 1 & & & & & & & \\
\hline$V_{c r v-\max }$ & $0.832 * *$ & $0.961 * *$ & 1 & & & & & & \\
\hline$V_{c r v-\min }$ & $0.798^{* *}$ & $0.966^{* *}$ & $0.919 * *$ & 1 & & & & & \\
\hline$a_{L}$ & $0.392 * *$ & $0.264 * *$ & $0.294 * *$ & $0.308^{* *}$ & 1 & & & & \\
\hline$R$ & 0.040 & $0.245^{* *}$ & $0.214 * *$ & $0.182 * *$ & $-0.547 * *$ & 1 & & & \\
\hline$e$ & -0.005 & $-0.131 * *$ & $-0.114 * *$ & $-0.074 * *$ & $0.388 * *$ & $-0.692 * *$ & 1 & & \\
\hline$\Delta$ & $-0.193 * *$ & $-0.292 * *$ & $-0.218^{* *}$ & $-0.322 * *$ & $0.222 * *$ & $-0.376^{* *}$ & $0.161^{* *}$ & 1 & \\
\hline$V_{\text {red }}^{\mathrm{a}}$ & $0.316^{* *}$ & $-0.338 * *$ & $-0.214 * *$ & $-0.278^{* *}$ & $0.143 * *$ & $-0.280^{* *}$ & $0.167^{* *}$ & $0.122^{* *}$ & 1 \\
\hline \multicolumn{10}{|c|}{$\begin{array}{l}V_{a p p}=\text { speed at approach tangent, } V_{M C}=\text { speed on curve, } V_{c r v-\max } \text { and } V_{c r v-\min }=\text { maximum and } \\
\text { minimum speed on curve respectively, } a_{L}=\text { comfort threshold, } R=\text { curve radius, } e=\text { superelevation } \\
\text { rate, } \Delta=\text { deflection angle, and } V_{\text {red }}=\text { speed reduction }=V_{a p p}-V_{M C} \text {. } \\
*=\text { correlation is significant at the } 0.05 \text { level (2-tailed). } \\
* *=\text { correlation is significant at the } 0.01 \text { level ( } 2 \text {-tailed). } \\
\text { a = consider only vehicles that decelerated on curves. }\end{array}$} \\
\hline
\end{tabular}




\subsection{Summary}

This chapter explained the preliminary analyses and tests that were conducted in order to provide the ground for model development. The analysis for speed choice and headway showed that the vehicle would be at free-flow conditions when having headway distance equal to or greater than $3 \mathrm{~s}$. This conclusion was reached after finding that the trend for the $85^{\text {th }}$ percentile of speed and mean speed, for different headway intervals, shows a slight increase of speed with increase of heading up to $3 \mathrm{~s}$. The One-Way-ANOVA showed that there is no significant difference between the mean of speed for the different headway intervals.

The ANOVA test was used to compare the difference among several speed and comfort parameters on curve and tangent. The results showed that there is no statistical difference between speed parameters at BC. Same results were obtained for MC, EC, and AT. Also, there were no significant difference between $V_{c m f-a v g}, V_{c m f-m i n}$, and $V_{c r v-a v g} ; V_{c r v-m i n}$ and $V_{c m f-m i n} ; V_{c m f-a v g}$ and $V_{M C} ; V_{c r v-a v g}, V_{c r v-m i n}$, and $V_{M C} ; V_{B C}$ and $V_{c r v-m a x}$; or between $V_{a p p-m a x}$ and $V_{a p p}$. Therefore, these parameters can be used as surrogate measures interchangeably. However, there was a significant difference between $V_{c r v-\max }$ and $V_{\text {crv-min }}$

Normality test was conducted and found that all extracted variables came from a normally-distributed population. Finally, the One-Way ANOVA was conducted to compare the speed on curve comfort threshold between left and right turn curve. It is found that the there is a significant difference between left and right for half of the curve for $a_{L}$ in rolling terrain and $100 \%$ in mountainous terrain. For speed on curve, the results showed 
that there is no significant difference for $\left(V_{M C}\right.$ and $\left.V_{c m f-a v g}\right)$ for approximately $92 \%$ and $100 \%$ of the curves in rolling terrain, and $50 \%$ and $75 \%$ in mountainous terrain, respectively. 


\section{CHAPTER FIVE}

\section{MODELS DEVELOPMENT}

\subsection{Introduction}

Many models are available in the literature to predict operating speed and other driver behaviour parameters on horizontal curves based on the highway's geometric features. The previous chapter provided information regarding several parameters to measure speed and comfort threshold. Understanding driving behaviour on curves would help in selecting speed parameters that better fit in model development and in the reliability analysis to evaluate different design criteria. This chapter is devoted to examining driving behaviour on horizontal curves, developing procedure to determine driver comfort threshold, and developing several models to predict speed on curve, comfort threshold, and available/demanded friction factor.

\subsection{Driver Behaviour Analysis}

The objective behind quantitatively evaluating driver behaviour lies in the ability to identify the critical locations on horizontal curves, determine proper design parameters, and develop more reliable models to predict maximum and minimum speed, and driver comfort threshold. Thus, reliable models can be developed for operating speed profiles, reliability analysis of horizontal curves, and design consistency evaluation (Dhahir and Hassan 2017). To achieve this objective, a methodology is developed to process the data files corresponding to NDS driver trips on horizontal curves to evaluate the different measures quantifying driver behaviour on horizontal curves. Frequency of these 
parameters are determined at different areas of the approach tangent and curve and are examined to determine the patterns of drivers traversing horizontal curves.

\subsubsection{General Speed State}

Selecting speed while traversing a horizontal curve differs from one driver to another, or even for the same driver within different circumstances. Figure 5.1 shows speed profiles for several trips. As shown in the figure, there are several driving patterns to traverse horizontal curves. Usually, drivers tend to decelerate at the approach tangent. The difference depends on several factors such as drivers' characteristics, horizontal curves geometry, and weather or pavement conditions. Each driving pattern has its own critical points such the maximum and minimum locations of the lateral friction demand.

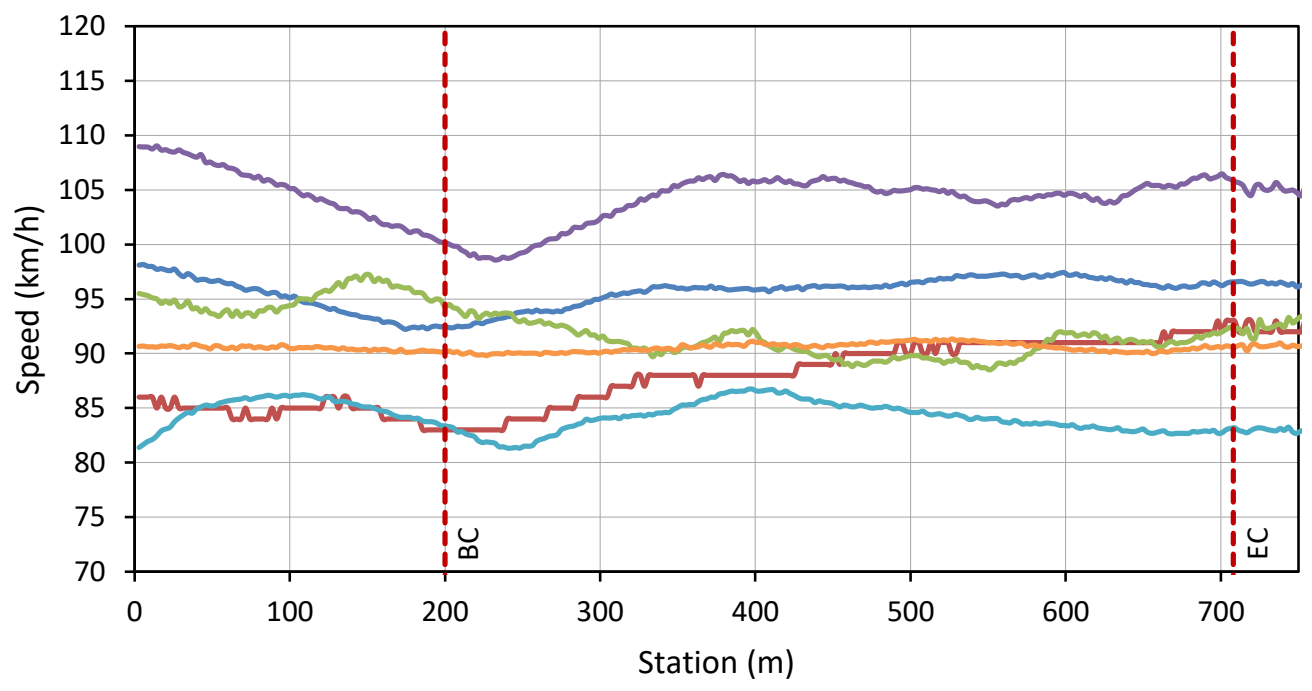

Figure 5.1: Different Speed Profiles on Curve MT 01.

To evaluate a driver's speed and longitudinal acceleration behaviour on curve and approach tangent, it is important to consider the acceleration and speed profiles. Figure 5.2 shows profiles for different parameters (speed, longitudinal and lateral acceleration) for a specific trip. Because of the high frequency of speed and acceleration measurements (10 
readings/s), an average acceleration based on the instantaneous measurements can be misleading. For example, by dividing the curve in Figure 5.2 into three equal- length zones, taking the average of the acceleration values at the middle zone would indicate a "deceleration" state whereas the speed profile shows a general "acceleration" trend. In addition, the high frequency of speed measurements can experience an instantaneous reduction in speed (deceleration) while the general trend indicates acceleration, or vice versa. The situation would be more critical when the instantaneous measurements are missing several observations. Therefore, the general speed trend or state of a specific trip should be evaluated based on the general trend of speed profile using multiple speed measurements. Furthermore, the speed trend can vary depending on the vehicle's relative location on the curve. Therefore, an analysis of general speed should be performed on curve segments or zones that can capture the general speed trend at various locations of the curve. It should be noted that the measured longitudinal acceleration may have been affected by the longitudinal grade. The downgrade curve, for instance, would increase the measured longitudinal acceleration value and vice versa.

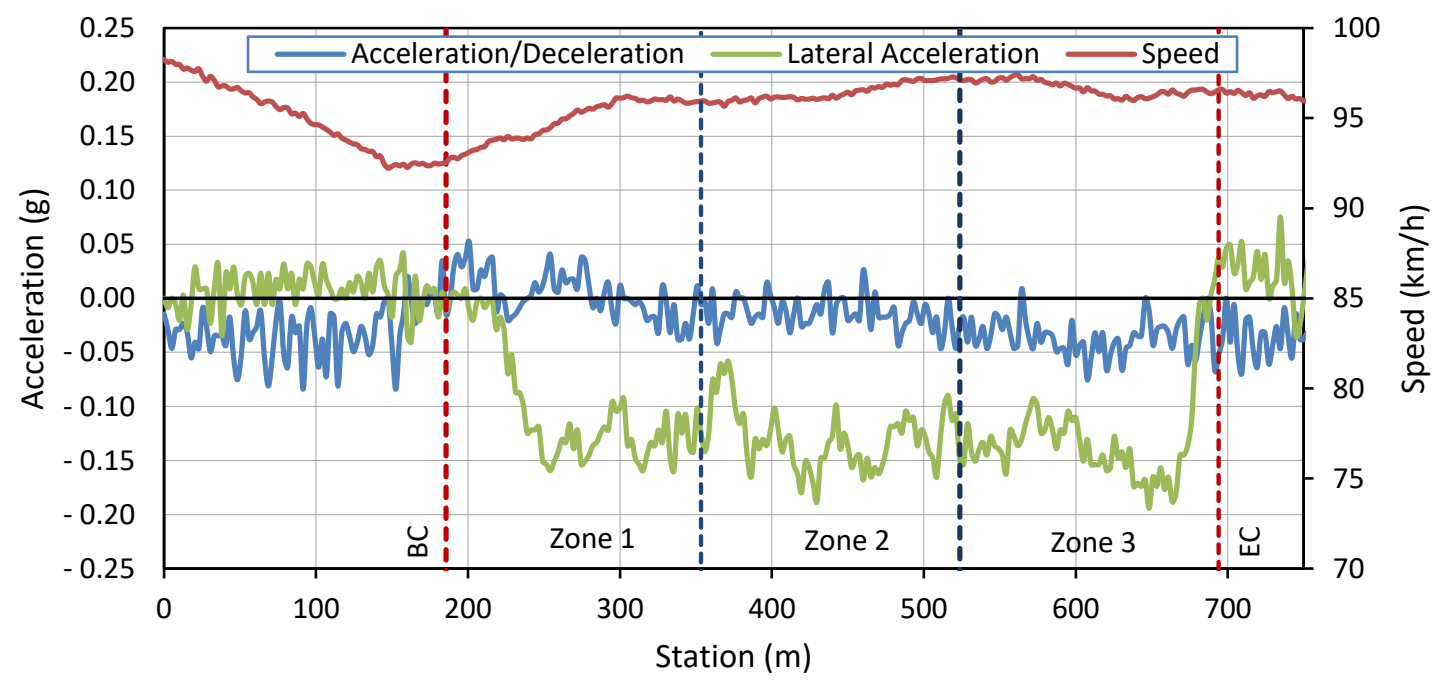

Figure 5.2: Example of Profiles of Speed and Longitudinal and Lateral Accelerations. 
Subsequently, a procedure was developed and coded in Matlab to divide the curve into a user-specified number of zones and determine the general speed state in each zone (Appendix E). The procedure involves the following steps:

1. Because of the different frequencies of speed and GPS measurements, the GPS data do not indicate the locations of all speed measurements. Therefore, the location of each speed measurement was determined using cumulative numerical integration of speed against over time. Because of the short time interval between each two speed measurements $(0.1 \mathrm{~s})$, cumulative trapezoid integration was deemed satisfactory. Additional distance was added for the first or last speed measurement if either point did not coincide with $\mathrm{BC}$ or EC, respectively, due to a gap in speed data. This distance was calculated as the speed at the first (or last) point multiplied by the time gap to BC (or EC). Accuracy of this calculation increases as the speed data gap decreases.

2. The curve is divided into zones of equal length and the speed measurements are assigned to the proper zone based on their calculated locations on the curve. It was found that some trips lacked speed measurements for a considerable length of one or more zones. If a trip was missing speed measurements on the first or last half of any zone length, it was thought that the trend of the remaining measurements might not accurately reflect the general speed trend, and the trip was deemed unusable for the analysis of speed state.

3. Linear regression was used to fit the speed measurements at each zone to a straight line versus time. Thus, the slope of speed line would represent the average trip acceleration in each zone. Subsequently, the trip speed state in each zone is 
classified as "acceleration", "deceleration", or "constant" if the speed line has a positive, negative, or zero slope, respectively. For practical reasons, the speed state was considered "constant" when the absolute value of the slope did not exceed a specific acceleration threshold (accThreshold) or if the slope as a regression coefficient was statistically insignificant (i.e. statistically not different from zero) at a specific level of significance threshold (pvalThreshold). A schematic for determining vehicle speed state is provided in Figure 5.3. The values assigned to these two parameters can affect the results of speed state.

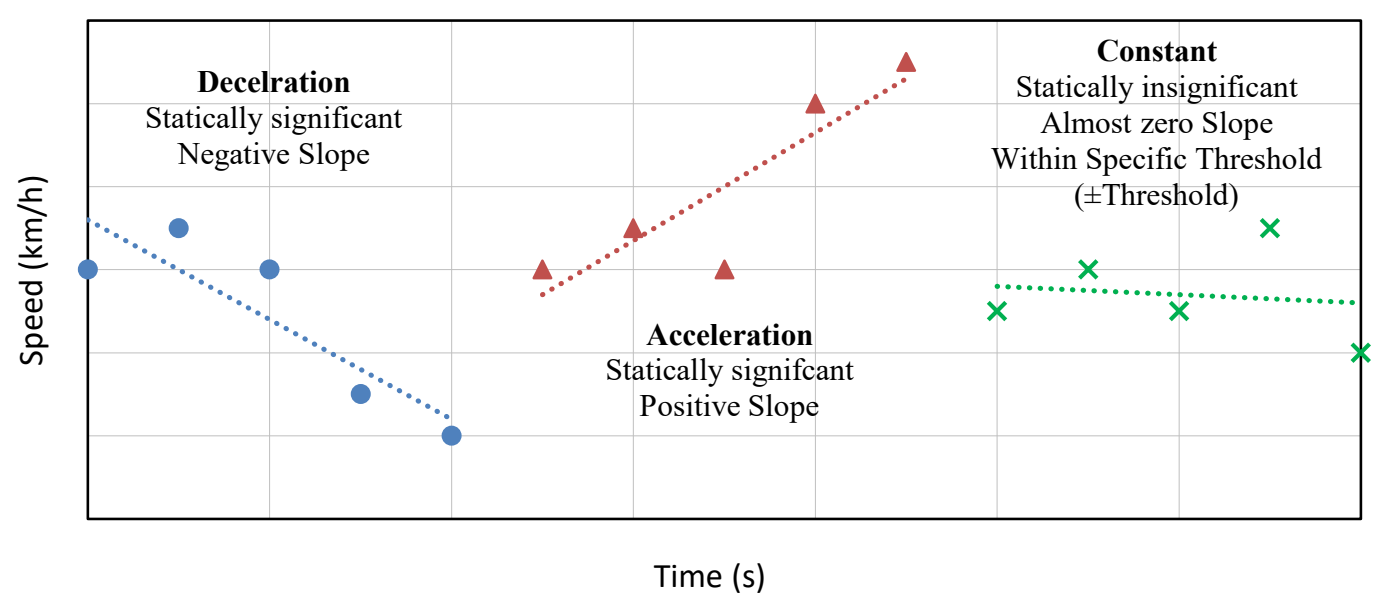

Figure 5.3: Schematic for Determining Vehicle Speed State.

\subsubsection{Curve Speed Measures}

The developed Matlab code also determines several measures of trip speed including speed on approach tangent; speed at beginning, middle, and end of curve; and minimum and maximum speeds on the curve $\left(V_{c r v-\min }\right.$ and $\left.V_{c r v-\max }\right)$ and their locations. In this research, the analysis focuses on the locations of $V_{c r v-\min }$ and $V_{c r v-\max }$ to examine the assumption of constant curve speed in most speed profile models, including the IHSDM's 
(Fitzpatrick et al. 1999). For easy display and aggregation of the results, the code divides the curve to segments or bins of equal length and determines the frequencies of occurrence of $V_{c r v-\min }$ and $V_{c r v-\max }$ in each bin. Because a trip can have multiple occurrences of $V_{c r v-\min }$ and/or $V_{c r v-\max }$, the frequencies presented in this research correspond to all occurrences of $V_{c r v-\min }$ and $V_{c r v-\max }$ of each trip. The results of this part of the procedure and Matlab code do not depend on any user-specified parameter, other than the number of bins, and can be determined to any trip with any number of speed measurements. However, it was decided to exclude the trips that were missing speed measurements to deem it unusable for the general speed state.

\subsubsection{Driver Comfort Threshold}

As explained in Section 2.4.1, the combined effects of lateral acceleration and vehicle's roll angle is a driver's sensation of forces pushing them to the outside of the curve. If the lateral acceleration exceeds a specific limit, the driver would reduce the speed on the curve to mitigate the effect of the developed lateral acceleration. This limit is commonly known as the "driver comfort threshold" (Dhahir and Hassan 2016), which varies from one driver to another. The current design guides adopt these comfort thresholds to set the design values of lateral friction coefficient in designing horizontal curves.

To determine comfort thresholds, it is important to consider the dynamic phases of a vehicle when negotiating a horizontal curve. As seen in Figure 5.2 and as explained in Chapter 2, the transient turn-entry phase starts at the beginning of the curve, where the lateral velocity (component of velocity in the direction of the lateral axis) develops from zero and changes with time. The second phase is the steady state cornering phase, where the vehicle moves on the curve with almost constant radius and lateral velocity. Finally, 
the transient turn-exit phase occurs at the end of the curve where the lateral velocity changes with time to return to zero (Milliken and Milliken 1995). The developed lateral acceleration has different values in these phases, with extreme values experienced usually in the transient phases. However, because of the very short driver exposure time to these extreme values, they will not likely prompt driver's speed adjustment and would not reflect the comfort threshold. Alternatively, the comfort threshold should correspond to the almost-constant value of lateral acceleration at the steady state cornering phase. Said et. al (2009) calculated comfort threshold for each driver on a specific curve as the average lateral acceleration after removing extreme observations. However, an average of all readings on the curve might not catch the actual comfort threshold for each driver unless the driver maintains a constant speed along the entire curve.

Considering the explanation provided in Section 2.4.1., drivers' response (accelerating or decelerating) would be affected by the applied lateral acceleration when travelling on a curved path. Drivers discomfort is correlated with centrifugal force sensation as reported by Merritt (1988). In other words, drivers tend to accelerate on curves when the applied lateral force is below their comfort threshold. In other words, they accelerate because they have not reached the discomfort threshold of the forces that pushing them to the outside of the curve. On the other hand, drivers tend to decelerate in order to mitigate the discomfort sensation of lateral force affect (side pitch). Therefore, it is assumed that drivers would maintain their constant speed if they travel at the comfort threshold. Based on this, an alternative methodology was developed in this research and coded in Matlab to evaluate the comfort threshold $\left(a_{L}\right)$ for each trip in the dataset by searching for the minimum value of average lateral acceleration measurements while the trip is at a constant speed state. To 
find this minimum, the user specifies the minimum length and minimum number of speed points to be used to establish this speed state. The larger distance based on these two parameters is set as a sliding window along the curve (Figure 5.4). Linear regression is used to find the speed state for each window location using the procedure explained earlier. If the speed state is "constant", the average lateral acceleration at that window is calculated and compared to any previous value for this trip and the minimum value is kept. At the end of the loop, the comfort threshold is determined as the minimum value of average lateral acceleration while the trip is at a state of constant speed. The code also determines the minimum $\left(V_{c m f-\min }\right)$ and mean $\left(V_{c m f-a v g}\right)$ speeds corresponding to the window corresponding to the comfort threshold.

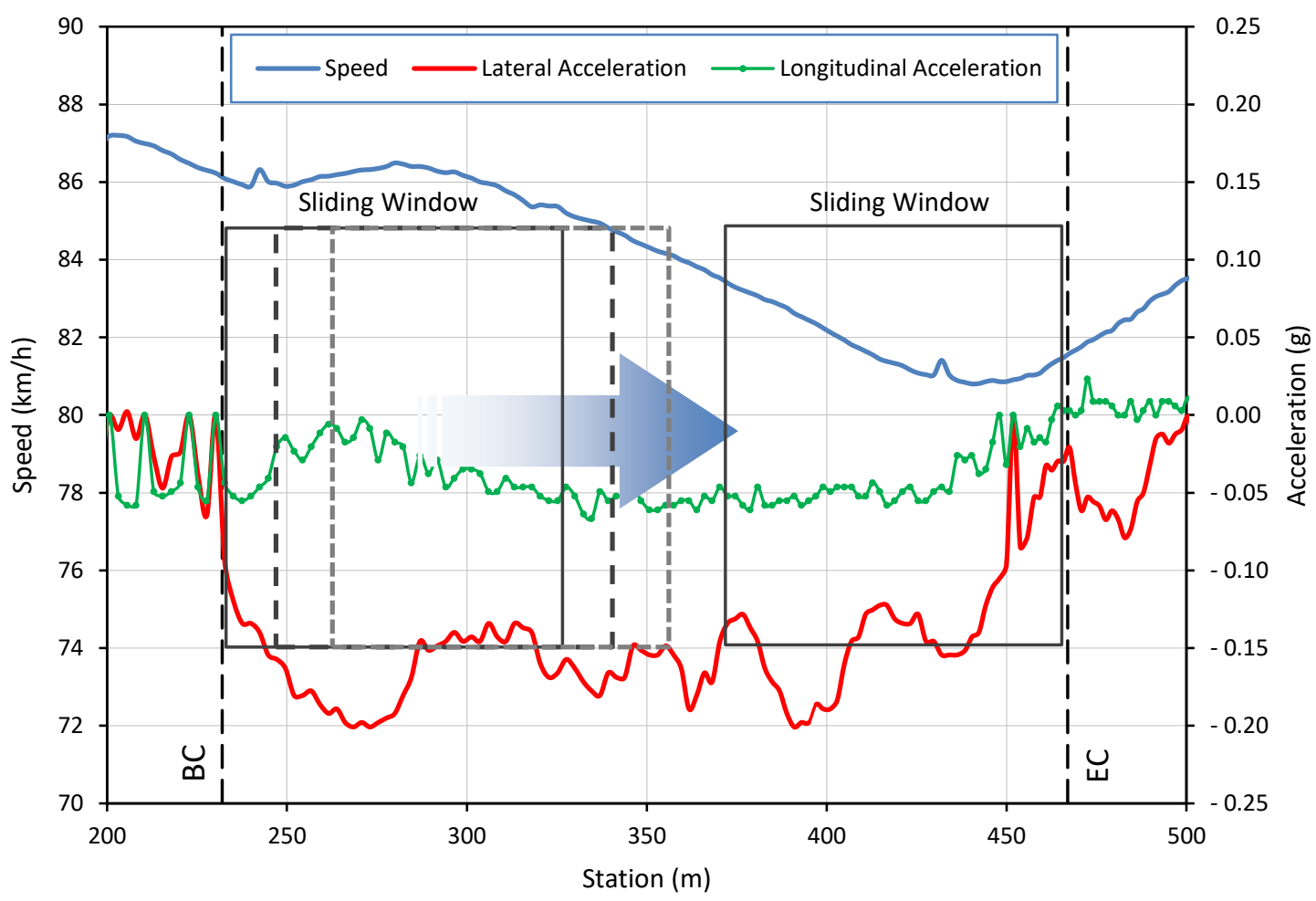

Figure 5.4: Sliding Window. 
The output of this procedure depends on the parameters accThreshold and pvalThreshold, which are used to establish the speed state. In addition, the output depends on the size of sliding window. It should be noted that a large window would increase the value of comfort threshold because of including speed points beyond where the minimum speed is reached. On the other hand, a very small window can deem many trips to be unusable if a gap equal to the window size is found in the speed data or if short-term speed fluctuations cause the small window to always be in "acceleration" or "deceleration" speed state.

\subsubsection{Sensitivity Analysis}

A sensitivity analysis was performed using the rolling terrain curves to assess the effect of three main parameters specified in the Matlab code on the results, which are pvalThreshold, accThreshold, and minimum size of sliding window (minSSWin). All these parameters would affect the calculations of comfort threshold while the general speed state is affected by pvalThreshold and accThreshold only. It should be noted that selecting the number of zones to examine driver acceleration behaviour on a specific curve should depend on the curve length. However, to compare and aggregate the results on different curves, the same number of zones is used for all curves in this research. As shown in Table $3-3$, around $85 \%$ of the rolling terrain curves had a length less than $300 \mathrm{~m}$. Therefore, the general speed states were evaluated by dividing each curve to three zones. Thus, each zone length was less than $100 \mathrm{~m}$ for most curves.

The first sensitivity analysis was run to examine the effect of pvalThreshold using accThreshold of $0.1 \mathrm{~m} / \mathrm{s}^{2}, \operatorname{minSSW}$ in of 0.3 , and three values of pvalThreshold of 0.01 , 0.05, and 0.1. As shown in Figure 5.5, the percentages of trips for the three speed states 
corresponding to all curves are almost constant for all values of pvalThreshold. In most cases, the maximum change in trip percentage for a specific state in a specific zone was between 2 and 3\%. The largest changes were less than 5.5\% and corresponded to the "constant" state which corresponded to over $50 \%$ of the trips. The same trend was observed for each curve individually.

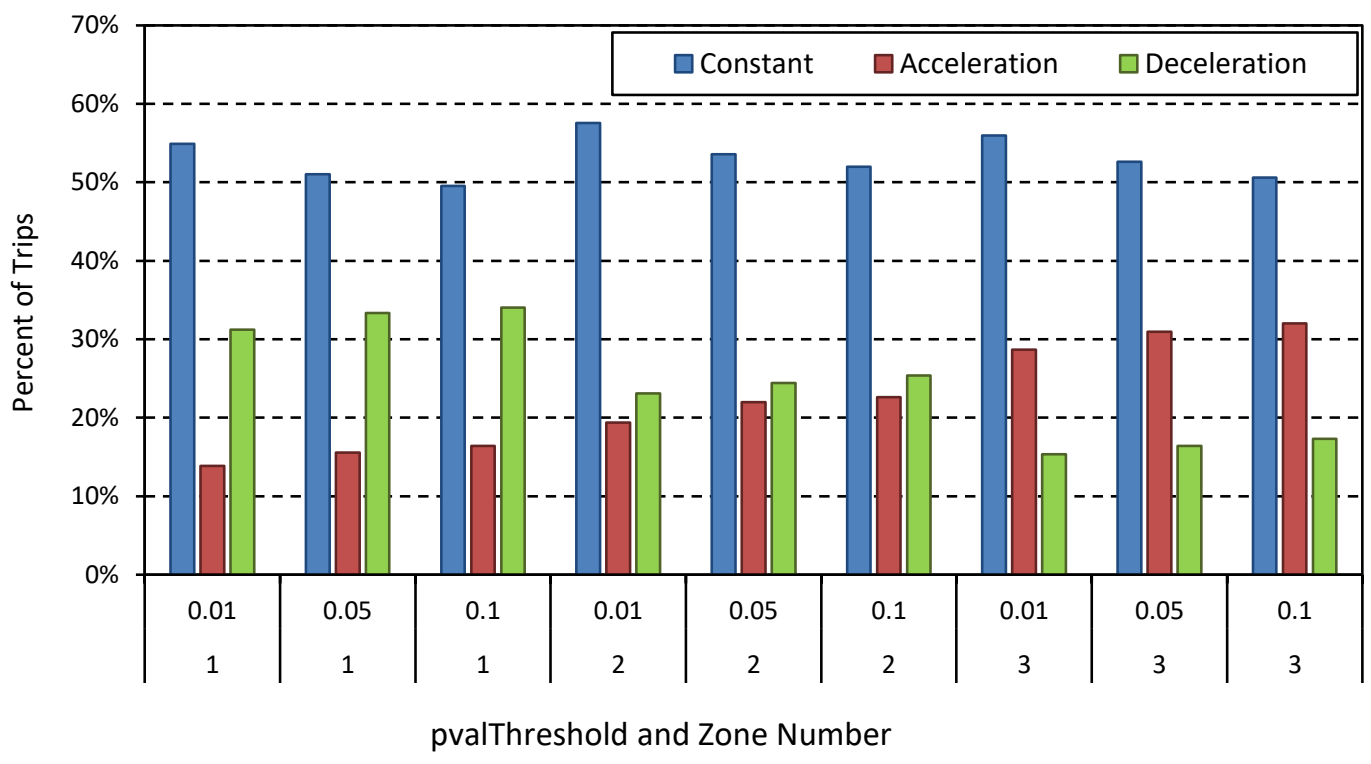

Figure 5.5: Change of Frequencies of General Speed State on All Curves with pvalThreshold.

As for the effect on comfort threshold, the absolute change in mean comfort threshold on each curve was less than $0.01 \mathrm{~g}$, and the percentage change for most curves was less than $2 \%$ (Figure 5.6). Similar findings were observed in relation to the standard deviation of comfort threshold where the maximum absolute change was approximately $0.005 \mathrm{~g}$, and the percentage change for most curves was less than 5\%. Therefore, the results are not sensitive to pvalThreshold, and a 5\% value was selected for the rest of this research. 


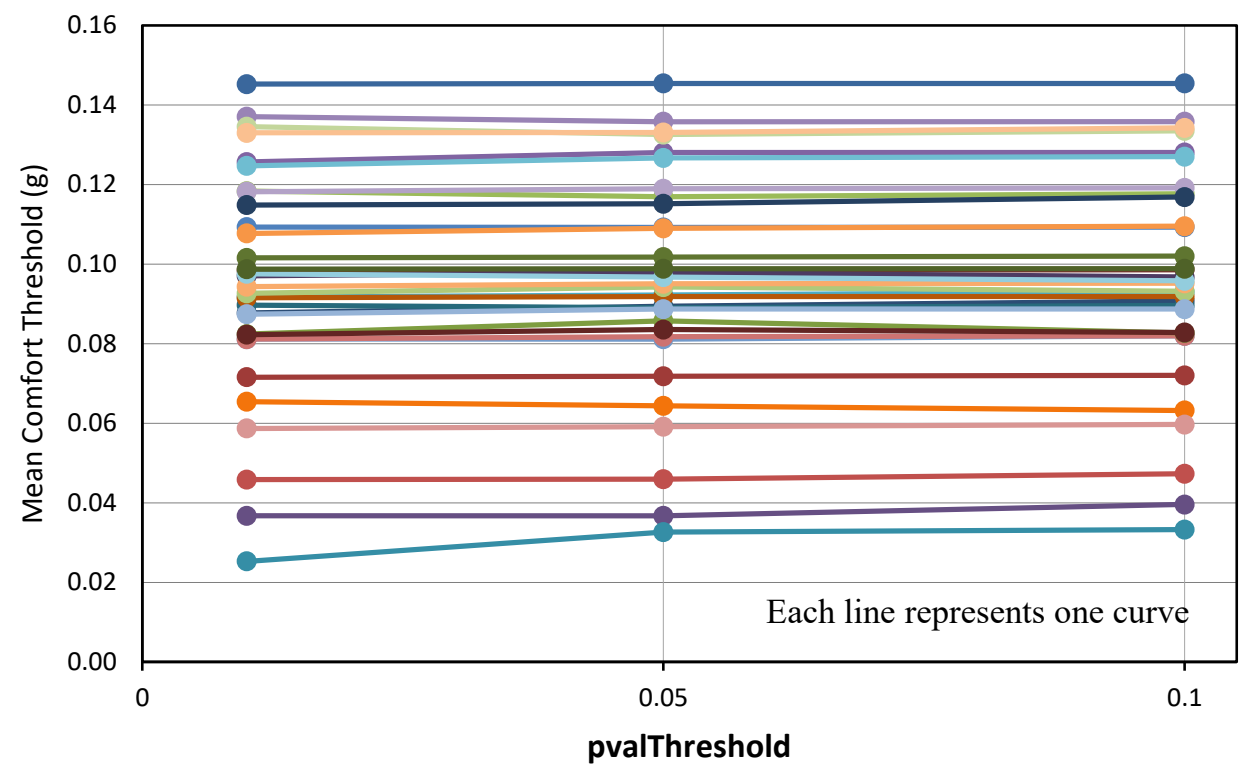

Figure 5.6: Change of Mean Comfort Threshold with pvalThreshold.

The sensitivity of accThreshold was analysed using five values ranging from 0.1 to 0.5 $\mathrm{m} / \mathrm{s}^{2}$, while setting pvalThreshold as 0.05 and minSSWin as 0.3 . The results of mean and standard deviation of comfort threshold showed lack of sensitivity to accThreshold. The maximum difference in mean and standard deviation values on any curve was $0.023 \mathrm{~g}$ and $0.018 \mathrm{~g}$, respectively. As for the general speed state, Figure 5.7 shows that the percentages of trips depend on the value of accThreshold with more drastic differences for accThreshold values below $0.3 \mathrm{~m} / \mathrm{s}^{2}$. The changes are relatively small for accThreshold greater than $0.3 \mathrm{~m} / \mathrm{s}^{2}$, but most trips in all zones get classified as "constant" speed state. 


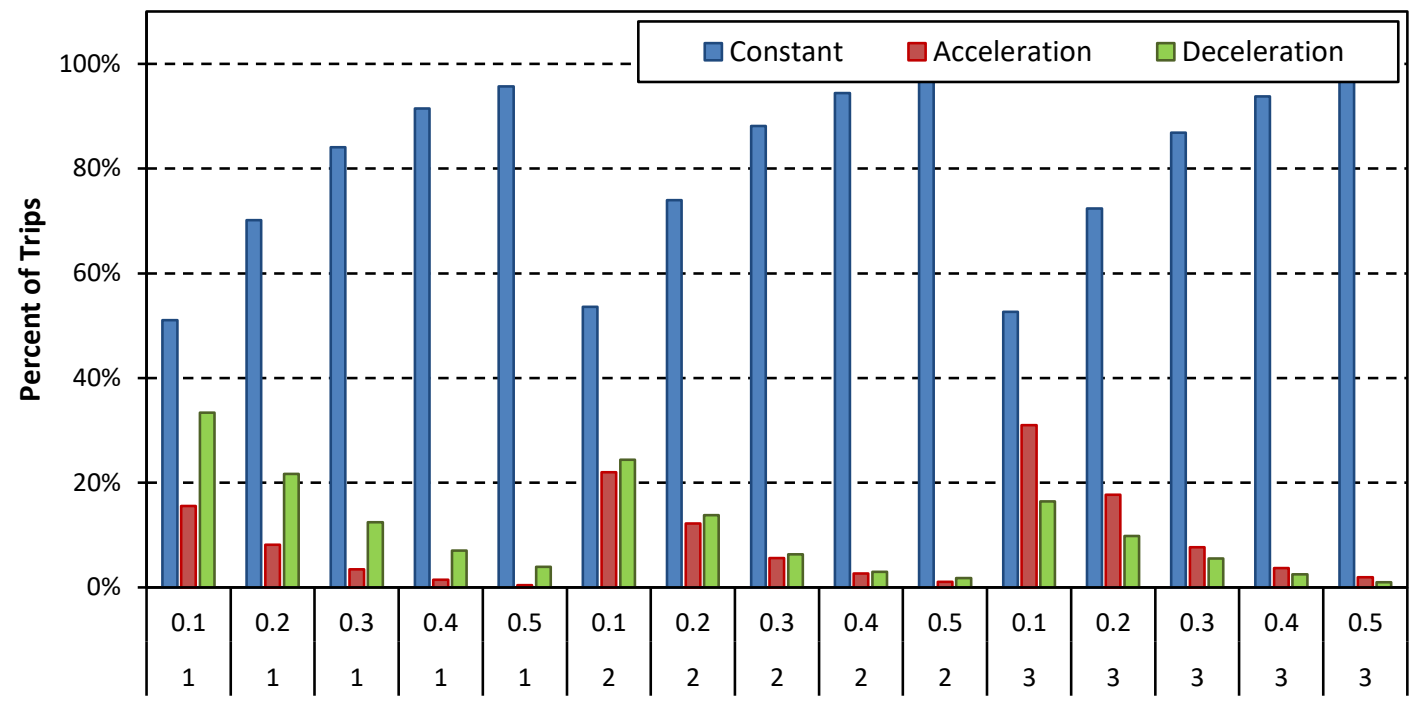

Longitudinal Acceleration Threshold $\left(\mathrm{m} / \mathrm{s}^{2}\right)$ and Zone Number

\section{Figure 5.7: Change of Frequencies of General Speed State on All Curves with} accThreshold.

Fitzpatrick et al. (1999) collected acceleration/deceleration data from 21 sites in 2 states in order to model speed on two-lane rural highways. They reported that a deceleration rate of $\left(-1.0 \mathrm{~m} / \mathrm{s}^{2}\right)$ is recommended for curves with radii less than $175 \mathrm{~m}$. A linear regression was developed to predict deceleration rates for curves with radii greater than $175 \mathrm{~m}$ and less than or equal to $436 \mathrm{~m}$. The prediction of declaration values ranges, in this radius interval, from -1 (radius $=175 \mathrm{~m})$ to zero $($ radius $=436 \mathrm{~m})$. On the other hand, a step-function was recommended for acceleration model based on the radius of the curve $(R)$. Constant acceleration values were recommended as follows (Fitzpatrick et al. 1999):

- $0.54 \mathrm{~m} / \mathrm{s}^{2}$ for curves $175 \mathrm{~m}<R<250 \mathrm{~m}$,

- $0.43 \mathrm{~m} / \mathrm{s}^{2}$ for curves $250 \mathrm{~m}<R<436 \mathrm{~m}$, 
- $0.21 \mathrm{~m} / \mathrm{s}^{2}$ for curves $436<R<875 \mathrm{~m}$, and

- $0.00 \mathrm{~m} / \mathrm{s}^{2}$ for curves with radii greater than $875 \mathrm{~m}$.

Based on the results of modelling longitudinal acceleration/deceleration rate for the Interactive Highway Safety Design Model (IHSDM) mentioned above, an accThreshold of $0.1 \mathrm{~m} / \mathrm{s}^{2}$ was used for the rest of this research.

Finally, the sensitivity analysis of comfort threshold to minSSWin was examined by comparing the mean and standard deviation values using pvalThreshold of 0.05 , accThreshold of $0.1 \mathrm{~m} / \mathrm{s}^{2}$, and five values of minSSWin ranging from 0.1 to 0.5 . As shown in Figure 5.8, the values of standard deviation were not affected by the change of minSSWin, with a maximum change in standard deviation value on any curve less than 0.02g. The figure shows that as expected the mean comfort threshold on each curve increases with the increase of minSSWin. However, the rate of increase decreases considerably for minSSWin of 0.3 and higher. It was judged that a minimum size of sliding window of 0.3 will provide a good compromise between the advantages of larger and smaller window sizes, and was used in the rest of this research. 


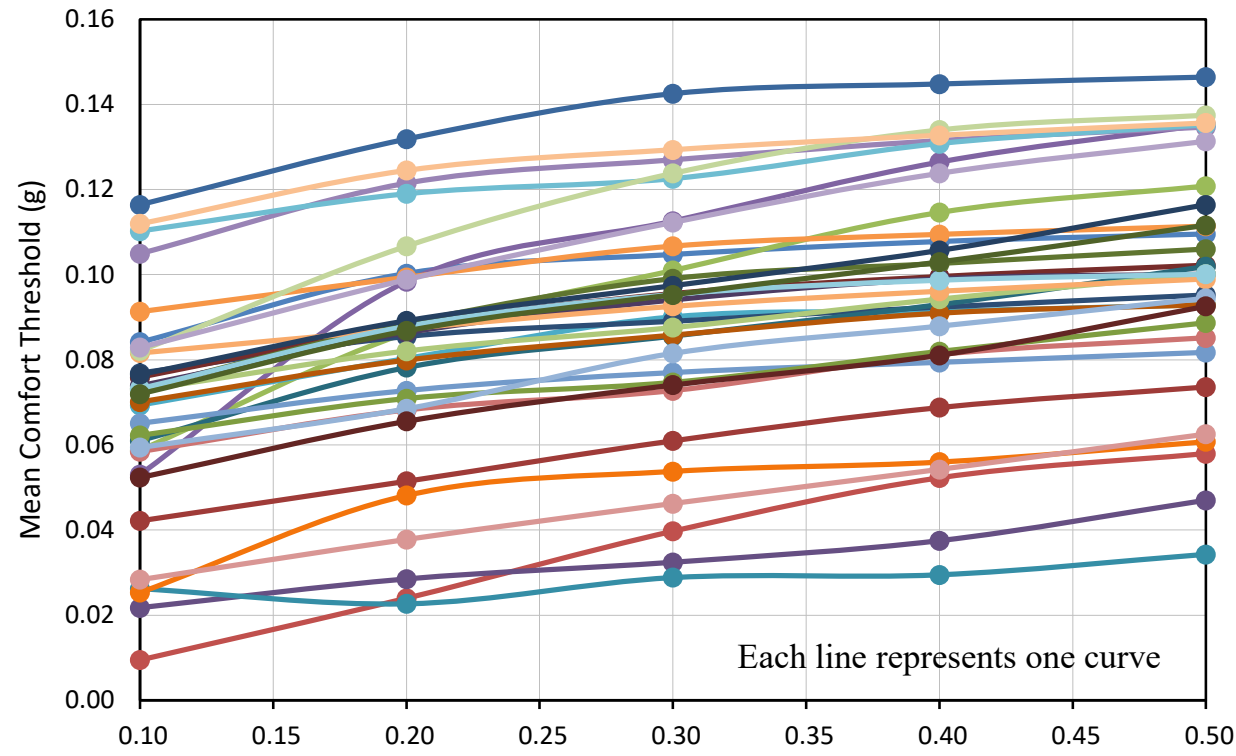

Size of Sliding Window

(a) Mean Comfort Threshold with minSSWin.

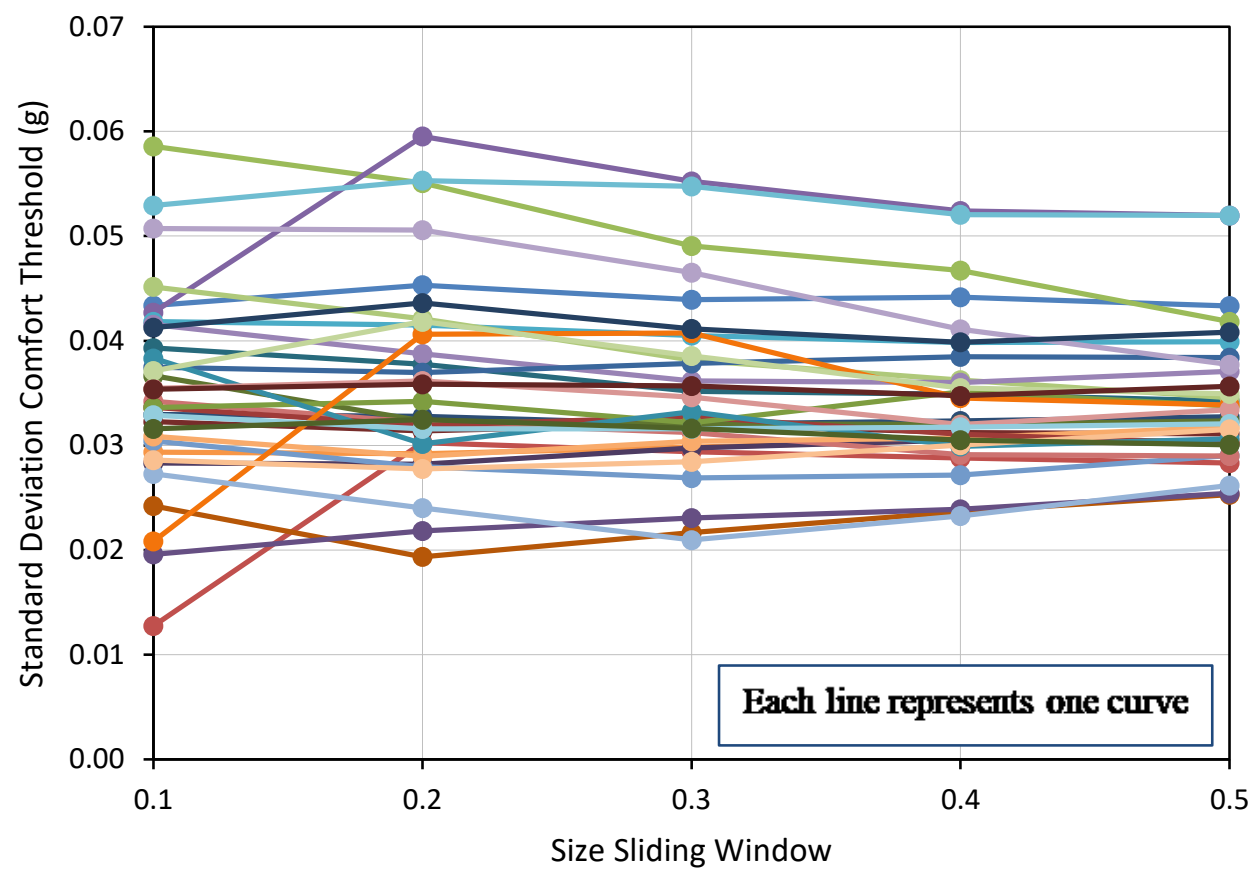

(b) Standard Deviation Comfort Threshold with minSSWin.

Figure 5.8: Change of Mean and Standard Deviation Comfort Threshold with minSSWin. 


\subsection{Models Development}

The previous sections and Chapter 3 prepared the foundation to develop models to predict speed and comfort threshold on horizontal curves. Three types of models were developed in this research for design and reliability analysis purposes. First, driver-level models (DLM) were developed using data of individual drivers, thus minimizing the amount of information lost due to data aggregation and accounting for the entire distribution of variable observed (speed/comfort threshold) at a location (Himes et al. 2013). DLM would predict the mean value of the dependent variable while the standard deviation is readily given as the standard error of estimation (SEE). Second, panel models (PM) were developed for speed/comfort threshold data in a panel form, thus modelling these variables with their dispersion. In this model form, the central tendency of variable estimation and the typical deviation of individual observations are separated (Bassani et al. 2015). The following PM form was used to predict different percentiles of the dependent variable (Medina and Tarko 2005):

$$
Y_{p}=\bar{Y}+Z_{p} \sigma+\varepsilon=\sum_{j} b_{j} X_{j}+Z_{p} \sum_{k} c_{k} X_{k}+\varepsilon
$$

Where $Y_{p}=$ value of dependent variable at any percentile $(p), \bar{Y}=$ mean value of the dependent variable, $Z_{p}=$ standardized normal variable corresponding to a selected percentile, $\sigma=$ standard deviation of the dependent variable, $b_{j}=$ coefficient representing the effect of the $X_{j}$ parameter on $\bar{Y}, c_{k}=$ coefficient representing the effect of $X_{k}$ parameter on $\sigma$.

The third model type is the traditional modelling of $85^{\text {th }}$ percentile speed/comfort threshold on curve. Such a curve level modelling is the common modelling approach in 
design consistency research (Hassan et al. 2011). SPSS forward stepwise regression analysis was used in developing the models. Consequently, several models of each type were developed, and the models with the highest coefficient of determination were selected. It should be noted that all independent variables in the developed models were significant at level of significance less than $5 \%$.

It should be noted that the fixed-effects were not considered in the development of models because drivers most likely drove once on each horizontal curve. Therefore, each trip has its own individual characteristics that may not influence the predictor variables. In other words, specific drivers are not assumed to impact or bias the predictor outcome variables.

\subsubsection{Speed models}

To serve curve design criteria and consistency evaluation, models were developed to predict several speed parameters on curves. As shown in Table 5-1 to Table 5-3, two sets of models for each dependent variable were developed based on the presence or absence of the speed at tangent parameter $\left(V_{a p p}\right)$. Based on the coefficient of determination, models that include $V_{a p p}$ provide better estimation; however, the other models are more applicable since their estimation depends only on geometrical characteristics of the horizontal curve. Also, it is noticed that the aggregated models provide better estimation than DLM models. However, DLM models provides the effect of the variable itself on the dependent variable and explain its contribution in the model and not their aggregated effect. In addition, it provided more information regarding the independent variables, in some models, such as the presence of spiral transition curves, grade, and superelevation rate. It is found that 
radius and presence of spiral transition curves have a direct relationship with all speed types.

Table 5-1: Speed Models at Driver Level.

\begin{tabular}{|c|c|c|c|c|c|}
\hline No. & Variable & $V_{a p p}$ & Model & $R_{a d j}^{2}$ & $S E E$ \\
\hline 1 & $V_{M C}$ & Yes & $36.144+0.692 V_{a p p}-\frac{3220.446}{R}+\frac{21.309}{\Delta}-\frac{0.091}{e}+2.056 S_{e}$ & 0.787 & 5.021 \\
\hline 2 & $V_{M C}$ & No & $\begin{array}{r}84.607-\frac{3837.089}{R}+\frac{72.520}{\Delta}-\frac{0.231}{e}+0.160 V_{L}+0.845 D \\
+2.422 S_{b}+1.600 S_{e}+5.862 T-0.222 G_{b}\end{array}$ & 0.512 & 7.629 \\
\hline 3 & $V_{c r v-\max }$ & Yes & $\begin{array}{c}41.770+0.683 V_{a p p}-\frac{2792.956}{R}-\frac{0.087}{\mathrm{e}}+0.562 S_{b}+1.439 S_{e} \\
-0.045 V_{L}+1.648 T\end{array}$ & 0.813 & 4.579 \\
\hline 4 & $V_{c r v-\max }$ & No & $\begin{array}{c}77.410-\frac{3319.586}{R}+\frac{50.898}{\Delta}+64.366 e+2.395 S_{b}+1.117 S_{e} \\
+0.171 V_{L}+6.000 T\end{array}$ & 0.500 & 7.476 \\
\hline 5 & $V_{c r v-\min }$ & Yes & $\begin{array}{c}31.367+0.701 V_{a p p}-\frac{2838.566}{R}+\frac{74.654}{\Delta}-\frac{0.129}{e}+0.880 S_{b} \\
+1.551 S_{e}\end{array}$ & 0.781 & 5.143 \\
\hline 6 & $V_{c r v-\min }$ & No & $\begin{array}{c}78.010-\frac{3396.151}{R}+\frac{128.358}{\Delta}-\frac{0.363}{e}+2.019 S_{b}+1.419 S_{e} \\
+0.208 V_{L}+5.246 T-0.186 G_{e}\end{array}$ & 0.497 & 7.782 \\
\hline 7 & $V_{B C}$ & Yes & $24.234+0.784 V_{a p p}-\frac{2151.497}{R}+1.552 S_{e}+0.073 G_{e}$ & 0.843 & 4.311 \\
\hline 8 & $V_{B C}$ & No & $\begin{array}{c}67.634-\frac{2276.890}{R}+\frac{73.402}{\Delta}+3.112 S_{b}+0.444 V_{L}+4.822 T \\
-0.316 G_{b}\end{array}$ & 0.476 & 7.889 \\
\hline
\end{tabular}

$R_{a d j}^{2}=$ adjusted coefficient of determination, $S E E=$ standard error of estimate, $V_{a p p}=$ speed at approach tangent $(\mathrm{km} / \mathrm{h})\left\{\right.$ Yes $=V_{a p p}$ is included as an independent variable; No $=V_{a p p}$ is not an independent variable $\}, R=$ radius $(\mathrm{m}), \Delta=$ deflection angle $\left({ }^{\circ}\right), e=$ superelevation rate $(\mathrm{m} / \mathrm{m}), V_{L}=$ speed limit $(\mathrm{km} / \mathrm{h})$, $D=$ turning direction $(0=$ left turn, $1=$ right turn $), T=$ terrain $(0=$ rolling, $1=$ mountainous $), S_{b}$ and $S_{e}=$ spiral curve transition at the beginning and end of the curve, respectively $(0=$ not used, $1=$ used $)$, and $G_{b}, G_{e}=$ grade at the beginning and end of the curve, respectively (\%). 
Table 5-2: Speed Models - Panel Models.

\begin{tabular}{|c|c|c|c|c|}
\hline No. & Variable & Model & $R_{a d j}^{2}$ & SEE \\
\hline 1 & $V_{M C, P}$ & $\begin{array}{c}75.236-\frac{3658.965}{R}+\frac{75.999}{\Delta}+53.567 e+0.184 V_{L} \\
+2.363 S_{b}+1.595 S_{e}+5.620 T \\
-0.292 G_{b}+6.149 Z_{p}-0.960 S_{b} Z_{p} \\
+0.926 Z_{p}\end{array}$ & 0.889 & 3.500 \\
\hline 2 & $V_{c r v-\max , P}$ & $\begin{array}{r}76.650-\frac{3113.024}{R}+\frac{53.326}{\Delta}+54.164 e+0.179 V_{L} \\
+2.183 S_{b}+1.315 S_{e}+6.210 T \\
-0.311 G_{b}+6.000 Z_{p}+0.882 T Z_{p}\end{array}$ & 0.895 & 3.280 \\
\hline 3 & $V_{c r v-\min , P}$ & $\begin{array}{c}66.145-\frac{3052.777}{R}+\frac{124.497}{\Delta}+63.035 e+0.222 V_{L} \\
+2.146 S_{b}+1.456 S_{e}+5.405 T \\
-0.251 G_{b}+6.225 Z_{p}+0.863 T Z_{p}\end{array}$ & 0.883 & 3.634 \\
\hline 4 & $V_{B C, P}$ & $\begin{array}{c}67.162-\frac{2591.811}{R}+\frac{79.653}{\Delta}+43.196 e+0.251 V_{L} \\
\quad+2.570 S_{b}+1.021 S_{e}+5.608 T \\
-0.322 G_{b}+6.533 Z_{p}-1.220 S_{b} Z_{p} \\
+0.790 Z_{p}\end{array}$ & 0.894 & 3.408 \\
\hline
\end{tabular}

Table 5-3: 85th Percentile Speed Models.

\begin{tabular}{|c|c|c|c|c|}
\hline \hline No. & Variable & Model & $R_{a d j}^{2}$ & $S E E$ \\
\hline 1 & $V_{M C, 85}$ & $80.352-\frac{3289.296}{R}+0.261 V_{L}+5.969 T$ & 0.829 & 3.782 \\
\hline 2 & $V_{B C, 85}$ & $74.853-\frac{2614.932}{R}+0.302 V_{L}+6.359 T$ & 0.820 & 3.916 \\
\hline 3 & $V_{c r v-\max , 85}$ & $83.711-\frac{2914.397}{R}+0.230 V_{L}$ & 0.829 & 3.733 \\
\hline 4 & $V_{c r v-\min , 85}$ & $69.153-\frac{2620.988}{R}+\frac{100.391}{\Delta}+0.230 V_{L}+5.158 T$ & 0.843 & 3.673 \\
\hline \hline
\end{tabular}




\subsubsection{Comfort Threshold Models}

Models developed to estimate driver comfort threshold considered two cases; all trips at free-flow conditions and those for drivers who decelerated on the horizontal curve under free-flow conditions. For applicability purposes, two sets of models were developed; with and without speed at tangent parameter $\left(V_{a p p}\right)$. Similar to speed models, models with $V_{a p p}$ provided better estimation based on coefficient of determination values. Also, the DLM models have the lowest coefficient of determination while the aggregated models provide better estimation (Table 5-4). However, DLM and panel model would provide the whole distribution of the dependent variable and more information regarding the effect of independent variables because these models consider the whole recorded data and do not lose information when aggregating the data into $85^{\text {th }}$ percentile value. In other words, the aggregated models provide estimation based on the general trend of the independent variables and, in some cases, the effect of relevant independent variables might be overlooked. For example, the comfort threshold DLM provides more information than $85^{\text {th }}$ percentile models regarding the effect of superelevation rate and presence of spiral curves. It should be noted that this represents the contribution of the independent variable in the model and not the aggregated value. However, all models showed that the comfort threshold has an inverse relationship with radius and a direct relationship with curve turning direction, which corresponds with the correlations among the variables in Section 4.8.1. Also, the panel models, which provide information regarding the variable with its dispersion, showed that radius, posted speed limit, and superelevation rate are significant variables affecting both the mean and variance of comfort threshold while the rest variables affecting only the mean. 
Table 5-4: Comfort Threshold Models.

\begin{tabular}{|c|c|c|c|c|c|}
\hline No. & Variable & $V_{a p p}$ & Model & $R_{a d j}^{2}$ & $S E E$ \\
\hline \multicolumn{6}{|c|}{ Driver Level Model } \\
\hline 1 & $a_{L}$ & Yes & $\begin{array}{c}-0.059+2 \times 10^{-4} R+3 \times 10^{-4} \Delta+0.002 V_{a p p}+0.029 D \\
+0.006 T-0.007 S_{b}+0.016 S_{e}\end{array}$ & 0.507 & 0.033 \\
\hline 2 & $a_{L}$ & No & $\begin{array}{c}-0.140-2 \times 10^{-4}+0.003 V_{a p p}-\frac{0.450}{\Delta}+0.027 D \\
-0.013 S_{b}+0.013 S_{e}\end{array}$ & 0.356 & 0.037 \\
\hline 3 & $a_{L} *$ & Yes & $\begin{array}{r}-0.313+0.003 V_{a p p}+\frac{32.115}{R}-\frac{0.672}{\Delta}+3 \times 10^{-4} V_{L} \\
+0.182 e+0.029 D-0.009 S_{b}+0.016 S_{e}\end{array}$ & 0.593 & 0.030 \\
\hline 4 & $a_{L} *$ & No & $\begin{array}{c}-0.145+\frac{28.100}{R}-\frac{0.500}{\Delta}+0.001 V_{L}+0.415 e+0.033 D \\
+0.016 S_{e}-0.001 G_{e}+0.017 T\end{array}$ & 0.376 & 0.037 \\
\hline \multicolumn{6}{|c|}{ Panel Model } \\
\hline 5 & $a_{L P} *$ & No & $\begin{aligned}-0.169+\frac{29.056}{R} & -\frac{0.529}{\Delta}+0.459 e+0.002 V_{L} \\
& -0.004 S_{b}+0.009 S_{e}+0.019 T \\
& -0.001 G_{b}+0.031 D-0.046 Z_{p} \\
& +8.132 R Z_{p}+0.079 e Z_{p}+0.001 V_{L} Z_{p}\end{aligned}$ & 0.778 & 0.021 \\
\hline 6 & $a_{L P}$ & No & $\begin{array}{rl}0.053-2 \times 10^{-4} & R-\frac{0.376}{\Delta}+0.401 e+0.001 V_{L} \\
& +0.008 S_{e}+0.026 T-0.001 G_{b} \\
& +0.030 D-0.033 Z_{p}-4 \times 10^{-5} R Z_{p} \\
& +0.137 e Z_{p}+0.004 T Z_{p}-4 \times 10^{-4} G_{b} Z_{p}\end{array}$ & 0.762 & 0.021 \\
\hline \multicolumn{6}{|c|}{$85^{\text {th }}$ Percentile Models } \\
\hline 7 & $a_{L 85} *$ & No & $\begin{array}{c}0.103+\frac{41.332}{R}-\frac{12.794}{V_{L}}+5 \times 10^{-4} \Delta+0.029 D \\
+0.021 T\end{array}$ & 0.652 & 0.026 \\
\hline 8 & $a_{L 85}$ & No & $0.127+\frac{37.769}{R}-\frac{10.101}{V_{L}}-\frac{0.707}{\Delta}+0.025 D+0.023 T$ & 0.642 & 0.024 \\
\hline
\end{tabular}

$*=$ only trips that decelerated on curves $\left(V_{a p p}-V_{M C}>0\right)$ were considered in the model.

The previous models were developed to predict speed and comfort threshold for trips that took place in the daytime, favourable weather, and dry pavement conditions. For the 
purpose of the research, models for speed on curve and comfort threshold were developed for conditions of raining and wet pavement conditions (Table 5-5). Since PM provided a good estimation as shown in the previous modes, it was used in developing the models in this table. Unfortunately, the data are not big enough to develop models for other conditions such as snowing, packed-snow or icy pavements, or for night trips. It should be noted that curves with low trips were removed from the database (29 curves were removed). The total number of trips used in the regression analysis was 941.

Table 5-5: Speed and Comfort Threshold Panel Models for Wet Pavement Conditions.

\begin{tabular}{|c|c|c|c|c|}
\hline No. & Type & Models (Rain-Wet) & $R^{2}$ & SEE \\
\hline 1 & $V_{M C-W e t, P}$ & $\begin{array}{c}84.738-\frac{1577.143}{R}+0.125 V_{L}-240.800 e+6.918 T \\
+283.450 e Z_{p}-\frac{2887.700}{R} Z_{p}\end{array}$ & 0.631 & 8.044 \\
\hline 2 & $V_{c m f-W e t, P}$ & $\begin{array}{c}76.354-\frac{3125.493}{R}+\frac{110.436}{\Delta}+0.161 V_{L}+2.035 S_{b}+4.007 S_{e} \\
-0.262 G_{e}+6.877 T+7.851 Z_{p}-1.832 S_{b} Z_{p}\end{array}$ & 0.592 & \begin{tabular}{|l|l} 
& 9.237
\end{tabular} \\
\hline 3 & $V_{c r v-m}$ & $\begin{array}{c}87.968-\frac{4611.070}{R}+0.148 V_{L}+2.367 T-0.494 G_{b}-\frac{232.664}{\Delta} \\
+0.051 V_{L} Z_{p}\end{array}$ & 0.887 & 3.110 \\
\hline 4 & $V_{B C-W e t, P}$ & $\begin{array}{c}76.096-\frac{2047.662}{R}+\frac{134.658}{\Delta}+0.131 V_{L}+7.439 T-0.583 G_{b} \\
+0.313 G_{e}+1.675 S_{b}+3.027 S_{e}-\frac{61.832}{\Delta} Z_{p} \\
+0.092 V_{L} Z_{p}-2.033 S_{b} Z_{p}+1.185 Z_{p}\end{array}$ & 0.691 & 6.569 \\
\hline 5 & $a_{L P-W e t} *$ & $\begin{array}{c}-0.107+\frac{24.803}{R}-\frac{1.524}{\Delta}+0.001 V_{L}-0.011 T-0.002 G_{b} \\
+0.024 D+0.017 Z_{p} T+\frac{12.401}{R} Z_{p} \\
-0.001 G_{b} Z_{p}+0.002 G_{e} Z_{p}\end{array}$ & 0.883 & $\mid$\begin{tabular}{|l}
$\mid c 016$ \\
\end{tabular} \\
\hline
\end{tabular}


It is noted that driving behaviour, in terms of speed $\left(V_{M C}\right)$ and comfort threshold, is influenced by the weather and pavement conditions (raining and wet pavement). The speed values (mean) on curve for such conditions were likely less than those in clear and dry pavement conditions as shown in Figure 5.9 a. Similarly, the mean comfort thresholds for clear weather conditions are most likely higher than those in raining conditions as shown in Figure $5.9 \mathrm{~b}$. That is, it is obvious that drivers tend to accept lower comfort threshold when driving in adverse weather conditions.

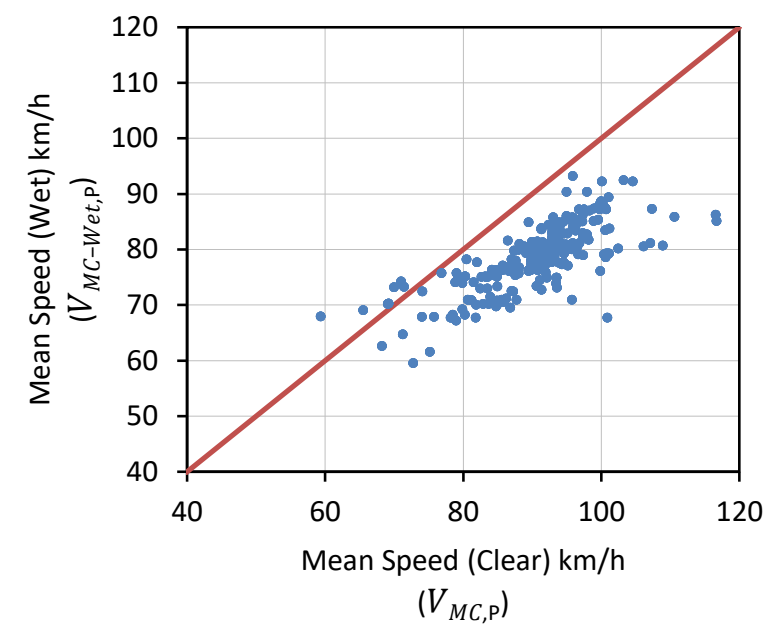

(a) Speed (Clear Weather vs. Wet)

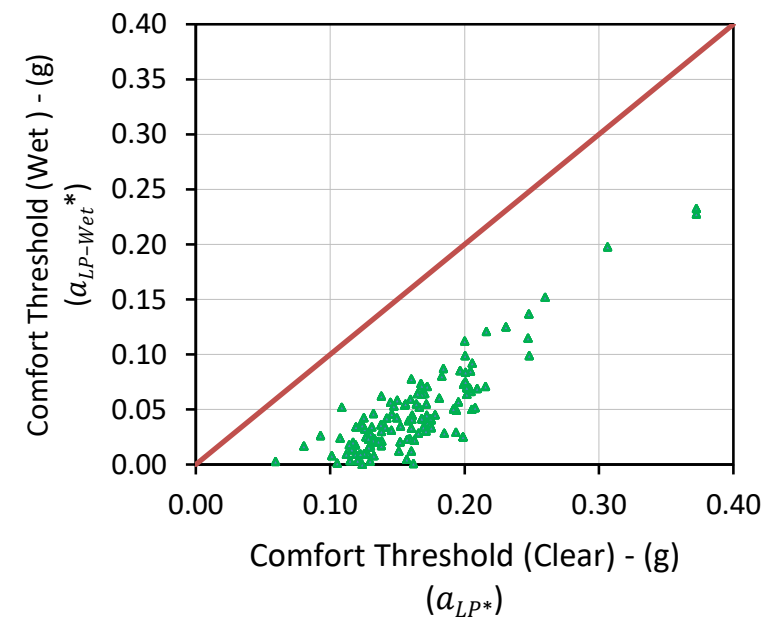

(b) Comfort Threshold Clear vs. Raining Weather Conditions.

Figure 5.9: Speed on Curve and Comfort Threshold for Different Weather Conditions. 


\subsubsection{Lateral Friction Demand Model}

In order to evaluate vehicle stability criterion using reliability analysis, the distribution of the lateral friction demand should be determined. The distribution of demand lateral friction was determined using CarSIM. Two typical types of vehicles were used in the analysis; sedan passenger car D-class and SUV D-class. As explained in Chapter 3, these vehicles were simulated to traverse horizontal curves designed according to the requirements of Green Book (A Policy 2011).

To examine the motion of the vehicle, 39 variables were observed at 0.1 second intervals. The variables included forces (longitudinal, lateral, and normal), angles (slip, steering, yaw, and roll), location of the vehicle (radius and offset from the predetermined path), accelerations (longitudinal and lateral), understeer gradient, and stability factor. The stability factor and understeer gradient were observed to determine the steering situation of the vehicle and to make sure that the vehicle maintains understeer or neutral situation. Thus, drivers either maintain the steering angle or increase the steering angle to keep the vehicle on the track. Otherwise, drivers in the oversteer situation need to decrease the steering angle with speed, which needs more driving skill. Therefore, a stability factor ranging from +0.0001 to +0.0008 and understeer gradient between 0.1 and +1.00 were adopted.

To develop a model to estimate the lateral friction demand in terms of road geometry and vehicle characteristics, the relationship between lateral friction demand and each parameter was first investigated. The outputs of CarSIM showed that the relationships between lateral friction and curves geometric parameters (radius and superelevation rate) are nonlinear as shown in Figure 5.10. 

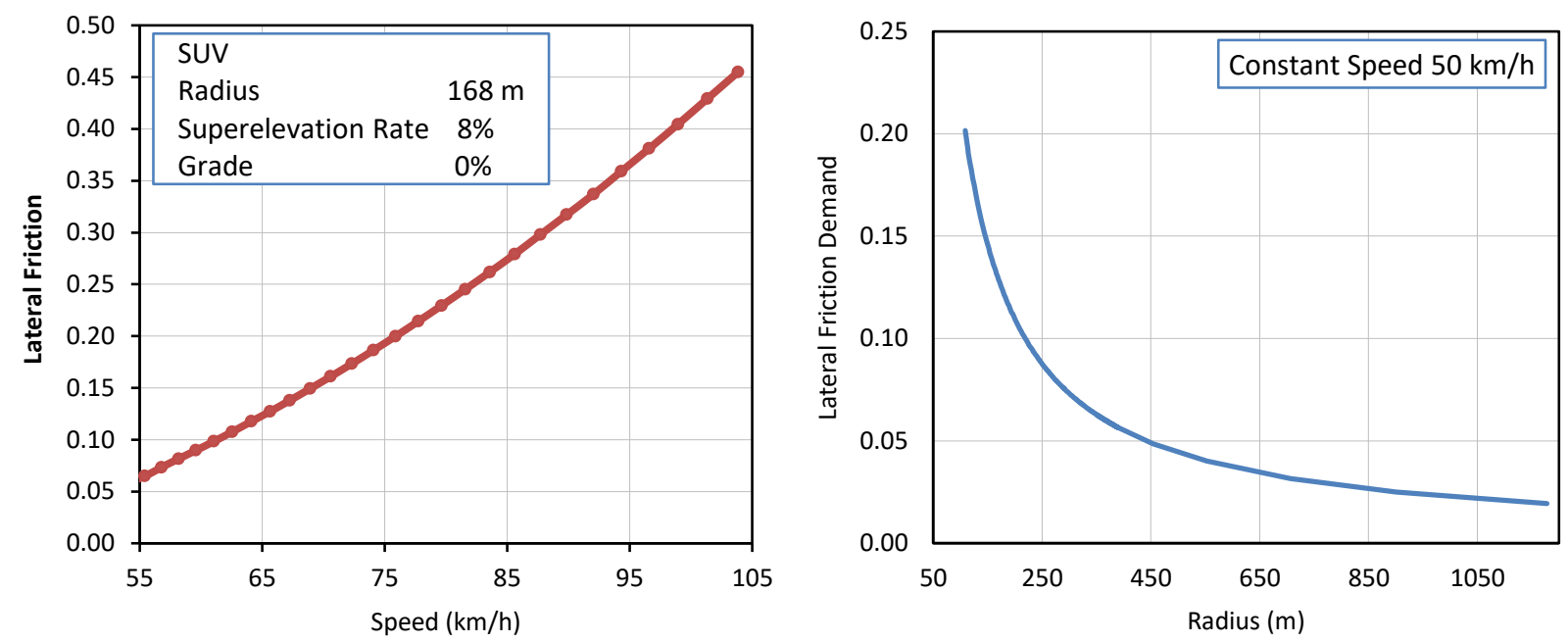

Figure 5.10: Relationship between Lateral Friction Demand and Speed and Radius.

Subsequently, a semi-empirical model was proposed to take the same form of the PMM model while having different coefficients to consider vehicle's characteristics implicitly. Thus, more accurate estimate for tire-road friction would be expected ( $\mathrm{Li}$ et al. 2006). Accordingly, the proposed non-linear model used in the regression analysis for estimating lateral friction is:

$f_{D}=\frac{\beta_{1} V^{\beta_{2}}}{R^{\beta_{3}}}+\beta_{4} e^{\beta_{5}}+\beta_{6} G$

Where $f_{D}=$ lateral friction demand, $V=$ vehicle speed $(\mathrm{km} / \mathrm{h}), R=$ curve radius $(\mathrm{m})$, $e=$ superelevation rate, $G=$ vertical grade, and $\beta_{1-6}=$ factor parameters.

In contrast to previous models mentioned in Chapter 2, the developed models in this research seek for the maximum lateral friction demand, which is expected in the transient phase as shown in Figure 5.11a. The most critical zones of a horizontal curve are the areas close to the beginning and end of the curve where the vehicle changes direction from straight to curve and vice versa. The peak value for lateral friction demand and lowest 
counterbalancing force, as the superelevation may have not been fully developed, are expected in these zones (Dhahir and Hassan 2015). The effect of having high value of peak friction demand can be mitigated using spiral curve transition as shown in Figure 5.11b.

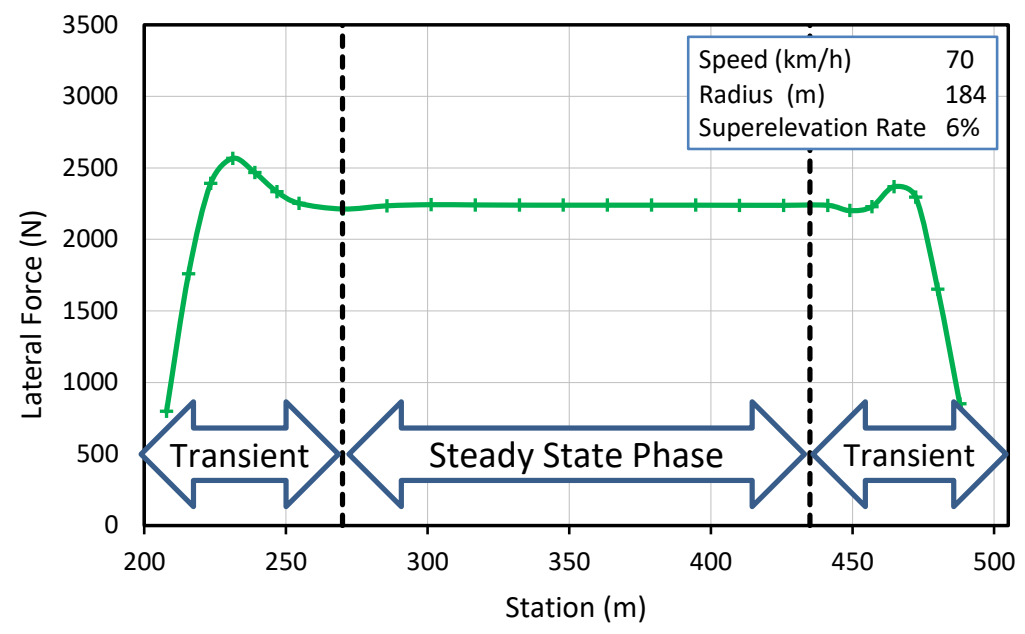

(a) Lateral Friction Demand on Different Wheels.

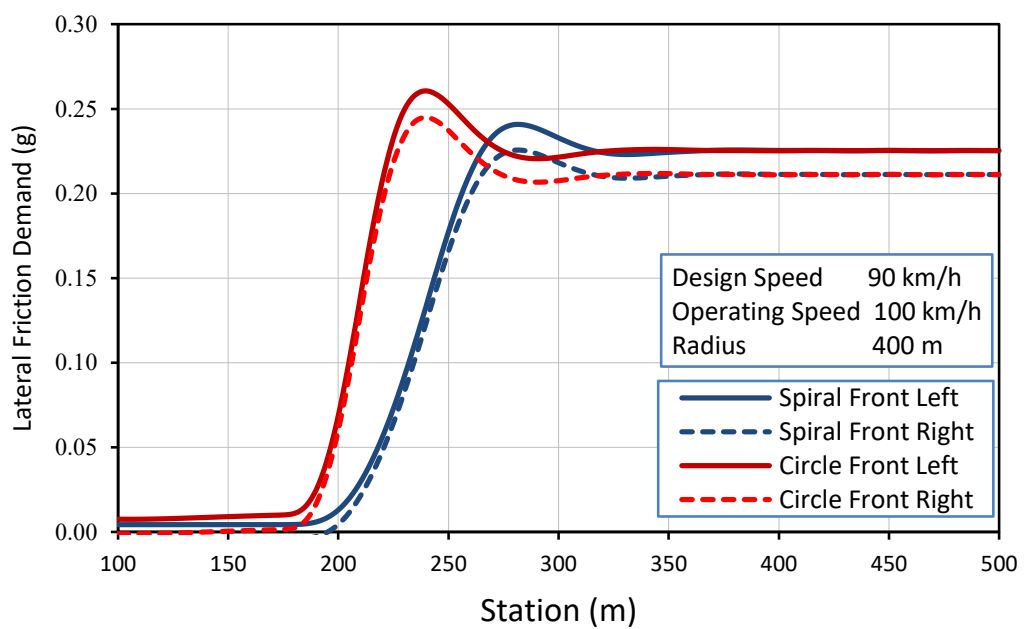

(b) Effect of Spiral Transition Curve on Lateral Friction Demand.

\section{Figure 5.11: Lateral Friction Demand on Horizontal Curve.}

Based on the results of CarSIM and using SPSS software for performing non-linear regression analysis, the model for $\mathrm{PC}$ and $\mathrm{SUV}$ for peak friction demand $\left(f_{D P}\right)$ are shown in Table 5-6. As shown in the table, the coefficients of determination of the developed 
models are high. This situation is expected because there is no random effect considered in the simulations. In other words, the outputs of the simulation will be the same when re-run the simulation. Therefore, the high values of coefficient of determination indicate that the variables and models' form selected in the regression capture the variation in the results accurately.

Table 5-6: Peak Lateral Friction Demand for Different Vehicle Types.

\begin{tabular}{|c|c|c|c|c|}
\hline Vehicle Type & Coefficient & Estimate & Standard Error & $\begin{array}{l}\text { Coefficient of } \\
\text { Determination }\end{array}$ \\
\hline \multicolumn{5}{|c|}{$f_{D}=\frac{\beta_{1} V^{\beta_{2}}}{R^{\beta_{3}}}+\beta_{4} e^{\beta_{5}}+\beta_{6} G$} \\
\hline \multirow{6}{*}{$\mathrm{PC}$} & $\overline{\beta_{1}}$ & 0.009 & $<0.001$ & \multirow{6}{*}{0.999} \\
\hline & $\beta_{2}$ & 1.879 & 0.013 & \\
\hline & $\beta_{3}$ & 0.886 & 0.006 & \\
\hline & $\beta_{4}$ & -0.947 & 0.071 & \\
\hline & $\beta_{5}$ & 0.928 & 0.035 & \\
\hline & $\beta_{6}$ & $2.6 \times 10^{-5}$ & $<0.001$ & \\
\hline \multirow{6}{*}{ SUV } & $\beta_{1}$ & 0.007 & 0.001 & \multirow{6}{*}{0.998} \\
\hline & $\beta_{2}$ & 2.021 & 0.029 & \\
\hline & $\beta_{3}$ & 0.970 & 0.013 & \\
\hline & $\beta_{4}$ & -0.979 & 0.105 & \\
\hline & $\beta_{5}$ & 1.049 & 0.057 & \\
\hline & $\beta_{6}$ & $1.2 \times 10^{-4}$ & $<0.001$ & \\
\hline
\end{tabular}

\subsubsection{Lateral Acceleration Model}

Along with driver comfort threshold, consideration of the driver comfort criterion requires the ability to estimate the lateral acceleration affecting vehicle's occupants while traveling on a horizontal curve. As shown in Figure 5.12, the lateral acceleration data showed a trend of initial peak similar to that observed in the lateral friction demand data. Because a steady state of lateral acceleration was quickly reached after the initial peak, it was relatively easy to estimate an average value of this steady state. The lateral acceleration 
affecting on vehicle occupants was taken as the sustained value at the middle of the curve and outside the two peaks at the curve beginning and end. Thus, the developed models estimate the applied lateral acceleration when the vehicle is in the steady state. It should be noted that the simulation outputs contained the total lateral acceleration including the portion counterbalanced by the weight component due to superelevation and the portion felt by the vehicle occupants. Because of the effects of vehicle body roll, this lateral acceleration is slightly different from the theoretical value of $v^{2} / R$, where $v=$ vehicle speed on the curve $(\mathrm{m} / \mathrm{s})$ and $R=$ curve radius $(\mathrm{m})$.

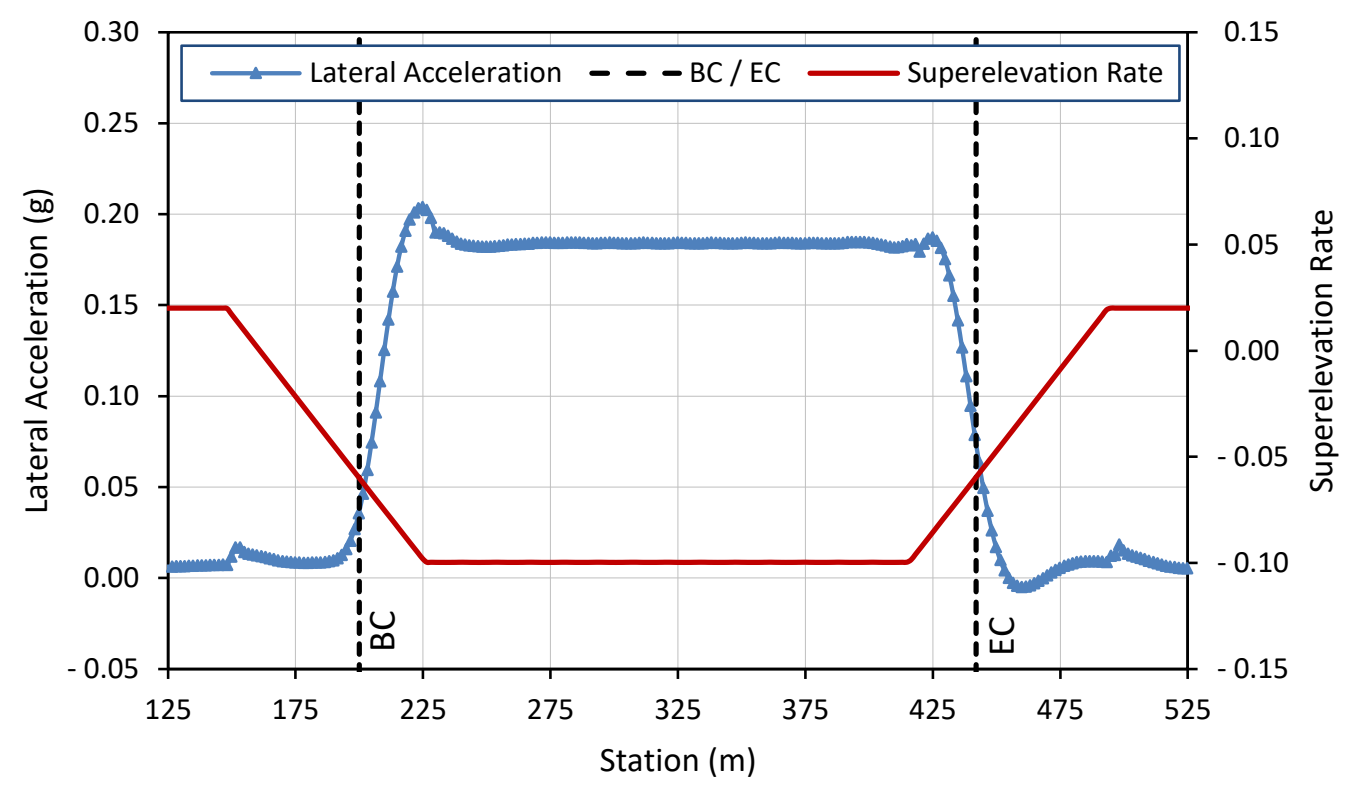

Figure 5.12: Lateral Acceleration Profile.

The outputs of CarSIM simulations were used in non-linear regression analysis using SPSS software to develop another model for the total lateral acceleration affecting a PC and SUV as shown in Table 5-7. The main difference between the equations is due to the suspension system characteristics of the vehicles. As mentioned earlier, the total lateral acceleration in this model includes the part of centripetal acceleration sustained by the 
weight component resulting from the superelevation. Therefore, the net lateral acceleration affecting the vehicle occupants would be the value predicted by the model minus the superelevation rate.

Table 5-7: Lateral Acceleration for Different Vehicle Types.

\begin{tabular}{|c|c|c|c|c|}
\hline $\begin{array}{l}\text { Vehicle } \\
\text { Type }\end{array}$ & Coefficient & Estimate & $\begin{array}{l}\text { Standard } \\
\text { Error }\end{array}$ & $\begin{array}{l}\text { Coefficient of } \\
\text { Determination }\end{array}$ \\
\hline \multicolumn{5}{|c|}{$a_{D}=\frac{\beta_{1} V^{\beta_{2}}}{R^{\beta_{3}}}+\beta_{4} e^{\beta_{5}}+\beta_{6} G$} \\
\hline \multirow{6}{*}{ PC } & $\beta_{1}$ & 0.011 & 0.006 & \multirow{6}{*}{0.957} \\
\hline & $\beta_{2}$ & 1.559 & 0.211 & \\
\hline & $\beta_{3}$ & 0.655 & 0.087 & \\
\hline & $\beta_{4}$ & -0.032 & 0.021 & \\
\hline & $\beta_{5}$ & 0.700 & 0.183 & \\
\hline & $\beta_{6}$ & $-4.3 \times 10^{-4}$ & $<0.001$ & \\
\hline \multirow{6}{*}{ SUV } & $\beta_{1}$ & 0.007 & 0.003 & \multirow{6}{*}{0.967} \\
\hline & $\beta_{2}$ & 1.702 & 0.136 & \\
\hline & $\beta_{3}$ & 0.703 & 0.054 & \\
\hline & $\beta_{4}$ & -0.019 & 0.010 & \\
\hline & $\beta_{5}$ & 0.876 & 0.167 & \\
\hline & $\beta_{6}$ & -0.001 & $<0.001$ & \\
\hline
\end{tabular}

$a_{D}=$ applied lateral acceleration $(\mathrm{g}), V=$ speed $(\mathrm{km} / \mathrm{h}), R=$ radius $(\mathrm{m}), e=$ superelevation rate (percent), $G=$ grade (percent).

\subsubsection{Available Friction Models}

Based on the data shown in Table 2-4 and Table 2-5, the equations provided by Olson et al. (1984) (2.41 and 2.42), and reduction factor of 0.925 provided by Lamm (1999) in Chapter 2, the distribution of friction data for PC and heavy trucks tires and pavement were calculated as shown in Table 5-8. The coefficient of variation (COV) of wet pavement was calculated and used to determine the standard deviation of dry pavement. This assumption was previously provided by Himes (2013). The available data of fiction for truck tires were assumed to be 70\% of the PC tires (Olson et al. 1984; Harwood and Mason 1994). 
Table 5-8: Friction Data Distribution for Passenger Car for Wet and Dry Pavement.

\begin{tabular}{|c|c|c|c|c|c|c|c|c|c|c|c|c|c|c|c|}
\hline \multirow[b]{2}{*}{$\begin{array}{c}\text { Speed } \\
(\mathrm{km} / \mathrm{h})\end{array}$} & \multicolumn{9}{|c|}{ Wet pavement } & \multicolumn{6}{|c|}{ Dry Pavement } \\
\hline & $\begin{array}{l}\text { Mean } \\
\text { Long. } \\
\text { Friction } \\
\text { (1) }\end{array}$ & $\begin{array}{l}\text { SD } \\
(2)\end{array}$ & $\begin{array}{c}\mathrm{COV} \\
(3)\end{array}$ & $\begin{array}{c}\text { Mean } \\
\text { Lateral } \\
\text { Friction } \\
\text { (4) }\end{array}$ & $\begin{array}{l}\text { SD } \\
(5)\end{array}$ & $\begin{array}{c}\mathrm{COV} \\
(6)\end{array}$ & $\begin{array}{c}\text { Mean } \\
\text { Peak } \\
\text { Friction } \\
\quad(7)\end{array}$ & $\begin{array}{l}\text { SD } \\
(8)\end{array}$ & $\begin{array}{c}\mathrm{COV} \\
(9)\end{array}$ & $\begin{array}{l}\text { Mean } \\
\text { Long. } \\
\text { Friction } \\
\text { (10) }\end{array}$ & $\begin{array}{l}\text { SD } \\
(11)\end{array}$ & $\begin{array}{c}\text { Mean } \\
\text { Lateral } \\
\text { Friction } \\
\text { (12) }\end{array}$ & $\begin{array}{l}\text { SD } \\
(13)\end{array}$ & $\begin{array}{c}\text { Mean } \\
\text { Peak } \\
\text { Friction } \\
\text { (14) }\end{array}$ & $\begin{array}{c}\text { SD } \\
(15)\end{array}$ \\
\hline 16.09 & 0.694 & 0.114 & 0.164 & 0.663 & 0.105 & 0.158 & 0.977 & 0.114 & 0.117 & 0.830 & 0.136 & 0.768 & 0.122 & 1.09 & 0.127 \\
\hline 24.14 & 0.648 & 0.117 & 0.181 & 0.597 & 0.108 & 0.181 & 0.926 & 0.117 & 0.126 & 0.826 & 0.149 & 0.764 & 0.138 & 1.088 & 0.137 \\
\hline 32.18 & 0.584 & 0.097 & 0.166 & 0.54 & 0.09 & 0.167 & 0.854 & 0.097 & 0.114 & 0.821 & 0.136 & 0.759 & 0.127 & 1.085 & 0.123 \\
\hline 40.23 & 0.526 & 0.084 & 0.160 & 0.487 & 0.077 & 0.158 & 0.789 & 0.084 & 0.106 & 0.815 & 0.130 & 0.754 & 0.119 & 1.073 & 0.114 \\
\hline 48.27 & 0.475 & 0.071 & 0.149 & 0.439 & 0.066 & 0.150 & 0.732 & 0.071 & 0.097 & 0.810 & 0.121 & 0.749 & 0.113 & 1.06 & 0.103 \\
\hline 56.32 & 0.429 & 0.063 & 0.147 & 0.397 & 0.058 & 0.146 & 0.68 & 0.063 & 0.093 & 0.805 & 0.118 & 0.745 & 0.109 & 1.048 & 0.097 \\
\hline 64.36 & 0.388 & 0.056 & 0.144 & 0.359 & 0.052 & 0.145 & 0.635 & 0.056 & 0.088 & 0.800 & 0.115 & 0.740 & 0.107 & 1.035 & 0.091 \\
\hline 72.41 & 0.351 & 0.052 & 0.148 & 0.324 & 0.048 & 0.148 & 0.593 & 0.052 & 0.088 & 0.796 & 0.118 & 0.736 & 0.109 & 1.028 & 0.090 \\
\hline 80.45 & 0.318 & 0.05 & 0.157 & 0.294 & 0.046 & 0.156 & 0.556 & 0.05 & 0.090 & 0.790 & 0.124 & 0.731 & 0.114 & 1.02 & 0.092 \\
\hline 88.50 & 0.289 & 0.048 & 0.166 & 0.268 & 0.044 & 0.164 & 0.524 & 0.048 & 0.092 & 0.786 & 0.131 & 0.727 & 0.119 & 1.003 & 0.092 \\
\hline 96.54 & 0.261 & 0.047 & 0.180 & 0.241 & 0.043 & 0.178 & 0.492 & 0.047 & 0.096 & 0.781 & 0.141 & 0.722 & 0.129 & 0.985 & 0.094 \\
\hline 104.59 & 0.237 & 0.047 & 0.198 & 0.219 & 0.043 & 0.196 & 0.465 & 0.047 & 0.101 & 0.775 & 0.154 & 0.717 & 0.141 & 0.968 & 0.098 \\
\hline 112.63 & 0.215 & 0.046 & 0.214 & 0.199 & 0.043 & 0.216 & 0.441 & 0.046 & 0.104 & 0.770 & 0.165 & 0.712 & 0.154 & 0.95 & 0.099 \\
\hline
\end{tabular}

$\mathrm{SD}=$ Standard deviation.

$\mathrm{COV}=$ Coefficient of determination.

Long. $=$ Longitudinal.

Data in Columns (1, 2, 4, 5, 7, 8, 12, and 14) were extracted from Himes (2013).

Column (3) = Column (2) / Column (1).

Column (6) = Column (5) / Column (4).

Column (9) = Column (8) / Column (7).

Column (10) $=$ Column (12) $/ 0.925$.

Column (11) = Column (10) x Column (3).

Column (13) $=$ Column (6) $\times$ Column (12).

Column (3) = Column (9) x Column (14). 
Based on the friction data in Table 5-8, the following non-linear models were developed to predict friction factors for different speeds for PC. Note that the highest value of standard error for model coefficients were 0.004. The developed models were used in the reliability analysis.

$$
\begin{array}{ll}
f_{P C W \text { mean }}=0.874 \mathrm{~V}^{-0.013} & R^{2}=0.999 \\
f_{P C W-S D}=1.56 \times 10^{-5} V^{2}-0.003 \mathrm{~V}+0.173 & R^{2}=0.994 \\
f_{P C L W \text { mean }}=0.807 \text { exp }^{-0.013 \mathrm{~V}} & R^{2}=0.999 \\
f_{P C L W-S D}=1.43 \times 10^{-5} V^{2}-0.003 \mathrm{~V}+0.160 & R^{2}=0.994 \\
f_{P C D-\text { mean }}=-6.0 \times 10^{-4} \mathrm{~V}+0.840 & R^{2}=0.999 \\
f_{P C D-S D}=2.16 \times 10^{-5} V^{2}-0.003 \mathrm{~V}+0.202 & R^{2}=0.994 \\
f_{P C L D-\text { mean }}=-6.0 \times 10^{-4} \mathrm{~V}+0.777 & R^{2}=0.999 \\
f_{P C L D-S D}=1.96 \times 10^{-5} \mathrm{~V}^{2}-0.002 \mathrm{~V}+0.187 & R^{2}=0.960
\end{array}
$$

Where $f_{P C W-\text { mean }}$ and $f_{P C W-S D}=$ mean and standard deviation of longitudinal friction factor for wet pavement, $f_{P C L W-m e a n}$ and $f_{P C L W-S D}=$ mean and standard deviation of the lateral friction for wet pavement, $f_{P C D-m e a n}$ and $f_{P C D-S D}=$ mean and standard deviation of the longitudinal friction for dry pavement, and $f_{P C L D-m e a n}$ and $f_{P C L D-S D}=$ mean and standard deviation of lateral friction for dry pavement.

For evaluating the performance of the horizontal curves in the study area in terms of vehicle stability and sight distance criteria, the following procedure was followed to calculate the available longitudinal and lateral friction factors. As explained in Section 3.6.2, the friction database includes route ID, average of skid number (SN) for the 
measured segment, peak value of $\mathrm{SN}$, test speed, date of measurement, and location of segment (mile post).

1. Based on the route ID and location of the curve (beginning and end points of the curve), the $\mathrm{SN}$ is determined. For example, for a curve located on route 002 (St. 71.54 - St. 71.86), the average SN for year 2008 was 53.5 measured at speed $62.75 \mathrm{~km} / \mathrm{h}(39 \mathrm{mph})$.

2. The average $\mathrm{SN}$ is calibrated using Wisconsin equation (Equation 3.22). Thus, the calibrated skid number $\left(F R_{40}\right)$ is:

$$
\begin{aligned}
& F R_{40}=F R S-0.57(40-S) \\
& F R_{40}=53.5-0.57(40-39) \\
& F R_{40}=52.93
\end{aligned}
$$

3. Although Olson et al. (1984) provided equations to estimate friction for PC and heavy truck tires (Equations 2.43 and 2.44), the skid number $\left(S N_{v}\right)$ cannot be calculated because the friction database lacked mean pavement depth values. Therefore, for the purpose of this research, the adjusted friction factor was assumed as the mean available longitudinal friction factor (sliding friction for wet pavement) for PC $\left(f_{P C W-m e a n}\right)$. And, the mean value of the heavy truck $\left(f_{T W-\text { mean }}\right)$ was assumed to be 70 percent of the PC friction factor.

$$
\begin{aligned}
& f_{P C W-\text { mean }}=F R_{40} / 100 \\
& f_{P C W \text {-mean }}=\left(\frac{52.93}{100}\right) \\
& f_{P C W \text {-mean }}=0.53
\end{aligned}
$$


$f_{T W-\text { mean }}=0.7 \times f_{P C W-\text { mean }}$

$f_{T W-\text { mean }}=0.7 \times 0.53$

$f_{T W-\text { mean }}=0.37$

4. The calibrated friction represents sliding friction factor for pavement because the $\mathrm{SN}$ is measured using locked-wheel technique. The peak value of the friction available for PC $\left(f_{P C W P-m e a n}\right)$ and heavy truck tires $\left(f_{P C W P-\text { mean }}\right)$ can be estimated using Olson et al. (1984) (Equations 2.41 and 2.42).

$f_{x, \max }=0.2+1.12 \times f_{x, \text { skidding }}$

$f_{P C W P-\text { mean }}=0.2+1.12(0.53)$

$f_{P W-\text { mean }}=0.79$

$f_{T W P-\text { mean }}=1.45(0.37)$

$f_{T W-\text { mean }}=0.53$

5. The available lateral friction is calculated using the reduction factor $(0.925)$ presented by Lamm et al. (1999). Thus, the available lateral friction factor for wet pavement for this curve for PC $\left(f_{P C L W-m e a n}\right)$ and heavy truck $\left(f_{T L W-m e a n}\right)$ tires are 0.49 and 0.34 , respectively.

6. Because the measurements were taken every two years, a linear interpolation was used to determine the friction for missing year measurements. Thus, friction measurements were calculated for the years 2009-2013 because the available database included measurements for years 2007-2014. 
As shown above, the calculations lacked data of friction factor for dry pavement. Thus, the distribution for dry pavement is unknown. It should be noted that transportation agencies do not conduct measurement for dry pavement. Therefore, to estimate the mean value of available friction factor for dry pavement, based on the available friction for wet pavement shown in Table 2-4, the difference between the mean values of the dry and wet pavement for every speed were calculated. Then the difference along with speed is modelled using non-linear regression. Thus, the relationship between the wet and dry pavement difference is developed as shown below.

For PC tires, the mean value of available longitudinal and lateral friction factor for dry pavement are shown in Equations 5.11 and 5.12, respectively.

$$
\begin{array}{ll}
f_{P C D \text {-mean }}=f_{P C W \text {-mean }}+\left(-3 \times 10^{-5} V^{2}+0.008 V+0.006\right) ; & R^{2}=0.999 \\
f_{L D \text {-mean }}=f_{P C L W \text {-mean }}+\left(-3 \times 10^{-5} V^{2}+0.008 V+0.012\right) ; & R^{2}=0.999
\end{array}
$$

For heavy truck tires, the mean value of available longitudinal and lateral friction factor for dry pavement are shown in Equations 5.13 and 5.14, respectively.

$$
\begin{gathered}
f_{T D \text {-mean }}=f_{P C W \text {-mean }}+\left(-2 \times 10^{-5} V^{2}+0.006 V+0.004\right) ; \quad R^{2}=0.999 \\
f_{T L D \text {-mean }}=f_{P C L W \text {-mean }}+\left(-2 \times 10^{-5} V^{2}+0.006 V+0.008\right) ; \quad R^{2}=0.999
\end{gathered}
$$

\subsection{Summary}

This chapter was devoted to developing models to be used in the reliability analysis based on the collected data. However, prior to developing these model, insight examination for driving behaviour was conducted in order to determine the most critical points on the horizontal curves. Thus, selecting the model that best fit the design criterion under 
evaluation in the reliability analysis. A procedure was developed and coded using Matlab to determine the general speed state. Thus, determining whether vehicles were accelerating, decelerating, or traversing the curve at a constant speed. Knowing the general vehicle speed state was used in determining the steady state of vehicle and then used to determine the comfort threshold for each trip.

Three types of models were developed to predict speed on curve and determine driver comfort threshold; driver level, panel model, and $85^{\text {th }}$ percentile. In general, the lowest values of coefficient of determination were observed in driver level models. Two sets of models were developed according to weather conditions. The outputs of the developed models showed that average speed and comfort threshold in favourable weather conditions were higher than those in raining weather conditions.

Several models were developed to estimate the peak value of lateral friction demand for PC and SUV. The models were developed at BC of the curve because it is expected to have the peak value of lateral friction demand. Models to determine lateral acceleration for these vehicles were developed as well. In addition, several models were developed to estimate available friction factors for wet and dry pavements for PC and heavy truck tires. 


\section{CHAPTER SIX}

\section{RELIABILITY ANALYSIS}

\subsection{Introduction}

Implementing surrogate measures of safety can overcome the problem of lacking collision data. This concept has been used in many traffic safety research areas to reduce or eliminate the dependency on collision data. Within this context, reliability analysis can be used to determine the probability of failure/noncompliance/hazard as a surrogate measure for safety on horizontal curves. Reliability analysis is used to evaluate horizontal curve design criteria based on the relationship between the supply and demand as explained in Chapter 2. This chapter focuses on implementing reliability methods to evaluate horizontal curves performance.

\subsection{Reliability Analysis}

Several software have been developed to estimate reliability index and probability of failure such as $R t$ software and Dakota. The $R t$ software is a part of the Inrisk software suite developed at the University of British Columbia by Mahsuli and Haukaas (2013). The software has the capability to perform FORM and SORM analyses and handle correlated variables using the Nataf Transformation procedure. However, the main shortcoming of the $R t$ software is that the data need to be uploaded into the program manually. To overcome this issue, a Matlab program was developed to extract horizontal curves data from spreadsheets (Excel file), conduct FORM analysis, and print the outputs in a spreadsheet file (Excel). The outputs of the developed program for many curves were 
compared with the outputs of $R t$ software and were found to be identical. It should be noted that difference between the outputs of FORM and SORM in evaluating horizontal curves design criteria was insignificant (Himes 2013).

The analysis method for FORM for correlated normal variables provided by Haldar and Mahadevan (2000) was used. This method includes the following steps:

1. Formulate correlation matrix and calculate Eigen values and vectors.

2. Define limit state performance function $(g)$.

3. Assume initial values for reliability index (5 was used).

4. Assume initial values for design points $\left(Y_{i}\right)$ on the failure surface (zero values were assumed).

5. Compute partial derivatives evaluated at the design points $\left(Y_{i}\right)$.

6. Compute the direction cosine $\alpha_{i}$ at the design points.

$$
\alpha_{i}=\frac{\frac{\partial g}{\partial Y_{i}}}{\sqrt{\sum \frac{\partial g}{\partial Y_{i}} \sigma_{i}}}
$$

Where $\sigma_{i}=$ standard deviation when the random variables are normally distributed; otherwise, the equivalent standard deviations at the checking point should be used for nonnormal random variables.

7. Compute the new values for checking points.

$$
Y_{i}=-\alpha_{i} \beta \sigma_{i}
$$

8. Substitute in the limit state function, g.

9. Solve to determine the new reliability index.

10. Repeat steps from 5 to 9 until reliability index converges to a predetermined 
tolerance level (0.001 was used).

The developed Matlab code is provided in Appendix (F).

\subsection{Probability of Failure (POF)}

Before going forward in implementing reliability analysis in evaluating horizontal curve design, it is important to understand how the probability of failure/ noncompliance/hazard changes with some parameters. FORM analysis was conducted to estimate the POF of vehicle stability with different values of available lateral friction and radius. Figure 6.1 shows that POF for a horizontal curve, which is designed according to Green Book (A Policy 2011) requirements for speed of $70 \mathrm{~km} / \mathrm{h}$ (radius $=184 \mathrm{~m}$, and superelevation rate $=6 \%$ ), with pavement surface of various available lateral friction. The analysis was conducted for PCs traversing the curve at different constant mean speeds (50, 60 , and $70 \mathrm{~km} / \mathrm{h})$. The standard deviation for speeds were $(6.73,7.42$, and $7.89 \mathrm{~km} / \mathrm{h})$, which were extracted from Himes (2013). The performance function of POF for PC used in the reliability analysis is shown in Equation 6.3.

$$
Z_{S}=f_{S}-f_{D}=f_{S}-\left(\frac{0.009 V^{1.879}}{R^{0.886}}-0.947 e^{0.928}+2.6 \times 10^{-5} G\right)
$$

The figure shows that the POF values are very low when having pavement with high available lateral friction (dry or wet pavement surface). However, the POF escalates drastically when having low values (packed-snow or icy pavement). However, it is expected that drivers tend to slow down when driving in adverse weather conditions to reduce lateral friction demand and increase the available lateral friction simultaneously. That is, the POF has an inverse relationship with available lateral friction factor. Also, it is readily seen that the vehicles travelling at lower speed has the lowest POF. It should be 
noted that friction factor ranges and pavement surface classification shown in Figure 6.1 were taken from Table 2-6.

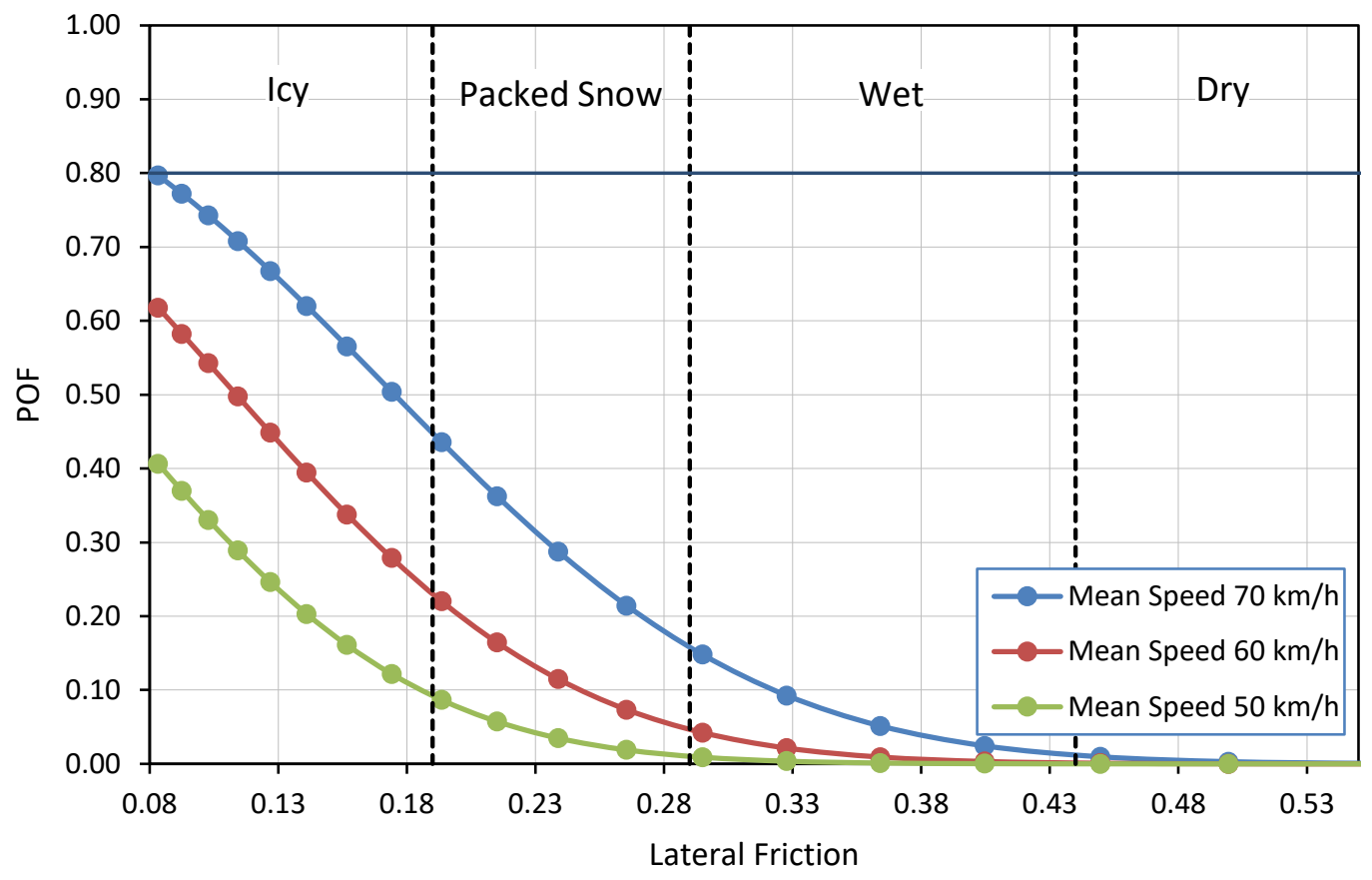

Figure 6.1: POF for PC with Different Available Lateral Friction Values.

To check the relationship between POF and curve radius and speed on curve, Figure 6.2 is developed. As shown in plot a, for a curve with a design speed of $50 \mathrm{~km} / \mathrm{h}$ (radius= $79 \mathrm{~m}$, superelevation rate $=6 \%$, and maximum friction $=0.19)$, the POF values were very low for mean speed on curve less than $50 \mathrm{~km} / \mathrm{h}$. However, the POF escalated drastically beyond the design speed. This concludes that POF has a direct relationship with speed, which is expected. The POF was also evaluated against different radii with a constant mean speed (60 and $70 \mathrm{~km} / \mathrm{h}$ ) on curve. As shown in the figure, the POF is very high when having a sharp curve. However, the POF decreases with radius. This indicates that POF has an inverse relationship with increase in radius. It should be noted that the lateral friction 
distribution was determined using models developed in Section 5.3.5 for wet pavement.

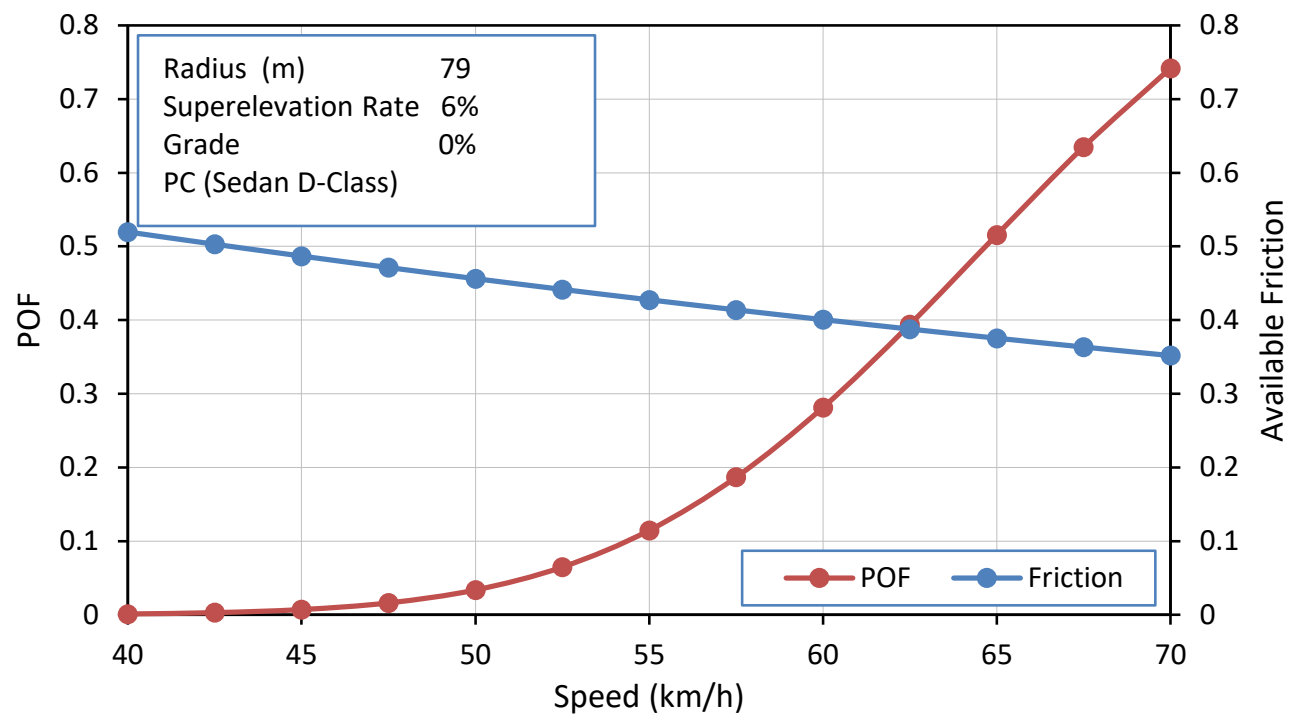

(a) Mean Speed vs. POF.

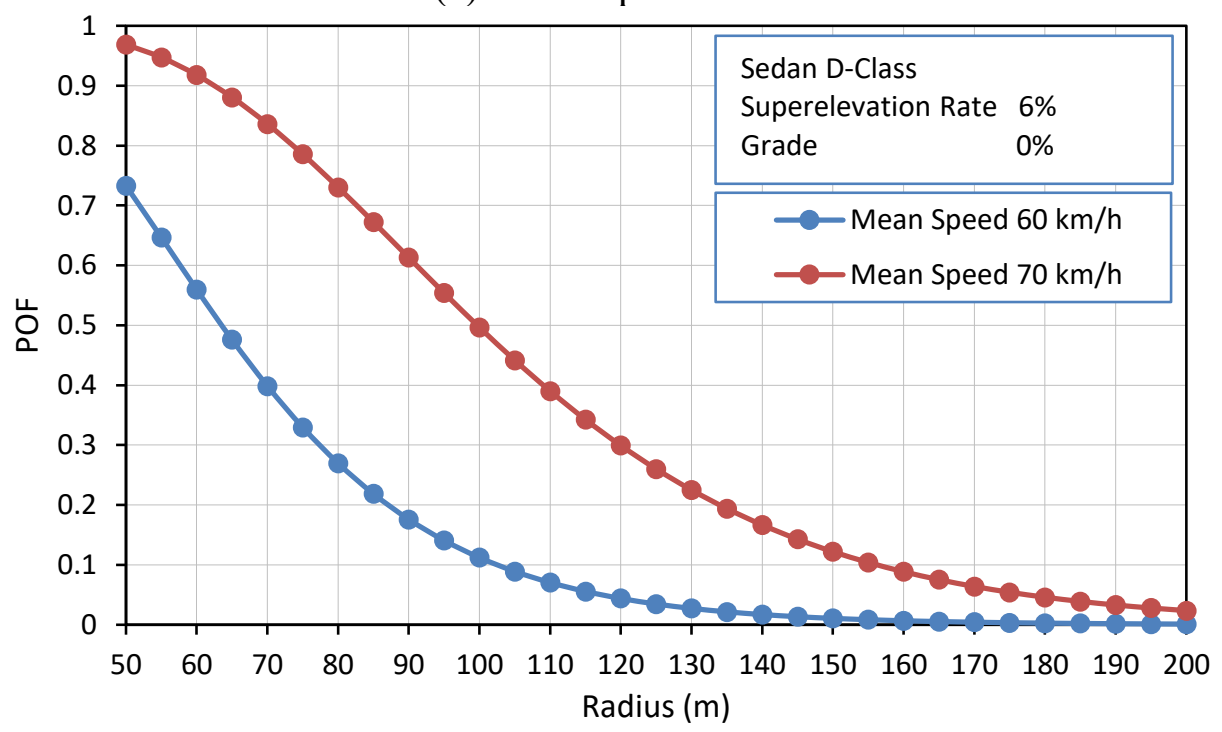

(b) Radius vs. POF.

Figure 6.2: Relationship between POF and Speed and Radius.

It is important to estimate the probability of failure for the horizontal curves designed according to Green Book (A Policy 2011) requirements. Figure 6.3 shows three POF for different radii for curves designed with superelevation of 4,8 , and $10 \%$. The dotted lines in the figure represent the minimum radii recommended by Green Book (A Policy 2011) 
for a design speed of $70 \mathrm{~km} / \mathrm{h}$. As shown in plot a (wet pavement conditions), the POF is different for the same design speed for different superelevation rates. However, the difference is very low and can be neglected. For dry pavement conditions, the difference is much lower. Thus, the POF for different superelevation rate for the same design speed can be assumed the same.

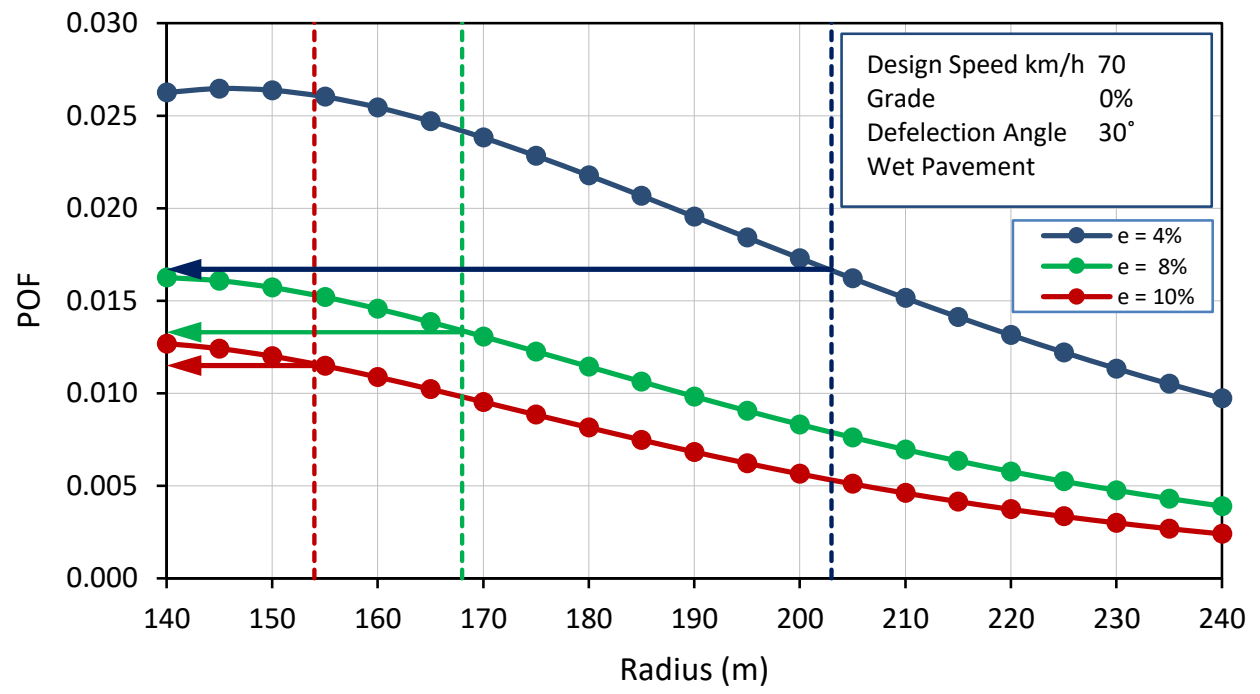

(a) POF for Wet Pavement.

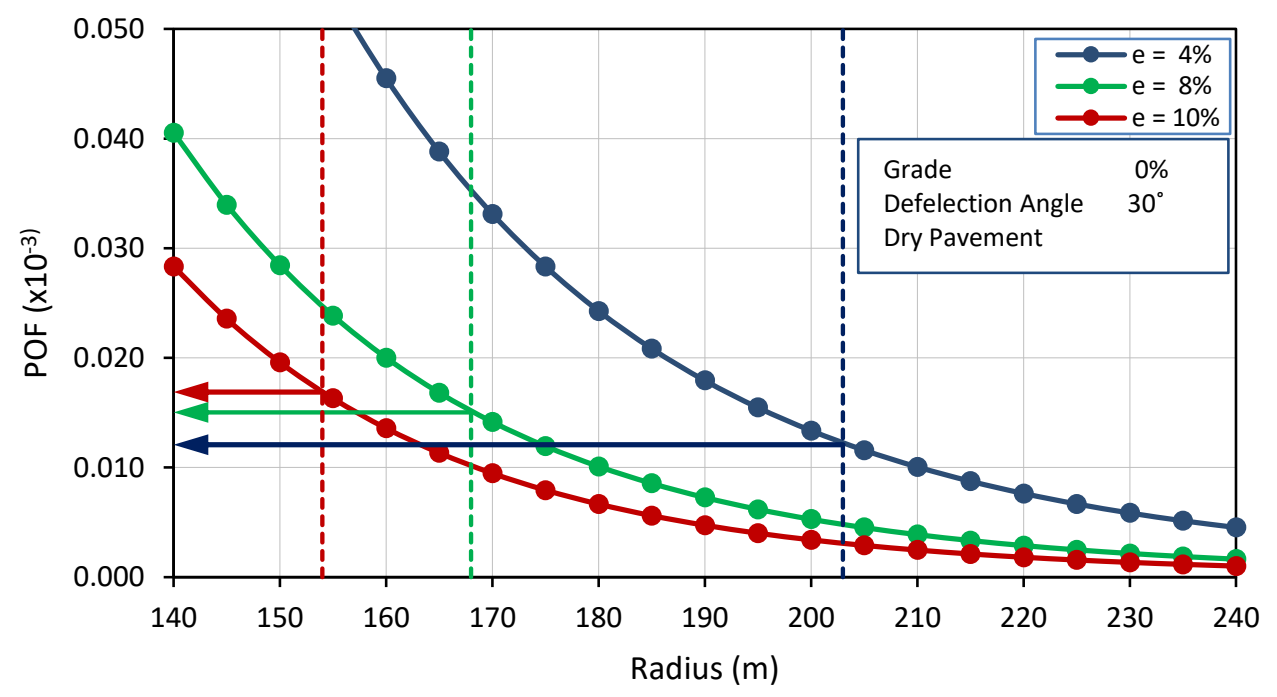

(b) FOF for Dry Pavement.

Figure 6.3: POF for Horizontal Curves with Different Superelevation Rates (e). 


\subsection{Probability of Noncompliance (PNC)}

As explained in Section 3.4, the performance function of driver comfort is the difference between the driver comfort threshold and the applied lateral acceleration. The performance function used in estimating PNC for PC is shown in Equation 6.2.

$$
Z_{C}=a_{L}-a_{a c t}=a_{L P}{ }^{*}-\left(\frac{0.011 V^{1.559}}{R^{0.655}}-0.032 e^{0.700}-4.3 \times 10^{-4} G\right)
$$

Where $a_{L P}{ }^{*}=$ model 5 in Table 5-4.

The relationship between probability of noncompliance (PNC) and driver comfort is more complicated than the POF. In estimating the PNC, the performance function depends on the relationship between the comfort threshold and applied lateral acceleration. As mentioned before, drivers tend to accept higher levels of comfort threshold (lateral acceleration) when traversing sharp horizontal curves. On such curves, it is expected to have low speed on curves and thus low lateral acceleration. As radius of curve increases, the mean speed on curve is expected to increase as well. Meanwhile, the mean comfort threshold and the applied lateral acceleration decrease but in different rates. Considering these issues, Figure 6.4, illustrates the relationships among PNC, radius, and mean speed on curve. The analysis was conducted for PC moving on horizontal curve in favourable weather conditions and dry pavement. As shown in the figure, the mean speed on curves increases with radius. For curves with radii less than $140 \mathrm{~m}$, the PNC is very low. Looking into the values of the developed lateral acceleration $\left(a_{D}\right)$ and the comfort threshold $\left(a_{L}\right)$ with radius of the curve would explain the PNC trend with radius. The $a_{D}$ within this radius range escalated slightly up to a point then it declined again. The comfort threshold was high at sharp curves and it declined with radius as well. Thus, the PNC values were very 
low within this range. Beyond radius of $140 \mathrm{~m}$, both $a_{D}$ and $a_{L}$ decreased simultaneously with radius but at different rates $\left(a_{L}\right.$ decreased at higher rate). The smallest difference between them was approximately at radius of $240 \mathrm{~m}$. Thus, it is expected to have the maximum PNC at this radius. The PNC declined again beyond this radius because the $a_{D}$ and the $a_{L}$ declined simultaneously at different rates ( $a_{D}$ decreased at higher rate).

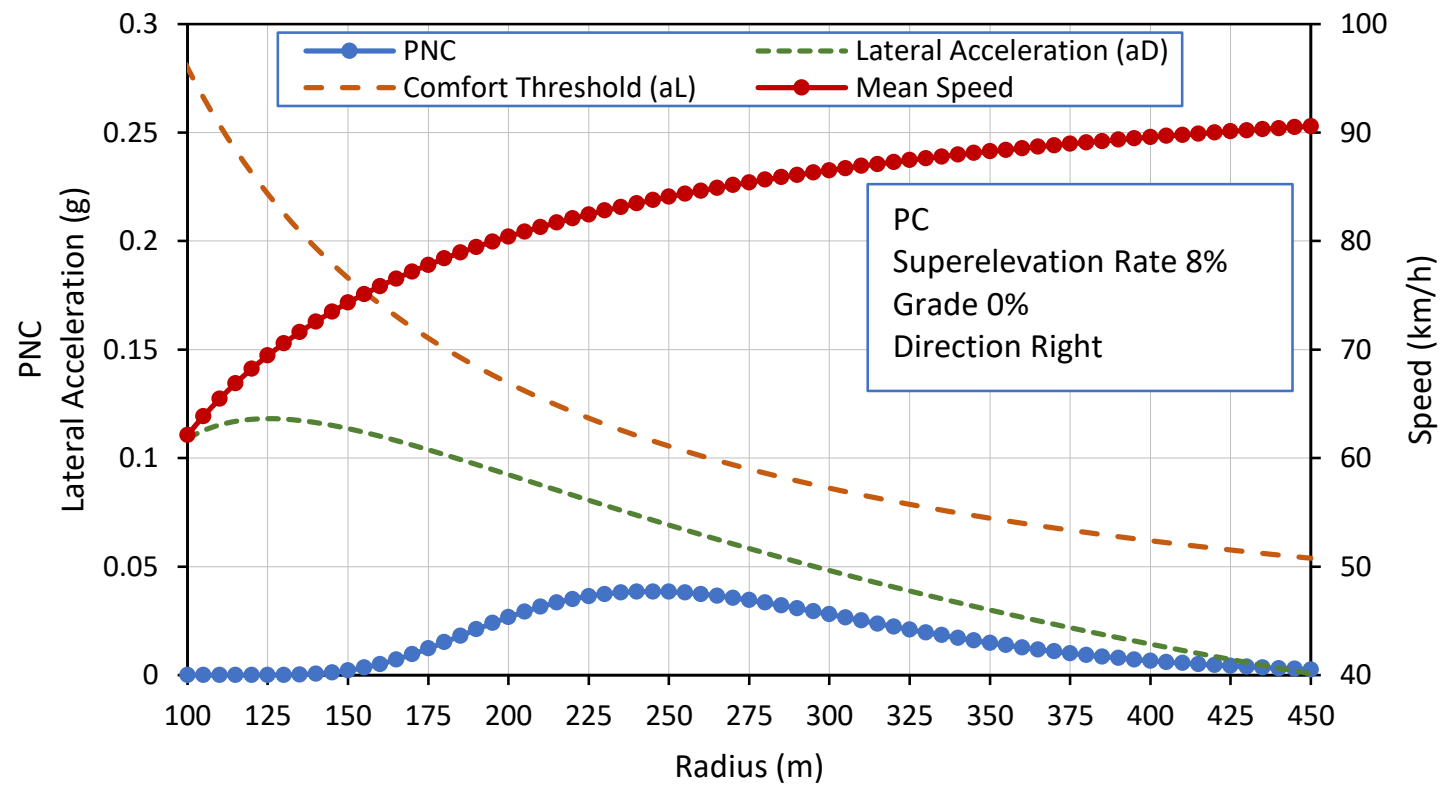

Figure 6.4: Relationship between PNC and Radius of Curve.

\subsection{Probability of Hazard (POH)}

As explained in Chapter 2, the performance function for sight distance criterion is the difference between available and demanded stopping sight distance (SSD). The SSD depends on the deceleration rate. Hussein et al (2014) used acceleration rate of $3.4 \mathrm{~m} / \mathrm{s}^{2}$ in determining the SSD in the limit state function. However, the situation would be critical when the longitudinal friction demand due to vehicle deceleration is higher than the available friction factor. For this reason, the longitudinal friction factor was used in the 
reliability analysis instead of deceleration rate. Thus, the performance function used in the analysis is:

$$
Z_{S D}=S_{a v i}-S_{D}=S_{a v i}-\left(0.278 V t+\frac{V^{2}}{254\left(f_{L} \mp G\right)}\right)
$$

Where $S_{D}=$ stopping sight distance $(\mathrm{m}), t=$ perception reaction time $(\mathrm{s})$, and $f_{L}=$ available longitudinal friction.

Also, the peak value for longitudinal friction factor was used in the analysis instead of sliding friction factor because the electronic stability control has been mandatory in vehicles in Canada and USA since 2012. Thus, it is assumed that tire-pavement slip ratio would be approximately $20 \%$ (friction peak value).

To evaluate sight distance criterion, the relationship between lateral clearance and $\mathrm{POH}$ is provided in Figure 6.5. It assumed that the lateral clearance is $1.0 \mathrm{~m}$ when the curve radius is $90 \mathrm{~m}$. There are no other sight distance limitations assumed in this example. The figure shows that the $\mathrm{POH}$ decreases with radius because the lateral clearance increases as well. It can be seen that the POH dropped considerably with radius for radii beyond 100 m.

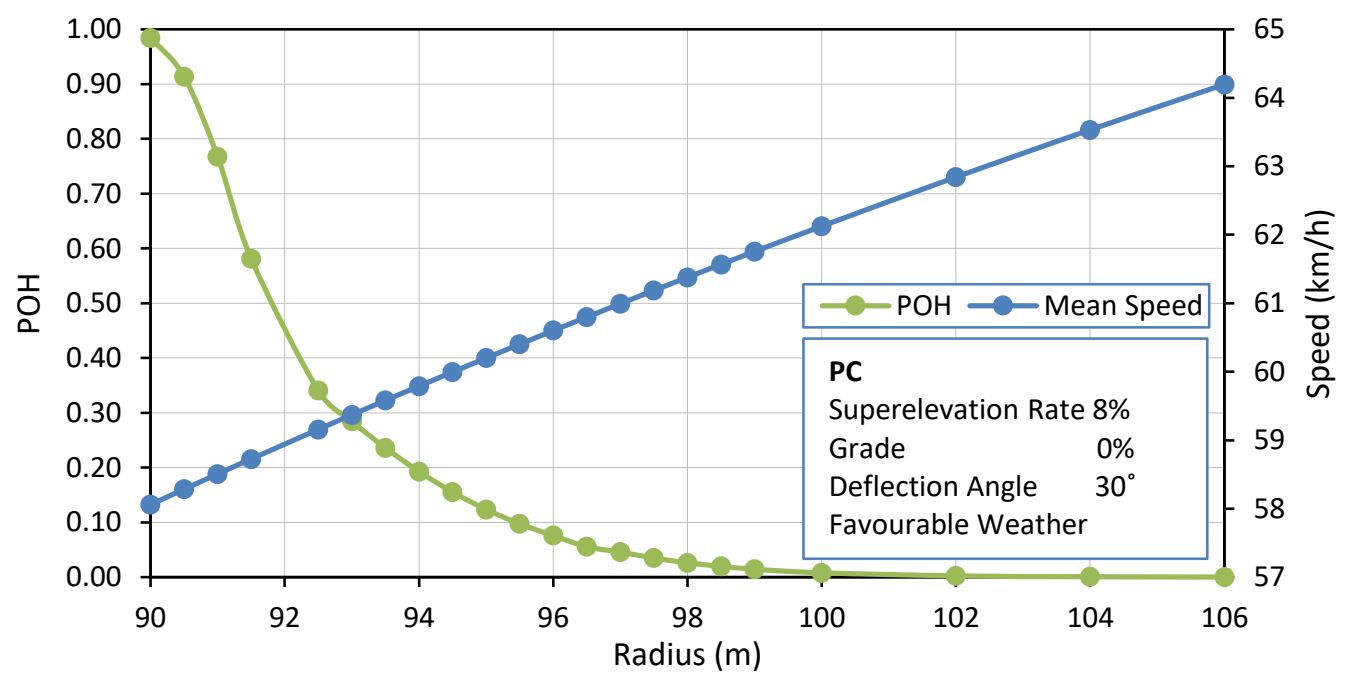

Figure 6.5: Relationship between POH and Radius of Curve. 


\subsection{Probability of Failure $\left(\mathrm{POF}_{\mathrm{R}}\right)$}

Vehicles would roll over when the overturning moment exceeds the counterbalancing moment. Also, the rollover needs pavement with high friction or any other mechanical tripping. Chapter 2 explained several ways to examine vehicles rolling over situation. However, the CarSIM was used in the research to determine the behaviour of sprung part of the vehicle. The outputs of the software were used to develop models to determine rolling angle based on speed of vehicle and horizontal curve geometry as shown in the following Equations.

$$
\begin{aligned}
\rho_{P C}=-5.024 & +0.005 V+\frac{967.234}{R}-59.617 e-0.003 G ; R_{a d j}=0.999, S E E \\
= & 0.044
\end{aligned}
$$

Where $\rho_{P C}=$ roll angle $\left(^{\circ}\right), V=$ speed $(\mathrm{km} / \mathrm{h}), R=$ radius $(\mathrm{m}), e=$ superelevation rate (percent), and $G=\operatorname{grade}(\%)$.

In this research, the forces affecting on vehicle's sprung and unsprung parts were considered in determining vehicle stability against turning over as shown in Figure 6.6. The change in roll angle has a great impact on vehicle stability. As speed of vehicle increases, the roll angle increases, and thus the counterbalance moment decreases. The performance function for rollover is shown in Equation 6.5. It should be noted that the deformation in vehicle's tires was ignored in the analysis.

$$
Z_{R}=M_{c}-M_{a}
$$

Where $M_{c}=$ counterbalance moment, and $M_{a}=$ applied moment. The calculations for determining $M_{c}$ and $M_{a}$ are shown in Appendix (A). 


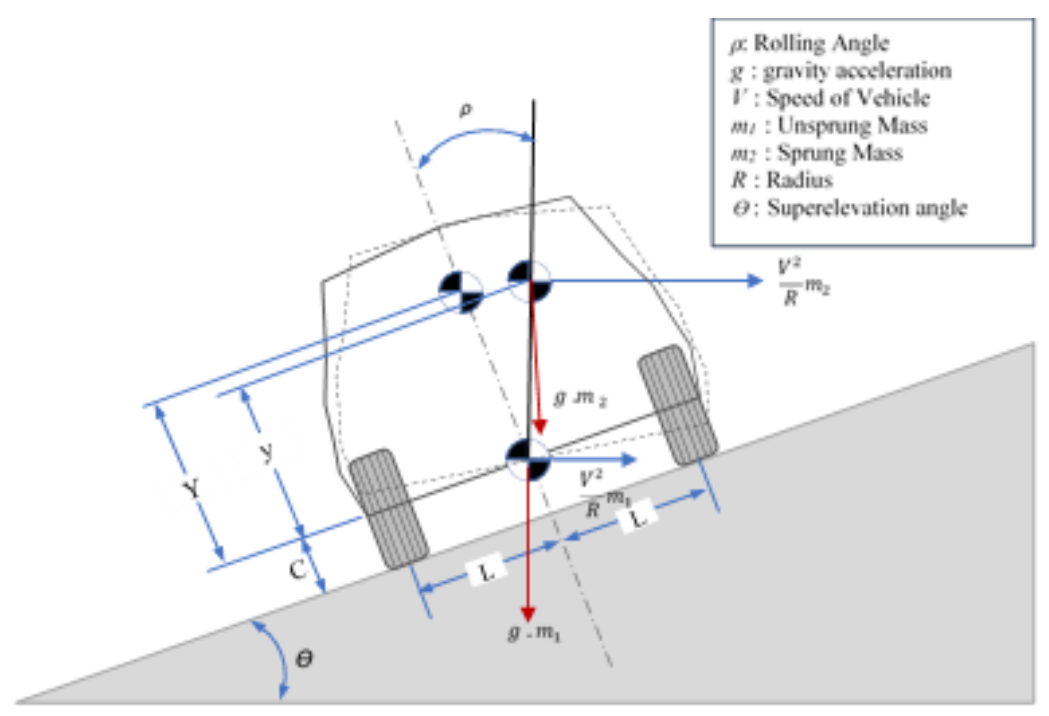

Figure 6.6: Forces Affecting on Sprung and Unsprung Vehicle Parts.

The probability of failure for Rollover $\left(P O F_{R}\right)$ was estimated for PCs moving on a horizontal curve designed for speed of $50 \mathrm{~km} / \mathrm{h}$ (radius $=86 \mathrm{~m}$ and superelevation $=4 \%$ ) as shown in Figure 6.7. The $\mathrm{POF}_{R}$ is very low even for mean speed greater than design speed by $40 \mathrm{~km} / \mathrm{h}$. This is expected since the height of centre of gravity of the sprung and unsprung parts are relatively low $(0.325 \mathrm{~m}$ and $0.520 \mathrm{~m}$, respectively).

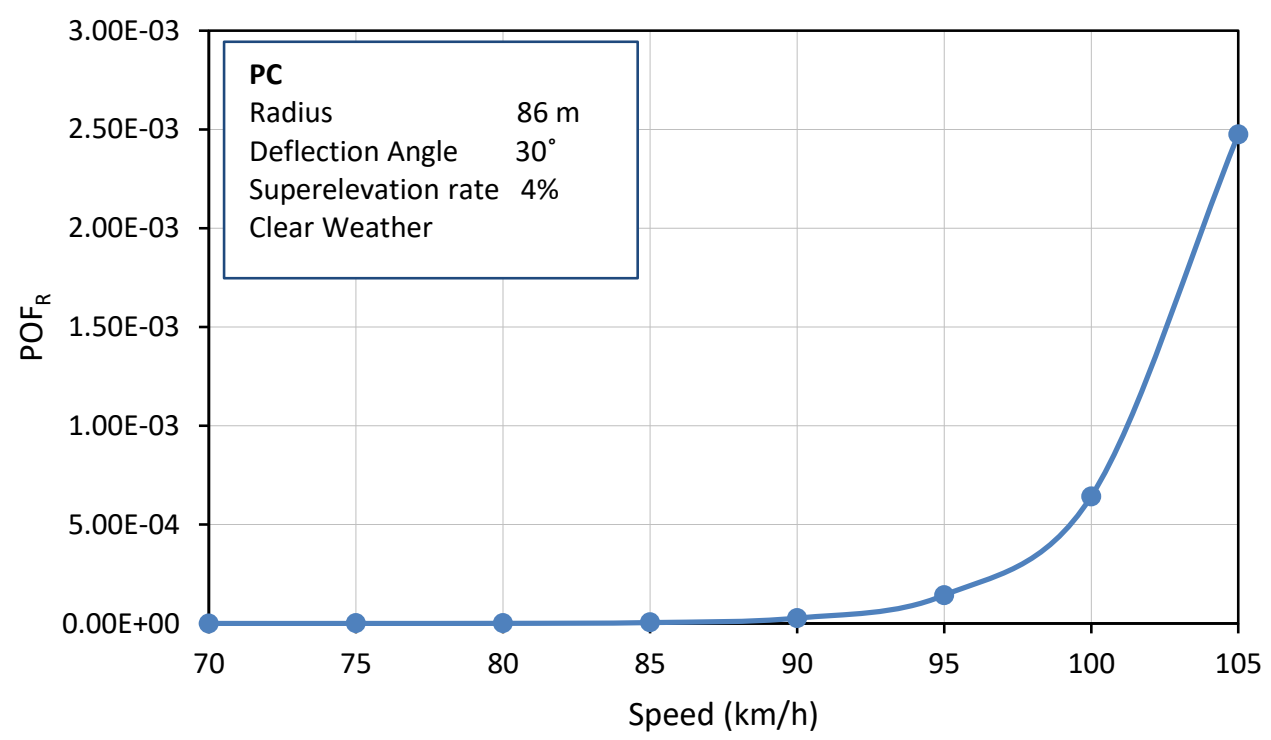

Figure 6.7: Relationship between POF (Rollover) and Mean Speed of Vehicle on Curve. 


\subsection{Two-Lane Highway Assessment}

There are 14,477 curves on two-lane highways in rolling and mountainous rural areas in the database provided by WSDOT. Since the data of these curves were in different datasets, a Matlab program was developed to build one database that includes all data of curves. However, many of these curves lacked some data such as superelevation rate and speed limit. The number of curves that have all geometric data and historical traffic data (AADT) from 2009-2013 was only 1,075. Unfortunately, not all of these curves had historical pavement frictional data because the data obtained from WSPMS were only for five routes in the state $(002,020,097,970$, and 104). Thus, the number of curves that had a completed set of data (radius, superelevation rate, deflection angle, friction data, collisions, ... etc) was relatively low. The total number of curves used in the analysis was 232. Figure 6.8 shows the locations of the segments where these curves are located. As

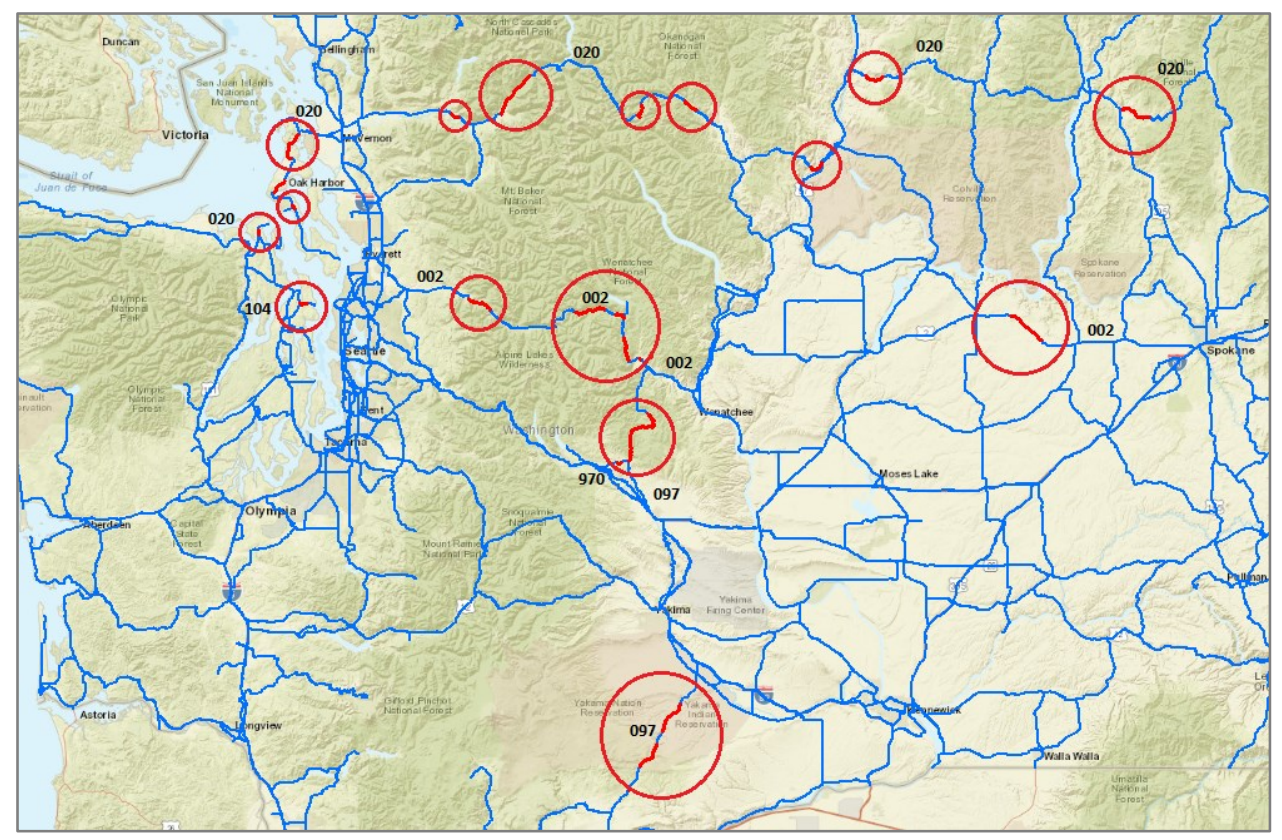

Figure 6.8: Locations of Horizontal Curves for Reliability Analysis. 
shown in the figure, these curves are in different locations throughout Washington State and thus might be subjected to different weather conditions.

The descriptive statistics for the extracted curves are shown in Table 6-1. As explained earlier, these curves have data for friction and AADT for five years (2009-2013). Thus, the database for five years includes 1,160 records.

Table 6-1: Descriptive Statistics for the Selected Curves.

\begin{tabular}{|l|c|c|c|c|c|}
\hline \multicolumn{1}{|c|}{ Variable } & $\begin{array}{c}\text { Number } \\
\text { of Curves }\end{array}$ & Minimum & Maximum & Mean & $\begin{array}{c}\text { Standard } \\
\text { Deviation }\end{array}$ \\
\hline Radius (m) & 232 & 107 & 1951 & 492.36 & 314.71 \\
\hline Angle (Degree) & 232 & 2.48 & 125.99 & 33.08 & 21.96 \\
\hline Superelevation Rate (m/m) & 232 & 0.03 & 0.14 & 0.06 & 0.01 \\
\hline Grade (Percent) & 232 & -7.03 & 7.07 & -0.07 & 3.00 \\
\hline Speed Limit (km/h) & 232 & 40.23 & 104.59 & 90.09 & 11.44 \\
\hline $\begin{array}{l}\text { Terrain (Rolling=0, } \\
\text { Mountainous=1) }\end{array}$ & 232 & 0.00 & 1.00 & 0.31 & 0.46 \\
\hline Collisions (5 years) & 232 & 0 & 22 & 2.35 & 3.09 \\
\hline \hline
\end{tabular}

The assessment was conducted for two-lane highways in Washington State considering four design criteria; vehicle stability, driver comfort, sight distance, and rollover. The reliability analysis was conducted for sedan passenger car only. Thus, the outputs for other types of vehicles would be different. It should be noted that the assessment in this research was developed using adjusted reliability indices. The adjustment was conducted for weather and pavements conditions. The dominant weather conditions for each curve in the database were extracted from various weather stations. Thus, number of precipitation days around the year was obtained from the closest weather station to the curve location. 


\subsubsection{Probability of Failure Adjustment}

As addressed earlier, each design criterion has its assumptions and requirements. Thus, the reliability index or probability of failure should be different from one criterion to the other for the same curve. Even the $\mathrm{POF} / \mathrm{PNC} / \mathrm{POH} / \mathrm{POF}_{\mathrm{R}}$ would be different for the same curve when having different circumstances such as traffic characteristics and weather conditions. As shown in Figure 6.9, each curve has four different reliability indices. It should be noted that these indices are calculated for sedan passenger car D-Class. The reliability indices would be slightly different for other types of vehicles. Furthermore, these indices are changing with the time (age of pavement), AADT, weather and light conditions, and even along the day. Thus, the overall assessment for horizontal curve performance should consider several factors that would affect each design criterion as explained in Section 3.3. For the purpose of the research and due to lack of data to cover all these factors (traffic characteristics, type of vehicles, time, and road classification), only weather and pavement surface conditions (wet and dry) were considered in calibrating the reliability indices. An application for the adjustment is provided in the next Chapter (Section 7.4.7). Accordingly, assessment for safety and overall performance of a horizontal curve would be more realistic and accurate. The adjusted indices would work as surrogate measures for curve evaluation and thus assist designers in the design of horizontal curves and help transportation agencies in developing more reliable maintenance policy. 


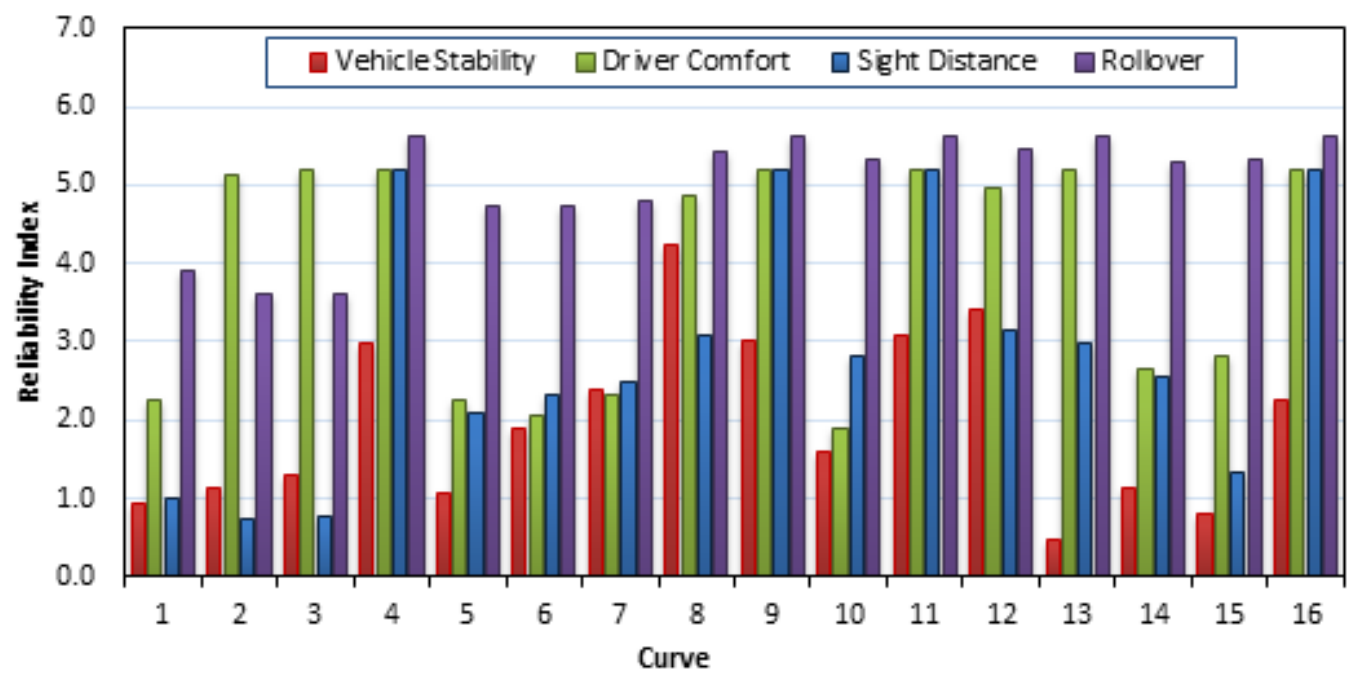

Figure 6.9: Reliability Indices for a Sample Curves.

\subsubsection{Vehicle Stability Assessment}

As explained earlier, the Washington State Pavement Management System (WSPMS) (Pavement Office) collects data on regular basis. Because of the variability inherent in a state-wide pavement network, the WSPMS does not use generalized models to predict pavement performance. Instead, the pavement performance is monitored every other year until the collected data show they are projected to reach the optimum time for rehabilitation. As mentioned earlier, the linear interpolation was used to determine the available friction for the years that lack measurements.

It should be noted that (as explained in Section 5.3.3) vehicle stability should consider the critical points where the peak lateral friction demand is expected. These points are located at the transient zones on the curves (at BC and EC) where the vehicle changes direction from straight to curve and vice versa. Therefore, speed parameter for vehicle stability should be taken at these critical points. $V_{B C}$ is selected over $V_{c r v-\max }$ because it is 
expected to have the peak lateral friction demand at $\mathrm{BC}$ since the superelevation rate is not fully reached at this point. In other words, $V_{c r v-\max }$ might not take place at the same point of the peak lateral friction demand. It should be noted that panel model equations for speed and comfort threshold were used in the reliability analysis.

Figure 6.10 shows number of curves according to their reliability index $\left(\beta_{V S}\right)$. Most of the curves have high $\beta_{V S}$, which means that these curves have low POF against failure due to sliding.

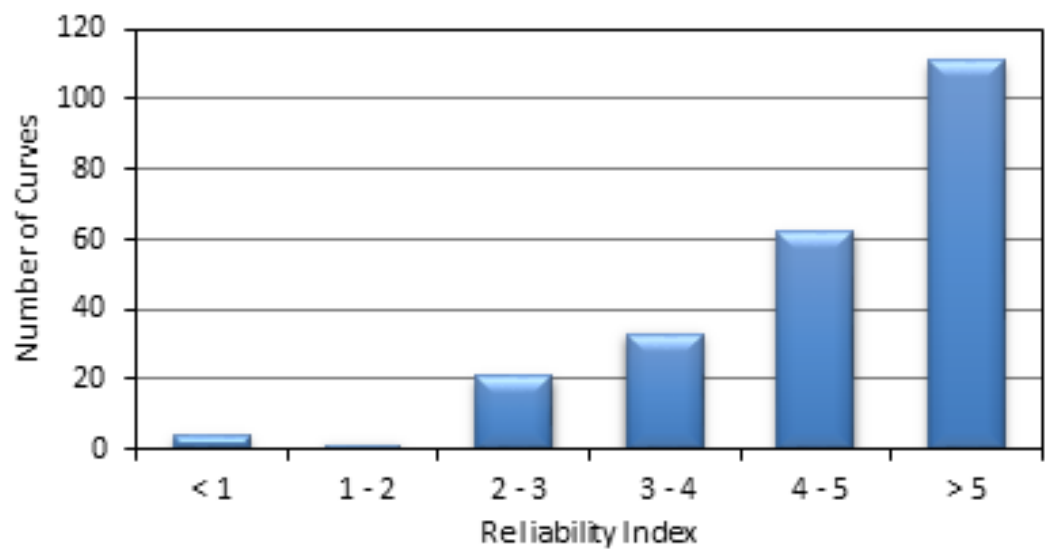

Figure 6.10: Distribution of Curves According to Reliability Index $\left(\beta_{V S}\right)$.

Combining values of $\beta_{V S}$ or POF with AADT and collision frequency would give another dimension for the performance of the curve. To illustrate the relationship among these parameters, Figure 6.11 is developed. Each circle in the figure represents a single curve and its size represents the POF value for vehicle stability criterion. Note that for illustration purpose only, curves with very small POF (less than 0.001) were given a value of 0.001 in order to be shown as a point in the figure. The figure shows that many of the circles are relatively small in size, which means that most of the curves have low POF and 
in turn relatively high $\beta_{V S}$ as explained earlier. This result is expected since the available friction for many curves was relatively moderate. In addition, the $\beta_{V S}$ values were taken as the average of five years and calibrated for weather conditions. Thus, the effect of dry pavement on POF was included. Number of curves with high collision frequency (greater than 5 per five years) is relatively low. However, many collisions happened on curves that have low POF. The high number of collisions on curves of high $\beta_{V S}$ does not help in explaining the relationship between $\beta_{V S}$ and safety performance of the curve. This situation raises several issues such as; the $\beta_{V S}$ is not a good measure to evaluate safety performance for the curve. Also, this situation would indicate that some of these collisions happened in adverse weather conditions, thus, vehicle stability situation is questionable due to low value of available friction factor. In addition, this situation might happen due to other reasons not related to vehicle stability, which would affect the safety performance of those curves as well.

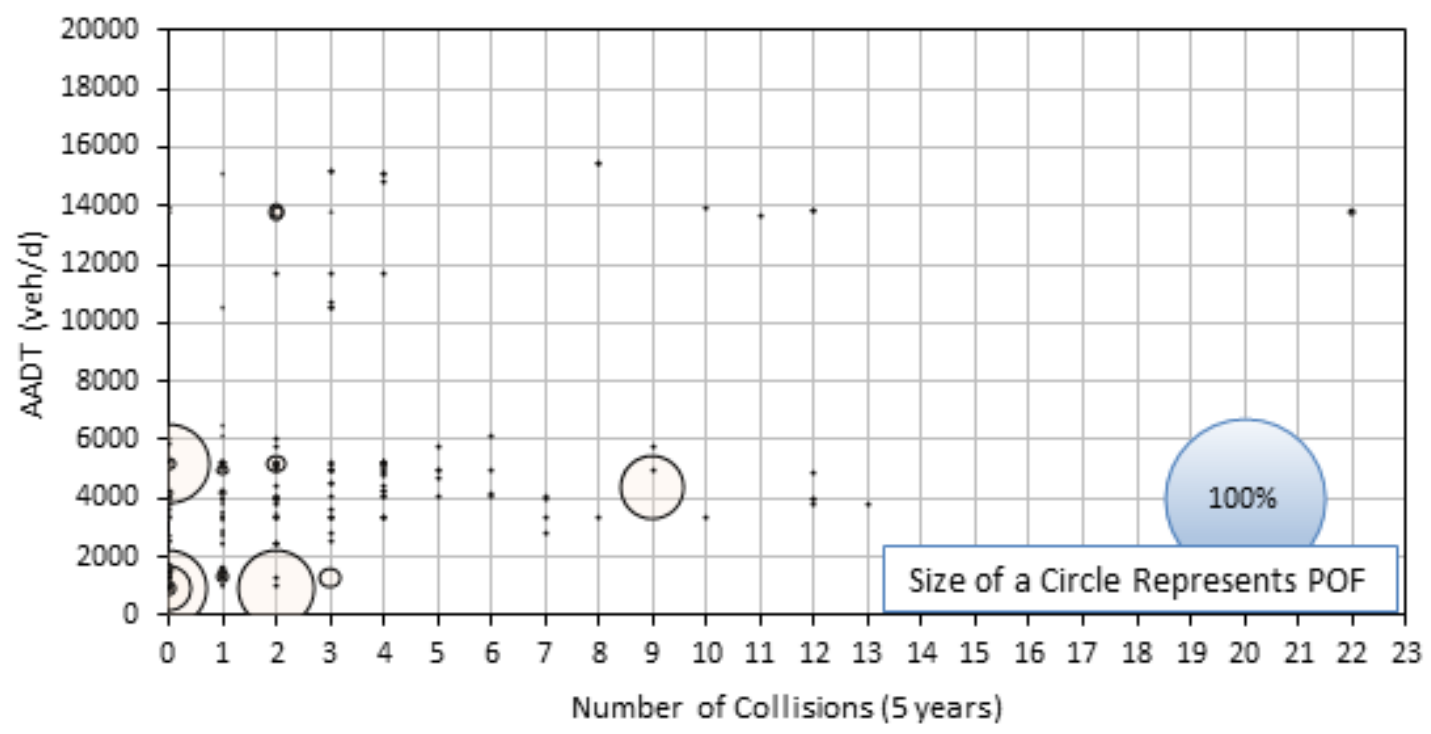

Figure 6.11: Relationship between POF, AADT, and Collisions. 
To investigate this issue, the $\beta_{V S}$ with AADT and collision frequency are tabulated into Table 6-2. The table provides additional quantitative information regarding the relationship among these parameters. As seen in the table, number of curves with $\beta_{V S}$ greater than 4.0 is 173 , which represents approximately $75 \%$ of the curves. Most of the curves serve AADT of 3,000-7,000 veh/d and about $9 \%$ of the curves serve AADT greater than 11,000 veh/d. It is expected to have high collision frequency with AADT.

Looking into the safety data provided in Section 3.6.6 would provide a good interpretation for this situation. As explained, that approximately $30 \%$ of the collisions took place in adverse weather conditions (raining, snowing, or foggy). About $40 \%$ of the collisions happened at night or insufficient light (dark). Finally, approximately $29 \%$ of collisions occurred on packed-snow and icy pavements. These figures would indicate that a considerable number of collisions occurred when the pavement had low friction. Also, this would indicate that other factors had impacts on collision frequency other than vehicle stability such limitations for sight distance. In addition, it is important to check the trend of number of collisions with $\beta_{V S}$ by mitigating the effect of these factors that might

Table 6-2: Number of Collisions, AADT, and Reliability Index $\left(\boldsymbol{\beta}_{V S}\right)$.

\begin{tabular}{|c|c|c|c|c|c|c|c|c|c|c|c|c|c|c|c|c|c|c|}
\hline \multirow{3}{*}{$\begin{array}{l}\text { Reliability } \\
\text { Index }\end{array}$} & \multicolumn{18}{|c|}{ AADT $\left(\mathrm{veh} / \mathrm{d} \times 10^{3}\right)$} \\
\hline & \multicolumn{2}{|c|}{$<1$} & \multicolumn{2}{|c|}{$1-3$} & \multicolumn{2}{|c|}{$3-5$} & \multicolumn{2}{|c|}{$5-7$} & \multicolumn{2}{|c|}{$7-9$} & \multicolumn{2}{|c|}{$9-11$} & \multicolumn{2}{|c|}{$11-13$} & \multicolumn{2}{|c|}{$13-15$} & \multicolumn{2}{|c|}{$>15$} \\
\hline & $\mathrm{Co}$ & $\mathrm{Nc}$ & $\mathrm{Co}$ & $\mathrm{Nc}$ & $\mathrm{Co}$ & $\mathrm{Nc}$ & $\mathrm{Co}$ & $\mathrm{Nc}$ & $\mathrm{Co}$ & $\mathrm{Nc}$ & $\mathrm{Co}$ & $\mathrm{Nc}$ & $\mathrm{Co}$ & $\mathrm{Nc}$ & $\mathrm{Co}$ & $\mathrm{Nc}$ & Co & $\mathrm{Nc}$ \\
\hline$<1$ & 2 & 2 & 0 & 0 & 9 & 1 & 0 & 1 & 0 & 0 & 0 & 0 & 0 & 0 & 0 & 0 & 0 & 0 \\
\hline $1-2$ & 0 & 1 & 0 & 0 & 0 & 0 & 0 & 0 & 0 & 0 & 0 & 0 & 0 & 0 & 0 & 0 & 0 & 0 \\
\hline $2-3$ & 0 & 2 & 8 & 8 & 2 & 3 & 11 & 5 & 0 & 0 & 0 & 0 & 0 & 0 & 26 & 3 & 0 & 0 \\
\hline $3-4$ & 0 & 1 & 1 & 6 & 21 & 7 & 24 & 11 & 0 & 0 & 4 & 2 & 4 & 1 & 24 & 3 & 11 & 2 \\
\hline $4-5$ & 0 & 1 & 6 & 9 & 93 & 28 & 35 & 14 & 0 & 0 & 6 & 2 & 3 & 1 & 24 & 4 & 15 & 3 \\
\hline$>5$ & 0 & 1 & 33 & 47 & 153 & 48 & 20 & 10 & 0 & 0 & 0 & 0 & 2 & 1 & 4 & 2 & 5 & 2 \\
\hline
\end{tabular}

$\mathrm{Co}=$ total number of collisions (5 years) on all curves within the indicated categories.

$\mathrm{Nc}=$ number of curves within the indicated categories. 
influence safety performance. Accordingly, the trend was taken for collisions that happened in dry and wet pavement conditions. The relationship between collision frequency and collision rate per curve (number of collisions/number of curves) for each reliability index category are calculated and plotted with $\beta_{V S}$ as shown in Figure 6.12. The figure shows that the frequency of collisions is expected to decline with $\beta_{V S}$. This result is expected because higher $\beta_{V S}$ means, in turn, higher value of available lateral friction. Based on that, $\beta_{V S}$ could be used as a surrogate measure for safety or as a predictor in the safety performance function.

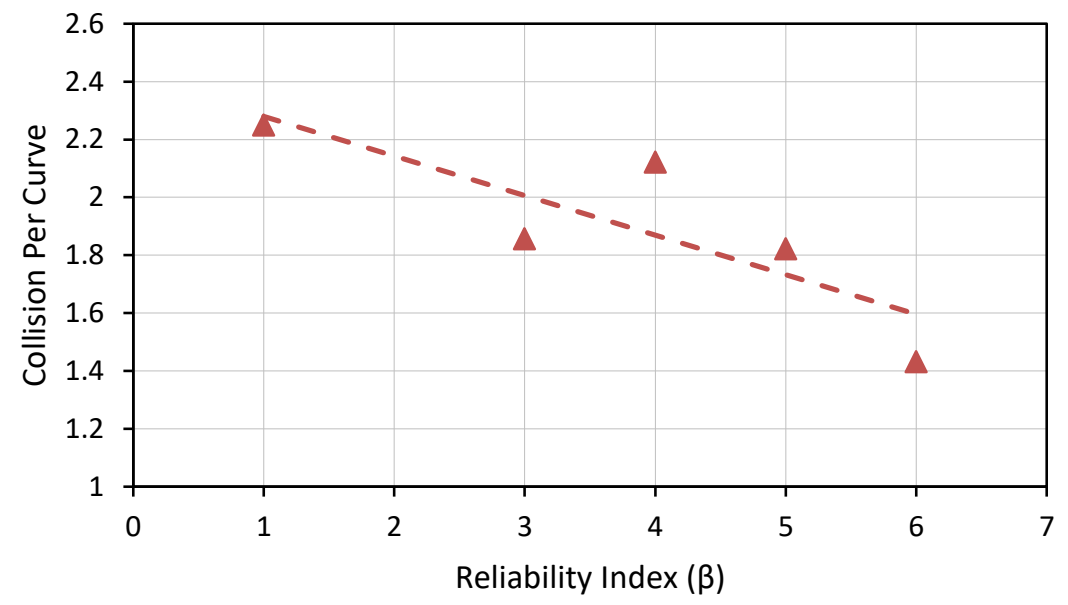

Figure 6.12: Trend of Average Collisions per Curve with Reliability Index $\left(\boldsymbol{\beta}_{V S}\right)$.

\subsubsection{Driver Comfort Assessment}

The same approach used to evaluate vehicle stability design criterion was used for assessing the driver comfort criterion. The speed on curve $\left(V_{c m f-a v g}\right)$ should be used in determining the applied lateral acceleration. However, the $V_{M C}$ was used in the analysis because the coefficient of determination is higher, the SEE is lower, and the ANOVA tests showed that there is no statistically significant difference between the two speed parameters. 
The estimated reliability index for driver comfort $\left(\beta_{c m f}\right)$ for most of the curves was less than 3.0, which means that these curves would have a high probability of noncompliance with this design criterion. Figure 6.13 shows number of curves for each reliability index criteria.

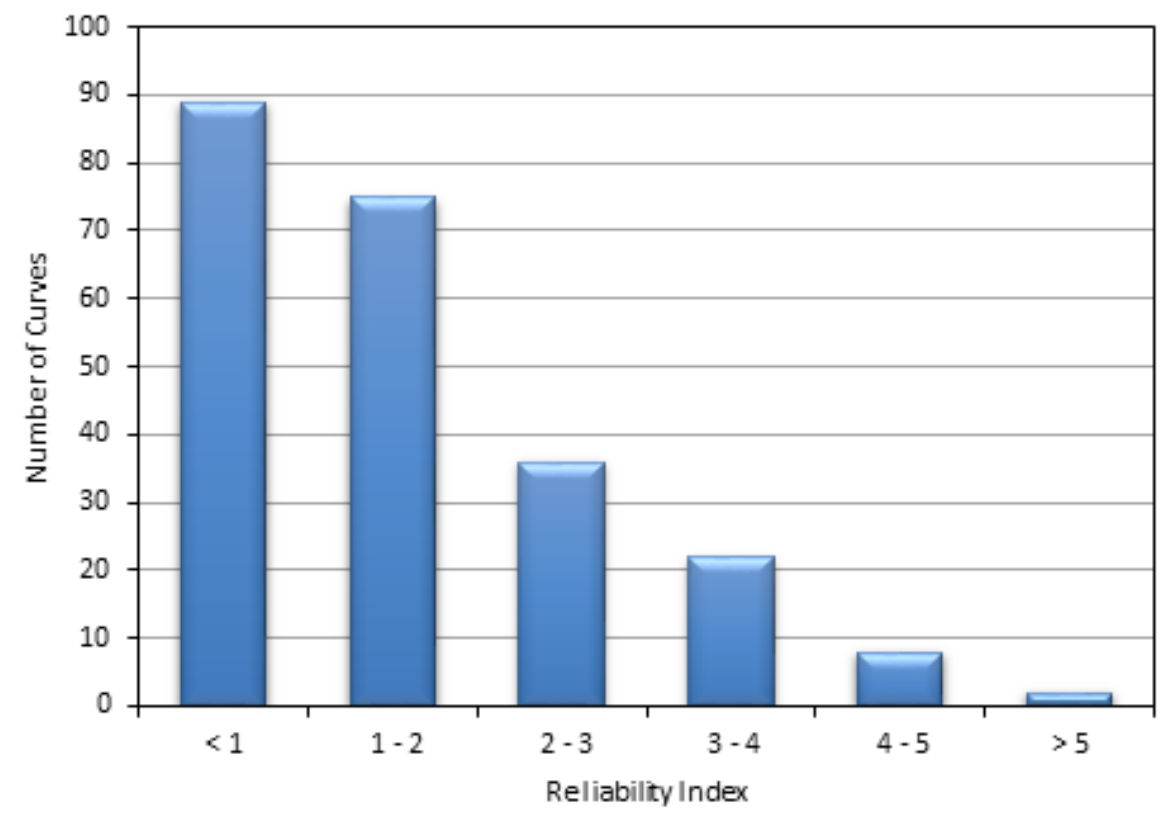

Figure 6.13: Number of Curves According Reliability Index $\left(\beta_{c m f}\right)$.

Figure 6.14 illustrates the relationship among the PNC, AADT, and number of collisions. The figure shows that most of the collisions occurred on curves that have high PNC values. The same result is obtained when considering only collisions that occurred in clear or raining weather conditions. This means that there is a strong relationship between PNC and number of collisions. To translate the figure illustrations into numbers, Table 6-3 is developed. 


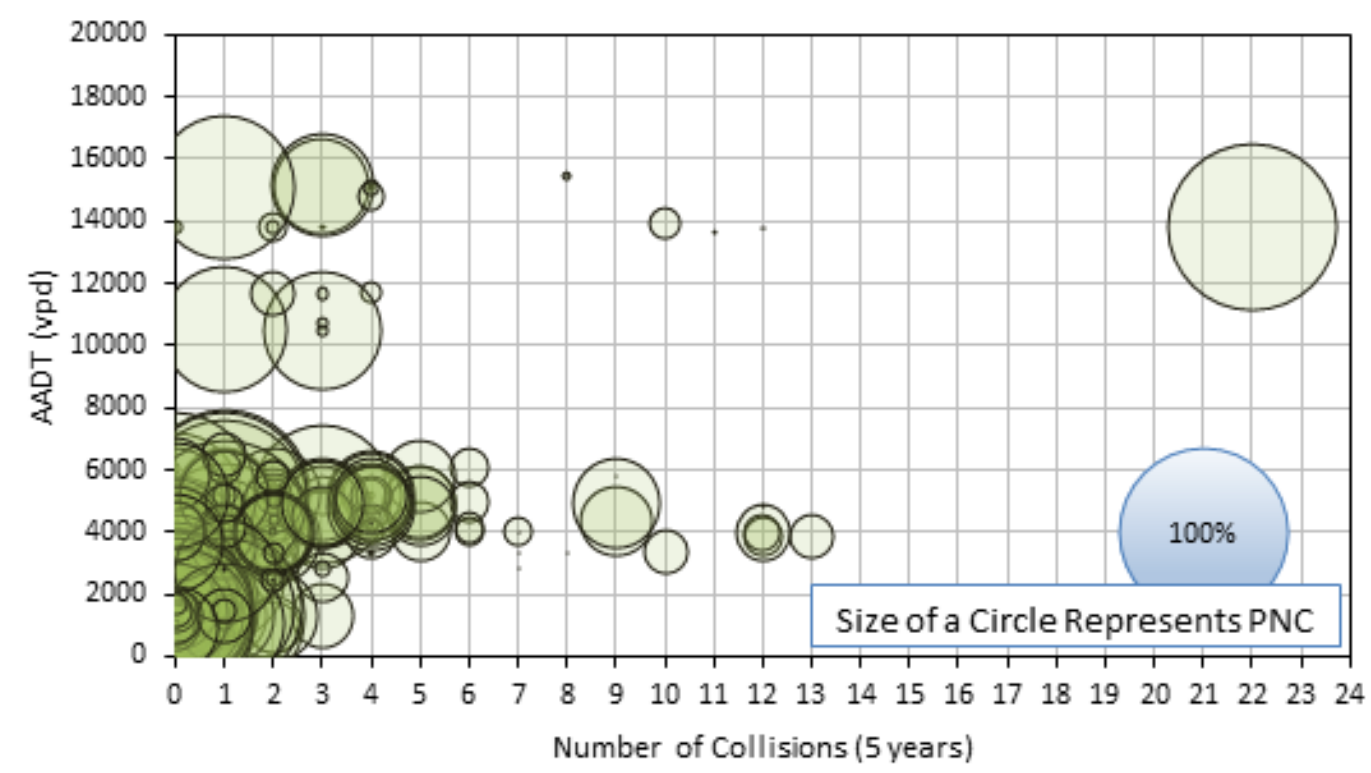

Figure 6.14: Relationship between Number of Collisions, AADT, and PNC.

Table 6-3: Number of Collisions on Curves within Specific Reliability Index ( $\left.\boldsymbol{\beta}_{\boldsymbol{c m} \boldsymbol{f}}\right)$ Interval.

\begin{tabular}{|c|c|c|c|c|c|c|c|c|c|c|c|c|c|c|c|c|c|c|}
\hline \multirow{3}{*}{$\begin{array}{l}\text { Reliability } \\
\text { Index }\end{array}$} & \multicolumn{18}{|c|}{ AADT $\left(\operatorname{vpd} \times 10^{3}\right)$} \\
\hline & \multicolumn{2}{|c|}{$<1$} & \multicolumn{2}{|c|}{$1-3$} & \multicolumn{2}{|c|}{$3-5$} & \multicolumn{2}{|c|}{$5-7$} & \multicolumn{2}{|c|}{$7-9$} & \multicolumn{2}{|c|}{$9-11$} & \multicolumn{2}{|c|}{$11-13$} & \multicolumn{2}{|c|}{$13-15$} & \multicolumn{2}{|c|}{$>15$} \\
\hline & $\mathrm{Co}$ & $\mathrm{Nc}$ & $\mathrm{Co}$ & $\mathrm{Nc}$ & $\mathrm{Co}$ & $\mathrm{Nc}$ & $\mathrm{Co}$ & $\mathrm{Nc}$ & $\mathrm{Co}$ & $\mathrm{Nc}$ & $\mathrm{Co}$ & $\mathrm{Nc}$ & $\mathrm{Co}$ & $\mathrm{Nc}$ & $\mathrm{Co}$ & $\mathrm{Nc}$ & $\mathrm{Co}$ & $\mathrm{Nc}$ \\
\hline$<1$ & 0 & 5 & 10 & 31 & 74 & 31 & 27 & 16 & 0 & 0 & 4 & 2 & 0 & 0 & 22 & 1 & 7 & 3 \\
\hline $1-2$ & 2 & 3 & 18 & 27 & 118 & 29 & 31 & 12 & 0 & 0 & 0 & 0 & 2 & 1 & 16 & 3 & 0 & 0 \\
\hline $2-3$ & 0 & 0 & 8 & 7 & 21 & 9 & 19 & 10 & 0 & 0 & 6 & 2 & 7 & 2 & 2 & 3 & 16 & 3 \\
\hline $3-4$ & 0 & 0 & 12 & 5 & 30 & 10 & 13 & 3 & 0 & 0 & 0 & 0 & 0 & 0 & 26 & 3 & 8 & 1 \\
\hline $4-5$ & 0 & 0 & 0 & 0 & 31 & 6 & 0 & 0 & 0 & 0 & 0 & 0 & 0 & 0 & 12 & 2 & 0 & 0 \\
\hline$>5$ & 0 & 0 & 0 & 0 & 4 & 2 & 0 & 0 & 0 & 0 & 0 & 0 & 0 & 0 & 0 & 0 & 0 & 0 \\
\hline
\end{tabular}

$\mathrm{Co}=$ total number of collisions ( 5 years) on all curves within the indicated categories.

$\mathrm{Nc}=$ number of curves within the indicated categories.

As seen in the table, most of the curves with high $\beta_{c m f}$, greater than 4.0 have low collision frequency throughout five years. However, there are some exceptions which indicate that other criteria would affect on the safety performance of the curves and should be considered in the overall assessment of horizontal curve design and conditions. Also, 
most of the collisions (approximately $75 \%$ of the total number) took place on curves with low $\beta_{c m f}$ (lower than 3.0) as shown earlier in the figure.

The relationship between the $\beta_{c m f}$ and percentage of collisions is shown in Figure 6.15. It is readily seen that collisions frequency has an inverse trend with $\beta_{c m f}$. The trend is the same whether the weather condiitions were clear or raining. However, the slope of the curve is relatively smoother when having raining weather conditions. This might happen because drivers tend to drive slower in adverse weather conditions as explained earlier in Section 5.3.2. This indicates that violating driver comfort criterion would put more pressure and workload on drivers. Accordingly, this would increase the probability of collision occurrence. This confirms the conclusion made by Sarhan and Hassan (2008) that increasing $\mathrm{POH}$ would not only increase probability of having a collision, but also reduce level of driver comfort.

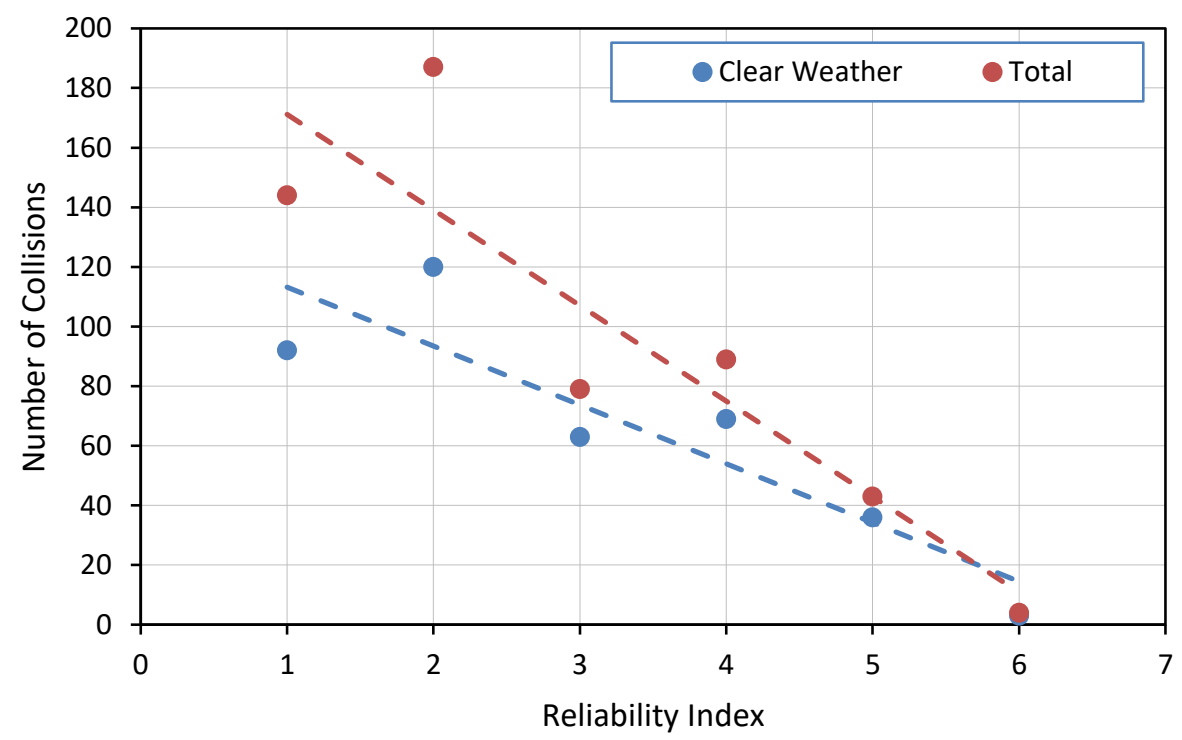

Figure 6.15: Trend of Collision Frequency with Reliability Index $\left(\boldsymbol{\beta}_{c m f}\right)$. 


\subsubsection{Sight Distance Assessment}

As explained earlier, the reliability analysis for sight distance design criteria depends on the available and demanded sight distance considering the case when having horizontal curve superimposed with a crest vertical curve or the sight distance is limited by an overpass. For the purpose of the research, the sight distance limitations in these cases were not considered in the reliability analysis. Due to lack of information regarding the interaction between vertical and horizontal curves, the sight distance was considered to be limited only by roadside obstructions such as trees. The performance function for sight distance criterion is the difference between the minimum available sight distance and demanded stopping sight distance.

$$
G=S_{a v i}-S_{D}=\frac{R}{28.65} \cos ^{-1}\left(1-\frac{C}{R}\right)-\left(0.278 V t+\frac{V^{2}}{254\left(f_{L} \mp G\right)}\right)
$$

Where $S_{a v i}$ and $S_{D}=$ available and demanded sight distances, $R=$ radius of the curve (m), $V=$ speed of the vehicle $(\mathrm{km} / \mathrm{h}), C=$ lateral clearance distance $(\mathrm{m}), t=$ perception reaction time (s), $f_{L}=$ longitudinal friction factor, and $G=$ the grade.

Although the roadways geometry dataset includes descriptions and measurements for the left and right shoulders, it lacks the distance to roadside barriers. Therefore, ArcMap, AutoCAD, and the State Route Tool (SRweb) were used to determine the lateral clearance and the minimum available sight distance in case of having the sight distance greater than the length of curve. The mean and standard deviation for the perception and brake reaction time were taken as 1.5 and $0.4 \mathrm{~s}$, respectively, as suggested by Lerner (1995).

The reliability index for sight distance criterion $\left(\beta_{S D}\right)$ is calculated. The frequency of curves according to $\beta_{S D}$ is shown in Figure 6.16. As shown in the figure, most of the curves 
have high $\beta_{S D}$.

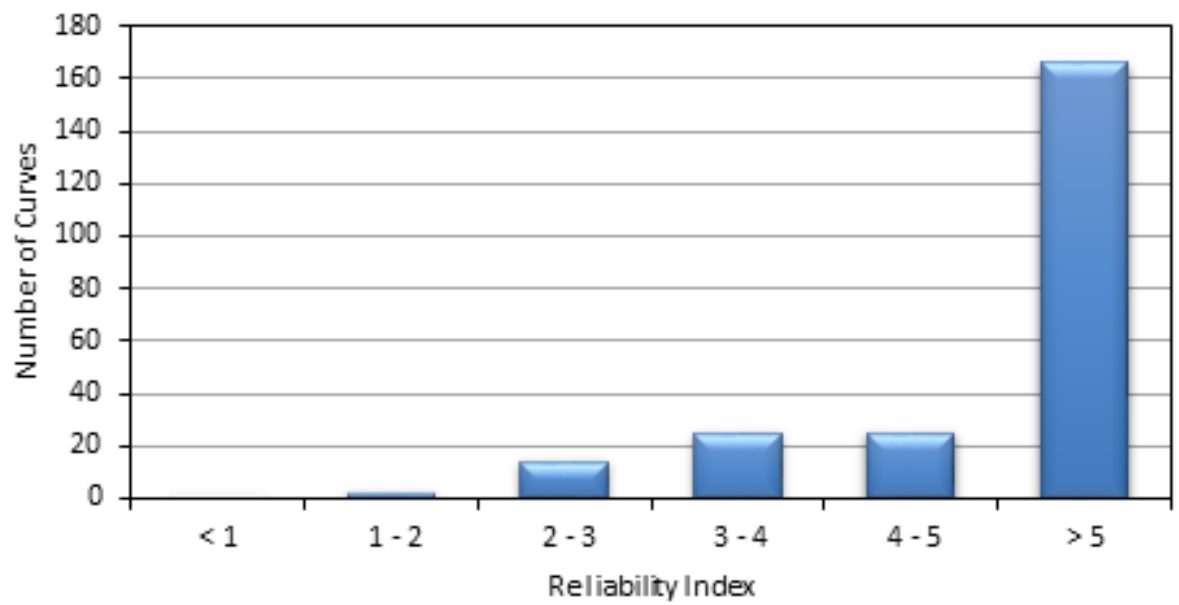

Figure 6.16: Number of Curves According Reliability Index $\left(\beta_{S D}\right)$.

The $\beta_{S D}$ is plotted along with AADT and total collisions on curve. Figure 6.17 shows that many curves have very small probability of Hazard (POH), especially for curves with zero and one collisions. Some curves with relatively high $\beta_{S D}$ had collisions; however, these curves serve relatively high AADT. It should be noted that was developed for total number of collisions.

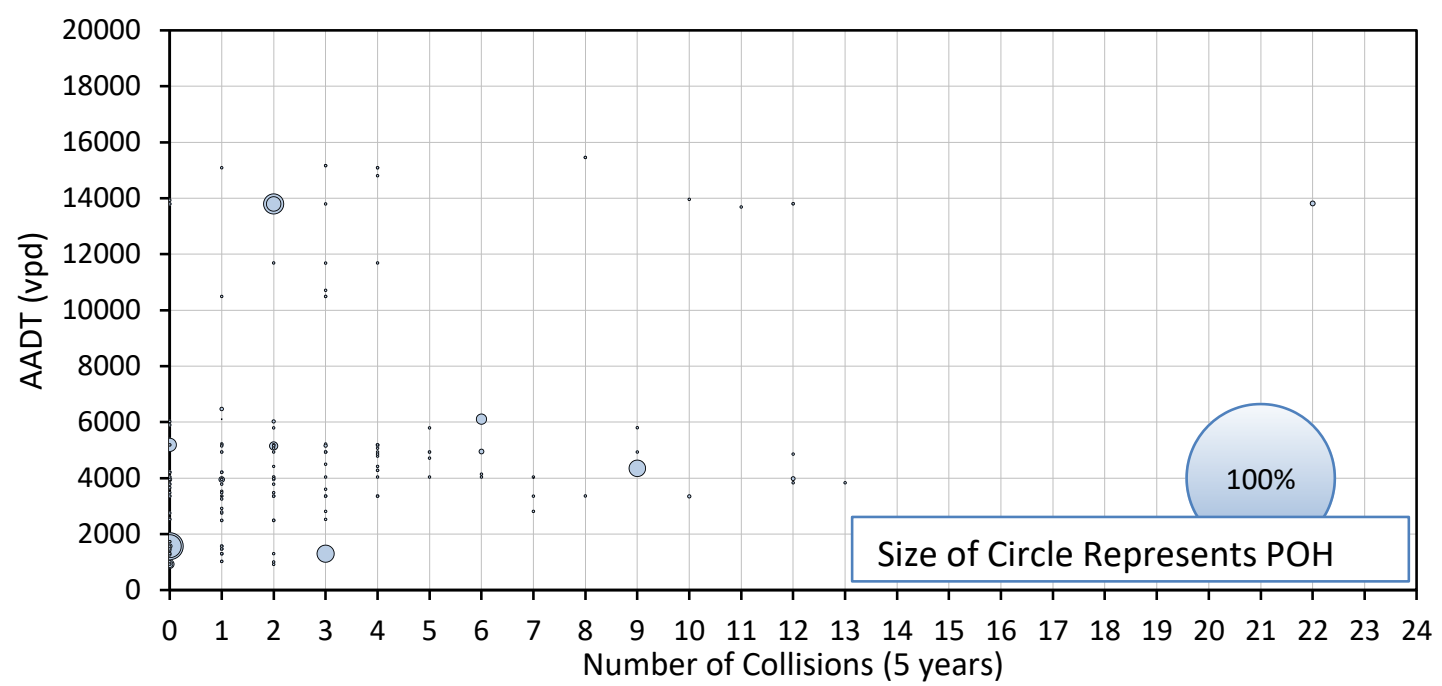

Figure 6.17: Relationship among Collisions, AADT, and POH of Sight Distance Criterion. 
The reliability analysis outputs are tabulated in Table 6-4. The results show that approximately $82 \%$ of the curves have very high value of $\beta_{S D}$ (greater than 4.0 ). The table also shows a high number of collisions occurred on curves with very high $\beta_{S D}$. This situation is similar to the vehicle stability criterion.

Table 6-4: Number of Collisions on Curves within Specific Reliability Index Interval POH.

\begin{tabular}{|c|c|c|c|c|c|c|c|c|c|c|c|c|c|c|c|c|c|c|}
\hline \multirow{3}{*}{$\begin{array}{l}\text { Reliability } \\
\text { Index }\end{array}$} & \multicolumn{18}{|c|}{ AADT $\left(\operatorname{vpd} \times 10^{3}\right)$} \\
\hline & \multicolumn{2}{|c|}{$<1$} & \multicolumn{2}{|c|}{$1-3$} & \multicolumn{2}{|c|}{$3-5$} & \multicolumn{2}{|c|}{$5-7$} & \multicolumn{2}{|c|}{$7-9$} & \multicolumn{2}{|c|}{$9-11$} & \multicolumn{2}{|c|}{$11-13$} & \multicolumn{2}{|c|}{$13-15$} & \multicolumn{2}{|c|}{$>15$} \\
\hline & $\mathrm{Co}$ & $\mathrm{Nc}$ & $\mathrm{Co}$ & $\mathrm{Nc}$ & $\mathrm{Co}$ & $\mathrm{Nc}$ & $\mathrm{Co}$ & $\mathrm{Nc}$ & Co & $\mathrm{Nc}$ & Co & $\mathrm{Nc}$ & Co & $\mathrm{Nc}$ & $\mathrm{Co}$ & $\mathrm{Nc}$ & $\mathrm{Co}$ & $\mathrm{Nc}$ \\
\hline$<1$ & 0 & 0 & 0 & 0 & 0 & 0 & 0 & 0 & 0 & 0 & 0 & 0 & 0 & 0 & 0 & 0 & 0 & 0 \\
\hline $1-2$ & 0 & 0 & 0 & 2 & 0 & 0 & 0 & 0 & 0 & 0 & 0 & 0 & 0 & 0 & 0 & 0 & 0 & 0 \\
\hline $2-3$ & 0 & 2 & 3 & 3 & 16 & 3 & 8 & 3 & 0 & 0 & 0 & 0 & 0 & 0 & 26 & 3 & 0 & 0 \\
\hline $3-4$ & 0 & 0 & 2 & 4 & 42 & 11 & 15 & 9 & 0 & 0 & 0 & 0 & 0 & 0 & 0 & 2 & 0 & 0 \\
\hline $4-5$ & 0 & 1 & 0 & 6 & 37 & 7 & 14 & 7 & 0 & 0 & 3 & 1 & 0 & 0 & 35 & 3 & 0 & 0 \\
\hline$>5$ & 2 & 5 & 43 & 55 & 183 & 66 & 53 & 22 & 0 & 0 & 7 & 3 & 9 & 3 & 17 & 4 & 31 & 7 \\
\hline
\end{tabular}

$\mathrm{Co}=$ total number of collisions ( 5 years) on all curves within the indicated categories.

$\mathrm{Nc}=$ number of curves within the indicated categories.

The trend between $\beta_{S D}$ and number of collision ratio is shown in Figure 6.18. The figure shows an inverse relationship between $\beta_{S D}$ and collision per curve for both total collisions and those occurring in clear weather conditions.

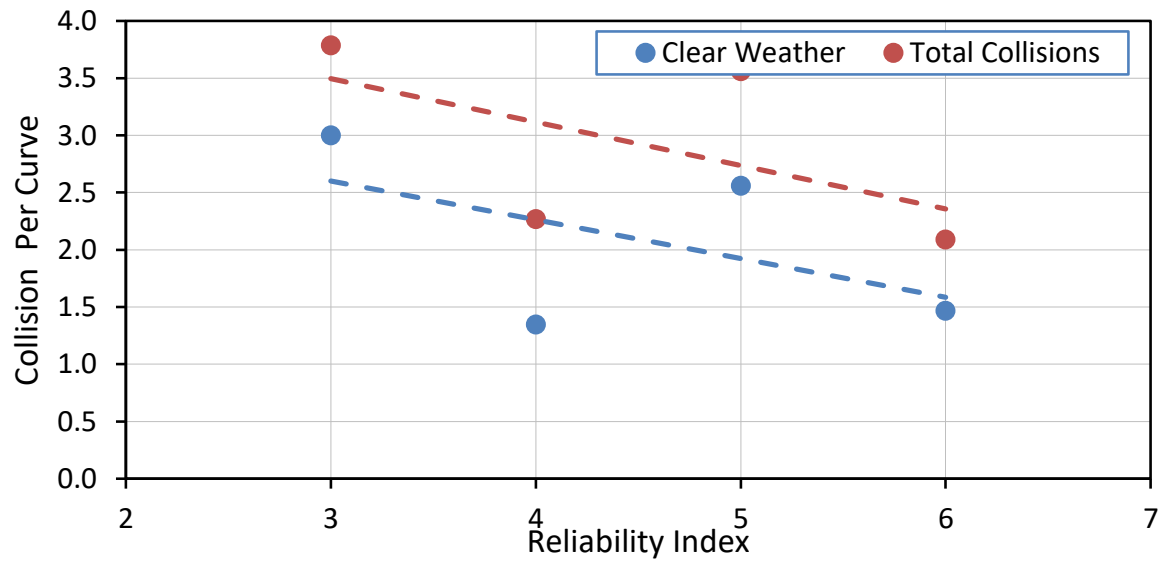

Figure 6.18: Relationship between Collisions per Curve and Reliability Index $\left(\boldsymbol{\beta}_{S D}\right)$. 


\subsubsection{Rollover Assessment}

Basically, vehicles start rolling over when the overturning moment, developed by the lateral force, exceeds the counterbalancing moment generated by the component of the weight of vehicle. It should be noted again that a vehicle requires sufficient lateral friction available or any means of tripping mechanism to rollover, otherwise the vehicle will skid off the road rather than rolling over. As explained in Section 6.6, the outputs of CarSIM were used to estimate the roll angle, and thus determine the overturning and counterbalancing moments considering the characteristics of vehicle.

Using PC to evaluate the curves against rollover criterion showed that all curves have high reliability index (greater than 3.0) as shown in Figure 6.19. It should be noted that the total number of rollover collisions was 50 . However, only 18 collisions took place on dry pavement conditions. Thus, approximately $97 \%$ of the rollover collisions most likely would have occurred after sliding failure. Unfortunately, the database does not provide information regarding the type of collided vehicles.

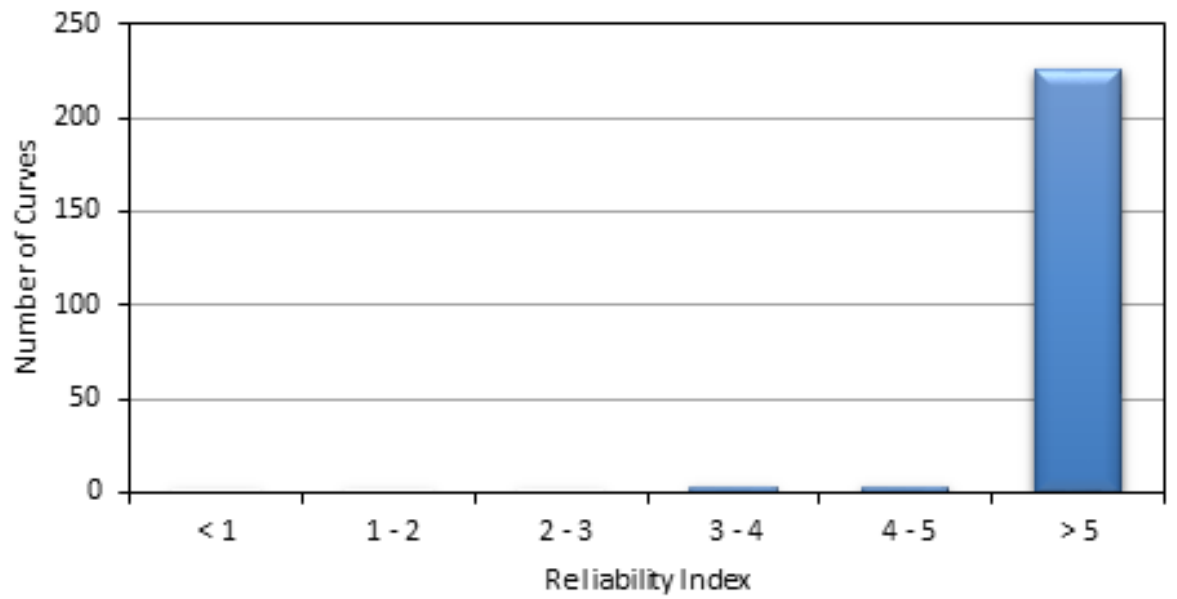

Figure 6.19: Relationship between Collisions Rate and Reliability Index $\left(\beta_{\text {Roll }}\right)$. 
Figure 6.20 shows the relationship among total collision frequency, AADT, and $\mathrm{POF}_{\mathrm{R}}$. As seen in the figure, all the curves have low POF. However, the reason behind these collisions for most of the cases might not related to violating the rollover criterion.

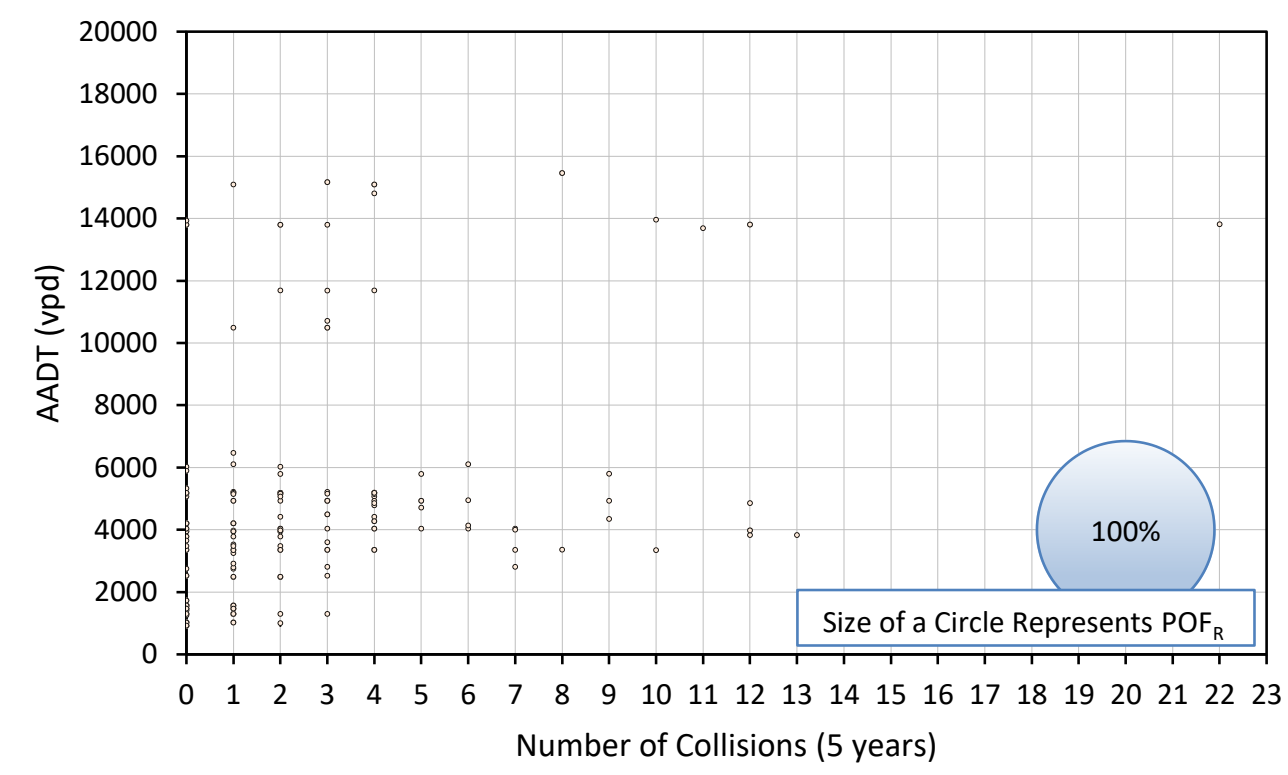

Figure 6.20: Relationship between Collisions Frequency, AADT, and POFR.

Considering all collisions and those occurred on only dry pavement surface, the relationship between $\beta_{\text {Roll }}$ and Collision per curve is shown in Figure 6.21. The figure shows that the total collisions decreases with $\beta_{\text {Roll }}$. Unfortunately, the trend between $\beta_{\text {Roll }}$ and rollover collisions only cannot be drawn because all these collisions have $\beta_{\text {Roll }}$ greater than 4 . 


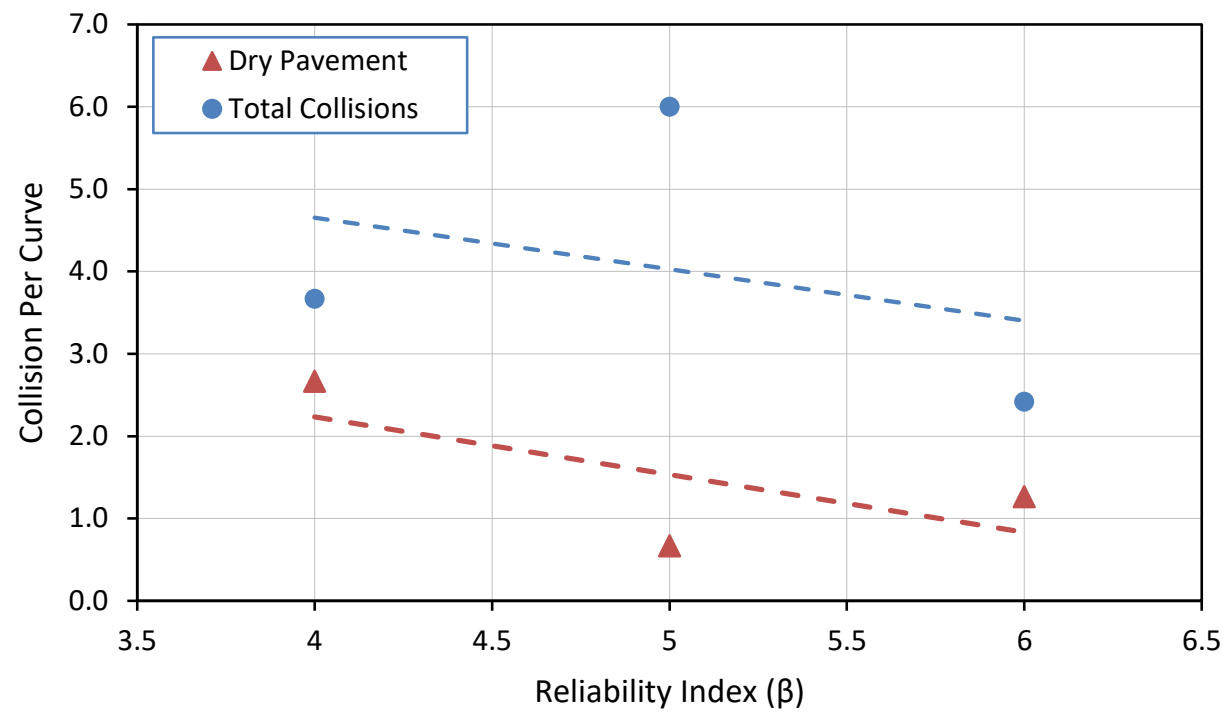

Figure 6.21: Relationship between Collisions per Curve and Reliability Index $\left(\boldsymbol{\beta}_{\text {Roll }}\right)$.

\subsection{Reliability Indices Correlations}

The bivariate (Pearson) correlation matrix among the reliability indices $\left(\beta_{V S}, \beta_{c m f}, \beta_{S D}\right.$ and $\beta_{\text {Roll }}$ ) was developed. The correlations of most indices are significant at $1 \%$ level of significance as shown in Table 6-5. As shown in the table, the correlation between $\beta_{V S}$ and $\beta_{\text {Roll }}$ is very high $(0.823)$.

Table 6-5: Bivariate (Pearson) Correlation Matrix for Reliability Indices.

\begin{tabular}{|c|c|c|c|c|}
\hline \hline & $\beta_{V S}$ & $\beta_{c m f}$ & $\beta_{S D}$ & $\beta_{\text {Roll }}$ \\
\hline$\beta_{V S}$ & 1.000 & & & \\
\hline$\beta_{c m f}$ & 0.236 & 1.000 & & \\
\hline$\beta_{S D}$ & 0.479 & 0.125 & 1.000 & \\
\hline$\beta_{\text {Roll }}$ & 0.823 & 0.082 & 0.497 & 1.000 \\
\hline \hline
\end{tabular}




\subsection{Summary}

This chapter illustrated the application of reliability analysis on evaluating horizontal curves performance. The POF, PNC, $\mathrm{POH}$, and $\mathrm{POF}_{\mathrm{R}}$ were calculated using FORM and the developed models in Chapter 5. The results showed that probability of failure has an inverse relationship with radius of curve and available friction factor and a direct relationship with speed of vehicles. The reliability analysis for the comfort threshold criterion showed that the PNC has low values for sharp curve because the applied lateral acceleration is relatively lower than driver comfort threshold. However, the general trend is that the PNC has an inverse relationship with radius because the applied lateral acceleration and driver comfort decrease at different rates. Also, the results showed that the POH decreases with lateral clearance when the analysis considers only 2-D case. Finally, the $\mathrm{POF}_{\mathrm{R}}$ was very low for $\mathrm{PC}$ and has an inverse relationship with radius.

The reliability indices $\left(\beta_{V S}, \beta_{c m f}, \beta_{S D}\right.$ and $\left.\beta_{\text {Roll }}\right)$ for 232 curves located in Washington State were calculated. The results showed that there is an inverse trend between these indices and collision frequency. Therefore, these indices could be used as surrogate measures to evaluate safety performance of horizontal curves. The results of the analysis showed that an evaluation of a horizontal curve should take into account all design criteria because violation for any design criterion would increase the probability of having a collision. 


\section{CHAPTER SEVEN}

\section{SAFETY PERFORMANCE FUNCTIONS}

\subsection{Introduction}

Several factors would affect the safety performance of horizontal curves. These factors could be internal such as radius, superelevation rate, or external such as the length of preceding approach tangent (TAC 2017). A considerable number of models have been developed to predict collision frequency or evaluate safety on horizontal curves. This chapter aims at developing safety performance functions (SPFs) considering reliability indices, as independent variables, and illustrating the new framework to design horizontal curves through a design example.

\subsection{Modelling Procedure}

Poisson and negative binomial (NB) regression models were used in developing the safety models in this research. The standard NB regression can be considered an extension to Poisson regression by considering the over-dispersion of the count data. The overdispersion in Poisson models occurs when the response variance is greater than the mean, which is caused by positive correlation between responses or by an excess variation between response probabilities or counts. The overdispersion should be manipulated because it may cause standard errors of estimates to be underestimated. However, the NB model might be overdispersed as well when the variance produced by the estimated model exceeds the negative binomial variance. The general formulation of Poisson and NB regression models is shown in Equation (7.1). 


$$
N=\exp ^{\left(\alpha+\beta_{1} X_{1}+\beta_{2} X_{2}+\cdots+\beta_{n} X_{n}\right)}
$$

Where $N=$ expected number of collisions, $\alpha=$ constant, $\beta_{i}=$ parameters to be estimated, and $X_{i}=$ predictor variables.

Zero-inflated Poisson (ZIP), zero-inflated negative binomial (ZINB), zero-truncated negative binomial, and other enhancements have been developed to accommodate excessive zero counts in Poisson and NB and to solve either a distributional or variance problem (Hilbe 2011). The zero-inflated models could be tested to determine whether they are different from their base model (Poisson or NB) by using Vuong test. The zero-inflated model will be preferred over the base model when having Vuong statistic value greater than 1.96, while the base model is preferred when the test value is less than -1.96 . Neither model is preferred over the other if the test value is between \pm 1.96 (Hilbe 2011). Regression analysis of the collision data was performed in this research using the Stata software. Selection of the best model that fits the data depends on several parameters such as Akaike Information Criterion (AIC) and Bayesian information criterion (BIC) and Pearson dispersion statistic.

The total collision frequency on horizontal curves was the dependent variable in the regression analysis while the main independent variables included curve geometric characteristics (radius and deflection angle), reliability indices $\left(\beta_{V S}, \beta_{c m f}, \beta_{S D}, \beta_{R o l l}\right)$, and posted speed limit. However, deflection angle and posted speed limit were insignificant in almost all modelling attempts. To account for the effect of traffic exposure on collision frequency, curve length $(L)$ and AADT were used as exposure variables in the regression models. These exposure factors can be combined in one exposure term or can have the values transferred using natural logarithm. The latter approach, taking the natural 
logarithm, was used because successful relations have been found between collision frequency and the natural logarithm of $L$ and AADT as reported by Anderson et al. (1999), Sawalha and Sayed (2001), and Ng and Sayed (2004).

To evaluate the contribution of reliability indices in the models, base models were developed for each regression model considering only the exposure variables (AADT and $L$ ) and radius of curve. Then, models were developed for each regression model considering the reliability indices. Then, the models were evaluated to determine whether or not the reliability indices have added more value to the base model. The evaluation of the models was based on the AIC and BIC tests.

\subsection{Safety Performance Functions}

Usually safety models are developed to predict collision frequency for 3 to 5 years. In this research, two sets of SPFs are developed considering one and five-year intervals. The reason behind that is the continuous change in pavement conditions and traffic volume, and in turn change in the reliability indices from one year to the other. For five-year interval, the average values of reliability indices, natural logarithm of $L$ and AADT are used in the regression analysis.

Considering the variation in curves characteristics shown in Table 6-1, the database was categorized into several groups to develop the SPFs. First set of models was developed using the data of all curves. The second set considered only curves with specific radius and curve length (i.e. radius $<1000 \mathrm{~m}$ and $L>100 \mathrm{~m}$ ), which are rounded values of the limits in the data used to develop the models in Chapters 5 and 6. Third set of models considered specific collision time and weather conditions (i.e. daytime and clear weather conditions), 
which correspond to the driving conditions used to develop the models in Chapter 5. The outputs of the regression analysis for all sets of models have almost the same trends. The regression outputs of using all curves are shown in this chapter, and the results of other models considering specific curve characteristics are shown in Appendix (G).

\subsubsection{SPFs of Annual Collision Frequency}

Considering the number of collisions per year, the statistics for collision frequency show that the mean and variance are 0.471 and 0.765 , respectively. For the base model (using only the natural logarithm of AADT and curve length), the Pearson statistic is 1.225 (Deviance $=0.996$ ) when conducting the Poisson generalized linear modelling, which means that some $22 \%$ extra dispersion exists in the data. The Pearson statistic dropped to 0.969 (Deviance $=0.769)$ when using NB generalized linear model. The AIC and BIC dropped as well as shown in Table 7-1. Thus, the NB model provides a better fit for the data than the Poisson model. The table also shows that the AIC and BIC dropped for Poisson and NB when considering the radius of the curve in the regression analysis. It should be noted that other curve characteristics (deflection angle, superelevation rate, and posted speed limit) were insignificant. 
Table 7-1: SPFs for Annual Collision Frequency (Base Models).

\begin{tabular}{|c|c|c|c|c|c|c|c|}
\hline $\begin{array}{l}\text { Regression } \\
\text { Model }\end{array}$ & Variable & Coef. & SEE & $\begin{array}{c}p \text { - } \\
\text { value }\end{array}$ & $\begin{array}{l}\text { Likelihood- } \\
\text { ratio test }\end{array}$ & AIC & $\mathrm{BIC}$ \\
\hline \multirow{3}{*}{ Poisson1-1 } & Log of (AADT) & 0.839 & 0.065 & $<0.001$ & \multirow{3}{*}{-- } & \multirow{3}{*}{1970.86} & \multirow{3}{*}{1986.03} \\
\hline & Log of $(L)$ & 0.738 & 0.075 & $<0.001$ & & & \\
\hline & Constant & -11.788 & 0.740 & $<0.001$ & & & \\
\hline \multirow{3}{*}{ NB1-1 } & Log of (AADT) & 0.864 & 0.078 & $<0.001$ & \multirow{3}{*}{$\begin{array}{c}40.01 \\
(p \text {-value } \\
<0.001)\end{array}$} & \multirow{3}{*}{1932.85} & \multirow{3}{*}{1953.07} \\
\hline & Log of $(L)$ & 0.755 & 0.087 & $<0.001$ & & & \\
\hline & Constant & -12.096 & 0.852 & $<0.001$ & & & \\
\hline \multirow{4}{*}{ Poisson1-2 } & Log of (AADT) & 0.738 & 0.067 & $<0.001$ & \multirow{4}{*}{-- } & \multirow{4}{*}{1947.75} & \multirow{4}{*}{1967.97} \\
\hline & $\log$ of $(L)$ & 0.892 & 0.083 & $<0.001$ & & & \\
\hline & $R$ & -0.001 & $<0.001$ & $<0.001$ & & & \\
\hline & Constant & -11.395 & 0.737 & $<0.001$ & & & \\
\hline \multirow{4}{*}{ NB1-2 } & Log of (AADT) & 0.768 & 0.079 & $<0.001$ & \multirow{4}{*}{$\begin{array}{c}34.60 \\
(p \text {-value } \\
<0.001)\end{array}$} & \multirow{4}{*}{1915.15} & \multirow{4}{*}{1940.43} \\
\hline & Log of $(L)$ & 0.913 & 0.096 & $<0.001$ & & & \\
\hline & $R$ & -0.001 & $<0.001$ & $<0.001$ & & & \\
\hline & Constant & -11.755 & 0.846 & $<0.001$ & & & \\
\hline
\end{tabular}

Coef. = coefficient, $S E E=$ standard error of estimate, AADT= average annual daily traffic (veh/d), $L=$ length of curve (m), and $R=$ Radius (m).

Figure 7.1 shows how total collisions fits the Poisson and NB distributions and readily shows that NB fits the data better. As shown in the figure, there is an obvious difference between Poisson model prediction and the observed collisions. On the other hand, NB provided more accurate prediction, which confirms the previous results.

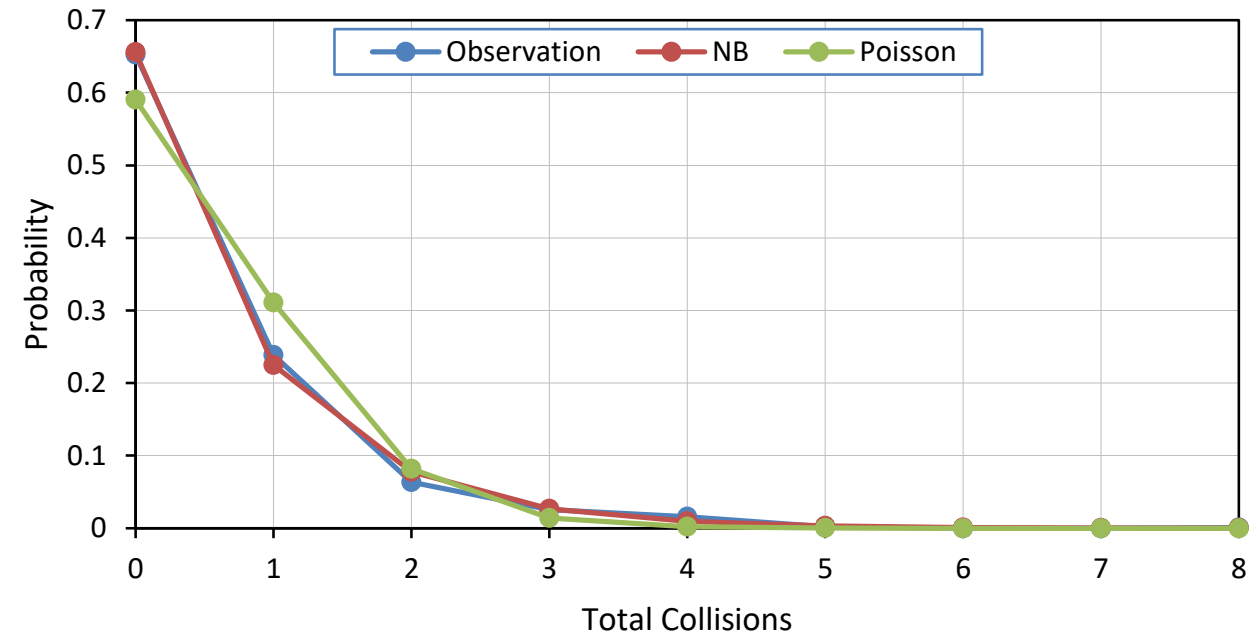

Figure 7.1: Observed Collisions (per year) Compared with Poisson and NB Distributions. 
Based on the previous discussion, the NB regression was selected to develop SPFs to predict annual collision frequency. The first set of SPFs considers each reliability index with exposure variables (AADT and $L$ ) as shown in Table 7-2. As shown in the table, all reliability indices (except for the comfort criterion) were significant at 5\% level of significance. The AIC and BIC of the new models are lower than those of the base model (NB1-1). Therefore, it could be concluded that considering the reliability index would add more value to the base model. Thus, the new models better fit the data than the base model.

Table 7-2: Annual Collision Frequency Models (Considering AADT and L).

\begin{tabular}{|c|c|c|c|c|c|c|c|}
\hline $\begin{array}{l}\text { Regression } \\
\text { Model }\end{array}$ & Variable & Coef. & SEE & $\begin{array}{c}p- \\
\text { value }\end{array}$ & $\begin{array}{l}\text { Likelihood- } \\
\text { ratio test }\end{array}$ & AIC & BIC \\
\hline \multirow{4}{*}{ NB1-3 } & Log of (AADT) & 0.076 & 10.730 & $<0.001$ & \multirow{4}{*}{$\begin{array}{l}34.96 \\
(p \text {-value } \\
<0.001)\end{array}$} & \multirow{4}{*}{1920.65} & \multirow{4}{*}{1945.93} \\
\hline & Log of $(L)$ & 0.860 & 0.091 & $<0.001$ & & & \\
\hline & $\beta_{V S}$ & -0.219 & 0.057 & $<0.001$ & & & \\
\hline & Constant & -11.320 & 0.848 & $<0.001$ & & & \\
\hline \multirow{4}{*}{ NB1-4 } & Log of (AADT) & 0.863 & 0.078 & $<0.001$ & \multirow{4}{*}{$\begin{array}{l}40.01 \\
(p \text {-value } \\
<0.001)\end{array}$} & \multirow{4}{*}{1934.84} & \multirow{4}{*}{1960.12} \\
\hline & Log of $(L)$ & 0.753 & 0.095 & $<0.001$ & & & \\
\hline & $\beta_{c m f}$ & 0.003 & 0.039 & 0.946 & & & \\
\hline & Constant & -12.082 & 0.880 & $<0.001$ & & & \\
\hline \multirow{4}{*}{ NB1-5 } & Log of (AADT) & 0.821 & 0.078 & $<0.001$ & \multirow{4}{*}{$\begin{array}{l}34.90 \\
(p \text {-value } \\
<0.001)\end{array}$} & \multirow{4}{*}{1922.99} & \multirow{4}{*}{1948.28} \\
\hline & Log of $(L)$ & 0.815 & 0.089 & $<0.001$ & & & \\
\hline & $\beta_{S D}$ & -0.091 & 0.026 & 0.001 & & & \\
\hline & Constant & -11.503 & 0.857 & $<0.001$ & & & \\
\hline \multirow{4}{*}{ NB1-6 } & Log of (AADT) & 0.837 & 0.077 & $<0.001$ & \multirow{4}{*}{$\begin{array}{c}36.48 \\
(p \text {-value } \\
<0.001)\end{array}$} & \multirow{4}{*}{1922.19} & \multirow{4}{*}{1947.47} \\
\hline & $\log$ of $(L)$ & 0.829 & 0.090 & $<0.001$ & & & \\
\hline & $\beta_{\text {Roll }}$ & -0.205 & 0.056 & $<0.001$ & & & \\
\hline & Constant & -10.701 & 0.906 & $<0.001$ & & & \\
\hline
\end{tabular}

Coef. = coefficient, $S E E=$ standard error of estimate, $\beta_{V S}, \beta_{c m f}, \beta_{S D}, \beta_{R o l l}=$ reliability indices for vehicle stability, driver comfort, sight distance, and rollover, respectively, and AADT= average annual daily traffic (veh/d). 
The second set of SPFs considers each reliability index, exposure variables (AADT and $L$ ), and radius of the curve as shown in Table 7-3. The table shows that the reliability index of vehicle stability improves the base model (NB1-2) in terms of AIC. However, the reliability indices of driver comfort, sight distance, and rollover were insignificant at 5\% level of significance.

Table 7-3: Annual Collision Frequency Models (Considering AADT, L, and R).

\begin{tabular}{|c|c|c|c|c|c|c|c|}
\hline $\begin{array}{c}\text { Regression } \\
\text { Model }\end{array}$ & Variable & Coef. & SEE & $\begin{array}{c}p- \\
\text { value }\end{array}$ & $\begin{array}{c}\text { Likelihood- } \\
\text { ratio test } \\
\end{array}$ & AIC & $\mathrm{BIC}$ \\
\hline \multirow{5}{*}{ NB1-7 } & Log of (AADT) & 0.765 & 0.018 & $<0.001$ & \multirow{5}{*}{$\begin{array}{l}32.99 \\
(p \text {-value } \\
<0.001)\end{array}$} & \multirow{5}{*}{1912.99} & \multirow{5}{*}{1943.33} \\
\hline & Log of $(L)$ & 0.941 & 0.097 & $<0.001$ & & & \\
\hline & $R$ & -0.0006 & $<0.001$ & 0.003 & & & \\
\hline & $\beta_{V S}$ & -0.131 & 0.064 & 0.039 & & & \\
\hline & Constant & -11.386 & 0.850 & $<0.001$ & & & \\
\hline \multirow{5}{*}{ NB1-8 } & Log of (AADT) & 0.759 & 0.080 & $<0.001$ & \multirow{5}{*}{$\begin{array}{c}34.42 \\
(p \text {-value } \\
<0.001)\end{array}$} & \multirow{5}{*}{1916.69} & \multirow{5}{*}{1947.03} \\
\hline & Log of $(L)$ & 0.892 & 0.101 & $<0.001$ & & & \\
\hline & $R$ & -0.001 & $<0.001$ & $<0.001$ & & & \\
\hline & $\beta_{c m f}$ & 0.026 & 0.039 & 0.500 & & & \\
\hline & Constant & -11.603 & 0.876 & $<0.001$ & & & \\
\hline \multirow{5}{*}{ NB1-9 } & Log of (AADT) & 0.763 & 0.079 & $<0.001$ & \multirow{5}{*}{$\begin{array}{l}32.82 \\
(p \text {-value } \\
<0.001)\end{array}$} & \multirow{5}{*}{1914.30} & \multirow{5}{*}{1944.64} \\
\hline & Log of $(L)$ & 0.915 & 0.096 & $<0.001$ & & & \\
\hline & $R$ & -0.001 & $<0.001$ & 0.002 & & & \\
\hline & $\beta_{S D}$ & -0.049 & 0.029 & 0.091 & & & \\
\hline & Constant & -11.496 & 0.854 & $<0.001$ & & & \\
\hline \multirow{5}{*}{ NB1-10 } & Log of (AADT) & 0.773 & 0.078 & $<0.001$ & \multirow{5}{*}{$\begin{array}{c}33.48 \\
(p \text {-value } \\
<0.001)\end{array}$} & \multirow{5}{*}{1913.83} & \multirow{5}{*}{1944.16} \\
\hline & Log of $(L)$ & 0.925 & 0.096 & $<0.001$ & & & \\
\hline & $R$ & -0.001 & $<0.001$ & 0.002 & & & \\
\hline & $\beta_{\text {Roll }}$ & -0.116 & 0.063 & 0.065 & & & \\
\hline & Constant & -11.057 & 0.913 & $<0.001$ & & & \\
\hline
\end{tabular}

The outputs of NB regression when considering all the reliability indices with the exposure variables (with and without considering the radius of curve) are shown in Table 7-4. The table shows that all the reliability indices were insignificant at 5\% level of significance. This would indicate that larger sample of curves and more data need to be collected. 
Table 7-4: SPFs for Annual Collision Frequency Considering All Reliability Indices.

\begin{tabular}{|c|c|c|c|c|c|c|c|}
\hline $\begin{array}{c}\text { Regression } \\
\text { Model }\end{array}$ & Variable & Coef. & SEE & $\begin{array}{c}p- \\
\text { value }\end{array}$ & $\begin{array}{c}\text { Likelihood- } \\
\text { ratio test }\end{array}$ & AIC & BIC \\
\hline \multirow{7}{*}{ NB1-11 } & Log of (AADT) & 0.078 & 10.280 & 0.649 & \multirow{7}{*}{$\begin{array}{l}33.03 \\
(p \text {-value } \\
<0.001)\end{array}$} & \multirow{7}{*}{1922.14} & \multirow{7}{*}{1962.59} \\
\hline & Log of $(L)$ & 0.853 & 0.098 & $<0.001$ & & & \\
\hline & $\beta_{V S}$ & -0.125 & 0.094 & 0.186 & & & \\
\hline & $\beta_{c m f}$ & 0.020 & 0.040 & 0.620 & & & \\
\hline & $\beta_{S D}$ & -0.054 & 0.030 & 0.070 & & & \\
\hline & $\beta_{\text {Roll }}$ & -0.055 & 0.092 & 0.548 & & & \\
\hline & Constant & -10.821 & 0.925 & $<0.001$ & & & \\
\hline \multirow{8}{*}{ NB1-12 } & Log of (AADT) & 0.752 & 0.079 & $<0.001$ & \multirow{8}{*}{$\begin{array}{l}31.90 \\
(p \text {-value } \\
<0.001)\end{array}$} & \multirow{8}{*}{1917.03} & \multirow{8}{*}{1962.53} \\
\hline & $\log$ of $(L)$ & 0.912 & 0.101 & $<0.001$ & & & \\
\hline & $R$ & -0.001 & $<0.001$ & 0.010 & & & \\
\hline & $\beta_{V S}$ & -0.101 & 0.095 & 0.288 & & & \\
\hline & $\beta_{c m f}$ & 0.032 & 0.041 & 0.426 & & & \\
\hline & $\beta_{S D}$ & -0.033 & 0.031 & 0.291 & & & \\
\hline & $\beta_{\text {Roll }}$ & -0.017 & 0.093 & 0.859 & & & \\
\hline & Constant & -11.018 & 0.930 & $<0.001$ & & & \\
\hline
\end{tabular}

The zero-inflated Poisson regression (ZIP) was used to develop SPFs. The outputs of ZIP regression for the base models, with and without considering the radius of the curve (ZIP1-1 and ZIP1-2), are shown in Table 7-5. The Vuong statistic was greater than +1.96 and significant at $5 \%$ level of significance, which means that the ZIP model is preferred over the base model (Poisson). Thus, the ZIP models succeeded in accommodating the excessive zero counts in Poisson and in solving either a distributional or variance problem. The AIC and BIC values of ZIP1-2 were lower than those of the ZIP1-1, which means that the former model better fits the data. In other words, considering the radius of the curve adds more value to the base model.

Zero-inflated negative binomial regression (ZINB) was used to develop SPFs as well as shown in Table 7-5. The Vuong statistic of the base models (with and without considering the radius of the curve) was not significant at $5 \%$ level of significance and the 
Table 7-5: ZIP and ZINB Models to Predict Annual Collision (Base Models).

\begin{tabular}{|c|c|c|c|c|c|c|c|}
\hline Model & Variable & Coef. & $S E E$ & $\begin{array}{c}p- \\
\text { value }\end{array}$ & $\begin{array}{l}\text { Vuong } \\
\text { statistic }\end{array}$ & AIC & $\mathrm{BIC}$ \\
\hline \multirow{7}{*}{ ZIP1-1 } & $\log (A A D T)$ & 0.707 & 0.132 & $<0.001$ & \multirow{7}{*}{$\begin{array}{c}2.30 \\
(p- \\
\text { value } \\
=0.011)\end{array}$} & \multirow{7}{*}{1948.19} & \multirow{7}{*}{1978.53} \\
\hline & $\log (L)$ & 0.402 & 0.130 & 0.002 & & & \\
\hline & Constant & -8.454 & 1.438 & $<0.001$ & & & \\
\hline & \multicolumn{4}{|l|}{ Inflate } & & & \\
\hline & $\log (A A D T)$ & -0.379 & 0.348 & 0.277 & & & \\
\hline & $\log (L)$ & -1.040 & 0.373 & 0.005 & & & \\
\hline & Constant & 8.003 & 3.043 & 0.009 & & & \\
\hline \multirow{8}{*}{ ZIP1-2 } & $\log (A A D T)$ & 0.526 & 0.136 & $<0.001$ & \multirow{8}{*}{$\begin{array}{c}2.12 \\
(p- \\
\text { value } \\
=0.017)\end{array}$} & \multirow{8}{*}{1931.67} & \multirow{8}{*}{1967.07} \\
\hline & $\log (L)$ & 0.603 & 0.141 & $<0.001$ & & & \\
\hline & $R$ & -0.0007 & $<0.001$ & $<0.001$ & & & \\
\hline & Constant & -7.675 & 1.404 & $<0.001$ & & & \\
\hline & \multicolumn{4}{|l|}{ Inflate } & & & \\
\hline & $\log (A A D T)$ & -0.704 & 0.400 & 0.079 & & & \\
\hline & $\log (L)$ & -0.879 & 0.367 & 0.017 & & & \\
\hline & Constant & 9.763 & 3.198 & 0.002 & & & \\
\hline \multirow{7}{*}{ ZINB1-1 } & $\log (A A D T)$ & 0.807 & 0.087 & $<0.001$ & \multirow{7}{*}{$\begin{array}{c}1.22 \\
(p- \\
\text { value } \\
=0.111)\end{array}$} & \multirow{7}{*}{1931.89} & \multirow{7}{*}{1967.28} \\
\hline & $\log (L)$ & 0.837 & 0.094 & $<0.001$ & & & \\
\hline & Constant & -12.025 & 0.921 & $<0.001$ & & & \\
\hline & \multicolumn{4}{|l|}{ Inflate } & & & \\
\hline & $\log (A A D T)$ & -4.286 & 1.757 & 0.015 & & & \\
\hline & $\log (L)$ & 4.342 & 2.147 & 0.043 & & & \\
\hline & Constant & 5.089 & 10.283 & 0.621 & & & \\
\hline \multirow{8}{*}{ ZINB1-2 } & $\log (A A D T)$ & 0.601 & 0.114 & $<0.001$ & \multirow{8}{*}{$\begin{array}{c}1.09 \\
(p- \\
\text { value } \\
=0.137)\end{array}$} & \multirow{8}{*}{1915.65} & \multirow{8}{*}{1956.09} \\
\hline & $\log (L)$ & 0.858 & 0.108 & $<0.001$ & & & \\
\hline & $R$ & -0.001 & $<0.001$ & $<0.001$ & & & \\
\hline & Constant & -9.952 & 1.279 & $<0.001$ & & & \\
\hline & \multicolumn{4}{|l|}{ Inflate } & & & \\
\hline & $\log (A A D T)$ & -3.300 & 1.303 & 0.011 & & & \\
\hline & $\log (L)$ & 0.056 & 1.036 & $\mathbf{0 . 9 5 7}$ & & & \\
\hline & Constant & 22.921 & 8.493 & 0.007 & & & \\
\hline
\end{tabular}

value was between \pm 1.96 , which means neither model is preferred over the other. However, by comparing the AIC of the NB and ZINB base models in Table 7-1 and Table 7-5, the ZINB base model without radius should be preferred over the corresponding NB model, while the two models with radius are basically similar. 
Table 7-6 shows the ZIP models that include reliability indices with AADT and $L$. The AIC and BIC for these models are less than those of the base model ZIP1-1 (except for the driver comfort), which means that the ZIP models better fit the data than the base model. It should be noted that all variables were significant at 5\% level of significance expect for the driver comfort reliability index.

Table 7-6: ZIP Models to Predict Annual Collision Frequency.

\begin{tabular}{|c|c|c|c|c|c|c|c|}
\hline Model & Variable & Coef. & $S E E$ & $p$-value & $\begin{array}{l}\text { Vuong } \\
\text { statistic }\end{array}$ & AIC & $\mathrm{BIC}$ \\
\hline \multirow{8}{*}{ ZIP1-3 } & $\log (A A D T)$ & 0.570 & 0.126 & $<0.001$ & \multirow{8}{*}{$\begin{array}{c}2.44 \\
(p \text {-value } \\
=0.007)\end{array}$} & \multirow{8}{*}{1930.68} & \multirow{8}{*}{1966.07} \\
\hline & $\log (L)$ & 0.506 & 0.134 & $<0.001$ & & & \\
\hline & $\beta_{V S}$ & -0.249 & 0.055 & $<0.001$ & & & \\
\hline & Constant & -6.728 & 1.362 & $<0.001$ & & & \\
\hline & \multicolumn{4}{|l|}{ Inflate } & & & \\
\hline & $\log (A A D T)$ & -0.653 & 0.331 & 0.049 & & & \\
\hline & $\log (L)$ & -1.018 & 0.365 & 0.005 & & & \\
\hline & Constant & 10.191 & 2.803 & $<0.001$ & & & \\
\hline \multirow{8}{*}{ ZIP1-4 } & $\log (A A D T)$ & 0.707 & 0.131 & $<0.001$ & \multirow{8}{*}{$\begin{aligned} & 2.25 \\
(p-\text { value } & \\
= & 0.012)\end{aligned}$} & \multirow{8}{*}{1950.13} & \multirow{8}{*}{1985.52} \\
\hline & $\log (L)$ & 0.410 & 0.133 & 0.002 & & & \\
\hline & $\beta_{c m f}$ & -0.009 & 0.036 & 0.803 & & & \\
\hline & Constant & -8.474 & 1.436 & $<0.001$ & & & \\
\hline & \multicolumn{4}{|l|}{ Inflate } & & & \\
\hline & $\log (A A D T)$ & -0.386 & 0.347 & 0.266 & & & \\
\hline & $\log (L)$ & -1.039 & 0.372 & 0.005 & & & \\
\hline & Constant & 8.065 & 3.037 & 0.008 & & & \\
\hline \multirow{8}{*}{ ZIP1-5 } & $\log (A A D T)$ & 0.635 & 0.130 & $<0.001$ & \multirow{8}{*}{$\begin{array}{c}2.27 \\
(p \text {-value } \\
=0.017)\end{array}$} & \multirow{8}{*}{1935.64} & \multirow{8}{*}{1971.04} \\
\hline & $\log (L)$ & 0.486 & 0.132 & $<0.001$ & & & \\
\hline & $\beta_{S D}$ & -0.097 & 0.025 & $<0.001$ & & & \\
\hline & Constant & -7.709 & 1.423 & $<0.001$ & & & \\
\hline & \multicolumn{4}{|l|}{ Inflate } & & & \\
\hline & $\log (A A D T)$ & -0.489 & 0.347 & 0.158 & & & \\
\hline & $\log (L)$ & -0.983 & 0.366 & 0.007 & & & \\
\hline & Constant & 8.584 & 3.022 & 0.005 & & & \\
\hline \multirow{8}{*}{ ZIP1-6 } & $\log (A A D T)$ & 0.616 & 0.134 & $<0.001$ & \multirow{8}{*}{$\begin{array}{c}2.21 \\
(p \text {-value } \\
=0.014)\end{array}$} & \multirow{8}{*}{1937.68} & \multirow{8}{*}{1973.07} \\
\hline & $\log (L)$ & 0.483 & 0.137 & $<0.001$ & & & \\
\hline & $\beta_{\text {Roll }}$ & -0.188 & 0.052 & $<0.001$ & & & \\
\hline & Constant & -6.692 & 1.449 & $<0.001$ & & & \\
\hline & \multicolumn{4}{|l|}{ Inflate } & & & \\
\hline & $\log (A A D T)$ & -0.575 & 0.374 & 0.124 & & & \\
\hline & $\log (L)$ & -1.006 & 0.400 & 0.012 & & & \\
\hline & Constant & 9.418 & 2.991 & 0.002 & & & \\
\hline
\end{tabular}


Table 7-6: ZIP Models to Predict Annual Collision Frequency.- Cont.

\begin{tabular}{|c|c|c|c|c|c|c|c|}
\hline Model & Variable & Coef. & $S E E$ & $p$-value & $\begin{array}{c}\text { Vuong } \\
\text { statistic }\end{array}$ & $\mathrm{AIC}$ & $\mathrm{BIC}$ \\
\hline \multirow{9}{*}{ ZIP1-7 } & $\log (A A D T)$ & 0.492 & 0.126 & $<0.001$ & \multirow{9}{*}{$\begin{array}{c}2.23 \\
(p \text {-value } \\
=0.013)\end{array}$} & \multirow{9}{*}{1925.41} & \multirow{9}{*}{1965.86} \\
\hline & $\log (L)$ & 0.608 & 0.139 & $<0.001$ & & & \\
\hline & $R$ & -0.001 & 0.000 & 0.009 & & & \\
\hline & $\beta_{V S}$ & -0.178 & 0.061 & 0.004 & & & \\
\hline & Constant & -6.725 & 1.355 & $<0.001$ & & & \\
\hline & \multicolumn{4}{|l|}{ Inflate } & & & \\
\hline & $\log (A A D T)$ & -0.785 & 0.361 & 0.030 & & & \\
\hline & $\log (L)$ & -0.941 & 0.360 & 0.009 & & & \\
\hline & Constant & 10.805 & 2.934 & $<0.001$ & & & \\
\hline \multirow{9}{*}{ ZIP1-8 } & $\log (A A D T)$ & 0.520 & 0.138 & $<0.001$ & \multirow{9}{*}{$\begin{array}{c}2.02 \\
(p \text {-value } \\
=0.021)\end{array}$} & \multirow{9}{*}{1933.53} & \multirow{9}{*}{1973.98} \\
\hline & $\log (L)$ & 0.596 & 0.143 & $<0.001$ & & & \\
\hline & $R$ & -0.001 & $<0.001$ & $<0.001$ & & & \\
\hline & $\beta_{c m f}$ & 0.013 & 0.036 & 0.710 & & & \\
\hline & Constant & -7.609 & 1.415 & $<0.001$ & & & \\
\hline & \multicolumn{4}{|l|}{ Inflate } & & & \\
\hline & $\log (A A D T)$ & -0.709 & 0.408 & 0.083 & & & \\
\hline & $\log (L)$ & -0.873 & 0.369 & 0.018 & & & \\
\hline & Constant & 9.769 & 3.222 & 0.002 & & & \\
\hline \multirow{9}{*}{ ZIP1-9 } & $\log (A A D T)$ & 0.533 & 0.134 & $<0.001$ & \multirow{9}{*}{$\begin{array}{c}2.11 \\
(p \text {-value } \\
=0.017)\end{array}$} & \multirow{9}{*}{1928.96} & \multirow{9}{*}{1969.41} \\
\hline & $\log (L)$ & 0.600 & 0.138 & $<0.001$ & & & \\
\hline & $R$ & -0.001 & $<0.001$ & 0.004 & & & \\
\hline & $\beta_{S D}$ & -0.060 & 0.028 & 0.030 & & & \\
\hline & Constant & -7.437 & 1.406 & $<0.001$ & & & \\
\hline & \multicolumn{4}{|l|}{ Inflate } & & & \\
\hline & $\log (A A D T)$ & -0.674 & 0.385 & 0.080 & & & \\
\hline & $\log (L)$ & -0.902 & 0.361 & 0.013 & & & \\
\hline & Constant & 9.636 & 3.153 & 0.002 & & & \\
\hline \multirow{9}{*}{ ZIP1-10 } & $\log (A A D T)$ & 0.506 & 0.133 & $<0.001$ & \multirow{9}{*}{$\begin{array}{c}2.14 \\
(p \text {-value } \\
=0.016)\end{array}$} & \multirow{9}{*}{1929.68} & \multirow{9}{*}{1970.13} \\
\hline & $\log (L)$ & 0.615 & 0.144 & $<0.001$ & & & \\
\hline & $R$ & -0.001 & $<0.001$ & 0.002 & & & \\
\hline & $\beta_{\text {Roll }}$ & -0.117 & 0.057 & 0.042 & & & \\
\hline & Constant & -6.766 & 1.415 & $<0.001$ & & & \\
\hline & \multicolumn{4}{|l|}{ Inflate } & & & \\
\hline & $\log (A A D T)$ & -0.781 & 0.414 & 0.059 & & & \\
\hline & $\log (L)$ & -0.893 & 0.387 & 0.021 & & & \\
\hline & Constant & 10.453 & 3.140 & 0.001 & & & \\
\hline
\end{tabular}


ZINB models were developed as well considering reliability indices and exposure variables (AADT and $L$ ) as shown in Table 7-7. The Vuong statistic was between \pm 1.96 , which means that there is no preference for the ZINB over the base model (NB). The AIC and BIC of the ZINB models are lower than those of ZIP models. Thus, the ZINB models would fit the data better than the ZIP models in terms of AIC and BIC.

A comparison was conducted between the ZIP and ZINB based on Vuong test. The Vuong statistic was calculated using Equation 7.1 and 7.2. In this case, the Vuong test is a comparison of the predicted fit values of ZINB and ZIP models, assessing if there is a significant difference between the two models (Hilbe 2011).

$$
V=\frac{\sqrt{n} \bar{u}}{S D\left(u_{i}\right)}
$$

And,

$$
u_{i}=\ln \left(\frac{\sum_{i} P_{Z I P}\left(y_{i} \mid x_{i}\right)}{\sum_{i} P_{Z I N B}\left(y_{i} \mid x_{i}\right)}\right)
$$

Where $V=$ Vuong statistic, $n=$ number of observations, $u=$ the $\log$ ratio of the sum of probabilities, $\bar{u}$ and $S D=$ mean and standard deviation of $u$, and $P_{Z I P}\left(y_{i} \mid x_{i}\right)$ and $P_{Z I N B}\left(y_{i} \mid x_{i}\right)=$ the probability of observing $y$ on the basis of $x$ in ZIP and ZINB models.

The Vuong statistic for the base models ZIP1-1 and ZINB1-1 was -0.287 ( $p$ value $=0.613$ ), which means that neither model is preferable over the other. While the Vuong statistic for ZIP1-2 and ZINB1-2 was 3.297 ( $p$-value $<0.001$ ), which means that ZIP1-2 is preferred over the ZINB1-2. Also, a comparison using Vuong test was conducted between the ZIP and ZINB models shown in Table 7-6 and Table 7-7. The Vuong statistic for all models was between \pm 1.96 , which means that there is no preference of each model over the other. 
Table 7-7: ZINB Models to Predict Annual Collision Frequency.

\begin{tabular}{|c|c|c|c|c|c|c|c|}
\hline Model & Variable & Coef. & $S E E$ & $\begin{array}{c}p- \\
\text { value }\end{array}$ & $\begin{array}{c}\text { Vuong } \\
\text { statistic }\end{array}$ & AIC & BIC \\
\hline \multirow{8}{*}{ ZINB3 } & $\log (A A D T)$ & 0.718 & 0.103 & $<0.001$ & \multirow{8}{*}{$\begin{array}{c}1.36 \\
(p \text {-value } \\
=0.087)\end{array}$} & \multirow{8}{*}{1918.05} & \multirow{8}{*}{1958.50} \\
\hline & $\log (L)$ & 0.946 & 0.099 & $<0.001$ & & & \\
\hline & $\beta_{V S}$ & -0.238 & 0.059 & $<0.001$ & & & \\
\hline & Constant & -10.785 & 1.103 & $<0.001$ & & & \\
\hline & \multicolumn{4}{|l|}{ Inflate } & & & \\
\hline & $\log (A A D T)$ & -4.102 & 1.615 & 0.011 & & & \\
\hline & $\log (L)$ & 3.300 & 2.082 & 0.113 & & & \\
\hline & Constant & 10.504 & 7.837 & 0.180 & & & \\
\hline \multirow{8}{*}{ ZINB4 } & $\log (A A D T)$ & 0.806 & 0.088 & $<0.001$ & \multirow{8}{*}{$\begin{array}{c}1.22 \\
(p \text {-value } \\
=0.112)\end{array}$} & \multirow{8}{*}{1933.88} & \multirow{8}{*}{1974.33} \\
\hline & $\log (L)$ & 0.834 & 0.102 & $<0.001$ & & & \\
\hline & $\beta_{c m f}$ & 0.003 & 0.038 & 0.929 & & & \\
\hline & Constant & -12.004 & 0.950 & $<0.001$ & & & \\
\hline & \multicolumn{4}{|l|}{ Inflate } & & & \\
\hline & $\log (A A D T)$ & -4.294 & 1.761 & 0.015 & & & \\
\hline & $\log (L)$ & 4.344 & 2.149 & 0.043 & & & \\
\hline & Constant & 5.145 & 10.270 & 0.616 & & & \\
\hline \multirow{8}{*}{ ZINB5 } & $\log (A A D T)$ & 0.769 & 0.087 & $<0.001$ & \multirow{8}{*}{$\begin{array}{c}1.14 \\
(p \text {-value } \\
=0.128)\end{array}$} & \multirow{8}{*}{1922.96} & \multirow{8}{*}{1963.41} \\
\hline & $\log (L)$ & 0.887 & 0.095 & $<0.001$ & & & \\
\hline & $\beta_{S D}$ & -0.086 & 0.026 & 0.001 & & & \\
\hline & Constant & -11.452 & 0.927 & $<0.001$ & & & \\
\hline & \multicolumn{4}{|l|}{ Inflate } & & & \\
\hline & $\log (A A D T)$ & -4.342 & 1.836 & 0.018 & & & \\
\hline & $\log (L)$ & 4.364 & 2.206 & 0.048 & & & \\
\hline & Constant & 5.310 & 10.795 & 0.623 & & & \\
\hline \multirow{8}{*}{ ZINB6 } & $\log (A A D T)$ & 0.754 & 0.093 & $<0.001$ & \multirow{8}{*}{$\begin{array}{c}1.27 \\
(p \text {-value } \\
=0.103)\end{array}$} & \multirow{8}{*}{1920.28} & \multirow{8}{*}{1960.73} \\
\hline & $\log (L)$ & 0.911 & 0.097 & $<0.001$ & & & \\
\hline & $\beta_{\text {Roll }}$ & -0.215 & 0.057 & $<0.001$ & & & \\
\hline & Constant & -10.328 & 1.058 & $<0.001$ & & & \\
\hline & \multicolumn{4}{|l|}{ Inflate } & & & \\
\hline & $\log (A A D T)$ & -4.435 & 1.675 & 0.008 & & & \\
\hline & $\log (L)$ & 3.871 & 2.040 & 0.058 & & & \\
\hline & Constant & 9.376 & 8.294 & 0.258 & & & \\
\hline
\end{tabular}


Table 7-7: ZINB Models to Predict Annual Collision Frequency.- Cont.

\begin{tabular}{|c|c|c|c|c|c|c|c|}
\hline Model & Variable & Coef. & SEE & $\begin{array}{c}p- \\
\text { value }\end{array}$ & $\begin{array}{l}\text { Vuong } \\
\text { statistic }\end{array}$ & AIC & BIC \\
\hline \multirow{9}{*}{ ZINB7 } & $\log (A A D T)$ & 0.578 & 0.112 & $<0.001$ & \multirow{9}{*}{$\begin{array}{c}1.30 \\
(p \text {-value } \\
=0.097)\end{array}$} & \multirow{9}{*}{1911.75} & \multirow{9}{*}{1967.25} \\
\hline & $\log (L)$ & 0.892 & 0.107 & $<0.001$ & & & \\
\hline & $R$ & -0.001 & $<0.001$ & 0.002 & & & \\
\hline & $\beta_{V S}$ & -0.160 & 0.065 & 0.014 & & & \\
\hline & Constant & -9.330 & 1.264 & $<0.001$ & & & \\
\hline & \multicolumn{4}{|l|}{ Inflate } & & & \\
\hline & $\log (A A D T)$ & -3.433 & 1.281 & 0.007 & & & \\
\hline & $\log (L)$ & 0.122 & 1.040 & 0.907 & & & \\
\hline & Constant & 23.643 & 8.677 & 0.006 & & & \\
\hline \multirow{9}{*}{ ZINB8 } & $\log (A A D T)$ & 0.591 & 0.114 & $<0.001$ & \multirow{9}{*}{$\begin{array}{c}1.09 \\
(p \text {-value } \\
=0.137)\end{array}$} & \multirow{9}{*}{1917.09} & \multirow{9}{*}{1962.59} \\
\hline & $\log (L)$ & 0.837 & 0.111 & $<0.001$ & & & \\
\hline & $R$ & -0.001 & 0.000 & $<0.001$ & & & \\
\hline & $\beta_{c m f}$ & 0.029 & 0.038 & 0.455 & & & \\
\hline & Constant & -9.795 & 1.289 & $<0.001$ & & & \\
\hline & \multicolumn{4}{|l|}{ Inflate } & & & \\
\hline & $\log (A A D T)$ & -3.346 & 1.302 & 0.010 & & & \\
\hline & $\log (L)$ & 0.108 & 1.040 & 0.918 & & & \\
\hline & Constant & 22.979 & 8.436 & 0.006 & & & \\
\hline \multirow{9}{*}{ ZINB9 } & $\log (A A D T)$ & 0.597 & 0.114 & $<0.001$ & \multirow{9}{*}{$\begin{array}{c}1.08 \\
(p \text {-value } \\
=0.140)\end{array}$} & \multirow{9}{*}{1915.01} & \multirow{9}{*}{1960.52} \\
\hline & $\log (L)$ & 0.858 & 0.108 & $<0.001$ & & & \\
\hline & $R$ & -0.001 & $<0.001$ & 0.001 & & & \\
\hline & $\beta_{S D}$ & -0.047 & 0.029 & 0.104 & & & \\
\hline & Constant & -9.702 & 1.299 & $<0.001$ & & & \\
\hline & \multicolumn{4}{|l|}{ Inflate } & & & \\
\hline & $\log (A A D T)$ & -3.208 & 1.306 & 0.014 & & & \\
\hline & $\log (L)$ & -0.021 & 1.041 & 0.984 & & & \\
\hline & Constant & 22.651 & 8.549 & 0.008 & & & \\
\hline \multirow{9}{*}{ ZINB10 } & $\log (A A D T)$ & 0.595 & 0.112 & $<0.001$ & \multirow{9}{*}{$\begin{array}{c}1.20 \\
(p \text {-value } \\
=0.115)\end{array}$} & \multirow{9}{*}{1913.22} & \multirow{9}{*}{1958.73} \\
\hline & $\log (L)$ & 0.871 & 0.107 & $<0.001$ & & & \\
\hline & $R$ & -0.001 & $<0.001$ & 0.001 & & & \\
\hline & $\beta_{\text {Roll }}$ & -0.136 & 0.064 & 0.033 & & & \\
\hline & Constant & -9.024 & 1.325 & $<0.001$ & & & \\
\hline & \multicolumn{4}{|l|}{ Inflate } & & & \\
\hline & $\log (A A D T)$ & -3.418 & 1.280 & 0.008 & & & \\
\hline & $\log (L)$ & 0.073 & 1.034 & 0.943 & & & \\
\hline & Constant & 23.735 & 8.625 & 0.006 & & & \\
\hline
\end{tabular}


Based on previous results, the NB and ZINB have approximately same AIC and BIC values without preference for one model over the other. In addition, the AIC and BIC values of the ZINB models were less than those of the ZIP models and the Vuong test did not show any preference for the ZIP over the ZINB. Therefore, the ZINB models shown in Table 7-7 were used in the coming analysis.

\subsubsection{SPFs of 5-Year Collisions}

The descriptive statistics for the five-year collision data show that the mean and variance for the total collisions are 2.353 and 9.597 , respectively. The Pearson statistic is $2.300($ Deviance $=2.104)$ when conducting the Poisson generalized linear modelling (base model), which means that some $130 \%$ extra dispersion exists in the data. Thus, the data distribution of total collisions is displaying obvious signs of over-dispersion. The Pearson statistic dropped to 1.058 (Deviance $=1.037)$ when conducting NB regression. Table 7-8 shows the base models of the SPF for Poisson and NB regressions.

Table 7-8: SPFs for Five-Year Collisions Frequency (Base Models).

\begin{tabular}{|c|c|c|c|c|c|c|c|}
\hline $\begin{array}{l}\text { Regression } \\
\text { Model }\end{array}$ & Variable & Coef. & $S E E$ & $\begin{array}{c}p- \\
\text { value }\end{array}$ & $\begin{array}{l}\text { Likelihood- } \\
\text { ratio test }\end{array}$ & AIC & BIC \\
\hline \multirow{3}{*}{ Poisson5-1 } & $\log$ of $(A A D T)$ & 0.837 & 0.065 & $<0.001$ & \multirow{3}{*}{--} & \multirow{3}{*}{940.32} & \multirow{3}{*}{950.66} \\
\hline & $\log$ of $(L)$ & 0.738 & 0.075 & $<0.001$ & & & \\
\hline & Constant & -10.165 & 0.740 & $<0.001$ & & & \\
\hline \multirow{3}{*}{ NB5-1 } & $\log$ of $(A A D T)$ & 0.904 & 0.105 & $<0.001$ & \multirow{3}{*}{$\begin{array}{l}101.46 \\
(p \text {-value } \\
<0.001)\end{array}$} & \multirow{3}{*}{840.86} & \multirow{3}{*}{854.64} \\
\hline & $\log$ of $(L)$ & 0.774 & 0.115 & $<0.001$ & & & \\
\hline & Constant & -10.923 & 1.087 & $<0.001$ & & & \\
\hline \multirow{4}{*}{ Poisson5-2 } & $\log$ of $(A A D T)$ & 0.067 & 10.900 & $<0.001$ & \multirow{4}{*}{--} & \multirow{4}{*}{917.08} & \multirow{4}{*}{930.86} \\
\hline & $\log$ of $(L)$ & 0.893 & 0.083 & $<0.001$ & & & \\
\hline & $R$ & -0.0008 & $<0.001$ & $<0.001$ & & & \\
\hline & Constant & -9.766 & 0.738 & $<0.001$ & & & \\
\hline \multirow{4}{*}{ NB5-2 } & $\log$ of $(A A D T)$ & 0.812 & 0.104 & $<0.001$ & \multirow{4}{*}{$\begin{array}{c}88.06 \\
(p \text {-value } \\
<0.001)\end{array}$} & \multirow{4}{*}{831.01} & \multirow{4}{*}{848.5} \\
\hline & $\log$ of $(L)$ & 0.930 & 0.123 & $<0.001$ & & & \\
\hline & $R$ & -0.0008 & $<0.001$ & $<0.001$ & & & \\
\hline & Constant & -10.609 & 1.066 & $<0.001$ & & & \\
\hline
\end{tabular}


Figure 7.2 shows that NB better fits the data. As shown in the figure, there is an obvious difference between Poisson model prediction and the observed collisions. Accordingly, NB provided more accurate prediction, which confirms the previous results.

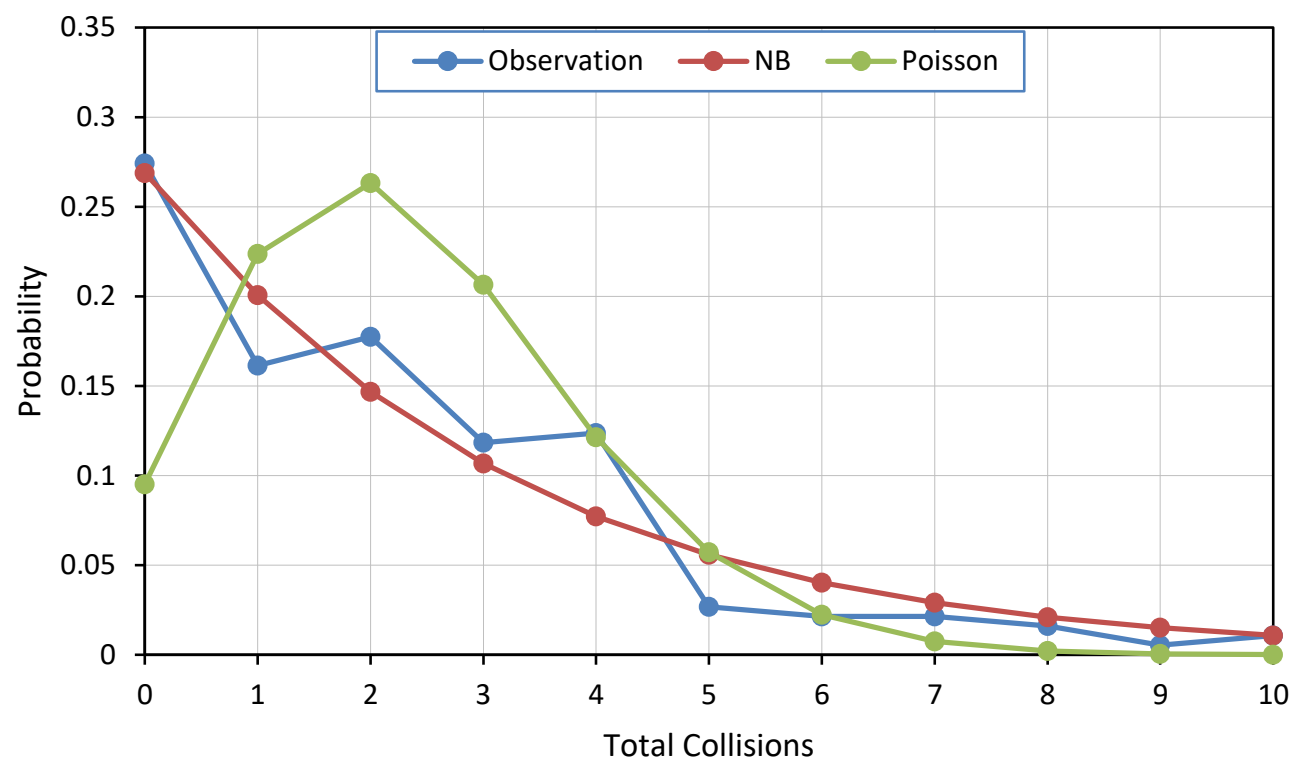

Figure 7.2: Observed Collisions (five-year) Compared with Poisson and NB Distributions.

Considering the reliability indices, exposure variables (AADT and $L$ ), and the radius of the curve $(R)$, Table 7-10 shows the outputs of the NB regression. All the reliability indices except for driver comfort index were significant at $5 \%$ level of significance. The AIC and BIC values of the first set of models in the table (without $R$ ) were less than those of the base model (NB5-1). Thus, it could be concluded that considering the reliability index to the model would add more value to the model and the model in this case better fits the data than the base models. On the other hand, the AIC and BIC of the second set of the models (with $R$ ) were slightly greater than the base model (NB5-2). Thus, considering reliability indices in this case did not add value to the model. Note that the reliability indices in the second set of the models were insignificant. 
Table 7-9: NB Models to Predict Five-Year Collisions Frequency.

\begin{tabular}{|c|c|c|c|c|c|c|c|}
\hline $\begin{array}{l}\text { Regression } \\
\text { Model }\end{array}$ & Variable & Coef. & SEE & $\begin{array}{c}p- \\
\text { value }\end{array}$ & $\begin{array}{l}\text { Likelihood- } \\
\text { ratio test }\end{array}$ & AIC & $\mathrm{BIC}$ \\
\hline \multirow{4}{*}{ NB5-3 } & Log of (AADT) & 0.857 & 0.104 & $<0.001$ & \multirow{4}{*}{$\begin{array}{c}94.24 \\
(p \text {-value } \\
<0.001)\end{array}$} & \multirow{4}{*}{836.45} & \multirow{4}{*}{853.68} \\
\hline & Log of $(L)$ & 0.886 & 0.123 & $<0.001$ & & & \\
\hline & $\beta_{V S}$ & -0.193 & 0.075 & 0.010 & & & \\
\hline & Constant & -10.295 & 1.094 & $<0.001$ & & & \\
\hline \multirow{4}{*}{ NB5-4 } & Log of (AADT) & 0.903 & 0.105 & $<0.001$ & \multirow{4}{*}{$\begin{array}{l}101.50 \\
(p \text {-value } \\
<0.001)\end{array}$} & \multirow{4}{*}{842.81} & \multirow{4}{*}{860.05} \\
\hline & Log of $(L)$ & 0.761 & 0.130 & $<0.001$ & & & \\
\hline & $\beta_{c m f}$ & 0.011 & 0.052 & 0.835 & & & \\
\hline & Constant & -10.864 & 1.123 & $<0.001$ & & & \\
\hline \multirow{4}{*}{ NB5-5 } & Log of (AADT) & 0.860 & 0.104 & $<0.001$ & \multirow{4}{*}{$\begin{array}{l}92.10 \\
(p \text {-value } \\
<0.001)\end{array}$} & \multirow{4}{*}{837.55} & \multirow{4}{*}{854.78} \\
\hline & Log of $(L)$ & 0.830 & 0.116 & $<0.001$ & & & \\
\hline & $\beta_{S D}$ & -0.080 & 0.035 & 0.020 & & & \\
\hline & Constant & -10.366 & 1.091 & $<0.001$ & & & \\
\hline \multirow{4}{*}{ NB5-6 } & Log of (AADT) & 0.893 & 0.103 & $<0.001$ & \multirow{4}{*}{$\begin{array}{l}93.56 \\
(p \text {-value } \\
<0.001)\end{array}$} & \multirow{4}{*}{834.50} & \multirow{4}{*}{851.73} \\
\hline & Log of $(L)$ & 0.854 & 0.118 & $<0.001$ & & & \\
\hline & $\beta_{\text {Roll }}$ & -0.216 & 0.074 & 0.004 & & & \\
\hline & Constant & -9.606 & 1.148 & $<0.001$ & & & \\
\hline \multirow{5}{*}{ NB5-7 } & Log of (AADT) & 0.807 & 0.104 & $<0.001$ & \multirow{5}{*}{$\begin{array}{c}87.19 \\
(p \text {-value } \\
<0.001)\end{array}$} & \multirow{5}{*}{831.91} & \multirow{5}{*}{852.59} \\
\hline & $\log$ of $(L)$ & 0.956 & 0.125 & $<0.001$ & & & \\
\hline & $R$ & -0.001 & $<0.001$ & 0.011 & & & \\
\hline & $\beta_{V S}$ & -0.089 & 0.084 & 0.292 & & & \\
\hline & Constant & -10.377 & 1.083 & $<0.001$ & & & \\
\hline \multirow{5}{*}{ NB5-8 } & Log of (AADT) & 0.807 & 0.105 & $<0.001$ & \multirow{5}{*}{$\begin{array}{c}87.92 \\
(p \text {-value } \\
<0.001)\end{array}$} & \multirow{5}{*}{832.51} & \multirow{5}{*}{853.19} \\
\hline & $\log$ of $(L)$ & 0.893 & 0.133 & $<0.001$ & & & \\
\hline & $R$ & -0.001 & $<0.001$ & $<0.001$ & & & \\
\hline & $\beta_{c m f}$ & 0.037 & 0.051 & 0.476 & & & \\
\hline & Constant & -10.410 & 1.103 & $<0.001$ & & & \\
\hline \multirow{5}{*}{ NB5-9 } & Log of (AADT) & 0.806 & 0.104 & $<0.001$ & \multirow{5}{*}{$\begin{array}{c}85.62 \\
(p \text {-value } \\
<0.001)\end{array}$} & \multirow{5}{*}{832.21} & \\
\hline & Log of $(L)$ & 0.933 & 0.122 & $<0.001$ & & & \\
\hline & $R$ & -0.001 & $<0.001$ & 0.007 & & & 852.89 \\
\hline & $\beta_{S D}$ & -0.034 & 0.038 & 0.370 & & & \\
\hline & Constant & -10.413 & 1.082 & $<0.001$ & & & \\
\hline & Log of (AADT) & 0.827 & 0.104 & $<0.001$ & & & \\
\hline & $\log$ of $(L)$ & 0.942 & 0.123 & $<0.001$ & & & \\
\hline NB5-10 & $R$ & -0.001 & $<0.001$ & 0.016 & ( $p$-value & 830.66 & 851.34 \\
\hline & $\beta_{\text {Roll }}$ & -0.126 & 0.082 & 0.124 & $<0.001)$ & & \\
\hline & Constant & -9.923 & 1.144 & $<0.001$ & & & \\
\hline
\end{tabular}

$\beta_{V S}, \beta_{c m f}, \beta_{S D}, \beta_{R o l l}=$ reliability indices for vehicle stability, driver comfort, sight distance, and rollover, respectively, $\mathrm{AADT}=$ average annual daily traffic (veh/d), and $R=$ radius (m). 
When considering all the reliability indices together in one model, all these indices were insignificant at 5\% level of significance as shown in Table 7-10.

Table 7-10: SPFs for Five-Year Collisions Frequency Considering All Reliability Indices.

\begin{tabular}{|c|c|c|c|c|c|c|c|}
\hline $\begin{array}{l}\text { Regression } \\
\text { Model }\end{array}$ & Variable & $\begin{array}{c}\text { Coefficie } \\
n t\end{array}$ & $\begin{array}{l}\text { Standard } \\
\text { Error }\end{array}$ & $p$-value & $\begin{array}{l}\text { Likelihood- } \\
\text { ratio test }\end{array}$ & AIC & $\mathrm{BIC}$ \\
\hline \multirow{7}{*}{ NB5-11 } & Log of (AADT) & 0.866 & 0.108 & $<0.001$ & \multirow{7}{*}{$\begin{array}{c}89.33 \\
(p \text {-value } \\
<0.001)\end{array}$} & \multirow{7}{*}{839.29} & \multirow{7}{*}{866.86} \\
\hline & Log of $(L)$ & 0.849 & 0.132 & $<0.001$ & & & \\
\hline & $\beta_{V S}$ & -0.019 & 0.146 & 0.894 & & & \\
\hline & $\beta_{c m f}$ & 0.020 & 0.054 & 0.710 & & & \\
\hline & $\beta_{S D}$ & -0.042 & 0.040 & 0.299 & & & \\
\hline & $\beta_{\text {Roll }}$ & -0.156 & 0.142 & 0.272 & & & \\
\hline & Constant & -9.515 & 1.187 & $<0.001$ & & & \\
\hline \multirow{8}{*}{ NB5-12 } & Log of (AADT) & 0.820 & 0.108 & $<0.001$ & \multirow{8}{*}{$\begin{array}{c}84.69 \\
(p \text {-value } \\
<0.001)\end{array}$} & \multirow{8}{*}{836.10} & \multirow{8}{*}{867.12} \\
\hline & Log of $(L)$ & 0.908 & 0.134 & $<0.001$ & & & \\
\hline & $R$ & -0.001 & 0.000 & 0.024 & & & \\
\hline & $\beta_{V S}$ & 0.020 & 0.145 & 0.892 & & & \\
\hline & $\beta_{c m f}$ & 0.031 & 0.054 & 0.570 & & & \\
\hline & $\beta_{S D}$ & -0.018 & 0.041 & 0.661 & & & \\
\hline & $\beta_{\text {Roll }}$ & -0.123 & 0.141 & 0.381 & & & \\
\hline & Constant & -9.720 & 1.181 & $<0.001$ & & & \\
\hline
\end{tabular}

The ZIP and ZINB regression were used to develop base models as shown in Table 7-11. The Vuong statistic for the ZIP model was greater than +1.96 , which means that the ZIP models better fit the data than the Poisson models. Also, considering the radius of curve in the regression analysis with the exposure variables would improve the models and add more value to the models based on the AIC and BIC. On the other hand, the Vuong statistic for the ZINB models was not significant at 5\% level of significance and the value was between \pm 1.96 , which means that there is no preference deducted between the base model (NB) and the zero-inflated model (ZINB). By comparing the AIC and BIC of the NB and ZINB base models in Table 7-8 and Table 7-11, the NB base models should be preferred over the ZINB models. 
Table 7-11: ZIP and ZINB Base Models to Predict Five-Year Collision Frequency.

\begin{tabular}{|c|c|c|c|c|c|c|c|}
\hline Model & Variable & Coef. & SEE & $\begin{array}{c}p- \\
\text { value }\end{array}$ & $\begin{array}{c}\text { Vuong } \\
\text { test }\end{array}$ & AIC & BIC \\
\hline \multirow{7}{*}{ ZIP5-1 } & $\log (A A D T)$ & 0.745 & 0.083 & $<0.001$ & \multirow{7}{*}{$\begin{array}{c}2.15 \\
(p- \\
\text { value } \\
=0.016)\end{array}$} & \multirow{7}{*}{913.85} & \multirow{7}{*}{934.53} \\
\hline & $\log (L)$ & 0.651 & 0.085 & $<0.001$ & & & \\
\hline & Constant & -8.756 & 0.972 & $<0.001$ & & & \\
\hline & \multicolumn{4}{|l|}{ Inflate } & & & \\
\hline & $\log (A A D T)$ & -0.813 & 0.424 & 0.055 & & & \\
\hline & $\log (L)$ & -0.376 & 0.388 & 0.333 & & & \\
\hline & Constant & 6.979 & 4.033 & 0.084 & & & \\
\hline \multirow{8}{*}{ ZIP5-2 } & $\log (A A D T)$ & 0.626 & 0.087 & $<0.001$ & \multirow{8}{*}{$\begin{array}{c}2.04 \\
(p- \\
\text { value } \\
=0.021)\end{array}$} & \multirow{8}{*}{897.36} & \multirow{8}{*}{921.49} \\
\hline & $\log (L)$ & 0.769 & 0.091 & $<0.001$ & & & \\
\hline & $R$ & -0.0008 & 0.000 & $<0.001$ & & & \\
\hline & Constant & -8.038 & 0.970 & $<0.001$ & & & \\
\hline & \multicolumn{4}{|l|}{ Inflate } & & & \\
\hline & $\log (A A D T)$ & -1.001 & 0.433 & 0.021 & & & \\
\hline & $\log (L)$ & -0.435 & 0.404 & 0.282 & & & \\
\hline & Constant & 8.780 & 3.893 & 0.024 & & & \\
\hline \multirow{7}{*}{ ZINB5-1 } & $\log (A A D T)$ & 0.859 & 0.110 & $<0.001$ & \multirow{7}{*}{$\begin{array}{c}0.68 \\
(p- \\
\text { value } \\
=0.247)\end{array}$} & \multirow{7}{*}{845.066} & \multirow{7}{*}{869.19} \\
\hline & $\log (L)$ & 0.763 & 0.114 & $<0.001$ & & & \\
\hline & Constant & -10.477 & 1.134 & $<0.001$ & & & \\
\hline & \multicolumn{4}{|l|}{ Inflate } & & & \\
\hline & $\log (A A D T)$ & -16.113 & 15.018 & 0.283 & & & \\
\hline & $\log (L)$ & 0.528 & 2.228 & 0.813 & & & \\
\hline & Constant & 108.025 & 104.575 & 0.302 & & & \\
\hline \multirow{8}{*}{ ZINB5-2 } & $\log (A A D T)$ & 0.753 & 0.109 & $<0.001$ & \multirow{8}{*}{$\begin{array}{c}0.86 \\
(p- \\
\text { value } \\
=0.137)\end{array}$} & \multirow{8}{*}{834.14} & \multirow{8}{*}{861.72} \\
\hline & $\log (L)$ & 0.923 & 0.122 & $<0.001$ & & & \\
\hline & $R$ & -0.0009 & $<0.001$ & $<0.001$ & & & \\
\hline & Constant & -10.040 & 1.112 & $<0.001$ & & & \\
\hline & \multicolumn{4}{|l|}{ Inflate } & & & \\
\hline & $\log (A A D T)$ & -16.191 & 14.244 & 0.256 & & & \\
\hline & $\log (L)$ & 0.665 & 1.935 & 0.731 & & & \\
\hline & Constant & 108.107 & 98.476 & 0.272 & & & \\
\hline
\end{tabular}

Using the ZIP regression considering the reliability indices with the AADT and $L$ improves the models based on the AIC and AIC values as shown in Table 7-12. All the reliability indices were significant at 5\% level of significance except for the driver comfort index. The Vuong statistic was greater than +1.96 and significant at $5 \%$ level of 
significance, which means that the ZIP is preferred over the base model (Poisson).

Table 7-12: ZIP Models to Predict Five-Year Collision Frequency.

\begin{tabular}{|c|c|c|c|c|c|c|c|}
\hline Model & Variable & Coef. & $S E E$ & $\begin{array}{c}p- \\
\text { value }\end{array}$ & $\begin{array}{c}\text { Vuong } \\
\text { test }\end{array}$ & AIC & BIC \\
\hline \multirow{8}{*}{ ZIP5-3 } & $\log (A A D T)$ & 0.655 & 0.083 & $<0.001$ & \multirow{8}{*}{$\begin{array}{c}2.21 \\
(p- \\
\text { value } \\
=0.014)\end{array}$} & \multirow{8}{*}{902.02} & \multirow{8}{*}{926.15} \\
\hline & $\log (L)$ & 0.724 & 0.086 & $<0.001$ & & & \\
\hline & $\beta_{V S}$ & -0.206 & 0.054 & $<0.001$ & & & \\
\hline & Constant & -7.478 & 0.975 & $<0.001$ & & & \\
\hline & \multicolumn{4}{|l|}{ Inflate } & & & \\
\hline & $\log (A A D T)$ & -0.952 & 0.395 & 0.016 & & & \\
\hline & $\log (L)$ & -0.441 & 0.377 & 0.243 & & & \\
\hline & Constant & 8.499 & 3.613 & 0.019 & & & \\
\hline \multirow{8}{*}{ ZIP5-4 } & $\log (A A D T)$ & 0.744 & 0.083 & $<0.001$ & \multirow{8}{*}{$\begin{array}{c}2.17 \\
(p- \\
\text { value } \\
=0.015)\end{array}$} & \multirow{8}{*}{915.80} & \multirow{8}{*}{939.92} \\
\hline & $\log (L)$ & 0.645 & 0.089 & $<0.001$ & & & \\
\hline & $\beta_{c m f}$ & 0.008 & 0.034 & 0.817 & & & \\
\hline & Constant & -8.734 & 0.978 & $<0.001$ & & & \\
\hline & \multicolumn{4}{|l|}{ Inflate } & & & \\
\hline & $\log (A A D T)$ & -0.806 & 0.427 & 0.059 & & & \\
\hline & $\log (L)$ & -0.372 & 0.389 & 0.339 & & & \\
\hline & Constant & 6.901 & 4.071 & 0.090 & & & \\
\hline \multirow{8}{*}{ ZIP5-5 } & $\log (A A D T)$ & 0.696 & 0.086 & $<0.001$ & \multirow{8}{*}{$\begin{array}{c}2.11 \\
(p- \\
\text { value } \\
=0.017)\end{array}$} & \multirow{8}{*}{903.01} & \multirow{8}{*}{927.14} \\
\hline & $\log (L)$ & 0.695 & 0.086 & $<0.001$ & & & \\
\hline & $\beta_{S D}$ & -0.086 & 0.024 & $<0.001$ & & & \\
\hline & Constant & -8.062 & 1.000 & $<0.001$ & & & \\
\hline & \multicolumn{4}{|l|}{ Inflate } & & & \\
\hline & $\log (A A D T)$ & -0.916 & 0.423 & 0.030 & & & \\
\hline & $\log (L)$ & -0.397 & 0.394 & 0.313 & & & \\
\hline & Constant & 7.914 & 3.915 & 0.043 & & & \\
\hline \multirow{8}{*}{ ZIP5-6 } & $\log (A A D T)$ & 0.690 & 0.083 & $<0.001$ & \multirow{8}{*}{$\begin{array}{c}2.17 \\
(p- \\
\text { value } \\
=0.015)\end{array}$} & \multirow{8}{*}{901.01} & \multirow{8}{*}{925.13} \\
\hline & $\log (L)$ & 0.725 & 0.086 & $<0.001$ & & & \\
\hline & $\beta_{\text {Roll }}$ & -0.197 & 0.049 & $<0.001$ & & & \\
\hline & Constant & -7.192 & 1.021 & $<0.001$ & & & \\
\hline & \multicolumn{4}{|l|}{ Inflate } & & & \\
\hline & $\log (A A D T)$ & -0.947 & 0.419 & 0.024 & & & \\
\hline & $\log (L)$ & -0.341 & 0.392 & $\mathbf{0 . 3 8 4}$ & & & \\
\hline & Constant & 7.868 & 3.840 & 0.040 & & & \\
\hline
\end{tabular}


Table 7-12: ZIP Models to Predict Five-Year Collision Frequency.- Cont.

\begin{tabular}{|c|c|c|c|c|c|c|c|}
\hline Model & Variable & Coef. & $S E E$ & $\begin{array}{c}p- \\
\text { value }\end{array}$ & $\begin{array}{c}\text { Vuong } \\
\text { test }\end{array}$ & AIC & $\mathrm{BIC}$ \\
\hline \multirow{9}{*}{ ZIP5-7 } & $\log (A A D T)$ & 0.602 & 0.086 & $<0.001$ & \multirow{9}{*}{$\begin{array}{c}2.03 \\
(p- \\
\text { value } \\
=0.021)\end{array}$} & \multirow{9}{*}{895.75} & \multirow{9}{*}{923.32} \\
\hline & $\log (L)$ & 0.786 & 0.090 & $<0.001$ & & & \\
\hline & $R$ & -0.001 & $<0.001$ & 0.006 & & & \\
\hline & $\beta_{V S}$ & -0.120 & 0.062 & 0.054 & & & \\
\hline & Constant & -7.477 & 0.980 & $<0.001$ & & & \\
\hline & \multicolumn{4}{|l|}{ Inflate } & & & \\
\hline & $\log (A A D T)$ & -1.047 & 0.416 & 0.012 & & & \\
\hline & $\log (L)$ & -0.444 & 0.400 & 0.268 & & & \\
\hline & Constant & 9.233 & 3.708 & 0.013 & & & \\
\hline \multirow{9}{*}{ ZIP5-4 } & $\log (A A D T)$ & 0.620 & 0.088 & $<0.001$ & \multirow{9}{*}{$\begin{array}{c}2.03 \\
(p- \\
\text { value } \\
=0.021)\end{array}$} & \multirow{9}{*}{898.70} & \multirow{9}{*}{926.28} \\
\hline & $\log (L)$ & 0.750 & 0.094 & $<0.001$ & & & \\
\hline & $R$ & -0.001 & $<0.001$ & $<0.001$ & & & \\
\hline & $\beta_{c m f}$ & 0.028 & 0.035 & 0.415 & & & \\
\hline & Constant & -7.924 & 0.982 & $<0.001$ & & & \\
\hline & \multicolumn{4}{|l|}{ Inflate } & & & \\
\hline & $\log (A A D T)$ & -0.978 & 0.433 & 0.024 & & & \\
\hline & $\log (L)$ & -0.433 & 0.406 & 0.286 & & & \\
\hline & Constant & 8.577 & 3.933 & 0.029 & & & \\
\hline \multirow{9}{*}{ ZIP5-5 } & $\log (A A D T)$ & 0.622 & 0.088 & $<0.001$ & \multirow{9}{*}{$\begin{array}{c}2.03 \\
(p- \\
\text { value } \\
=0.021)\end{array}$} & \multirow{9}{*}{895.79} & \multirow{9}{*}{923.37} \\
\hline & $\log (L)$ & 0.768 & 0.090 & $<0.001$ & & & \\
\hline & $R$ & -0.001 & $<0.001$ & 0.003 & & & \\
\hline & $\beta_{S D}$ & -0.050 & 0.027 & 0.059 & & & \\
\hline & Constant & -7.769 & 0.987 & $<0.001$ & & & \\
\hline & \multicolumn{4}{|l|}{ Inflate } & & & \\
\hline & $\log (A A D T)$ & -1.029 & 0.429 & 0.017 & & & \\
\hline & $\log (L)$ & -0.435 & 0.407 & 0.285 & & & \\
\hline & Constant & 9.007 & 3.856 & 0.019 & & & \\
\hline \multirow{9}{*}{ ZIP5-6 } & $\log (A A D T)$ & 0.619 & 0.086 & $<0.001$ & \multirow{9}{*}{$\begin{array}{c}2.17 \\
(p- \\
\text { value } \\
=0.015)\end{array}$} & \multirow{9}{*}{901.01} & \multirow{9}{*}{925.13} \\
\hline & $\log (L)$ & 0.792 & 0.091 & $<0.001$ & & & \\
\hline & $R$ & -0.001 & $<0.001$ & 0.005 & & & \\
\hline & $\beta_{\text {Roll }}$ & -0.129 & 0.055 & 0.020 & & & \\
\hline & Constant & -7.210 & 1.013 & $<0.001$ & & & \\
\hline & \multicolumn{4}{|l|}{ Inflate } & & & \\
\hline & $\log (A A D T)$ & -1.055 & 0.430 & 0.014 & & & \\
\hline & $\log (L)$ & -0.386 & 0.407 & 0.342 & & & \\
\hline & Constant & 8.953 & 3.815 & 0.019 & & & \\
\hline
\end{tabular}


The ZINB regression was used to develop SPFs considering reliability indices as shown in Table 7-13. The Vuong statistic for all ZINB models was between \pm 1.96 , which means that there is no preference for the ZINB over the base model (NB). However, the Vuong test was insignificant.

Table 7-13: ZINB Models to Predict Five-Year Collision Frequency.

\begin{tabular}{|c|c|c|c|c|c|c|c|}
\hline Model & Variable & Coef. & $S E E$ & $\begin{array}{c}p- \\
\text { value }\end{array}$ & $\begin{array}{c}\text { Vuong } \\
\text { test }\end{array}$ & AIC & BIC \\
\hline \multirow{8}{*}{ ZINB5-3 } & $\log (A A D T)$ & 0.789 & 0.110 & $<0.001$ & \multirow{8}{*}{$\begin{array}{c}0.98 \\
(p- \\
\text { value } \\
=0.163)\end{array}$} & \multirow{8}{*}{838.77} & \multirow{8}{*}{866.35} \\
\hline & $\log (L)$ & 0.891 & 0.122 & $<0.001$ & & & \\
\hline & $\beta_{V S}$ & -0.226 & 0.078 & 0.004 & & & \\
\hline & Constant & -9.584 & 1.146 & $<0.001$ & & & \\
\hline & \multicolumn{4}{|l|}{ Inflate } & & & \\
\hline & $\log (A A D T)$ & -18.892 & 16.008 & 0.238 & & & \\
\hline & $\log (L)$ & 0.526 & 2.054 & 0.798 & & & \\
\hline & Constant & 127.423 & 111.217 & 0.252 & & & \\
\hline \multirow{8}{*}{ ZINB5-4 } & $\log (A A D T)$ & 0.858 & 0.110 & $<0.001$ & \multirow{8}{*}{$\begin{array}{c}0.68 \\
(p- \\
\text { value } \\
=0.247)\end{array}$} & \multirow{8}{*}{847.03} & \multirow{8}{*}{874.61} \\
\hline & $\log (L)$ & 0.752 & 0.129 & $<0.001$ & & & \\
\hline & $\beta_{c m f}$ & 0.009 & 0.052 & 0.858 & & & \\
\hline & Constant & -10.427 & 1.169 & $<0.001$ & & & \\
\hline & \multicolumn{4}{|l|}{ Inflate } & & & \\
\hline & $\log (A A D T)$ & -16.072 & 14.987 & 0.284 & & & \\
\hline & $\log (L)$ & 0.532 & 2.227 & 0.811 & & & \\
\hline & Constant & 107.719 & 104.354 & 0.302 & & & \\
\hline \multirow{8}{*}{ ZINB5-5 } & $\log (A A D T)$ & 0.771 & 0.130 & $<0.001$ & \multirow{8}{*}{$\begin{array}{c}0.72 \\
(p- \\
\text { value } \\
=0.235)\end{array}$} & \multirow{8}{*}{841.64} & \multirow{8}{*}{869.21} \\
\hline & $\log (L)$ & 0.814 & 0.121 & $<0.001$ & & & \\
\hline & $\beta_{S D}$ & -0.085 & 0.035 & 0.014 & & & \\
\hline & Constant & -9.474 & 1.372 & $<0.001$ & & & \\
\hline & \multicolumn{4}{|l|}{ Inflate } & & & \\
\hline & $\log (A A D T)$ & -3.915 & 3.654 & 0.284 & & & \\
\hline & $\log (L)$ & 0.562 & 1.783 & 0.753 & & & \\
\hline & Constant & 23.679 & 25.993 & 0.362 & & & \\
\hline \multirow{8}{*}{ ZINB5-6 } & $\log (A A D T)$ & 0.833 & 0.108 & $<0.001$ & \multirow{8}{*}{$\begin{array}{c}0.95 \\
(p- \\
\text { value } \\
=0.171)\end{array}$} & \multirow{8}{*}{836.87} & \multirow{8}{*}{864.45} \\
\hline & $\log (L)$ & 0.850 & 0.117 & $<0.001$ & & & \\
\hline & $\beta_{\text {Roll }}$ & -0.246 & 0.077 & 0.001 & & & \\
\hline & Constant & -8.844 & 1.206 & $<0.001$ & & & \\
\hline & \multicolumn{4}{|l|}{ Inflate } & & & \\
\hline & $\log (A A D T)$ & -19.193 & 16.173 & 0.235 & & & \\
\hline & $\log (L)$ & 0.529 & 2.120 & 0.803 & & & \\
\hline & Constant & 129.435 & 112.412 & 0.250 & & & \\
\hline
\end{tabular}


Table 7-13: ZINB Models to Predict Five-Year Collision Frequency.

\begin{tabular}{|c|c|c|c|c|c|c|c|}
\hline Model & Variable & Coef. & $S E E$ & $\begin{array}{c}p- \\
\text { value }\end{array}$ & $\begin{array}{c}\text { Vuong } \\
\text { test }\end{array}$ & AIC & BIC \\
\hline \multirow{9}{*}{ ZINB5-7 } & $\log (A A D T)$ & 0.738 & 0.109 & $<0.001$ & \multirow{9}{*}{$\begin{array}{c}0.98 \\
(p- \\
\text { value } \\
=0.163)\end{array}$} & \multirow{9}{*}{834.19} & \multirow{9}{*}{865.21} \\
\hline & $\log (L)$ & 0.959 & 0.125 & $<0.001$ & & & \\
\hline & $R$ & -0.001 & $<0.001$ & 0.011 & & & \\
\hline & $\beta_{V S}$ & -0.122 & 0.087 & 0.160 & & & \\
\hline & Constant & -9.656 & 1.135 & $<0.001$ & & & \\
\hline & \multicolumn{4}{|l|}{ Inflate } & & & \\
\hline & $\log (A A D T)$ & -17.601 & 14.666 & 0.230 & & & \\
\hline & $\log (L)$ & 0.630 & 1.915 & 0.742 & & & \\
\hline & Constant & 118.069 & 101.396 & 0.244 & & & \\
\hline \multirow{9}{*}{ ZINB5-8 } & $\log (A A D T)$ & 0.747 & 0.110 & $<0.001$ & \multirow{9}{*}{$\begin{array}{c}0.85 \\
(p- \\
\text { value } \\
=0.197)\end{array}$} & \multirow{9}{*}{835.65} & \multirow{9}{*}{866.67} \\
\hline & $\log (L)$ & 0.888 & 0.132 & $<0.001$ & & & \\
\hline & $R$ & -0.001 & $<0.001$ & $<0.001$ & & & \\
\hline & $\beta_{c m f}$ & 0.036 & 0.051 & 0.483 & & & \\
\hline & Constant & -9.843 & 1.148 & $<0.001$ & & & \\
\hline & \multicolumn{4}{|l|}{ Inflate } & & & \\
\hline & $\log (A A D T)$ & -16.054 & 14.187 & 0.258 & & & \\
\hline & $\log (L)$ & 0.681 & 1.927 & 0.724 & & & \\
\hline & Constant & 107.081 & 98.072 & 0.275 & & & \\
\hline \multirow{9}{*}{ ZINB5-9 } & $\log (A A D T)$ & 0.711 & 0.135 & $<0.001$ & \multirow{9}{*}{$\begin{array}{c}0.85 \\
(p- \\
\text { value } \\
=0.196)\end{array}$} & \multirow{9}{*}{835.53} & \multirow{9}{*}{866.55} \\
\hline & $\log (L)$ & 0.920 & 0.126 & $<0.001$ & & & \\
\hline & $R$ & -0.001 & $<0.001$ & 0.005 & & & \\
\hline & $\beta_{S D}$ & -0.037 & 0.038 & $\mathbf{0 . 3 3 0}$ & & & \\
\hline & Constant & -9.485 & 1.443 & $<0.001$ & & & \\
\hline & \multicolumn{4}{|l|}{ Inflate } & & & \\
\hline & $\log (A A D T)$ & -5.112 & 3.654 & 0.415 & & & \\
\hline & $\log (L)$ & 0.589 & 1.783 & 0.726 & & & \\
\hline & Constant & 32.018 & 25.993 & 0.422 & & & \\
\hline \multirow{9}{*}{ ZINB5-10 } & $\log (A A D T)$ & 0.763 & 0.109 & $<0.001$ & \multirow{9}{*}{$\begin{array}{c}0.98 \\
(p- \\
\text { value } \\
=0.165)\end{array}$} & \multirow{9}{*}{832.79} & \multirow{9}{*}{863.81} \\
\hline & $\log (L)$ & 0.938 & 0.122 & $<0.001$ & & & \\
\hline & $R$ & -0.001 & $<0.001$ & 0.014 & & & \\
\hline & $\beta_{\text {Roll }}$ & -0.153 & 0.084 & 0.067 & & & \\
\hline & Constant & -9.139 & 1.198 & $<0.001$ & & & \\
\hline & \multicolumn{4}{|l|}{ Inflate } & & & \\
\hline & $\log (A A D T)$ & -18.058 & 14.689 & 0.219 & & & \\
\hline & $\log (L)$ & 0.627 & 1.954 & 0.748 & & & \\
\hline & Constant & 121.215 & 101.567 & 0.233 & & & \\
\hline
\end{tabular}


Finally, the Vuong test was used to compare the base models of the ZIP (ZIP5-1 and ZIP5-2) to the ZINB (ZINB5-1 and ZINB5-2) using Equations (7.1) and (7.2). The Vuong statistic was $2.479(p$-value $=0.007)$ and $2.732(p$-value $=0.003)$ for the base models without and with the radius of curve, respectively. This means that the ZIP base models for predicting the five-year collision frequency are preferred over the ZINB base models. Also, a comparison was conducted between the ZIP and ZINB models shown in Table 7-12 and Table 7-13. The Vuong statistic for all models was greater than +1.96 and significant at $5 \%$ level of significance. Thus, the ZIP models are preferred over the ZINB models. Since the AIC and BIC are lower for the NB models than ZIP and ZINB models and the Vuong test did not show a preference for ZINB over the base model (NB), the NB models are used in the coming analysis.

\subsection{Summary of SPFs}

Based on the discussion in the previous sections, the following equations summarize the models recommended to predict collision frequency on a horizontal curve for one-year and five-year periods.

$$
\begin{aligned}
& S P F 1_{V S}=A A D T^{0.718} L^{0.946} e^{\left(-10.785-0.238 \beta_{V S}\right)} \\
& S P F 1_{S D}=A A D T^{0.769} L^{0.887} e^{\left(-11.452-0.086 \beta_{S D}\right)} \\
& S P F 1_{\text {Roll }}=A A D T^{0.754} L^{0.911} e^{\left(-10.328-0.215 \beta_{\text {Roll }}\right)} \\
& S P F 5_{V S}=A A D T^{0.857} L^{0886} e^{\left(-10.295-0.193 \beta_{V S}\right)} \\
& S P F 5_{S D}=A A D T^{0.860} L^{0.830} e^{\left(-10.366-0.080 \beta_{S D}\right)} \\
& S P F 5_{R o l l}=A A D T^{0.893} L^{0.854} e^{\left(-9.606-0.216 \beta_{R o l l}\right)}
\end{aligned}
$$


Where subscript $V S, S D$, and Roll= vehicle stability, sight distance, and rollover, respectively, 1 and 5= annual and five-year collision frequency, respectively, AADT= average annual daily traffic, $L=$ length of curve, and $\beta=$ reliability index.

\subsection{Horizontal Curve Design Procedure}

As mentioned in Chapter 1, the proposed framework to design horizontal curve is an iterative process that starts with selecting an initial design speed. The next steps are:

1. Select initial values for horizontal curve geometry (radius and superelevation rate) and determine the lateral clearance (available sight distance). Understanding the typical weather conditions of the region and the expected AADT will help in calibrating reliability indices and reaching the final design.

2. Determine the distribution of curve speed $\left(V_{M C}\right.$ and $\left.V_{B C}\right)$ using the developed models provided in Chapter 5.

3. Implement the above information and the friction models mentioned in Section 5.3.5 in conducting reliability analysis (FORM) to estimate POF, PNC, $\mathrm{POF}_{\mathrm{R}}$, and $\mathrm{POH}$.

4. Adjust POF, $\mathrm{PNC}, \mathrm{POF}_{\mathrm{R}}$, and $\mathrm{POH}$ to reflect the effect of weather and pavement conditions, traffic volume and other factors mentioned earlier.

5. Use SPFs mentioned earlier to estimate the expected collision frequency on the curve.

6. Conduct design consistency analysis (out of the scope of the research).

7. Finalize the design of the horizontal curve if the safety analysis outputs are within acceptable limits. 
A Matlab program was developed to perform the procedure of the design. The following design example for a horizontal curve explains how the program works and the expected outputs.

It is required to design a horizontal curve on a two-lane highway in rolling terrain in a rural area. The design speed of the curve is selected $80 \mathrm{~km} / \mathrm{h}$. The curve should connect two tangents with a deflection angle of $40^{\circ}$. It is expected to have 60 days of rain throughout the year. The expected AADT is 6,000 veh/d. The lateral clearance distance is $15 \mathrm{~m}$ when having radius of curve of $150 \mathrm{~m}$. The curve will be constructed in a fill section. There is no vertical curve superimposed with horizontal curve.

The following analyses were conducted for PC (sedan D-Class). It should be noted that driving in favourable weather conditions was assumed to be on dry pavement and driving on wet pavement was assumed to be travelling in raining conditions.

\subsubsection{Initial Values Selection}

The first step was determining initial values for horizontal curve parameters. The source to select initials values are the design guides; the TAC (TAC 2017) and the Green Book (A Policy 2011). For illustration purposes, the initial values for this example are; Radius $=150 \mathrm{~m}$, and superelevation rate $=6 \%$. It is intended to keep the superelevation rate constant and evaluate the change in expected collision frequency with the change in radius. Therefore, several radii were considered in the analysis from 150 to $350 \mathrm{~m}$. The posted speed limit was assumed to be $70 \mathrm{~km} / \mathrm{h}$.

\subsubsection{Determine Speed Distributions}

Based on the information provided in the design example, the reliability analysis 
(FORM) was conducted considering two situations; driving in clear and raining weather conditions, thus, driving on dry and wet pavement conditions. Therefore, four speed models were used in FORM to predict speed at MC and BC. The speed panel models provided in Table 5-2 and Table 5-5 were used to predict speed distributions at $\mathrm{BC}$ and MC for favourable and raining weather conditions. The predicted speeds using these models for different radii are shown in Figure 7.3. The figure shows that the speed at BC in adverse weather conditions is higher than favourable conditions. However, the difference in speed is relatively small. Drivers likely do not recognize the beginning of the curve especially when having adverse weather conditions. Thus, drivers would enter the curve with a high speed at $\mathrm{BC}$ then they decelerate after feeling the effect of lateral acceleration.

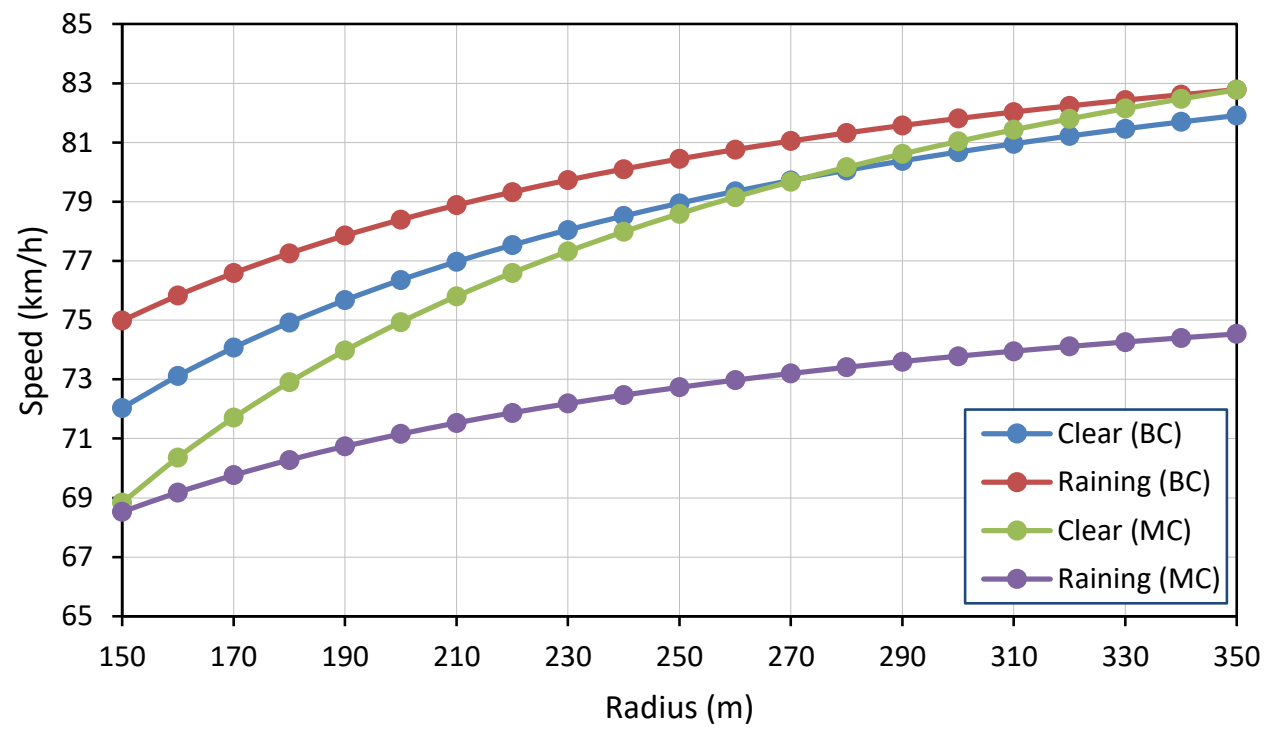

Figure 7.3: Predicted Mean Speed at MC and BC for Different Weather Conditions. 


\subsubsection{POF Calculation}

To calculate the POF for vehicle stability, speed at BC (favourable and raining weather conditions) and lateral friction factor distributions (dry and wet pavement) should be calculated. Equations provided in Section 5.3.5. were used to estimate mean and standard deviation for the available lateral friction factor. The demanded lateral friction was determined using PC model (sedan D-Class model) provided in Table 5-6.

The developed Matlab code mentioned earlier to conduct reliability analysis using FORM method was used to estimate the POF. The outputs of POF for vehicle stability criterion for different radii are shown in Figure 7.4. As shown in the figure, the POF is almost zero when having dry pavement (for radii greater than $150 \mathrm{~m}$ ) and low values for wet pavement. As expected and mentioned before, the POF decreases with radius.

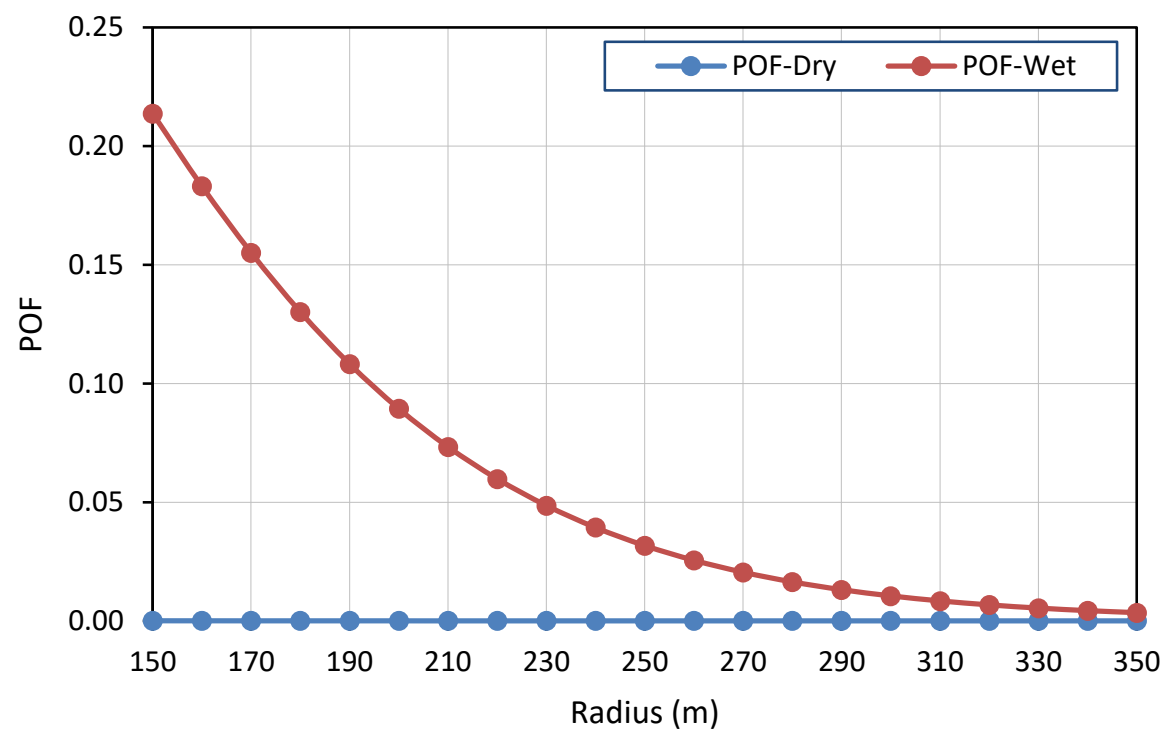

Figure 7.4: Radius Versus POF for Dry and Wet Pavement Conditions. 


\subsubsection{PNC Calculation}

The speed distribution at MC mentioned in Section 7.5.2 is considered in evaluating driver comfort criterion. Thus, Model 1 in Table 5-2 and Table 5-5 were used to predict speeds on curve for favourable and raining weather conditions, respectively. For determining comfort threshold, Model 5 in both Table 5-4 and Table 5-5 were used for clear and raining weather conditions, respectively. The predicted driver comfort thresholds of these models with different radii are shown in Figure 7.5. It is readily seen that comfort thresholds for favourable weather conditions are higher than those in raining conditions.

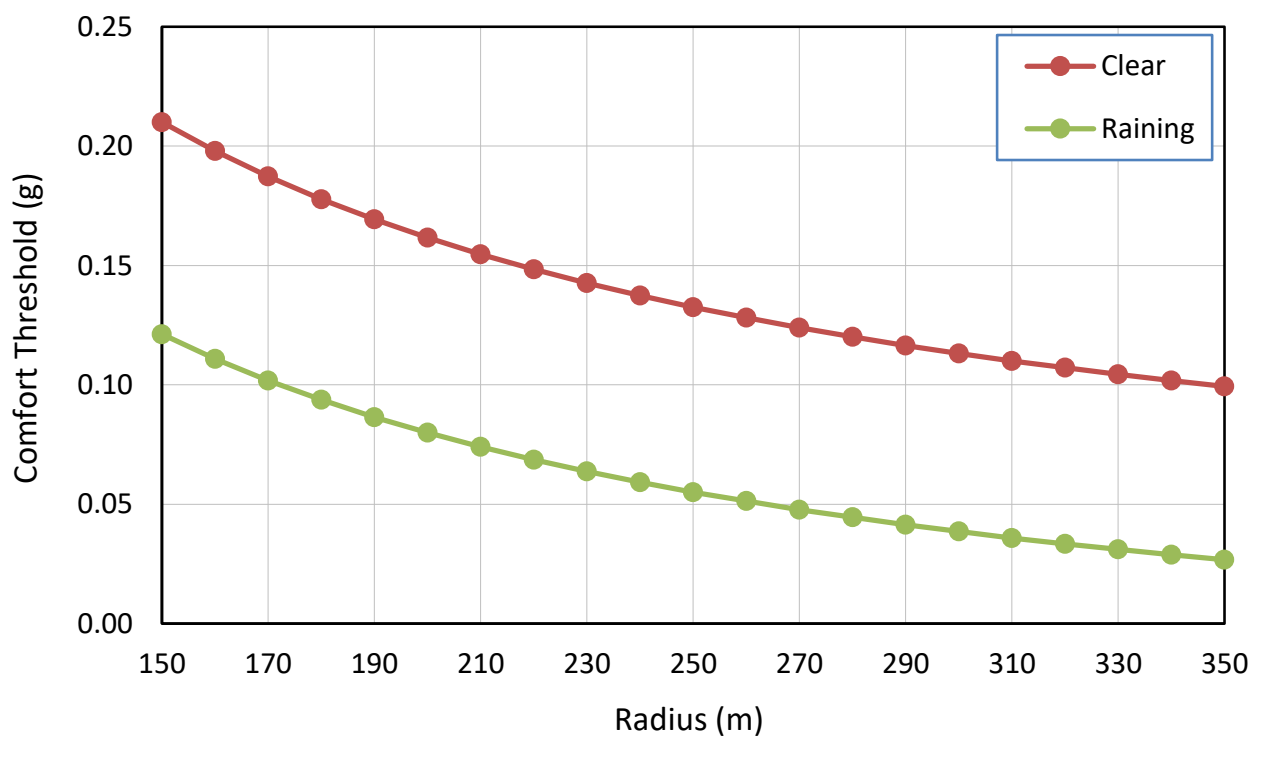

Figure 7.5: Mean Comfort Threshold for Different Radii.

The outputs of the reliability analysis for evaluating driver comfort criterion are shown in Figure 7.6. As shown in the figure, the PNC is very low when driving in the favourable weather conditions. However, the PNC is very high when driving in adverse weather conditions and having wet pavement, especially for sharp curves. The PNC for both weather conditions escalates with radius until reaching the peak value (approximately at 
radius $=200 \mathrm{~m}$ for wet pavement) then decreases with radius. However, this trend is obvious for trips taking place at rainy weather conditions. This trend (increasing in PNC then decreasing) is expected because both the comfort threshold and the applied lateral acceleration decrease with radius at different rates as explained earlier in Section 6.4,

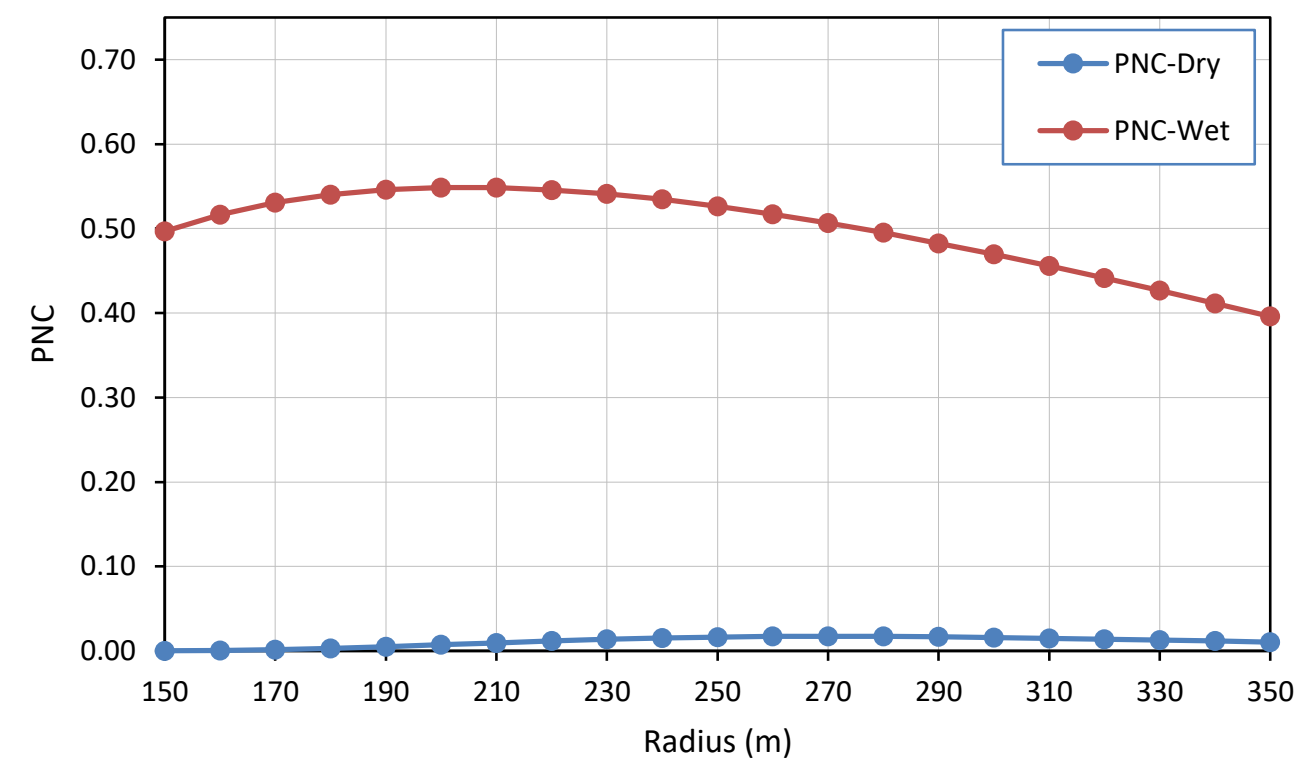

Figure 7.6: Radius Versus PNC for different Weather and Pavement Conditions.

\subsubsection{POH Calculation}

Several factors could affect the available sight distance such as the highway visibility (weather conditions), vertical curves, and lateral clearance (LC) on horizontal curves. The LC was considered in the design example; however, other sight distance limitations such as the case of having horizontal curve superimposed with a crest vertical curve were not considered. Thus, 2-D approach was used to determine the available sight distance. The minimum LC distance was calculated for each radius considering that the deflection angle and centreline of the curve tangents are not being changed. For example, the minimum LC 
distance will be $6.72 \mathrm{~m}$ for horizontal curve of radius $280 \mathrm{~m}$ (available sight distance is $122.99 \mathrm{~m}$ ). The $\mathrm{LC}$ will drop to $5.45 \mathrm{~m}$ for curve of radius $300 \mathrm{~m}$ (available sight distance is $114.58 \mathrm{~m}$ ). As mentioned earlier, AutoCAD and Excel formula were used to determine the available sight distance when having the sight distance greater than the length of curve as shown in Figure 7.7.

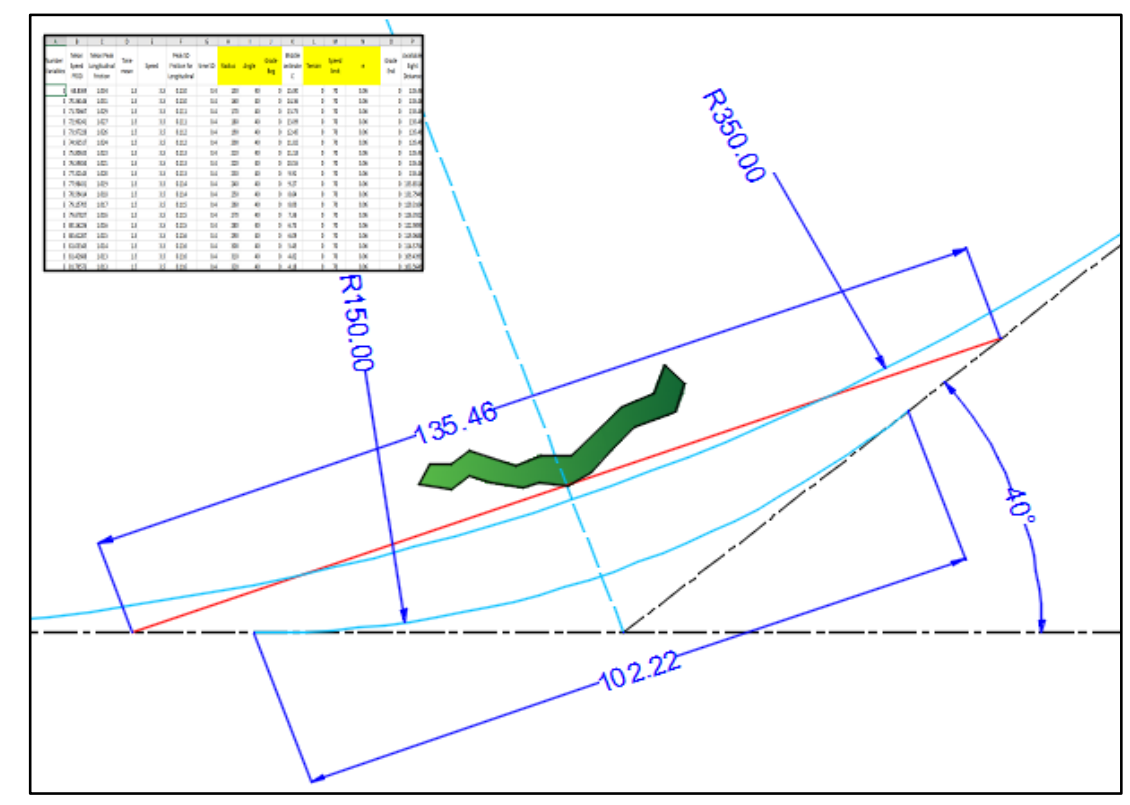

Figure 7.7: Determining Available Sight Distance.

The mean available sight distance $\left(S_{a v i}\right)$ and the mean demanded stopping sight distance (SSD) for dry and wet pavement are shown in Figure 7.8. Note that the SSD was calculated using Equation 3.36. The $S_{a v i}$ decreases with radius because the deflection angle is constant, and the location of lateral object is fixed. It is assumed that $S_{a v i}$ is not affected by the rain. The SSD increases with radius because the $V_{B C}$ increases and, as a result, the available longitudinal friction decreases. 


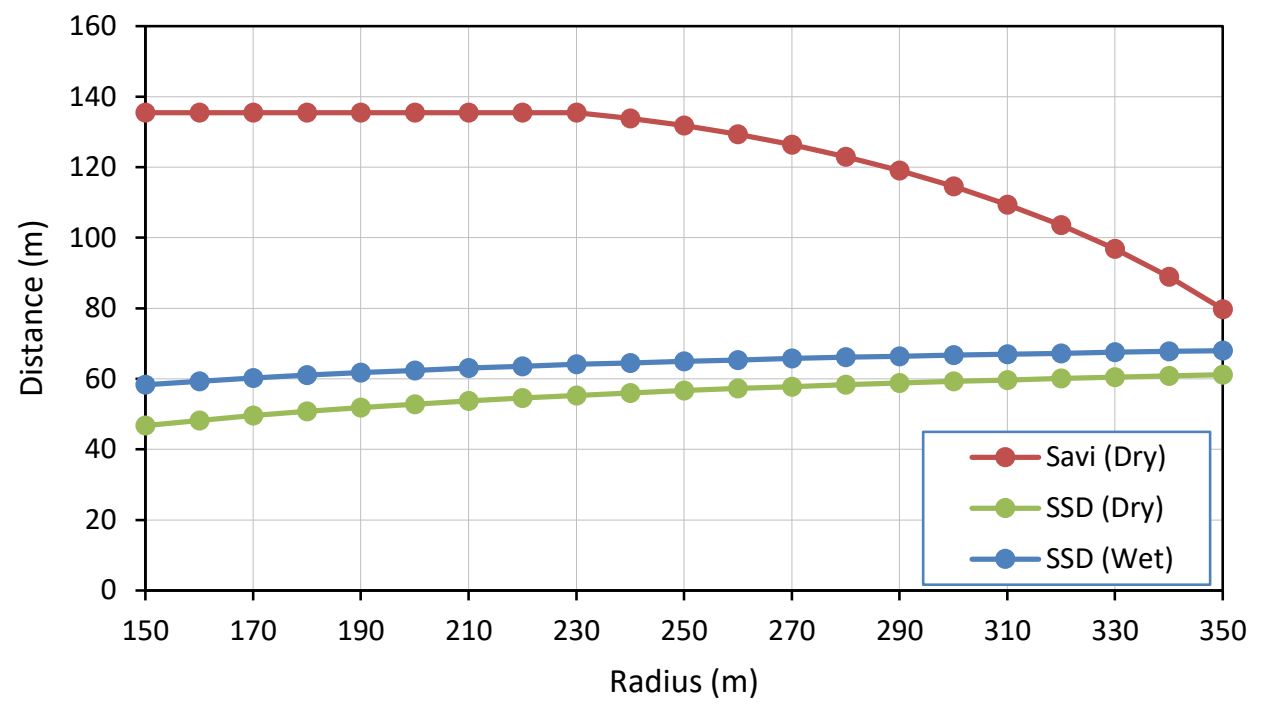

Figure 7.8: Mean Sight Distance and Stopping Sight Distance.

The outputs for the POH are shown in Figure 7.9. As shown in the figure, the POH is very low for dry pavement and relatively low for wet pavement for radii less than 280 $\mathrm{m}$. However, the $\mathrm{POH}$ increases dramatically for radius greater than 300 and $330 \mathrm{~m}$ for wet and dry pavement, respectively. This is expected because the $S_{a v i}$ decreases due to reducing the minimum lateral clearance. Meanwhile, the demanded stopping distance increases with speed.

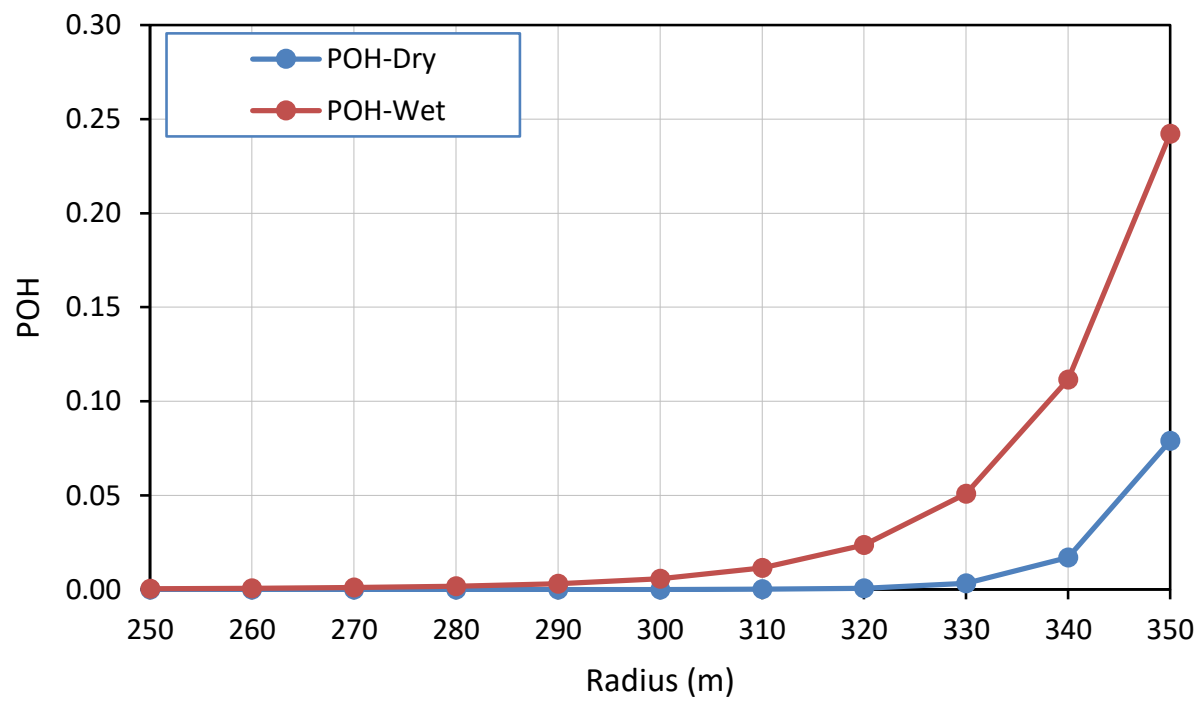

Figure 7.9: Radius Versus POH for Dry and Wet Pavement. 


\subsection{6. $\mathrm{POF}_{\mathrm{R}}$ Calculation}

As explained in Section 6.6, several factors affecting on vehicle stability against turning over such as suspension system characteristics and speed of vehicle. The evaluation of vehicle rollover criterion for $\mathrm{PC}$ shows that the $\mathrm{POF}_{\mathrm{R}}$ values were very low for dry and wet pavements as shown in Figure 7.10. This is expected since the centre of gravity for PC height, from the ground, is very low. The figure shows that $P_{\mathrm{R}}$ values for wet pavement are relatively higher than those for dry pavement although the overturning moment in the latter situation would be higher (due to higher speed). This situation would be expected because the $S E E$ (standard deviation) of the speed on curve for raining conditions $\left(V_{B C}\right)$ is approximately two times the SEE of the model for favourable weather conditions. However, the difference between the two cases is still very low.

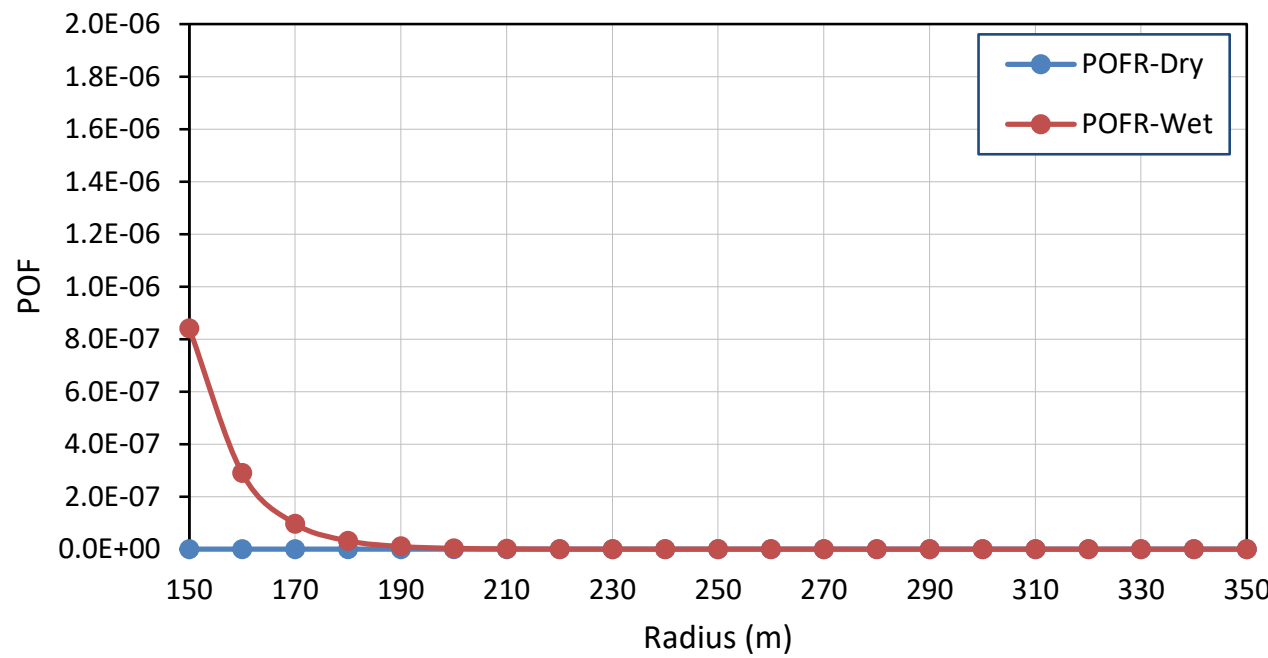

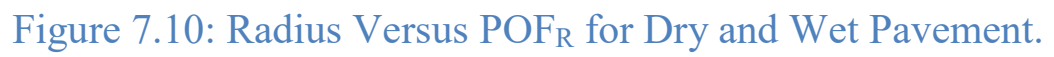

It should be noted that the minimum radius for a design speed of $80 \mathrm{~km} / \mathrm{h}$ and superelevation rate of $6 \%$ according to the Green Book (A Policy 2011) is $252 \mathrm{~m}$. By comparing the values of $\mathrm{POF}, \mathrm{PNC}, \mathrm{POH}$, and $\mathrm{POF}_{\mathrm{R}}$ (of the example) corresponding to this radius, it could be concluded that the requirements stated in the Green Book (A Policy 
2011) would provide very low values of failure for dry/favourable and wet/raining pavement/weather conditions (Figure 7.11) except for driver comfort criterion. The probabilities of failure for wet pavement and raining weather conditions were $\mathrm{POF}=0.03$, $\mathrm{PNC}=0.05, \mathrm{POH}<0.001$, and $\mathrm{POF}<0.001)$. It should be noted that these results correspond to the inputs of the design example. However, this may not necessarily be the case when considering other conditions such as the different vehicle types and different weather and pavement conditions.

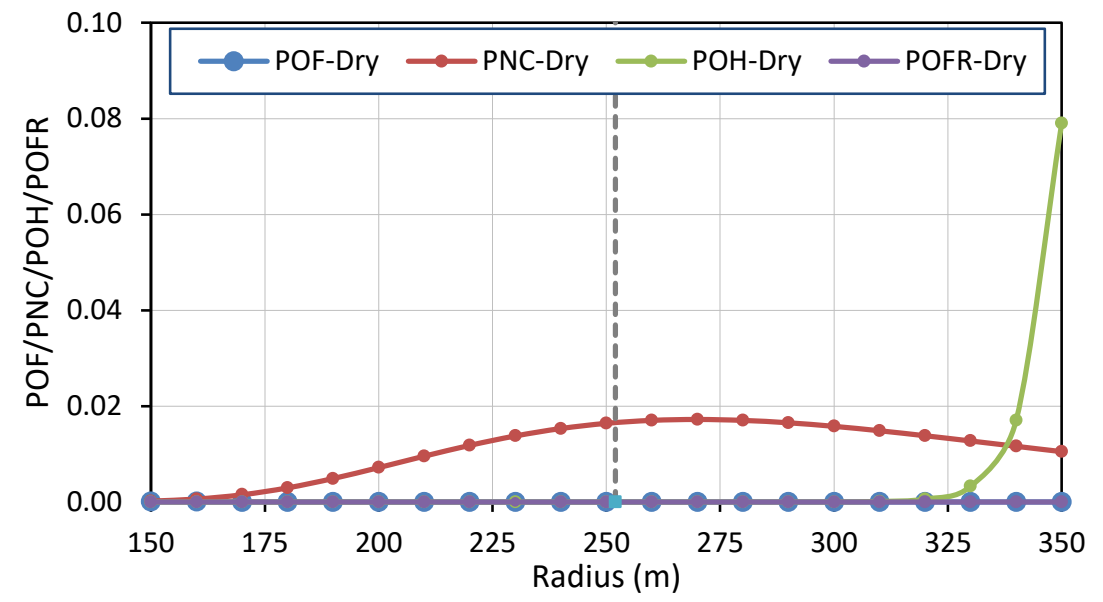

(a) Dry Pavement.

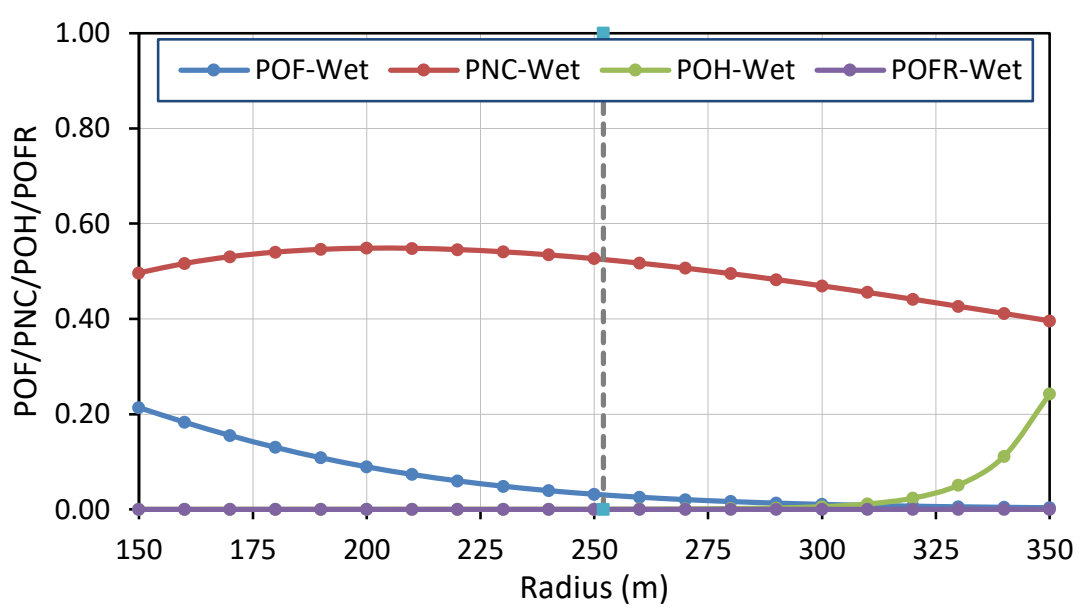

(b) Wet Pavement.

Figure 7.11: Radius Versus POF, PNC, POH, and POFR for Dry and Wet Pavement. 


\subsubsection{Overall Probability of Failure}

As mentioned in the design example, the curve is neither always dry nor having clear weather conditions. Vehicles are not always at free-flow conditions or very high traffic volume. That is, it is required to adjust the effect of these conditions on the overall horizontal curve performance as explained in Section 3.4. The adjustment should take into account traffic characteristics, weather and pavement conditions, and types of vehicles simultaneously. However, for the purpose of illustrating the calibration procedure and because of lack of the data to fill cells in this perspective, the calculation is conducted only to adjust the effect of weather and pavement conditions on the overall performance of the curve. The adjustments, for POF, for pavement and weather conditions for the first year and for a curve with radius of $200 \mathrm{~m}$ is shown below. Same procedure was followed for the other design criteria.

$$
\begin{aligned}
& P O F_{\text {avg }, 1}=\frac{P O F_{d r y} \times 305+P O F_{w e t} \times 60}{365} \\
& P O F_{\text {avg }, 1}=\frac{0 \times 305+0.047 \times 60}{365} \\
& P O F_{\text {avg }, 1}=0.007
\end{aligned}
$$

The results of probability of failures for other radii are plotted in Figure 7.12 and tabulated in Table 7-14. 


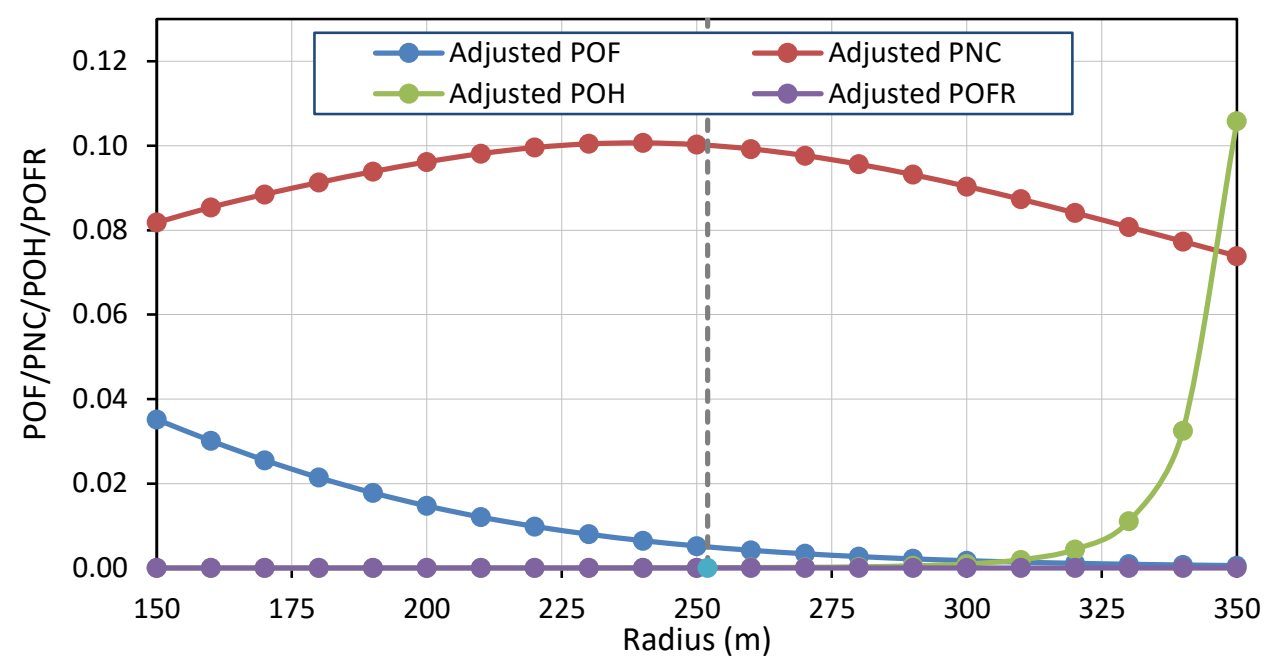

Figure 7.12: Adjusted POF, PNC, POH, and POFR for Different Radii.

Table 7-14: Adjusted POF, PNC, POH and $\mathrm{POF}_{\mathrm{R}}$ for Weather and Pavement Conditions.

\begin{tabular}{|c|c|c|c|c|}
\hline \hline Radius & $\begin{array}{c}\text { Adjusted } \\
\text { POF }\end{array}$ & $\begin{array}{c}\text { Adjusted } \\
\text { PNC }\end{array}$ & $\begin{array}{c}\text { Adjusted } \\
\text { POH }\end{array}$ & $\begin{array}{c}\text { Adjusted POF } \\
\left(\times 10^{-6}\right)\end{array}$ \\
\hline 150 & 0.0352 & 0.0818 & 0.0000 & 0.1383 \\
\hline 160 & 0.0301 & 0.0854 & 0.0000 & 0.0476 \\
\hline 170 & 0.0255 & 0.0885 & 0.0000 & 0.0159 \\
\hline 180 & 0.0214 & 0.0913 & 0.0000 & 0.0052 \\
\hline 190 & 0.0178 & 0.0939 & 0.0000 & 0.0017 \\
\hline 200 & 0.0147 & 0.0962 & 0.0000 & 0.0005 \\
\hline 210 & 0.0121 & 0.0981 & 0.0000 & 0.0002 \\
\hline 220 & 0.0098 & 0.0996 & 0.0000 & 0.0000 \\
\hline 230 & 0.0080 & 0.1004 & 0.0000 & 0.0000 \\
\hline 240 & 0.0065 & 0.1007 & 0.0000 & 0.0000 \\
\hline 250 & 0.0052 & 0.1003 & 0.0001 & 0.0000 \\
\hline 260 & 0.0042 & 0.0992 & 0.0001 & 0.0000 \\
\hline 270 & 0.0034 & 0.0977 & 0.0002 & 0.0000 \\
\hline 280 & 0.0027 & 0.0956 & 0.0003 & 0.0000 \\
\hline 290 & 0.0022 & 0.0931 & 0.0005 & 0.0000 \\
\hline 300 & 0.0017 & 0.0904 & 0.0010 & 0.0000 \\
\hline 310 & 0.0014 & 0.0873 & 0.0020 & 0.0000 \\
\hline 320 & 0.0011 & 0.0841 & 0.0044 & 0.0000 \\
\hline 330 & 0.0009 & 0.0808 & 0.0111 & 0.0000 \\
\hline 340 & 0.0007 & 0.0773 & 0.0325 & 0.0000 \\
\hline 350 & 0.0006 & 0.0739 & 0.1058 & 0.0000 \\
\hline \hline
\end{tabular}

The values shown in the table are given for a precision of 4 decimal digits. 


\subsubsection{Safety Performance Evaluation}

Based on the adjusted probability of failures mentioned earlier, the adjusted reliability indices were calculated by computing the standard normal inverse cumulative distribution (mean equals to zero and standard deviation equals to 1.0). Figure 7.13 shows the trend patterns for adjusted reliability indices with radius (for the design example). As shown in the figure, the $\beta_{V S}$ increases with radius, which means that vehicle stability increases with speed. The $\beta_{c m f}$ decreases before radii less than approximately $250 \mathrm{~m}$ then increases with radius. This radius is equal to the minimum radius stated in design guides (TAC and Green Book). On the other hand, $\beta_{S D}$ decreases significantly for radii greater than $290 \mathrm{~m}$. This is expected since the lateral clearance decreases with radius as explained earlier. Finally, the adjusted $\beta_{\text {Roll }}$ increases with radius because the overturning moment decreases with radius. The different trends of the reliability indices indicate the importance of considering all design criteria simultaneously. Also, changing one of the curve parameters, i.e. increase the radius of the curve, might not end up with lower probability of failure.

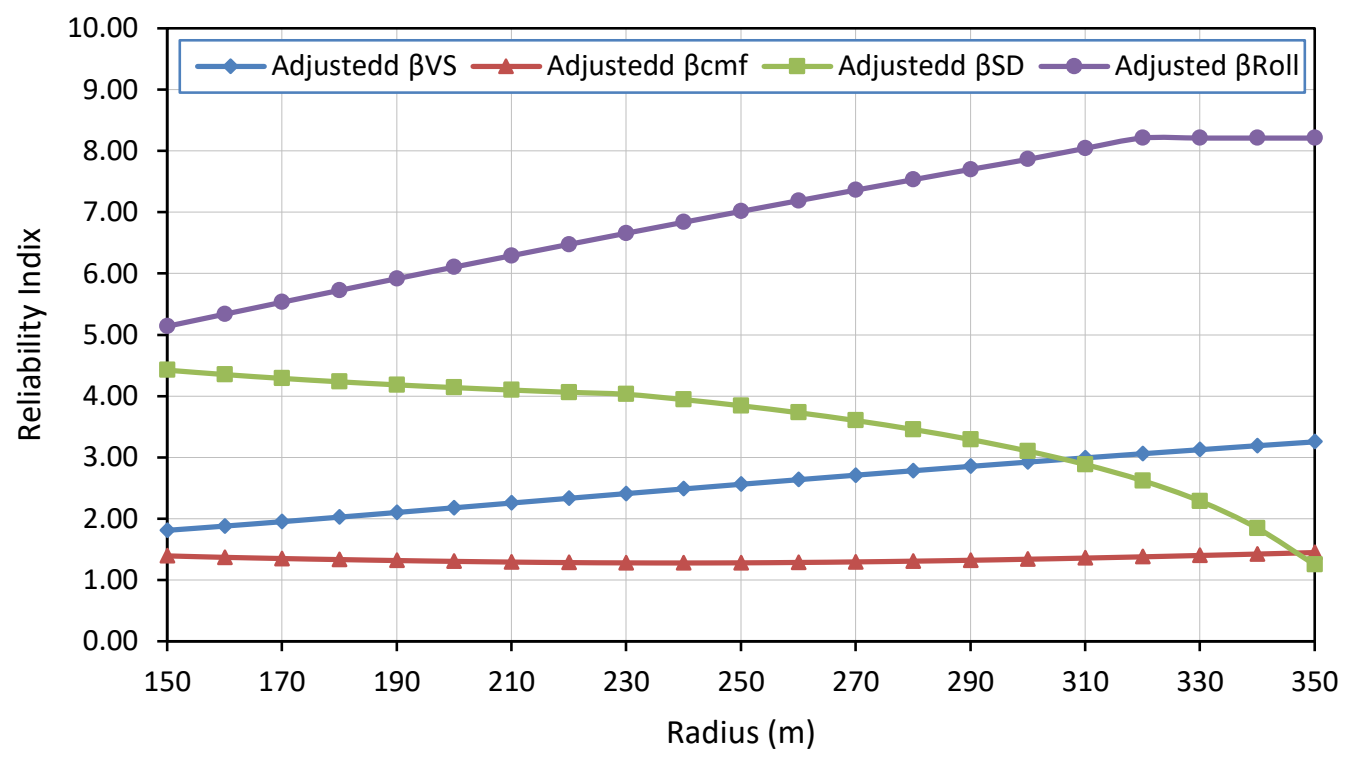

Figure 7.13: Radius Versus Adjusted Reliability Indices. 
The SPFs provided in Section 7.4 were used to predict annual and 5-year collision frequency, respectively. The SPF of the driver comfort was not used because the reliability index was insignificant. Based on the calculated adjusted reliability indices, the predicted number of collisions according to the radius of the curve is shown in Figure 7.14. The SPFs based on vehicle stability criterion (ZINB3 and NB5-3) show that the collision frequency decreases with increase of radius. This is expected since the lateral friction demand has an inverse relationship with radius. On the other hand, the collision frequency based on rollover criterion (ZINB6 and NB5-6) decreases with radius up to point (at radius $=320 \mathrm{~m}$ ) then it starts to increase. The situation can be explained by understanding the relationship between the overturning moment with the speed on curve, which is expected to increase on flatter curves, and with rolling angle. Finally, the collision frequency based on sight distance criterion increases with radius. This is expected because the lateral clearance decreases with radius as explained in the example.

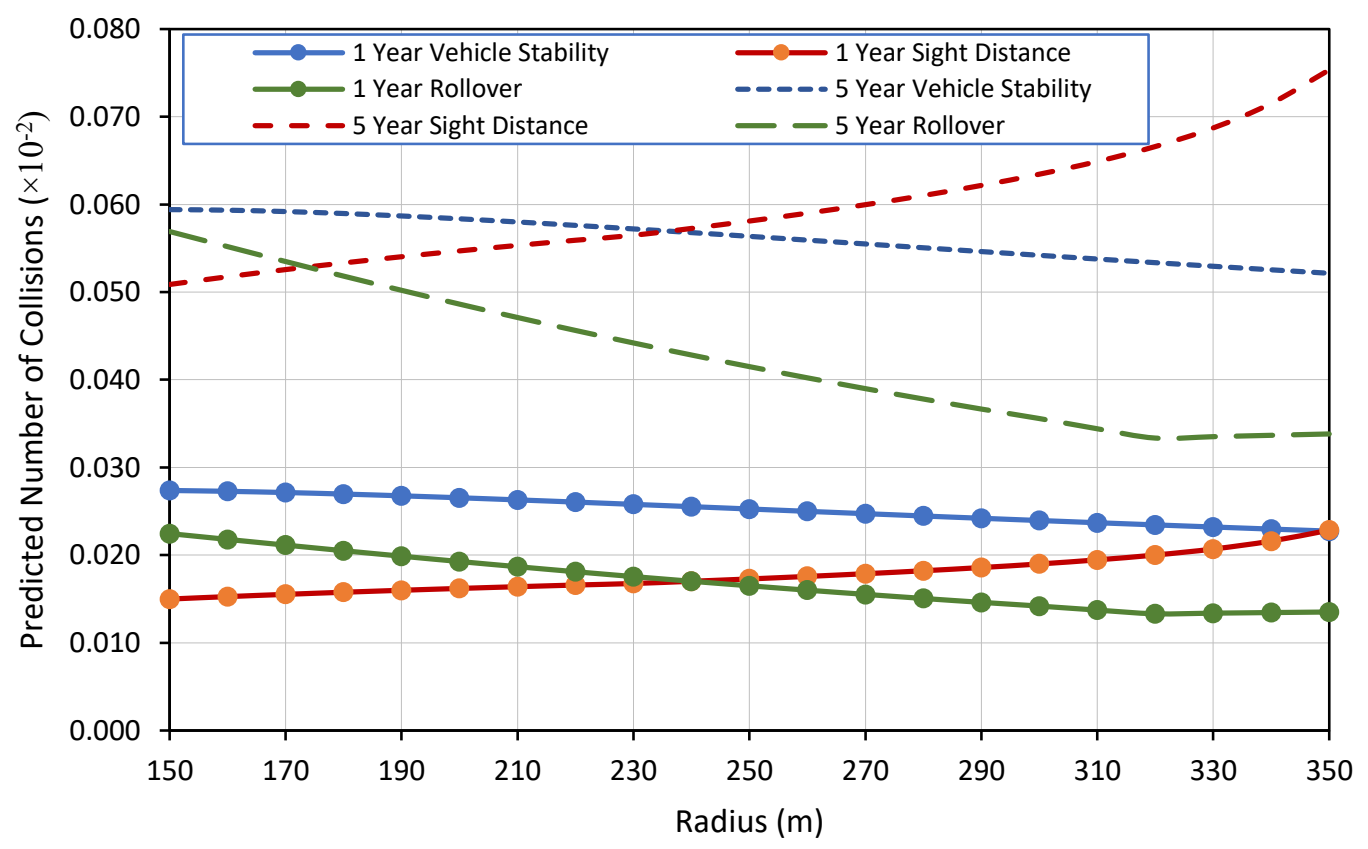

Figure 7.14: Predicted Annual and 5-Year Collisions Frequency. 
From this design example it could be concluded that although there is an improvement in vehicle stability and vehicle rollover (by increasing the radius of the curve), the safety performance of the curve was influenced by the sight distance criterion. This situation supports the idea of checking all design criteria in order to evaluate the performance of the curve.

\subsection{Summary}

This chapter discussed developing safety performance functions and explained the procedure used to develop models that would fit the data. Several SPFs were developed considering horizontal curve characteristics and reliability indices. These models provided predictions of collision frequency for one and five-year intervals. Four types of regression models were used to develop SPFs; Poisson, negative binomial (NB), zero-inflated Poisson (ZIP) and zero-inflated negative binomial (ZINB). The developed SPFs were evaluated based on AIC, BIC, and Vuong test. Based on the AIC and BIC tests, the reliability indices added more value to the models and they were significant except for the driver comfort. Thus, the models with reliability indices better fit the data. However, considering all the reliability indices in one model ended up with insignificant models. Therefore, more data are required to be collected.

This chapter provided the application of the new framework to design horizontal curves. A design example was provided to illustrate the new procedure to design horizontal curve considering four design criteria; vehicle stability, driver comfort, sight distance, and vehicle rollover. Adjustment for the reliability indices was conducted considering only weather and pavement conditions. To consider the effect of other factors (such as vehicle 
type, time of the day, and traffic characteristics) on reliability indices, more data need to be collected. The reliability indices (or probability of failures) would have different trends when changing one of horizontal curve parameters such as the radius. Therefore, it is important to examine all design criteria simultaneously. 


\section{CHAPTER EIGHT}

\section{DISCUSSION AND CONCLUSIONS}

\subsection{Summary}

This research focused on providing a new approach to design horizontal curves using reliability analysis and naturalistic driving study (NDS). Reliability analysis concept is a promising technique that can be used in evaluating different transportation areas. The evaluation can be implemented in the design phase or in evaluating the performance of an existing horizontal curve as long as the performance functions (the relationship between the supply/available and demand/applied) are set correctly. Reliability concept would overcome problems inherently associated with the deterministic approach such as overlooking the effect of variation of design factors. Using data extracted from NDS would overcome several issues associated with traditional technique in data collection procedure. The following were covered in the research:

- The research was planned to provide a comprehensive literature review to cover five topics related to the new framework to design horizontal curves; vehicle dynamics, vehicle stability and control, horizontal curve design criteria, friction phenomenon, and reliability concept. Thus, the movement of a vehicle on a curved path was comprehended through illustrating the forces/moments that affect the movement along with understanding the interaction between vehicle's tires and pavement conditions. Also, the design criteria currently used in design guides to ensure safe and comfort travel for vehicle occupants were reviewed. In addition, 
the main areas of reliability analysis, concept, performance functions, and methods, were covered.

- A methodology to implement the new framework to design horizontal curves was presented. The methodology comprises four phases; ( $i)$ determining the distributions of the speed, driver comfort, lateral acceleration, and available friction, (ii) conduct reliability analysis, (iii) adjust the reliability indices considering pavement and weather conditions, $(i v)$ and conduct safety analysis.

- The data were collected from several sources. The NDS provided data for 36 and 29 horizontal curves on two-lane highways in rolling and mountainous in Washington State. The data were collected along three years for different weather conditions and daytime of the day. Drivers participated in this program came from different backgrounds. Only curves with trips higher than 30 were included in the analysis.

- Matlab scripts were developed to extract several variables. For speed, three parameters were calculated based on the instantaneous measurement and the average of measurements over a short distance or time at different locations on the curve (BC, MC, EC, and AT). Also, a new approach to determine driver comfort threshold was developed and coded using Matlab.

- Matlab scripts were developed to determine vehicle speed state (deceleration, acceleration, or constant). Based on that, a new approach to determine comfort threshold was provided. The Matlab scripts seek the lowest lateral acceleration within a specific sliding window when the vehicle is in "constant" speed state. 
- Based on the extracted data from the NDS database, geometric parameters, and CarSIM outputs, several models were developed to determine the distribution of speed parameters (max, min, at $\mathrm{BC}$, and at $\mathrm{MC}$ ) and driver comfort threshold. Three types of models were developed; DLM, panel, and $85^{\text {th }}$ percentile. Aggregated models provided better estimation in terms of coefficient of determination. These models were used in the reliability analysis. Also, it is found that the mean speed and comfort threshold values for favourable weather conditions (dry pavement) are higher than those in raining conditions (wet pavement).

- Based on several studies, the distributions of available longitudinal and lateral friction factor for dry and wet pavement were modelled. The analysis showed that the beginning of the curve is the most critical point for vehicle stability. Thus, the developed models to estimate the lateral friction demand considered the friction at BC. In addition, models were developed to determine lateral acceleration.

\subsection{Discussion and Conclusions}

The second task in the research was collecting the required data. However, prior to use the data in the analyses, the extracted variables were subjected to several statistical analyses. Following are the main findings:

- The results showed that speed parameters and driver comfort threshold were come from normally distributed population. Also, the assumption of homogeneity of variance was not violated as well. In addition, the ANOVA tests showed no significant difference between the instantaneous and mean speed parameters at BC, MC, EC, and AT. Thus, the instantaneous speed is an acceptable speed parameter 
that is not significantly affected by the fluctuations of speed observations along the trip. A statistically significant difference was found between the means of minimum and maximum speeds on most curves, which indicates that drivers do not follow a constant speed on the curve.

- A right-turn curve will likely have higher $V_{M C}$ and $a_{L}$ than the left-turn for the same curve. However, the statistical analysis does not provide a strong evidence whether this difference is statistically significant or insignificant.

- The results showed a trend of slight increase of speed with the increase of headway, in particular for headway intervals less than 3s. This would indicate that headway can be a factor affecting speed choice up to a headway of $3 \mathrm{~s}$. Therefore, trips with headway equal to or greater than $3 \mathrm{~s}$ were considered to correspond to traveling under free-flow conditions.

- Evaluation for horizontal curves performance were conducted considering four design criteria. The POF, PNC, POH, and $\mathrm{POF}_{\mathrm{R}}$ were calculated using FORM and the developed models in this research. The results showed that POF has an inverse relationship with radius and available friction factor and a direct relationship with mean speed. The PNC has different trend with radius. The PNC would be low for sharp curves because of having low speed and high driver comfort threshold. However, the PNC decreases with radius because the comfort threshold and applied lateral acceleration decreases at different rates. The reliability analysis showed that the POH decreases with lateral clearance. The analysis was conducted considering only 2-dimensions case. Also, the results showed that $\mathrm{POF}_{\mathrm{R}}$ decreases with radius. 
- The reliability analysis was conducted to evaluate the performance of 232 curves in Washington State. The four design criteria were considered in the analysis. The results showed that reliability indices $\left(\beta_{V S}, \beta_{c m f}, \beta_{S D}\right.$ and $\left.\beta_{\text {Roll }}\right)$ has an inverse relationship with collision frequency. Therefore, these indices could be used as surrogate measures to evaluate safety performance of horizontal curves. Also, the results showed that the values of reliability index differ from one criterion another.

- The probability of failures of all design criteria would change with environment, time of day, traffic characteristics, etc.). Therefore, an adjustment should be conducted to obtain realistic evaluation. However, because the data lacked for some necessary components, only pavement and weather conditions were considered in the analysis.

- Several safety performance functions were developed considering the reliability indices as independent variables (predictors). Four types of regression models were used to develop models; Poisson, NB, ZIP, and ZINB. Based on the AIC, BIC, and Vuong tests, the ZINB was found to be better fit the annual collision data and thus was used to predict annual collision frequency. On the other hand, the NB was found to be better fit the 5-year collision data and thus was used to predict 5-year collision frequency.

- To explain the new framework to design horizontal curve, a design example was provided. The probability of failure for four design criteria were calculated and adjusted according to example requirements. The SPFs showed that the safety performance of the horizontal curves generally would be improved with radius. 
Based on previous discussion, the new framework provides a procedure to evaluate the design performance of horizontal curves in terms of probabilities of failure and collision frequency. The developed safety performance functions considering the reliability indices and the developed Matlab codes to predict probability of failure for each design criterion could be used to develop design aids to design horizontal curves. These design aids will be good tools for the designers to evaluate the design performance and be capable of indicating the problems associated with the design criteria such as the effect of weather and pavement conditions. Accordingly, they could help in mitigating the impact of these problems and thus reduce collision frequency.

\subsection{Recommendations for Future Research}

The research tried to quantify the performance of horizontal curves in terms of probability of failure. Considering the new framework to design horizontal curves, the research covered several topics, collected different types of data, developed many models, and provided the procedure and tool to conduct the analysis. However, the following would be recommended for future studies:

- The developed models for speed and comfort threshold in this research covered horizontal curves within a range of radii between 146 and $437 \mathrm{~m}$ for rolling terrain and between 279 and $873 \mathrm{~m}$ for mountainous terrain. As a result, prediction for speed and comfort threshold out of this range would be extrapolation. Therefore, it is recommended to expand the sample size to include wider range of radii, curve length, deflection angle, and posted speed limit in order to develop models with better prediction. 
- To reach more realistic evaluation for horizontal curve performance, additional factors should be considered such as driving at night time, pavement conditions (i.e. packed-snow and icy), driving at adverse weather conditions (i.e. snowing) and traffic characteristics.

- Although the research developed models for two types of vehicles, the evaluation for horizontal curves performance was conducted for PC only. It is recommended to consider other vehicle types in the evaluation.

- It recommended to develop models to predict speed and comfort threshold for non-independent approach tangent.

- The consistency evaluation between approach tangent and horizontal curve was mentioned in the design framework. Therefore, the future work should consider conducting the evaluation before finalizing the design.

- The research showed that every curve would have different values of reliability index for each design criterion (vehicle stability, driver comfort, sight distance, and rollover). Therefore, it is recommended to study this issue in order to determine a proper threshold for each design criterion that could be used in the design phase of horizontal curve. Also, these thresholds could be used in pavement management system by transportation agencies to monitor horizontal curve safety performance. 


\section{REFERENCES}

Anderson, I., M. Bauer, D. Harwood, K. Fitzpatrick. 1999. Relationship to Safety of Geometric Design Consistency Measures for Rural Two-Lane Highways. Transportation Research Record 1658, TRB, National Research Council, Washington, D.C., pp. 43-51.

A policy on geometric design of highways and streets 2011. American Association of State Highway and Transportation Officials (AASHTO), Washington, DC.

Allen, R. W., Szostak, H., Klyde, D., Rosenthal, T., and Owens, K. 1992. Vehicle Dynamic Stability. Department of Transportation, National Highway Traffic Safety Administration, Report number DOT HS 807 956, Washington D.C.

Allen, R.W., Rosenthal, T.J. and Chrstos, J.P., 1998. Applying Vehicle Dynamics Analysis and Visualization to Roadway and Roadside Studies, Washington D.C.

Al-Qadi, I., Loulizi, A., Flintsch, W., Roosevelt, D., and Decker, R., Wambold, J. Nixon, W. 2002. Feasibility of Using Friction Indicators to Improve Winter Maintenance Operations and Mobility. NCHRP Web Document 53 (Project 6-14). National Cooperative Highway Research Program, Transportation Research Board of the National Academies.

ASTM. 2011. Standard E1960-07: Standard Practice for Calculating International Friction Index of a Pavement Surface. ASTM International, West Conshohocken, PA.

ASTM. 2012. Standard E867-06: Standard Terminology Relating to Vehicle-Pavement Systems; Designation. ASTM International, West Conshohocken, PA.

Awadallah, F., 2005. Theoretical Analysis for Horizontal Curves Based on Actual Discomfort Speed. Journal of Transportation Engineering ASCE, 131(November), pp.843-850. 
Barnett, J., 1936. Safe Side Friction and Superelevation Design. Highway Research Board, Proceeding of Annual Meeting-Highway Research Board, Washington, D.C., pp. 69-76.

Ben-Akiva, M., Hirsh, M., and Prashkar, J. 1985. Probabilistic and Economic Factors in Highway Geometric Design. Transportation Science, Vol. 19, No. 1, Feb. 1985.

Bonneson, J.A., 2001. Controls for Horizontal Curve Design. Transportation Research Record, (1751), pp.82-89.

Bonneson, J.A., 2000. Kinematic Approach to Horizontal Curve Transition Design. Transportation Research Record, (1737), pp.1-8.

Chang, T. H., 2001. Effect of Vehicles' Suspension on Highway Horizontal Curve Design. Journal of transportation engineering, 127(1), pp.89-90.

Clark, S.K., 1971. Mechanics of pneumatic Tires. National Bureau of Standards. Washington D.C.

Dimaiuta, M., Donnell, E., Himes, S., and Porter, Richard. (Editors) (2011). "Modeling operating speed synthesis report." Transportation Research Circular E-C151, Transportation Research Board, Washington, DC.

Dhahir, B. and Hassan, Y. 2015. Using Vehicle Simulation in Reliability Analysis of Horizontal Curves Based on Vehicle Stability and Driver Comfort. Proceedings, Road and Safety Simulation Conference. Orlando, FL.

Dhahir, B., and Hassan, Y. 2016. Reliability-based design of horizontal curves on two lane rural highways. Transportation Research Record, 2588, pp. 22-31.

Dhahir, B., and Hassan, Y. (2017). Relationship between traffic safety and speed differential on horizontal curves based on naturalistic driving studies. Proceedings, Road Safety \& Simulation RSS2017 Conference, October 17-19, 2017, The Hague, The Netherlands. 
Dhahir, B., and Hassan, Y. 2018. Studying driving behavior on horizontal curves using naturalistic driving study data. Proceedings, Annual Meeting of the Transportation Research Board, Paper 2018-02863, January 8-11, 2018, Washington DC.

De Solminihac, H., Echaveguren, T., and Vargas, S. 2007. Friction Reliability Criteria Applied to Horizontal Curve Design of Low-Volume Roads. Transportation Research Record, 1(1989), pp. 138-147.

Echaveguren, T., Bustos, M. and Solminihac, H. De, 2005. Assessment of horizontal curves of an existing road using reliability concepts. Canadian Journal of Civil Engineering, 32, pp.1030-1038.

Essa, M., Sayed, T., and Hussein, M., 2015. Application of System Reliability Analysis in Geometric Design: A Case Study of Horizontal Curves on the Sea to Sky Highway. 5th International Symposium on Highway Geometric Design. Vancouver, BC.

El-Bassiouni S., and Sayed, T., 2011. Developing safety performance functions incorporating reliability-based risk measures. Accident Analysis \& Prevention, Volume 43, issue 6, pp. 2153-2159.

Felipe, E. and Navin, F., 1998. Automobiles on Horizontal Curves: Experiments and Observations. Transportation Research Record, 1628(98), pp.50-56.

FHWA, 2015. IHSDM. Available https://www.fhwa.dot.gov/research/tfhrc/projects/safety/comprehensive/ihsdm/ (September 27, 2015).

Fitzpatrick, K. and Carlson, P., (2002). Selection of Design Speed Values. Transportation Research Record, 1796 (1), pp. 3-11.

Fitzpatrick, K., Elefteriadou, L., Harwood, D., Collins, M. McFadden, J., Anderson, I., Krammes, R., Irizarry, N., Parma, K., Bauer, K., and Passetti, K., 1999. Speed Prediction for Two-Lane Rural Highways. Report No. FHWA-RD-99-171, Federal Highway Administration, Washington, DC. 
Flintsch, G.W., De León, E., Mcghee, K., and Al-Qadi, I., 2003. Pavement Surface Macrotexture Measurement and Applications. Transportation Research Record, 1860(3), pp.168-177.

Gillespie, T.D., 1992. Fundamentals of vehicle dynamics. Society of Automotive Engineers, Warrendale, PA.

Glen, F., 2002. Vehicle Stability on Combined Horizontal and Vertical Alignment. MASc. thesis, Department of Civil and Environmental Engineering, Carleton University. Ottawa, Canada.

Glennon, J.C., Neuman, T.R., and Leisch, J. E., 1985. Safety and Operational Considerations for Design of Rural Highway Curves, Report number FHWA/RD86/035, Mclean, Virginia.

Gokhale, S. and Bischoff, D., 2012. State-wide Pavement Friction Testing, Wisconsin Department of Transportation, WisDOT Report \# WI-06-12, Green Bay, WI, USA.

Haldar, A. and Sankaran, M., 2000. Probability, Reliability, and Statistical Methods in Engineering Design, John Wiley and Sons, Inc. New York, NY.

Hall, J. W., Smith, K. L., and Littlton, P., 2009. Guide for Pavement Friction. American Association of State Highway and Transportation Officials, transportation research board, Washington, D.C., USA.

Harwood, D. and Mason, J., 1994. Horizontal Curve design for passenger cars and trucks. Transportation Research Board, (1445), pp.22-33.

Hassan, Y., 2004. Highway Design Consistency: Refining the State of Knowledge and Practice. Transportation Research Record, 1881(1), pp.63-71.

Hassan, Y., M. Sarhan, and M. Salehi. Probabilistic Model for Design of Freeway Acceleration Speed-Change Lanes. In Transportation Research Record: Journal of the Transportation Research Board, No. 2309, Transportation Research Board of the National Academies, Washington, D.C., 2012, pp. 3-11. 
Haukaas, T., 2014. Notes - System Reliability, http://www.inrisk.ubc.ca/files/2012/ 11/System Reliability2.pdf. (October 2016).

Henry, J., 2000. Evaluation of Pavement Friction Characteristics: A Synthesis of Highway Practice: NCHRP Synthesis 291. National Cooperative Highway Research Program, Washington, D.C.

Hilbe, J., 2011. Negative Binomial Regression. $2^{\text {nd }}$ edition, Cambridge University Press, New York, NY.

Himes, S., 2013. Reliability Based Design of Horizontal Curves Considering the Effects of Grades. PhD thesis, Pennsylvania State University, State College, PA.

Himes, S.C., and Donnell, E.T., 2014. Reliability Approach to Horizontal Curve Design. Transportation Research Record, 2436, pp. 51-59.

Hussein, M., Sayed, T., Ismail, K., and Van Espan, A. 2014. Calibrating Road Design Guides Using Risk-Based Reliability Analysis, Journal of Transportation Engineering, ASCE, 1988, pp. 1-6.

Jazar, R.N., 2014. Vehicle Dynamics: Theory and Application. $2^{\text {nd }}$ edition., Springer, New York, NY.

Kapur, K.C. and Lamberson, L.R., 1977. Reliability in Engineering Design, John Wiley \& Sons Inc., New York. NY.

Karnopp, D., 2004. Vehicle Stability, Marcel Dekker Inc., New York, NY.

Kontaratos, M., Psarianos, B. and Yotis, A., 1994. Minimum Horizontal Curve Radius as Function of Grade Incurred by Vehicle Motion in Driving Mode. Transportation Research Record, (1445), pp.86-93.

Lamm, R., Basil, P. and Theodor, M., 1999. Highway Design and Traffic Safety Engineering Handbook, McGraw-Hill companies, New York. NY. 
Lerner, N., 1995. Age and driver perception-reaction time for sight distance design requirements. Proceedings, 1995 Compendium of Technical Papers. Institute of Transportation Engineers 65th Annual Meeting. Denver, CO, pp. 624-628.

Li, L., Wang, F.Y. and Zhou, Q., 2006. Integrated Longitudinal and Lateral Tire/Road Friction Modelling and Monitoring for Vehicle Motion Control. Transactions on Intelligent Transportation Systems, 7(1), pp.1-19.

Lu, Q., Steven, B. and Davis, U.C., 2008. Friction Testing of Pavement Preservation Treatments: Literature Review. http://www.its.ucdavis.edu/research/publications/publicationdetail/?pub_id=1475.

MacIsaac, J. and Riley, G., 2002. Preliminary Findings of the Effect of Tire Inflation Pressure on the Peak and Slide Coefficients of Friction. National Highway Traffic Safety Administration, Report NHTSA/NRD-50, Washington, D.C.

Marvin, R. and Arnljot, H., 2004. System Reliability Theory, Models, Statistical Methods, and Applications. $2^{\text {nd }}$ edition, Hoboken, Wiley-Intercedence, $\mathrm{NJ}$.

Masato, A. 2009. Vehicle Handling Dynamics: Theory and Application. ButterworthHeinemann, Norwood, MA, 2009.

$\begin{array}{lll}\text { Mechanical Simulation, } & 2014 . & \text { CarSIM. }\end{array}$ http://www.carsim.com/products/carsim/index.php (March 15, 2014).

Merritt, D.R., 1988. Safe Speeds on Curves: A Historical Perspective of the Ball Bank Indicator. ITE Journal, Institute of Transportation Engineers, 58(9), pp.15-19.

Messer, C.J., 1989. Methodology for evaluating geometric design consistency. Transportation Research Record, 757, pp.17-14.

Milliken, W.F. \& Milliken, D.L., 1995. Race Car Vehicle Dynamics. SAE International, USA.

Morrall F, J., and Talarico J, R. 1994. Side Friction Demanded and Margins of Safety on Horizontal Curves. Transportation Research Record, 1435, pp. 145-152. 
Ng, J., and Sayed, T. 2003. Quantifying the relationship between geometric design consistency and road safety. $85^{\text {th }}$ Transportation Research Board Annual Meeting, Paper No. 03-2363. Washington, D.C.

Nujjetty, A., Mohamedshah, Y. and Council, F., 2014. Guidebook for State Data Files. Federal Highway Administration, Washington D.C.

Olson, P., Fancher, P. and Schneider, L., 1984. Parameters Affecting Stopping Sight Distance, Final Report, National Cooperative Highway Research Program, Transportation Research Board, National Research Council, Jun, Washington, D.C.

Rajamani, R., 2006. Vehicle Dynamics and Control. Springer Science, USA.

Richl, L. and Sayed, T., 2006. Evaluating the Safety Risk of Narrow Medians Using Reliability Analysis. Journal of Transportation Engineering, 132(5), pp.366-375.

Robert Bosch GmbH, 2006. Safety, Comfort and Convenience Systems, John Wiley and Sons Ltd. England.

Rudin-Brown, C., Jenkins, R., Whithead, T., and Burns, P. 2009. Could ESC (Electronic Stability Control) change the way we drive? Traffic injury prevention, 10(4), pp.340-7.

Said, D., 2008. Development of the Interrelationship Between Driver Behaviour and Highway. Department of Civil and Environmental Engineering, Carleton University, Ottawa, Canada.

Sarhan, M. and Hassan, Y., 2008. Three-Dimensional, Probabilistic Highway Design: Sight Distance Application. Transportation Research Record: Journal of the Transportation Research Board, No. 2060, pp. 10-18.

Sawalha, Z. and Sayed, T., 2006. Traffic accident modelling: some statistical issues. Canadian Journal of Civil Engineering, 33(9), pp.1115-1124.

Sawalha, Z., and Sayed, T., 2001. Evaluating safety of urban arterial roadways. Journal of Transportation Engineering, 127(2), 151-158. 
Shuo, L., Samy, N. and Karen, Z.S., 2003. Upgrading the INDOT Pavement Friction Testing Program. Department of Transportation Federal Highway Administration, Report number FHWA/IN/JTRP-2003/23, Indiana, USA.

Singh, V.P., Jain, S.K. and Aditya, T., 2007. Risk and Reliability Analysis: A handbook for Civil and Environmental Engineers. ASCE Press, Reston, Virginia.

TAC, 2017. Geometric Design Guide for Canadian Roads. Transportation Association of Canada, Ottawa, Canada.

Thambirajah, M.S., 2011. Developmental Assessment of the School-Aged Child with Developmental Disabilities: A Clinician's Guide, Jessica Kingsley Publishers, London, UK.

Torbic, D. et al., 2014. Superelevation Criteria for Sharp Horizontal Curves on Steep Grades. Transportation research board, NCHRP Report 774, Washington D.C.

Tyagi, A. and Haan, C.T., 2001. Uncertainty analysis using corrected approximation method. Water Resources Research, 37(6), pp.1847-1858.

Vieira, R.S., Nicolazzi, L.C. and Roqueiro, N., 2012. Four-wheel vehicle kinematic and geometric constraints for definition of tire slip angle. International Journal of Automotive Technolog, 13(4), pp.553-562.

Wolshon, B., 2004. Geometric Design of Streets and Highways. Kutz, M. (Ed), Handbook of Transportation Engineering, McGraw-Hill Companies, New York.

Wong, J.Y., 2008. Theory of Ground Vehicles. $4^{\text {th }}$ edition, John Wiley and Sons, Inc. New Jersey, USA.

$\begin{array}{lllll}\text { WSDOT, } & 2017 . & \text { WSDOT } & - & \text { SRweb }\end{array}$ http://www.wsdot.wa.gov/mapsdata/tools/srweb.htm (November, 2017).

You, K. and Sun, L., 2013. Reliability Analysis of Vehicle Stability on Combined Horizontal and Vertical Alignments. Driving Safety Perspective. Journal of Transportation Engineering, ASCE, pp.804-813. 
Zheng, Z., 1997. Application of Reliability Theory to Highway Geometric Design. PhD thesis, Department of Civil Engineering, University of British Columbia, B.C., Canada. 


\section{APPENDIX A}

Typical Sedan and SUV 


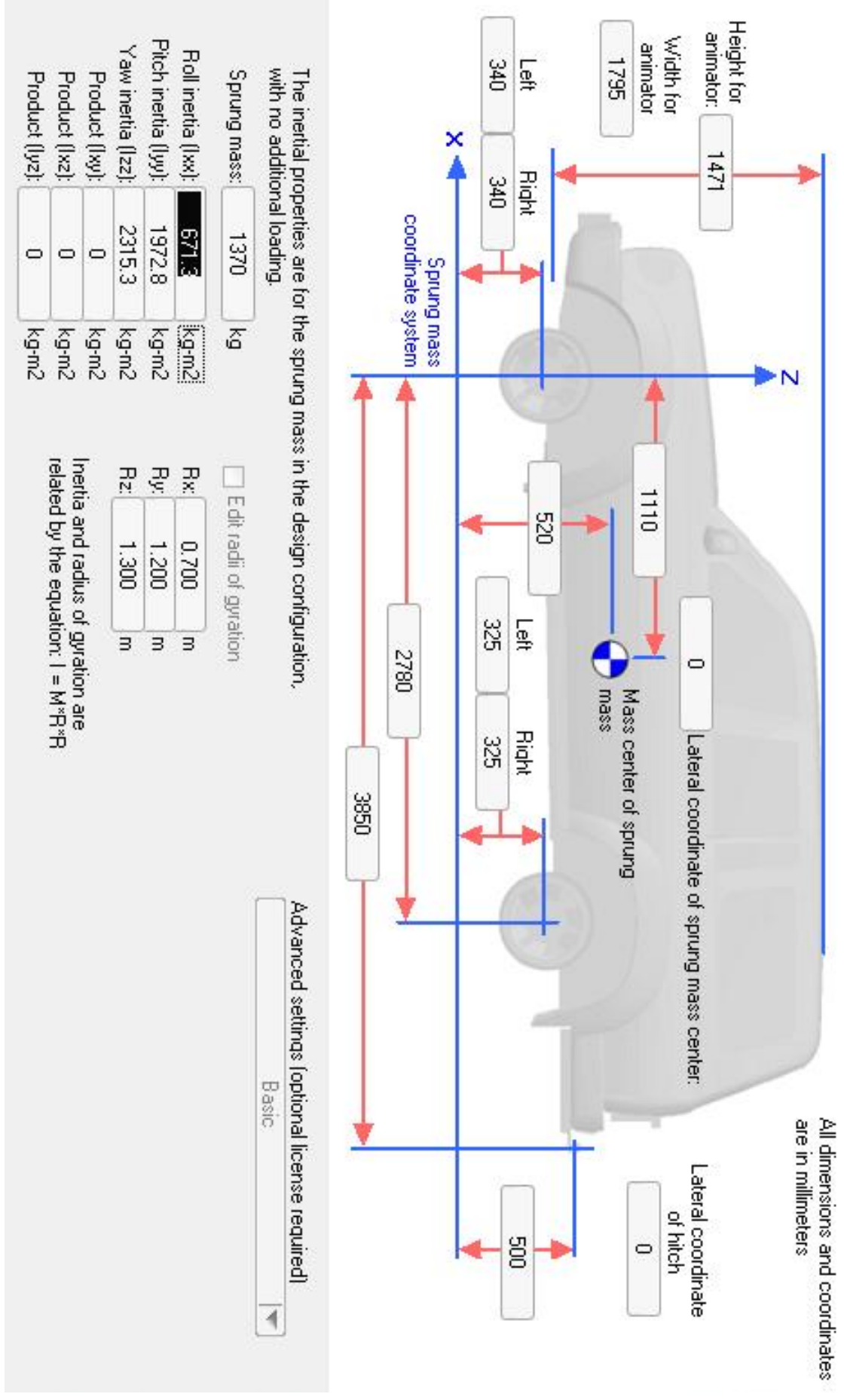

A-1: Sedan D-Class. 


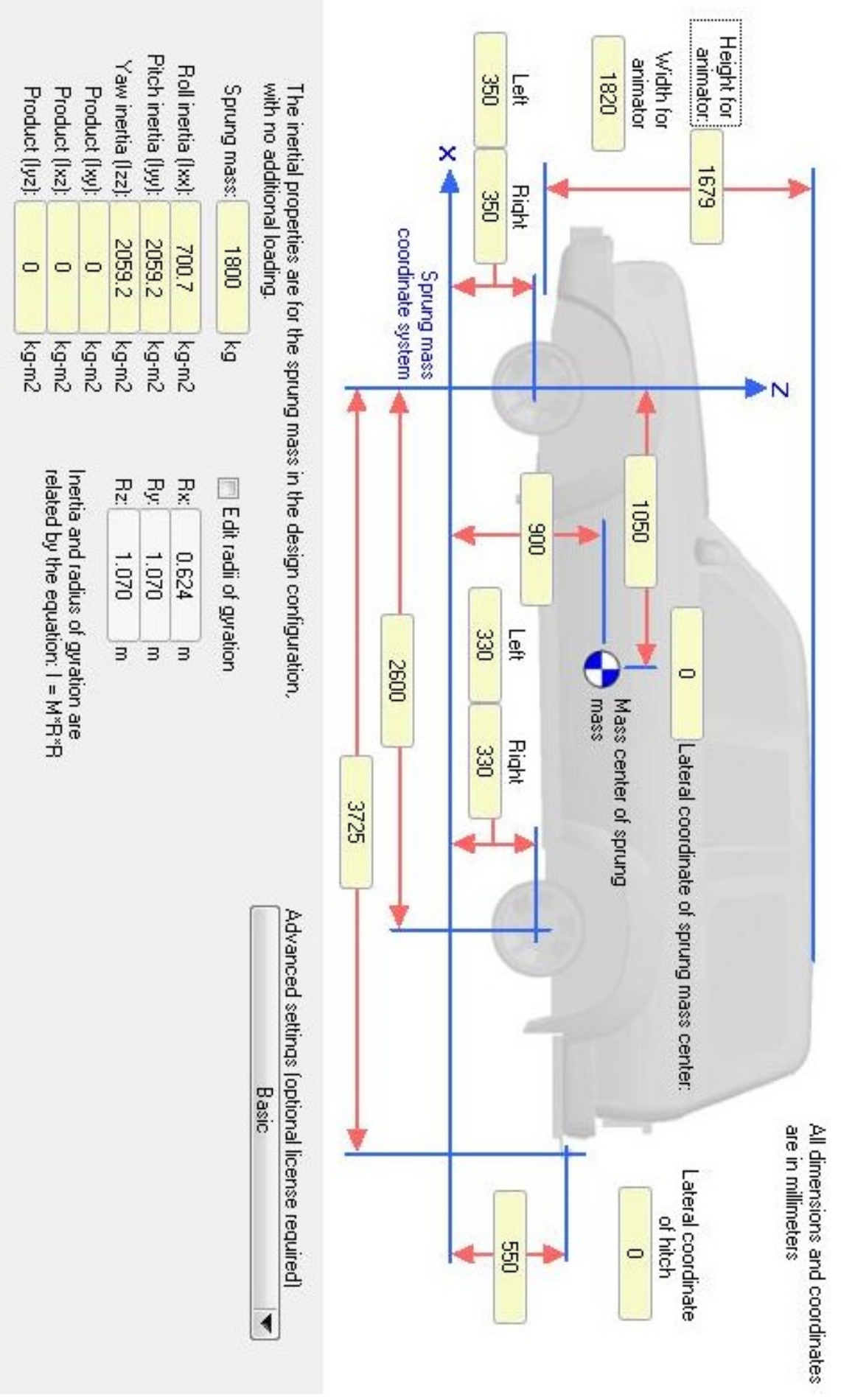

A-2: Typical SUV D-Class. 


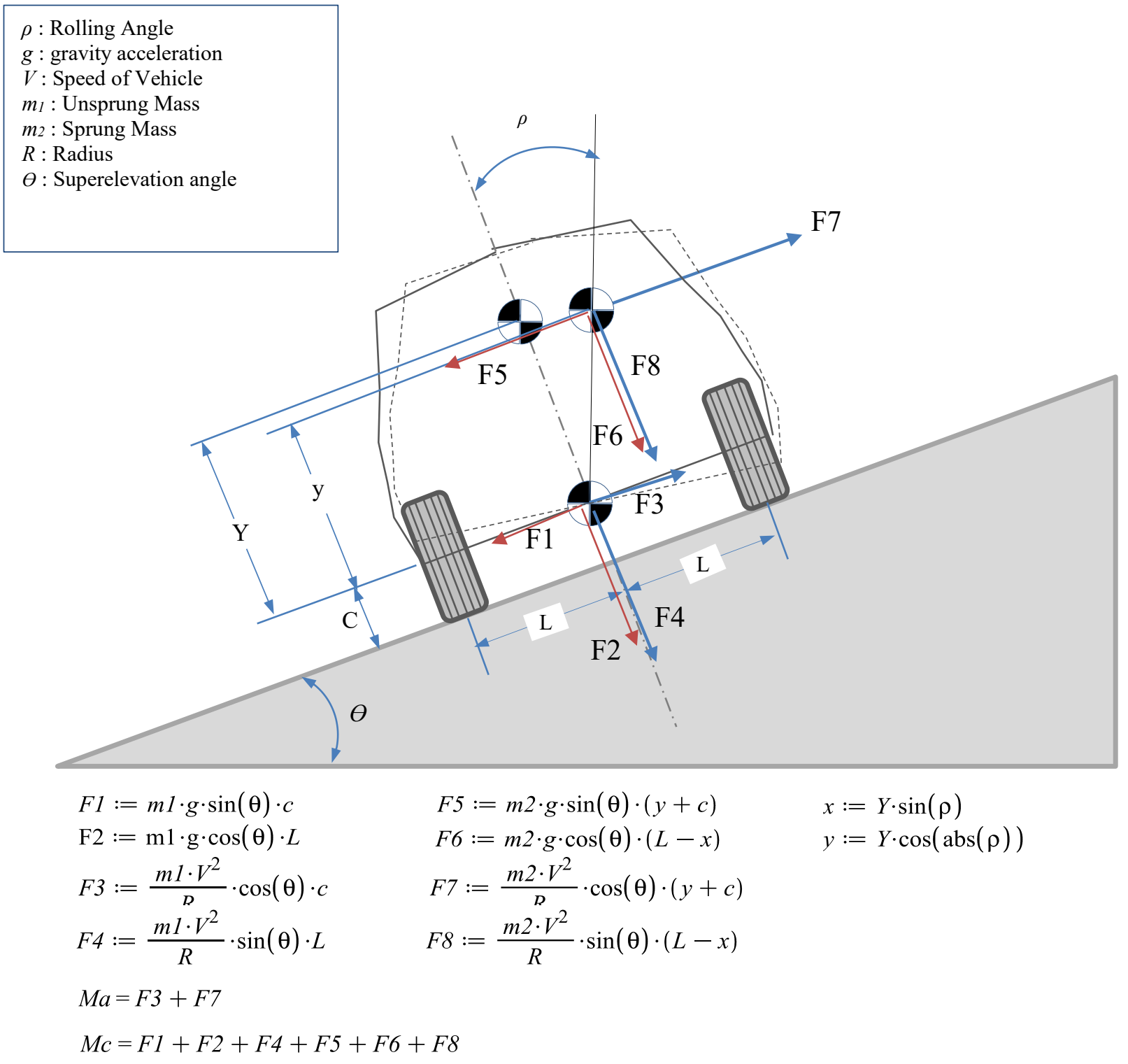

(The Fs are the moments caused by these forces).

A-3: The Applied and Counterbalance Moments. 


\section{$\underline{\text { APPENDIX B }}$}

Relationship between Mean and 85th percentile of Speed and Headway in Mountainous Terrain 


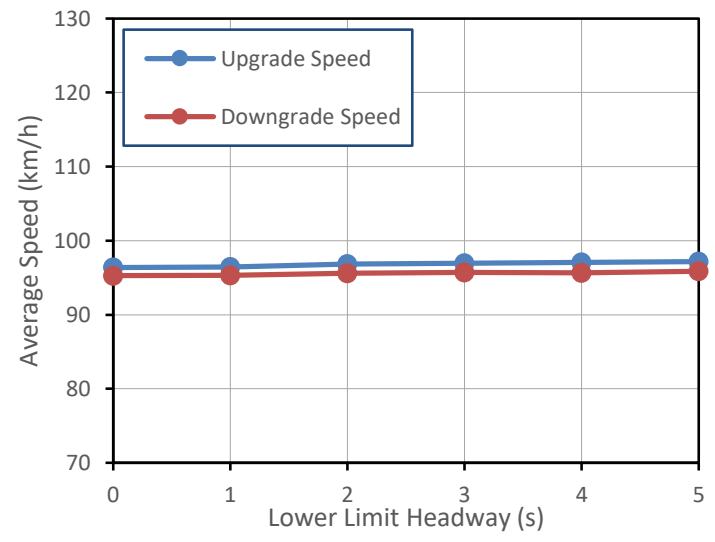

(a) Mean $V_{D M C}$.

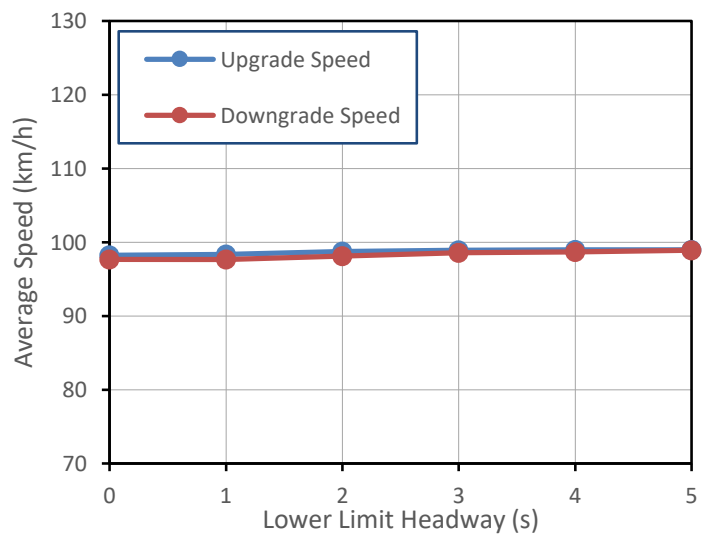

(c) Mean $V_{a p p}$.

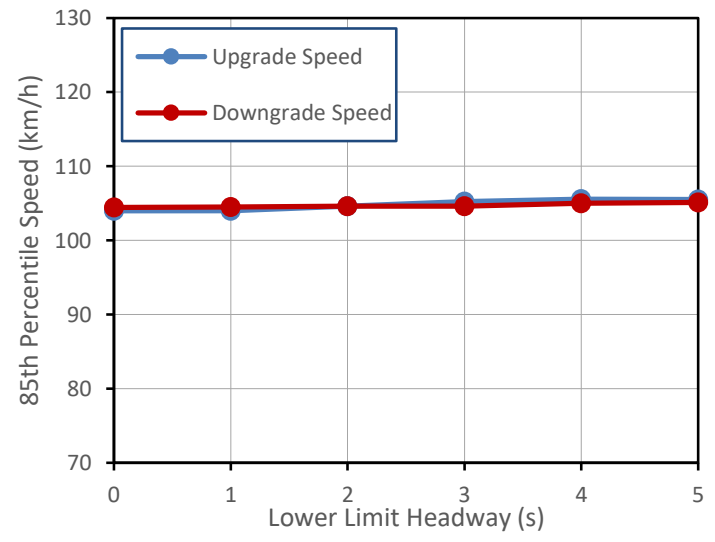

(b) $85^{\text {th }}$ Percentile $V_{D M C}$.

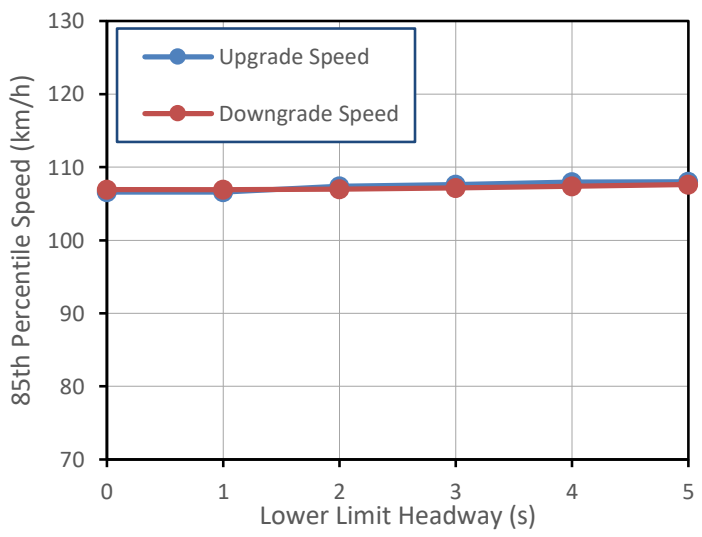

(d) 85th Percentile $V_{\text {app }}$.

B-1: Relationship between Mean and 85th percentile of Speed and Headway (Mountainous Terrain). 


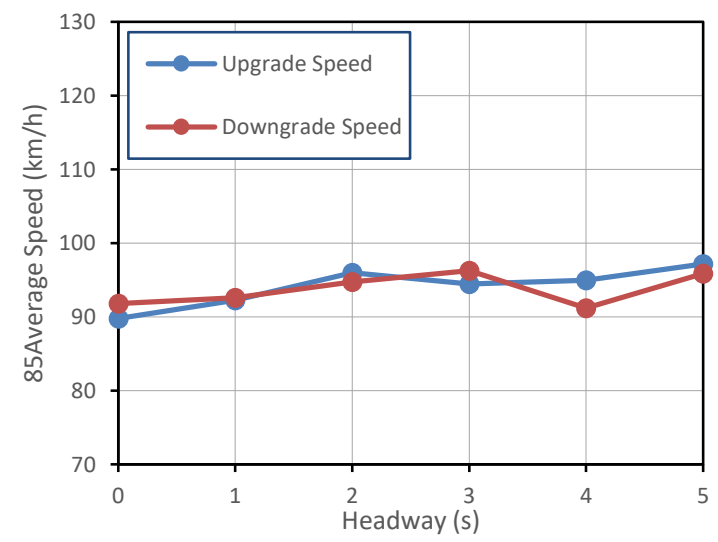

(a) Mean $V_{D M C}$.

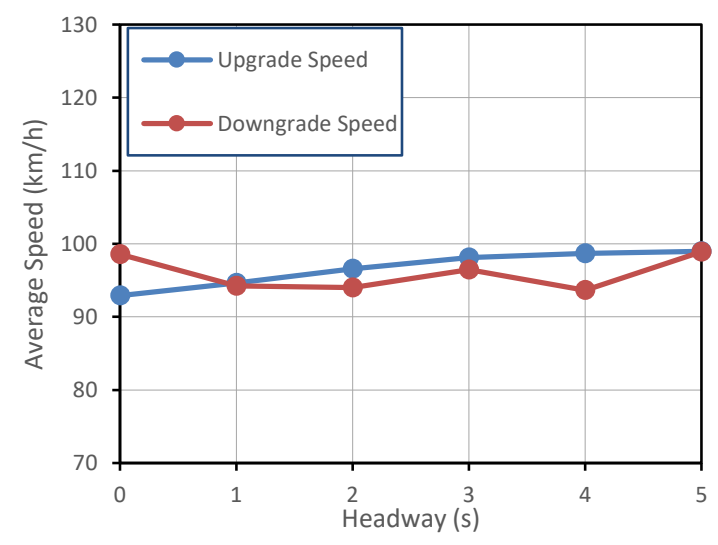

(c) Mean $V_{a p p}$.

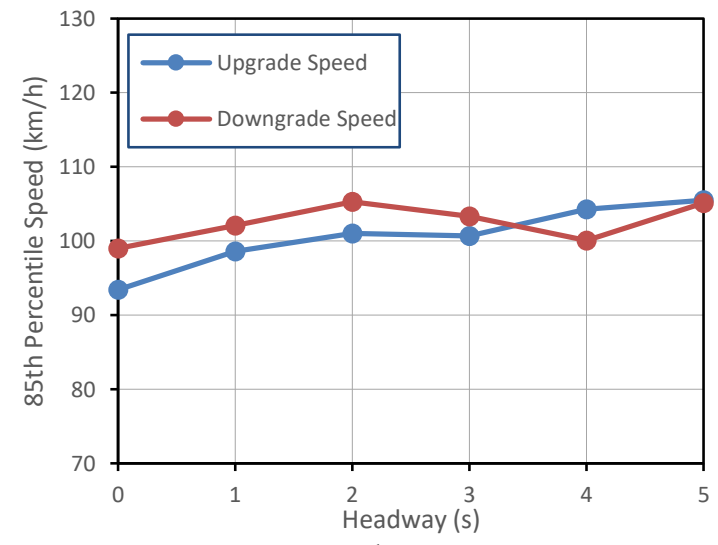

(b) $85^{\text {th }}$ Percentile $V_{D M C}$.

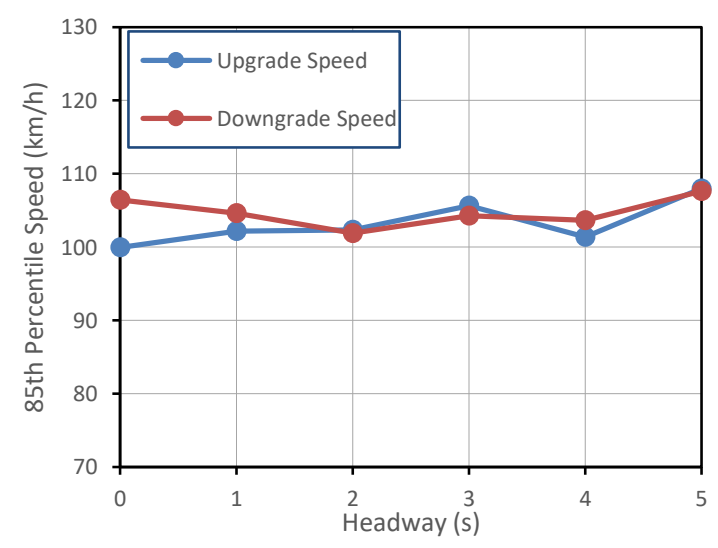

(d) 85th Percentile $V_{a p p}$.

B-2: Mean and 85th percentile of Speed vs. Specific Headway Intervals on Curve and at Approach Tangent (Mountainous Terrain). 


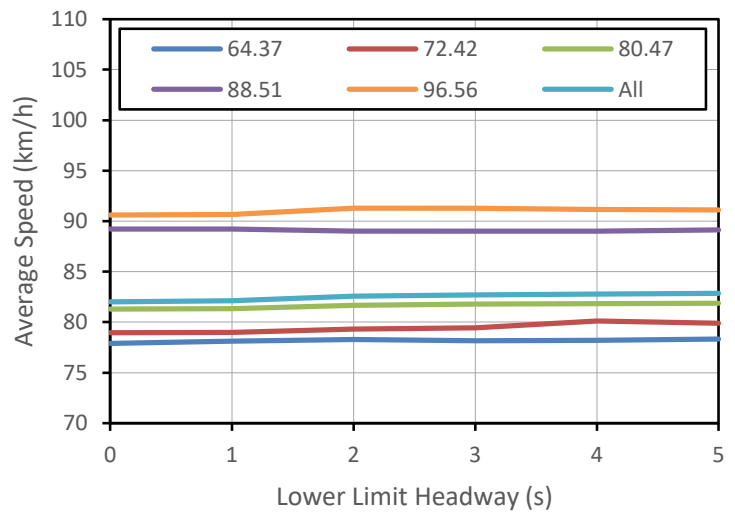

(a) Mean $V_{c r v-a v g}$.

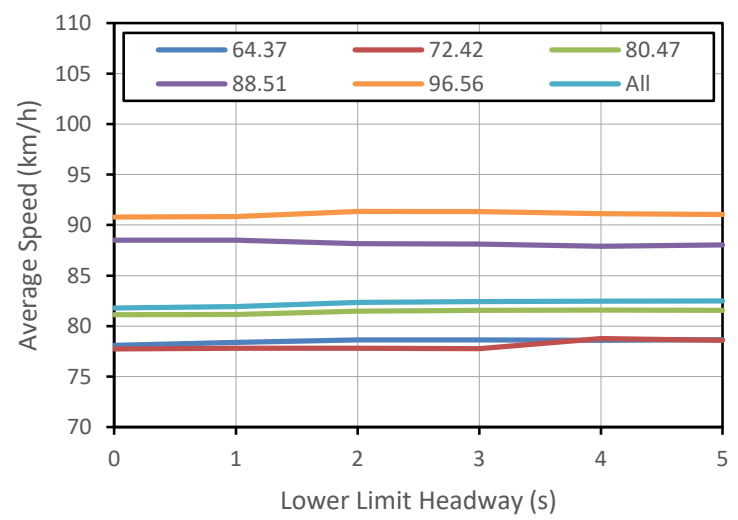

(c) Mean $V_{M C}$.

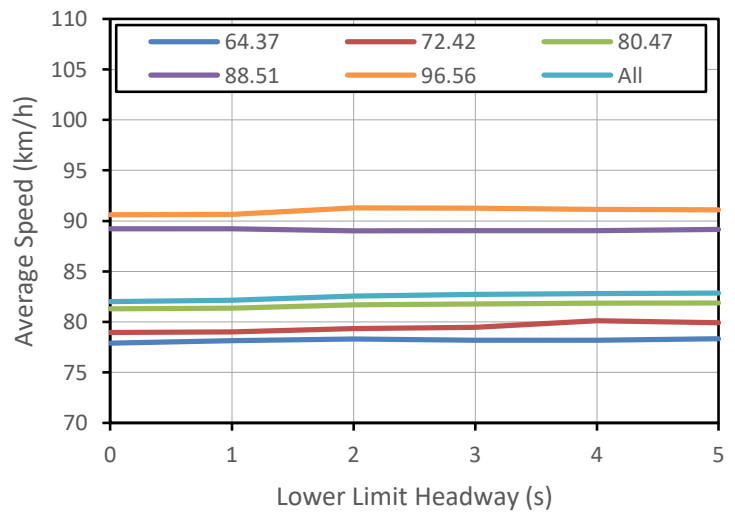

(e) Mean $V_{M C}$.

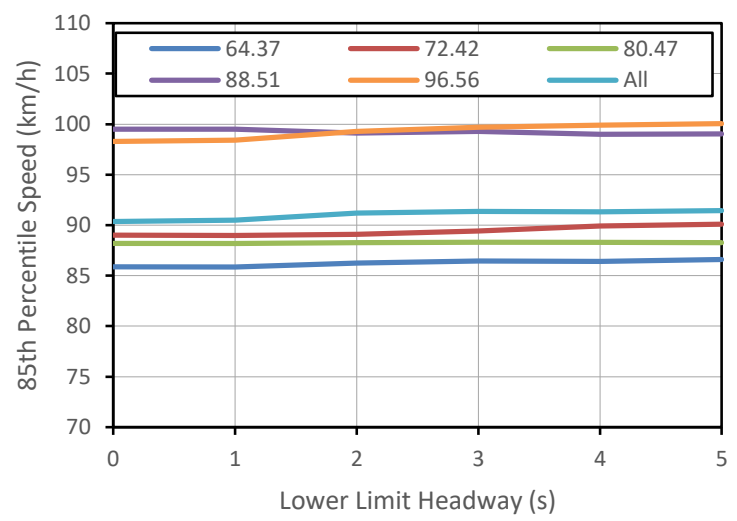

(b) $85^{\text {th }}$ Percentile $V_{c r v-a v g}$.

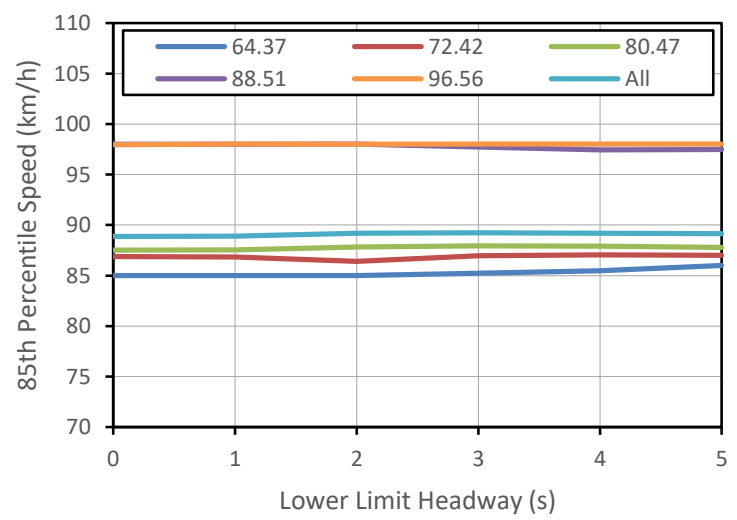

(d) 85th Percentile $V_{M C}$.

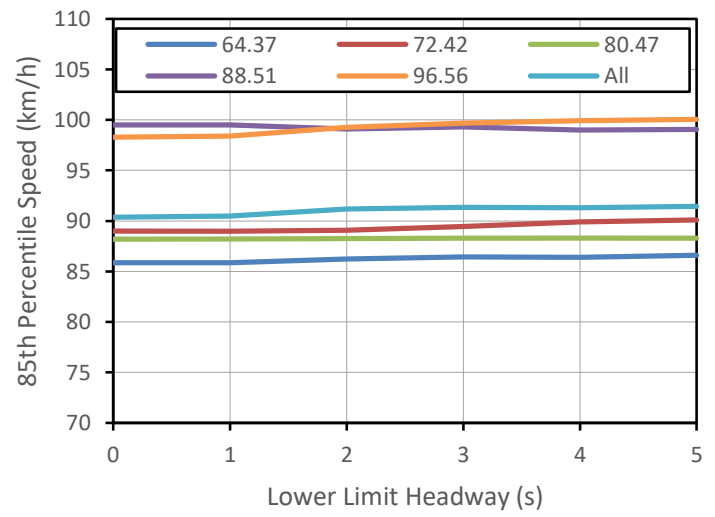

(f) 85th Percentile $V_{M C}$.

B-3: Relationship between Mean and 85th percentile of Speed Parameters $V_{c r v-a v g}, V_{M C}$, and $V_{T M C}$, and Headway (Rolling Terrain). 

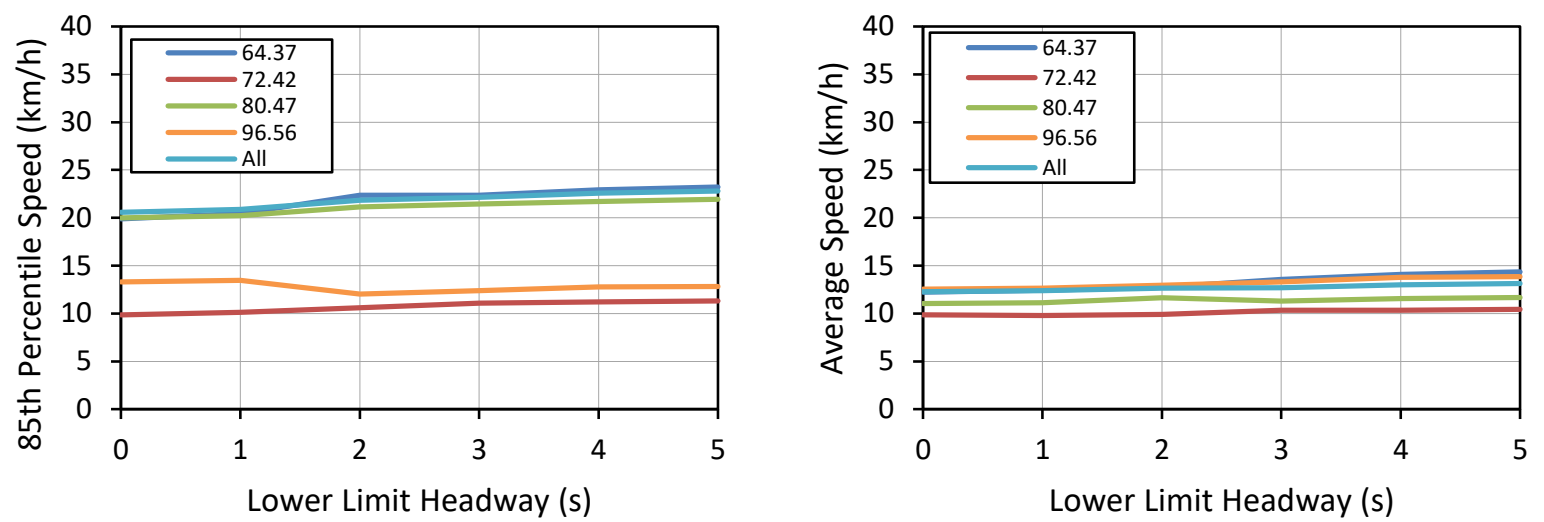

Standard Deviation for $V_{a p p}$ (Headway Category) Standard Deviation for $V_{a p p}$ (headway Interval)
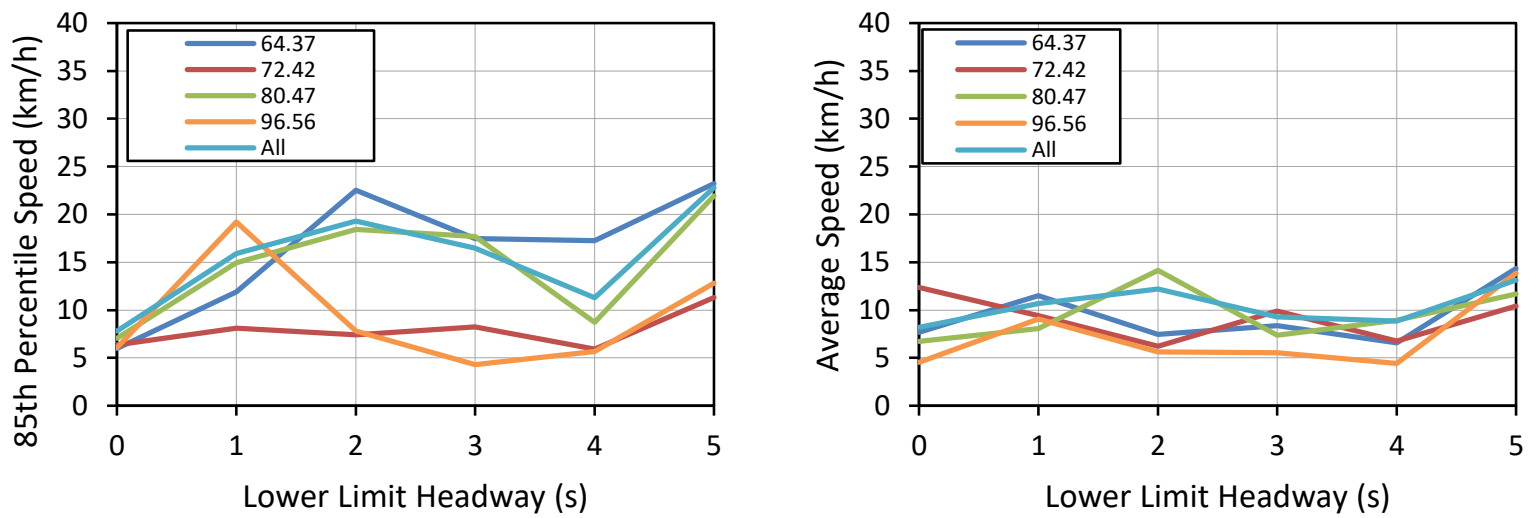

Standard Deviation for $V_{D M C}$ (Headway Category) Standard Deviation for $V_{D M C}$ (headway Interval)

B-4: Standard Deviation of Speed vs. Specific Headway Intervals on Curve and at Approach Tangent (Rolling Terrain) 


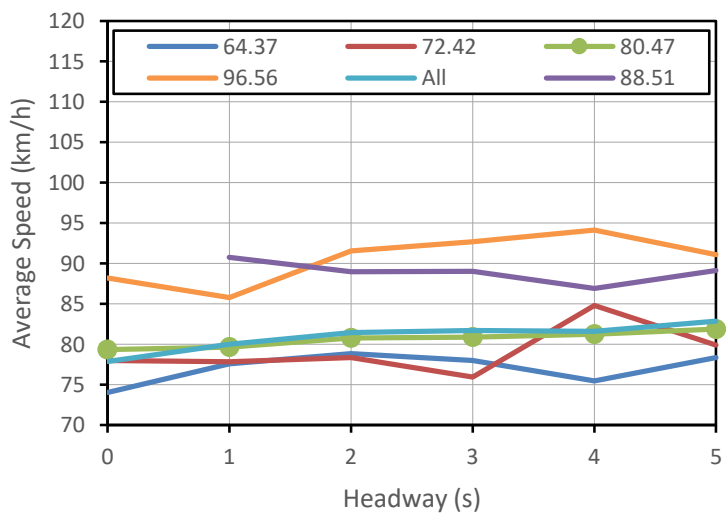

(a) Mean $V_{c r v-a v g}$.

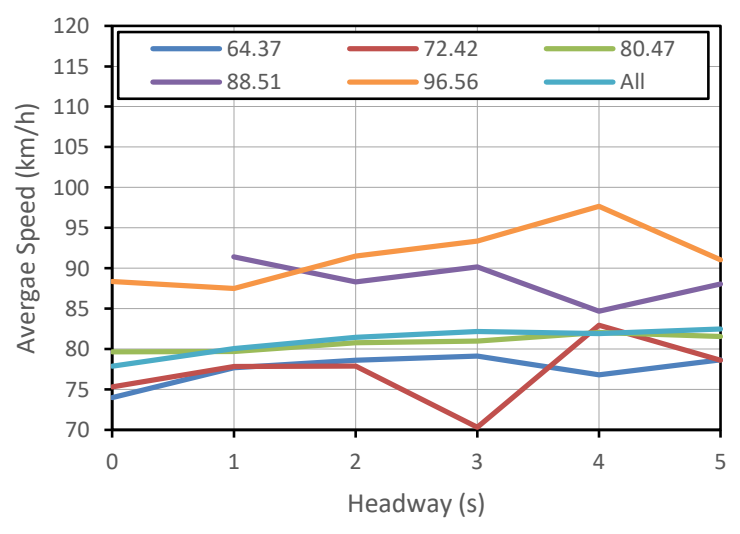

(c) Mean $V_{M C}$.

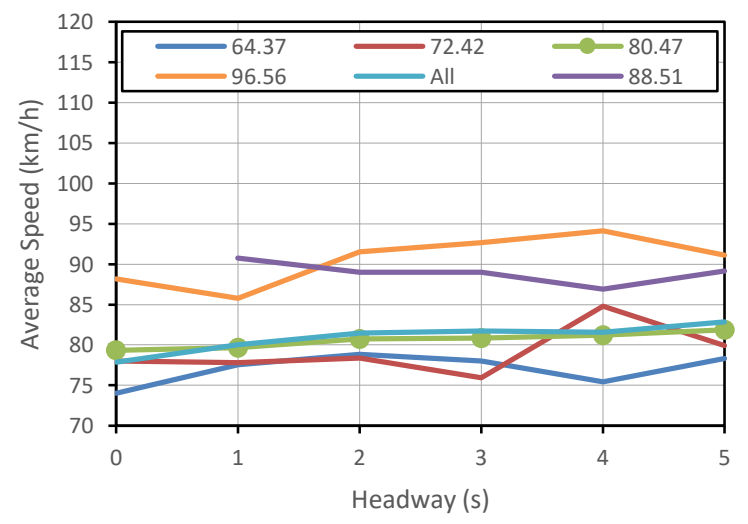

(e) Mean $V_{M C}$.

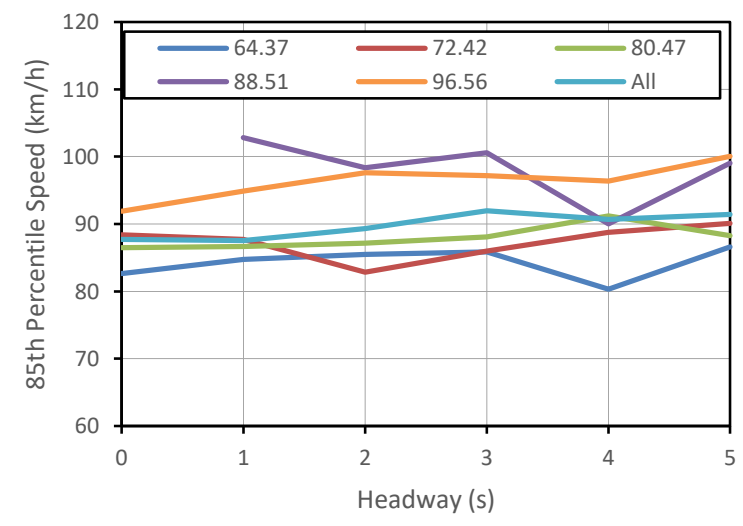

(b) $85^{\text {th }}$ Percentile $V_{c r v-a v g}$.

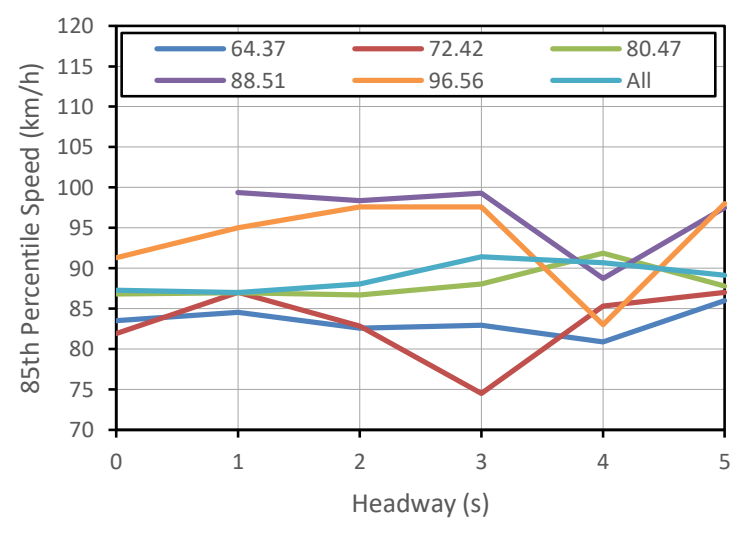

(d) 85 th Percentile $V_{M C}$.

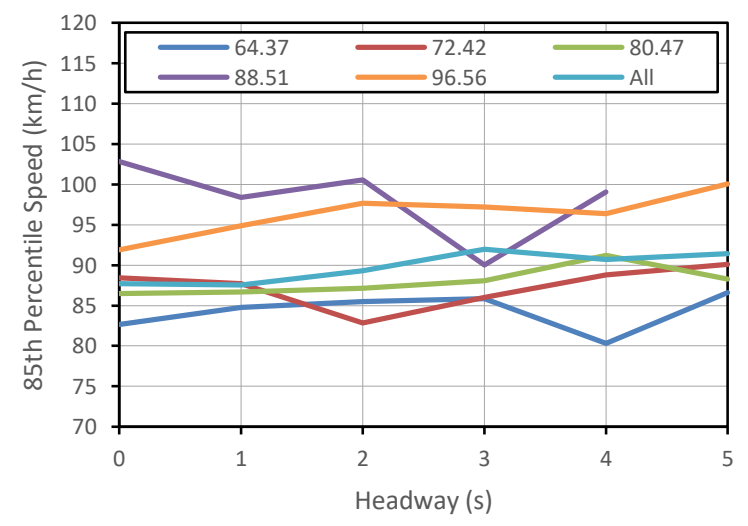

(f) 85th Percentile $V_{M C}$.

B-5: Relationship between Mean and 85th percentile of Speed Parameters $V_{c r v-a v g}, V_{M C}$, and $V_{T M C}$, and Specific Headway Intervals (Rolling Terrain). 


\title{
APPENDIX C
}

\author{
Normality Test Results
}


Table C1: Kolmogorov-Smirnov and Shapiro-Wilk tests for Rolling Terrain for Headway 3 s.

\begin{tabular}{|c|c|c|c|c|c|c|c|c|c|c|c|c|c|c|c|c|}
\hline $\begin{array}{c}\text { Curve } \\
\text { ID }\end{array}$ & Test & $V_{c r v-a v g}$ & $V_{c r v-\min }$ & $V_{c r v-\max }$ & $V_{B C}$ & $V_{T B C}$ & $V_{D B C}$ & $V_{M C}$ & $V_{T M C}$ & $V_{D M C}$ & $V_{E C}$ & $V_{T E C}$ & $V_{D E C}$ & $a_{L}$ & $\mid V_{c m f-a v g}$ & $V_{c m f-m i n}$ \\
\hline \multirow[t]{4}{*}{ RT 01} & K-S (Statistic) & 0.060 & 0.074 & 0.054 & 0.056 & 0.059 & 0.063 & 0.072 & 0.073 & 0.073 & 0.054 & 0.046 & 0.069 & 0.110 & 0.072 & 0.071 \\
\hline & K-S (p-value) & $0.200^{*}$ & $0.200^{*}$ & $0.200^{*}$ & $0.200^{*}$ & $0.200^{*}$ & $0.200^{*}$ & $0.200^{*}$ & $0.200^{*}$ & $0.200^{*}$ & $0.200^{*}$ & $0.200^{*}$ & $0.200^{*}$ & $0.200^{*}$ & $0.200^{*}$ & $0.200^{*}$ \\
\hline & S-W (Statistic) & 0.991 & 0.987 & 0.992 & 0.991 & 0.992 & 0.992 & 0.984 & 0.984 & 0.984 & 0.988 & 0.988 & 0.988 & 0.975 & 0.985 & 0.985 \\
\hline & S-W (p-value) & 0.975 & 0.887 & 0.990 & 0.983 & 0.991 & 0.990 & 0.795 & 0.783 & 0.801 & 0.910 & 0.929 & 0.917 & 0.433 & 0.840 & 0.846 \\
\hline \multirow[t]{4}{*}{ RT 02} & K-S (Statistic) & 0.076 & 0.078 & 0.082 & 0.084 & 0.080 & 0.087 & 0.086 & 0.082 & 0.084 & 0.080 & 0.083 & 0.080 & 0.094 & 0.072 & 0.077 \\
\hline & K-S (p-value) & $0.200^{*}$ & $0.200^{*}$ & $0.200^{*}$ & $0.200^{*}$ & $0.200^{*}$ & $0.200^{*}$ & $0.200^{*}$ & $0.200^{*}$ & $0.200^{*}$ & $0.200^{*}$ & $0.200^{*}$ & $0.200^{*}$ & $0.200^{*}$ & $0.200^{*}$ & $0.200^{*}$ \\
\hline & S-W (Statistic) & 0.976 & 0.977 & 0.979 & 0.983 & 0.984 & 0.982 & 0.976 & 0.976 & 0.977 & 0.983 & 0.983 & 0.979 & 0.939 & 0.984 & 0.984 \\
\hline & S-W (p-value) & 0.411 & 0.452 & 0.500 & 0.666 & 0.714 & 0.638 & 0.396 & 0.414 & 0.421 & 0.699 & 0.667 & 0.492 & 0.012 & 0.746 & 0.735 \\
\hline \multirow[t]{4}{*}{ RT 03} & K-S (Statistic) & 0.097 & 0.074 & 0.151 & 0.142 & 0.130 & 0.124 & 0.073 & 0.076 & 0.078 & 0.075 & 0.079 & 0.086 & 0.139 & 0.083 & 0.081 \\
\hline & K-S (p-value) & $0.200^{*}$ & $0.200^{*}$ & 0.013 & 0.027 & 0.059 & 0.086 & $0.200^{*}$ & $0.200^{*}$ & $0.200^{*}$ & $0.200^{*}$ & $0.200^{*}$ & $0.200^{*}$ & 0.032 & $0.200^{*}$ & $0.200^{*}$ \\
\hline & S-W (Statistic) & 0.969 & 0.975 & 0.954 & 0.945 & 0.947 & 0.950 & 0.971 & 0.968 & 0.968 & 0.984 & 0.981 & 0.972 & 0.939 & 0.977 & 0.977 \\
\hline & S-W (p-value) & 0.279 & 0.450 & 0.077 & 0.037 & 0.042 & 0.056 & 0.322 & 0.264 & 0.253 & 0.788 & 0.674 & 0.363 & 0.022 & 0.506 & 0.522 \\
\hline \multirow[t]{4}{*}{ RT 04} & K-S (Statistic) & 0.131 & 0.137 & 0.118 & 0.164 & 0.169 & 0.180 & 0.110 & 0.103 & 0.104 & 0.091 & 0.111 & 0.141 & 0.113 & 0.110 & 0.115 \\
\hline & K-S (p-value) & $0.200^{*}$ & 0.194 & $0.200^{*}$ & 0.052 & 0.040 & 0.020 & $0.200^{*}$ & $0.200^{*}$ & $0.200^{*}$ & $0.200^{*}$ & $0.200^{*}$ & 0.160 & $0.200^{*}$ & $0.200^{*}$ & $0.200^{*}$ \\
\hline & S-W (Statistic) & 0.945 & 0.955 & 0.942 & 0.936 & 0.937 & 0.937 & 0.953 & 0.955 & 0.952 & 0.976 & 0.971 & 0.953 & 0.981 & 0.954 & 0.956 \\
\hline & S-W (p-value) & 0.150 & 0.260 & 0.125 & 0.086 & 0.095 & 0.093 & 0.238 & 0.262 & 0.224 & 0.734 & 0.605 & 0.233 & 0.879 & 0.243 & 0.280 \\
\hline \multirow[t]{4}{*}{ RT 05} & K-S (Statistic) & 0.089 & 0.116 & 0.073 & 0.088 & 0.066 & 0.066 & 0.084 & 0.086 & 0.077 & 0.135 & 0.135 & 0.092 & 0.087 & 0.086 & 0.083 \\
\hline & K-S (p-value) & $0.200^{*}$ & 0.064 & $0.200^{*}$ & $0.200^{*}$ & $0.200^{*}$ & $0.200^{*}$ & $0.200^{*}$ & $0.200^{*}$ & $0.200^{*}$ & 0.014 & 0.014 & $0.200^{*}$ & $0.200^{*}$ & $0.200^{*}$ & $0.200^{*}$ \\
\hline & S-W (Statistic) & 0.975 & 0.960 & 0.979 & 0.986 & 0.987 & 0.984 & 0.972 & 0.972 & 0.973 & 0.946 & 0.949 & 0.973 & 0.980 & 0.976 & 0.970 \\
\hline & S-W (p-value) & 0.309 & 0.062 & 0.453 & 0.747 & 0.793 & 0.689 & 0.218 & 0.227 & 0.256 & 0.015 & 0.021 & 0.253 & 0.504 & 0.323 & 0.193 \\
\hline \multirow[t]{4}{*}{ RT 06} & K-S (Statistic) & 0.086 & 0.085 & 0.082 & 0.08 & 0.105 & 0.107 & 0.099 & 0.10 & 0.094 & 0.124 & 0.120 & 0.073 & 0.102 & 0.102 & 0.098 \\
\hline & K-S ( $p$-value) & $0.200^{*}$ & $0.200^{*}$ & $0.200^{*}$ & $0.200^{*}$ & $0.200^{*}$ & $0.200^{*}$ & $0.200^{*}$ & $0.200^{*}$ & $0.200^{*}$ & 0.0 & 0.064 & $0.200^{*}$ & $0.200^{*}$ & $0.200^{*}$ & $0.200^{*}$ \\
\hline & S-n & & & 0.97 & & 0.9 & & & & & & 0.9 & & 0.959 & 0.9 & 0.968 \\
\hline & S-W & 0. & 0.394 & 0.216 & 0.42 & 0.296 & 0.258 & 0.549 & 0.56 & 0.562 & 0.218 & 0.4 & 0.683 & 0.076 & 0.239 & 0.184 \\
\hline \multirow[t]{4}{*}{ RT 07} & K-S (Statistic) & 0.165 & 0.180 & 0.106 & 0.1 & 0.159 & 0.173 & 0.140 & 0.144 & 0.147 & 0.128 & 0.121 & 0.160 & 0.169 & 0.149 & 0.140 \\
\hline & K-S (p-value) & $0.200^{*}$ & $0.200^{*}$ & $0.200^{*}$ & $0.200^{*}$ & $0.200^{*}$ & $0.200^{*}$ & $0.200^{*}$ & $0.200^{*}$ & $0.200^{*}$ & $0.200^{*}$ & $0.200^{*}$ & $0.200^{*}$ & $0.200^{*}$ & $0.200^{*}$ & $0.200^{*}$ \\
\hline & S-W (Statistic) & 0.926 & 0.928 & 0.969 & 0.909 & 0.912 & 0.914 & 0.944 & 0.939 & 0.935 & 0.980 & 0.985 & 0.937 & 0.940 & 0.920 & 0.934 \\
\hline & S-W (p-value) & 0.303 & 0.325 & 0.880 & 0.177 & 0.194 & 0.210 & 0.516 & 0.450 & 0.391 & 0.977 & 0.995 & 0.415 & 0.460 & 0.248 & 0.387 \\
\hline RT 08 & K-S (Statistic) & 0.155 & 0.141 & 0.135 & 0.155 & 0.141 & 0.130 & 0.160 & 0.165 & 0.177 & 0.140 & 0.154 & 0.147 & 0.173 & 0.138 & 0.158 \\
\hline
\end{tabular}


Table C1: Kolmogorov-Smirnov and Shapiro-Wilk tests for Rolling Terrain for Headway 3 s- Cont.

\begin{tabular}{|c|c|c|c|c|c|c|c|c|c|c|c|c|c|c|c|c|}
\hline $\begin{array}{l}\text { Curve } \\
\text { ID }\end{array}$ & Test & $V_{c r v-a v g}$ & $V_{c r v-\min }$ & $V_{c r v-\max }$ & $V_{B C}$ & $V_{T B C}$ & $V_{D B C}$ & $V_{M C}$ & $V_{T M C}$ & $V_{D M C}$ & $V_{E C}$ & $V_{T E C}$ & $V_{D E C}$ & $a_{L}$ & $V_{c m f-a v g}$ & $V_{c m f-\min }$ \\
\hline & K-S (p-value) & $0.200^{*}$ & $0.200^{*}$ & $0.200^{*}$ & $0.200^{*}$ & $0.200^{*}$ & $0.200^{*}$ & $0.200^{*}$ & $0.200^{*}$ & $0.200^{*}$ & $0.200^{*}$ & $0.200^{*}$ & $0.200^{*}$ & $0.200^{*}$ & $0.200^{*}$ & $0.200^{*}$ \\
\hline & S-W (Statistic) & 0.918 & 0.923 & 0.938 & 0.931 & 0.931 & 0.934 & 0.912 & 0.917 & 0.918 & 0.940 & 0.937 & 0.911 & 0.933 & 0.945 & 0.945 \\
\hline & S-W (p-value) & 0.205 & 0.245 & 0.395 & 0.318 & 0.314 & 0.345 & 0.171 & 0.201 & 0.203 & 0.413 & 0.383 & 0.165 & 0.337 & 0.487 & 0.481 \\
\hline \multirow[t]{4}{*}{ RT 09} & K-S (Statistic) & 0.175 & 0.220 & 0.175 & 0.146 & 0.137 & 0.130 & 0.151 & 0.150 & 0.163 & 0.185 & 0.173 & 0.213 & 0.207 & 0.185 & 0.209 \\
\hline & K-S (p-value) & $0.200^{*}$ & 0.086 & $0.200^{*}$ & $0.200^{*}$ & $0.200^{*}$ & $0.200^{*}$ & $0.200^{*}$ & $0.200^{*}$ & $0.200^{*}$ & $0.200^{*}$ & $0.200^{*}$ & 0.110 & 0.131 & $0.200^{*}$ & 0.125 \\
\hline & S-W (Statistic) & 0.942 & 0.952 & 0.911 & 0.938 & 0.945 & 0.949 & 0.957 & 0.958 & 0.956 & 0.910 & 0.930 & 0.946 & 0.941 & 0.947 & 0.950 \\
\hline & S-W (p-value) & 0.490 & 0.634 & 0.187 & 0.430 & 0.524 & 0.577 & 0.703 & 0.724 & 0.684 & 0.182 & 0.345 & 0.544 & 0.464 & 0.557 & 0.599 \\
\hline \multirow[t]{4}{*}{ RT 10} & K-S (Statistic) & 0.144 & 0.179 & 0.111 & 0.094 & 0.121 & 0.136 & 0.146 & 0.141 & 0.139 & 0.158 & 0.145 & 0.147 & 0.122 & 0.157 & 0.180 \\
\hline & K-S (p-value) & 0.009 & 0.000 & 0.155 & $0.200^{*}$ & 0.056 & 0.018 & 0.007 & 0.011 & 0.014 & 0.002 & 0.008 & 0.007 & 0.053 & 0.003 & 0.000 \\
\hline & S-W (Statistic) & 0.885 & 0.898 & 0.877 & 0.927 & 0.924 & 0.918 & 0.863 & 0.866 & 0.870 & 0.890 & 0.892 & 0.881 & 0.922 & 0.884 & 0.885 \\
\hline & S-W (p-value) & 0.000 & 0.000 & 0.000 & 0.003 & 0.003 & 0.002 & 0.000 & 0.000 & 0.000 & 0.000 & 0.000 & 0.000 & 0.002 & 0.000 & 0.000 \\
\hline \multirow[t]{4}{*}{ RT 11} & K-S (Statistic) & 0.098 & 0.094 & 0.103 & 0.080 & 0.085 & 0.096 & 0.125 & 0.126 & 0.107 & 0.123 & 0.125 & 0.108 & 0.099 & 0.127 & 0.112 \\
\hline & K-S (p-value) & $0.200^{*}$ & $0.200^{*}$ & $0.200^{*}$ & $0.200^{*}$ & $0.200^{*}$ & $0.200^{*}$ & 0.080 & 0.079 & $0.200^{*}$ & 0.091 & 0.084 & $0.200^{*}$ & $0.200^{*}$ & 0.072 & $0.200^{*}$ \\
\hline & S-W (Statistic) & 0.958 & 0.956 & 0.969 & 0.961 & 0.964 & 0.967 & 0.952 & 0.951 & 0.954 & 0.952 & 0.958 & 0.956 & 0.970 & 0.960 & 0.955 \\
\hline & S-W (p-value) & 0.113 & 0.095 & 0.276 & 0.143 & 0.179 & 0.231 & 0.067 & 0.062 & 0.076 & 0.066 & 0.105 & 0.093 & 0.297 & 0.130 & 0.083 \\
\hline \multirow[t]{4}{*}{ RT 12} & K-S (Statistic) & 0.128 & 0.158 & 0.123 & 0.109 & 0.097 & 0.092 & 0.125 & 0.125 & 0.126 & 0.115 & 0.106 & 0.119 & 0.126 & 0.129 & 0.131 \\
\hline & K-S (p-value) & $0.200^{*}$ & 0.186 & $0.200^{*}$ & $0.200^{*}$ & $0.200^{*}$ & $0.200^{*}$ & $0.200^{*}$ & $0.200^{*}$ & $0.200^{*}$ & $0.200^{*}$ & $0.200^{*}$ & $0.200^{*}$ & $0.200^{*}$ & $0.200^{*}$ & $0.200^{*}$ \\
\hline & S-W (Statistic) & 0.945 & 0.956 & 0.969 & 0.978 & 0.979 & 0.968 & 0.953 & 0.953 & 0.953 & 0.949 & 0.955 & 0.943 & 0.952 & 0.969 & 0.959 \\
\hline & S-W (p-value) & 0.268 & 0.435 & 0.706 & 0.895 & 0.914 & 0.684 & 0.385 & 0.392 & 0.392 & 0.331 & 0.425 & 0.252 & 0.367 & 0.707 & 0.500 \\
\hline \multirow[t]{4}{*}{ RT 13} & K-S (Statistic) & 0.149 & 0.183 & 0.145 & 0.155 & 0.154 & 0.160 & 0.111 & 0.107 & 0.109 & 0.126 & 0.125 & 0.131 & 0.180 & 0.157 & 0.150 \\
\hline & K-S (p-value) & $0.200^{*}$ & 0.189 & $0.200^{*}$ & $0.200^{*}$ & $0.200^{*}$ & $0.200^{*}$ & $0.200^{*}$ & $0.200^{*}$ & $0.200^{*}$ & $0.200^{*}$ & $0.200^{*}$ & $0.200^{*}$ & $0.200^{\star}$ & $0.200^{*}$ & $0.200^{*}$ \\
\hline & S-W (Statistic) & 0.953 & 0.935 & 0.958 & 0.940 & 0.951 & 0.954 & 0.971 & 0.970 & 0.969 & 0.983 & 0.971 & 0.968 & 0.877 & 0.969 & 0.970 \\
\hline & S-W (p-value) & 0.578 & 0.319 & 0.666 & 0.386 & 0.542 & 0.592 & 0.869 & 0.861 & 0.841 & 0.985 & 0.876 & 0.820 & 0.043 & 0.838 & 0.856 \\
\hline \multirow[t]{4}{*}{ RT 14} & K-S (Statistic) & 0.132 & 0.141 & 0.162 & 0.214 & 0.178 & 0.112 & 0.161 & 0.150 & 0.152 & 0.117 & 0.123 & 0.157 & 0.158 & 0.130 & 0.151 \\
\hline & K-S (p-value) & $0.200^{*}$ & $0.200^{*}$ & $0.200^{*}$ & 0.037 & 0.159 & $0.200^{*}$ & $0.200^{*}$ & $0.200^{*}$ & $0.200^{*}$ & $0.200^{*}$ & $0.200^{*}$ & $0.200^{*}$ & $0.200^{*}$ & $0.200^{*}$ & $0.200^{*}$ \\
\hline & S-W (Statistic) & 0.971 & 0.962 & 0.959 & 0.902 & 0.923 & 0.949 & 0.948 & 0.949 & 0.947 & 0.967 & 0.963 & 0.955 & 0.933 & 0.973 & 0.968 \\
\hline & S-W (p-value) & 0.830 & 0.660 & 0.621 & 0.075 & 0.169 & 0.447 & 0.423 & 0.434 & 0.415 & 0.757 & 0.687 & 0.549 & 0.243 & 0.864 & 0.790 \\
\hline \multirow[t]{2}{*}{ RT 15} & K-S (Statistic) & 0.136 & 0.157 & 0.127 & 0.115 & 0.128 & 0.139 & 0.138 & 0.139 & 0.133 & 0.145 & 0.150 & 0.139 & 0.096 & 0.138 & 0.145 \\
\hline & K-S (p-value) & 0.020 & 0.003 & 0.039 & 0.091 & 0.036 & 0.015 & 0.016 & 0.015 & 0.024 & 0.009 & 0.006 & 0.016 & $0.200^{*}$ & 0.017 & 0.009 \\
\hline
\end{tabular}


Table C1: Kolmogorov-Smirnov and Shapiro-Wilk tests for Rolling Terrain for Headway 3 s- Cont.

\begin{tabular}{|c|c|c|c|c|c|c|c|c|c|c|c|c|c|c|c|c|}
\hline $\begin{array}{l}\text { Curve } \\
\text { ID }\end{array}$ & Test & $V_{c r v-a v g}$ & $V_{c r v-\min }$ & $V_{c r v-\max }$ & $V_{B C}$ & $V_{T B C}$ & $V_{D B C}$ & $V_{M C}$ & $V_{T M C}$ & $V_{D M C}$ & $V_{E C}$ & $V_{T E C}$ & $V_{D E C}$ & $a_{L}$ & $V_{c m f-a v g}$ & $V_{c m f-\min }$ \\
\hline \multirow{5}{*}{ RT 16} & S-W (Statistic) & 0.884 & 0.873 & 0.896 & 0.929 & 0.929 & 0.928 & 0.869 & 0.869 & 0.873 & 0.838 & 0.848 & 0.863 & 0.972 & 0.871 & 0.867 \\
\hline & S-W (p-value) & 0.000 & 0.000 & 0.000 & 0.004 & 0.005 & 0.004 & 0.000 & 0.000 & 0.000 & 0.000 & 0.000 & 0.000 & 0.259 & 0.000 & 0.000 \\
\hline & K-S (Statistic) & 0.145 & 0.226 & 0.081 & 0.135 & 0.139 & 0.134 & 0.104 & 0.113 & 0.110 & 0.304 & 0.286 & 0.121 & 0.118 & 0.140 & 0.139 \\
\hline & K-S (p-value) & 0.170 & 0.001 & $0.200^{*}$ & $0.200^{*}$ & $0.200^{*}$ & $0.200^{*}$ & $0.200^{*}$ & $0.200^{*}$ & $0.200^{*}$ & 0.000 & 0.000 & $0.200^{*}$ & $0.200^{*}$ & $0.200^{*}$ & $0.200^{*}$ \\
\hline & S-W (Statistic) & 0.949 & 0.701 & 0.983 & 0.966 & 0.962 & 0.959 & 0.979 & 0.979 & 0.978 & 0.605 & 0.621 & 0.945 & 0.978 & 0.958 & 0.952 \\
\hline \multirow{3}{*}{ RT 17} & S-W (p-value) & 0.221 & 0.000 & 0.934 & 0.533 & 0.433 & 0.373 & 0.858 & 0.841 & 0.840 & 0.000 & 0.000 & 0.177 & 0.825 & 0.361 & 0.263 \\
\hline & K-S (Statistic) & 0.153 & 0.154 & 0.165 & 0.165 & 0.173 & 0.173 & 0.145 & 0.143 & 0.143 & 0.180 & 0.168 & 0.147 & 0.060 & 0.134 & 0.140 \\
\hline & K-S (p-value) & 0.012 & 0.011 & 0.004 & 0.004 & 0.002 & 0.002 & 0.021 & 0.024 & 0.025 & 0.001 & 0.003 & 0.018 & $0.200^{\star}$ & 0.045 & 0.030 \\
\hline \multirow{5}{*}{ RT 18} & S-W (Statistic) & 0.870 & 0.875 & 0.867 & 0.841 & 0.845 & 0.858 & 0.868 & 0.870 & 0.874 & 0.872 & 0.874 & 0.876 & 0.990 & 0.881 & 0.875 \\
\hline & S-W (p-value) & 0.000 & 0.000 & 0.000 & 0.000 & 0.000 & 0.000 & 0.000 & 0.000 & 0.000 & 0.000 & 0.000 & 0.000 & 0.972 & 0.000 & 0.000 \\
\hline & K-S (Statistic) & 0.116 & 0.124 & 0.110 & 0.089 & 0.106 & 0.113 & 0.145 & 0.144 & 0.139 & 0.111 & 0.115 & 0.112 & 0.117 & 0.155 & 0.145 \\
\hline & K-S (p-value) & $0.200^{*}$ & $0.200^{*}$ & $0.200^{*}$ & $0.200^{*}$ & $0.200^{*}$ & $0.200^{*}$ & 0.151 & 0.161 & 0.193 & $0.200^{*}$ & $0.200^{*}$ & $0.200^{*}$ & $0.200^{*}$ & 0.093 & 0.155 \\
\hline & S-W (Statistic) & 0.944 & 0.949 & 0.959 & 0.961 & 0.954 & 0.945 & 0.939 & 0.940 & 0.941 & 0.972 & 0.973 & 0.956 & 0.961 & 0.942 & 0.941 \\
\hline \multirow{3}{*}{ RT 19} & S-W (p-value) & 0.153 & 0.207 & 0.343 & 0.386 & 0.268 & 0.161 & 0.116 & 0.121 & 0.128 & 0.663 & 0.687 & 0.299 & 0.383 & 0.139 & 0.131 \\
\hline & K-S (Statistic) & 0.130 & 0.123 & 0.125 & 0.117 & 0.121 & 0.112 & 0.117 & 0.116 & 0.109 & 0.141 & 0.167 & 0.126 & 0.194 & 0.136 & 0.130 \\
\hline & K-S (p-value) & $0.200^{*}$ & $0.200^{*}$ & $0.200^{*}$ & $0.200^{*}$ & $0.200^{*}$ & $0.200^{*}$ & $0.200^{*}$ & $0.200^{*}$ & $0.200^{*}$ & 0.196 & 0.060 & $0.200^{*}$ & 0.013 & $0.200^{*}$ & $0.200^{*}$ \\
\hline \multirow{5}{*}{ RT 20} & S-W (Statistic) & 0.970 & 0.954 & 0.941 & 0.943 & 0.938 & 0.947 & 0.960 & 0.960 & 0.963 & 0.901 & 0.905 & 0.966 & 0.938 & 0.959 & 0.958 \\
\hline & S-W (p-value) & 0.619 & 0.281 & 0.142 & 0.162 & 0.122 & 0.202 & 0.391 & 0.394 & 0.448 & 0.017 & 0.020 & 0.532 & 0.119 & 0.371 & 0.360 \\
\hline & K-S (Statistic) & 0.131 & 0.173 & 0.137 & 0.184 & 0.176 & 0.172 & 0.125 & 0.125 & 0.125 & 0.123 & 0.127 & 0.134 & 0.092 & 0.140 & 0.152 \\
\hline & K-S (p-value) & 0.188 & 0.019 & 0.144 & 0.009 & 0.015 & 0.020 & $0.200^{*}$ & $0.200^{*}$ & $0.200^{*}$ & $0.200^{*}$ & $0.200^{*}$ & 0.168 & $0.200^{*}$ & 0.128 & 0.067 \\
\hline & S-W (Statistic) & 0.967 & 0.952 & 0.966 & 0.942 & 0.946 & 0.948 & 0.968 & 0.968 & 0.966 & 0.974 & 0.973 & 0.971 & 0.972 & 0.957 & 0.958 \\
\hline \multirow{3}{*}{ RT 21} & S-W (p-value) & 0.442 & 0.173 & 0.407 & 0.095 & 0.124 & 0.140 & 0.469 & 0.477 & 0.414 & 0.628 & 0.606 & 0.540 & 0.561 & 0.241 & 0.256 \\
\hline & K-S (Statistic) & 0.103 & 0.103 & 0.125 & 0.114 & 0.131 & 0.114 & 0.111 & 0.120 & 0.121 & 0.113 & 0.116 & 0.105 & 0.116 & 0.103 & 0.109 \\
\hline & K-S (p-value) & $0.200^{*}$ & $0.200^{*}$ & 0.053 & 0.130 & 0.035 & 0.142 & 0.174 & 0.075 & 0.072 & 0.159 & 0.097 & $0.200^{*}$ & 0.094 & $0.200^{*}$ & 0.197 \\
\hline \multirow{5}{*}{ RT 22} & S-W (Statistic) & 0.932 & 0.947 & 0.931 & 0.941 & 0.939 & 0.938 & 0.934 & 0.933 & 0.931 & 0.955 & 0.952 & 0.932 & 0.965 & 0.937 & 0.935 \\
\hline & S-W (p-value) & 0.008 & 0.028 & 0.007 & 0.016 & 0.013 & 0.012 & 0.008 & 0.008 & 0.006 & 0.057 & 0.044 & 0.007 & 0.159 & 0.011 & 0.010 \\
\hline & K-S (Statistic) & 0.125 & 0.107 & 0.119 & 0.115 & 0.107 & 0.117 & 0.105 & 0.109 & 0.112 & 0.112 & 0.115 & 0.133 & 0.119 & 0.127 & 0.118 \\
\hline & K-S (p-value) & 0.080 & $0.200^{*}$ & 0.125 & 0.172 & $0.200^{*}$ & 0.153 & $0.200^{*}$ & $0.200^{*}$ & $0.200^{*}$ & $0.200^{*}$ & 0.172 & 0.049 & 0.134 & 0.072 & 0.144 \\
\hline & S-W (Statistic) & 0.980 & 0.984 & 0.968 & 0.981 & 0.982 & 0.984 & 0.986 & 0.985 & 0.985 & 0.974 & 0.975 & 0.982 & 0.953 & 0.982 & 0.986 \\
\hline
\end{tabular}


Table C1: Kolmogorov-Smirnov and Shapiro-Wilk tests for Rolling Terrain for Headway $3 \mathrm{~s}-$ Cont.

\begin{tabular}{|c|c|c|c|c|c|c|c|c|c|c|c|c|c|c|c|c|}
\hline $\begin{array}{c}\text { Curve } \\
\text { ID }\end{array}$ & Test & $V_{c r v-a v g}$ & $V_{c r v-\min }$ & $V_{c r v-\max }$ & $V_{B C}$ & $V_{T B C}$ & $V_{D B C}$ & $V_{M C}$ & $V_{T M C}$ & $V_{D M C}$ & $V_{E C}$ & $V_{T E C}$ & $V_{D E C}$ & $a_{L}$ & $V_{c m f-a v g}$ & $V_{c m f-\min }$ \\
\hline & S-W (p-value) & 0.632 & 0.784 & 0.266 & 0.687 & 0.728 & 0.780 & 0.864 & 0.837 & 0.823 & 0.423 & 0.459 & 0.717 & 0.074 & 0.727 & 0.867 \\
\hline \multirow[t]{4}{*}{ RT 23} & K-S (Statistic) & 0.119 & 0.119 & 0.131 & 0.119 & 0.100 & 0.078 & 0.133 & 0.130 & 0.128 & 0.134 & 0.124 & 0.098 & 0.127 & 0.079 & 0.096 \\
\hline & K-S (p-value) & $0.200^{*}$ & $0.200^{*}$ & $0.200^{*}$ & $0.200^{*}$ & $0.200^{*}$ & $0.200^{*}$ & $0.200^{*}$ & $0.200^{*}$ & $0.200^{*}$ & $0.200^{*}$ & $0.200^{*}$ & $0.200^{*}$ & $0.200^{*}$ & $0.200^{*}$ & $0.200^{*}$ \\
\hline & S-W (Statistic) & 0.979 & 0.976 & 0.962 & 0.948 & 0.953 & 0.971 & 0.963 & 0.962 & 0.969 & 0.943 & 0.950 & 0.973 & 0.981 & 0.977 & 0.974 \\
\hline & S-W (p-value) & 0.924 & 0.873 & 0.589 & 0.341 & 0.415 & 0.775 & 0.609 & 0.579 & 0.735 & 0.273 & 0.366 & 0.816 & 0.941 & 0.886 & 0.828 \\
\hline \multirow[t]{4}{*}{ RT 24} & K-S (Statistic) & 0.135 & 0.162 & 0.170 & 0.154 & 0.147 & 0.176 & 0.151 & 0.153 & 0.144 & 0.161 & 0.163 & 0.158 & 0.106 & 0.119 & 0.128 \\
\hline & K-S (p-value) & $0.200^{*}$ & 0.092 & 0.062 & 0.128 & 0.173 & 0.045 & 0.143 & 0.135 & 0.191 & 0.092 & 0.084 & 0.110 & $0.200^{*}$ & $0.200^{*}$ & $0.200^{*}$ \\
\hline & S-W (Statistic) & 0.934 & 0.931 & 0.942 & 0.916 & 0.931 & 0.943 & 0.925 & 0.925 & 0.929 & 0.954 & 0.953 & 0.931 & 0.964 & 0.949 & 0.949 \\
\hline & S-W (p-value) & 0.105 & 0.090 & 0.165 & 0.042 & 0.090 & 0.170 & 0.067 & 0.068 & 0.084 & 0.300 & 0.293 & 0.092 & 0.494 & 0.237 & 0.240 \\
\hline \multirow[t]{4}{*}{ RT 25} & K-S (Statistic) & 0.108 & 0.168 & 0.083 & 0.095 & 0.116 & 0.108 & 0.108 & 0.101 & 0.107 & 0.171 & 0.125 & 0.119 & 0.117 & 0.132 & 0.135 \\
\hline & K-S (p-value) & $0.200^{*}$ & 0.077 & $0.200^{*}$ & $0.200^{*}$ & $0.200^{*}$ & $0.200^{*}$ & $0.200^{*}$ & $0.200^{*}$ & $0.200^{*}$ & 0.068 & $0.200^{*}$ & $0.200^{*}$ & $0.200^{*}$ & $0.200^{*}$ & $0.200^{*}$ \\
\hline & S-W (Statistic) & 0.971 & 0.882 & 0.983 & 0.980 & 0.978 & 0.971 & 0.961 & 0.962 & 0.963 & 0.939 & 0.946 & 0.969 & 0.949 & 0.953 & 0.907 \\
\hline & S-W (p-value) & 0.690 & 0.009 & 0.944 & 0.887 & 0.861 & 0.693 & 0.451 & 0.474 & 0.501 & 0.152 & 0.224 & 0.643 & 0.252 & 0.319 & 0.031 \\
\hline \multirow[t]{4}{*}{ RT 26} & K-S (Statistic) & 0.150 & 0.184 & 0.158 & 0.120 & 0.113 & 0.134 & 0.152 & 0.151 & 0.175 & 0.187 & 0.201 & 0.160 & 0.099 & 0.157 & 0.154 \\
\hline & K-S (p-value) & 0.153 & 0.029 & 0.107 & $0.200^{*}$ & $0.200^{*}$ & $0.200^{*}$ & 0.139 & 0.143 & 0.047 & 0.024 & 0.010 & 0.099 & $0.200^{*}$ & 0.112 & 0.129 \\
\hline & S-W (Statistic) & 0.912 & 0.818 & 0.973 & 0.978 & 0.977 & 0.963 & 0.869 & 0.868 & 0.865 & 0.864 & 0.867 & 0.904 & 0.958 & 0.852 & 0.837 \\
\hline & S-W (p-value) & 0.033 & 0.000 & 0.709 & 0.841 & 0.812 & 0.481 & 0.004 & 0.004 & 0.003 & 0.003 & 0.004 & 0.022 & 0.370 & 0.002 & 0.001 \\
\hline \multirow[t]{4}{*}{ RT 27} & K-S (Statistic) & 0.145 & 0.122 & 0.151 & 0.109 & 0.103 & 0.113 & 0.117 & 0.122 & 0.126 & 0.112 & 0.103 & 0.115 & 0.155 & 0.134 & 0.116 \\
\hline & K-S (p-value) & $0.200^{*}$ & $0.200^{*}$ & $0.200^{*}$ & $0.200^{*}$ & $0.200^{*}$ & $0.200^{*}$ & $0.200^{*}$ & $0.200^{*}$ & $0.200^{*}$ & $0.200^{*}$ & $0.200^{*}$ & $0.200^{*}$ & $0.200^{*}$ & $0.200^{*}$ & $0.200^{*}$ \\
\hline & S-W (Statistic) & 0.956 & 0.968 & 0.965 & 0.978 & 0.977 & 0.979 & 0.964 & 0.963 & 0.960 & 0.972 & 0.972 & 0.969 & 0.880 & 0.962 & 0.967 \\
\hline & S-W (p-value) & 0.497 & 0.745 & 0.673 & 0.920 & 0.898 & 0.934 & 0.645 & 0.637 & 0.578 & 0.811 & 0.823 & 0.754 & 0.021 & 0.604 & 0.712 \\
\hline \multirow[t]{4}{*}{ RT 28} & K-S (Statistic) & 0.151 & 0.149 & 0.147 & 0.204 & 0.203 & 0.186 & 0.138 & 0.135 & 0.133 & 0.154 & 0.159 & 0.155 & 0.118 & 0.189 & 0.188 \\
\hline & K-S (p-value) & 0.129 & 0.142 & 0.154 & 0.007 & 0.007 & 0.021 & $0.200^{*}$ & $0.200^{*}$ & $0.200^{*}$ & 0.117 & 0.090 & 0.111 & $0.200^{*}$ & 0.017 & 0.019 \\
\hline & S-W (Statistic) & 0.925 & 0.924 & 0.908 & 0.915 & 0.916 & 0.921 & 0.938 & 0.937 & 0.937 & 0.913 & 0.913 & 0.924 & 0.949 & 0.918 & 0.918 \\
\hline & S-W (p-value) & 0.059 & 0.057 & 0.023 & 0.034 & 0.036 & 0.048 & 0.118 & 0.115 & 0.111 & 0.030 & 0.032 & 0.056 & 0.218 & 0.041 & 0.040 \\
\hline \multirow[t]{4}{*}{ RT 29} & K-S (Statistic) & 0.138 & 0.100 & 0.124 & 0.178 & 0.163 & 0.153 & 0.093 & 0.090 & 0.092 & 0.126 & 0.135 & 0.154 & 0.106 & 0.138 & 0.188 \\
\hline & K-S (p-value) & $0.200^{*}$ & $0.200^{*}$ & $0.200^{*}$ & $0.200^{*}$ & $0.200^{*}$ & $0.200^{*}$ & $0.200^{*}$ & $0.200^{*}$ & $0.200^{*}$ & $0.200^{*}$ & $0.200^{*}$ & $0.200^{*}$ & $0.200^{*}$ & $0.200^{*}$ & $0.200^{*}$ \\
\hline & S-W (Statistic) & 0.950 & 0.972 & 0.956 & 0.915 & 0.924 & 0.939 & 0.966 & 0.967 & 0.967 & 0.962 & 0.953 & 0.953 & 0.972 & 0.956 & 0.947 \\
\hline & S-W (p-value) & 0.594 & 0.919 & 0.691 & 0.216 & 0.280 & 0.450 & 0.840 & 0.856 & 0.853 & 0.785 & 0.644 & 0.649 & 0.919 & 0.692 & 0.549 \\
\hline
\end{tabular}


Table C1: Kolmogorov-Smirnov and Shapiro-Wilk tests for Rolling Terrain for Headway $3 \mathrm{~s}-$ Cont.

\begin{tabular}{|c|c|c|c|c|c|c|c|c|c|c|c|c|c|c|c|c|}
\hline $\begin{array}{l}\text { Curve } \\
\text { ID }\end{array}$ & Test & $V_{c r v-a v g}$ & $V_{c r v-\min }$ & $V_{c r v-\max }$ & $V_{B C}$ & $V_{T B C}$ & $V_{D B C}$ & $V_{M C}$ & $V_{T M C}$ & $V_{D M C}$ & $V_{E C}$ & $V_{T E C}$ & $V_{D E C}$ & $a_{L}$ & $V_{c m f-a v g}$ & $V_{c m f-\min }$ \\
\hline \multirow[t]{4}{*}{ RT 30} & K-S (Statistic) & 0.110 & 0.124 & 0.117 & 0.126 & 0.133 & 0.129 & 0.105 & 0.106 & 0.103 & 0.114 & 0.111 & 0.104 & 0.118 & 0.117 & 0.110 \\
\hline & K-S (p-value) & $0.200^{*}$ & 0.136 & 0.198 & 0.122 & 0.079 & 0.098 & $0.200^{*}$ & $0.200^{*}$ & $0.200^{*}$ & $0.200^{*}$ & $0.200^{*}$ & $0.200^{*}$ & 0.188 & 0.198 & $0.200^{*}$ \\
\hline & S-W (Statistic) & 0.976 & 0.971 & 0.971 & 0.953 & 0.958 & 0.965 & 0.976 & 0.977 & 0.977 & 0.971 & 0.975 & 0.977 & 0.935 & 0.972 & 0.973 \\
\hline & S-W (p-value) & 0.543 & 0.406 & 0.413 & 0.103 & 0.153 & 0.263 & 0.570 & 0.594 & 0.593 & 0.394 & 0.520 & 0.584 & 0.025 & 0.432 & 0.476 \\
\hline \multirow[t]{4}{*}{ RT 31} & K-S (Statistic) & 0.124 & 0.180 & 0.137 & 0.107 & 0.127 & 0.123 & 0.085 & 0.087 & 0.088 & 0.178 & 0.172 & 0.132 & 0.129 & 0.108 & 0.097 \\
\hline & K-S (p-value) & $0.200^{*}$ & 0.052 & $0.200^{*}$ & $0.200^{*}$ & $0.200^{*}$ & 0.200 * & $0.200^{*}$ & $0.200^{*}$ & $0.200^{*}$ & 0.058 & 0.075 & $0.200^{*}$ & $0.200^{*}$ & $0.200^{*}$ & $0.200^{*}$ \\
\hline & S-W (Statistic) & 0.981 & 0.957 & 0.955 & 0.972 & 0.971 & 0.966 & 0.981 & 0.979 & 0.978 & 0.909 & 0.921 & 0.978 & 0.975 & 0.972 & 0.970 \\
\hline & S-W (p-value) & 0.922 & 0.412 & 0.368 & 0.733 & 0.709 & 0.602 & 0.915 & 0.882 & 0.876 & 0.038 & 0.071 & 0.863 & 0.800 & 0.748 & 0.689 \\
\hline \multirow[t]{4}{*}{ RT 32} & K-S (Statistic) & 0.095 & 0.103 & 0.095 & 0.125 & 0.134 & 0.113 & 0.118 & 0.110 & 0.121 & 0.092 & 0.117 & 0.104 & 0.087 & 0.128 & 0.134 \\
\hline & K-S (p-value) & $0.200^{*}$ & $0.200^{*}$ & $0.200^{*}$ & $0.200^{*}$ & $0.200^{*}$ & $0.200^{*}$ & $0.200^{*}$ & $0.200^{*}$ & $0.200^{*}$ & $0.200^{*}$ & $0.200^{*}$ & $0.200^{*}$ & $0.200^{*}$ & $0.200^{*}$ & $0.200^{*}$ \\
\hline & S-W (Statistic) & 0.974 & 0.968 & 0.966 & 0.967 & 0.964 & 0.966 & 0.978 & 0.979 & 0.975 & 0.962 & 0.956 & 0.975 & 0.975 & 0.957 & 0.955 \\
\hline & S-W (p-value) & 0.742 & 0.588 & 0.547 & 0.564 & 0.493 & 0.555 & 0.832 & 0.856 & 0.774 & 0.456 & 0.347 & 0.779 & 0.775 & 0.364 & 0.317 \\
\hline \multirow[t]{4}{*}{ RT 33} & K-S (Statistic) & 0.078 & 0.064 & 0.086 & 0.078 & 0.078 & 0.081 & 0.083 & 0.083 & 0.084 & 0.111 & 0.098 & 0.086 & 0.129 & 0.065 & 0.065 \\
\hline & K-S (p-value) & $0.200^{*}$ & $0.200^{*}$ & $0.200^{*}$ & $0.200^{*}$ & $0.200^{*}$ & $0.200^{*}$ & $0.200^{*}$ & $0.200^{*}$ & $0.200^{*}$ & $0.200^{*}$ & $0.200^{*}$ & $0.200^{*}$ & 0.100 & $0.200^{*}$ & $0.200^{*}$ \\
\hline & S-W (Statistic) & 0.993 & 0.992 & 0.991 & 0.991 & 0.994 & 0.994 & 0.991 & 0.991 & 0.991 & 0.982 & 0.986 & 0.990 & 0.951 & 0.994 & 0.993 \\
\hline & S-W (p-value) & 0.997 & 0.994 & 0.987 & 0.985 & 0.998 & 0.998 & 0.986 & 0.986 & 0.985 & 0.769 & 0.894 & 0.981 & 0.089 & 0.999 & 0.997 \\
\hline
\end{tabular}


Table C2: Kolmogorov-Smirnov and Shapiro-Wilk tests for Mountainous Terrain for Headway $3 \mathrm{~s}$

\begin{tabular}{|c|c|c|c|c|c|c|c|c|c|c|c|c|c|c|c|c|}
\hline $\begin{array}{l}\text { Curve } \\
\text { ID }\end{array}$ & Test & $V_{c r v-a v g}$ & $V_{c r v-m i n}$ & $V_{c r v-\max }$ & $V_{B C}$ & $V_{T B C}$ & $V_{D B C}$ & $V_{M C}$ & $V_{T M C}$ & $V_{D M C}$ & $V_{E C}$ & $V_{T E C}$ & $V_{D E C}$ & $a_{L}$ & $V_{c m f-a v g}$ & $V_{c m f-\min }$ \\
\hline \multirow[t]{4}{*}{ MT 01} & K-S (Statistic) & 0.125 & 0.106 & 0.114 & 0.092 & 0.100 & 0.116 & 0.108 & 0.106 & 0.105 & 0.105 & 0.115 & 0.125 & 0.072 & 0.090 & 0.108 \\
\hline & K-S (p-value) & 0.032 & 0.184 & 0.073 & $0.200^{*}$ & $0.200^{*}$ & 0.062 & 0.168 & 0.188 & 0.197 & 0.194 & 0.070 & 0.032 & $0.200^{*}$ & $0.200^{*}$ & 0.160 \\
\hline & S-W (Statistic) & 0.969 & 0.972 & 0.975 & 0.979 & 0.978 & 0.974 & 0.980 & 0.980 & 0.979 & 0.966 & 0.967 & 0.969 & 0.988 & 0.978 & 0.967 \\
\hline & S-W (p-value) & 0.163 & 0.234 & 0.320 & 0.439 & 0.408 & 0.290 & 0.505 & 0.477 & 0.433 & 0.127 & 0.130 & 0.167 & 0.869 & 0.401 & 0.133 \\
\hline \multirow[t]{4}{*}{ MT 02} & K-S (Statistic) & 0.120 & 0.115 & 0.095 & 0.110 & 0.125 & 0.122 & 0.104 & 0.105 & 0.106 & 0.078 & 0.087 & 0.112 & 0.118 & 0.104 & 0.098 \\
\hline & K-S (p-value) & 0.051 & 0.070 & $0.200^{*}$ & 0.152 & 0.036 & 0.045 & $0.200^{*}$ & $0.200^{*}$ & 0.195 & $0.200^{*}$ & $0.200^{*}$ & 0.089 & 0.060 & $0.200^{*}$ & $0.200^{*}$ \\
\hline & S-W (Statistic) & 0.931 & 0.943 & 0.940 & 0.935 & 0.929 & 0.925 & 0.932 & 0.932 & 0.930 & 0.959 & 0.960 & 0.936 & 0.966 & 0.927 & 0.926 \\
\hline & S-W (p-value) & 0.004 & 0.013 & 0.010 & 0.006 & 0.003 & 0.002 & 0.005 & 0.005 & 0.004 & 0.064 & 0.067 & 0.007 & 0.126 & 0.003 & 0.003 \\
\hline \multirow[t]{4}{*}{ MT 03} & K-S (Statistic) & 0.097 & 0.077 & 0.160 & 0.091 & 0.097 & 0.098 & 0.106 & 0.107 & 0.107 & 0.122 & 0.115 & 0.100 & 0.102 & 0.081 & 0.091 \\
\hline & K-S (p-value) & $0.200^{*}$ & $0.200^{*}$ & 0.001 & $0.200^{*}$ & $0.200^{*}$ & $0.200^{*}$ & 0.173 & 0.159 & 0.163 & 0.033 & 0.060 & $0.200^{*}$ & $0.200^{*}$ & $0.200^{*}$ & $0.200^{*}$ \\
\hline & S-W (Statistic) & 0.951 & 0.984 & 0.888 & 0.984 & 0.983 & 0.982 & 0.907 & 0.907 & 0.906 & 0.922 & 0.917 & 0.947 & 0.974 & 0.955 & 0.966 \\
\hline & S-W (p-value) & 0.023 & 0.671 & 0.000 & 0.671 & 0.582 & 0.563 & 0.000 & 0.000 & 0.000 & 0.001 & 0.001 & 0.014 & 0.248 & 0.034 & 0.108 \\
\hline \multirow[t]{4}{*}{ MT 04} & K-S (Statistic) & 0.121 & 0.102 & 0.133 & 0.155 & 0.167 & 0.155 & 0.120 & 0.125 & 0.127 & 0.118 & 0.121 & 0.105 & 0.095 & 0.090 & 0.096 \\
\hline & K-S (p-value) & $0.200^{*}$ & $0.200^{*}$ & $0.200^{*}$ & 0.082 & 0.044 & 0.082 & $0.200^{*}$ & $0.200^{*}$ & $0.200^{*}$ & $0.200^{*}$ & $0.200^{*}$ & $0.200^{*}$ & $0.200^{*}$ & $0.200^{*}$ & $0.200^{*}$ \\
\hline & S-W (Statistic) & 0.954 & 0.968 & 0.948 & 0.922 & 0.921 & 0.921 & 0.955 & 0.954 & 0.952 & 0.966 & 0.963 & 0.957 & 0.946 & 0.962 & 0.962 \\
\hline & S-W (p-value) & 0.254 & 0.522 & 0.176 & 0.040 & 0.037 & 0.037 & 0.263 & 0.244 & 0.226 & 0.481 & 0.418 & 0.302 & 0.159 & 0.385 & 0.379 \\
\hline \multirow[t]{4}{*}{ MT 05} & K-S (Statistic) & 0.090 & 0.067 & 0.093 & 0.134 & 0.117 & 0.115 & 0.083 & 0.082 & 0.075 & 0.078 & 0.066 & 0.094 & 0.124 & 0.096 & 0.075 \\
\hline & K-S (p-value) & $0.200^{*}$ & $0.200^{*}$ & $0.200^{*}$ & 0.168 & $0.200^{*}$ & $0.200^{*}$ & $0.200^{*}$ & $0.200^{*}$ & $0.200^{*}$ & $0.200^{*}$ & $0.200^{*}$ & $0.200^{*}$ & $0.200^{*}$ & $0.200^{*}$ & $0.200^{*}$ \\
\hline & S-W (Statistic) & 0.978 & 0.980 & 0.983 & 0.968 & 0.975 & 0.979 & 0.978 & 0.978 & 0.979 & 0.974 & 0.977 & 0.978 & 0.973 & 0.979 & 0.979 \\
\hline & S-W (p-value) & 0.755 & 0.800 & 0.892 & 0.464 & 0.678 & 0.791 & 0.761 & 0.769 & 0.775 & 0.623 & 0.730 & 0.760 & 0.605 & 0.772 & 0.798 \\
\hline \multirow[t]{4}{*}{ MT 06} & K-S (Statistic) & 0.116 & 0.091 & 0.130 & 0.129 & 0.117 & 0.114 & 0.120 & 0.111 & 0.112 & 0.160 & 0.124 & 0.107 & 0.129 & 0.075 & 0.080 \\
\hline & K-S (p-value) & $0.200^{*}$ & $0.200^{*}$ & $0.200^{*}$ & $0.200^{*}$ & $0.200^{*}$ & $0.200^{*}$ & $0.200^{*}$ & $0.200^{*}$ & $0.200^{*}$ & 0.149 & $0.200^{*}$ & $0.200^{*}$ & $0.200^{*}$ & $0.200^{*}$ & $0.200^{*}$ \\
\hline & S-W (Statistic) & 0.955 & 0.976 & 0.943 & 0.942 & 0.947 & 0.951 & 0.960 & 0.960 & 0.959 & 0.954 & 0.960 & 0.966 & 0.980 & 0.969 & 0.968 \\
\hline & S-W (p-value) & 0.402 & 0.845 & 0.228 & 0.219 & 0.279 & 0.330 & 0.488 & 0.495 & 0.474 & 0.383 & 0.482 & 0.619 & 0.923 & 0.682 & 0.666 \\
\hline \multirow[t]{4}{*}{ MT 07} & K-S (Statistic) & 0.141 & 0.138 & 0.102 & 0.121 & 0.110 & 0.125 & 0.155 & 0.154 & 0.157 & 0.098 & 0.097 & 0.133 & 0.095 & 0.137 & 0.126 \\
\hline & K-S (p-value) & $0.200^{*}$ & $0.200^{*}$ & $0.200^{*}$ & $0.200^{*}$ & $0.200^{*}$ & $0.200^{*}$ & $0.200^{*}$ & $0.200^{*}$ & 0.192 & $0.200^{*}$ & $0.200^{*}$ & $0.200^{*}$ & $0.200^{*}$ & $0.200^{*}$ & $0.200^{*}$ \\
\hline & S-W (Statistic) & 0.961 & 0.956 & 0.980 & 0.967 & 0.968 & 0.971 & 0.954 & 0.956 & 0.957 & 0.985 & 0.984 & 0.966 & 0.965 & 0.950 & 0.948 \\
\hline & S-W (p-value) & 0.543 & 0.434 & 0.924 & 0.674 & 0.695 & 0.760 & 0.400 & 0.434 & 0.457 & 0.979 & 0.971 & 0.654 & 0.613 & 0.345 & 0.314 \\
\hline MT 08 & K-S (Statistic) & 0.130 & 0.163 & 0.116 & 0.112 & 0.111 & 0.109 & 0.160 & 0.160 & 0.148 & 0.117 & 0.119 & 0.121 & 0.144 & 0.205 & 0.204 \\
\hline
\end{tabular}


Table C2: Kolmogorov-Smirnov and Shapiro-Wilk tests for Mountainous Terrain for Headway 3 s- Cont.

\begin{tabular}{|c|c|c|c|c|c|c|c|c|c|c|c|c|c|c|c|c|}
\hline $\begin{array}{c}\text { Curve } \\
\text { ID }\end{array}$ & Test & $V_{c r v-a v g}$ & $V_{c r v-\min }$ & $V_{c r v-\max }$ & $V_{B C}$ & $V_{T B C}$ & $V_{D B C}$ & $V_{M C}$ & $V_{T M C}$ & $V_{D M C}$ & $V_{E C}$ & $V_{T E C}$ & $V_{D E C}$ & $a_{L}$ & $V_{c m f-a v g}$ & $V_{c m f-m i n}$ \\
\hline & K-S (p-value) & $0.200^{*}$ & 0.170 & $0.200^{*}$ & $0.200^{*}$ & $0.200^{*}$ & $0.200^{*}$ & 0.196 & 0.192 & $0.200^{*}$ & $0.200^{*}$ & $0.200^{*}$ & $0.200^{*}$ & $0.200^{*}$ & 0.028 & 0.029 \\
\hline & S-W (Statistic) & 0.951 & 0.965 & 0.936 & 0.962 & 0.964 & 0.963 & 0.946 & 0.945 & 0.949 & 0.963 & 0.967 & 0.951 & 0.946 & 0.932 & 0.936 \\
\hline & S-W (p-value) & 0.376 & 0.653 & $0.200^{*}$ & 0.587 & 0.634 & 0.613 & 0.305 & 0.294 & 0.345 & 0.609 & 0.697 & 0.386 & 0.309 & 0.170 & 0.204 \\
\hline \multirow[t]{4}{*}{ MT 09} & K-S (Statistic) & 0.170 & 0.184 & 0.148 & 0.184 & 0.180 & 0.182 & 0.190 & 0.174 & 0.176 & 0.154 & 0.155 & 0.164 & 0.126 & 0.185 & 0.197 \\
\hline & K-S (p-value) & 0.153 & 0.088 & $0.200^{*}$ & 0.091 & 0.107 & 0.098 & 0.070 & 0.131 & 0.123 & $0.200^{*}$ & $0.200^{*}$ & 0.193 & $0.200^{*}$ & 0.086 & 0.051 \\
\hline & S-W (Statistic) & 0.933 & 0.932 & 0.946 & 0.942 & 0.942 & 0.939 & 0.928 & 0.929 & 0.930 & 0.935 & 0.936 & 0.930 & 0.968 & 0.929 & 0.927 \\
\hline & S-W (p-value) & 0.193 & 0.188 & 0.343 & 0.290 & 0.286 & 0.255 & 0.161 & 0.166 & 0.176 & 0.215 & 0.227 & 0.171 & 0.745 & 0.167 & 0.152 \\
\hline \multirow[t]{4}{*}{ MT 10} & K-S (Statistic) & 0.155 & 0.190 & 0.133 & 0.146 & 0.150 & 0.145 & 0.164 & 0.160 & 0.159 & 0.184 & 0.181 & 0.170 & 0.143 & 0.156 & 0.159 \\
\hline & K-S (p-value) & $0.200^{*}$ & 0.084 & $0.200^{*}$ & $0.200^{*}$ & $0.200^{*}$ & $0.200^{*}$ & $0.200^{*}$ & $0.200^{*}$ & $0.200^{*}$ & 0.108 & 0.122 & 0.182 & $0.200^{*}$ & $0.200^{*}$ & $0.200^{*}$ \\
\hline & S-W (Statistic) & 0.897 & 0.882 & 0.920 & 0.889 & 0.896 & 0.900 & 0.896 & 0.900 & 0.902 & 0.894 & 0.900 & 0.901 & 0.947 & 0.925 & 0.923 \\
\hline & S-W (p-value) & 0.052 & 0.028 & 0.129 & 0.036 & 0.048 & 0.057 & 0.049 & 0.058 & 0.061 & 0.045 & 0.058 & 0.059 & 0.374 & 0.157 & 0.149 \\
\hline \multirow[t]{4}{*}{ MT 11} & K-S (Statistic) & 0.162 & 0.144 & 0.129 & 0.148 & 0.140 & 0.138 & 0.116 & 0.112 & 0.109 & 0.112 & 0.127 & 0.165 & 0.130 & 0.154 & 0.149 \\
\hline & K-S (p-value) & 0.090 & 0.189 & $0.200^{*}$ & 0.163 & $0.200^{*}$ & $0.200^{*}$ & $0.200^{*}$ & $0.200^{*}$ & $0.200^{*}$ & $0.200^{*}$ & $0.200^{*}$ & 0.078 & $0.200^{*}$ & 0.127 & 0.160 \\
\hline & S-W (Statistic) & 0.943 & 0.933 & 0.960 & 0.923 & 0.926 & 0.926 & 0.963 & 0.964 & 0.964 & 0.969 & 0.968 & 0.947 & 0.970 & 0.934 & 0.940 \\
\hline & S-W (p-value) & 0.174 & 0.102 & 0.420 & 0.061 & 0.069 & 0.070 & 0.480 & 0.496 & 0.490 & 0.608 & 0.589 & 0.218 & 0.648 & 0.110 & 0.145 \\
\hline \multirow[t]{4}{*}{ MT 12} & K-S (Statistic) & 0.254 & 0.278 & 0.199 & 0.172 & 0.182 & 0.212 & 0.273 & 0.274 & 0.271 & 0.246 & 0.247 & 0.269 & 0.094 & 0.277 & 0.284 \\
\hline & K-S (p-value) & 0.001 & 0.000 & 0.023 & 0.089 & 0.056 & 0.011 & 0.000 & 0.000 & 0.000 & 0.001 & 0.001 & 0.000 & $0.200^{*}$ & 0.000 & 0.000 \\
\hline & S-W (Statistic) & 0.726 & 0.676 & 0.848 & 0.869 & 0.856 & 0.806 & 0.689 & 0.687 & 0.692 & 0.749 & 0.745 & 0.698 & 0.971 & 0.678 & 0.665 \\
\hline & S-W (p-value) & 0.000 & 0.000 & 0.003 & 0.008 & 0.004 & 0.001 & 0.000 & 0.000 & 0.000 & 0.000 & 0.000 & 0.000 & 0.745 & 0.000 & 0.000 \\
\hline \multirow[t]{4}{*}{ MT 13} & K-S (Statistic) & 0.163 & 0.182 & 0.119 & 0.141 & 0.140 & 0.139 & 0.173 & 0.172 & 0.171 & 0.165 & 0.165 & 0.165 & 0.099 & 0.171 & 0.162 \\
\hline & K-S (p-value) & 0.084 & 0.032 & $0.200^{*}$ & $0.200^{*}$ & $0.200^{*}$ & $0.200^{*}$ & 0.051 & 0.054 & 0.059 & 0.078 & 0.079 & 0.078 & $0.200^{*}$ & 0.058 & 0.089 \\
\hline & S-W (Statistic) & 0.904 & 0.867 & 0.947 & 0.952 & 0.947 & 0.938 & 0.916 & 0.915 & 0.917 & 0.865 & 0.871 & 0.896 & 0.965 & 0.890 & 0.878 \\
\hline & S-W (p-value) & 0.022 & 0.004 & 0.217 & 0.273 & 0.210 & 0.131 & 0.041 & 0.039 & 0.043 & 0.003 & 0.004 & 0.015 & 0.519 & 0.011 & 0.006 \\
\hline \multirow[t]{4}{*}{ MT 14} & K-S (Statistic) & 0.136 & 0.118 & 0.140 & 0.116 & 0.121 & 0.124 & 0.119 & 0.115 & 0.129 & 0.154 & 0.147 & 0.133 & 0.119 & 0.159 & 0.144 \\
\hline & K-S (p-value) & $0.200^{*}$ & $0.200^{*}$ & $0.200^{*}$ & $0.200^{*}$ & $0.200^{*}$ & $0.200^{*}$ & $0.200^{*}$ & $0.200^{*}$ & $0.200^{*}$ & $0.200^{*}$ & $0.200^{*}$ & $0.200^{*}$ & $0.200^{*}$ & 0.176 & $0.200^{*}$ \\
\hline & S-W (Statistic) & 0.920 & 0.928 & 0.908 & 0.931 & 0.925 & 0.921 & 0.923 & 0.922 & 0.920 & 0.910 & 0.914 & 0.918 & 0.948 & 0.919 & 0.920 \\
\hline & S-W (p-value) & 0.086 & 0.124 & 0.050 & 0.146 & 0.107 & 0.092 & 0.099 & 0.097 & 0.085 & 0.055 & 0.066 & 0.080 & 0.306 & 0.083 & 0.087 \\
\hline \multirow[t]{2}{*}{ MT 15} & K-S (Statistic) & 0.076 & 0.085 & 0.116 & 0.058 & 0.059 & 0.059 & 0.114 & 0.114 & 0.114 & 0.078 & 0.083 & 0.082 & 0.062 & 0.075 & 0.071 \\
\hline & K-S (p-value) & $0.200^{*}$ & $0.200^{*}$ & 0.080 & $0.200^{*}$ & $0.200^{*}$ & $0.200^{*}$ & 0.092 & 0.089 & 0.092 & $0.200^{*}$ & $0.200^{*}$ & $0.200^{*}$ & $0.200^{*}$ & $0.200^{*}$ & $0.200^{*}$ \\
\hline
\end{tabular}


Table C2: Kolmogorov-Smirnov and Shapiro-Wilk tests for Mountainous Terrain for Headway 3 s- Cont.

\begin{tabular}{|c|c|c|c|c|c|c|c|c|c|c|c|c|c|c|c|c|}
\hline $\begin{array}{c}\text { Curve } \\
\text { ID }\end{array}$ & Test & $V_{c r v-a v g}$ & $V_{c r v-\min }$ & $V_{c r v-\max }$ & $V_{B C}$ & $V_{T B C}$ & $V_{D B C}$ & $V_{M C}$ & $V_{T M C}$ & $V_{D M C}$ & $V_{E C}$ & $V_{T E C}$ & $V_{D E C}$ & $a_{L}$ & $V_{c m f-a v g}$ & $V_{c m f-m i n}$ \\
\hline \multirow{5}{*}{ MT 16} & S-W (Statistic) & 0.972 & 0.985 & 0.920 & 0.992 & 0.992 & 0.992 & 0.952 & 0.952 & 0.953 & 0.957 & 0.956 & 0.968 & 0.989 & 0.977 & 0.980 \\
\hline & S-W (p-value) & 0.247 & 0.761 & 0.002 & 0.974 & 0.975 & 0.985 & 0.034 & 0.036 & 0.037 & 0.055 & 0.054 & 0.173 & 0.914 & 0.411 & 0.524 \\
\hline & K-S (Statistic) & $0.200^{*}$ & 0.151 & 0.102 & 0.116 & 0.109 & 0.102 & 0.158 & 0.158 & 0.157 & 0.166 & 0.184 & 0.171 & 0.114 & 0.177 & 0.165 \\
\hline & K-S (p-value) & 0.009 & 0.130 & $0.200^{*}$ & $0.200^{*}$ & $0.200^{*}$ & $0.200^{*}$ & 0.093 & 0.093 & 0.099 & 0.064 & 0.024 & 0.048 & $0.200^{*}$ & 0.035 & 0.066 \\
\hline & S-W (Statistic) & 0.941 & 0.942 & 0.979 & 0.980 & 0.981 & 0.982 & 0.949 & 0.949 & 0.948 & 0.940 & 0.939 & 0.944 & 0.957 & 0.942 & 0.938 \\
\hline \multirow{3}{*}{ MT 17} & S-W (p-value) & 0.144 & 0.152 & 0.860 & 0.874 & 0.902 & 0.914 & 0.217 & 0.218 & 0.209 & 0.131 & 0.125 & 0.166 & 0.331 & 0.151 & 0.121 \\
\hline & K-S (Statistic) & 0.166 & 0.180 & 0.148 & 0.208 & 0.215 & 0.216 & 0.166 & 0.169 & 0.168 & 0.133 & 0.133 & 0.161 & 0.179 & 0.158 & 0.154 \\
\hline & K-S (p-value) & 0.063 & 0.030 & 0.147 & 0.005 & 0.003 & 0.003 & 0.062 & 0.055 & 0.058 & $0.200^{*}$ & $0.200^{*}$ & 0.080 & 0.032 & 0.095 & 0.117 \\
\hline \multirow{5}{*}{ MT 18} & S-W (Statistic) & 0.902 & 0.889 & 0.927 & 0.892 & 0.894 & 0.893 & 0.904 & 0.903 & 0.902 & 0.929 & 0.926 & 0.906 & 0.960 & 0.900 & 0.893 \\
\hline & S-W (p-value) & 0.018 & 0.009 & 0.066 & 0.011 & 0.011 & 0.011 & 0.019 & 0.018 & 0.017 & 0.073 & 0.061 & 0.022 & 0.393 & 0.016 & 0.011 \\
\hline & K-S (Statistic) & 0.170 & 0.184 & 0.148 & 0.184 & 0.180 & 0.182 & 0.190 & 0.174 & 0.176 & 0.154 & 0.155 & 0.164 & 0.126 & 0.185 & 0.197 \\
\hline & K-S (p-value) & 0.153 & 0.088 & $0.200^{*}$ & 0.091 & 0.107 & 0.098 & 0.070 & 0.131 & 0.123 & $0.200^{*}$ & $0.200^{*}$ & 0.193 & $0.200^{*}$ & 0.086 & 0.051 \\
\hline & S-W (Statistic) & 0.933 & 0.932 & 0.946 & 0.942 & 0.942 & 0.939 & 0.928 & 0.929 & 0.930 & 0.935 & 0.936 & 0.930 & 0.968 & 0.929 & 0.927 \\
\hline \multirow{3}{*}{ MT 19} & S-W (p-value) & 0.193 & 0.188 & 0.343 & 0.290 & 0.286 & 0.255 & 0.161 & 0.166 & 0.176 & 0.215 & 0.227 & 0.171 & 0.745 & 0.167 & 0.152 \\
\hline & K-S (Statistic) & 0.082 & 0.093 & 0.093 & 0.115 & 0.116 & 0.100 & 0.101 & 0.113 & 0.110 & 0.115 & 0.094 & 0.088 & 0.061 & 0.103 & 0.102 \\
\hline & K-S (p-value) & $0.200^{*}$ & $0.200^{*}$ & $0.200^{*}$ & $0.200^{*}$ & $0.200^{*}$ & $0.200^{*}$ & $0.200^{*}$ & $0.200^{*}$ & $0.200^{*}$ & $0.200^{*}$ & $0.200^{*}$ & $0.200^{*}$ & $0.200^{*}$ & $0.200^{*}$ & $0.200^{*}$ \\
\hline \multirow{5}{*}{ MT 20} & S-W (Statistic) & 0.970 & 0.975 & 0.964 & 0.951 & 0.959 & 0.963 & 0.972 & 0.971 & 0.971 & 0.971 & 0.972 & 0.971 & 0.993 & 0.970 & 0.970 \\
\hline & S-W (p-value) & 0.393 & 0.557 & 0.265 & 0.093 & 0.172 & 0.234 & 0.438 & 0.416 & 0.410 & 0.413 & 0.454 & 0.415 & 0.997 & 0.387 & 0.381 \\
\hline & K-S (Statistic) & 0.078 & 0.097 & 0.116 & 0.065 & 0.076 & 0.076 & 0.096 & 0.096 & 0.091 & 0.084 & 0.088 & 0.088 & 0.121 & 0.089 & 0.088 \\
\hline & K-S (p-value) & $0.200^{*}$ & $0.200^{*}$ & 0.162 & $0.200^{*}$ & $0.200^{*}$ & $0.200^{*}$ & $0.200^{*}$ & $0.200^{*}$ & $0.200^{*}$ & $0.200^{*}$ & $0.200^{*}$ & $0.200^{*}$ & 0.112 & $0.200^{*}$ & $0.200^{*}$ \\
\hline & S-W (Statistic) & 0.984 & 0.985 & 0.979 & 0.991 & 0.991 & 0.991 & 0.976 & 0.977 & 0.978 & 0.978 & 0.979 & 0.981 & 0.965 & 0.978 & 0.976 \\
\hline \multirow{3}{*}{ MT 21} & S-W (p-value) & 0.788 & 0.832 & 0.612 & 0.983 & 0.983 & 0.983 & 0.471 & 0.520 & 0.574 & 0.564 & 0.604 & 0.667 & 0.199 & 0.568 & 0.476 \\
\hline & K-S (Statistic) & 0.065 & 0.077 & 0.060 & 0.075 & 0.069 & 0.064 & 0.076 & 0.071 & 0.070 & 0.068 & 0.064 & 0.071 & 0.067 & 0.054 & 0.070 \\
\hline & K-S (p-value) & $0.200^{*}$ & $0.200^{*}$ & $0.200^{*}$ & $0.200^{*}$ & $0.200^{*}$ & $0.200^{*}$ & $0.200^{*}$ & $0.200^{*}$ & $0.200^{*}$ & $0.200^{*}$ & $0.200^{*}$ & $0.200^{*}$ & $0.200^{*}$ & $0.200^{*}$ & $0.200^{*}$ \\
\hline \multirow{5}{*}{ MT 22} & S-W (Statistic) & 0.986 & 0.982 & 0.992 & 0.986 & 0.986 & 0.989 & 0.985 & 0.986 & 0.986 & 0.985 & 0.985 & 0.986 & 0.985 & 0.988 & 0.988 \\
\hline & S-W (p-value) & 0.852 & 0.687 & 0.988 & 0.824 & 0.838 & 0.926 & 0.813 & 0.838 & 0.844 & 0.796 & 0.810 & 0.850 & 0.797 & 0.917 & 0.894 \\
\hline & K-S (Statistic) & 0.091 & 0.105 & 0.138 & 0.097 & 0.089 & 0.107 & 0.109 & 0.105 & 0.108 & 0.140 & 0.142 & 0.088 & 0.088 & 0.129 & 0.117 \\
\hline & K-S (p-value) & $0.200^{*}$ & $0.200^{*}$ & 0.123 & $0.200^{*}$ & $0.200^{*}$ & $0.200^{*}$ & $0.200^{*}$ & $0.200^{*}$ & $0.200^{*}$ & 0.111 & 0.098 & $0.200^{*}$ & $0.200^{*}$ & 0.188 & $0.200^{*}$ \\
\hline & S-W (Statistic) & 0.974 & 0.977 & 0.969 & 0.988 & 0.988 & 0.987 & 0.966 & 0.968 & 0.968 & 0.947 & 0.948 & 0.973 & 0.945 & 0.961 & 0.961 \\
\hline
\end{tabular}


Table C2: Kolmogorov-Smirnov and Shapiro-Wilk tests for Mountainous Terrain for Headway 3 s- Cont.

\begin{tabular}{|c|c|c|c|c|c|c|c|c|c|c|c|c|c|c|c|c|}
\hline $\begin{array}{l}\text { Curve } \\
\text { ID }\end{array}$ & Test & $V_{c r v-a v g}$ & $V_{c r v-m i n}$ & $V_{c r v-\max }$ & $V_{B C}$ & $V_{T B C}$ & $V_{D B C}$ & $V_{M C}$ & $V_{T M C}$ & $V_{D M C}$ & $V_{E C}$ & $V_{T E C}$ & $V_{D E C}$ & $a_{L}$ & $V_{c m f-a v g}$ & $V_{c m f-m i n}$ \\
\hline & S-W (p-value) & 0.607 & 0.705 & 0.485 & 0.973 & 0.970 & 0.955 & 0.392 & 0.436 & 0.443 & 0.116 & 0.129 & 0.583 & 0.105 & 0.301 & 0.288 \\
\hline \multirow{4}{*}{ MT 23} & K-S (Statistic) & 0.130 & 0.153 & 0.120 & 0.160 & 0.157 & 0.143 & 0.143 & 0.143 & 0.142 & 0.114 & 0.113 & 0.126 & 0.106 & 0.116 & 0.124 \\
\hline & K-S (p-value) & 0.194 & 0.062 & $0.200^{*}$ & 0.042 & 0.051 & 0.105 & 0.107 & 0.105 & 0.115 & $0.200^{*}$ & $0.200^{*}$ & $0.200^{*}$ & $0.200^{*}$ & $0.200^{*}$ & $0.200^{*}$ \\
\hline & S-W (Statistic) & 0.944 & 0.918 & 0.949 & 0.956 & 0.954 & 0.954 & 0.940 & 0.942 & 0.943 & 0.927 & 0.930 & 0.942 & 0.964 & 0.948 & 0.939 \\
\hline & S-W (p-value) & 0.107 & 0.021 & 0.150 & 0.221 & $0.200^{*}$ & 0.202 & 0.083 & 0.092 & 0.098 & 0.037 & 0.044 & 0.095 & 0.362 & 0.136 & 0.076 \\
\hline \multirow[t]{4}{*}{ MT 24} & K-S (Statistic) & 0.088 & 0.136 & 0.125 & 0.117 & 0.131 & 0.146 & 0.091 & 0.090 & 0.093 & 0.125 & 0.122 & 0.100 & 0.117 & 0.110 & 0.122 \\
\hline & K-S (p-value) & $0.200^{*}$ & $0.200^{*}$ & $0.200^{*}$ & $0.200^{*}$ & $0.200^{*}$ & 0.146 & $0.200^{*}$ & $0.200^{*}$ & $0.200^{*}$ & $0.200^{*}$ & $0.200^{*}$ & $0.200^{*}$ & $0.200^{*}$ & $0.200^{*}$ & $0.200^{*}$ \\
\hline & S-W (Statistic) & 0.975 & 0.964 & 0.979 & 0.980 & 0.982 & 0.983 & 0.972 & 0.971 & 0.971 & 0.981 & 0.983 & 0.972 & 0.978 & 0.969 & 0.969 \\
\hline & S-W (p-value) & 0.735 & 0.464 & 0.839 & 0.864 & 0.913 & 0.922 & 0.645 & 0.620 & 0.629 & 0.890 & 0.930 & 0.667 & 0.823 & 0.567 & 0.570 \\
\hline \multirow[t]{4}{*}{ MT 25} & K-S (Statistic) & 0.095 & 0.091 & 0.090 & 0.072 & 0.069 & 0.070 & 0.100 & 0.100 & 0.099 & 0.073 & 0.077 & 0.095 & 0.064 & 0.103 & 0.104 \\
\hline & K-S (p-value) & $0.200^{*}$ & $0.200^{*}$ & $0.200^{\star}$ & $0.200^{*}$ & $0.200^{*}$ & $0.200^{*}$ & $0.200^{*}$ & $0.200^{*}$ & $0.200^{*}$ & $0.200^{*}$ & $0.200^{*}$ & $0.200^{*}$ & $0.200^{*}$ & $0.200^{*}$ & $0.200^{*}$ \\
\hline & S-W (Statistic) & 0.983 & 0.979 & 0.984 & 0.979 & 0.981 & 0.981 & 0.973 & 0.973 & 0.975 & 0.990 & 0.991 & 0.981 & 0.982 & 0.980 & 0.977 \\
\hline & S-W (p-value) & 0.819 & 0.686 & 0.858 & 0.696 & 0.763 & 0.759 & 0.481 & 0.484 & 0.545 & 0.976 & 0.985 & 0.763 & 0.787 & 0.711 & 0.608 \\
\hline \multirow[t]{4}{*}{ MT 26} & K-S (Statistic) & 0.098 & 0.106 & 0.161 & 0.166 & 0.166 & 0.171 & 0.086 & 0.086 & 0.086 & 0.173 & 0.153 & 0.113 & 0.208 & 0.117 & 0.118 \\
\hline & K-S (p-value) & $0.200^{*}$ & $0.200^{*}$ & 0.163 & 0.133 & 0.137 & 0.108 & $0.200^{*}$ & $0.200^{*}$ & $0.200^{*}$ & 0.101 & $0.200^{*}$ & $0.200^{*}$ & 0.018 & $0.200^{*}$ & $0.200^{*}$ \\
\hline & S-W (Statistic) & 0.974 & 0.978 & 0.956 & 0.936 & 0.940 & 0.944 & 0.977 & 0.978 & 0.977 & 0.957 & 0.959 & 0.975 & 0.934 & 0.974 & 0.973 \\
\hline & S-W (p-value) & 0.824 & 0.901 & 0.447 & 0.185 & 0.217 & 0.260 & 0.878 & 0.891 & 0.870 & 0.454 & 0.489 & 0.835 & 0.165 & 0.809 & 0.793 \\
\hline \multirow[t]{4}{*}{ MT 27} & K-S (Statistic) & 0.139 & 0.136 & 0.131 & 0.118 & 0.120 & 0.123 & 0.145 & 0.145 & 0.135 & 0.117 & 0.121 & 0.136 & 0.085 & 0.139 & 0.137 \\
\hline & K-S (p-value) & 0.195 & $0.200^{*}$ & $0.200^{*}$ & $0.200^{*}$ & $0.200^{*}$ & $0.200^{*}$ & 0.152 & 0.150 & $0.200^{*}$ & $0.200^{*}$ & $0.200^{*}$ & $0.200^{*}$ & $0.200^{*}$ & 0.193 & $0.200^{*}$ \\
\hline & S-W (Statistic) & 0.946 & 0.940 & 0.955 & 0.958 & 0.955 & 0.948 & 0.942 & 0.941 & 0.943 & 0.951 & 0.949 & 0.947 & 0.977 & 0.938 & 0.938 \\
\hline & S-W (p-value) & 0.170 & 0.119 & 0.286 & 0.327 & 0.276 & 0.195 & 0.134 & 0.132 & 0.148 & 0.227 & 0.204 & 0.177 & 0.781 & 0.108 & 0.106 \\
\hline \multirow[t]{4}{*}{ MT 28} & K-S (Statistic) & 0.159 & 0.161 & 0.131 & 0.143 & 0.149 & 0.150 & 0.130 & 0.132 & 0.134 & 0.178 & 0.179 & 0.168 & 0.120 & 0.154 & 0.155 \\
\hline & K-S (p-value) & 0.030 & 0.026 & 0.147 & 0.077 & 0.053 & 0.049 & 0.155 & 0.142 & 0.129 & 0.008 & 0.007 & 0.016 & $0.200^{*}$ & 0.041 & 0.036 \\
\hline & S-W (Statistic) & 0.940 & 0.906 & 0.958 & 0.945 & 0.943 & 0.941 & 0.955 & 0.955 & 0.954 & 0.911 & 0.918 & 0.942 & 0.977 & 0.950 & 0.942 \\
\hline & S-W (p-value) & 0.062 & 0.007 & 0.217 & 0.089 & 0.076 & 0.065 & 0.175 & 0.173 & 0.165 & 0.009 & 0.014 & 0.069 & 0.682 & 0.119 & 0.069 \\
\hline \multirow[t]{4}{*}{ MT 29} & K-S (Statistic) & 0.202 & 0.205 & $0.200^{\star}$ & 0.194 & 0.184 & 0.185 & 0.249 & 0.248 & 0.228 & 0.209 & 0.207 & 0.231 & 0.188 & 0.254 & 0.247 \\
\hline & K-S (p-value) & 0.000 & 0.000 & 0.000 & 0.001 & 0.002 & 0.002 & 0.000 & 0.000 & 0.000 & 0.000 & 0.000 & 0.000 & 0.001 & 0.000 & 0.000 \\
\hline & S-W (Statistic) & 0.831 & 0.828 & 0.800 & 0.853 & 0.851 & 0.851 & 0.813 & 0.813 & 0.815 & 0.806 & 0.807 & 0.821 & 0.848 & 0.811 & 0.806 \\
\hline & S-W (p-value) & 0.000 & 0.000 & 0.000 & 0.000 & 0.000 & 0.000 & 0.000 & 0.000 & 0.000 & 0.000 & 0.000 & 0.000 & 0.000 & 0.000 & 0.000 \\
\hline
\end{tabular}


Table C3: Kolmogorov-Smirnov and Shapiro-Wilk tests for Rolling Terrain for Headway 5 s.

\begin{tabular}{|c|c|c|c|c|c|c|c|c|c|c|c|c|c|c|c|c|}
\hline $\begin{array}{c}\text { Curve } \\
\text { ID }\end{array}$ & Test & $V_{c r v-a v g}$ & $V_{c r v-\min }$ & $V_{c r v-\max }$ & $V_{B C}$ & $V_{T B C}$ & $V_{D B C}$ & $V_{M C}$ & $V_{T M C}$ & $V_{D M C}$ & $V_{E C}$ & $V_{T E C}$ & $V_{D E C}$ & $a_{L}$ & $\mid V_{c m f-a v g}$ & $V_{c m f-m i n}$ \\
\hline \multirow[t]{4}{*}{ RT 01} & K-S (Statistic) & 0.069 & 0.083 & 0.049 & 0.066 & 0.068 & 0.079 & 0.085 & 0.086 & 0.087 & 0.070 & 0.061 & 0.080 & 0.132 & 0.077 & 0.068 \\
\hline & K-S (p-value) & $0.200^{*}$ & $0.200^{*}$ & $0.200^{*}$ & $0.200^{*}$ & $0.200^{*}$ & $0.200^{*}$ & $0.200^{*}$ & $0.200^{*}$ & $0.200^{*}$ & $0.200^{*}$ & $0.200^{*}$ & $0.200^{*}$ & 0.083 & $0.200^{*}$ & $0.200^{*}$ \\
\hline & S-W (Statistic) & 0.985 & 0.984 & 0.986 & 0.988 & 0.988 & 0.986 & 0.984 & 0.984 & 0.983 & 0.987 & 0.986 & 0.983 & 0.961 & 0.984 & 0.984 \\
\hline & S-W (p-value) & 0.877 & 0.847 & 0.893 & 0.942 & 0.947 & 0.905 & 0.834 & 0.834 & 0.799 & 0.915 & 0.888 & 0.806 & 0.188 & 0.854 & 0.852 \\
\hline \multirow[t]{4}{*}{ RT 02} & K-S (Statistic) & 0.077 & 0.082 & 0.090 & 0.097 & 0.093 & 0.101 & 0.087 & 0.086 & 0.085 & 0.080 & 0.075 & 0.091 & 0.114 & 0.072 & 0.086 \\
\hline & K-S (p-value) & $0.200^{*}$ & $0.200^{*}$ & $0.200^{*}$ & $0.200^{*}$ & $0.200^{*}$ & $0.200^{*}$ & $0.200^{*}$ & $0.200^{*}$ & $0.200^{*}$ & $0.200^{*}$ & $0.200^{*}$ & $0.200^{*}$ & 0.164 & $0.200^{*}$ & $0.200^{*}$ \\
\hline & S-W (Statistic) & 0.973 & 0.977 & 0.972 & 0.978 & 0.979 & 0.977 & 0.970 & 0.971 & 0.972 & 0.984 & 0.983 & 0.975 & 0.930 & 0.981 & 0.981 \\
\hline & S-W (p-value) & 0.347 & 0.488 & 0.329 & 0.533 & 0.577 & 0.485 & 0.283 & 0.299 & 0.322 & 0.767 & 0.735 & 0.428 & 0.009 & 0.640 & .650 \\
\hline \multirow[t]{4}{*}{ RT 03} & K-S (Statistic) & 0.102 & 0.095 & 0.158 & 0.166 & 0.137 & 0.142 & 0.077 & 0.074 & 0.088 & 0.076 & 0.087 & 0.094 & 0.169 & 0.090 & 0.084 \\
\hline & K-S (p-value) & $0.200^{*}$ & $0.200^{*}$ & 0.015 & 0.008 & 0.063 & 0.047 & $0.200^{*}$ & $0.200^{*}$ & $0.200^{*}$ & $0.200^{*}$ & $0.200^{*}$ & $0.200^{*}$ & 0.007 & $0.200^{*}$ & $0.200^{*}$ \\
\hline & S-W (Statistic) & 0.962 & 0.971 & 0.946 & 0.937 & 0.940 & 0.943 & 0.967 & 0.965 & 0.964 & 0.978 & 0.974 & 0.966 & 0.940 & 0.972 & 0.973 \\
\hline & S-W (p-value) & 0.205 & 0.414 & 0.062 & 0.029 & 0.037 & 0.046 & 0.311 & 0.260 & 0.234 & 0.621 & 0.504 & 0.279 & 0.039 & 0.427 & 0.470 \\
\hline \multirow[t]{4}{*}{ RT 04} & K-S (Statistic) & 0.139 & 0.151 & 0.131 & 0.172 & 0.177 & 0.188 & 0.105 & 0.103 & 0.108 & 0.099 & 0.121 & 0.156 & 0.130 & 0.124 & 0.142 \\
\hline & K-S (p-value) & $0.200^{*}$ & 0.146 & $0.200^{*}$ & 0.055 & 0.041 & 0.023 & $0.200^{*}$ & $0.200^{*}$ & $0.200^{*}$ & $0.200^{*}$ & $0.200^{*}$ & 0.121 & $0.200^{*}$ & $0.200^{*}$ & $0.200^{*}$ \\
\hline & S-W (Statistic) & 0.944 & 0.943 & 0.946 & 0.907 & 0.908 & 0.908 & 0.949 & 0.951 & 0.948 & 0.974 & 0.969 & 0.951 & 0.968 & 0.927 & 0.934 \\
\hline & S-W (p-value) & 0.182 & 0.177 & 0.202 & 0.026 & 0.028 & 0.028 & 0.243 & 0.269 & 0.225 & 0.746 & 0.628 & 0.265 & 0.596 & 0.074 & 0.105 \\
\hline \multirow[t]{4}{*}{ RT 05} & K-S (Statistic) & 0.080 & 0.111 & 0.073 & 0.091 & 0.077 & 0.074 & 0.093 & 0.089 & 0.087 & 0.134 & 0.124 & 0.090 & 0.112 & 0.082 & 0.078 \\
\hline & K-S (p-value) & $0.200^{*}$ & 0.181 & $0.200^{*}$ & $0.200^{*}$ & $0.200^{*}$ & $0.200^{*}$ & $0.200^{*}$ & $0.200^{*}$ & $0.200^{*}$ & 0.029 & 0.058 & $0.200^{*}$ & 0.170 & $0.200^{*}$ & $0.200^{*}$ \\
\hline & S-W (Statistic) & 0.977 & 0.955 & 0.977 & 0.979 & 0.980 & 0.978 & 0.975 & 0.975 & 0.975 & 0.944 & 0.948 & 0.973 & 0.974 & 0.979 & 0.975 \\
\hline & S-W (p-value) & 0.430 & 0.059 & 0.449 & 0.507 & 0.563 & 0.475 & 0.367 & 0.372 & 0.393 & 0.022 & 0.030 & 0.328 & 0.351 & 0.522 & 0.371 \\
\hline \multirow[t]{4}{*}{ RT 06} & K-S (Statistic) & 0.084 & 0.086 & 0.098 & 0.08 & 0.104 & 0.10 & 0.100 & 0.102 & 0.094 & 0.1 & 0.114 & 0.090 & 0.106 & 0.105 & 0.098 \\
\hline & K-S ( $p$-value) & $0.200^{*}$ & $0.200^{*}$ & $0.200^{*}$ & $0.200^{*}$ & $0.200^{*}$ & $0.200^{*}$ & $0.200^{*}$ & $0.200^{*}$ & $0.200^{*}$ & 0.099 & 0.153 & $0.200^{*}$ & $0.200^{*}$ & $0.200^{*}$ & $0.200^{*}$ \\
\hline & S-n & & $0.9 \varepsilon$ & 0.96 & 0.9 & 0.9 & 0.9 & & 0.9 & $0 . c$ & 0.971 & 0.978 & 0. & 0.957 & 0.972 & 0.971 \\
\hline & S-W & 0.638 & 0.564 & 0.234 & 0.50 & 0.368 & 0.329 & 0.631 & 0.64 & 0.649 & 0.278 & 0.481 & 0.764 & 0.078 & 0.301 & 0.272 \\
\hline \multirow[t]{4}{*}{ RT 07} & K-S (Statistic) & 0.151 & 0.172 & 0.113 & 0.165 & 0.166 & 0.171 & 0.146 & 0.150 & 0.153 & 0.116 & 0.111 & 0.143 & 0.161 & 0.155 & 0.146 \\
\hline & K-S (p-value) & $0.200^{*}$ & $0.200^{*}$ & $0.200^{*}$ & $0.200^{*}$ & $0.200^{*}$ & $0.200^{*}$ & $0.200^{*}$ & $0.200^{*}$ & $0.200^{*}$ & $0.200^{*}$ & $0.200^{*}$ & $0.200^{*}$ & $0.200^{*}$ & $0.200^{*}$ & $0.200^{*}$ \\
\hline & S-W (Statistic) & 0.925 & 0.917 & 0.968 & 0.913 & 0.914 & 0.913 & 0.944 & 0.939 & 0.934 & 0.982 & 0.985 & 0.937 & 0.943 & 0.913 & 0.925 \\
\hline & S-W (p-value) & 0.330 & 0.265 & 0.883 & 0.235 & 0.243 & 0.232 & 0.548 & 0.483 & 0.420 & 0.990 & 0.997 & 0.455 & 0.541 & 0.230 & 0.328 \\
\hline RT 08 & K-S (Statistic) & 0.190 & 0.180 & 0.234 & 0.243 & 0.234 & 0.228 & 0.190 & 0.179 & 0.183 & 0.179 & 0.193 & 0.183 & 0.148 & 0.202 & 0.209 \\
\hline
\end{tabular}


Table C3: Kolmogorov-Smirnov and Shapiro-Wilk tests for Rolling Terrain for Headway 5 s- Cont.

\begin{tabular}{|c|c|c|c|c|c|c|c|c|c|c|c|c|c|c|c|c|}
\hline $\begin{array}{l}\text { Curve } \\
\text { ID }\end{array}$ & Test & $V_{c r v-a v g}$ & $V_{c r v-\min }$ & $V_{c r v-\max }$ & $V_{B C}$ & $V_{T B C}$ & $V_{D B C}$ & $V_{M C}$ & $V_{T M C}$ & $V_{D M C}$ & $V_{E C}$ & $V_{T E C}$ & $V_{D E C}$ & $a_{L}$ & $V_{c m f-a v g}$ & $V_{c m f-\min }$ \\
\hline & K-S (p-value) & $0.200^{*}$ & $0.200^{*}$ & 0.094 & 0.069 & 0.095 & 0.115 & $0.200^{*}$ & $0.200^{*}$ & $0.200^{*}$ & $0.200^{*}$ & $0.200^{*}$ & $0.200^{*}$ & $0.200^{*}$ & $0.200^{*}$ & 0.197 \\
\hline & S-W (Statistic) & 0.896 & 0.900 & 0.905 & 0.894 & 0.896 & 0.900 & 0.900 & 0.906 & 0.906 & 0.925 & 0.927 & 0.897 & 0.930 & 0.922 & 0.921 \\
\hline & S-W (p-value) & 0.164 & 0.186 & 0.212 & 0.155 & 0.167 & 0.184 & 0.186 & 0.221 & 0.216 & 0.361 & 0.383 & 0.170 & 0.414 & 0.335 & 0.330 \\
\hline \multirow[t]{4}{*}{ RT 09} & K-S (Statistic) & 0.251 & 0.320 & 0.191 & 0.150 & 0.175 & 0.180 & 0.267 & 0.265 & 0.275 & 0.250 & 0.253 & 0.299 & 0.197 & 0.296 & 0.320 \\
\hline & K-S (p-value) & 0.147 & 0.016 & $0.200^{*}$ & $0.200^{*}$ & $0.200^{*}$ & $0.200^{*}$ & 0.097 & 0.102 & 0.077 & 0.150 & 0.140 & 0.034 & $0.200^{*}$ & 0.038 & 0.016 \\
\hline & S-W (Statistic) & 0.857 & 0.862 & 0.865 & 0.935 & 0.912 & 0.874 & 0.850 & 0.856 & 0.854 & 0.876 & 0.895 & 0.880 & 0.908 & 0.865 & 0.862 \\
\hline & S-W (p-value) & 0.111 & 0.126 & 0.135 & 0.558 & 0.366 & 0.167 & 0.094 & 0.110 & 0.105 & 0.172 & 0.260 & 0.187 & 0.343 & 0.135 & 0.126 \\
\hline \multirow[t]{4}{*}{ RT 10} & K-S (Statistic) & 0.147 & 0.182 & 0.121 & 0.104 & 0.129 & 0.146 & 0.153 & 0.148 & 0.146 & 0.149 & 0.167 & 0.149 & 0.133 & 0.161 & 0.184 \\
\hline & K-S (p-value) & 0.009 & 0.000 & 0.067 & $0.200^{*}$ & 0.036 & 0.009 & 0.005 & 0.008 & 0.010 & 0.007 & 0.001 & 0.007 & 0.027 & 0.002 & 0.000 \\
\hline & S-W (Statistic) & 0.886 & 0.895 & 0.875 & 0.921 & 0.918 & 0.913 & 0.863 & 0.866 & 0.870 & 0.891 & 0.892 & 0.882 & 0.915 & 0.880 & 0.880 \\
\hline & S-W (p-value) & 0.000 & 0.000 & 0.000 & 0.002 & 0.002 & 0.001 & 0.000 & 0.000 & 0.000 & 0.000 & 0.000 & 0.000 & 0.002 & 0.000 & 0.000 \\
\hline \multirow[t]{4}{*}{ RT 11} & K-S (Statistic) & 0.117 & 0.099 & 0.118 & 0.109 & 0.114 & 0.110 & 0.117 & 0.118 & 0.121 & 0.123 & 0.116 & 0.122 & 0.112 & 0.128 & 0.118 \\
\hline & K-S (p-value) & 0.184 & $0.200^{*}$ & 0.166 & $0.200^{*}$ & $0.200^{*}$ & $0.200^{*}$ & 0.176 & 0.170 & 0.140 & 0.128 & 0.186 & 0.137 & $0.200^{*}$ & 0.098 & 0.173 \\
\hline & S-W (Statistic) & 0.955 & 0.949 & 0.968 & 0.949 & 0.949 & 0.955 & 0.952 & 0.951 & 0.952 & 0.949 & 0.955 & 0.954 & 0.952 & 0.959 & 0.951 \\
\hline & S-W (p-value) & 0.110 & 0.069 & 0.302 & 0.072 & 0.072 & 0.114 & 0.092 & 0.080 & 0.088 & 0.073 & 0.114 & 0.103 & 0.086 & 0.155 & 0.079 \\
\hline \multirow[t]{4}{*}{ RT 12} & K-S (Statistic) & 0.109 & 0.143 & 0.116 & 0.121 & 0.118 & 0.120 & 0.093 & 0.094 & 0.094 & 0.112 & 0.103 & 0.107 & 0.153 & 0.113 & 0.117 \\
\hline & K-S (p-value) & $0.200^{*}$ & $0.200^{*}$ & $0.200^{*}$ & $0.200^{*}$ & $0.200^{*}$ & $0.200^{*}$ & $0.200^{*}$ & $0.200^{*}$ & $0.200^{*}$ & $0.200^{*}$ & $0.200^{*}$ & $0.200^{*}$ & $0.200^{*}$ & $0.200^{*}$ & $0.200^{*}$ \\
\hline & S-W (Statistic) & 0.950 & 0.950 & 0.973 & 0.973 & 0.970 & 0.952 & 0.960 & 0.959 & 0.960 & 0.952 & 0.958 & 0.950 & 0.922 & 0.970 & 0.962 \\
\hline & S-W (p-value) & 0.426 & 0.427 & 0.857 & 0.850 & 0.805 & 0.449 & 0.596 & 0.590 & 0.607 & 0.455 & 0.570 & 0.421 & 0.142 & 0.791 & 0.633 \\
\hline \multirow[t]{4}{*}{ RT 13} & K-S (Statistic) & 0.149 & 0.183 & 0.145 & 0.155 & 0.154 & 0.160 & 0.111 & 0.107 & 0.109 & 0.126 & 0.125 & 0.131 & 0.180 & 0.157 & 0.150 \\
\hline & K-S (p-value) & $0.200^{*}$ & 0.189 & $0.200^{*}$ & $0.200^{*}$ & $0.200^{*}$ & 0.200 * & $0.200^{*}$ & 0.200 * & $0.200^{*}$ & $0.200^{*}$ & $0.200^{\star}$ & $0.200^{*}$ & $0.200^{*}$ & $0.200^{*}$ & $0.200^{*}$ \\
\hline & S-W (Statistic) & 0.953 & 0.935 & 0.958 & 0.940 & 0.951 & 0.954 & 0.971 & 0.970 & 0.969 & 0.983 & 0.971 & 0.968 & 0.877 & 0.969 & 0.970 \\
\hline & S-W (p-value) & 0.578 & 0.319 & 0.666 & 0.386 & 0.542 & 0.592 & 0.869 & 0.861 & 0.841 & 0.985 & 0.876 & 0.820 & 0.043 & 0.838 & 0.856 \\
\hline \multirow[t]{4}{*}{ RT 14} & K-S (Statistic) & 0.196 & 0.172 & 0.235 & 0.156 & 0.158 & 0.171 & 0.229 & 0.228 & 0.235 & 0.181 & 0.181 & 0.240 & 0.151 & 0.147 & 0.169 \\
\hline & K-S (p-value) & 0.181 & $0.200^{*}$ & 0.048 & $0.200^{*}$ & $0.200^{*}$ & $0.200^{*}$ & 0.062 & 0.064 & 0.048 & $0.200^{*}$ & $0.200^{*}$ & 0.040 & $0.200^{*}$ & $0.200^{*}$ & $0.200^{*}$ \\
\hline & S-W (Statistic) & 0.940 & 0.936 & 0.919 & 0.883 & 0.892 & 0.912 & 0.920 & 0.918 & 0.914 & 0.930 & 0.926 & 0.915 & 0.947 & 0.949 & 0.949 \\
\hline & S-W (p-value) & 0.463 & 0.406 & 0.244 & 0.079 & 0.103 & 0.194 & 0.254 & 0.233 & 0.207 & 0.345 & 0.301 & 0.215 & 0.560 & 0.589 & 0.591 \\
\hline \multirow[t]{2}{*}{$\mathrm{RT} 15$} & K-S (Statistic) & 0.151 & 0.143 & 0.157 & 0.132 & 0.132 & 0.141 & 0.148 & 0.150 & 0.146 & 0.168 & 0.170 & 0.151 & 0.101 & 0.147 & 0.149 \\
\hline & K-S (p-value) & 0.015 & 0.027 & 0.010 & 0.060 & 0.057 & 0.031 & 0.019 & 0.017 & 0.022 & 0.004 & 0.003 & 0.015 & $0.200^{*}$ & 0.021 & 0.017 \\
\hline
\end{tabular}


Table C3: Kolmogorov-Smirnov and Shapiro-Wilk tests for Rolling Terrain for Headway 5 s- Cont.

\begin{tabular}{|c|c|c|c|c|c|c|c|c|c|c|c|c|c|c|c|c|}
\hline $\begin{array}{l}\text { Curve } \\
\text { ID }\end{array}$ & Test & $V_{c r v-a v g}$ & $V_{c r v-\min }$ & $V_{c r v-\max }$ & $V_{B C}$ & $V_{T B C}$ & $V_{D B C}$ & $V_{M C}$ & $V_{T M C}$ & $V_{D M C}$ & $V_{E C}$ & $V_{T E C}$ & $V_{D E C}$ & $a_{L}$ & $V_{c m f-a v g}$ & $V_{c m f-\min }$ \\
\hline \multirow{5}{*}{ RT 16} & S-W (Statistic) & 0.839 & 0.840 & 0.842 & 0.876 & 0.881 & 0.884 & 0.825 & 0.826 & 0.829 & 0.796 & 0.808 & 0.822 & 0.961 & 0.834 & 0.827 \\
\hline & S-W (p-value) & 0.000 & 0.000 & 0.000 & 0.000 & 0.000 & 0.000 & 0.000 & 0.000 & 0.000 & 0.000 & 0.000 & 0.000 & 0.156 & 0.000 & 0.000 \\
\hline & K-S (Statistic) & 0.136 & 0.254 & 0.102 & 0.132 & 0.162 & 0.156 & 0.104 & 0.114 & 0.110 & 0.327 & 0.309 & 0.133 & 0.131 & 0.111 & 0.119 \\
\hline & K-S (p-value) & $0.200^{*}$ & 0.001 & $0.200^{*}$ & $0.200^{*}$ & 0.137 & 0.172 & $0.200^{*}$ & $0.200^{*}$ & $0.200^{*}$ & 0.000 & 0.000 & $0.200^{*}$ & $0.200^{*}$ & $0.200^{*}$ & $0.200^{*}$ \\
\hline & S-W (Statistic) & 0.944 & 0.648 & 0.962 & 0.971 & 0.964 & 0.962 & 0.974 & 0.973 & 0.973 & 0.554 & 0.569 & 0.936 & 0.986 & 0.973 & 0.967 \\
\hline \multirow{3}{*}{ RT 17} & S-W (p-value) & 0.245 & 0.000 & 0.521 & 0.738 & 0.581 & 0.531 & 0.807 & 0.776 & 0.782 & 0.000 & 0.000 & 0.166 & 0.980 & 0.775 & 0.638 \\
\hline & K-S (Statistic) & 0.153 & 0.168 & 0.173 & 0.175 & 0.180 & 0.176 & 0.165 & 0.149 & 0.150 & 0.197 & 0.177 & 0.152 & 0.080 & 0.148 & 0.157 \\
\hline & K-S (p-value) & 0.020 & 0.006 & 0.004 & 0.004 & 0.002 & 0.003 & 0.008 & 0.026 & 0.024 & 0.000 & 0.003 & 0.021 & $0.200^{*}$ & 0.028 & 0.014 \\
\hline \multirow{5}{*}{ RT 18} & S-W (Statistic) & 0.866 & 0.870 & 0.859 & 0.833 & 0.840 & 0.855 & 0.864 & 0.865 & 0.869 & 0.867 & 0.871 & 0.872 & 0.986 & 0.882 & 0.876 \\
\hline & S-W (p-value) & 0.000 & 0.000 & 0.000 & 0.000 & 0.000 & 0.000 & 0.000 & 0.000 & 0.000 & 0.000 & 0.000 & 0.000 & 0.889 & 0.001 & 0.000 \\
\hline & K-S (Statistic) & 0.140 & 0.151 & 0.110 & 0.107 & 0.107 & 0.126 & 0.177 & 0.175 & 0.171 & 0.142 & 0.146 & 0.149 & 0.119 & 0.184 & 0.178 \\
\hline & K-S (p-value) & $0.200^{*}$ & 0.190 & $0.200^{*}$ & $0.200^{*}$ & $0.200^{*}$ & $0.200^{*}$ & 0.060 & 0.064 & 0.078 & $0.200^{*}$ & $0.200^{*}$ & $0.200^{*}$ & $0.200^{*}$ & 0.042 & 0.056 \\
\hline & S-W (Statistic) & 0.933 & 0.937 & 0.951 & 0.948 & 0.942 & 0.940 & 0.918 & 0.919 & 0.922 & 0.961 & 0.960 & 0.935 & 0.965 & 0.922 & 0.921 \\
\hline \multirow{3}{*}{ RT 19} & S-W (p-value) & 0.126 & 0.154 & 0.306 & 0.260 & 0.200 & 0.177 & 0.060 & 0.063 & 0.073 & 0.474 & 0.459 & 0.143 & 0.572 & 0.073 & 0.070 \\
\hline & K-S (Statistic) & 0.129 & 0.149 & 0.129 & 0.135 & 0.131 & 0.141 & 0.111 & 0.111 & 0.109 & 0.140 & 0.173 & 0.126 & $0.200^{*}$ & 0.162 & 0.157 \\
\hline & K-S (p-value) & $0.200^{*}$ & 0.183 & $0.200^{*}$ & $0.200^{*}$ & $0.200^{*}$ & $0.200^{*}$ & $0.200^{*}$ & $0.200^{*}$ & $0.200^{*}$ & $0.200^{*}$ & 0.062 & $0.200^{*}$ & 0.014 & 0.104 & 0.131 \\
\hline \multirow{5}{*}{ RT 20} & S-W (Statistic) & 0.969 & 0.949 & 0.936 & 0.938 & 0.933 & 0.941 & 0.961 & 0.962 & 0.964 & 0.897 & 0.900 & 0.966 & 0.931 & 0.955 & 0.954 \\
\hline & S-W (p-value) & 0.636 & 0.252 & 0.134 & 0.150 & 0.113 & 0.174 & 0.460 & 0.474 & 0.531 & 0.019 & 0.021 & 0.562 & 0.101 & 0.343 & 0.336 \\
\hline & K-S (Statistic) & 0.137 & 0.172 & 0.139 & 0.205 & 0.198 & 0.192 & 0.133 & 0.136 & 0.138 & 0.108 & 0.104 & 0.133 & 0.090 & 0.136 & 0.149 \\
\hline & K-S (p-value) & $0.200^{*}$ & 0.039 & 0.193 & 0.005 & 0.008 & 0.012 & $0.200^{*}$ & $0.200^{*}$ & $0.200^{*}$ & $0.200^{*}$ & $0.200^{*}$ & $0.200^{*}$ & $0.200^{*}$ & $0.200^{*}$ & 0.127 \\
\hline & S-W (Statistic) & 0.969 & 0.956 & 0.967 & 0.933 & 0.937 & 0.942 & 0.970 & 0.970 & 0.967 & 0.981 & 0.983 & 0.974 & 0.960 & 0.965 & 0.965 \\
\hline \multirow{3}{*}{ RT 21} & S-W (p-value) & 0.587 & 0.296 & 0.536 & 0.083 & 0.105 & 0.138 & 0.591 & 0.596 & 0.524 & 0.888 & 0.915 & 0.719 & 0.378 & 0.477 & 0.484 \\
\hline & K-S (Statistic) & 0.097 & 0.113 & 0.123 & 0.111 & 0.128 & 0.109 & 0.104 & 0.104 & 0.105 & 0.102 & 0.109 & 0.099 & 0.132 & 0.091 & 0.110 \\
\hline & K-S (p-value) & $0.200^{*}$ & $0.200^{*}$ & 0.100 & $0.200^{*}$ & 0.076 & $0.200^{*}$ & $0.200^{*}$ & $0.200^{*}$ & $0.200^{*}$ & $0.200^{*}$ & $0.200^{*}$ & $0.200^{*}$ & 0.059 & $0.200^{*}$ & $0.200^{*}$ \\
\hline \multirow{5}{*}{ RT 22} & S-W (Statistic) & 0.943 & 0.961 & 0.938 & 0.956 & 0.955 & 0.954 & 0.944 & 0.943 & 0.941 & 0.961 & 0.958 & 0.940 & 0.966 & 0.956 & 0.954 \\
\hline & S-W (p-value) & 0.034 & 0.150 & 0.022 & 0.103 & 0.092 & 0.083 & 0.035 & 0.034 & 0.029 & 0.155 & 0.121 & 0.026 & 0.224 & 0.099 & 0.083 \\
\hline & K-S (Statistic) & 0.144 & 0.111 & 0.153 & 0.144 & 0.140 & 0.151 & 0.119 & 0.124 & 0.130 & 0.112 & 0.115 & 0.154 & 0.127 & 0.145 & 0.136 \\
\hline & K-S (p-value) & 0.044 & $0.200^{*}$ & 0.025 & 0.046 & 0.059 & 0.029 & 0.192 & 0.145 & 0.103 & $0.200^{*}$ & $0.200^{*}$ & 0.023 & 0.128 & 0.043 & 0.075 \\
\hline & S-W (Statistic) & 0.970 & 0.978 & 0.951 & 0.950 & 0.953 & 0.957 & 0.971 & 0.969 & 0.970 & 0.974 & 0.974 & 0.972 & 0.954 & 0.963 & 0.965 \\
\hline
\end{tabular}


Table C3: Kolmogorov-Smirnov and Shapiro-Wilk tests for Rolling Terrain for Headway 5 s- Cont.

\begin{tabular}{|c|c|c|c|c|c|c|c|c|c|c|c|c|c|c|c|c|}
\hline $\begin{array}{l}\text { Curve } \\
\text { ID }\end{array}$ & Test & $V_{c r v-a v g}$ & $V_{c r v-\min }$ & $V_{c r v-\max }$ & $V_{B C}$ & $V_{T B C}$ & $V_{D B C}$ & $V_{M C}$ & $V_{T M C}$ & $V_{D M C}$ & $V_{E C}$ & $V_{T E C}$ & $V_{D E C}$ & $a_{L}$ & $V_{c m f-a v g}$ & $V_{c m f-\min }$ \\
\hline & S-W (p-value) & 0.401 & 0.649 & 0.094 & 0.089 & 0.112 & 0.152 & 0.416 & 0.373 & 0.384 & 0.505 & 0.506 & 0.445 & 0.123 & 0.237 & 0.279 \\
\hline \multirow[t]{4}{*}{ RT 23} & K-S (Statistic) & 0.119 & 0.119 & 0.131 & 0.119 & 0.100 & 0.078 & 0.133 & 0.130 & 0.128 & 0.134 & 0.124 & 0.098 & 0.127 & 0.079 & 0.096 \\
\hline & K-S (p-value) & $0.200^{*}$ & $0.200^{*}$ & $0.200^{*}$ & $0.200^{*}$ & $0.200^{*}$ & $0.200^{*}$ & $0.200^{*}$ & $0.200^{*}$ & $0.200^{*}$ & $0.200^{*}$ & $0.200^{*}$ & $0.200^{*}$ & $0.200^{*}$ & $0.200^{*}$ & $0.200^{*}$ \\
\hline & S-W (Statistic) & 0.979 & 0.976 & 0.962 & 0.948 & 0.953 & 0.971 & 0.963 & 0.962 & 0.969 & 0.943 & 0.950 & 0.973 & 0.981 & 0.977 & 0.974 \\
\hline & S-W (p-value) & 0.924 & 0.873 & 0.589 & 0.341 & 0.415 & 0.775 & 0.609 & 0.579 & 0.735 & 0.273 & 0.366 & 0.816 & 0.941 & 0.886 & 0.828 \\
\hline \multirow[t]{4}{*}{ RT 24} & K-S (Statistic) & 0.159 & 0.160 & 0.140 & 0.155 & 0.143 & 0.143 & 0.184 & 0.187 & 0.177 & 0.139 & 0.171 & 0.166 & 0.137 & 0.139 & 0.127 \\
\hline & K-S (p-value) & 0.178 & 0.171 & $0.200^{*}$ & $0.200^{*}$ & $0.200^{*}$ & $0.200^{*}$ & 0.062 & 0.054 & 0.087 & $0.200^{*}$ & 0.109 & 0.136 & $0.200^{*}$ & $0.200^{*}$ & $0.200^{*}$ \\
\hline & S-W (Statistic) & 0.933 & 0.936 & 0.948 & 0.923 & 0.937 & 0.946 & 0.920 & 0.919 & 0.923 & 0.959 & 0.960 & 0.926 & 0.951 & 0.946 & 0.948 \\
\hline & S-W (p-value) & 0.158 & 0.182 & 0.316 & 0.102 & 0.194 & 0.284 & 0.088 & 0.084 & 0.099 & 0.506 & 0.512 & 0.117 & 0.353 & 0.286 & 0.309 \\
\hline \multirow[t]{4}{*}{ RT 25} & K-S (Statistic) & 0.115 & 0.167 & 0.093 & 0.089 & 0.105 & 0.111 & 0.122 & 0.119 & 0.116 & 0.173 & 0.128 & 0.130 & 0.099 & 0.130 & 0.151 \\
\hline & K-S (p-value) & $0.200^{*}$ & 0.115 & $0.200^{*}$ & $0.200^{*}$ & $0.200^{*}$ & $0.200^{*}$ & $0.200^{*}$ & $0.200^{*}$ & $0.200^{*}$ & 0.086 & $0.200^{*}$ & $0.200^{*}$ & $0.200^{*}$ & $0.200^{*}$ & $0.200^{*}$ \\
\hline & S-W (Statistic) & 0.961 & 0.889 & 0.980 & 0.979 & 0.978 & 0.970 & 0.960 & 0.962 & 0.961 & 0.935 & 0.942 & 0.960 & 0.944 & 0.943 & 0.907 \\
\hline & S-W (p-value) & 0.513 & 0.018 & 0.915 & 0.896 & 0.877 & 0.710 & 0.488 & 0.523 & 0.517 & 0.154 & 0.213 & 0.489 & 0.235 & 0.231 & 0.041 \\
\hline \multirow[t]{4}{*}{ RT 26} & K-S (Statistic) & 0.191 & 0.252 & 0.168 & 0.167 & 0.175 & 0.191 & 0.223 & 0.231 & 0.228 & 0.177 & 0.198 & 0.215 & 0.136 & 0.226 & 0.254 \\
\hline & K-S (p-value) & 0.146 & 0.011 & $0.200^{*}$ & $0.200^{*}$ & $0.200^{*}$ & 0.146 & 0.043 & 0.030 & 0.035 & $0.200^{*}$ & 0.118 & 0.059 & $0.200^{*}$ & 0.038 & 0.010 \\
\hline & S-W (Statistic) & 0.829 & 0.771 & 0.946 & 0.949 & 0.944 & 0.926 & 0.789 & 0.787 & 0.785 & 0.942 & 0.933 & 0.827 & 0.927 & 0.789 & 0.774 \\
\hline & S-W (p-value) & 0.009 & 0.002 & 0.458 & 0.511 & 0.436 & 0.234 & 0.003 & 0.003 & 0.002 & 0.413 & 0.303 & 0.008 & 0.246 & 0.003 & 0.002 \\
\hline \multirow[t]{4}{*}{ RT 27} & K-S (Statistic) & 0.168 & 0.135 & 0.175 & 0.116 & 0.115 & 0.132 & 0.151 & 0.146 & 0.146 & 0.130 & 0.121 & 0.136 & 0.156 & 0.141 & 0.130 \\
\hline & K-S (p-value) & $0.200^{*}$ & $0.200^{*}$ & 0.173 & $0.200^{*}$ & $0.200^{*}$ & $0.200^{*}$ & $0.200^{*}$ & $0.200^{*}$ & $0.200^{*}$ & $0.200^{*}$ & $0.200^{*}$ & $0.200^{*}$ & $0.200^{*}$ & $0.200^{*}$ & $0.200^{*}$ \\
\hline & S-W (Statistic) & 0.945 & 0.968 & 0.945 & 0.974 & 0.973 & 0.974 & 0.955 & 0.955 & 0.951 & 0.967 & 0.969 & 0.959 & 0.900 & 0.965 & 0.970 \\
\hline & S-W (p-value) & 0.378 & 0.784 & 0.388 & 0.888 & 0.864 & 0.883 & 0.549 & 0.542 & 0.474 & 0.762 & 0.806 & 0.614 & 0.069 & 0.735 & 0.817 \\
\hline \multirow[t]{4}{*}{ RT 28} & K-S (Statistic) & 0.166 & 0.204 & 0.161 & 0.211 & 0.209 & 0.191 & 0.158 & 0.154 & 0.157 & 0.166 & 0.174 & 0.165 & 0.149 & 0.198 & 0.211 \\
\hline & K-S (p-value) & 0.118 & 0.017 & 0.143 & 0.012 & 0.014 & 0.037 & 0.164 & 0.189 & 0.171 & 0.117 & 0.081 & 0.120 & $0.200^{*}$ & 0.024 & 0.012 \\
\hline & S-W (Statistic) & 0.879 & 0.872 & 0.853 & 0.856 & 0.858 & 0.870 & 0.897 & 0.896 & 0.894 & 0.859 & 0.861 & 0.879 & 0.931 & 0.864 & 0.866 \\
\hline & S-W (p-value) & 0.012 & 0.008 & 0.004 & 0.004 & 0.005 & 0.008 & 0.026 & 0.024 & 0.023 & 0.005 & 0.005 & 0.011 & 0.129 & 0.006 & 0.007 \\
\hline \multirow[t]{4}{*}{ RT 29} & K-S (Statistic) & 0.138 & 0.100 & 0.124 & 0.178 & 0.163 & 0.153 & 0.093 & 0.090 & 0.092 & 0.126 & 0.135 & 0.154 & 0.106 & 0.138 & 0.188 \\
\hline & K-S (p-value) & $0.200^{*}$ & $0.200^{*}$ & $0.200^{*}$ & $0.200^{*}$ & $0.200^{*}$ & $0.200^{*}$ & $0.200^{*}$ & $0.200^{*}$ & $0.200^{*}$ & $0.200^{*}$ & $0.200^{*}$ & $0.200^{*}$ & $0.200^{*}$ & $0.200^{*}$ & $0.200^{*}$ \\
\hline & S-W (Statistic) & 0.950 & 0.972 & 0.956 & 0.915 & 0.924 & 0.939 & 0.966 & 0.967 & 0.967 & 0.962 & 0.953 & 0.953 & 0.972 & 0.956 & 0.947 \\
\hline & S-W (p-value) & 0.594 & 0.919 & 0.691 & 0.216 & 0.280 & 0.450 & 0.840 & 0.856 & 0.853 & 0.785 & 0.644 & 0.649 & 0.919 & 0.692 & 0.549 \\
\hline
\end{tabular}


Table C3: Kolmogorov-Smirnov and Shapiro-Wilk tests for Rolling Terrain for Headway 5 s- Cont.

\begin{tabular}{|c|c|c|c|c|c|c|c|c|c|c|c|c|c|c|c|c|}
\hline $\begin{array}{l}\text { Curve } \\
\text { ID }\end{array}$ & Test & $V_{c r v-a v g}$ & $V_{c r v-\min }$ & $V_{c r v-\max }$ & $V_{B C}$ & $V_{T B C}$ & $V_{D B C}$ & $V_{M C}$ & $V_{T M C}$ & $V_{D M C}$ & $V_{E C}$ & $V_{T E C}$ & $V_{D E C}$ & $a_{L}$ & $V_{c m f-a v g}$ & $V_{c m f-\min }$ \\
\hline \multirow[t]{4}{*}{ RT 30} & K-S (Statistic) & 0.106 & 0.126 & 0.104 & 0.116 & 0.125 & 0.121 & 0.101 & 0.104 & 0.101 & 0.111 & 0.109 & 0.101 & 0.116 & 0.117 & 0.112 \\
\hline & K-S (p-value) & $0.200^{*}$ & 0.178 & $0.200^{*}$ & $0.200^{*}$ & 0.187 & $0.200^{*}$ & $0.200^{*}$ & $0.200^{*}$ & $0.200^{*}$ & $0.200^{*}$ & $0.200^{*}$ & $0.200^{*}$ & $0.200^{*}$ & $0.200^{*}$ & $0.200^{*}$ \\
\hline & S-W (Statistic) & 0.979 & 0.978 & 0.969 & 0.950 & 0.955 & 0.962 & 0.982 & 0.983 & 0.983 & 0.978 & 0.982 & 0.984 & 0.932 & 0.977 & 0.980 \\
\hline & S-W (p-value) & 0.727 & 0.692 & 0.413 & 0.112 & 0.161 & 0.259 & 0.823 & 0.861 & 0.846 & 0.698 & 0.825 & 0.876 & 0.031 & 0.668 & 0.774 \\
\hline \multirow[t]{4}{*}{ RT 31} & K-S (Statistic) & 0.186 & 0.196 & 0.174 & 0.150 & 0.177 & 0.172 & 0.139 & 0.140 & 0.140 & 0.210 & 0.208 & 0.195 & 0.153 & 0.128 & 0.135 \\
\hline & K-S (p-value) & 0.082 & 0.052 & 0.134 & $0.200^{*}$ & 0.121 & 0.141 & $0.200^{*}$ & $0.200^{*}$ & $0.200^{*}$ & 0.027 & 0.030 & 0.055 & $0.200^{*}$ & $0.200^{*}$ & $0.200^{*}$ \\
\hline & S-W (Statistic) & 0.960 & 0.945 & 0.901 & 0.969 & 0.964 & 0.956 & 0.977 & 0.977 & 0.979 & 0.873 & 0.886 & 0.951 & 0.949 & 0.974 & 0.977 \\
\hline & S-W (p-value) & 0.580 & 0.320 & 0.050 & 0.754 & 0.659 & 0.491 & 0.909 & 0.908 & 0.924 & 0.016 & 0.027 & 0.406 & 0.376 & 0.857 & 0.905 \\
\hline \multirow[t]{4}{*}{ RT 32} & K-S (Statistic) & 0.113 & 0.132 & 0.079 & 0.106 & 0.112 & 0.126 & 0.124 & 0.116 & 0.127 & 0.092 & 0.104 & 0.111 & 0.087 & 0.146 & 0.141 \\
\hline & K-S (p-value) & $0.200^{*}$ & $0.200^{*}$ & $0.200^{*}$ & $0.200^{*}$ & $0.200^{*}$ & $0.200^{*}$ & $0.200^{*}$ & $0.200^{*}$ & $0.200^{*}$ & $0.200^{*}$ & $0.200^{*}$ & $0.200^{*}$ & $0.200^{*}$ & $0.200^{*}$ & $0.200^{*}$ \\
\hline & S-W (Statistic) & 0.974 & 0.961 & 0.966 & 0.974 & 0.972 & 0.971 & 0.976 & 0.976 & 0.974 & 0.958 & 0.952 & 0.974 & 0.977 & 0.951 & 0.946 \\
\hline & S-W (p-value) & 0.806 & 0.504 & 0.622 & 0.791 & 0.748 & 0.743 & 0.836 & 0.853 & 0.795 & 0.448 & 0.346 & 0.796 & 0.868 & 0.331 & 0.261 \\
\hline \multirow[t]{4}{*}{ RT 33} & K-S (Statistic) & 0.089 & 0.075 & 0.091 & 0.082 & 0.083 & 0.092 & 0.094 & 0.094 & 0.096 & 0.108 & 0.092 & 0.089 & 0.119 & 0.071 & 0.075 \\
\hline & K-S (p-value) & $0.200^{*}$ & $0.200^{*}$ & $0.200^{*}$ & $0.200^{*}$ & $0.200^{*}$ & $0.200^{*}$ & $0.200^{*}$ & $0.200^{*}$ & $0.200^{*}$ & $0.200^{*}$ & $0.200^{*}$ & $0.200^{*}$ & $0.200^{*}$ & $0.200^{*}$ & $0.200^{*}$ \\
\hline & S-W (Statistic) & 0.988 & 0.989 & 0.985 & 0.986 & 0.988 & 0.990 & 0.985 & 0.985 & 0.985 & 0.973 & 0.978 & 0.984 & 0.948 & 0.988 & 0.986 \\
\hline & S-W (p-value) & 0.957 & 0.977 & 0.884 & 0.923 & 0.951 & 0.982 & 0.896 & 0.894 & 0.898 & 0.506 & 0.671 & 0.867 & 0.091 & 0.952 & 0.927 \\
\hline
\end{tabular}


Table C4: Kolmogorov-Smirnov and Shapiro-Wilk tests for Mountainous Terrain for Headway $5 \mathrm{~s}$

\begin{tabular}{|c|c|c|c|c|c|c|c|c|c|c|c|c|c|c|c|c|}
\hline $\begin{array}{c}\text { Curve } \\
\text { ID }\end{array}$ & Test & $V_{c r v-a v g}$ & $V_{c r v-m i n}$ & $V_{c r v-\max }$ & $V_{B C}$ & $V_{T B C}$ & $V_{D B C}$ & $V_{M C}$ & $V_{T M C}$ & $V_{D M C}$ & $V_{E C}$ & $V_{T E C}$ & $V_{D E C}$ & $a_{L}$ & $V_{c m f-a v g}$ & $V_{c m f-\min }$ \\
\hline \multirow[t]{4}{*}{ MT 01} & K-S (Statistic) & 0.148 & 0.125 & 0.135 & 0.100 & 0.109 & 0.122 & 0.116 & 0.114 & 0.113 & 0.138 & 0.143 & 0.149 & 0.073 & 0.094 & 0.113 \\
\hline & K-S (p-value) & 0.010 & 0.060 & 0.028 & $0.200^{*}$ & $0.200^{*}$ & 0.073 & 0.120 & 0.157 & 0.163 & 0.023 & 0.015 & 0.009 & $0.200^{*}$ & $0.200^{*}$ & 0.161 \\
\hline & S-W (Statistic) & 0.958 & 0.964 & 0.966 & 0.968 & 0.968 & 0.965 & 0.973 & 0.972 & 0.971 & 0.956 & 0.956 & 0.959 & 0.989 & 0.973 & 0.961 \\
\hline & S-W (p-value) & 0.083 & 0.148 & 0.169 & 0.213 & 0.218 & 0.165 & 0.344 & 0.314 & 0.279 & 0.073 & 0.068 & 0.091 & 0.927 & 0.339 & 0.106 \\
\hline \multirow[t]{4}{*}{ MT 02} & K-S (Statistic) & 0.108 & 0.109 & 0.094 & 0.107 & 0.120 & 0.119 & 0.107 & 0.107 & 0.109 & 0.075 & 0.076 & 0.100 & 0.111 & 0.110 & 0.103 \\
\hline & K-S (p-value) & $0.200^{*}$ & $0.200^{*}$ & $0.200^{*}$ & $0.200^{*}$ & 0.075 & 0.081 & $0.200^{*}$ & $0.200^{*}$ & $0.200^{*}$ & $0.200^{*}$ & $0.200^{*}$ & $0.200^{*}$ & 0.176 & 0.191 & $0.200^{*}$ \\
\hline & S-W (Statistic) & 0.935 & 0.947 & 0.944 & 0.938 & 0.932 & 0.929 & 0.935 & 0.935 & 0.932 & 0.961 & 0.963 & 0.940 & 0.968 & 0.929 & 0.928 \\
\hline & S-W (p-value) & 0.010 & 0.028 & 0.021 & 0.012 & 0.007 & 0.005 & 0.009 & 0.009 & 0.007 & 0.108 & 0.127 & 0.015 & 0.208 & 0.006 & 0.005 \\
\hline \multirow[t]{4}{*}{ MT 03} & K-S (Statistic) & 0.095 & 0.075 & 0.158 & 0.095 & 0.099 & 0.100 & 0.105 & 0.107 & 0.106 & 0.120 & 0.112 & 0.102 & 0.107 & 0.079 & 0.089 \\
\hline & K-S (p-value) & $0.200^{*}$ & $0.200^{*}$ & 0.003 & $0.200^{*}$ & $0.200^{*}$ & $0.200^{*}$ & $0.200^{*}$ & $0.200^{*}$ & $0.200^{*}$ & 0.066 & 0.141 & $0.200^{*}$ & $0.200^{*}$ & $0.200^{*}$ & $0.200^{*}$ \\
\hline & S-W (Statistic) & 0.944 & 0.983 & 0.874 & 0.982 & 0.980 & 0.981 & 0.902 & 0.901 & 0.900 & 0.908 & 0.902 & 0.939 & 0.971 & 0.956 & 0.968 \\
\hline & S-W (p-value) & 0.018 & 0.679 & 0.000 & 0.623 & 0.526 & 0.570 & 0.000 & 0.000 & 0.000 & 0.001 & 0.000 & 0.011 & 0.233 & 0.057 & 0.175 \\
\hline \multirow[t]{4}{*}{ MT 04} & K-S (Statistic) & 0.124 & 0.107 & 0.127 & 0.145 & 0.156 & 0.150 & 0.126 & 0.131 & 0.133 & 0.127 & 0.130 & 0.109 & 0.100 & 0.095 & 0.102 \\
\hline & K-S (p-value) & $0.200^{*}$ & $0.200^{*}$ & $0.200^{*}$ & 0.153 & 0.090 & 0.123 & $0.200^{*}$ & $0.200^{*}$ & $0.200^{*}$ & $0.200^{*}$ & $0.200^{*}$ & $0.200^{*}$ & $0.200^{*}$ & $0.200^{*}$ & $0.200^{*}$ \\
\hline & S-W (Statistic) & 0.954 & 0.965 & 0.952 & 0.926 & 0.925 & 0.926 & 0.954 & 0.952 & 0.951 & 0.965 & 0.962 & 0.956 & 0.946 & 0.958 & 0.958 \\
\hline & S-W (p-value) & 0.274 & 0.485 & 0.236 & 0.056 & 0.051 & 0.055 & 0.263 & 0.246 & 0.229 & 0.482 & 0.410 & 0.304 & 0.170 & 0.324 & 0.328 \\
\hline \multirow[t]{4}{*}{ MT 05} & K-S (Statistic) & 0.096 & 0.071 & 0.106 & 0.110 & 0.109 & 0.105 & 0.104 & 0.104 & 0.091 & 0.073 & 0.070 & 0.099 & 0.138 & 0.109 & 0.093 \\
\hline & K-S (p-value) & $0.200^{*}$ & $0.200^{*}$ & $0.200^{\star}$ & $0.200^{*}$ & $0.200^{*}$ & $0.200^{*}$ & $0.200^{*}$ & $0.200^{*}$ & $0.200^{*}$ & $0.200^{*}$ & $0.200^{*}$ & $0.200^{*}$ & $0.200^{*}$ & $0.200^{*}$ & $0.200^{*}$ \\
\hline & S-W (Statistic) & 0.966 & 0.969 & 0.972 & 0.967 & 0.971 & 0.974 & 0.965 & 0.966 & 0.966 & 0.966 & 0.970 & 0.966 & 0.966 & 0.961 & 0.967 \\
\hline & S-W (p-value) & 0.513 & 0.606 & 0.671 & 0.539 & 0.657 & 0.716 & 0.495 & 0.513 & 0.513 & 0.528 & 0.611 & 0.513 & 0.534 & 0.419 & 0.536 \\
\hline \multirow[t]{4}{*}{ MT 06} & K-S (Statistic) & 0.127 & 0.102 & 0.133 & 0.138 & 0.127 & 0.116 & 0.146 & 0.138 & 0.138 & 0.150 & 0.121 & 0.116 & 0.145 & 0.088 & 0.092 \\
\hline & K-S (p-value) & $0.200^{*}$ & $0.200^{*}$ & $0.200^{*}$ & $0.200^{*}$ & $0.200^{*}$ & $0.200^{*}$ & $0.200^{*}$ & $0.200^{*}$ & $0.200^{*}$ & $0.200^{*}$ & $0.200^{*}$ & $0.200^{*}$ & $0.200^{*}$ & $0.200^{*}$ & $0.200^{*}$ \\
\hline & S-W (Statistic) & 0.955 & 0.976 & 0.949 & 0.946 & 0.950 & 0.953 & 0.954 & 0.955 & 0.954 & 0.960 & 0.964 & 0.966 & 0.976 & 0.966 & 0.965 \\
\hline & S-W (p-value) & 0.477 & 0.885 & 0.373 & 0.338 & 0.394 & 0.437 & 0.457 & 0.473 & 0.463 & 0.579 & 0.654 & 0.695 & 0.892 & 0.691 & 0.667 \\
\hline \multirow[t]{4}{*}{ MT 07} & K-S (Statistic) & 0.118 & 0.138 & 0.079 & 0.102 & 0.101 & 0.105 & 0.135 & 0.129 & 0.132 & 0.093 & 0.099 & 0.128 & 0.089 & 0.139 & 0.128 \\
\hline & K-S (p-value) & $0.200^{*}$ & $0.200^{*}$ & $0.200^{*}$ & $0.200^{*}$ & $0.200^{*}$ & $0.200^{*}$ & $0.200^{*}$ & $0.200^{*}$ & $0.200^{*}$ & $0.200^{*}$ & $0.200^{*}$ & $0.200^{*}$ & $0.200^{*}$ & $0.200^{*}$ & $0.200^{*}$ \\
\hline & S-W (Statistic) & 0.960 & 0.955 & 0.986 & 0.971 & 0.972 & 0.975 & 0.953 & 0.955 & 0.956 & 0.986 & 0.985 & 0.964 & 0.968 & 0.953 & 0.952 \\
\hline & S-W (p-value) & 0.571 & 0.483 & 0.988 & 0.790 & 0.808 & 0.865 & 0.441 & 0.475 & 0.498 & 0.988 & 0.985 & 0.649 & 0.730 & 0.446 & 0.429 \\
\hline MT 08 & K-S (Statistic) & 0.147 & 0.166 & 0.125 & 0.145 & 0.147 & 0.149 & 0.156 & 0.156 & 0.151 & 0.133 & 0.125 & 0.125 & 0.140 & 0.212 & 0.207 \\
\hline
\end{tabular}


Table C4: Kolmogorov-Smirnov and Shapiro-Wilk tests for Mountainous Terrain for Headway 5 s- Cont.

\begin{tabular}{|c|c|c|c|c|c|c|c|c|c|c|c|c|c|c|c|c|}
\hline $\begin{array}{c}\text { Curve } \\
\text { ID }\end{array}$ & Test & $V_{c r v-a v g}$ & $V_{c r v-\min }$ & $V_{c r v-\max }$ & $V_{B C}$ & $V_{T B C}$ & $V_{D B C}$ & $V_{M C}$ & $V_{T M C}$ & $V_{D M C}$ & $V_{E C}$ & $V_{T E C}$ & $V_{D E C}$ & $a_{L}$ & $V_{c m f-a v g}$ & $V_{c m f-m i n}$ \\
\hline & K-S (p-value) & $0.200^{*}$ & $0.200^{*}$ & $0.200^{*}$ & $0.200^{*}$ & $0.200^{*}$ & $0.200^{*}$ & $0.200^{*}$ & $0.200^{*}$ & $0.200^{*}$ & $0.200^{*}$ & $0.200^{*}$ & $0.200^{*}$ & $0.200^{*}$ & 0.032 & 0.040 \\
\hline & S-W (Statistic) & 0.937 & 0.960 & 0.917 & 0.944 & 0.946 & 0.945 & 0.932 & 0.931 & 0.935 & 0.953 & 0.958 & 0.938 & 0.947 & 0.917 & 0.924 \\
\hline & S-W (p-value) & 0.254 & 0.601 & 0.116 & 0.337 & 0.371 & 0.352 & 0.210 & 0.204 & 0.235 & 0.475 & 0.572 & 0.273 & 0.386 & 0.116 & 0.150 \\
\hline \multirow[t]{4}{*}{ MT 09} & K-S (Statistic) & 0.159 & 0.175 & 0.137 & 0.167 & 0.164 & 0.166 & 0.174 & 0.159 & 0.161 & 0.151 & 0.144 & 0.153 & 0.133 & 0.169 & 0.180 \\
\hline & K-S (p-value) & $0.200^{*}$ & 0.149 & $0.200^{*}$ & 0.198 & $0.200^{*}$ & $0.200^{*}$ & 0.156 & $0.200^{*}$ & $0.200^{*}$ & $0.200^{*}$ & $0.200^{*}$ & $0.200^{*}$ & $0.200^{*}$ & 0.188 & 0.126 \\
\hline & S-W (Statistic) & 0.946 & 0.944 & 0.957 & 0.954 & 0.954 & 0.951 & 0.941 & 0.942 & 0.943 & 0.945 & 0.947 & 0.942 & 0.962 & 0.942 & 0.940 \\
\hline & S-W (p-value) & 0.361 & 0.341 & 0.549 & 0.493 & 0.494 & 0.447 & 0.307 & 0.315 & 0.332 & 0.354 & 0.376 & 0.318 & 0.643 & 0.317 & 0.294 \\
\hline \multirow[t]{4}{*}{ MT 10} & K-S (Statistic) & 0.155 & 0.190 & 0.133 & 0.146 & 0.150 & 0.145 & 0.164 & 0.160 & 0.159 & 0.184 & 0.181 & 0.170 & 0.143 & 0.156 & 0.159 \\
\hline & K-S (p-value) & $0.200^{*}$ & 0.084 & $0.200^{*}$ & $0.200^{*}$ & $0.200^{*}$ & $0.200^{*}$ & $0.200^{*}$ & $0.200^{*}$ & $0.200^{*}$ & 0.108 & 0.122 & 0.182 & $0.200^{*}$ & $0.200^{*}$ & $0.200^{*}$ \\
\hline & S-W (Statistic) & 0.897 & 0.882 & 0.920 & 0.889 & 0.896 & 0.900 & 0.896 & 0.900 & 0.902 & 0.894 & 0.900 & 0.901 & 0.947 & 0.925 & 0.923 \\
\hline & S-W (p-value) & 0.052 & 0.028 & 0.129 & 0.036 & 0.048 & 0.057 & 0.049 & 0.058 & 0.061 & 0.045 & 0.058 & 0.059 & 0.374 & 0.157 & 0.149 \\
\hline \multirow[t]{4}{*}{ MT 11} & K-S (Statistic) & 0.156 & 0.149 & 0.126 & 0.164 & 0.156 & 0.154 & 0.115 & 0.116 & 0.115 & 0.114 & 0.129 & 0.158 & 0.135 & 0.165 & 0.159 \\
\hline & K-S (p-value) & 0.151 & 0.200 & $0.200^{*}$ & 0.112 & 0.154 & 0.165 & $0.200^{*}$ & $0.200^{*}$ & $0.200^{*}$ & $0.200^{*}$ & $0.200^{*}$ & 0.141 & $0.200^{*}$ & 0.104 & 0.138 \\
\hline & S-W (Statistic) & 0.926 & 0.922 & 0.944 & 0.900 & 0.903 & 0.899 & 0.949 & 0.950 & 0.949 & 0.957 & 0.956 & 0.932 & 0.965 & 0.922 & 0.930 \\
\hline & S-W (p-value) & 0.088 & 0.073 & 0.217 & 0.026 & 0.029 & 0.024 & 0.280 & 0.290 & 0.283 & 0.408 & 0.396 & 0.123 & 0.579 & 0.075 & 0.107 \\
\hline \multirow[t]{4}{*}{ MT 12} & K-S (Statistic) & 0.254 & 0.273 & 0.202 & 0.167 & 0.178 & 0.209 & 0.274 & 0.275 & 0.272 & 0.247 & 0.249 & 0.270 & 0.111 & 0.279 & 0.285 \\
\hline & K-S (p-value) & 0.002 & 0.000 & 0.032 & 0.147 & 0.097 & 0.022 & 0.000 & 0.000 & 0.000 & 0.002 & 0.002 & 0.000 & $0.200^{*}$ & 0.000 & 0.000 \\
\hline & S-W (Statistic) & 0.741 & 0.687 & 0.860 & 0.875 & 0.863 & 0.815 & 0.705 & 0.703 & 0.708 & 0.766 & 0.761 & 0.715 & 0.974 & 0.696 & 0.683 \\
\hline & S-W (p-value) & 0.000 & 0.000 & 0.008 & 0.014 & 0.009 & 0.001 & 0.000 & 0.000 & 0.000 & 0.000 & 0.000 & 0.000 & 0.829 & 0.000 & 0.000 \\
\hline \multirow[t]{4}{*}{ MT 13} & K-S (Statistic) & $0.200^{*}$ & 0.214 & 0.149 & 0.175 & 0.175 & 0.176 & 0.180 & 0.180 & 0.180 & 0.187 & 0.182 & 0.202 & 0.120 & 0.177 & 0.177 \\
\hline & K-S (p-value) & 0.017 & 0.007 & $0.200^{*}$ & 0.065 & 0.064 & 0.062 & 0.052 & 0.051 & 0.051 & 0.037 & 0.047 & 0.016 & $0.200^{*}$ & 0.059 & 0.059 \\
\hline & S-W (Statistic) & 0.900 & 0.866 & 0.939 & 0.941 & 0.939 & 0.931 & 0.916 & 0.915 & 0.917 & 0.853 & 0.861 & 0.893 & 0.962 & 0.894 & 0.883 \\
\hline & S-W (p-value) & 0.025 & 0.005 & 0.174 & 0.188 & 0.168 & 0.118 & 0.056 & 0.053 & 0.057 & 0.003 & 0.004 & 0.018 & 0.504 & 0.019 & 0.011 \\
\hline \multirow[t]{4}{*}{ MT 14} & K-S (Statistic) & 0.147 & 0.128 & 0.149 & 0.126 & 0.131 & 0.134 & 0.129 & 0.125 & 0.140 & 0.163 & 0.157 & 0.144 & 0.123 & 0.172 & 0.155 \\
\hline & K-S (p-value) & $0.200^{*}$ & $0.200^{*}$ & $0.200^{*}$ & $0.200^{*}$ & $0.200^{*}$ & $0.200^{*}$ & $0.200^{*}$ & $0.200^{*}$ & $0.200^{*}$ & 0.170 & $0.200^{*}$ & $0.200^{*}$ & $0.200^{*}$ & 0.125 & $0.200^{*}$ \\
\hline & S-W (Statistic) & 0.912 & 0.924 & 0.904 & 0.929 & 0.923 & 0.920 & 0.912 & 0.912 & 0.909 & 0.909 & 0.912 & 0.908 & 0.946 & 0.905 & 0.907 \\
\hline & S-W (p-value) & 0.071 & 0.120 & 0.049 & 0.148 & 0.114 & 0.099 & 0.070 & 0.069 & 0.061 & 0.061 & 0.070 & 0.060 & 0.315 & 0.052 & 0.055 \\
\hline \multirow[t]{2}{*}{ MT 15} & K-S (Statistic) & 0.083 & 0.078 & 0.126 & 0.066 & 0.060 & 0.060 & 0.121 & 0.121 & 0.121 & 0.086 & 0.092 & 0.089 & 0.054 & 0.082 & 0.072 \\
\hline & K-S (p-value) & $0.200^{*}$ & $0.200^{*}$ & 0.056 & $0.200^{*}$ & $0.200^{*}$ & $0.200^{*}$ & 0.077 & 0.074 & 0.077 & $0.200^{*}$ & $0.200^{*}$ & $0.200^{*}$ & $0.200^{*}$ & $0.200^{*}$ & $0.200^{*}$ \\
\hline
\end{tabular}


Table C4: Kolmogorov-Smirnov and Shapiro-Wilk tests for Mountainous Terrain for Headway 5 s- Cont.

\begin{tabular}{|c|c|c|c|c|c|c|c|c|c|c|c|c|c|c|c|c|}
\hline $\begin{array}{l}\text { Curve } \\
\text { ID }\end{array}$ & Test & $V_{c r v-a v g}$ & $V_{c r v-\min }$ & $V_{c r v-\max }$ & $V_{B C}$ & $V_{T B C}$ & $V_{D B C}$ & $V_{M C}$ & $V_{T M C}$ & $V_{D M C}$ & $V_{E C}$ & $V_{T E C}$ & $V_{D E C}$ & $a_{L}$ & $V_{c m f-a v g}$ & $V_{c m f-\min }$ \\
\hline \multirow{6}{*}{ MT 16} & S-W (Statistic) & 0.973 & 0.987 & 0.917 & 0.990 & 0.991 & 0.992 & 0.951 & 0.952 & 0.953 & 0.951 & 0.952 & 0.968 & 0.992 & 0.978 & 0.981 \\
\hline & S-W (p-value) & 0.319 & 0.866 & 0.002 & 0.962 & 0.974 & 0.981 & 0.045 & 0.049 & 0.052 & 0.043 & 0.047 & 0.214 & 0.983 & 0.504 & 0.618 \\
\hline & K-S (Statistic) & 0.164 & 0.128 & 0.107 & 0.154 & 0.149 & 0.142 & 0.128 & 0.125 & 0.127 & 0.118 & 0.132 & 0.139 & 0.132 & 0.138 & 0.144 \\
\hline & K-S (p-value) & 0.128 & $0.200^{*}$ & $0.200^{*}$ & 0.188 & $0.200^{*}$ & $0.200^{*}$ & $0.200^{*}$ & $0.200^{*}$ & $0.200^{*}$ & 0.200 * & $0.200^{*}$ & $0.200^{*}$ & $0.200^{*}$ & $0.200^{*}$ & $0.200^{*}$ \\
\hline & S-W (Statistic) & 0.956 & 0.960 & 0.979 & 0.970 & 0.972 & 0.974 & 0.969 & 0.969 & 0.968 & 0.957 & 0.957 & 0.962 & 0.947 & 0.959 & 0.954 \\
\hline & S-W (p-value) & 0.411 & 0.493 & 0.897 & 0.702 & 0.759 & 0.803 & 0.682 & 0.688 & 0.676 & 0.424 & 0.433 & 0.529 & 0.271 & 0.462 & 0.374 \\
\hline \multirow[t]{4}{*}{ MT 17} & K-S (Statistic) & 0.210 & 0.228 & 0.166 & 0.237 & 0.244 & 0.247 & 0.213 & 0.216 & 0.215 & 0.137 & 0.138 & 0.197 & 0.197 & 0.187 & 0.194 \\
\hline & K-S (p-value) & 0.012 & 0.004 & 0.117 & 0.002 & 0.001 & 0.001 & 0.010 & 0.009 & 0.009 & $0.200^{*}$ & $0.200^{*}$ & 0.026 & 0.027 & 0.045 & 0.030 \\
\hline & S-W (Statistic) & 0.894 & 0.887 & 0.924 & 0.882 & 0.882 & 0.882 & 0.896 & 0.896 & 0.895 & 0.930 & 0.927 & 0.902 & 0.957 & 0.896 & 0.890 \\
\hline & S-W (p-value) & 0.023 & 0.017 & 0.090 & 0.013 & 0.013 & 0.013 & 0.025 & 0.024 & 0.024 & 0.125 & 0.107 & 0.032 & 0.424 & 0.024 & 0.018 \\
\hline \multirow[t]{4}{*}{ MT 18} & K-S (Statistic) & 0.159 & 0.175 & 0.137 & 0.167 & 0.164 & 0.166 & 0.174 & 0.159 & 0.161 & 0.151 & 0.144 & 0.153 & 0.133 & 0.169 & 0.180 \\
\hline & K-S (p-value) & $0.200^{*}$ & 0.149 & $0.200^{*}$ & 0.198 & $0.200^{*}$ & $0.200^{*}$ & 0.156 & $0.200^{*}$ & $0.200^{*}$ & $0.200^{*}$ & $0.200^{*}$ & $0.200^{*}$ & $0.200^{*}$ & 0.188 & 0.126 \\
\hline & S-W (Statistic) & 0.946 & 0.944 & 0.957 & 0.954 & 0.954 & 0.951 & 0.941 & 0.942 & 0.943 & 0.945 & 0.947 & 0.942 & 0.962 & 0.942 & 0.940 \\
\hline & S-W (p-value) & 0.361 & 0.341 & 0.549 & 0.493 & 0.494 & 0.447 & 0.307 & 0.315 & 0.332 & 0.354 & 0.376 & 0.318 & 0.643 & 0.317 & 0.294 \\
\hline \multirow[t]{4}{*}{ MT 19} & K-S (Statistic) & 0.097 & 0.098 & 0.105 & 0.129 & 0.132 & 0.109 & 0.106 & 0.118 & 0.115 & 0.134 & 0.110 & 0.097 & 0.062 & 0.095 & 0.095 \\
\hline & K-S (p-value) & $0.200^{*}$ & $0.200^{*}$ & $0.200^{*}$ & 0.162 & 0.142 & $0.200^{*}$ & $0.200^{*}$ & $0.200^{*}$ & $0.200^{*}$ & 0.130 & $0.200^{*}$ & $0.200^{*}$ & $0.200^{*}$ & $0.200^{*}$ & $0.200^{*}$ \\
\hline & S-W (Statistic) & 0.967 & 0.975 & 0.958 & 0.940 & 0.950 & 0.958 & 0.970 & 0.969 & 0.969 & 0.966 & 0.968 & 0.969 & 0.992 & 0.968 & 0.968 \\
\hline & S-W (p-value) & 0.391 & 0.622 & 0.216 & 0.060 & 0.127 & 0.206 & 0.457 & 0.434 & 0.429 & 0.363 & 0.421 & 0.436 & 0.995 & 0.418 & 0.416 \\
\hline \multirow[t]{4}{*}{ MT 20} & K-S (Statistic) & 0.078 & 0.099 & 0.121 & 0.068 & 0.079 & 0.078 & 0.095 & 0.094 & 0.089 & 0.083 & 0.086 & 0.086 & 0.121 & 0.077 & 0.089 \\
\hline & K-S ( $p$-value) & $0.200^{*}$ & $0.200^{*}$ & 0.145 & $0.200^{*}$ & $0.200^{*}$ & $0.200^{*}$ & $0.200^{*}$ & $0.200^{*}$ & $0.200^{*}$ & $0.200^{*}$ & $0.200^{*}$ & $0.200^{*}$ & 0.141 & $0.200^{*}$ & $0.200^{*}$ \\
\hline & S-W (Statistic) & 0.984 & 0.987 & 0.976 & 0.989 & 0.989 & 0.989 & 0.976 & 0.978 & 0.979 & 0.979 & 0.979 & 0.981 & 0.966 & 0.981 & 0.980 \\
\hline & S-W (p-value) & 0.820 & 0.928 & 0.547 & 0.960 & 0.964 & 0.965 & 0.553 & 0.606 & 0.639 & 0.641 & 0.669 & 0.718 & 0.260 & 0.723 & 0.676 \\
\hline \multirow[t]{4}{*}{ MT 21} & K-S (Statistic) & 0.081 & 0.101 & 0.076 & 0.078 & 0.077 & 0.074 & 0.092 & 0.088 & 0.087 & 0.093 & 0.088 & 0.083 & 0.060 & 0.068 & 0.095 \\
\hline & K-S (p-value) & $0.200^{*}$ & $0.200^{*}$ & $0.200^{*}$ & $0.200^{*}$ & $0.200^{*}$ & $0.200^{*}$ & $0.200^{*}$ & $0.200^{*}$ & $0.200^{*}$ & $0.200^{*}$ & $0.200^{*}$ & $0.200^{*}$ & $0.200^{*}$ & $0.200^{*}$ & $0.200^{*}$ \\
\hline & S-W (Statistic) & 0.984 & 0.971 & 0.993 & 0.986 & 0.986 & 0.991 & 0.984 & 0.985 & 0.985 & 0.978 & 0.976 & 0.984 & 0.984 & 0.984 & 0.982 \\
\hline & S-W (p-value) & 0.843 & 0.392 & 0.997 & 0.898 & 0.906 & 0.981 & 0.833 & 0.862 & 0.866 & 0.602 & 0.561 & 0.826 & 0.837 & 0.816 & 0.755 \\
\hline \multirow[t]{3}{*}{ MT 22} & K-S (Statistic) & 0.092 & 0.116 & 0.128 & 0.089 & 0.085 & 0.103 & 0.099 & 0.097 & 0.099 & 0.134 & 0.136 & 0.091 & 0.110 & 0.130 & 0.117 \\
\hline & K-S (p-value) & $0.200^{*}$ & $0.200^{*}$ & $0.200^{*}$ & $0.200^{*}$ & $0.200^{*}$ & $0.200^{*}$ & $0.200^{*}$ & $0.200^{*}$ & $0.200^{*}$ & 0.196 & 0.183 & $0.200^{*}$ & $0.200^{*}$ & $0.200^{*}$ & $0.200^{*}$ \\
\hline & S-W (Statistic) & 0.972 & 0.972 & 0.970 & 0.984 & 0.984 & 0.983 & 0.966 & 0.967 & 0.968 & 0.945 & 0.947 & 0.972 & 0.951 & 0.963 & 0.963 \\
\hline
\end{tabular}


Table C4: Kolmogorov-Smirnov and Shapiro-Wilk tests for Mountainous Terrain for Headway 5 s- Cont.

\begin{tabular}{|c|c|c|c|c|c|c|c|c|c|c|c|c|c|c|c|c|}
\hline $\begin{array}{c}\text { Curve } \\
\text { ID }\end{array}$ & Test & $V_{c r v-a v g}$ & $V_{c r v-\min }$ & $V_{c r v-\max }$ & $V_{B C}$ & $V_{T B C}$ & $V_{D B C}$ & $V_{M C}$ & $V_{T M C}$ & $V_{D M C}$ & $V_{E C}$ & $V_{T E C}$ & $V_{D E C}$ & $a_{L}$ & $V_{c m f-a v g}$ & $V_{c m f-m i n}$ \\
\hline & S-W (p-value) & 0.616 & 0.628 & 0.572 & 0.923 & 0.922 & 0.913 & 0.454 & 0.492 & 0.496 & 0.139 & 0.156 & 0.611 & 0.192 & 0.394 & 0.388 \\
\hline \multirow[t]{4}{*}{ MT 23} & K-S (Statistic) & 0.146 & 0.176 & 0.156 & 0.195 & 0.192 & 0.176 & 0.167 & 0.167 & 0.167 & 0.115 & 0.117 & 0.131 & 0.087 & 0.141 & 0.143 \\
\hline & K-S (p-value) & 0.182 & 0.043 & 0.116 & 0.015 & 0.018 & 0.045 & 0.071 & 0.070 & 0.072 & $0.200^{*}$ & $0.200^{*}$ & $0.200^{*}$ & $0.200^{*}$ & $0.200^{*}$ & 0.198 \\
\hline & S-W (Statistic) & 0.922 & 0.894 & 0.928 & 0.924 & 0.922 & 0.924 & 0.912 & 0.914 & 0.915 & 0.928 & 0.930 & 0.920 & 0.966 & 0.928 & 0.918 \\
\hline & S-W (p-value) & 0.056 & 0.014 & 0.078 & 0.062 & 0.058 & 0.062 & 0.034 & 0.038 & 0.039 & 0.080 & 0.086 & 0.052 & 0.557 & 0.078 & 0.046 \\
\hline \multirow[t]{4}{*}{ MT 24} & K-S (Statistic) & 0.097 & 0.145 & 0.111 & 0.105 & 0.117 & 0.130 & 0.106 & 0.109 & 0.111 & 0.118 & 0.115 & 0.098 & 0.117 & 0.118 & 0.131 \\
\hline & K-S (p-value) & $0.200^{*}$ & $0.200^{*}$ & $0.200^{*}$ & $0.200^{*}$ & $0.200^{*}$ & $0.200^{*}$ & $0.200^{*}$ & $0.200^{*}$ & $0.200^{*}$ & $0.200^{*}$ & $0.200^{*}$ & $0.200^{*}$ & $0.200^{*}$ & $0.200^{*}$ & $0.200^{*}$ \\
\hline & S-W (Statistic) & 0.973 & 0.955 & 0.980 & 0.976 & 0.978 & 0.977 & 0.968 & 0.967 & 0.967 & 0.984 & 0.985 & 0.971 & 0.977 & 0.960 & 0.960 \\
\hline & S-W (p-value) & 0.729 & 0.353 & 0.903 & 0.805 & 0.865 & 0.838 & 0.623 & 0.588 & 0.594 & 0.951 & 0.968 & 0.701 & 0.842 & 0.437 & 0.441 \\
\hline \multirow[t]{4}{*}{ MT 25} & K-S (Statistic) & 0.072 & 0.090 & 0.101 & 0.072 & 0.087 & 0.079 & 0.101 & 0.103 & 0.098 & 0.094 & 0.058 & 0.079 & 0.066 & 0.086 & 0.100 \\
\hline & K-S (p-value) & $0.200^{*}$ & $0.200^{*}$ & $0.200^{*}$ & $0.200^{*}$ & $0.200^{*}$ & $0.200^{*}$ & $0.200^{*}$ & $0.200^{*}$ & $0.200^{*}$ & $0.200^{*}$ & $0.200^{*}$ & $0.200^{*}$ & $0.200^{*}$ & $0.200^{*}$ & $0.200^{*}$ \\
\hline & S-W (Statistic) & 0.984 & 0.980 & 0.982 & 0.977 & 0.979 & 0.981 & 0.974 & 0.974 & 0.976 & 0.990 & 0.993 & 0.983 & 0.979 & 0.983 & 0.981 \\
\hline & S-W (p-value) & 0.888 & 0.775 & 0.842 & 0.677 & 0.729 & 0.808 & 0.595 & 0.593 & 0.650 & 0.985 & 0.998 & 0.869 & 0.728 & 0.866 & 0.817 \\
\hline \multirow[t]{4}{*}{ MT 26} & K-S (Statistic) & 0.123 & 0.130 & 0.154 & 0.173 & 0.168 & 0.170 & 0.111 & 0.109 & 0.112 & 0.164 & 0.145 & 0.118 & 0.229 & 0.112 & 0.128 \\
\hline & K-S (p-value) & $0.200^{*}$ & $0.200^{*}$ & $0.200^{*}$ & $0.200^{*}$ & $0.200^{*}$ & $0.200^{*}$ & $0.200^{*}$ & $0.200^{*}$ & $0.200^{*}$ & $0.200^{*}$ & $0.200^{*}$ & $0.200^{*}$ & 0.025 & $0.200^{*}$ & $0.200^{*}$ \\
\hline & S-W (Statistic) & 0.967 & 0.968 & 0.953 & 0.914 & 0.925 & 0.930 & 0.966 & 0.967 & 0.966 & 0.960 & 0.960 & 0.969 & 0.935 & 0.969 & 0.967 \\
\hline & S-W (p-value) & 0.792 & 0.803 & 0.531 & 0.134 & 0.202 & 0.246 & 0.771 & 0.790 & 0.777 & 0.656 & 0.668 & 0.818 & 0.289 & 0.825 & 0.781 \\
\hline \multirow[t]{4}{*}{ MT 27} & K-S (Statistic) & 0.154 & 0.151 & 0.147 & 0.134 & 0.136 & 0.139 & 0.160 & 0.160 & 0.150 & 0.124 & 0.131 & 0.150 & 0.089 & 0.154 & 0.151 \\
\hline & K-S (p-value) & 0.145 & 0.166 & 0.196 & $0.200^{*}$ & $0.200^{*}$ & $0.200^{*}$ & 0.116 & 0.114 & 0.175 & $0.200^{*}$ & $0.200^{*}$ & 0.172 & $0.200^{*}$ & 0.147 & 0.163 \\
\hline & S-W (Statistic) & 0.943 & 0.934 & 0.953 & 0.952 & 0.949 & 0.943 & 0.941 & 0.940 & 0.943 & 0.952 & 0.950 & 0.946 & 0.983 & 0.937 & 0.936 \\
\hline & S-W (p-value) & 0.191 & 0.119 & 0.308 & 0.303 & 0.257 & 0.193 & 0.170 & 0.167 & 0.189 & 0.298 & 0.268 & 0.223 & 0.938 & 0.139 & 0.134 \\
\hline \multirow[t]{4}{*}{ MT 28} & K-S (Statistic) & 0.143 & 0.153 & 0.117 & 0.127 & 0.140 & 0.141 & 0.114 & 0.115 & 0.118 & 0.166 & 0.167 & 0.152 & 0.124 & 0.142 & 0.145 \\
\hline & K-S (p-value) & 0.121 & 0.073 & $0.200^{*}$ & $0.200^{*}$ & 0.137 & 0.129 & $0.200^{*}$ & $0.200^{*}$ & $0.200^{*}$ & 0.034 & 0.032 & 0.074 & $0.200^{*}$ & 0.124 & 0.108 \\
\hline & S-W (Statistic) & 0.952 & 0.920 & 0.964 & 0.955 & 0.954 & 0.953 & 0.965 & 0.965 & 0.965 & 0.923 & 0.929 & 0.954 & 0.977 & 0.958 & 0.954 \\
\hline & S-W (p-value) & 0.189 & 0.026 & 0.380 & 0.231 & 0.216 & 0.199 & 0.418 & 0.423 & 0.413 & 0.032 & 0.047 & 0.213 & 0.746 & 0.282 & 0.212 \\
\hline \multirow[t]{4}{*}{ MT 29} & K-S (Statistic) & 0.197 & 0.199 & 0.197 & 0.180 & 0.175 & 0.179 & 0.245 & 0.244 & 0.225 & 0.202 & 0.200 & 0.229 & 0.192 & 0.250 & 0.245 \\
\hline & K-S (p-value) & 0.001 & 0.001 & 0.001 & 0.005 & 0.008 & 0.006 & 0.000 & 0.000 & 0.000 & 0.001 & 0.001 & 0.000 & 0.002 & 0.000 & 0.000 \\
\hline & S-W (Statistic) & 0.852 & 0.846 & 0.823 & 0.873 & 0.870 & 0.869 & 0.831 & 0.830 & 0.833 & 0.828 & 0.829 & 0.842 & 0.861 & 0.830 & 0.825 \\
\hline & S-W (p-value) & 0.000 & 0.000 & 0.000 & 0.001 & 0.001 & 0.001 & 0.000 & 0.000 & 0.000 & 0.000 & 0.000 & 0.000 & 0.000 & 0.000 & 0.000 \\
\hline
\end{tabular}




\section{APPENDIX D}

One-Way-ANOVA and homogeneity of variance tests for Speed on Curve at

$\mathrm{BC}, \mathrm{MC}$, and $\mathrm{EC}$ 
D-1: One-Way-ANOVA Test for Rolling and Mountainous Terrains.

\begin{tabular}{|c|c|c|c|c|c|c|c|}
\hline \multicolumn{4}{|c|}{ Rolling Terrain } & \multicolumn{4}{|c|}{ Mountainous Terrain } \\
\hline Curve & Point & $\mathrm{F}$ & Sig. & Curve & Point & $\mathrm{F}$ & Sig. \\
\hline \multirow[t]{3}{*}{ RT 01} & $\mathrm{BC}$ & 0.355 & 0.702 & \multirow[t]{3}{*}{ MT 01} & $\mathrm{BC}$ & 0.050 & 0.951 \\
\hline & $\mathrm{MC}$ & 0.093 & 0.911 & & $\mathrm{MC}$ & 0.055 & 0.947 \\
\hline & $\mathrm{EC}$ & 0.036 & 0.964 & & $\mathrm{EC}$ & 0.079 & 0.924 \\
\hline \multirow[t]{3}{*}{ RT 02} & $\mathrm{BC}$ & 0.002 & 0.998 & \multirow[t]{3}{*}{ MT 02} & $\mathrm{BC}$ & 0.063 & 0.939 \\
\hline & $\mathrm{MC}$ & 0.048 & 0.953 & & $\mathrm{MC}$ & 0.005 & 0.995 \\
\hline & $\mathrm{EC}$ & 0.216 & 0.806 & & $\mathrm{EC}$ & 0.009 & 0.991 \\
\hline \multirow[t]{3}{*}{ RT 03} & $\mathrm{BC}$ & 0.083 & 0.920 & \multirow[t]{3}{*}{ MT 03} & $\mathrm{BC}$ & 0.026 & 0.974 \\
\hline & $\mathrm{MC}$ & 0.151 & 0.860 & & $\mathrm{MC}$ & 0.000 & 1.000 \\
\hline & EC & 0.174 & 0.841 & & $\mathrm{EC}$ & 0.106 & 0.899 \\
\hline \multirow[t]{3}{*}{ RT 04} & $\mathrm{BC}$ & 0.022 & 0.979 & \multirow[t]{3}{*}{ MT 04} & $\mathrm{BC}$ & 0.253 & 0.777 \\
\hline & $\mathrm{MC}$ & 0.203 & 0.817 & & $\mathrm{MC}$ & 0.135 & 0.874 \\
\hline & $\mathrm{EC}$ & 0.029 & 0.971 & & $\mathrm{EC}$ & 0.003 & 0.997 \\
\hline \multirow[t]{3}{*}{ RT 05} & $\mathrm{BC}$ & 0.049 & 0.952 & \multirow[t]{3}{*}{ MT 05} & $\mathrm{BC}$ & 0.011 & 0.989 \\
\hline & $\mathrm{MC}$ & 0.208 & 0.813 & & $\mathrm{MC}$ & 0.029 & 0.972 \\
\hline & EC & 2.470 & 0.090 & & $\mathrm{EC}$ & 2.270 & 0.107 \\
\hline \multirow[t]{3}{*}{ RT 06} & $\mathrm{BC}$ & 0.031 & 0.970 & \multirow[t]{3}{*}{ MT 06} & $\mathrm{BC}$ & 0.238 & 0.788 \\
\hline & $\mathrm{MC}$ & 0.046 & 0.955 & & $\mathrm{MC}$ & 0.005 & 0.995 \\
\hline & EC & 0.253 & 0.777 & & $\mathrm{EC}$ & 0.996 & 0.371 \\
\hline \multirow[t]{3}{*}{ RT 07} & $\mathrm{BC}$ & 0.000 & 1.000 & \multirow[t]{3}{*}{ MT 07} & $\mathrm{BC}$ & 0.020 & 0.980 \\
\hline & $\mathrm{MC}$ & 0.534 & 0.588 & & $\mathrm{MC}$ & 0.076 & 0.927 \\
\hline & $\mathrm{EC}$ & 0.433 & 0.650 & & $\mathrm{EC}$ & 0.088 & 0.916 \\
\hline \multirow[t]{3}{*}{ RT 08} & BC & 0.004 & 0.996 & \multirow[t]{3}{*}{ MT 08} & $\mathrm{BC}$ & 0.051 & 0.950 \\
\hline & $\mathrm{MC}$ & 0.050 & 0.951 & & $\mathrm{MC}$ & 0.043 & 0.958 \\
\hline & EC & 0.165 & 0.848 & & EC & 0.036 & 0.964 \\
\hline \multirow[t]{3}{*}{ RT 09} & BC & 0.000 & 1.000 & \multirow[t]{3}{*}{ MT 09} & $\mathrm{BC}$ & 0.013 & 0.987 \\
\hline & $\mathrm{MC}$ & 0.001 & 0.999 & & $\mathrm{MC}$ & 0.068 & 0.934 \\
\hline & $\mathrm{EC}$ & 0.003 & 0.997 & & $\mathrm{EC}$ & 1.125 & 0.328 \\
\hline \multirow[t]{3}{*}{ RT 10} & $\mathrm{BC}$ & 0.005 & 0.995 & \multirow[t]{3}{*}{ MT 10} & $\mathrm{BC}$ & 0.001 & 0.999 \\
\hline & $\mathrm{MC}$ & 0.001 & 0.999 & & $\mathrm{MC}$ & 0.261 & 0.771 \\
\hline & $\mathrm{EC}$ & 0.066 & 0.936 & & EC & 0.610 & 0.546 \\
\hline \multirow[t]{3}{*}{ RT 11} & $\mathrm{BC}$ & 0.007 & 0.993 & \multirow[t]{3}{*}{ MT 11} & $\mathrm{BC}$ & 0.008 & 0.992 \\
\hline & $\mathrm{MC}$ & 0.064 & 0.938 & & $\mathrm{MC}$ & 0.009 & 0.991 \\
\hline & $\mathrm{EC}$ & 0.186 & 0.831 & & EC & 0.062 & 0.940 \\
\hline \multirow[t]{3}{*}{ RT 12} & $\mathrm{BC}$ & 0.065 & 0.937 & \multirow[t]{3}{*}{ MT 12} & $\mathrm{BC}$ & 0.005 & 0.995 \\
\hline & $\mathrm{MC}$ & 0.076 & 0.927 & & $\mathrm{MC}$ & 0.007 & 0.993 \\
\hline & $\mathrm{EC}$ & 0.589 & 0.558 & & $\mathrm{EC}$ & 0.307 & 0.736 \\
\hline
\end{tabular}


D-1: One-Way-ANOVA Test for Rolling and Mountainous Terrains - Cont.

\begin{tabular}{|c|c|c|c|c|c|c|c|}
\hline \multicolumn{4}{|c|}{ Rolling Terrain } & \multicolumn{4}{|c|}{ Mountainous Terrain } \\
\hline Curve & Point & $\mathrm{F}$ & Sig. & Curve & Point & $\mathrm{F}$ & Sig. \\
\hline \multirow[t]{3}{*}{ RT 13} & $\mathrm{BC}$ & 0.033 & 0.968 & \multirow[t]{3}{*}{ MT 13} & $\mathrm{BC}$ & 0.129 & 0.879 \\
\hline & $\mathrm{MC}$ & 0.402 & 0.671 & & $\mathrm{MC}$ & 0.281 & 0.756 \\
\hline & $\mathrm{EC}$ & 1.548 & 0.220 & & $\mathrm{EC}$ & 0.219 & 0.804 \\
\hline \multirow[t]{3}{*}{ RT 14} & $\mathrm{BC}$ & 0.199 & 0.820 & \multirow[t]{3}{*}{ MT 14} & $\mathrm{BC}$ & 0.020 & 0.980 \\
\hline & $\mathrm{MC}$ & 0.140 & 0.870 & & $\mathrm{MC}$ & 0.144 & 0.866 \\
\hline & $\mathrm{EC}$ & 0.017 & 0.983 & & $\mathrm{EC}$ & 0.035 & 0.966 \\
\hline \multirow[t]{3}{*}{ RT 15} & $\mathrm{BC}$ & 0.038 & 0.963 & \multirow[t]{3}{*}{ MT 15} & $\mathrm{BC}$ & 0.004 & 0.996 \\
\hline & $\mathrm{MC}$ & 0.032 & 0.969 & & $\mathrm{MC}$ & 0.012 & 0.989 \\
\hline & $\mathrm{EC}$ & 0.512 & 0.601 & & $\mathrm{EC}$ & 0.048 & 0.953 \\
\hline \multirow[t]{3}{*}{ RT 16} & $\mathrm{BC}$ & 0.130 & 0.878 & \multirow[t]{3}{*}{ MT 16} & $\mathrm{BC}$ & 0.035 & 0.965 \\
\hline & $\mathrm{MC}$ & 0.325 & 0.724 & & $\mathrm{MC}$ & 0.012 & 0.988 \\
\hline & $\mathrm{EC}$ & 0.081 & 0.923 & & $\mathrm{EC}$ & 0.078 & 0.925 \\
\hline \multirow[t]{3}{*}{ RT 17} & $\mathrm{BC}$ & 0.094 & 0.910 & \multirow[t]{3}{*}{ MT 17} & $\mathrm{BC}$ & 0.228 & 0.796 \\
\hline & $\mathrm{MC}$ & 0.203 & 0.817 & & $\mathrm{MC}$ & 0.000 & 1.000 \\
\hline & $\mathrm{EC}$ & 0.090 & 0.914 & & $\mathrm{EC}$ & 0.112 & 0.894 \\
\hline \multirow[t]{3}{*}{ RT 18} & $\mathrm{BC}$ & 0.307 & 0.737 & \multirow[t]{3}{*}{ MT 18} & $\mathrm{BC}$ & 0.079 & 0.924 \\
\hline & $\mathrm{MC}$ & 0.055 & 0.947 & & $\mathrm{MC}$ & 0.004 & 0.996 \\
\hline & $\mathrm{EC}$ & 1.090 & 0.341 & & $\mathrm{EC}$ & 0.000 & 1.000 \\
\hline \multirow[t]{3}{*}{ RT 19} & $\mathrm{BC}$ & 0.040 & 0.960 & \multirow[t]{3}{*}{ MT 19} & $\mathrm{BC}$ & 0.113 & 0.893 \\
\hline & $\mathrm{MC}$ & 0.012 & 0.988 & & $\mathrm{MC}$ & 0.150 & 0.861 \\
\hline & EC & 0.109 & 0.897 & & $\mathrm{EC}$ & 0.357 & 0.701 \\
\hline \multirow[t]{3}{*}{ RT 20} & $\mathrm{BC}$ & 0.027 & 0.974 & \multirow[t]{3}{*}{ MT 20} & $\mathrm{BC}$ & 0.038 & 0.963 \\
\hline & $\mathrm{MC}$ & 0.000 & 1.000 & & $\mathrm{MC}$ & 0.020 & 0.980 \\
\hline & EC & 0.022 & 0.979 & & EC & 0.075 & 0.928 \\
\hline \multirow[t]{3}{*}{ RT 21} & $\mathrm{BC}$ & 0.041 & 0.960 & \multirow[t]{3}{*}{ MT 21} & $\mathrm{BC}$ & 0.001 & 0.999 \\
\hline & $\mathrm{MC}$ & 0.027 & 0.973 & & $\mathrm{MC}$ & 0.022 & 0.978 \\
\hline & $\mathrm{EC}$ & 0.986 & 0.378 & & EC & 0.065 & 0.937 \\
\hline \multirow[t]{3}{*}{ RT 22} & $\mathrm{BC}$ & 0.003 & 0.997 & \multirow[t]{3}{*}{ MT 22} & $\mathrm{BC}$ & 0.001 & 0.999 \\
\hline & $\mathrm{MC}$ & 0.060 & 0.942 & & $\mathrm{MC}$ & 0.024 & 0.976 \\
\hline & EC & 0.199 & 0.820 & & $\mathrm{EC}$ & 0.003 & 0.997 \\
\hline \multirow[t]{3}{*}{ RT 23} & $\mathrm{BC}$ & 0.030 & 0.971 & \multirow[t]{3}{*}{ MT 23} & $\mathrm{BC}$ & 0.052 & 0.949 \\
\hline & MC & 0.066 & 0.936 & & $\mathrm{MC}$ & 0.192 & 0.825 \\
\hline & EC & 0.001 & 0.999 & & EC & 0.442 & 0.644 \\
\hline \multirow[t]{3}{*}{ RT 24} & $\mathrm{BC}$ & 0.018 & 0.982 & \multirow[t]{3}{*}{ MT 24} & $\mathrm{BC}$ & 0.128 & 0.880 \\
\hline & MC & 0.032 & 0.968 & & $\mathrm{MC}$ & 0.262 & 0.770 \\
\hline & $\mathrm{EC}$ & 0.174 & 0.841 & & EC & 0.394 & 0.675 \\
\hline
\end{tabular}


D-1: One-Way-ANOVA Test for Rolling and Mountainous Terrains - Cont.

\begin{tabular}{|c|c|c|c|c|c|c|c|}
\hline \multicolumn{4}{|c|}{ Rolling Terrain } & \multicolumn{4}{|c|}{ Mountainous Terrain } \\
\hline Curve & Point & $\mathrm{F}$ & Sig. & Curve & Point & $\mathrm{F}$ & Sig. \\
\hline \multirow[t]{3}{*}{ RT 26} & $\mathrm{BC}$ & 0.163 & 0.850 & \multirow{3}{*}{ MT 26} & $\mathrm{BC}$ & 0.044 & 0.957 \\
\hline & $\mathrm{MC}$ & 0.412 & 0.664 & & $\mathrm{MC}$ & 0.273 & 0.762 \\
\hline & $\mathrm{EC}$ & 0.227 & 0.798 & & $\mathrm{EC}$ & 0.011 & 0.989 \\
\hline \multirow[t]{3}{*}{ RT 27} & $\mathrm{BC}$ & 0.057 & 0.944 & \multirow[t]{3}{*}{ MT 27} & $\mathrm{BC}$ & 0.082 & 0.922 \\
\hline & $\mathrm{MC}$ & 0.036 & 0.965 & & $\mathrm{MC}$ & 0.110 & 0.896 \\
\hline & $\mathrm{EC}$ & 0.080 & 0.923 & & $\mathrm{EC}$ & 0.628 & 0.536 \\
\hline \multirow[t]{3}{*}{ RT 28} & $\mathrm{BC}$ & 0.000 & 1.000 & \multirow[t]{3}{*}{ MT 28} & $\mathrm{BC}$ & 0.012 & 0.989 \\
\hline & $\mathrm{MC}$ & 0.001 & 0.999 & & $\mathrm{MC}$ & 0.011 & 0.989 \\
\hline & $\mathrm{EC}$ & 0.083 & 0.921 & & $\mathrm{EC}$ & 0.113 & 0.894 \\
\hline \multirow[t]{3}{*}{ RT 29} & $\mathrm{BC}$ & 0.006 & 0.994 & \multirow[t]{3}{*}{ MT 29} & $\mathrm{BC}$ & 0.004 & 0.996 \\
\hline & $\mathrm{MC}$ & 0.044 & 0.957 & & $\mathrm{MC}$ & 0.012 & 0.989 \\
\hline & $\mathrm{EC}$ & 0.059 & 0.943 & & $\mathrm{EC}$ & 0.048 & 0.953 \\
\hline \multirow[t]{3}{*}{ RT 30} & $\mathrm{BC}$ & 0.003 & 0.997 & & & & \\
\hline & $\mathrm{MC}$ & 0.023 & 0.977 & & & & \\
\hline & EC & 0.560 & 0.573 & & & & \\
\hline \multirow[t]{3}{*}{ RT 31} & $\mathrm{BC}$ & 0.183 & 0.833 & & & & \\
\hline & $\mathrm{MC}$ & 0.210 & 0.811 & & & & \\
\hline & $\mathrm{EC}$ & 0.017 & 0.983 & & & & \\
\hline \multirow[t]{3}{*}{ RT 32} & $\mathrm{BC}$ & 0.015 & 0.985 & & & & \\
\hline & $\mathrm{MC}$ & 0.005 & 0.995 & & & & \\
\hline & $\mathrm{EC}$ & 0.025 & 0.975 & & & & \\
\hline \multirow[t]{3}{*}{ RT 33} & $\mathrm{BC}$ & 0.033 & 0.968 & & & & \\
\hline & $\mathrm{MC}$ & 0.037 & 0.964 & & & & \\
\hline & $\mathrm{EC}$ & 0.007 & 0.993 & & & & \\
\hline
\end{tabular}


D-2: Homogeneity of Variance for Rolling and Mountainous Terrains.

\begin{tabular}{|c|c|c|c|c|c|c|c|}
\hline \multicolumn{4}{|c|}{ Rolling Terrain } & \multicolumn{4}{|c|}{ Mountainous Terrain } \\
\hline Curve & Point & $\begin{array}{l}\text { Levene } \\
\text { Statistic } \\
\end{array}$ & $p$-value & Curve & Point & $\begin{array}{c}\text { Levene } \\
\text { Statistic }\end{array}$ & $p$-value \\
\hline \multirow[t]{3}{*}{$\overline{\text { RT } 01}$} & $\mathrm{BC}$ & 0.002 & 0.998 & \multirow[t]{3}{*}{ MT 01} & $\mathrm{BC}$ & 0.013 & 0.987 \\
\hline & $\mathrm{MC}$ & 0.249 & 0.780 & & $\mathrm{MC}$ & 0.065 & 0.937 \\
\hline & $\mathrm{EC}$ & 0.283 & 0.754 & & EC & 0.295 & 0.745 \\
\hline \multirow[t]{3}{*}{ RT 02} & $\mathrm{BC}$ & 0.029 & 0.972 & \multirow[t]{3}{*}{ MT 02} & $\mathrm{BC}$ & 0.027 & 0.973 \\
\hline & $\mathrm{MC}$ & 0.054 & 0.947 & & $\mathrm{MC}$ & 0.008 & 0.992 \\
\hline & $\mathrm{EC}$ & 0.121 & 0.886 & & EC & 0.011 & 0.989 \\
\hline \multirow[t]{3}{*}{ RT 03} & $\mathrm{BC}$ & 0.050 & 0.951 & \multirow[t]{3}{*}{ MT 03} & $\mathrm{BC}$ & 0.014 & 0.986 \\
\hline & $\mathrm{MC}$ & 0.031 & 0.970 & & $\mathrm{MC}$ & 0.070 & 0.932 \\
\hline & EC & 0.107 & 0.899 & & $\mathrm{EC}$ & 0.100 & 0.905 \\
\hline \multirow[t]{3}{*}{ RT 04} & $\mathrm{BC}$ & 0.025 & 0.976 & \multirow[t]{3}{*}{ MT 04} & $\mathrm{BC}$ & 0.022 & 0.978 \\
\hline & $\mathrm{MC}$ & 0.038 & 0.962 & & $\mathrm{MC}$ & 0.006 & 0.994 \\
\hline & $\mathrm{EC}$ & 0.156 & 0.856 & & $\mathrm{EC}$ & 0.078 & 0.925 \\
\hline \multirow[t]{3}{*}{ RT 05} & $\mathrm{BC}$ & 0.001 & 0.999 & \multirow[t]{3}{*}{ MT 05} & $\mathrm{BC}$ & 0.007 & 0.993 \\
\hline & $\mathrm{MC}$ & 0.006 & 0.994 & & $\mathrm{MC}$ & 0.027 & 0.973 \\
\hline & EC & 0.007 & 0.993 & & $\mathrm{EC}$ & 0.123 & 0.884 \\
\hline \multirow[t]{3}{*}{ RT 06} & $\mathrm{BC}$ & 0.012 & 0.988 & \multirow[t]{3}{*}{ MT 06} & $\mathrm{BC}$ & 0.051 & 0.950 \\
\hline & $\mathrm{MC}$ & 0.011 & 0.989 & & $\mathrm{MC}$ & 0.000 & 1.000 \\
\hline & EC & 0.081 & 0.922 & & $\mathrm{EC}$ & 0.064 & 0.938 \\
\hline \multirow[t]{3}{*}{ RT 07} & $\mathrm{BC}$ & 0.036 & 0.965 & \multirow[t]{3}{*}{ MT 07} & $\mathrm{BC}$ & 0.002 & 0.998 \\
\hline & $\mathrm{MC}$ & 0.210 & 0.811 & & $\mathrm{MC}$ & 0.006 & 0.994 \\
\hline & EC & 0.001 & 0.999 & & EC & 0.094 & 0.911 \\
\hline \multirow[t]{3}{*}{ RT 08} & $\mathrm{BC}$ & 0.001 & 0.999 & \multirow[t]{3}{*}{ MT 08} & $\mathrm{BC}$ & 0.009 & 0.991 \\
\hline & $\mathrm{MC}$ & 0.018 & 0.982 & & $\mathrm{MC}$ & 0.100 & 0.905 \\
\hline & EC & 0.003 & 0.997 & & $\mathrm{EC}$ & 0.048 & 0.953 \\
\hline \multirow[t]{3}{*}{ RT 09} & $\mathrm{BC}$ & 0.024 & 0.976 & \multirow[t]{3}{*}{ MT 09} & $\mathrm{BC}$ & 0.006 & 0.994 \\
\hline & $\mathrm{MC}$ & 0.095 & 0.909 & & $\mathrm{MC}$ & 0.008 & 0.992 \\
\hline & EC & 0.017 & 0.983 & & $\mathrm{EC}$ & 0.173 & 0.842 \\
\hline \multirow[t]{3}{*}{ RT 10} & $\mathrm{BC}$ & 0.030 & 0.970 & \multirow[t]{3}{*}{ MT 10} & $\mathrm{BC}$ & 0.054 & 0.948 \\
\hline & $\mathrm{MC}$ & 0.003 & 0.997 & & $\mathrm{MC}$ & 1.347 & 0.266 \\
\hline & EC & 0.026 & 0.975 & & $\mathrm{EC}$ & 0.929 & 0.399 \\
\hline \multirow[t]{3}{*}{ RT 11} & $\mathrm{BC}$ & 0.002 & 0.998 & \multirow[t]{3}{*}{ MT 11} & $\mathrm{BC}$ & 0.000 & 1.000 \\
\hline & $\mathrm{MC}$ & 0.005 & 0.995 & & $\mathrm{MC}$ & 0.008 & 0.992 \\
\hline & EC & 0.042 & 0.959 & & $\mathrm{EC}$ & 0.123 & 0.884 \\
\hline \multirow[t]{3}{*}{ RT 12} & $\mathrm{BC}$ & 0.003 & 0.997 & \multirow[t]{3}{*}{ MT 12} & $\mathrm{BC}$ & 0.014 & 0.986 \\
\hline & $\mathrm{MC}$ & 0.154 & 0.858 & & $\mathrm{MC}$ & 0.057 & 0.945 \\
\hline & $\mathrm{EC}$ & 0.008 & 0.992 & & $\mathrm{EC}$ & 0.012 & 0.988 \\
\hline
\end{tabular}


D-2: Homogeneity of Variance for Rolling and Mountainous Terrains - Cont.

\begin{tabular}{|c|c|c|c|c|c|c|c|}
\hline \multicolumn{4}{|c|}{ Rolling Terrain } & \multicolumn{4}{|c|}{ Mountainous Terrain } \\
\hline Curve & Point & $\begin{array}{l}\text { Levene } \\
\text { Statistic }\end{array}$ & $p$-value & Curve & Point & $\begin{array}{l}\text { Levene } \\
\text { Statistic }\end{array}$ & $p$-value \\
\hline \multirow{3}{*}{ RT 13} & $\mathrm{BC}$ & 0.035 & 0.965 & \multirow[t]{3}{*}{ MT 13} & $\mathrm{BC}$ & 0.006 & 0.994 \\
\hline & $\mathrm{MC}$ & 0.491 & 0.614 & & $\mathrm{MC}$ & 0.001 & 0.999 \\
\hline & $\mathrm{EC}$ & 0.393 & 0.677 & & $\mathrm{EC}$ & 0.018 & 0.983 \\
\hline \multirow[t]{3}{*}{ RT 14} & $\mathrm{BC}$ & 0.013 & 0.987 & \multirow[t]{3}{*}{ MT 14} & $\mathrm{BC}$ & 0.001 & 0.999 \\
\hline & $\mathrm{MC}$ & 0.035 & 0.966 & & $\mathrm{MC}$ & 0.001 & 0.999 \\
\hline & $\mathrm{EC}$ & 0.071 & 0.932 & & $\mathrm{EC}$ & 0.045 & 0.956 \\
\hline \multirow[t]{3}{*}{ RT 15} & $\mathrm{BC}$ & 0.136 & 0.873 & \multirow[t]{3}{*}{ MT 15} & $\mathrm{BC}$ & 0.000 & 1.000 \\
\hline & $\mathrm{MC}$ & 0.150 & 0.861 & & $\mathrm{MC}$ & 0.055 & 0.946 \\
\hline & $\mathrm{EC}$ & 0.226 & 0.798 & & $\mathrm{EC}$ & 0.005 & 0.995 \\
\hline \multirow[t]{3}{*}{ RT 16} & $\mathrm{BC}$ & 0.084 & 0.919 & \multirow[t]{3}{*}{ MT 16} & $\mathrm{BC}$ & 0.012 & 0.988 \\
\hline & $\mathrm{MC}$ & 0.125 & 0.883 & & $\mathrm{MC}$ & 0.004 & 0.996 \\
\hline & $\mathrm{EC}$ & 0.017 & 0.983 & & $\mathrm{EC}$ & 0.021 & 0.979 \\
\hline \multirow[t]{3}{*}{ RT 17} & $\mathrm{BC}$ & 0.019 & 0.982 & \multirow[t]{3}{*}{ MT 17} & $\mathrm{BC}$ & 0.280 & 0.756 \\
\hline & $\mathrm{MC}$ & 0.006 & 0.994 & & $\mathrm{MC}$ & 0.098 & 0.907 \\
\hline & $\mathrm{EC}$ & 0.031 & 0.970 & & $\mathrm{EC}$ & 0.016 & 0.984 \\
\hline \multirow[t]{3}{*}{ RT 18} & $\mathrm{BC}$ & 0.154 & 0.858 & \multirow[t]{3}{*}{ MT 18} & $\mathrm{BC}$ & 0.028 & 0.972 \\
\hline & $\mathrm{MC}$ & 0.002 & 0.998 & & $\mathrm{MC}$ & 0.054 & 0.948 \\
\hline & $\mathrm{EC}$ & 0.067 & 0.935 & & $\mathrm{EC}$ & 0.027 & 0.973 \\
\hline \multirow[t]{3}{*}{ RT 19} & $\mathrm{BC}$ & 0.004 & 0.996 & \multirow[t]{3}{*}{ MT 19} & $\mathrm{BC}$ & 0.006 & 0.994 \\
\hline & $\mathrm{MC}$ & 0.002 & 0.998 & & $\mathrm{MC}$ & 0.182 & 0.834 \\
\hline & $\mathrm{EC}$ & 0.039 & 0.962 & & $\mathrm{EC}$ & 0.058 & 0.944 \\
\hline \multirow[t]{3}{*}{ RT 20} & $\mathrm{BC}$ & 0.003 & 0.997 & \multirow[t]{3}{*}{ MT 20} & $\mathrm{BC}$ & 0.002 & 0.998 \\
\hline & $\mathrm{MC}$ & 0.000 & 1.000 & & $\mathrm{MC}$ & 0.017 & 0.983 \\
\hline & $\mathrm{EC}$ & 0.002 & 0.998 & & $\mathrm{EC}$ & 0.046 & 0.955 \\
\hline \multirow[t]{3}{*}{ RT 21} & $\mathrm{BC}$ & 0.022 & 0.979 & \multirow[t]{3}{*}{ MT 21} & $\mathrm{BC}$ & 0.008 & 0.992 \\
\hline & $\mathrm{MC}$ & 0.001 & 0.999 & & $\mathrm{MC}$ & 0.099 & 0.906 \\
\hline & $\mathrm{EC}$ & 0.101 & 0.904 & & $\mathrm{EC}$ & 0.074 & 0.929 \\
\hline \multirow[t]{3}{*}{ RT 22} & $\mathrm{BC}$ & 0.022 & 0.978 & \multirow[t]{3}{*}{ MT 22} & $\mathrm{BC}$ & 0.088 & 0.916 \\
\hline & $\mathrm{MC}$ & 0.004 & 0.996 & & $\mathrm{MC}$ & 0.138 & 0.871 \\
\hline & $\mathrm{EC}$ & 0.042 & 0.959 & & $\mathrm{EC}$ & 0.092 & 0.912 \\
\hline \multirow[t]{3}{*}{ RT 23} & $\mathrm{BC}$ & 0.200 & 0.819 & \multirow[t]{3}{*}{ MT 23} & $\mathrm{BC}$ & 0.059 & 0.943 \\
\hline & $\mathrm{MC}$ & 0.005 & 0.995 & & $\mathrm{MC}$ & 0.321 & 0.726 \\
\hline & $\mathrm{EC}$ & 0.011 & 0.989 & & $\mathrm{EC}$ & 0.298 & 0.743 \\
\hline \multirow[t]{3}{*}{ RT 24} & $\mathrm{BC}$ & 0.045 & 0.956 & MT 24 & $\mathrm{BC}$ & 0.075 & 0.928 \\
\hline & $\mathrm{MC}$ & 0.162 & 0.850 & & $\mathrm{MC}$ & 0.001 & 0.999 \\
\hline & $\mathrm{EC}$ & 0.009 & 0.991 & & $\mathrm{EC}$ & 0.148 & 0.862 \\
\hline RT 25 & $\mathrm{BC}$ & 0.009 & 0.991 & MT 25 & $\mathrm{BC}$ & 0.049 & 0.952 \\
\hline & $\mathrm{MC}$ & 0.004 & 0.996 & & $\mathrm{MC}$ & 0.012 & 0.988 \\
\hline & $\mathrm{EC}$ & 0.005 & 0.995 & & $\mathrm{EC}$ & 0.158 & 0.854 \\
\hline
\end{tabular}


D-2: Homogeneity of Variance for Rolling and Mountainous Terrains - Cont.

\begin{tabular}{|c|c|c|c|c|c|c|c|}
\hline \multicolumn{4}{|c|}{ Rolling Terrain } & \multicolumn{4}{|c|}{ Mountainous Terrain } \\
\hline Curve & Point & $\begin{array}{l}\text { Levene } \\
\text { Statistic }\end{array}$ & $p$-value & \multirow{3}{*}{$\begin{array}{l}\text { Curve } \\
\text { MT } 26\end{array}$} & Point & $\begin{array}{l}\text { Levene } \\
\text { Statistic }\end{array}$ & $p$-value \\
\hline \multirow[t]{3}{*}{ RT 26} & $\mathrm{BC}$ & 0.023 & 0.977 & & $\mathrm{BC}$ & 0.017 & 0.983 \\
\hline & $\mathrm{MC}$ & 1.536 & 0.226 & & $\mathrm{MC}$ & 0.001 & 0.999 \\
\hline & EC & 0.085 & 0.918 & \multirow{4}{*}{ MT 27} & EC & 0.057 & 0.945 \\
\hline \multirow[t]{3}{*}{ RT 27} & $\mathrm{BC}$ & 0.019 & 0.982 & & $\mathrm{BC}$ & 0.067 & 0.935 \\
\hline & $\mathrm{MC}$ & 0.000 & 1.000 & & $\mathrm{MC}$ & 0.158 & 0.854 \\
\hline & $\mathrm{EC}$ & 0.119 & 0.888 & & $\mathrm{EC}$ & 0.083 & 0.921 \\
\hline \multirow[t]{3}{*}{ RT 28} & $\mathrm{BC}$ & 0.036 & 0.965 & \multirow[t]{3}{*}{ MT 28} & $\mathrm{BC}$ & 0.124 & 0.884 \\
\hline & $\mathrm{MC}$ & 0.173 & 0.841 & & $\mathrm{MC}$ & 0.189 & 0.828 \\
\hline & $\mathrm{EC}$ & 0.000 & 1.000 & & $\mathrm{EC}$ & 0.065 & 0.937 \\
\hline \multirow[t]{3}{*}{ RT 29} & $\mathrm{BC}$ & 0.045 & 0.956 & \multirow[t]{3}{*}{ MT 29} & $\mathrm{BC}$ & 0.000 & 1.000 \\
\hline & $\mathrm{MC}$ & 0.027 & 0.974 & & $\mathrm{MC}$ & 0.055 & 0.946 \\
\hline & $\mathrm{EC}$ & 0.017 & 0.983 & & $\mathrm{EC}$ & 0.005 & 0.995 \\
\hline \multirow[t]{3}{*}{ RT 30} & $\mathrm{BC}$ & 0.066 & 0.936 & & & & \\
\hline & $\mathrm{MC}$ & 0.092 & 0.912 & & & & \\
\hline & $\mathrm{EC}$ & 0.523 & 0.595 & & & & \\
\hline \multirow[t]{3}{*}{ RT 31} & $\mathrm{BC}$ & 0.062 & 0.940 & & & & \\
\hline & $\mathrm{MC}$ & 0.123 & 0.885 & & & & \\
\hline & $\mathrm{EC}$ & 0.027 & 0.973 & & & & \\
\hline \multirow[t]{3}{*}{ RT 32} & $\mathrm{BC}$ & 0.003 & 0.997 & & & & \\
\hline & $\mathrm{MC}$ & 0.001 & 0.999 & & & & \\
\hline & $\mathrm{EC}$ & 0.007 & 0.993 & & & & \\
\hline \multirow[t]{3}{*}{ RT 33} & $\mathrm{BC}$ & 0.001 & 0.999 & & & & \\
\hline & $\mathrm{MC}$ & 0.018 & 0.983 & & & & \\
\hline & $\mathrm{EC}$ & 0.004 & 0.996 & & & & \\
\hline
\end{tabular}


D-3: One-Way-ANOVA Test for Tangents on Rolling and Mountainous Terrains.

\begin{tabular}{|c|c|c|c|c|c|}
\hline \multicolumn{3}{|c|}{ Rolling Terrain } & \multicolumn{3}{|c|}{ Mountainous Terrain } \\
\hline Curve & $F$ & $p$-value & Curve & $F$ & $p$-value \\
\hline RT 01 & 0.378 & 0.686 & MT 01 & 4.132 & 0.018 \\
\hline RT 02 & 0.000 & 1.000 & MT 02 & 0.735 & 0.481 \\
\hline RT 03 & 0.087 & 0.917 & MT 03 & 0.680 & 0.508 \\
\hline RT 04 & 0.025 & 0.976 & MT 04 & 0.496 & 0.610 \\
\hline RT 05 & 0.093 & 0.912 & MT 05 & 0.181 & 0.835 \\
\hline RT 06 & 0.034 & 0.967 & MT 06 & 2.453 & 0.089 \\
\hline RT 07 & 0.001 & 0.999 & MT 07 & 1.391 & 0.253 \\
\hline RT 08 & 0.003 & 0.997 & MT 08 & 1.352 & 0.263 \\
\hline RT 09 & 0.000 & 1.000 & MT 09 & 1.696 & 0.188 \\
\hline RT 10 & 0.005 & 0.995 & MT 10 & 1.128 & 0.328 \\
\hline RT 11 & 0.009 & 0.991 & MT 11 & 0.235 & 0.791 \\
\hline RT 12 & 0.065 & 0.937 & MT 12 & 0.851 & 0.431 \\
\hline RT 13 & 0.040 & 0.961 & MT 13 & 0.565 & 0.570 \\
\hline RT 14 & 0.199 & 0.820 & MT 14 & 0.451 & 0.638 \\
\hline RT 15 & 0.031 & 0.969 & MT 15 & 0.215 & 0.807 \\
\hline RT 16 & 0.124 & 0.884 & MT 16 & 0.219 & 0.803 \\
\hline RT 17 & 0.099 & 0.906 & MT 17 & 2.946 & 0.057 \\
\hline $\begin{array}{ll}\text { RT } 18 \\
\end{array}$ & 0.280 & 0.757 & MT 18 & 0.665 & 0.516 \\
\hline RT 19 & 0.051 & 0.950 & MT 19 & 0.228 & 0.797 \\
\hline RT 20 & 0.030 & 0.970 & MT 20 & 0.087 & 0.917 \\
\hline RT 21 & 0.003 & 0.997 & MT 21 & 0.440 & 0.645 \\
\hline RT 22 & 0.003 & 0.997 & MT 22 & 2.514 & 0.086 \\
\hline RT 23 & 0.030 & 0.971 & MT 23 & 1.163 & 0.316 \\
\hline RT 24 & 0.024 & 0.976 & MT 24 & 0.637 & 0.531 \\
\hline RT 25 & 0.154 & 0.858 & MT 25 & 1.927 & 0.151 \\
\hline RT 26 & 0.171 & 0.843 & MT 26 & 1.137 & 0.323 \\
\hline RT 27 & 0.055 & 0.946 & MT 27 & 0.190 & 0.827 \\
\hline RT 28 & 0.000 & 1.000 & MT 28 & 0.050 & 0.951 \\
\hline RT 29 & 0.010 & 0.990 & MT 29 & 0.215 & 0.807 \\
\hline RT 30 & 0.004 & 0.996 & & & \\
\hline RT 31 & 0.234 & 0.792 & & & \\
\hline RT 32 & 0.012 & 0.988 & & & \\
\hline RT 33 & 0.033 & 0.968 & & & \\
\hline
\end{tabular}


D-4: Homogeneity of Variance for Speed on Tangents (Rolling and Mountainous Terrains).

\begin{tabular}{|c|c|c|c|c|c|}
\hline \multicolumn{3}{|c|}{ Rolling Terrain } & \multicolumn{3}{c|}{ Mountainous Terrain } \\
\hline Curve & $\begin{array}{c}\text { Levene } \\
\text { Statistic }\end{array}$ & $p$-value & Curve & $\begin{array}{c}\text { Levene } \\
\text { Statistic }\end{array}$ & $p$-value \\
\hline RT 01 & 0.003 & 0.997 & MT 01 & 0.185 & 0.831 \\
\hline RT 02 & 0.004 & 0.996 & MT 02 & 0.080 & 0.924 \\
\hline RT 03 & 0.021 & 0.979 & MT 03 & 0.052 & 0.950 \\
\hline RT 04 & 0.046 & 0.955 & MT 04 & 0.097 & 0.908 \\
\hline RT 05 & 0.003 & 0.997 & MT 05 & 0.040 & 0.961 \\
\hline RT 06 & 0.013 & 0.987 & MT 06 & 0.407 & 0.666 \\
\hline RT 07 & 0.034 & 0.967 & MT 07 & 0.012 & 0.988 \\
\hline RT 08 & 0.008 & 0.992 & MT 08 & 0.030 & 0.971 \\
\hline RT 09 & 0.020 & 0.980 & MT 09 & 0.016 & 0.984 \\
\hline RT 10 & 0.036 & 0.965 & MT 10 & 0.540 & 0.584 \\
\hline RT 11 & 0.003 & 0.997 & MT 11 & 0.042 & 0.959 \\
\hline RT 12 & 0.003 & 0.997 & MT 12 & 0.005 & 0.995 \\
\hline RT 13 & 0.014 & 0.986 & MT 13 & 0.208 & 0.813 \\
\hline RT 14 & 0.013 & 0.987 & MT 14 & 0.019 & 0.981 \\
\hline RT 15 & 0.148 & 0.863 & MT 15 & 0.031 & 0.970 \\
\hline RT 16 & 0.076 & 0.927 & MT 16 & 0.039 & 0.962 \\
\hline RT 17 & 0.012 & 0.988 & MT 17 & 0.130 & 0.879 \\
\hline RT 18 & 0.095 & 0.910 & MT 18 & 0.032 & 0.968 \\
\hline RT 19 & 0.009 & 0.991 & MT 19 & 0.007 & 0.993 \\
\hline RT 20 & 0.009 & 0.991 & MT 20 & 0.023 & 0.977 \\
\hline RT 21 & 0.001 & 0.999 & MT 21 & 0.013 & 0.987 \\
\hline RT 22 & 0.022 & 0.978 & MT 22 & 0.069 & 0.933 \\
\hline RT 23 & 0.200 & 0.819 & MT 23 & 0.253 & 0.777 \\
\hline RT 24 & 0.042 & 0.959 & MT 24 & 0.215 & 0.806 \\
\hline RT 25 & 0.017 & 0.983 & MT 25 & 0.097 & 0.907 \\
\hline RT 26 & 0.027 & 0.973 & MT 26 & 0.051 & 0.950 \\
\hline RT 27 & 0.014 & 0.986 & MT 27 & 0.061 & 0.941 \\
\hline RT 28 & 0.035 & 0.966 & MT 28 & 0.200 & 0.819 \\
\hline RT 29 & 0.050 & 0.951 & MT 29 & 0.031 & 0.970 \\
\hline RT 30 & 0.067 & 0.935 & & & \\
\hline RT 31 & 0.102 & 0.903 & & & \\
\hline RT 32 & 0.004 & 0.996 & & & \\
\hline RT 33 & 0.001 & 0.999 & & & \\
\hline
\end{tabular}


D-5: One-Way-ANOVA Test for $V_{c m f-a v g}$ and $V_{c m f-\min }$ on Rolling and Mountainous Terrains.

\begin{tabular}{|c|c|c|c|c|c|}
\hline \multicolumn{3}{|c|}{ Rolling Terrain } & \multicolumn{3}{|c|}{ Mountainous Terrain } \\
\hline Curve & $F$ & $p$-value & Curve & $F$ & $p$-value \\
\hline RT 01 & 0.243 & 0.623 & MT 01 & 0.572 & 0.451 \\
\hline RT 02 & 0.094 & 0.760 & MT 02 & 0.120 & 0.730 \\
\hline RT 03 & 0.199 & 0.657 & MT 03 & 0.173 & 0.678 \\
\hline RT 04 & 0.116 & 0.734 & MT 04 & 0.361 & 0.549 \\
\hline RT 05 & 0.297 & 0.588 & MT 05 & 0.618 & 0.433 \\
\hline RT 06 & 0.325 & 0.570 & MT 06 & 0.355 & 0.552 \\
\hline RT 07 & 0.140 & 0.710 & MT 07 & 0.195 & 0.660 \\
\hline RT 08 & 0.296 & 0.587 & MT 08 & 0.329 & 0.568 \\
\hline RT 09 & 0.418 & 0.519 & MT 09 & 0.183 & 0.670 \\
\hline RT 10 & 0.506 & 0.478 & MT 10 & 0.201 & 0.656 \\
\hline RT 11 & 0.277 & 0.600 & MT 11 & 0.145 & 0.705 \\
\hline RT 12 & 0.147 & 0.703 & MT 12 & 0.164 & 0.687 \\
\hline RT 13 & 0.134 & 0.715 & MT 13 & 0.124 & 0.726 \\
\hline RT 14 & 0.136 & 0.715 & MT 14 & 0.274 & 0.602 \\
\hline RT 15 & 0.104 & 0.748 & MT 15 & 0.064 & 0.801 \\
\hline RT 16 & 0.085 & 0.773 & MT 16 & 0.026 & 0.874 \\
\hline RT 17 & 0.368 & 0.546 & MT 17 & 0.312 & 0.578 \\
\hline RT 18 & 0.251 & 0.618 & MT 18 & 0.459 & 0.500 \\
\hline RT 19 & 0.212 & 0.646 & MT 19 & 0.171 & 0.681 \\
\hline RT 20 & 0.153 & 0.696 & MT 20 & 0.015 & 0.903 \\
\hline RT 21 & 0.097 & 0.756 & MT 21 & 0.026 & 0.872 \\
\hline RT 22 & 0.234 & 0.631 & MT 22 & 0.288 & 0.593 \\
\hline RT 23 & 0.054 & 0.819 & MT 23 & 0.181 & 0.672 \\
\hline RT 24 & 0.030 & 0.863 & MT 24 & 0.111 & 0.740 \\
\hline RT 25 & 0.276 & 0.602 & MT 25 & 0.221 & 0.640 \\
\hline RT 26 & 0.163 & 0.689 & MT 26 & 0.329 & 0.567 \\
\hline RT 27 & 0.087 & 0.769 & MT 27 & 0.182 & 0.671 \\
\hline RT 28 & 0.235 & 0.629 & MT 28 & 0.125 & 0.724 \\
\hline RT 29 & 0.250 & 0.619 & MT 29 & 0.064 & 0.801 \\
\hline RT 30 & 0.215 & 0.644 & & & \\
\hline RT 31 & 0.455 & 0.503 & & & \\
\hline RT 32 & 0.068 & 0.794 & & & \\
\hline RT 33 & 0.131 & 0.718 & & & \\
\hline
\end{tabular}


D-6: One-Way-ANOVA Test for $V_{c m f-a v g}$ and $V_{c r v-a v g}$ on Rolling and Mountainous

Terrains.

\begin{tabular}{|c|c|c|c|c|c|}
\hline \multicolumn{3}{|c|}{ Rolling Terrain } & \multicolumn{3}{|c|}{ Mountainous Terrain } \\
\hline Curve & $F$ & $p$-value & Curve & $F$ & $p$-value \\
\hline RT 01 & 0.819 & 0.367 & MT 01 & 0.060 & 0.807 \\
\hline RT 02 & 0.049 & 0.826 & MT 02 & 0.031 & 0.861 \\
\hline RT 03 & 1.440 & 0.233 & MT 03 & 0.185 & 0.668 \\
\hline RT 04 & 0.095 & 0.759 & MT 04 & 0.137 & 0.712 \\
\hline RT 05 & 0.088 & 0.767 & MT 05 & 0.006 & 0.940 \\
\hline RT 06 & 0.055 & 0.815 & MT 06 & 0.105 & 0.746 \\
\hline RT 07 & 0.000 & 0.995 & MT 07 & 0.794 & 0.376 \\
\hline RT 08 & 1.012 & 0.317 & MT 08 & 0.009 & 0.924 \\
\hline RT 09 & 0.019 & 0.892 & MT 09 & 0.175 & 0.677 \\
\hline RT 10 & 0.743 & 0.390 & MT 10 & 0.003 & 0.960 \\
\hline RT 11 & 0.106 & 0.746 & MT 11 & 0.000 & 0.996 \\
\hline RT 12 & 0.012 & 0.912 & MT 12 & 0.191 & 0.664 \\
\hline RT 13 & 0.017 & 0.897 & MT 13 & 0.001 & 0.975 \\
\hline RT 14 & 0.529 & 0.471 & MT 14 & 0.174 & 0.678 \\
\hline RT 15 & 0.128 & 0.722 & MT 15 & 0.010 & 0.921 \\
\hline RT 16 & 0.040 & 0.842 & MT 16 & 0.001 & 0.981 \\
\hline RT 17 & 0.045 & 0.833 & MT 17 & 0.458 & 0.501 \\
\hline RT 18 & 0.147 & 0.702 & MT 18 & 0.135 & 0.714 \\
\hline RT 19 & 0.008 & 0.929 & MT 19 & 0.271 & 0.605 \\
\hline RT 20 & 0.102 & 0.750 & MT 20 & 0.383 & 0.538 \\
\hline RT 21 & 0.021 & 0.884 & MT 21 & 0.000 & 0.996 \\
\hline RT 22 & 0.258 & 0.615 & MT 22 & 0.024 & 0.878 \\
\hline RT 23 & 0.066 & 0.799 & MT 23 & 0.030 & 0.862 \\
\hline RT 24 & 0.057 & 0.812 & MT 24 & 0.867 & 0.354 \\
\hline RT 25 & 0.135 & 0.715 & MT 25 & 0.109 & 0.742 \\
\hline RT 26 & 0.012 & 0.913 & MT 26 & 0.370 & 0.544 \\
\hline RT 27 & 0.188 & 0.665 & MT 27 & 0.119 & 0.731 \\
\hline RT 28 & 0.107 & 0.745 & MT 28 & 0.704 & 0.405 \\
\hline RT 29 & 0.037 & 0.847 & MT 29 & 0.894 & 0.349 \\
\hline RT 30 & 0.009 & 0.923 & & & \\
\hline RT 31 & 0.159 & 0.691 & & & \\
\hline RT 32 & 0.430 & 0.514 & & & \\
\hline RT 33 & 0.030 & 0.864 & & & \\
\hline
\end{tabular}


D-7: One-Way-ANOVA Test for $V_{c r v-\min }$ and $V_{c r v-\max }$ on Rolling and Mountainous Terrains.

\begin{tabular}{|c|c|c|c|c|c|}
\hline \multicolumn{3}{|c|}{ Rolling Terrain } & \multicolumn{3}{|c|}{ Mountainous Terrain } \\
\hline Curve & $F$ & $p$-value & Curve & $F$ & $p$-value \\
\hline RT 01 & 22.326 & $<0.001$ & MT 01 & 15.528 & $<0.001$ \\
\hline RT 02 & 6.877 & 0.010 & MT 02 & 13.297 & $<0.001$ \\
\hline RT 03 & 17.594 & $<0.001$ & MT 03 & 11.660 & 0.001 \\
\hline RT 04 & 32.864 & $<0.001$ & MT 04 & 6.812 & 0.010 \\
\hline RT 05 & 3.254 & 0.075 & MT 05 & 1.230 & 0.270 \\
\hline RT 06 & 7.861 & 0.006 & MT 06 & 9.042 & 0.003 \\
\hline RT 07 & 5.502 & 0.021 & MT 07 & 6.466 & 0.013 \\
\hline RT 08 & 14.778 & $<0.001$ & MT 08 & 16.068 & 0.000 \\
\hline RT 09 & 12.924 & $<0.001$ & MT 09 & 2.212 & 0.141 \\
\hline RT 10 & 40.805 & $<0.001$ & MT 10 & 12.663 & 0.001 \\
\hline RT 11 & 9.004 & 0.003 & MT 11 & 5.560 & 0.021 \\
\hline RT 12 & 3.795 & 0.058 & MT 12 & 11.514 & 0.001 \\
\hline RT 13 & 4.182 & 0.046 & MT 13 & 2.846 & 0.097 \\
\hline RT 14 & 4.116 & 0.049 & MT 14 & 3.371 & 0.070 \\
\hline RT 15 & 15.186 & 0.000 & MT 15 & 4.427 & 0.039 \\
\hline RT 16 & 9.635 & 0.003 & MT 16 & 7.997 & 0.006 \\
\hline RT 17 & 11.207 & 0.001 & MT 17 & 12.204 & 0.001 \\
\hline RT 18 & 6.782 & 0.012 & MT 18 & 1.814 & 0.181 \\
\hline RT 19 & 9.428 & 0.003 & MT 19 & 6.439 & 0.014 \\
\hline RT 20 & 7.817 & 0.006 & MT 20 & 6.925 & 0.011 \\
\hline RT 21 & 6.530 & 0.013 & MT 21 & 4.179 & 0.045 \\
\hline RT 22 & 2.539 & 0.118 & MT 22 & 3.946 & 0.051 \\
\hline RT 23 & 2.120 & 0.154 & MT 23 & 3.827 & 0.054 \\
\hline RT 24 & 4.835 & 0.031 & MT 24 & 2.390 & 0.125 \\
\hline RT 25 & 5.292 & 0.026 & MT 25 & 5.684 & 0.020 \\
\hline RT 26 & 10.314 & 0.003 & MT 26 & 10.572 & 0.001 \\
\hline RT 27 & 18.682 & $<0.001$ & MT 27 & 1.937 & 0.168 \\
\hline RT 28 & 16.599 & $<0.001$ & MT 28 & 1.500 & 0.224 \\
\hline RT 29 & 11.492 & 0.001 & MT 29 & 8.762 & 0.004 \\
\hline RT 30 & 4.695 & 0.034 & & & \\
\hline RT 31 & 2.560 & 0.114 & & & \\
\hline RT 32 & 7.736 & 0.006 & & & \\
\hline RT 33 & 2.557 & 0.113 & & & \\
\hline
\end{tabular}


D-8: One-Way-ANOVA Test for $V_{c r v-\min }$ and $V_{c r v-a v g}$ on Rolling and Mountainous

Terrains.

\begin{tabular}{|c|c|c|c|c|c|}
\hline \multicolumn{3}{|c|}{ Rolling Terrain } & \multicolumn{3}{|c|}{ Mountainous Terrain } \\
\hline Curve & $F$ & $p$-value & Curve & $F$ & $p$-value \\
\hline RT 01 & 4.011 & 0.047 & MT 01 & 4.364 & 0.039 \\
\hline RT 02 & 1.772 & 0.185 & MT 02 & 2.599 & 0.110 \\
\hline RT 03 & 3.344 & 0.070 & MT 03 & 2.387 & 0.125 \\
\hline RT 04 & 8.677 & 0.004 & MT 04 & 1.058 & 0.306 \\
\hline RT 05 & 0.614 & 0.436 & MT 05 & 0.257 & 0.613 \\
\hline RT 06 & 1.357 & 0.248 & MT 06 & 2.028 & 0.157 \\
\hline RT 07 & 1.381 & 0.243 & MT 07 & 1.928 & 0.169 \\
\hline RT 08 & 2.807 & 0.096 & MT 08 & 4.078 & 0.047 \\
\hline RT 09 & 3.020 & 0.085 & MT 09 & 0.497 & 0.483 \\
\hline RT 10 & 8.588 & 0.004 & MT 10 & 2.180 & 0.145 \\
\hline RT 11 & 1.930 & 0.168 & MT 11 & 1.420 & 0.237 \\
\hline RT 12 & 0.823 & 0.369 & MT 12 & 2.116 & 0.151 \\
\hline RT 13 & 0.895 & 0.349 & MT 13 & 0.698 & 0.407 \\
\hline RT 14 & 0.917 & 0.343 & MT 14 & 0.671 & 0.415 \\
\hline RT 15 & 4.112 & 0.047 & MT 15 & 1.325 & 0.254 \\
\hline RT 16 & 2.544 & 0.118 & MT 16 & 1.934 & 0.169 \\
\hline RT 17 & 2.855 & 0.096 & MT 17 & 0.109 & 0.742 \\
\hline RT 18 & 1.253 & 0.267 & MT 18 & 0.434 & 0.512 \\
\hline RT 19 & 2.238 & 0.137 & MT 19 & 1.464 & 0.231 \\
\hline RT 20 & 2.201 & 0.142 & MT 20 & 1.215 & 0.274 \\
\hline RT 21 & 1.450 & 0.234 & MT 21 & 0.910 & 0.343 \\
\hline RT 22 & 0.471 & 0.496 & MT 22 & 0.707 & 0.403 \\
\hline RT 23 & 0.477 & 0.494 & MT 23 & 0.738 & 0.393 \\
\hline RT 24 & 0.879 & 0.352 & MT 24 & 0.563 & 0.454 \\
\hline RT 25 & 1.035 & 0.314 & MT 25 & 0.965 & 0.329 \\
\hline RT 26 & 2.729 & 0.107 & MT 26 & 2.019 & 0.158 \\
\hline RT 27 & 3.262 & 0.073 & MT 27 & 0.472 & 0.494 \\
\hline RT 28 & 1.712 & 0.194 & MT 28 & 0.369 & 0.545 \\
\hline RT 29 & 2.348 & 0.130 & MT 29 & 2.174 & 0.145 \\
\hline RT 30 & 0.886 & 0.350 & & & \\
\hline RT 31 & 0.555 & 0.459 & & & \\
\hline RT 32 & 1.401 & 0.239 & & & \\
\hline RT 33 & 0.507 & 0.478 & & & \\
\hline
\end{tabular}


D-9: One-Way-ANOVA Test for $V_{c r v-\max }$ and $V_{B C}$ on Rolling and Mountainous

Terrains.

\begin{tabular}{|c|c|c|c|c|c|}
\hline \multicolumn{3}{|c|}{ Rolling Terrain } & \multicolumn{3}{|c|}{ Mountainous Terrain } \\
\hline Curve & $F$ & $p$-value & Curve & $F$ & $p$-value \\
\hline RT 01 & 0.410 & 0.523 & MT 01 & 5.245 & 0.024 \\
\hline RT 02 & 0.724 & 0.397 & MT 02 & 0.474 & 0.493 \\
\hline RT 03 & 0.429 & 0.514 & MT 03 & 0.693 & 0.407 \\
\hline RT 04 & 1.155 & 0.286 & MT 04 & 0.137 & 0.712 \\
\hline RT 05 & 14.010 & $<0.001$ & MT 05 & 0.933 & 0.336 \\
\hline RT 06 & 0.500 & 0.482 & MT 06 & 2.523 & 0.115 \\
\hline RT 07 & 1.334 & 0.251 & MT 07 & 2.217 & 0.141 \\
\hline RT 08 & 2.678 & 0.104 & MT 08 & 2.063 & 0.155 \\
\hline RT 09 & 1.360 & 0.246 & MT 09 & 2.385 & 0.127 \\
\hline RT 10 & 1.253 & 0.265 & MT 10 & 1.493 & 0.227 \\
\hline RT 11 & 0.520 & 0.472 & MT 11 & 0.251 & 0.618 \\
\hline RT 12 & 6.694 & 0.014 & MT 12 & 1.002 & 0.321 \\
\hline RT 13 & 2.828 & 0.099 & MT 13 & 0.277 & 0.601 \\
\hline RT 14 & 0.001 & 0.974 & MT 14 & 0.380 & 0.539 \\
\hline RT 15 & 4.013 & 0.050 & MT 15 & 0.389 & 0.535 \\
\hline RT 16 & 0.608 & 0.440 & MT 16 & 0.130 & 0.720 \\
\hline RT 17 & 3.756 & 0.058 & MT 17 & 3.428 & 0.069 \\
\hline RT 18 & 1.903 & 0.173 & MT 18 & 0.579 & 0.449 \\
\hline RT 19 & 1.712 & 0.193 & MT 19 & 0.044 & 0.834 \\
\hline RT 20 & 3.890 & 0.052 & MT 20 & 0.046 & 0.830 \\
\hline RT 21 & 4.112 & 0.047 & MT 21 & 0.703 & 0.405 \\
\hline RT 22 & 2.159 & 0.149 & MT 22 & 4.254 & 0.043 \\
\hline RT 23 & 1.096 & 0.303 & MT 23 & 1.424 & 0.236 \\
\hline RT 24 & 1.412 & 0.239 & MT 24 & 0.660 & 0.418 \\
\hline RT 25 & 2.185 & 0.146 & MT 25 & 2.579 & 0.113 \\
\hline RT 26 & 0.556 & 0.461 & MT 26 & 1.323 & 0.252 \\
\hline RT 27 & 0.825 & 0.366 & MT 27 & 0.068 & 0.795 \\
\hline RT 28 & 0.903 & 0.344 & MT 28 & 0.037 & 0.849 \\
\hline RT 29 & 1.628 & 0.206 & MT 29 & 0.389 & 0.535 \\
\hline RT 30 & 2.663 & 0.108 & & & \\
\hline RT 31 & 1.712 & 0.195 & & & \\
\hline RT 32 & 0.302 & 0.584 & & & \\
\hline RT 33 & 0.296 & 0.588 & & & \\
\hline
\end{tabular}


D-10: One-Way-ANOVA Test for $V_{c r v-a v g}$ and $V_{c r v-m i n}$ on Rolling and Mountainous

Terrains.

\begin{tabular}{|c|c|c|c|c|c|}
\hline \multicolumn{3}{|c|}{ Rolling Terrain } & \multicolumn{3}{|c|}{ Mountainous Terrain } \\
\hline Curve & $F$ & $p$-value & Curve & $F$ & $p$-value \\
\hline RT 01 & 4.011 & 0.047 & MT 01 & 4.364 & 0.039 \\
\hline RT 02 & 1.772 & 0.185 & MT 02 & 1.158 & 0.285 \\
\hline RT 03 & 3.344 & 0.070 & MT 03 & 1.691 & 0.196 \\
\hline RT 04 & 1.305 & 0.257 & MT 04 & 2.420 & 0.123 \\
\hline RT 05 & 3.955 & 0.049 & MT 05 & 8.955 & 0.003 \\
\hline RT 06 & 1.659 & 0.202 & MT 06 & 3.725 & 0.056 \\
\hline RT 07 & 1.615 & 0.207 & MT 07 & 1.522 & 0.221 \\
\hline RT 08 & 2.180 & 0.142 & MT 08 & 2.920 & 0.091 \\
\hline RT 09 & 2.341 & 0.129 & MT 09 & 0.153 & 0.697 \\
\hline RT 10 & 2.793 & 0.097 & MT 10 & 1.011 & 0.319 \\
\hline RT 11 & 1.533 & 0.218 & MT 11 & 0.911 & 0.343 \\
\hline RT 12 & 2.046 & 0.160 & MT 12 & 1.261 & 0.266 \\
\hline RT 13 & 1.367 & 0.248 & MT 13 & 0.736 & 0.394 \\
\hline RT 14 & 1.068 & 0.307 & MT 14 & 1.004 & 0.320 \\
\hline RT 15 & 0.963 & 0.331 & MT 15 & 0.352 & 0.555 \\
\hline RT 16 & 1.559 & 0.218 & MT 16 & 0.662 & 0.419 \\
\hline RT 17 & 7.316 & 0.009 & MT 17 & 2.878 & 0.094 \\
\hline RT 18 & 2.620 & 0.111 & MT 18 & 2.036 & 0.157 \\
\hline RT 19 & 1.476 & 0.227 & MT 19 & 0.839 & 0.363 \\
\hline RT 20 & 1.853 & 0.177 & MT 20 & 0.136 & 0.713 \\
\hline RT 21 & 1.603 & 0.211 & MT 21 & 0.228 & 0.634 \\
\hline RT 22 & 1.463 & 0.233 & MT 22 & 2.201 & 0.143 \\
\hline RT 23 & 0.398 & 0.532 & MT 23 & 1.461 & 0.230 \\
\hline RT 24 & 0.789 & 0.377 & MT 24 & 0.652 & 0.421 \\
\hline RT 25 & 2.948 & 0.092 & MT 25 & 1.096 & 0.299 \\
\hline RT 26 & 1.397 & 0.245 & MT 26 & 2.192 & 0.141 \\
\hline RT 27 & 1.448 & 0.231 & MT 27 & 1.344 & 0.250 \\
\hline RT 28 & 1.269 & 0.263 & MT 28 & 0.705 & 0.404 \\
\hline RT 29 & 2.306 & 0.133 & MT 29 & 0.352 & 0.555 \\
\hline RT 30 & 0.478 & 0.492 & & & \\
\hline RT 31 & 2.436 & 0.123 & & & \\
\hline RT 32 & 0.402 & 0.527 & & & \\
\hline RT 33 & 0.507 & 0.478 & & & \\
\hline
\end{tabular}


D-11: One-Way-ANOVA Test for $V_{M C}$ and $V_{c m f-a v g}$ on Rolling and Mountainous

Terrains.

\begin{tabular}{|c|c|c|c|c|c|}
\hline \multicolumn{3}{|c|}{ Rolling Terrain } & \multicolumn{3}{|c|}{ Mountainous Terrain } \\
\hline Curve & $F$ & $p$-value & Curve & $F$ & $p$-value \\
\hline RT 01 & $<0.001$ & 0.993 & MT 01 & 0.572 & 0.451 \\
\hline RT 02 & $<0.001$ & 0.983 & MT 02 & 0.055 & 0.816 \\
\hline RT 03 & 0.057 & 0.811 & MT 03 & 0.059 & 0.808 \\
\hline RT 04 & 0.053 & 0.819 & MT 04 & 0.460 & 0.499 \\
\hline RT 05 & 0.004 & 0.952 & MT 05 & 0.001 & 0.976 \\
\hline RT 06 & 0.034 & 0.855 & MT 06 & 0.165 & 0.686 \\
\hline RT 07 & 1.010 & 0.318 & MT 07 & 0.095 & 0.759 \\
\hline RT 08 & 0.007 & 0.933 & MT 08 & 0.437 & 0.511 \\
\hline RT 09 & 0.003 & 0.956 & MT 09 & 0.159 & 0.691 \\
\hline RT 10 & 0.033 & 0.857 & MT 10 & 0.122 & 0.728 \\
\hline RT 11 & 0.001 & 0.971 & MT 11 & 0.001 & 0.979 \\
\hline RT 12 & 0.232 & 0.633 & MT 12 & 0.002 & 0.964 \\
\hline RT 13 & 0.473 & 0.495 & MT 13 & 0.507 & 0.480 \\
\hline RT 14 & 0.204 & 0.654 & MT 14 & 0.067 & 0.797 \\
\hline RT 15 & 0.307 & 0.582 & MT 15 & 0.290 & 0.593 \\
\hline RT 16 & 0.192 & 0.664 & MT 16 & 0.552 & 0.462 \\
\hline RT 17 & 1.305 & 0.258 & MT 17 & 0.109 & 0.742 \\
\hline RT 18 & 0.185 & 0.669 & MT 18 & 0.008 & 0.927 \\
\hline RT 19 & 0.344 & 0.559 & MT 19 & 0.222 & 0.639 \\
\hline RT 20 & 0.003 & 0.957 & MT 20 & 0.041 & 0.841 \\
\hline RT 21 & 0.006 & 0.941 & MT 21 & 0.091 & 0.764 \\
\hline RT 22 & 0.049 & 0.825 & MT 22 & 0.196 & 0.659 \\
\hline RT 23 & 0.115 & 0.737 & MT 23 & 0.428 & 0.515 \\
\hline RT 24 & 0.005 & 0.946 & MT 24 & 0.033 & 0.857 \\
\hline RT 25 & 0.204 & 0.654 & MT 25 & 0.113 & 0.738 \\
\hline RT 26 & 0.929 & 0.342 & MT 26 & 0.447 & 0.505 \\
\hline RT 27 & 0.001 & 0.970 & MT 27 & 0.078 & 0.781 \\
\hline RT 28 & 0.023 & 0.879 & MT 28 & 0.060 & 0.807 \\
\hline RT 29 & 0.112 & 0.739 & MT 29 & 0.290 & 0.593 \\
\hline RT 30 & 0.103 & 0.749 & & & \\
\hline RT 31 & 0.051 & 0.822 & & & \\
\hline RT 32 & 0.011 & 0.918 & & & \\
\hline RT 33 & 0.040 & 0.842 & & & \\
\hline
\end{tabular}




\section{APPENDIX E}

Matlab Code for Determining Vehicle Speed State and Comfort Threshold 
clc; clear

tic ostart stopwatch

odeclare global variables

global accThreshold curveNo distBME maxZonelength minNumPtsCurve ... minNumPtsTang200 minNumPtsTang250 minNumZonePts minPtComfort ... minPropZoneLength nZonesCurve nZonesTang pvalThreshold ... sizeslidingWindow timeInterval timeBME

codeVer $=8$; $\frac{0}{2}$ ersion of the matlab code; uses distance on curve dataRead $=1 ;$ oif $=0$ extract data from Excel file; otherwise get data from mat file

odefine the data and analysis prameters

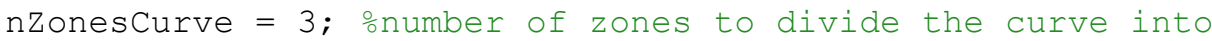

nZonesTang $=3$; $\frac{\circ}{0}$ number of zones to divide the tangent $(0-200)$ into maxzonelength $=50$; omax length $(\mathrm{m})$ of zone 1 or last zone on curve timeInterval $=0.1 ;$ otime interval for speed measurement in seconds

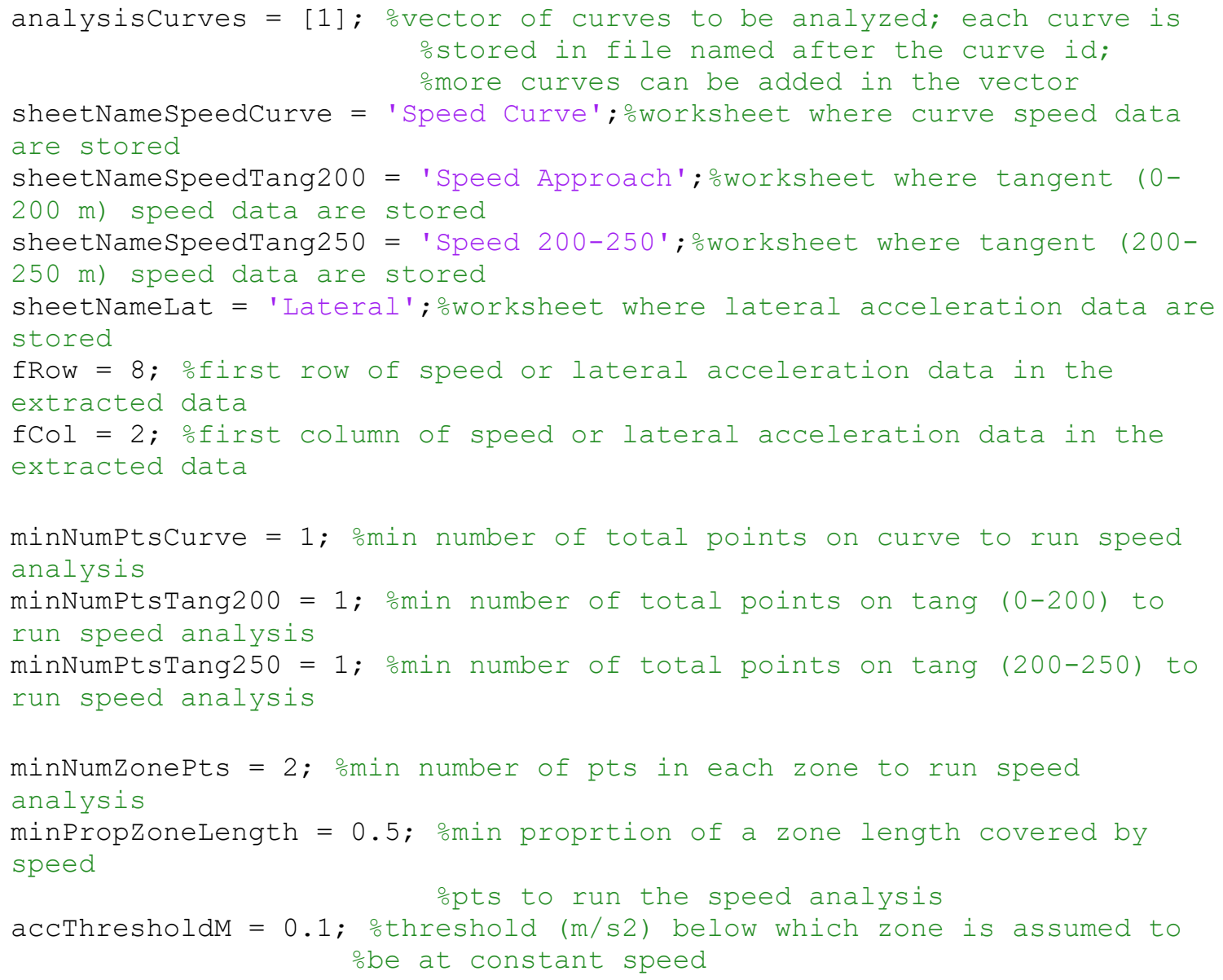




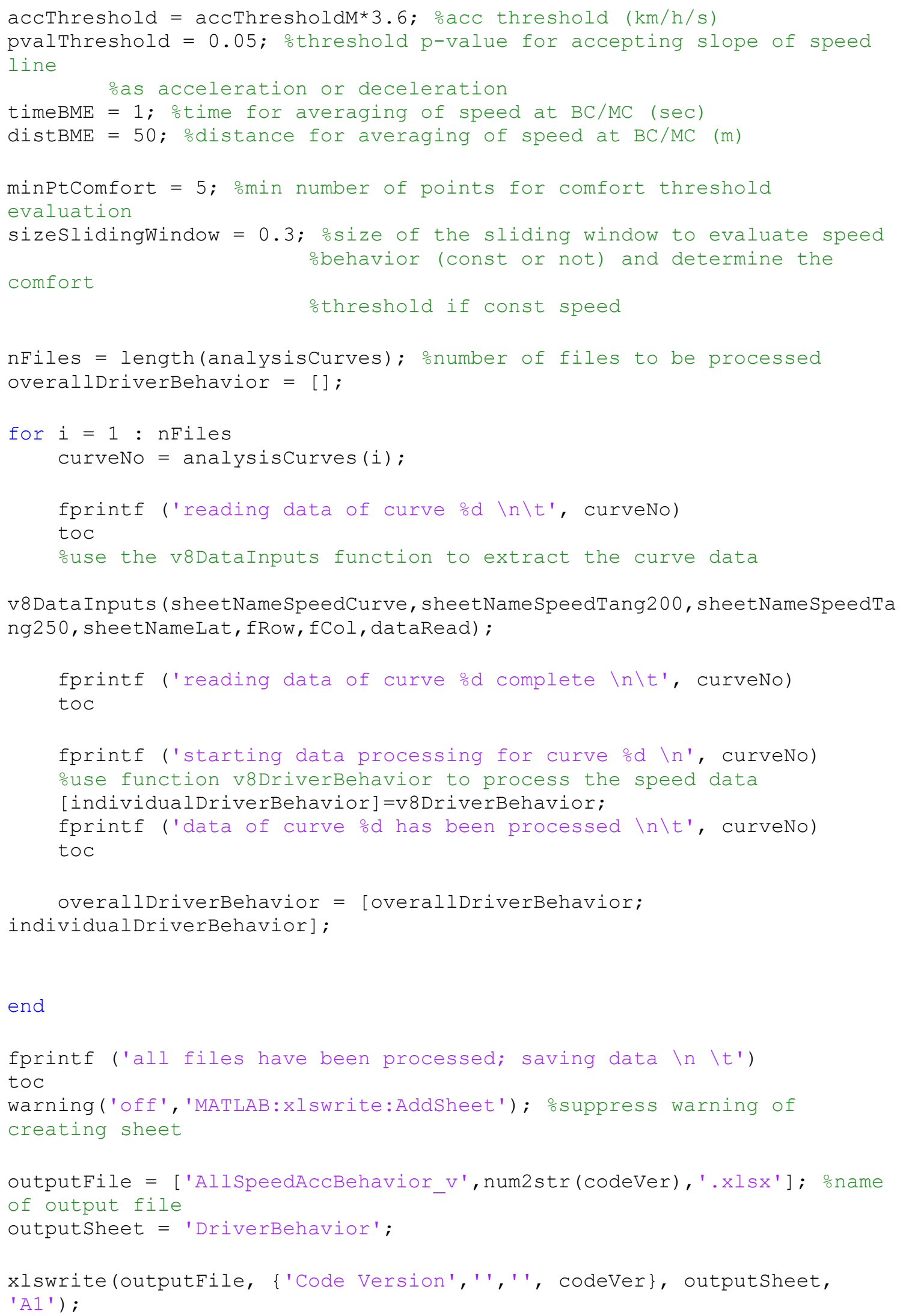


xlswrite (outputfile, \{'Min number of pts for speed on curve, tang200, and tang250',' ', ' ',' ', ' ', minNumptsCurve, minNumPtsTang200,

minNumPtsTang250\}, outputsheet, 'A3');

xlswrite (outputfile, \{'Min number of speed pts per zone',' ',' ', minNumZonePts\}, outputsheet, 'A4');

xlswrite (outputfile, \{'Min proportion of zone covered by speed pts',' ',' ', minPropZoneLength\}, outputSheet, 'A5');

xlswrite (outputFile, \{'Dist for calculating speed at BC/MC/EC',' ',' ', distBME\}, outputsheet, 'A6');

xlswrite (outputFile, \{'Time for calculating speed at BC/MC/EC',' ', '', timeBME\}, outputsheet, 'A7');

xlswrite (outputfile, \{'For acc/dec state: $-1=$ dec; 0 =const; $\left.1=a c c^{\prime}\right\}$, outputsheet, 'A8');

xlswrite (outputFile, \{'Long acc threshold','','', accThreshold, 'km/h/s',' ', accThresholdM, 'm/s2'\}, outputsheet, 'A10'); xlswrite (outputfile, \{'p-value threshold',' ',' ', pvalThreshold\}, outputsheet, 'A11');

xlswrite (outputfile, \{'Min number of pts for comfort threshold', ' ', ' ', minPtComfort\}, outputsheet, 'A12');

xlswrite (outputfile, \{'Size of sliding window',' ',' ', sizeSlidingWindow\}, outputsheet, 'A13');

xlswrite (outputfile, \{'Below are the data of each driver with data pts:'\}, outputsheet, 'A15');

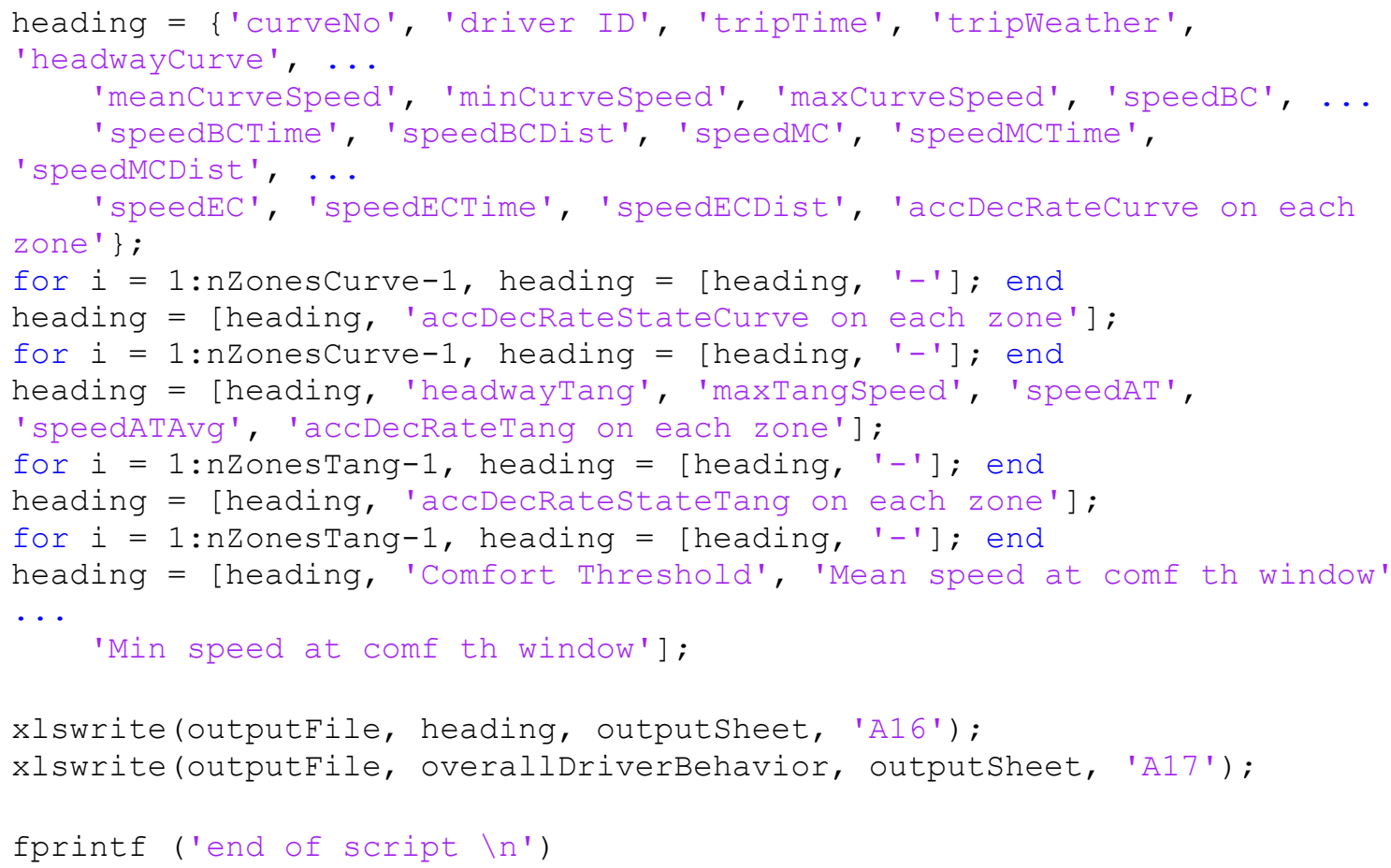




\section{v8ZonesAcc.m}

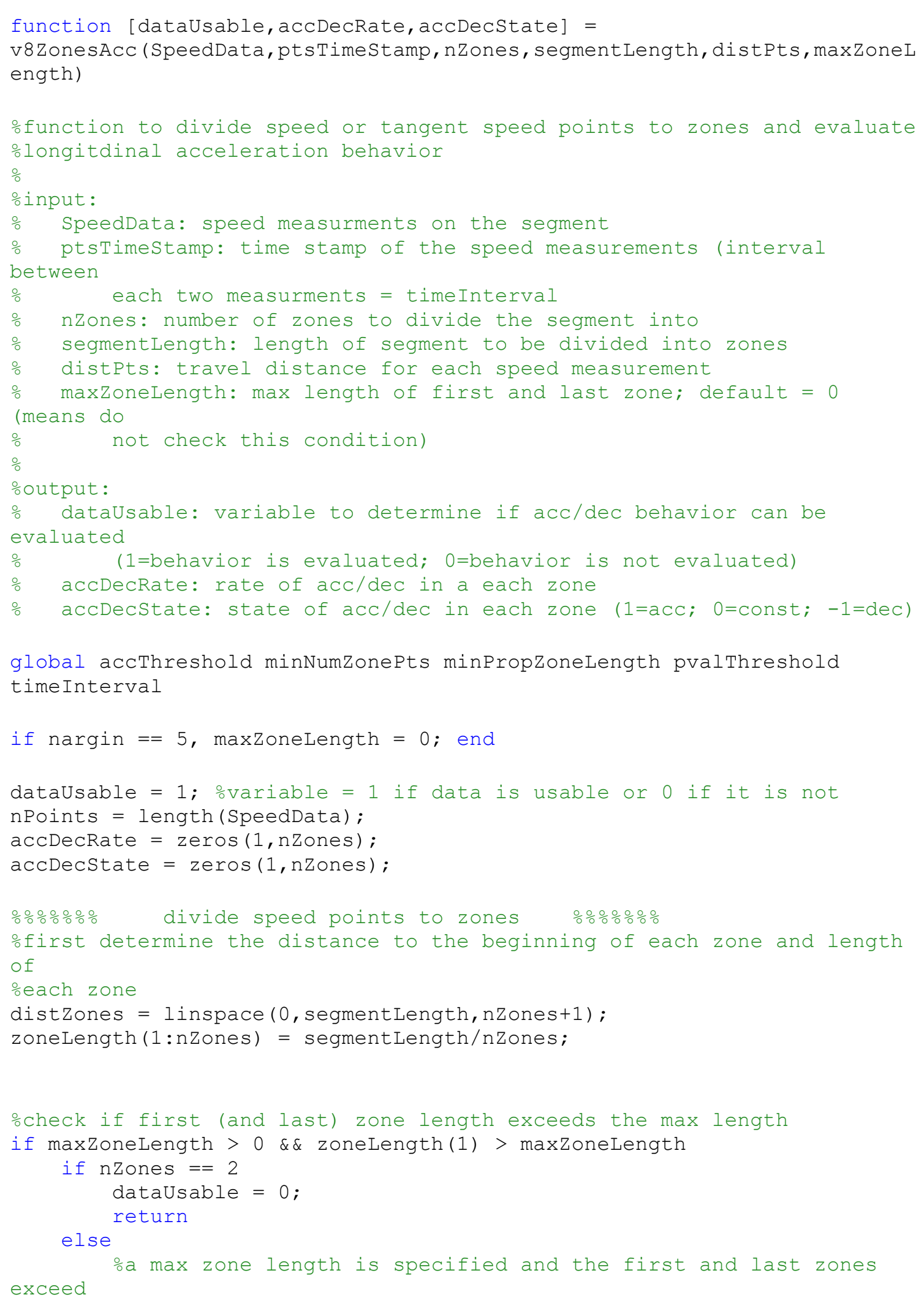




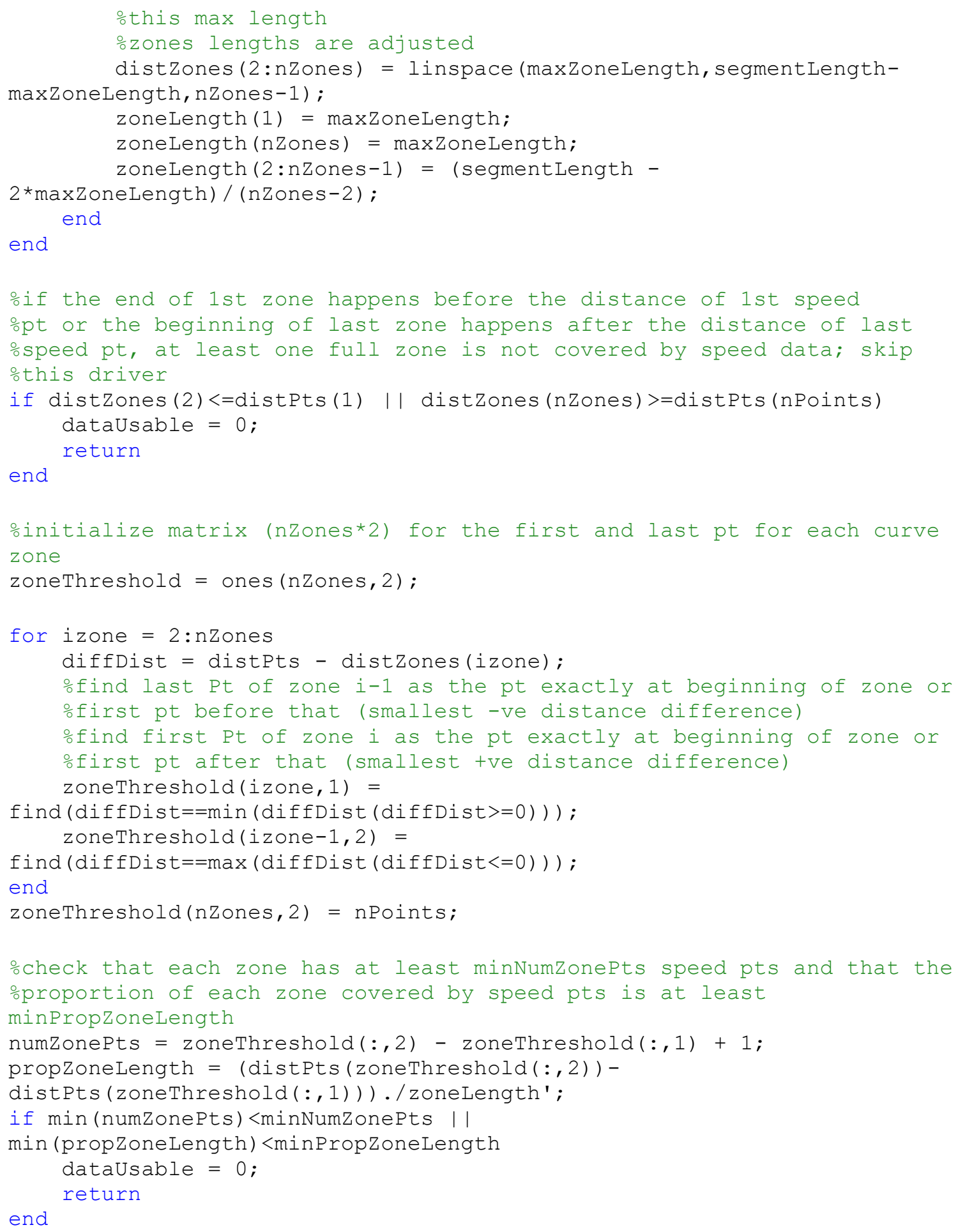




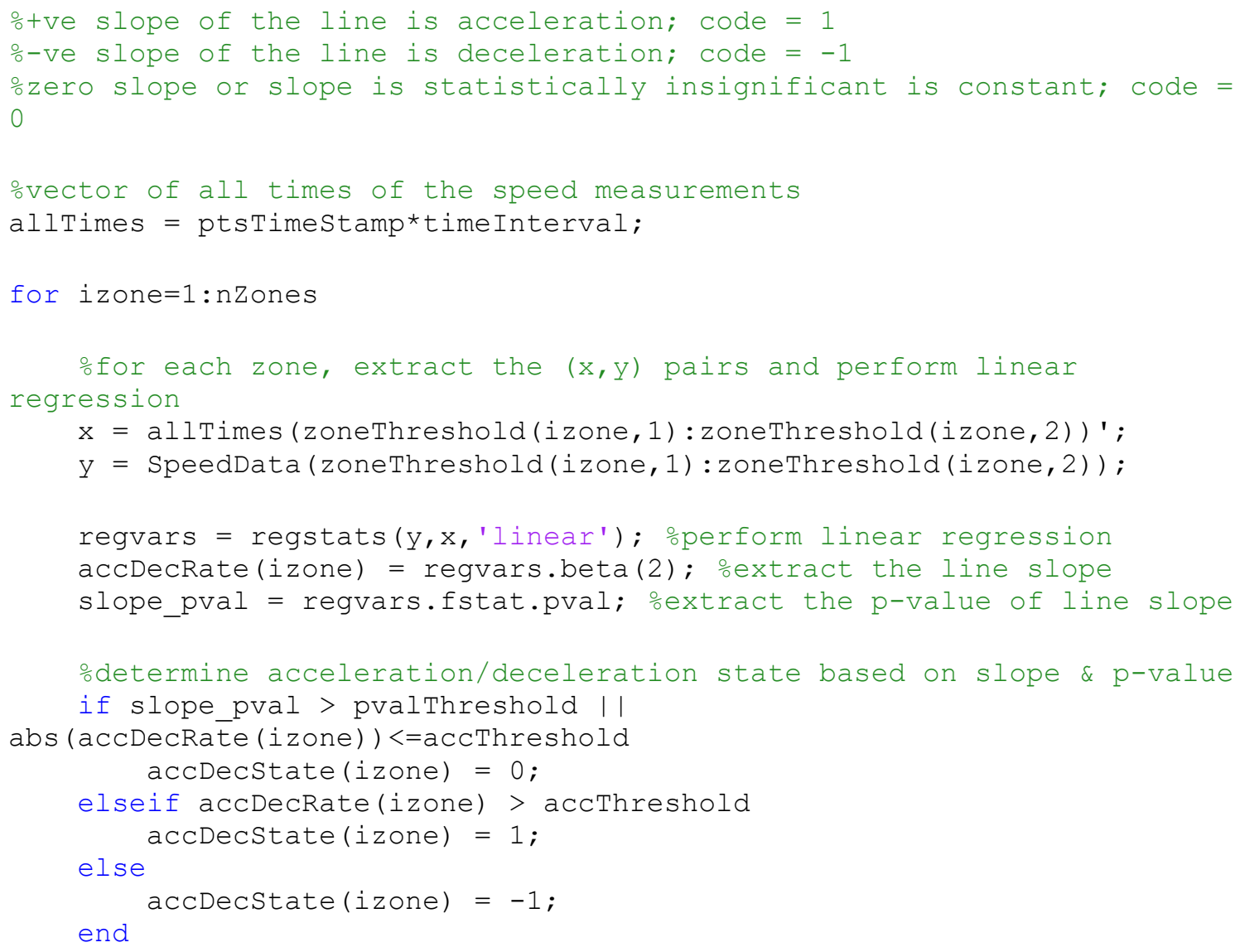

end 


\section{v8DriverBehavior.m}

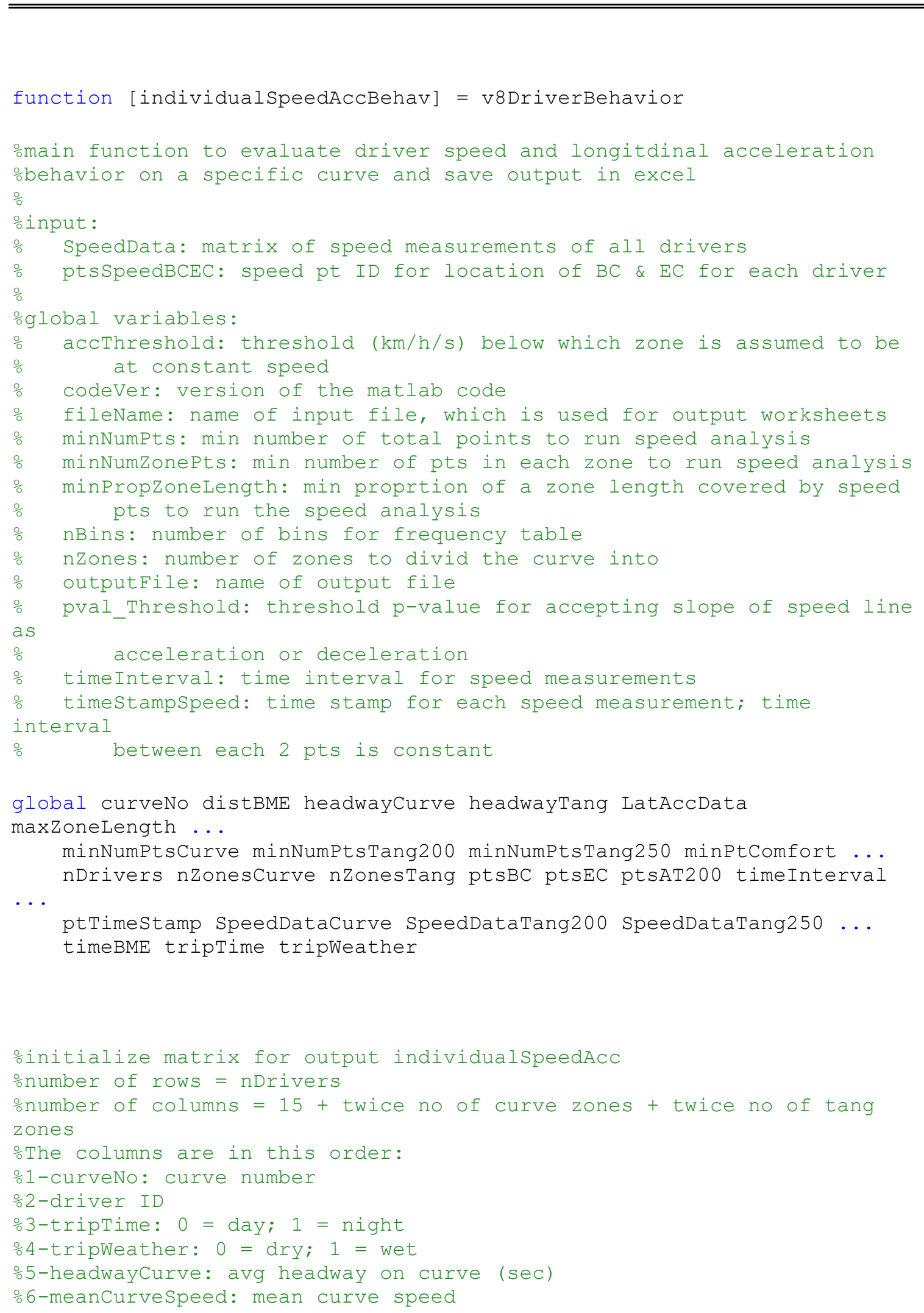


7-mincurvespeed: min curve speed

\%8-maxCurvespeed: max curve speed

-9-speedBC: instantaneous speed at BC or closest point within

dist\&timeBME

\%10-speedBCTime: BC speed based on time

\%11-speedBCDist: BC speed based on distance

o12-speedMC: instantaneous speed at BC or closest point within

dist\&timeBME

\%13-speedMCTime: MC speed based on time

\%14-speedMCDist: MC speed based on distance

\%15-speedEC: instantaneous speed at BC or closest point within

dist\&timeBME

o16-speedECTime: EC speed based on time

\%17-speedECDist: EC speed based on distance

\%18-accDecRateCurve: acc/dec rate on each of curve zone (m/s2)

o19-accDecStateCurve: acc/dec state on each of curve zone (1=acc;

$0=$ const ;

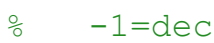

\%20-headwayTang: avg headway on tang ( $\mathrm{sec}$ )

\%21-maxTangspeed: max tangent speed $(0-200 \mathrm{~m})$

\%22-speedAT: AT speed (instantaneous at AT200 or 1st preceding point on

tang 200-250 m)

\%23-speedATAvg: AT speed (average from 200-250 m)

24-accDecRateTang: acc/dec rate on each of tang zone (m/s2)

\%25-accDecstateTang: acc/dec state on each of tang zone (1=acc;

$0=$ const ;

$\div \quad-1=\mathrm{dec}$

26-Comfort Threshold: estimated using function v8ComfortThreshold

-27-Mean speed at comfort threshold window: estimated using function

v8ComfortThreshold

\%28-Min speed at comfort threshold window: estimated using function

v8ComfortThreshold

individualspeedAccBehav =

zeros (nDrivers, (21+2*nZonesCurve+2*nZonesTang+3));

oprocess data for each driver for driver i ranging from 1 to nDrivers

for $i=1$ : nDrivers 250)

oextract profile of speed data on curve, tang(0-200), and tang(200-

speedDriverCurve = SpeedDataCurve $(:$, i $)$;

speedDriverTang200 = SpeedDataTang200 (:,i);

speedDriverTang250 = SpeedDataTang250 (:,i);

\author{
oxtract time stamp for speed data by removing NaN data from the \\ ogeneral time stamps (time stamp data for tang250 are not required) \\ oremove NaN from speed data \\ timeStampSpeedCurve = ptTimeStamp ( isnan (speedDriverCurve)); \\ speedDriverCurve (isnan (speedDriverCurve)) = [ ] ; \\ timeStampspeedTang200 = ptTimeStamp ( isnan (speedDriverTang200));
}




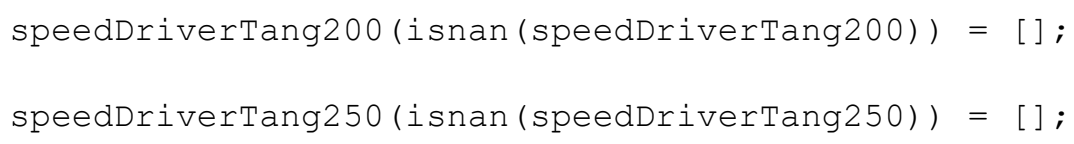




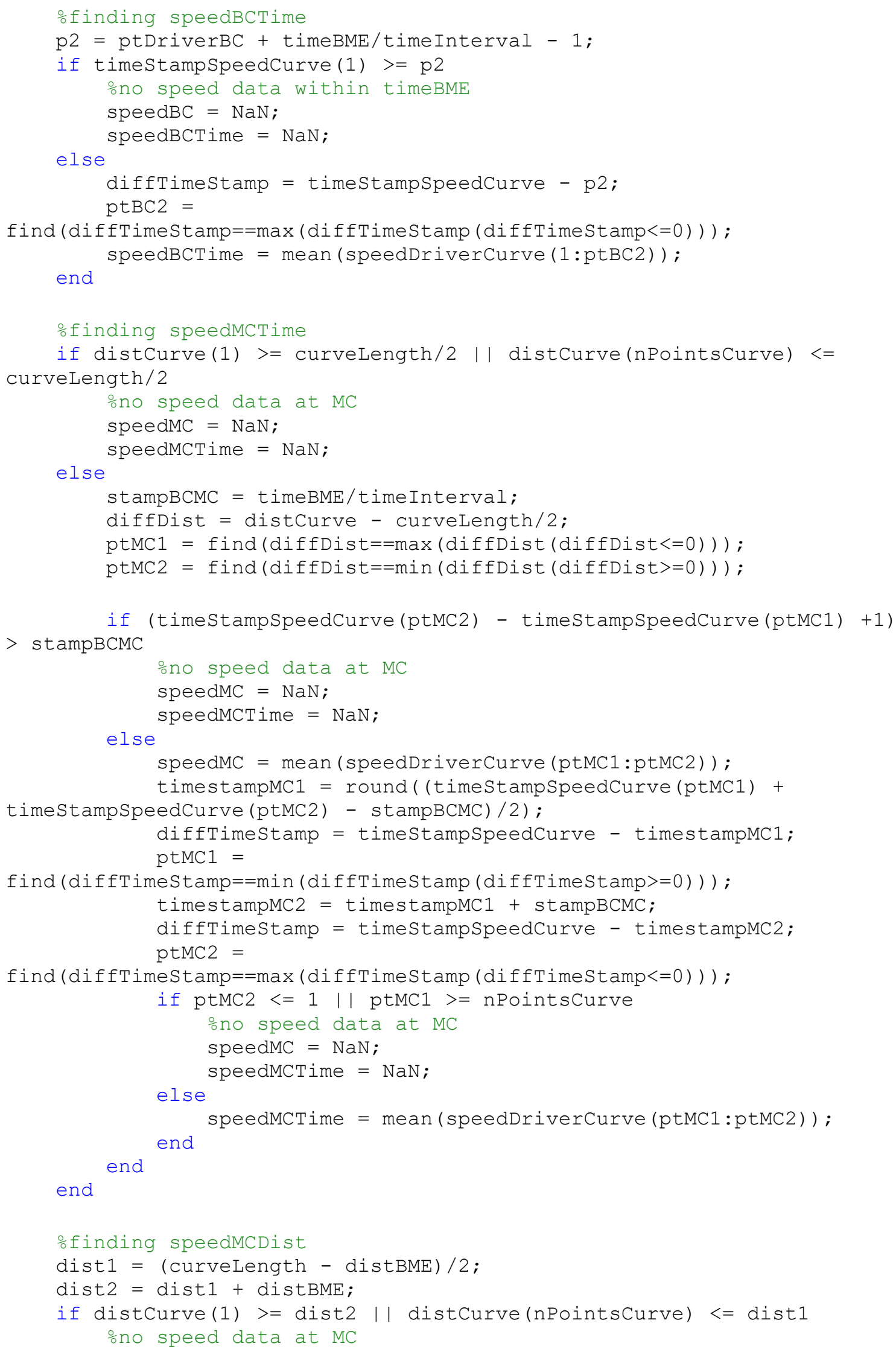




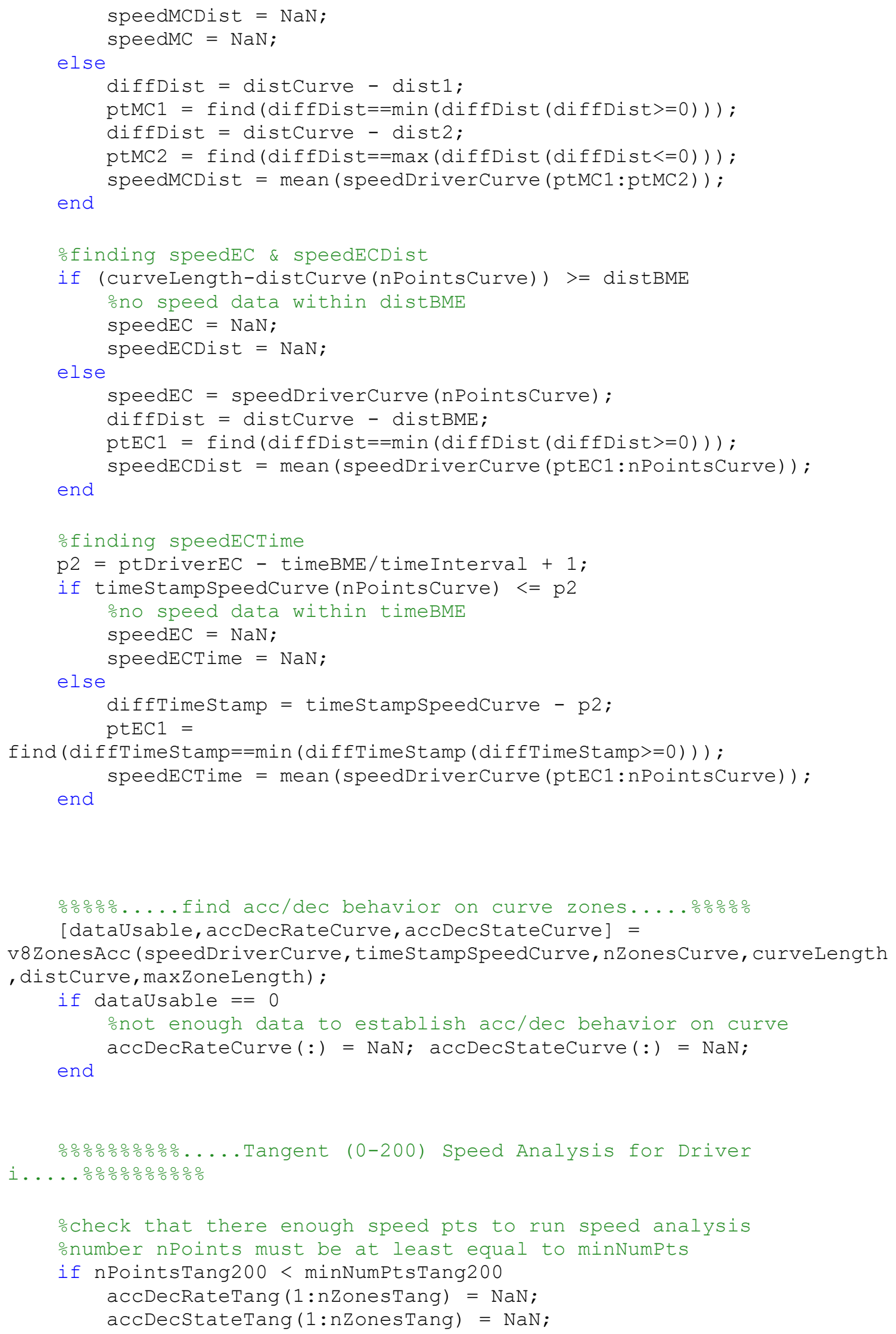




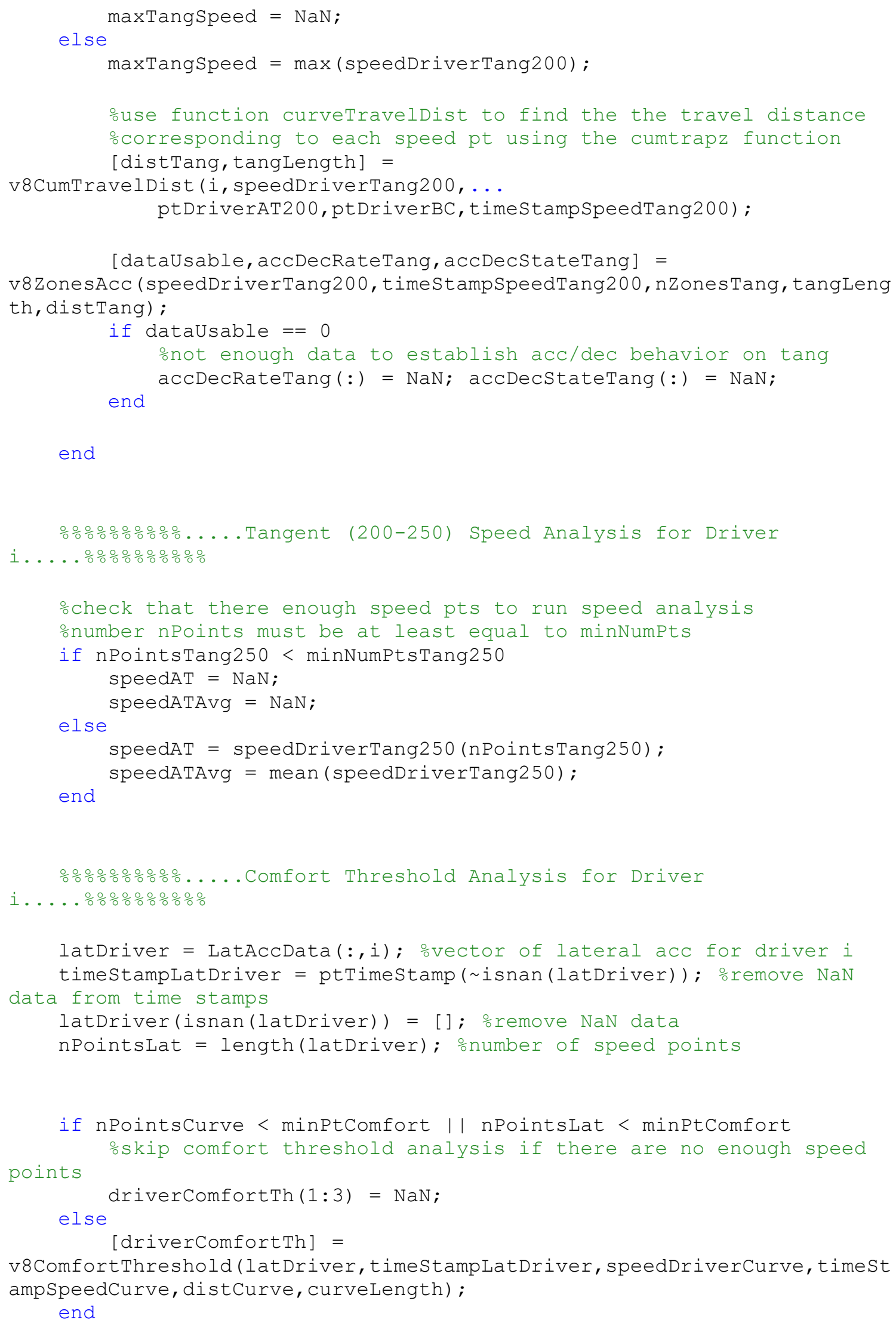




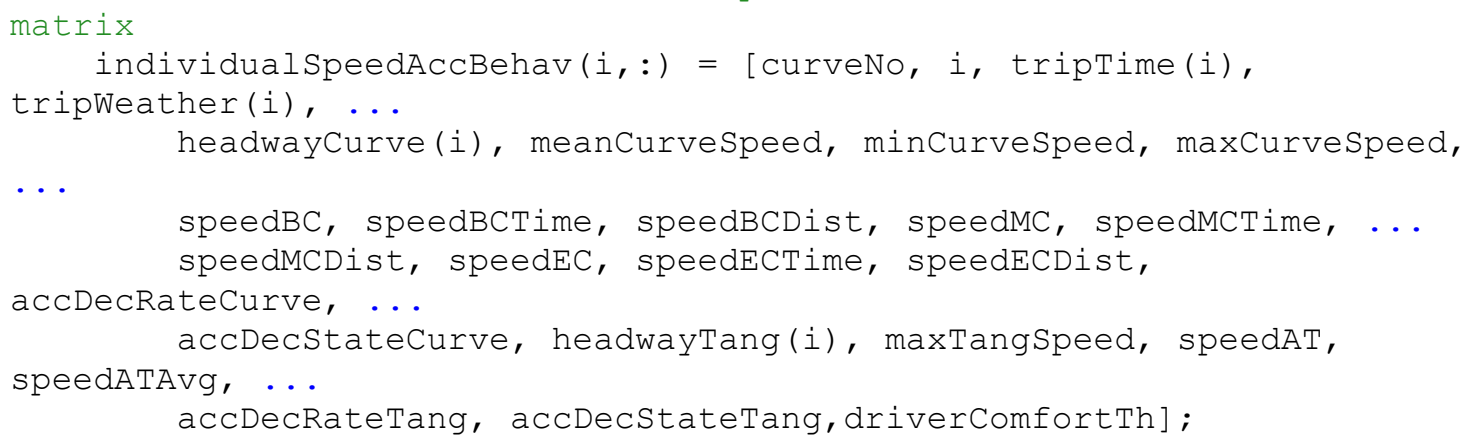

end

oremove from the main matrix rows of drivers with no data

individualSpeedAccBehav ( (individualSpeedAcCBehav $(:, 2)==0$ ), : ) = [ ] ; 
v8DataInputs.m

function v8DataInputs (sheetNameSpeedCurve, sheetNamespeedTang200,... sheetNameSpeedTang250, sheetNameLat, fRow, fCol, dataRead)

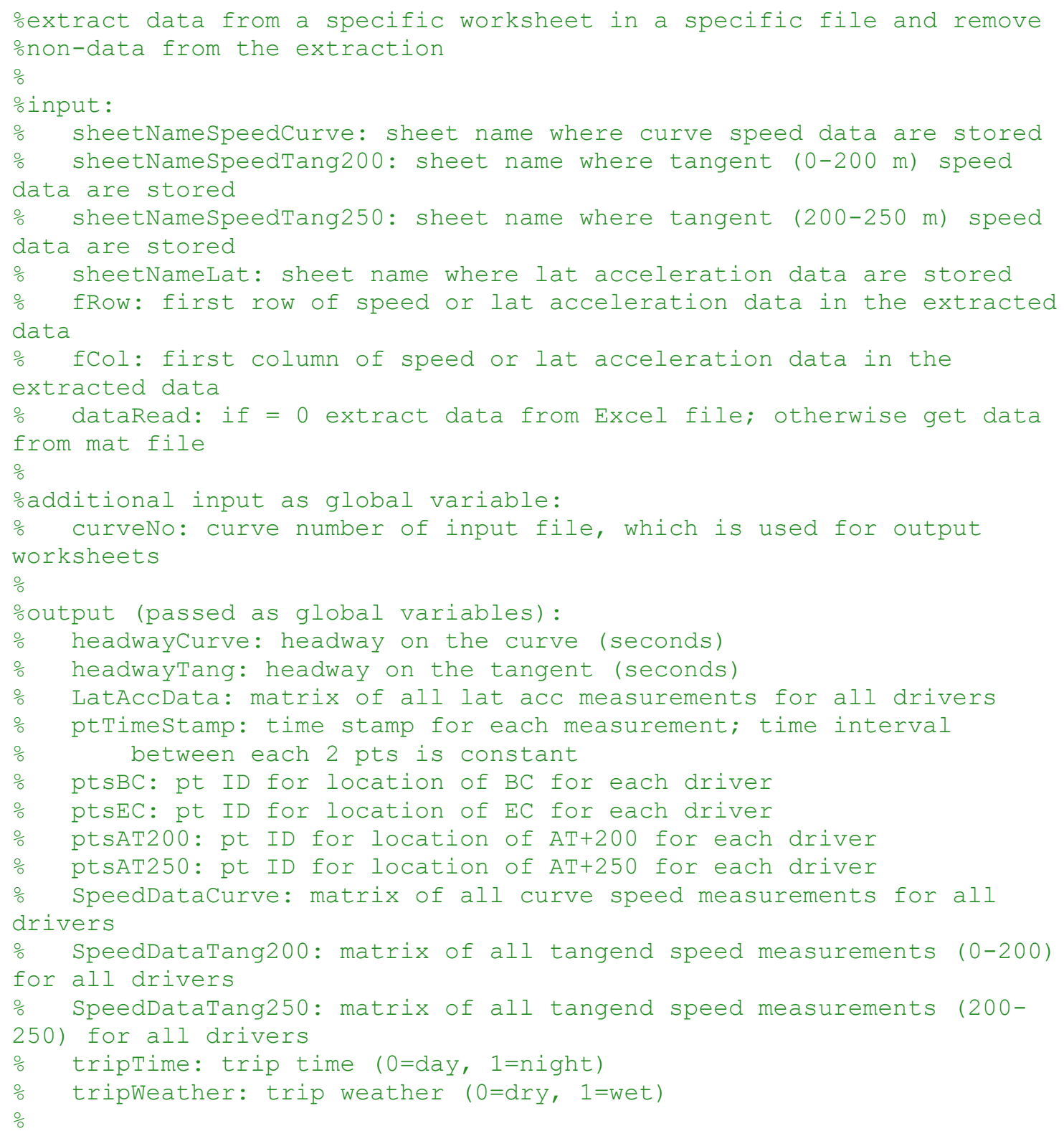

global curveNo nDrivers headwayCurve headwayTang ptTimestamp ... SpeedDataCurve SpeedDataTang200 speedDataTang250 LatAccData... ptsBC ptsEC ptsAT200 ptsAT250 triptime tripWeather ...

matFileName = ['CurveTangent', num2str(curveNo), '.mat'];

if dataRead $==0$ 


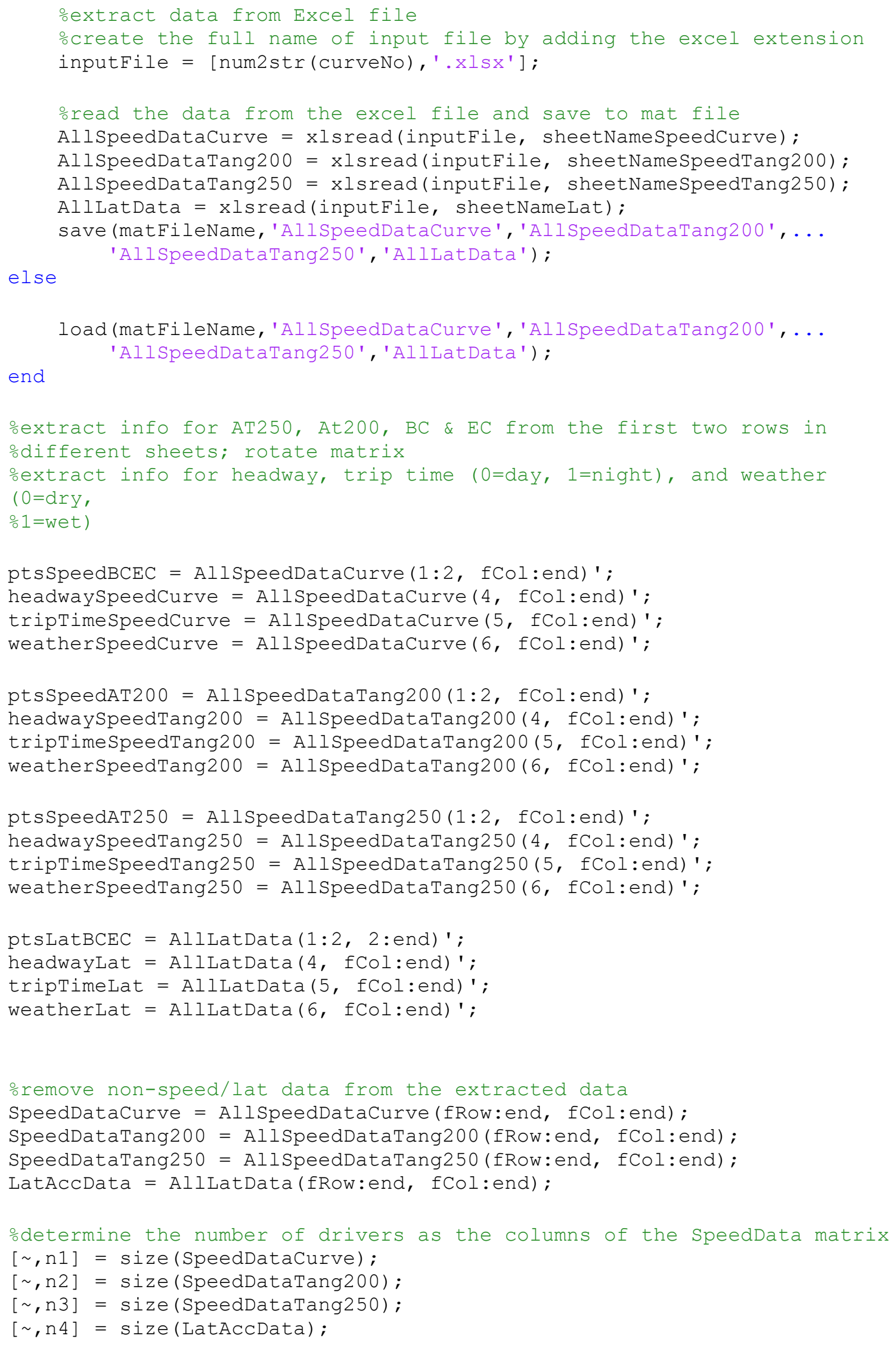




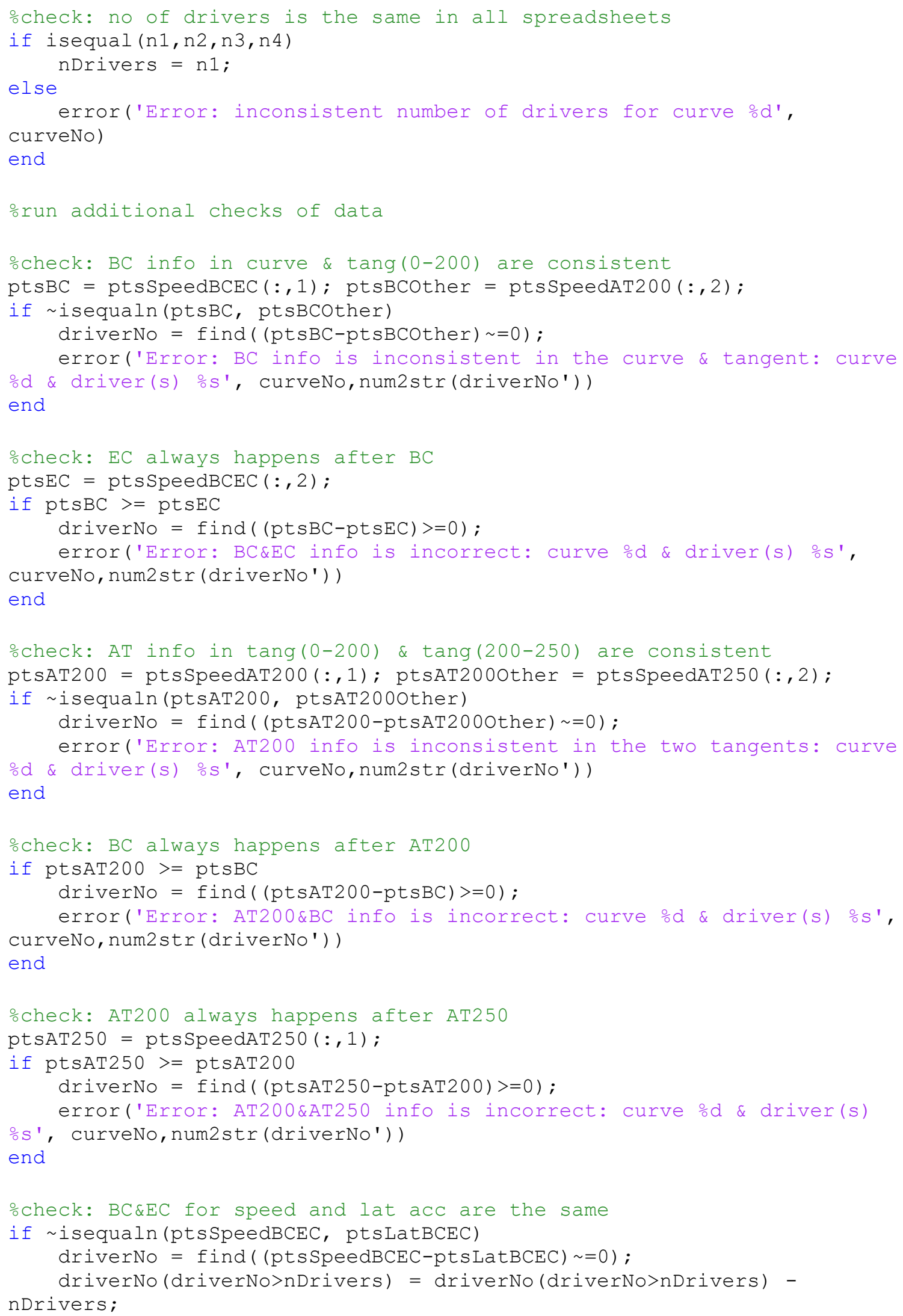


error('Error: BC/EC info is inconsistent in speed \& lat acc data: curve d \& driver(s) \%s', curveNo, num2str(driverNo'))

end

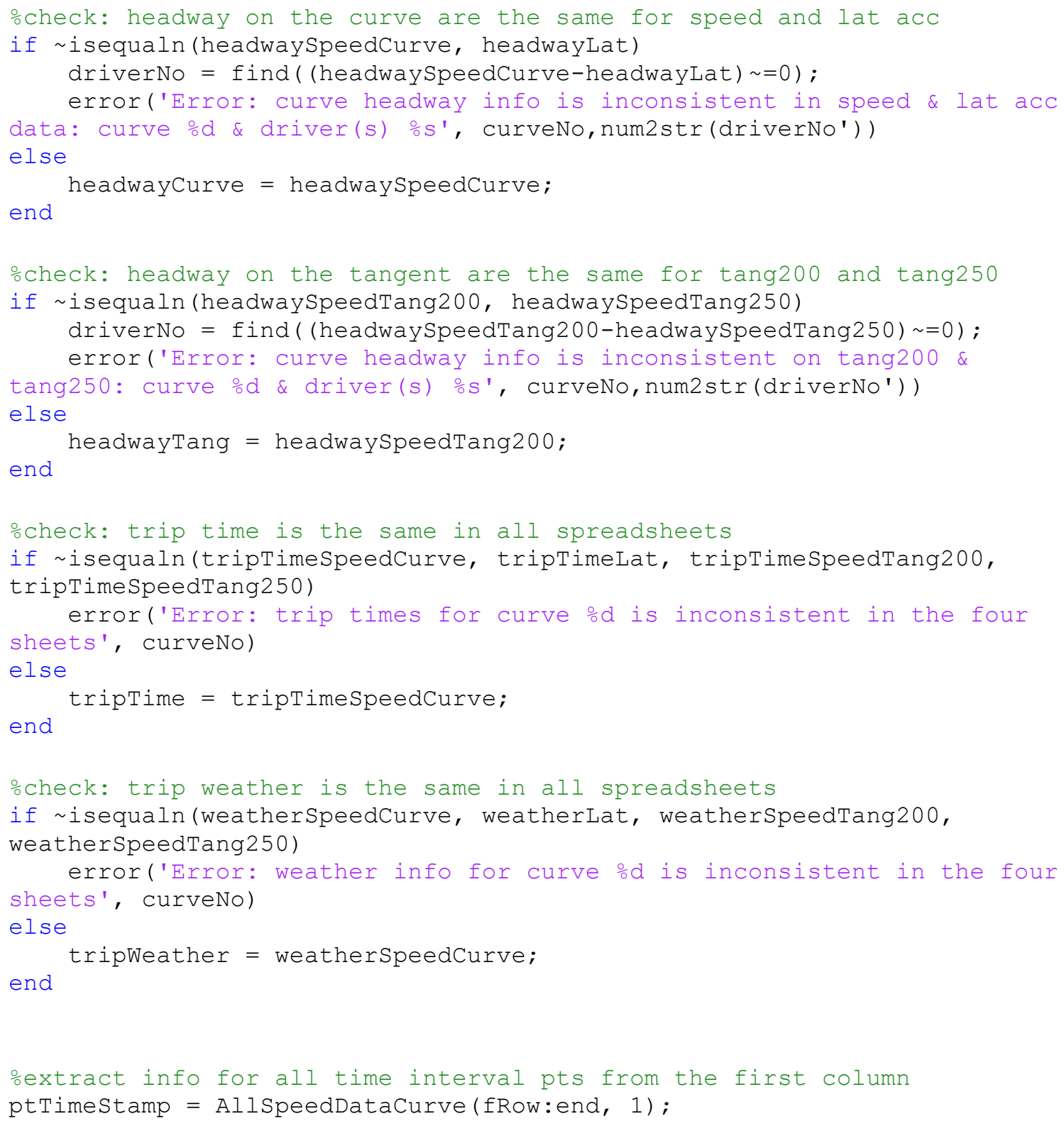




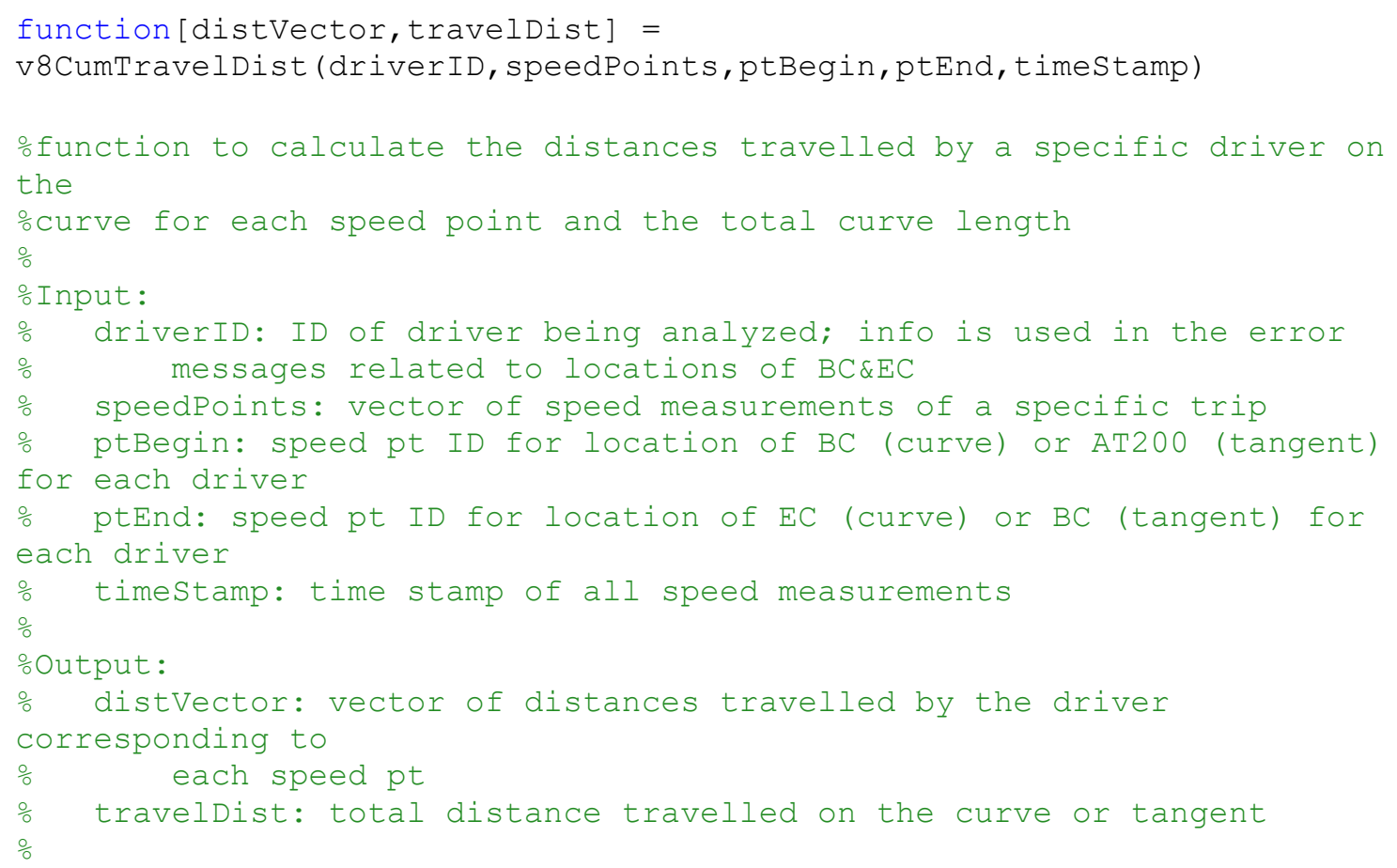




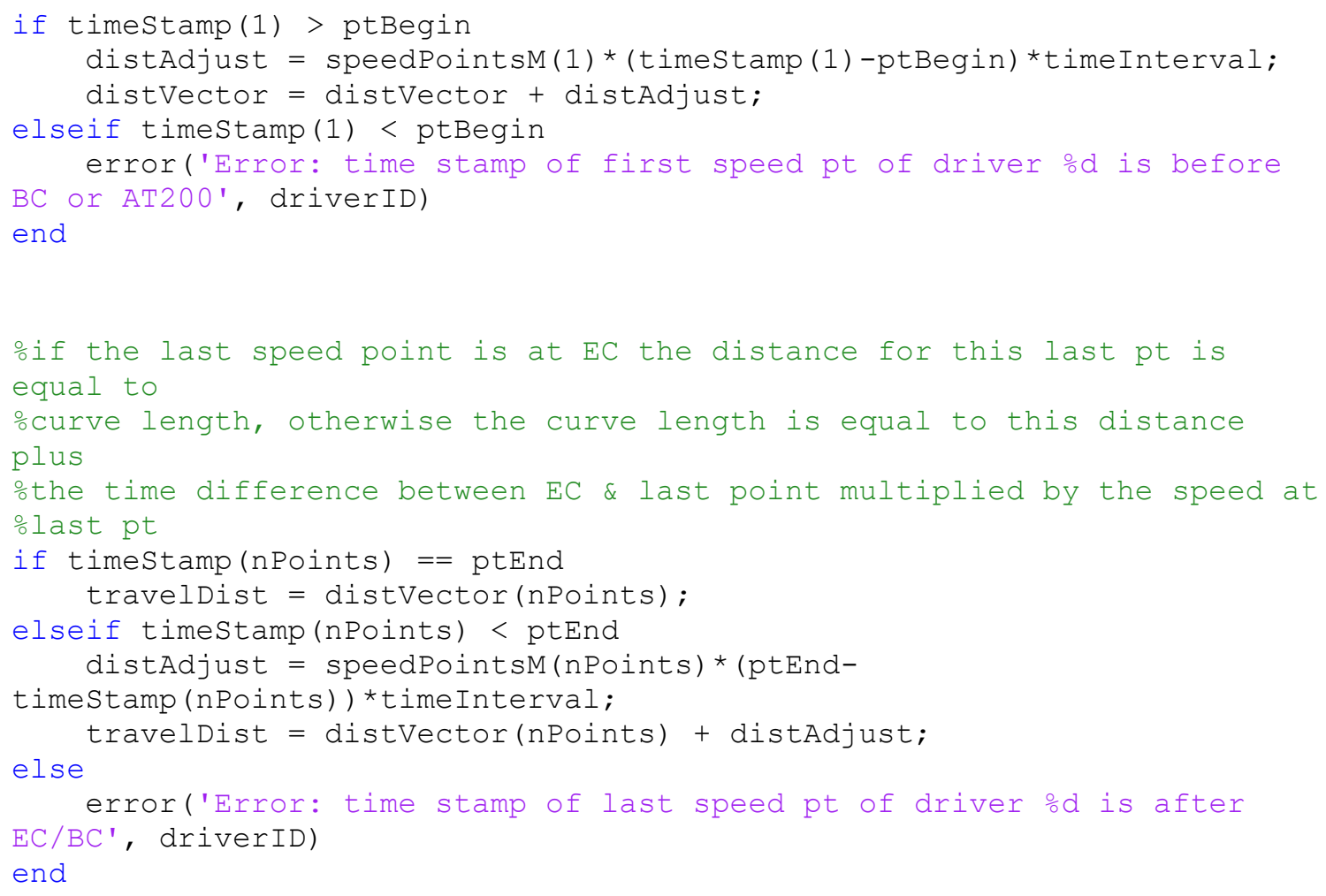




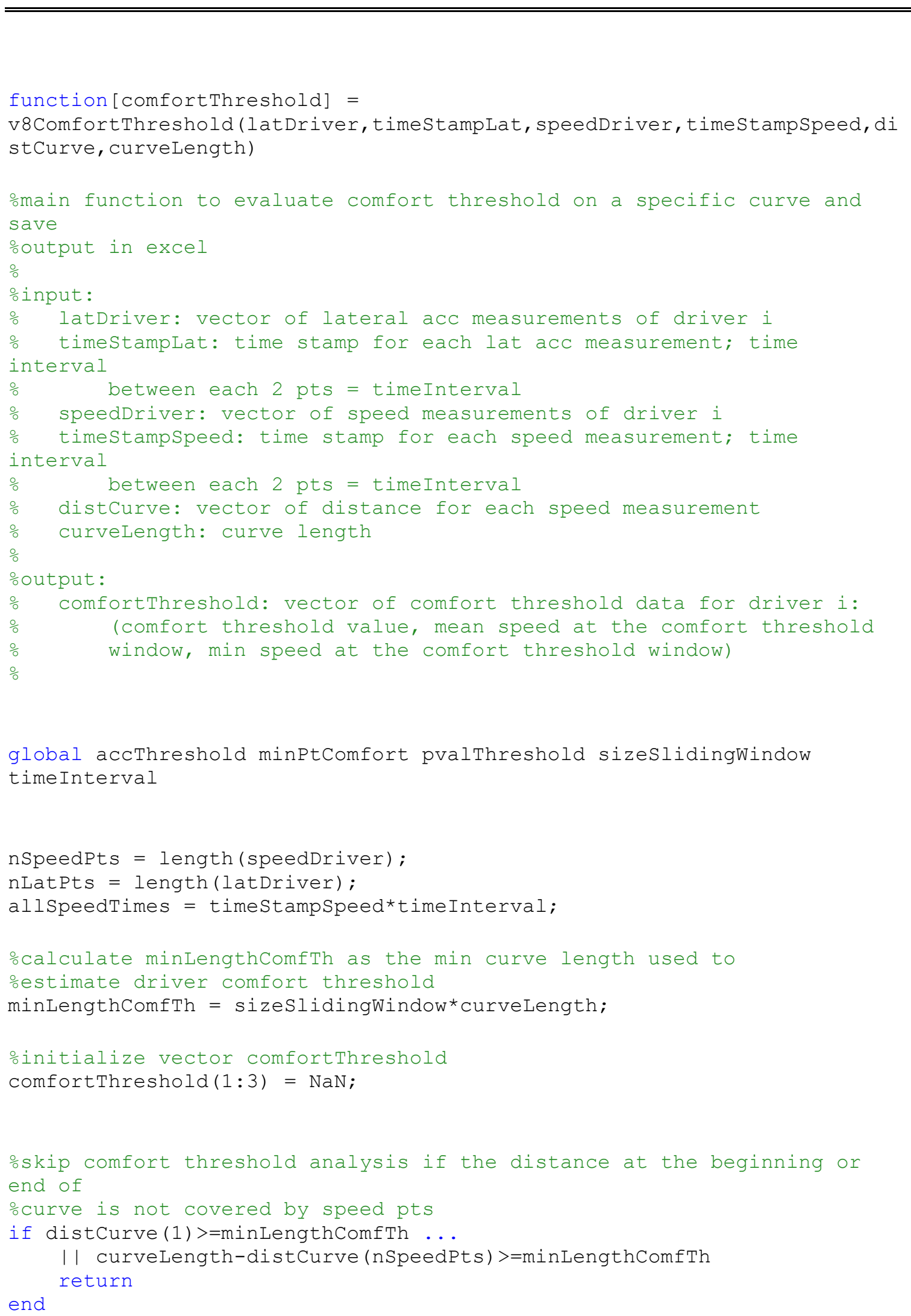


oinitialize variables related to moving segment to estimate comfort threshold

op1: first speed pt in the moving segment; initial value =1

opn: last pt in the moving segment; does not need to be initialized odist1: distance from BC to pl; does not need to be initialized odist2: distance from BC to pn; initial value = minLengthComfTh

$\mathrm{p} 1=1 ;$

dist2 = minLengthComfTh;

while dist2 <= distCurve(nSpeedPts)

oif speed pts happen before the first lat acc pt, skip the ofirst iterations and find the first speed pt that has the same otime stamp as the first lat acc pt or just after it

if timestampspeed (p1) < timeStampLat (1)

timeStampDiff0 = timeStampspeed - timestampLat(1);

$\mathrm{p} 1=$

find ( timestampDiff0==min (timeStampDiff0 (timeStampDiff0>=0)) ) ;

end

ofind diff in distance between each speed pt and dist2 distDiff $=$ distCurve - dist2;

opts before dist2 will have -ve distDiff; therefore the first opossible pn is the pt with 0 or smallest +ve distDiff value obut pn must be a specific number of pts from p1

oso, find pn as the max of (p1+ min no of pts -1) and the pt owith zero or smallest +ve distDiff value $\mathrm{pn}=\max (\mathrm{p} 1+\operatorname{minPtComfort}-$

1, find (distDiff==min (distDiff (distDiff $>=0)))$ );

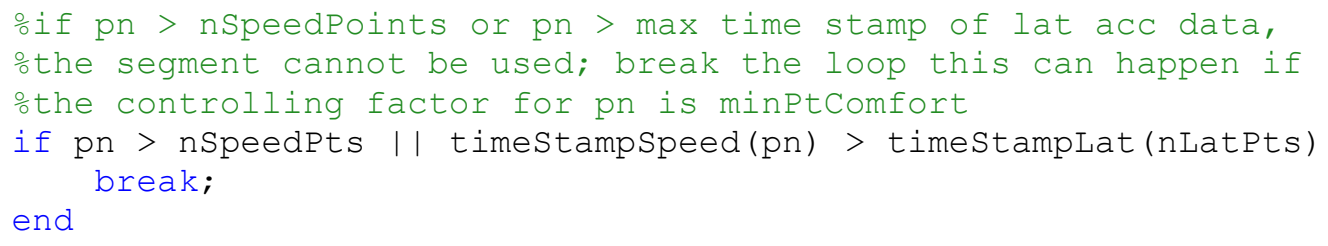


oplL is the pt with zero time stamp diff or smallest -ve value

opnL is the pt with zero time stamp diff or smallest +ve value timeStampDiffl = timeStampLat - timeStampSpeed (p1);

$\mathrm{p} 1 \mathrm{~L}=$

find (timeStampDiffl==max (timeStampDiffl (timeStampDiff1<=0)) );

timeStampDiff2 = timeStampLat - timeStampSpeed (pn);

pnL

find (timeStampDiff2==min (timeStampDiff2(timeStampDiff2>=0) )) ;

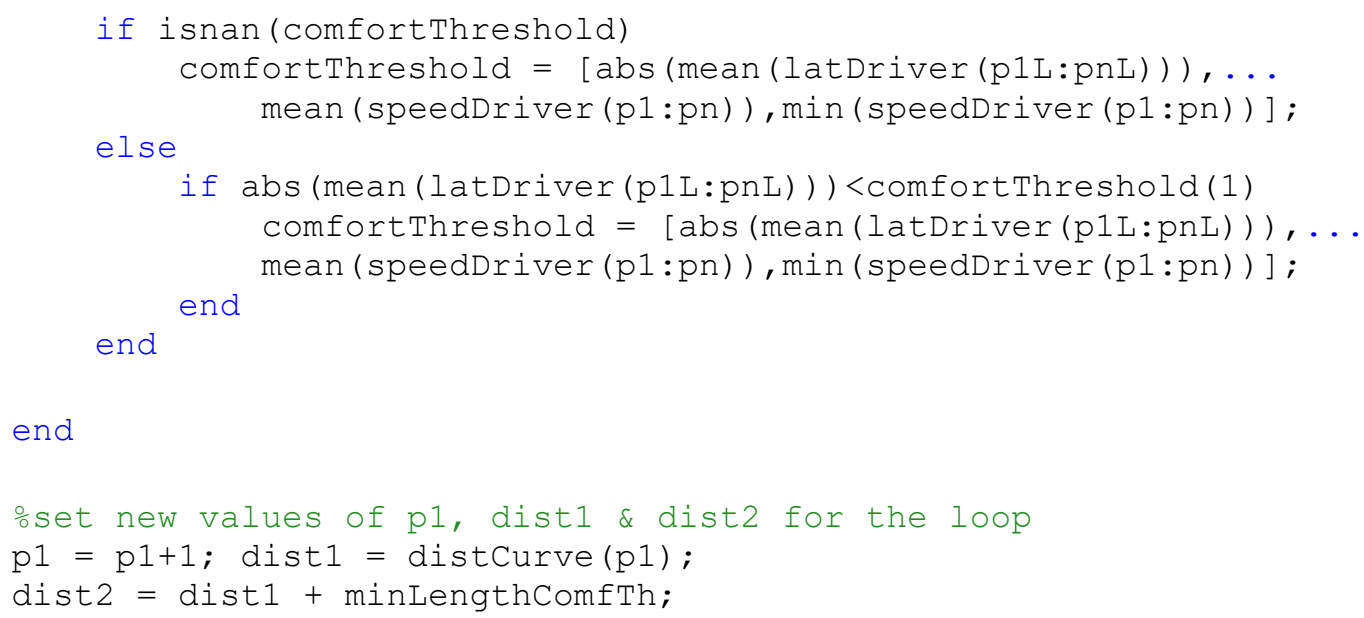




\section{APPENDIX F}

Matlab Code for Reliability Analysis (FORM) 


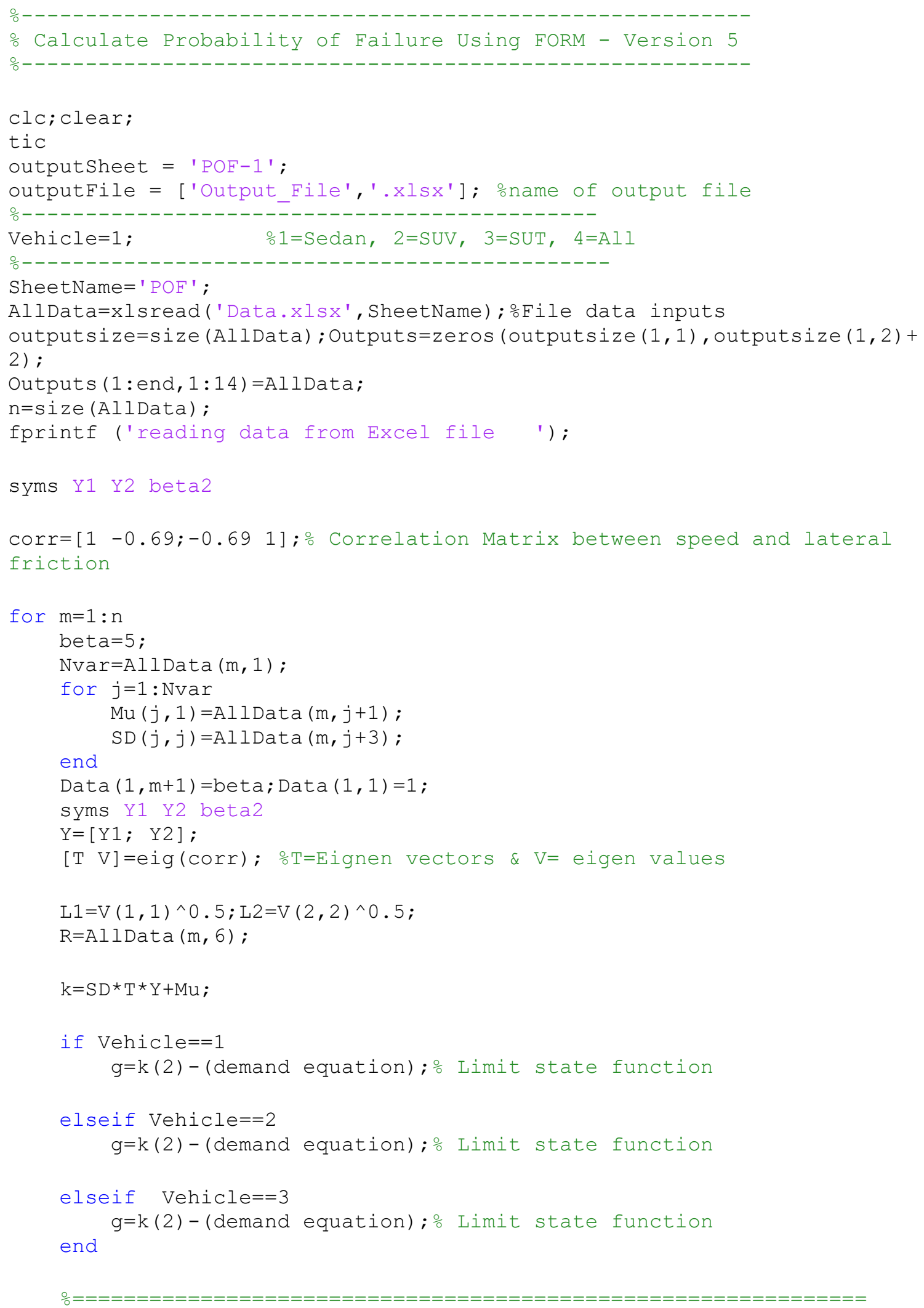




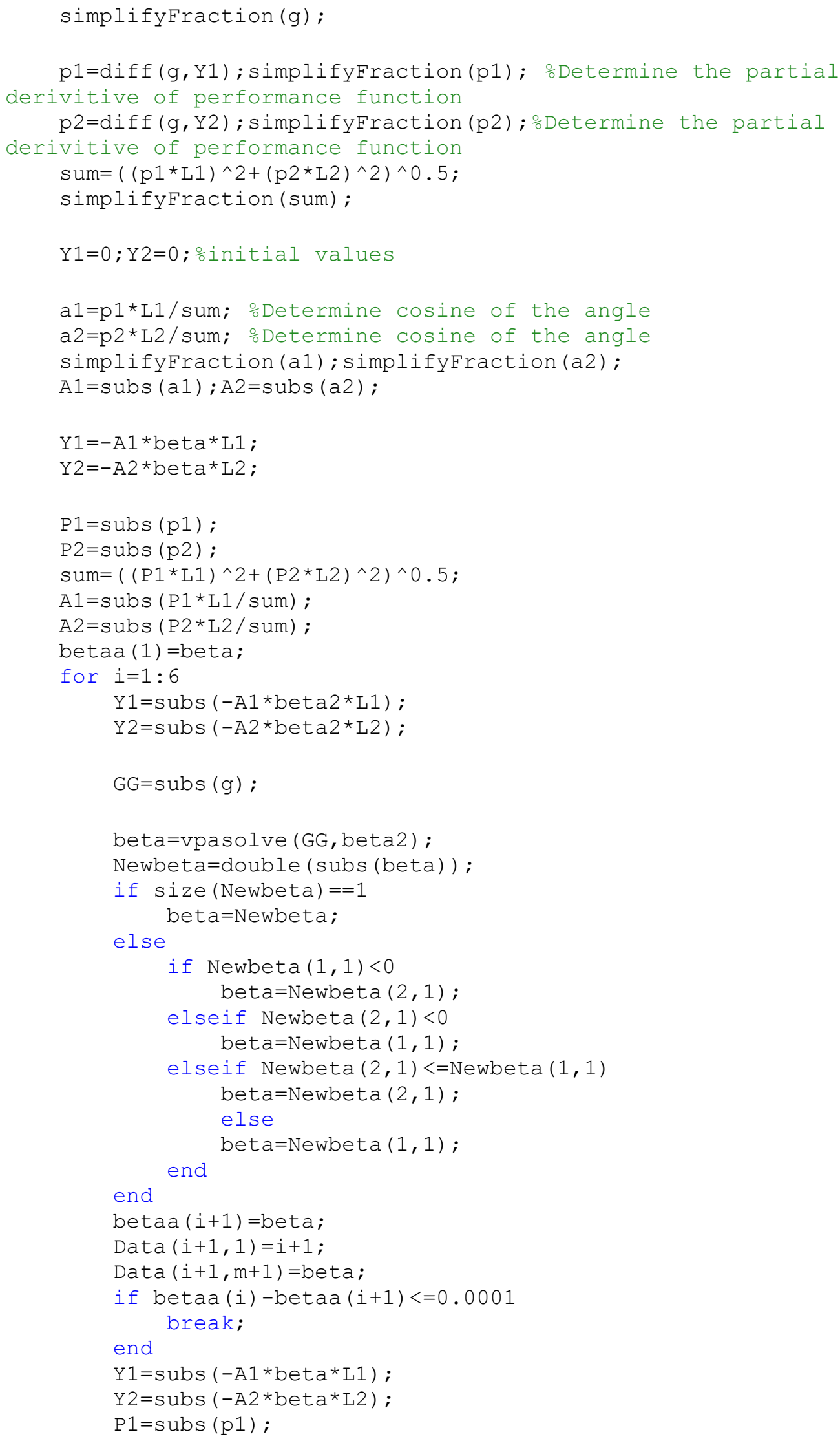




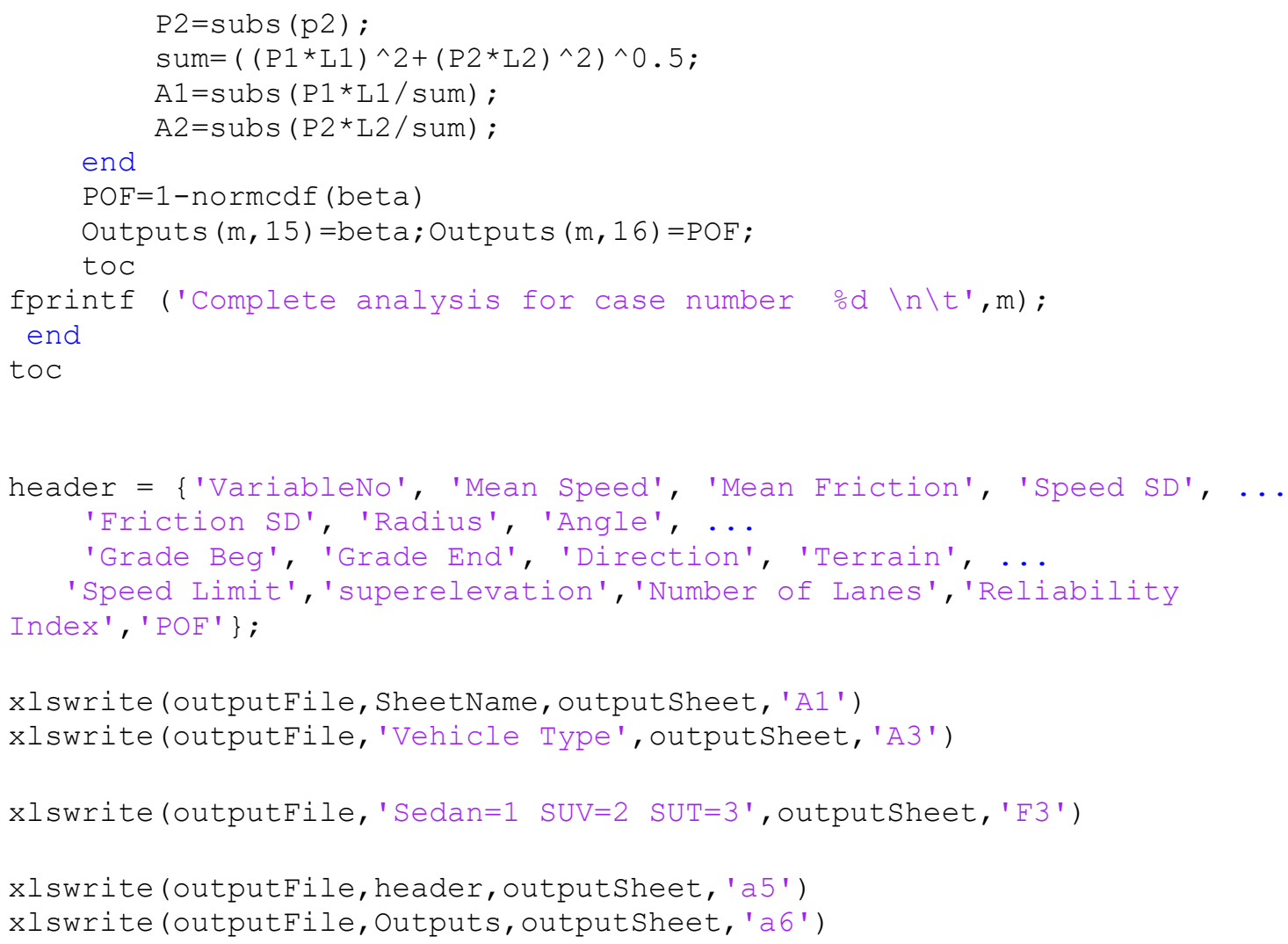




\section{$\underline{\text { APPENDIX G }}$}

Safety Performance Functions for Specific Radius and Curves' Length 
Table G-1: SPFs for Annual Collision Frequency (Base Models).

\begin{tabular}{|c|c|c|c|c|c|c|c|}
\hline $\begin{array}{c}\text { Regression } \\
\text { Model }\end{array}$ & Variable & Coef. & $S E E$ & $\begin{array}{c}p \text { - } \\
\text { value }\end{array}$ & $\begin{array}{l}\text { Likelihood- } \\
\text { ratio test }\end{array}$ & AIC & $\mathrm{BIC}$ \\
\hline \multirow{3}{*}{ Poisson1-1a } & $\begin{array}{l}\text { Log of } \\
\text { (AADT) }\end{array}$ & 0.807 & 0.069 & $<0.001$ & \multirow{3}{*}{--} & \multirow{3}{*}{1709.07} & \multirow{3}{*}{1723.58} \\
\hline & Log of $(L)$ & 0.804 & 0.096 & $<0.001$ & & & \\
\hline & Constant & -11.870 & 0.873 & $<0.001$ & & & \\
\hline \multirow{3}{*}{ NB1-1a } & $\begin{array}{l}\begin{array}{c}\text { Log of } \\
\text { (AADT) }\end{array}\end{array}$ & 0.832 & 0.081 & $<0.001$ & \multirow{3}{*}{$\begin{array}{l}30.22 \\
(p \text {-value } \\
<0.001)\end{array}$} & \multirow{3}{*}{1680.85} & \multirow{3}{*}{1700.19} \\
\hline & $\log$ of $(L)$ & 0.832 & 0.113 & $<0.001$ & & & \\
\hline & Constant & -12.241 & 1.007 & $<0.001$ & & & \\
\hline \multirow{4}{*}{ Poisson1-2a } & $\begin{array}{l}\text { Log of } \\
\text { (AADT) }\end{array}$ & 0.713 & 0.072 & $<0.001$ & \multirow{4}{*}{--} & \multirow{4}{*}{1693.86} & \multirow{4}{*}{1713.20} \\
\hline & Log of $(L)$ & 0.909 & 0.101 & $<0.001$ & & & \\
\hline & $R$ & -0.001 & $<0.001$ & $<0.001$ & & & \\
\hline & Constant & -11.200 & 0.869 & $<0.001$ & & & \\
\hline \multirow{4}{*}{ NB1-2a } & $\begin{array}{c}\text { Log of } \\
\text { (AADT) }\end{array}$ & 0.742 & 0.083 & $<0.001$ & \multirow{4}{*}{$\begin{array}{c}26.02 \\
(p \text {-value } \\
<0.001)\end{array}$} & \multirow{4}{*}{1669.84} & \multirow{4}{*}{1694.02} \\
\hline & $\log$ of $(L)$ & 0.940 & 0.117 & $<0.001$ & & & \\
\hline & $R$ & -0.001 & 0.000 & $<0.001$ & & & \\
\hline & Constant & -11.629 & 0.998 & $<0.001$ & & & \\
\hline
\end{tabular}

Coef. = coefficient, $S E E=$ standard error of estimate, AADT= average annual daily traffic (veh/d), $L=$ length of curve (m), and $R=$ Radius (m). 
Table G-2: Annual Collision Frequency Models (Considering AADT and $L$ ).

\begin{tabular}{|c|c|c|c|c|c|c|c|}
\hline $\begin{array}{c}\text { Regression } \\
\text { Model }\end{array}$ & Variable & Coef. & SEE & $\begin{array}{c}p- \\
\text { value }\end{array}$ & $\begin{array}{l}\text { Likelihood- } \\
\text { ratio test }\end{array}$ & AIC & $\mathrm{BIC}$ \\
\hline \multirow{4}{*}{ NB1-3a } & Log of (AADT) & 0.796 & 0.080 & $<0.001$ & \multirow{4}{*}{$\begin{array}{c}26.92 \\
(p \text {-value } \\
<0.001)\end{array}$} & \multirow{4}{*}{1675.46} & \multirow{4}{*}{1699.64} \\
\hline & $\log$ of $(L)$ & 0.889 & 0.114 & $<0.001$ & & & \\
\hline & $\beta_{V S}$ & -0.172 & 0.062 & 0.005 & & & \\
\hline & Constant & -11.466 & 1.013 & $<0.001$ & & & \\
\hline \multirow{4}{*}{ NB1-4a } & Log of (AADT) & 0.830 & 0.081 & $<0.001$ & \multirow{4}{*}{$\begin{array}{c}30.08 \\
(p \text {-value } \\
<0.001)\end{array}$} & \multirow{4}{*}{1682.73} & \multirow{4}{*}{1706.91} \\
\hline & $\log$ of $(L)$ & 0.824 & 0.115 & $<0.001$ & & & \\
\hline & $\beta_{c m f}$ & 0.014 & 0.039 & 0.731 & & & \\
\hline & Constant & -12.198 & 1.015 & $<0.001$ & & & \\
\hline \multirow{4}{*}{ NB1-5a } & Log of (AADT) & 0.797 & 0.081 & $<0.001$ & \multirow{4}{*}{$\begin{array}{c}26.56 \\
(p \text {-value } \\
<0.001)\end{array}$} & \multirow{4}{*}{1675.44} & \multirow{4}{*}{1699.61} \\
\hline & $\log$ of $(L)$ & 0.877 & 0.113 & $<0.001$ & & & \\
\hline & $\beta_{S D}$ & -0.076 & 0.028 & 0.006 & & & \\
\hline & Constant & -11.726 & 1.011 & $<0.001$ & & & \\
\hline \multirow{4}{*}{ NB1-6a } & Log of (AADT) & 0.810 & 0.080 & $<0.001$ & \multirow{4}{*}{$\begin{array}{c}27.87 \\
(p \text {-value } \\
<0.001)\end{array}$} & \multirow{4}{*}{1677.81} & \multirow{4}{*}{1701.99} \\
\hline & $\log$ of $(L)$ & 0.881 & 0.115 & $<0.001$ & & & \\
\hline & $\beta_{\text {Roll }}$ & -0.142 & 0.062 & 0.022 & & & \\
\hline & Constant & -11.235 & 1.073 & $<0.001$ & & & \\
\hline
\end{tabular}


Table G-3: Annual Collision Frequency Models (Considering AADT, L, and R).

\begin{tabular}{|c|c|c|c|c|c|c|c|}
\hline $\begin{array}{c}\text { Regression } \\
\text { Model }\end{array}$ & Variable & Coef. & $S E E$ & $\begin{array}{c}p- \\
\text { value }\end{array}$ & $\begin{array}{l}\text { Likelihood- } \\
\text { ratio test }\end{array}$ & AIC & $\mathrm{BIC}$ \\
\hline \multirow{5}{*}{$\mathrm{NB} 1-7 \mathrm{a}$} & Log of (AADT) & 0.744 & 0.082 & $<0.001$ & \multirow{5}{*}{$\begin{array}{c}25.58 \\
(p \text {-value } \\
<0.001)\end{array}$} & \multirow{5}{*}{1671.25} & \multirow{5}{*}{1700.27} \\
\hline & Log of $(L)$ & 0.944 & 0.117 & $<0.001$ & & & \\
\hline & $R$ & -0.001 & 0.000 & 0.014 & & & \\
\hline & $\beta_{V S}$ & -0.059 & 0.077 & 0.442 & & & \\
\hline & Constant & -11.468 & 1.012 & $<0.001$ & & & \\
\hline \multirow{5}{*}{ NB1-8a } & Log of (AADT) & 0.729 & 0.084 & $<0.001$ & \multirow{5}{*}{$\begin{array}{c}25.82 \\
(p \text {-value } \\
<0.001)\end{array}$} & \multirow{5}{*}{1670.82} & \multirow{5}{*}{1699.84} \\
\hline & Log of $(L)$ & 0.922 & 0.119 & $<0.001$ & & & \\
\hline & $R$ & -0.001 & 0.000 & $<0.001$ & & & \\
\hline & $\beta_{c m f}$ & 0.041 & 0.041 & 0.313 & & & \\
\hline & Constant & -11.467 & 1.011 & $<0.001$ & & & \\
\hline \multirow{5}{*}{ NB1-9a } & Log of (AADT) & 0.740 & 0.083 & $<0.001$ & \multirow{5}{*}{$\begin{array}{c}24.90 \\
(p \text {-value } \\
<0.001)\end{array}$} & \multirow{5}{*}{1670.37} & \multirow{5}{*}{1699.38} \\
\hline & Log of $(L)$ & 0.943 & 0.117 & $<0.001$ & & & \\
\hline & $R$ & -0.001 & 0.000 & 0.009 & & & \\
\hline & $\beta_{S D}$ & -0.038 & 0.031 & 0.224 & & & \\
\hline & Constant & -11.478 & 1.003 & $<0.001$ & & & \\
\hline \multirow{5}{*}{ NB1-10a } & Log of (AADT) & 0.744 & 0.083 & $<0.001$ & \multirow{5}{*}{$\begin{array}{l}1671.80 \\
(p \text {-value } \\
<0.001)\end{array}$} & \multirow{5}{*}{1700.81} & \multirow{5}{*}{1944.16} \\
\hline & $\log$ of $(L)$ & 0.941 & 0.117 & $<0.001$ & & & \\
\hline & $R$ & -0.001 & 0.000 & 0.006 & & & \\
\hline & $\beta_{\text {Roll }}$ & -0.016 & 0.076 & 0.837 & & & \\
\hline & Constant & -11.546 & 1.076 & $<0.001$ & & & \\
\hline
\end{tabular}


Table G-4: SPFs for Annual Collision Frequency Considering All Reliability Indices.

\begin{tabular}{|c|c|c|c|c|c|c|c|}
\hline $\begin{array}{l}\text { Regression } \\
\text { Model }\end{array}$ & Variable & Coef. & SEE & $\begin{array}{c}p- \\
\text { value }\end{array}$ & $\begin{array}{l}\text { Likelihood- } \\
\text { ratio test }\end{array}$ & AIC & $\mathrm{BIC}$ \\
\hline \multirow{7}{*}{ NB1-11a } & Log of (AADT) & 0.772 & 0.081 & $<0.001$ & \multirow{7}{*}{$\begin{array}{c}24.63 \\
(p \text {-value } \\
<0.001)\end{array}$} & \multirow{7}{*}{1677.71} & \multirow{7}{*}{1716.39} \\
\hline & Log of $(L)$ & 0.883 & 0.117 & $<0.001$ & & & \\
\hline & $\beta_{V S}$ & -0.136 & 0.099 & 0.172 & & & \\
\hline & $\beta_{c m f}$ & 0.036 & 0.041 & $\mathbf{0 . 3 7 7}$ & & & \\
\hline & $\beta_{S D}$ & -0.055 & 0.031 & 0.076 & & & \\
\hline & $\beta_{\text {Roll }}$ & 0.012 & 0.097 & 0.902 & & & \\
\hline & Constant & -11.216 & 1.068 & $<0.001$ & & & \\
\hline \multirow{8}{*}{ NB1-12a } & Log of (AADT) & 0.719 & 0.084 & $<0.001$ & \multirow{8}{*}{$\begin{array}{c}24.63 \\
(p \text {-value } \\
<0.001)\end{array}$} & \multirow{8}{*}{1674.37} & \multirow{8}{*}{1717.89} \\
\hline & $\log$ of $(L)$ & 0.919 & 0.118 & $<0.001$ & & & \\
\hline & $R$ & -0.001 & 0.000 & 0.023 & & & \\
\hline & $\beta_{V S}$ & -0.092 & 0.100 & 0.357 & & & \\
\hline & $\beta_{c m f}$ & 0.053 & 0.042 & $\mathbf{0 . 2 0 7}$ & & & \\
\hline & $\beta_{S D}$ & -0.036 & 0.032 & 0.251 & & & \\
\hline & $\beta_{\text {Roll }}$ & 0.076 & 0.101 & 0.450 & & & \\
\hline & Constant & -11.419 & 1.076 & $<0.001$ & & & \\
\hline
\end{tabular}


Table G-5: ZIP and ZINB Models to Predict Annual Collision.

\begin{tabular}{|c|c|c|c|c|c|c|c|}
\hline Model & Variable & Coef. & $S E E$ & $\begin{array}{c}p- \\
\text { value }\end{array}$ & $\begin{array}{c}\text { Vuong } \\
\text { test }\end{array}$ & AIC & $\mathrm{BIC}$ \\
\hline \multirow{7}{*}{ ZIP1-1a } & $\log (A A D T)$ & 0.685 & 0.130 & $<0.001$ & \multirow{7}{*}{$\begin{array}{c}2.04 \\
(p- \\
\text { value } \\
=0.020)\end{array}$} & \multirow{7}{*}{1692.73} & \multirow{7}{*}{1721.74} \\
\hline & $\log (L)$ & 0.389 & 0.143 & 0.007 & & & \\
\hline & Constant & -8.211 & 1.568 & $<0.001$ & & & \\
\hline & \multicolumn{4}{|l|}{ Inflate } & & & \\
\hline & $\log (A A D T)$ & -0.423 & 0.359 & 0.238 & & & \\
\hline & $\log (L)$ & -1.752 & 0.558 & 0.002 & & & \\
\hline & Constant & 12.088 & 3.811 & 0.002 & & & \\
\hline \multirow{8}{*}{ ZIP1-2a } & $\log (A A D T)$ & 0.490 & 0.133 & $<0.001$ & \multirow{8}{*}{$\begin{array}{c}2.06 \\
(p- \\
\text { value } \\
=0.019)\end{array}$} & \multirow{8}{*}{1680.05} & \multirow{8}{*}{1713.90} \\
\hline & $\log (L)$ & 0.519 & 0.150 & $<0.001$ & & & \\
\hline & $R$ & -0.001 & $<0.001$ & $<0.001$ & & & \\
\hline & Constant & -6.812 & 1.499 & $<0.001$ & & & \\
\hline & \multicolumn{4}{|l|}{ Inflate } & & & \\
\hline & $\log (A A D T)$ & -0.777 & 0.383 & 0.043 & & & \\
\hline & $\log (L)$ & -1.617 & 0.545 & 0.003 & & & \\
\hline & Constant & 14.296 & 3.727 & 0.000 & & & \\
\hline \multirow{7}{*}{ ZINB1-1a } & $\log (A A D T)$ & 0.673 & 0.117 & $<0.001$ & \multirow{7}{*}{$\begin{array}{c}0.99 \\
(p- \\
\text { value } \\
=0.162)\end{array}$} & \multirow{7}{*}{1682.61} & \multirow{7}{*}{1716.46} \\
\hline & $\log (L)$ & 0.712 & 0.136 & $<0.001$ & & & \\
\hline & Constant & -10.156 & 1.503 & $<0.001$ & & & \\
\hline & \multicolumn{4}{|l|}{ Inflate } & & & \\
\hline & $\log (A A D T)$ & -2.693 & 1.382 & 0.051 & & & \\
\hline & $\log (L)$ & -1.346 & 1.922 & 0.484 & & & \\
\hline & Constant & 25.930 & 10.621 & 0.015 & & & \\
\hline \multirow{8}{*}{ ZINB1-2a } & $\log (A A D T)$ & 0.541 & 0.118 & $<0.001$ & \multirow{8}{*}{$\begin{array}{c}\begin{array}{c}1.21 \\
(p- \\
\text { value }\end{array} \\
=0.114)\end{array}$} & \multirow{8}{*}{1669.34} & \multirow{8}{*}{1708.02} \\
\hline & $\log (L)$ & 0.808 & 0.136 & $<0.001$ & & & \\
\hline & $R$ & -0.001 & $<0.001$ & $<0.001$ & & & \\
\hline & Constant & -9.075 & 1.477 & $<0.001$ & & & \\
\hline & \multicolumn{4}{|l|}{ Inflate } & & & \\
\hline & $\log (A A D T)$ & -2.894 & 1.298 & 0.026 & & & \\
\hline & $\log (L)$ & -1.335 & 1.457 & 0.360 & & & \\
\hline & Constant & 27.564 & 9.593 & 0.004 & & & \\
\hline
\end{tabular}


Table G-6: ZIP Models to Predict Annual Collision Frequency.

\begin{tabular}{|c|c|c|c|c|c|c|c|}
\hline Model & Variable & Coef. & $S E E$ & $p$-value & Vuong test & AIC & $\mathrm{BIC}$ \\
\hline \multirow{8}{*}{ ZIP1-3a } & $\log (A A D T)$ & 0.546 & 0.126 & $<0.001$ & \multirow{8}{*}{$\begin{aligned} & 2.19 \\
(p \text {-value } & \\
= & 0.014)\end{aligned}$} & \multirow{8}{*}{1681.78} & \multirow{8}{*}{1715.62} \\
\hline & $\log (L)$ & 0.414 & 0.142 & 0.003 & & & \\
\hline & $\beta_{V S}$ & -0.224 & 0.062 & $<0.001$ & & & \\
\hline & Constant & -6.135 & 1.543 & $<0.001$ & & & \\
\hline & \multicolumn{4}{|l|}{ Inflate } & & & \\
\hline & $\log (A A D T)$ & -0.710 & 0.327 & 0.030 & & & \\
\hline & $\log (L)$ & -1.804 & 0.536 & 0.001 & & & \\
\hline & Constant & 14.838 & 3.573 & $<0.001$ & & & \\
\hline \multirow{8}{*}{ ZIP1-4a } & $\log (A A D T)$ & 0.687 & 0.130 & $<0.001$ & \multirow{8}{*}{$\begin{array}{c}1.97 \\
(p \text {-value } \\
=0.024)\end{array}$} & \multirow{8}{*}{1694.63} & \multirow{8}{*}{1728.48} \\
\hline & $\log (L)$ & 0.383 & 0.144 & 0.008 & & & \\
\hline & $\beta_{c m f}$ & 0.011 & 0.037 & 0.757 & & & \\
\hline & Constant & -8.225 & 1.571 & $<0.001$ & & & \\
\hline & \multicolumn{4}{|l|}{ Inflate } & & & \\
\hline & $\log (A A D T)$ & -0.408 & 0.365 & 0.263 & & & \\
\hline & $\log (L)$ & -1.769 & 0.562 & 0.002 & & & \\
\hline & Constant & 12.044 & 3.825 & 0.002 & & & \\
\hline \multirow{8}{*}{ ZIP1-5a } & $\log (A A D T)$ & 0.625 & 0.127 & $<0.001$ & \multirow{8}{*}{$\begin{aligned} & 2.05 \\
&(p \text {-value } \\
&=0.020)\end{aligned}$} & \multirow{8}{*}{1684.02} & \multirow{8}{*}{1717.86} \\
\hline & $\log (L)$ & 0.433 & 0.142 & 0.001 & & & \\
\hline & $\beta_{S D}$ & -0.088 & 0.027 & 0.002 & & & \\
\hline & Constant & -7.420 & 1.545 & $<0.001$ & & & \\
\hline & \multicolumn{4}{|l|}{ Inflate } & & & \\
\hline & $\log (A A D T)$ & -0.505 & 0.343 & 0.141 & & & \\
\hline & $\log (L)$ & -1.790 & 0.556 & 0.001 & & & \\
\hline & Constant & 12.962 & 3.742 & 0.001 & & & \\
\hline \multirow{8}{*}{ ZIP1-6a } & $\log (A A D T)$ & 0.616 & 0.129 & $<0.001$ & \multirow{8}{*}{$\begin{array}{c}2.03 \\
(p \text {-value } \\
=0.021)\end{array}$} & \multirow{8}{*}{1687.79} & \multirow{8}{*}{1721.63} \\
\hline & $\log (L)$ & 0.421 & 0.141 & 0.003 & & & \\
\hline & $\beta_{\text {Roll }}$ & -0.156 & 0.058 & 0.007 & & & \\
\hline & Constant & -6.612 & 1.623 & $<0.001$ & & & \\
\hline & \multicolumn{4}{|l|}{ Inflate } & & & \\
\hline & $\log (A A D T)$ & -0.565 & 0.352 & 0.109 & & & \\
\hline & $\log (L)$ & -1.890 & 0.565 & 0.001 & & & \\
\hline & Constant & 13.987 & 3.749 & $<0.001$ & & & \\
\hline
\end{tabular}


Table G-6: ZIP Models to Predict Annual Collision Frequency - Cont.

\begin{tabular}{|c|c|c|c|c|c|c|c|}
\hline Model & Variable & Coef. & $S E E$ & $p$-value & $\begin{array}{l}\text { Vuong } \\
\text { statistic }\end{array}$ & AIC & $\mathrm{BIC}$ \\
\hline \multirow{9}{*}{ ZIP1-7a } & $\log (A A D T)$ & 0.475 & 0.128 & $<0.001$ & \multirow{9}{*}{$\begin{array}{c}2.08 \\
(p \text {-value } \\
=0.019)\end{array}$} & \multirow{9}{*}{1678.94} & \multirow{9}{*}{1717.62} \\
\hline & $\log (L)$ & 0.494 & 0.149 & 0.001 & & & \\
\hline & $R$ & -0.001 & $<0.001$ & 0.030 & & & \\
\hline & $\beta_{V S}$ & -0.131 & 0.074 & 0.077 & & & \\
\hline & Constant & -6.084 & 1.510 & $<0.001$ & & & \\
\hline & \multicolumn{4}{|l|}{ Inflate } & & & \\
\hline & $\log (A A D T)$ & -0.818 & 0.350 & 0.019 & & & \\
\hline & $\log (L)$ & -1.708 & 0.537 & 0.001 & & & \\
\hline & Constant & 15.173 & 3.613 & $<0.001$ & & & \\
\hline \multirow{9}{*}{ ZIP1-8a } & $\log (A A D T)$ & 0.479 & 0.135 & $<0.001$ & \multirow{9}{*}{$\begin{array}{c}1.96 \\
(p \text {-value } \\
=0.025)\end{array}$} & \multirow{9}{*}{1681.28} & \multirow{9}{*}{1719.96} \\
\hline & $\log (L)$ & 0.509 & 0.151 & 0.001 & & & \\
\hline & $R$ & -0.001 & $<0.001$ & $<0.001$ & & & \\
\hline & $\beta_{c m f}$ & 0.033 & 0.038 & 0.377 & & & \\
\hline & Constant & -6.714 & 1.504 & $<0.001$ & & & \\
\hline & \multicolumn{4}{|l|}{ Inflate } & & & \\
\hline & $\log (A A D T)$ & -0.772 & 0.389 & 0.048 & & & \\
\hline & $\log (L)$ & -1.634 & 0.544 & 0.003 & & & \\
\hline & Constant & 14.332 & 3.711 & $<0.001$ & & & \\
\hline \multirow{9}{*}{ ZIP1-9a } & $\log (A A D T)$ & 0.508 & 0.132 & $<0.001$ & \multirow{9}{*}{$\begin{array}{c}2.03 \\
(p \text {-value } \\
=0.021)\end{array}$} & \multirow{9}{*}{1679.14} & \multirow{9}{*}{1717.83} \\
\hline & $\log (L)$ & 0.510 & 0.147 & 0.001 & & & \\
\hline & $R$ & -0.001 & $<0.001$ & 0.009 & & & \\
\hline & $\beta_{S D}$ & -0.051 & 0.030 & 0.089 & & & \\
\hline & Constant & -6.721 & 1.509 & $<0.001$ & & & \\
\hline & \multicolumn{4}{|l|}{ Inflate } & & & \\
\hline & $\log (A A D T)$ & -0.715 & 0.365 & 0.050 & & & \\
\hline & $\log (L)$ & -1.696 & 0.547 & 0.002 & & & \\
\hline & Constant & 14.204 & 3.708 & 0.002 & & & \\
\hline \multirow{9}{*}{ ZIP1-10a } & $\log (A A D T)$ & 0.493 & 0.132 & $<0.001$ & \multirow{9}{*}{$\begin{array}{c}2.08 \\
(p \text {-value } \\
=0.019)\end{array}$} & \multirow{9}{*}{1681.72} & \multirow{9}{*}{1720.40} \\
\hline & $\log (L)$ & 0.515 & 0.150 & 0.001 & & & \\
\hline & $R$ & -0.001 & $<0.001$ & 0.005 & & & \\
\hline & $\beta_{\text {Roll }}$ & -0.042 & 0.072 & 0.562 & & & \\
\hline & Constant & -6.547 & 1.559 & $<0.001$ & & & \\
\hline & \multicolumn{4}{|l|}{ Inflate } & & & \\
\hline & $\log (A A D T)$ & -0.776 & 0.377 & 0.040 & & & \\
\hline & $\log (L)$ & -1.668 & 0.554 & 0.003 & & & \\
\hline & Constant & 14.553 & 3.728 & $<0.001$ & & & \\
\hline
\end{tabular}


Table G-7: ZINB Models to Predict Annual Collision Frequency.

\begin{tabular}{|c|c|c|c|c|c|c|c|}
\hline Model & Variable & Coef. & $S E E$ & $\begin{array}{c}p- \\
\text { value }\end{array}$ & $\begin{array}{l}\text { Vuong } \\
\text { statistic }\end{array}$ & AIC & $\mathrm{BIC}$ \\
\hline \multirow{8}{*}{ ZINB1-3a } & $\log (A A D T)$ & 0.579 & 0.115 & $<0.001$ & \multirow{8}{*}{$\begin{array}{c}1.37 \\
(p \text {-value } \\
=0.085)\end{array}$} & \multirow{8}{*}{1673.90} & \multirow{8}{*}{1712.58} \\
\hline & $\log (L)$ & 0.754 & 0.133 & $<0.001$ & & & \\
\hline & $\beta_{V S}$ & -0.216 & 0.065 & 0.001 & & & \\
\hline & Constant & -8.605 & 1.511 & $<0.001$ & & & \\
\hline & \multicolumn{4}{|l|}{ Inflate } & & & \\
\hline & $\log (A A D T)$ & -2.955 & 1.283 & 0.021 & & & \\
\hline & $\log (L)$ & -1.281 & 1.418 & 0.366 & & & \\
\hline & Constant & 27.814 & 9.681 & 0.004 & & & \\
\hline \multirow{8}{*}{ ZINB1-4a } & $\log (A A D T)$ & 0.672 & 0.117 & $<0.001$ & \multirow{8}{*}{$\begin{array}{c}0.98 \\
(p \text {-value } \\
=0.163)\end{array}$} & \multirow{8}{*}{1684.57} & \multirow{8}{*}{1723.25} \\
\hline & $\log (L)$ & 0.708 & 0.137 & $<0.001$ & & & \\
\hline & $\beta_{c m f}$ & 0.009 & 0.039 & 0.824 & & & \\
\hline & Constant & -10.146 & 1.502 & $<0.001$ & & & \\
\hline & \multicolumn{4}{|l|}{ Inflate } & & & \\
\hline & $\log (A A D T)$ & -2.701 & 1.390 & 0.052 & & & \\
\hline & $\log (L)$ & -1.320 & 1.938 & 0.496 & & & \\
\hline & Constant & 25.850 & 10.618 & 0.015 & & & \\
\hline \multirow{8}{*}{ ZINB1-5a } & $\log (A A D T)$ & 0.635 & 0.119 & $<0.001$ & \multirow{8}{*}{$\begin{array}{c}1.06 \\
(p \text {-value } \\
=0.144)\end{array}$} & \multirow{8}{*}{1676.89} & \multirow{8}{*}{1715.57} \\
\hline & $\log (L)$ & 0.743 & 0.141 & $<0.001$ & & & \\
\hline & $\beta_{S D}$ & -0.077 & 0.028 & 0.005 & & & \\
\hline & Constant & -9.529 & 1.553 & $<0.001$ & & & \\
\hline & \multicolumn{4}{|l|}{ Inflate } & & & \\
\hline & $\log (A A D T)$ & -2.470 & 1.453 & 0.089 & & & \\
\hline & $\log (L)$ & -1.739 & 1.996 & 0.383 & & & \\
\hline & Constant & 26.384 & 11.017 & 0.017 & & & \\
\hline \multirow{8}{*}{ ZINB1-6a } & $\log (A A D T)$ & 0.624 & 0.115 & $<0.001$ & \multirow{8}{*}{$\begin{array}{c}1.16 \\
(p \text {-value } \\
=0.122)\end{array}$} & \multirow{8}{*}{1677.89} & \multirow{8}{*}{1716.57} \\
\hline & $\log (L)$ & 0.760 & 0.134 & $<0.001$ & & & \\
\hline & $\beta_{\text {Roll }}$ & -0.169 & 0.064 & 0.008 & & & \\
\hline & Constant & -8.707 & 1.542 & $<0.001$ & & & \\
\hline & \multicolumn{4}{|l|}{ Inflate } & & & \\
\hline & $\log (A A D T)$ & -2.949 & 1.332 & 0.027 & & & \\
\hline & $\log (L)$ & -1.281 & 1.627 & 0.431 & & & \\
\hline & Constant & 27.590 & 10.254 & 0.007 & & & \\
\hline
\end{tabular}


Table G-7: ZINB Models to Predict Annual Collision Frequency - Cont.

\begin{tabular}{|c|c|c|c|c|c|c|c|}
\hline Model & Variable & Coef. & SEE & $\begin{array}{c}p- \\
\text { value }\end{array}$ & $\begin{array}{l}\text { Vuong } \\
\text { statistic }\end{array}$ & AIC & $\mathrm{BIC}$ \\
\hline \multirow{9}{*}{ ZINB1-7a } & $\log (A A D T)$ & 0.527 & 0.117 & $<0.001$ & \multirow{9}{*}{$\begin{array}{c}1.36 \\
(p \text {-value } \\
=0.087)\end{array}$} & \multirow{9}{*}{1669.66} & \multirow{9}{*}{1713.17} \\
\hline & $\log (L)$ & 0.808 & 0.135 & $<0.001$ & & & \\
\hline & $R$ & -0.001 & $<0.001$ & 0.014 & & & \\
\hline & $\beta_{V S}$ & -0.103 & 0.079 & 0.192 & & & \\
\hline & Constant & -8.597 & 1.501 & $<0.001$ & & & \\
\hline & \multicolumn{4}{|l|}{ Inflate } & & & \\
\hline & $\log (A A D T)$ & -2.979 & 1.282 & 0.020 & & & \\
\hline & $\log (L)$ & -1.323 & 1.366 & 0.333 & & & \\
\hline & Constant & 28.214 & 9.502 & 0.003 & & & \\
\hline \multirow{9}{*}{ ZINB1-8a } & $\log (A A D T)$ & 0.529 & 0.118 & $<0.001$ & \multirow{9}{*}{$\begin{array}{c}1.19 \\
(p \text {-value } \\
=0.116)\end{array}$} & \multirow{9}{*}{1670.41} & \multirow{9}{*}{1713.93} \\
\hline & $\log (L)$ & 0.796 & 0.136 & $<0.001$ & & & \\
\hline & $R$ & -0.001 & $<0.001$ & $<0.001$ & & & \\
\hline & $\beta_{c m f}$ & 0.039 & 0.040 & 0.333 & & & \\
\hline & Constant & -8.959 & 1.478 & $<0.001$ & & & \\
\hline & \multicolumn{4}{|l|}{ Inflate } & & & \\
\hline & $\log (A A D T)$ & -2.943 & 1.308 & 0.024 & & & \\
\hline & $\log (L)$ & -1.237 & 1.467 & 0.399 & & & \\
\hline & Constant & 27.404 & 9.492 & 0.004 & & & \\
\hline \multirow{9}{*}{ ZINB1-9a } & $\log (A A D T)$ & 0.543 & 0.119 & $<0.001$ & \multirow{9}{*}{$\begin{array}{c}1.20 \\
(p \text {-value } \\
=0.115)\end{array}$} & \multirow{9}{*}{1670.08} & \multirow{9}{*}{1713.60} \\
\hline & $\log (L)$ & 0.808 & 0.137 & $<0.001$ & & & \\
\hline & $R$ & -0.001 & $<0.001$ & 0.003 & & & \\
\hline & $\beta_{S D}$ & -0.035 & 0.031 & 0.261 & & & \\
\hline & Constant & -8.949 & 1.496 & $<0.001$ & & & \\
\hline & \multicolumn{4}{|l|}{ Inflate } & & & \\
\hline & $\log (A A D T)$ & -2.795 & 1.319 & 0.034 & & & \\
\hline & $\log (L)$ & -1.457 & 1.512 & 0.335 & & & \\
\hline & Constant & 27.471 & 9.776 & 0.005 & & & \\
\hline \multirow{9}{*}{ ZINB1-10a } & $\log (A A D T)$ & 0.542 & 0.117 & $<0.001$ & \multirow{9}{*}{$\begin{array}{c}1.23 \\
(p \text {-value } \\
=0.110)\end{array}$} & \multirow{9}{*}{1671.11} & \multirow{9}{*}{1714.63} \\
\hline & $\log (L)$ & 0.811 & 0.136 & $<0.001$ & & & \\
\hline & $R$ & -0.001 & $<0.001$ & 0.004 & & & \\
\hline & $\beta_{\text {Roll }}$ & -0.037 & 0.078 & 0.632 & & & \\
\hline & Constant & -8.853 & 1.539 & $<0.001$ & & & \\
\hline & \multicolumn{4}{|l|}{ Inflate } & & & \\
\hline & $\log (A A D T)$ & -2.934 & 1.300 & 0.024 & & & \\
\hline & $\log (L)$ & -1.330 & 1.449 & 0.359 & & & \\
\hline & Constant & 27.842 & 9.649 & 0.004 & & & \\
\hline
\end{tabular}


Table G-8: SPFs for Five-Year Collisions Frequency (Base Models).

\begin{tabular}{|c|c|c|c|c|c|c|c|}
\hline $\begin{array}{l}\text { Regression } \\
\text { Model }\end{array}$ & Variable & Coef. & $S E E$ & $\begin{array}{c}p- \\
\text { value }\end{array}$ & $\begin{array}{l}\text { Likelihood- } \\
\text { ratio test }\end{array}$ & AIC & BIC \\
\hline \multirow{3}{*}{ Poisson5-1a } & Log of $(A A D T)$ & 0.806 & 0.069 & $<0.001$ & \multirow{3}{*}{-- } & \multirow{3}{*}{796.05} & \multirow{3}{*}{805.76} \\
\hline & $\log$ of $(L)$ & 0.804 & 0.096 & $<0.001$ & & & \\
\hline & Constant & -10.251 & 0.874 & $<0.001$ & & & \\
\hline \multirow{3}{*}{ NB5-1a } & Log of $(A A D T)$ & 0.876 & 0.109 & $<0.001$ & \multirow{3}{*}{$\begin{array}{l}80.25 \\
(p \text {-value } \\
<0.001)\end{array}$} & \multirow{3}{*}{717.83} & \multirow{3}{*}{730.74} \\
\hline & $\log$ of $(L)$ & 0.874 & 0.153 & $<0.001$ & & & \\
\hline & Constant & -11.228 & 1.310 & $<0.001$ & & & \\
\hline \multirow{4}{*}{ Poisson5-2a } & Log of $(A A D T)$ & 0.711 & 0.072 & $<0.001$ & \multirow{4}{*}{-- } & \multirow{4}{*}{780.77} & \multirow{4}{*}{793.67} \\
\hline & $\log$ of $(L)$ & 0.909 & 0.101 & $<0.001$ & & & \\
\hline & $R$ & -0.001 & $<0.001$ & $<0.001$ & & & \\
\hline & Constant & $\begin{array}{l}-9.572 \\
\end{array}$ & 0.870 & $<0.001$ & & & \\
\hline \multirow{4}{*}{ NB5-2a } & Log of $(A A D T)$ & 0.790 & 0.109 & $<0.001$ & \multirow{4}{*}{$\begin{array}{l}70.50 \\
(p \text {-value } \\
<0.001)\end{array}$} & \multirow{4}{*}{712.27} & \multirow{4}{*}{728.40} \\
\hline & $\log$ of $(L)$ & 0.991 & 0.156 & $<0.001$ & & & \\
\hline & $R$ & -0.001 & $<0.001$ & $<0.001$ & & & \\
\hline & Constant & -10.713 & 1.289 & $<0.001$ & & & \\
\hline
\end{tabular}


Table G-9: NB Models to Predict Five-Year Collisions Frequency.

\begin{tabular}{|c|c|c|c|c|c|c|c|}
\hline $\begin{array}{l}\text { Regression } \\
\text { Model }\end{array}$ & Variable & Coef. & $S E E$ & $\begin{array}{c}p- \\
\text { value }\end{array}$ & $\begin{array}{l}\text { Likelihood- } \\
\text { ratio test }\end{array}$ & AIC & $\mathrm{BIC}$ \\
\hline \multirow{4}{*}{ NB5-3a } & Log of (AADT) & 0.842 & 0.109 & $<0.001$ & \multirow{4}{*}{$\begin{array}{l}76.15 \\
(p \text {-value } \\
<0.001)\end{array}$} & \multirow{4}{*}{717.20} & \multirow{4}{*}{733.33} \\
\hline & Log of $(L)$ & 0.933 & 0.157 & $<0.001$ & & & \\
\hline & $\beta_{V S}$ & -0.138 & 0.084 & 0.101 & & & \\
\hline & Constant & -10.657 & 1.333 & $<0.001$ & & & \\
\hline \multirow{4}{*}{ NB5-4a } & Log of (AADT) & 0.875 & 0.109 & $<0.001$ & \multirow{4}{*}{$\begin{array}{c}80.08 \\
(p \text {-value } \\
<0.001)\end{array}$} & \multirow{4}{*}{719.74} & \multirow{4}{*}{735.86} \\
\hline & Log of $(L)$ & 0.859 & 0.159 & $<0.001$ & & & \\
\hline & $\beta_{c m f}$ & 0.016 & 0.053 & 0.755 & & & \\
\hline & Constant & -11.172 & 1.322 & $<0.001$ & & & \\
\hline \multirow{4}{*}{ NB5-5a } & Log of (AADT) & 0.839 & 0.109 & $<0.001$ & \multirow{4}{*}{$\begin{array}{c}73.71 \\
(p \text {-value } \\
<0.001)\end{array}$} & \multirow{4}{*}{717.08} & \multirow{4}{*}{733.20} \\
\hline & Log of $(L)$ & 0.912 & 0.153 & $<0.001$ & & & \\
\hline & $\beta_{S D}$ & -0.062 & 0.037 & 0.094 & & & \\
\hline & Constant & -10.760 & 1.318 & $<0.001$ & & & \\
\hline \multirow{4}{*}{ NB5-6a } & Log of (AADT) & 0.865 & 0.108 & $<0.001$ & \multirow{4}{*}{$\begin{array}{l}75.82 \\
(p \text {-value } \\
<0.001)\end{array}$} & \multirow{4}{*}{716.84} & \multirow{4}{*}{732.97} \\
\hline & Log of $(L)$ & 0.920 & 0.155 & $<0.001$ & & & \\
\hline & $\beta_{\text {Roll }}$ & -0.148 & 0.085 & 0.082 & & & \\
\hline & Constant & -10.261 & 1.394 & $<0.001$ & & & \\
\hline \multirow{5}{*}{ NB5-3a } & Log of (AADT) & 0.790 & 0.109 & $<0.001$ & \multirow{5}{*}{$\begin{array}{c}70.49 \\
(p \text {-value } \\
<0.001)\end{array}$} & \multirow{5}{*}{714.26} & \multirow{5}{*}{733.61} \\
\hline & Log of $(L)$ & 0.989 & 0.157 & $<0.001$ & & & \\
\hline & $R$ & -0.001 & $<0.001$ & 0.026 & & & \\
\hline & $\beta_{V S}$ & 0.010 & 0.106 & 0.925 & & & \\
\hline & Constant & -10.740 & 1.321 & $<0.001$ & & & \\
\hline \multirow{5}{*}{ NB5-4a } & Log of (AADT) & 0.780 & 0.110 & $<0.001$ & \multirow{5}{*}{$\begin{array}{c}70.31 \\
(p \text {-value } \\
<0.001)\end{array}$} & \multirow{5}{*}{713.21} & \multirow{5}{*}{732.56} \\
\hline & Log of $(L)$ & 0.954 & 0.160 & $<0.001$ & & & \\
\hline & $R$ & -0.001 & 0.000 & 0.003 & & & \\
\hline & $\beta_{c m f}$ & 0.055 & 0.054 & 0.305 & & & \\
\hline & Constant & -10.488 & 1.308 & $<0.001$ & & & \\
\hline \multirow{5}{*}{ NB5-5a } & Log of (AADT) & 0.787 & 0.109 & $<0.001$ & \multirow{5}{*}{$\begin{array}{c}69.08 \\
(p \text {-value } \\
<0.001)\end{array}$} & \multirow{5}{*}{714.08} & \\
\hline & Log of $(L)$ & 0.992 & 0.156 & $<0.001$ & & & \\
\hline & $R$ & -0.001 & $<0.001$ & 0.025 & & & 733.43 \\
\hline & $\beta_{S D}$ & -0.018 & 0.041 & 0.661 & & & \\
\hline & Constant & -10.619 & 1.303 & $<0.001$ & & & \\
\hline & Log of (AADT) & 0.793 & 0.110 & $<0.001$ & & & \\
\hline & Log of $(L)$ & 0.991 & 0.156 & $<0.001$ & 70.37 & & \\
\hline NB5-6a & $R$ & -0.001 & $<0.001$ & 0.031 & ( $p$-value & 714.24 & 733.60 \\
\hline & $\overline{\beta_{\text {Roll }}}$ & -0.017 & 0.103 & 0.869 & $<0.001)$ & & \\
\hline & Constant & -10.625 & 1.393 & $<0.001$ & & & \\
\hline
\end{tabular}


Table G-10: SPFs for Five-Year Collisions Frequency Considering All Reliability Indices.

\begin{tabular}{|c|c|c|c|c|c|c|c|}
\hline $\begin{array}{c}\text { Regression } \\
\text { Model } \\
\end{array}$ & Variable & $\begin{array}{c}\text { Coefficie } \\
\mathrm{nt}\end{array}$ & $\begin{array}{c}\text { Standard } \\
\text { Error }\end{array}$ & $p$-value & $\begin{array}{c}\text { Likelihood- } \\
\text { ratio test }\end{array}$ & AIC & BIC \\
\hline \multirow{7}{*}{ NB5-5a } & Log of (AADT) & 0.831 & 0.112 & $<0.001$ & \multirow{7}{*}{$\begin{array}{l}72.15 \\
(p \text {-value } \\
<0.001)\end{array}$} & \multirow{7}{*}{721.40} & \multirow{7}{*}{747.20} \\
\hline & Log of $(L)$ & 0.914 & 0.160 & $<0.001$ & & & \\
\hline & $\beta_{V S}$ & -0.057 & 0.156 & 0.715 & & & \\
\hline & $\beta_{c m f}$ & 0.034 & 0.056 & 0.541 & & & \\
\hline & $\beta_{S D}$ & -0.043 & 0.042 & 0.305 & & & \\
\hline & $\beta_{\text {Roll }}$ & -0.059 & 0.152 & 0.697 & & & \\
\hline & Constant & -10.165 & 1.406 & $<0.001$ & & & \\
\hline \multirow[t]{8}{*}{ NB5-5a } & Log of (AADT) & 0.774 & 0.113 & $<0.001$ & \multirow{8}{*}{$\begin{array}{c}68.74 \\
(p \text {-value } \\
<0.001)\end{array}$} & \multirow[t]{8}{*}{718.95} & \multirow[t]{8}{*}{747.98} \\
\hline & Log of $(L)$ & 0.954 & 0.160 & $<0.001$ & & & \\
\hline & $R$ & -0.001 & 0.001 & 0.035 & & & \\
\hline & $\beta_{V S}$ & 0.008 & 0.156 & 0.960 & & & \\
\hline & $\beta_{c m f}$ & 0.057 & 0.057 & 0.319 & & & \\
\hline & $\beta_{S D}$ & -0.022 & 0.043 & 0.610 & & & \\
\hline & $\beta_{\text {Roll }}$ & 0.008 & 0.153 & 0.959 & & & \\
\hline & Constant & -10.429 & 1.406 & $<0.001$ & & & \\
\hline
\end{tabular}


Table G-11: ZIP and ZINB Base Models to Predict Five-Year Collision Frequency.

\begin{tabular}{|c|c|c|c|c|c|c|c|}
\hline Model & Variable & Coef. & $S E E$ & $\begin{array}{c}p- \\
\text { value }\end{array}$ & $\begin{array}{c}\text { Vuong } \\
\text { test }\end{array}$ & AIC & BIC \\
\hline \multirow{7}{*}{ ZIP5-1a } & $\log (A A D T)$ & 0.685 & 0.085 & $<0.001$ & \multirow{7}{*}{$\begin{array}{c}2.21 \\
(p- \\
\text { value } \\
=0.014)\end{array}$} & \multirow{7}{*}{771.57} & \multirow{7}{*}{790.93} \\
\hline & $\log (L)$ & 0.617 & 0.105 & $<0.001$ & & & \\
\hline & Constant & -8.032 & 1.082 & $<0.001$ & & & \\
\hline & \multicolumn{4}{|l|}{ Inflate } & & & \\
\hline & $\log (A A D T)$ & -0.919 & 0.388 & 0.018 & & & \\
\hline & $\log (L)$ & -1.232 & 0.600 & 0.040 & & & \\
\hline & Constant & 12.568 & 4.390 & 0.004 & & & \\
\hline \multirow{8}{*}{ ZIP5-2a } & $\log (A A D T)$ & 0.574 & 0.089 & $<0.001$ & \multirow{8}{*}{$\begin{array}{c}2.20 \\
(p- \\
\text { value } \\
=0.014)\end{array}$} & \multirow{8}{*}{759.04} & \multirow{8}{*}{781.62} \\
\hline & $\log (L)$ & 0.715 & 0.110 & $<0.001$ & & & \\
\hline & $R$ & -0.001 & 0.000 & $<0.001$ & & & \\
\hline & Constant & -7.187 & 1.072 & $<0.001$ & & & \\
\hline & \multicolumn{4}{|l|}{ Inflate } & & & \\
\hline & $\log (A A D T)$ & -1.047 & 0.401 & 0.009 & & & \\
\hline & $\log (L)$ & -1.275 & 0.626 & 0.042 & & & \\
\hline & Constant & 13.801 & 4.427 & 0.002 & & & \\
\hline \multirow{7}{*}{ ZINB5-1a } & $\log (A A D T)$ & 0.751 & 0.135 & $<0.001$ & \multirow{7}{*}{$\begin{array}{c}0.75 \\
(p- \\
\text { value } \\
=\mathbf{0 . 2 2 6})\end{array}$} & \multirow{7}{*}{721.55} & \multirow{7}{*}{744.13} \\
\hline & $\log (L)$ & 0.784 & 0.168 & $<0.001$ & & & \\
\hline & Constant & -9.640 & 1.705 & $<0.001$ & & & \\
\hline & \multicolumn{4}{|l|}{ Inflate } & & & \\
\hline & $\log (A A D T)$ & -3.024 & 2.296 & 0.188 & & & \\
\hline & $\log (L)$ & -1.207 & 2.076 & 0.561 & & & \\
\hline & Constant & 26.977 & 16.274 & 0.097 & & & \\
\hline \multirow{8}{*}{ ZINB5-2a } & $\log (A A D T)$ & 0.642 & 0.134 & $<0.001$ & \multirow{8}{*}{$\begin{array}{c}0.87 \\
(p- \\
\text { value } \\
=\mathbf{0 . 1 9 1})\end{array}$} & \multirow{8}{*}{714.98} & \multirow{8}{*}{740.79} \\
\hline & $\log (L)$ & 0.899 & 0.169 & $<0.001$ & & & \\
\hline & $R$ & -0.001 & $<0.001$ & 0.003 & & & \\
\hline & Constant & -8.876 & 1.667 & $<0.001$ & & & \\
\hline & \multicolumn{4}{|l|}{ Inflate } & & & \\
\hline & $\log (A A D T)$ & -3.116 & 2.144 & 0.146 & & & \\
\hline & $\log (L)$ & -1.082 & 1.822 & 0.552 & & & \\
\hline & Constant & 27.161 & 14.991 & 0.070 & & & \\
\hline
\end{tabular}


Table G-12: ZIP Models to Predict Five-Year Collision Frequency.

\begin{tabular}{|c|c|c|c|c|c|c|c|}
\hline Model & Variable & Coef. & SEE & $\begin{array}{c}p- \\
\text { value }\end{array}$ & $\begin{array}{c}\text { Vuong } \\
\text { test }\end{array}$ & AIC & BIC \\
\hline \multirow{8}{*}{ ZIP5-3a } & $\log (A A D T)$ & 0.615 & 0.086 & $<0.001$ & \multirow{8}{*}{$\begin{array}{c}2.28 \\
(p- \\
\text { value } \\
=0.011)\end{array}$} & \multirow{8}{*}{765.77} & \multirow{8}{*}{788.35} \\
\hline & $\log (L)$ & 0.655 & 0.104 & $<0.001$ & & & \\
\hline & $\beta_{V S}$ & -0.166 & 0.058 & 0.004 & & & \\
\hline & Constant & -6.900 & 1.097 & $<0.001$ & & & \\
\hline & \multicolumn{4}{|l|}{ Inflate } & & & \\
\hline & $\log (A A D T)$ & -1.008 & 0.376 & 0.007 & & & \\
\hline & $\log (L)$ & -1.281 & 0.600 & 0.033 & & & \\
\hline & Constant & 13.585 & 4.248 & 0.001 & & & \\
\hline \multirow{8}{*}{ ZIP5-4a } & $\log (A A D T)$ & 0.686 & 0.085 & $<0.001$ & \multirow{8}{*}{$\begin{array}{c}2.12 \\
(p- \\
\text { value } \\
=0.017)\end{array}$} & \multirow{8}{*}{773.49} & \multirow{8}{*}{796.07} \\
\hline & $\log (L)$ & 0.613 & 0.106 & $<0.001$ & & & \\
\hline & $\beta_{c m f}$ & 0.010 & 0.036 & 0.777 & & & \\
\hline & Constant & -8.036 & 1.083 & $<0.001$ & & & \\
\hline & \multicolumn{4}{|l|}{ Inflate } & & & \\
\hline & $\log (A A D T)$ & -0.911 & 0.391 & 0.020 & & & \\
\hline & $\log (L)$ & -1.234 & 0.604 & 0.041 & & & \\
\hline & Constant & 12.506 & 4.424 & 0.005 & & & \\
\hline \multirow{8}{*}{ ZIP5-5a } & $\log (A A D T)$ & 0.649 & 0.088 & $<0.001$ & \multirow{8}{*}{$\begin{array}{c}2.18 \\
(p- \\
\text { value } \\
=0.015)\end{array}$} & \multirow{8}{*}{765.35} & \multirow{8}{*}{787.93} \\
\hline & $\log (L)$ & 0.651 & 0.106 & $<0.001$ & & & \\
\hline & $\beta_{S D}$ & -0.073 & 0.026 & 0.004 & & & \\
\hline & Constant & -7.474 & 1.106 & $<0.001$ & & & \\
\hline & \multicolumn{4}{|l|}{ Inflate } & & & \\
\hline & $\log (A A D T)$ & -0.974 & 0.393 & 0.013 & & & \\
\hline & $\log (L)$ & -1.280 & 0.621 & 0.039 & & & \\
\hline & Constant & 13.256 & 4.428 & 0.003 & & & \\
\hline \multirow{8}{*}{ ZIP5-6a } & $\log (A A D T)$ & 0.650 & 0.086 & $<0.001$ & \multirow{8}{*}{$\begin{array}{c}2.21 \\
(p- \\
\text { value } \\
=0.014)\end{array}$} & \multirow{8}{*}{766.99} & \multirow{8}{*}{789.57} \\
\hline & $\log (L)$ & 0.672 & 0.107 & $<0.001$ & & & \\
\hline & $\beta_{\text {Roll }}$ & -0.144 & 0.055 & 0.008 & & & \\
\hline & Constant & -6.939 & 1.138 & $<0.001$ & & & \\
\hline & \multicolumn{4}{|l|}{ Inflate } & & & \\
\hline & $\log (A A D T)$ & -0.972 & 0.386 & 0.012 & & & \\
\hline & $\log (L)$ & -1.238 & 0.606 & 0.041 & & & \\
\hline & Constant & 13.030 & 4.345 & 0.003 & & & \\
\hline
\end{tabular}


Table G-12: ZIP Models to Predict Five-Year Collision Frequency - Cont.

\begin{tabular}{|c|c|c|c|c|c|c|c|}
\hline Model & Variable & Coef. & SEE & $\begin{array}{c}p- \\
\text { value }\end{array}$ & $\begin{array}{c}\text { Vuong } \\
\text { test }\end{array}$ & AIC & BIC \\
\hline \multirow{9}{*}{ ZIP5-7a } & $\log (A A D T)$ & 0.568 & 0.089 & $<0.001$ & \multirow{9}{*}{$\begin{array}{c}2.13 \\
(p- \\
\text { value } \\
=0.016)\end{array}$} & \multirow{9}{*}{760.70} & \multirow{9}{*}{786.50} \\
\hline & $\log (L)$ & 0.715 & 0.109 & $<0.001$ & & & \\
\hline & $R$ & -0.001 & $<0.001$ & 0.010 & & & \\
\hline & $\beta_{V S}$ & -0.045 & 0.075 & 0.554 & & & \\
\hline & Constant & -6.993 & 1.109 & $<0.001$ & & & \\
\hline & \multicolumn{4}{|l|}{ Inflate } & & & \\
\hline & $\log (A A D T)$ & -1.057 & 0.397 & 0.008 & & & \\
\hline & $\log (L)$ & -1.282 & 0.623 & 0.040 & & & \\
\hline & Constant & 13.937 & 4.391 & 0.002 & & & \\
\hline \multirow{9}{*}{ ZIP5-4a } & $\log (A A D T)$ & 0.565 & 0.090 & $<0.001$ & \multirow{9}{*}{$\begin{array}{c}2.13 \\
(p- \\
\text { value } \\
=0.016)\end{array}$} & \multirow{9}{*}{760.07} & \multirow{9}{*}{785.88} \\
\hline & $\log (L)$ & 0.705 & 0.111 & $<0.001$ & & & \\
\hline & $R$ & -0.001 & 0.000 & $<0.001$ & & & \\
\hline & $\beta_{c m f}$ & 0.037 & 0.037 & 0.321 & & & \\
\hline & Constant & -7.106 & 1.078 & $<0.001$ & & & \\
\hline & \multicolumn{4}{|l|}{ Inflate } & & & \\
\hline & $\log (A A D T)$ & -1.028 & 0.402 & 0.011 & & & \\
\hline & $\log (L)$ & -1.298 & 0.638 & 0.042 & & & \\
\hline & Constant & 13.750 & 4.496 & 0.002 & & & \\
\hline \multirow{9}{*}{ ZIP5-5a } & $\log (A A D T)$ & 0.573 & 0.090 & $<0.001$ & \multirow{9}{*}{$\begin{array}{c}2.18 \\
(p- \\
\text { value } \\
=0.014)\end{array}$} & \multirow{9}{*}{759.35} & \multirow{9}{*}{785.16} \\
\hline & $\log (L)$ & 0.715 & 0.109 & $<0.001$ & & & \\
\hline & $R$ & -0.001 & $<0.001$ & 0.006 & & & \\
\hline & $\beta_{S D}$ & -0.037 & 0.028 & 0.194 & & & \\
\hline & Constant & -7.035 & 1.086 & $<0.001$ & & & \\
\hline & \multicolumn{4}{|l|}{ Inflate } & & & \\
\hline & $\log (A A D T)$ & -1.059 & 0.401 & 0.008 & & & \\
\hline & $\log (L)$ & -1.294 & 0.633 & 0.041 & & & \\
\hline & Constant & 13.993 & 4.444 & 0.002 & & & \\
\hline \multirow{9}{*}{ ZIP5-6a } & $\log (A A D T)$ & 0.576 & 0.089 & $<0.001$ & \multirow{9}{*}{$\begin{array}{c}2.21 \\
(p- \\
\text { value } \\
=0.013)\end{array}$} & \multirow{9}{*}{760.76} & \multirow{9}{*}{786.56} \\
\hline & $\log (L)$ & 0.721 & 0.110 & $<0.001$ & & & \\
\hline & $R$ & -0.001 & $<0.001$ & 0.006 & & & \\
\hline & $\beta_{\text {Roll }}$ & -0.036 & 0.067 & 0.591 & & & \\
\hline & Constant & -7.000 & 1.123 & $<0.001$ & & & \\
\hline & \multicolumn{4}{|l|}{ Inflate } & & & \\
\hline & $\log (A A D T)$ & -1.049 & 0.400 & 0.009 & & & \\
\hline & $\log (L)$ & -1.271 & 0.626 & 0.042 & & & \\
\hline & Constant & 13.803 & 4.415 & 0.002 & & & \\
\hline
\end{tabular}


Table G-13: ZINB Models to Predict Five-Year Collision Frequency.

\begin{tabular}{|c|c|c|c|c|c|c|c|}
\hline Model & Variable & Coef. & $S E E$ & $\begin{array}{c}p- \\
\text { value }\end{array}$ & $\begin{array}{c}\text { Vuong } \\
\text { test }\end{array}$ & AIC & $\mathrm{BIC}$ \\
\hline \multirow{8}{*}{ ZINB5-3a } & $\log (A A D T)$ & 0.694 & 0.135 & $<0.001$ & \multirow{8}{*}{$\begin{array}{c}0.93 \\
(p- \\
\text { value } \\
=0.177)\end{array}$} & \multirow{8}{*}{719.95} & \multirow{8}{*}{745.76} \\
\hline & $\log (L)$ & 0.847 & 0.171 & $<0.001$ & & & \\
\hline & $\beta_{V S}$ & -0.166 & 0.087 & 0.057 & & & \\
\hline & Constant & -8.759 & 1.741 & $<0.001$ & & & \\
\hline & \multicolumn{4}{|l|}{ Inflate } & & & \\
\hline & $\log (A A D T)$ & -3.175 & 2.447 & 0.238 & & & \\
\hline & $\log (L)$ & -1.129 & 1.888 & 0.798 & & & \\
\hline & Constant & 27.790 & 16.787 & 0.252 & & & \\
\hline \multirow{8}{*}{ ZINB5-4a } & $\log (A A D T)$ & 0.752 & 0.135 & $<0.001$ & \multirow{8}{*}{$\begin{array}{c}0.76 \\
(p- \\
\text { value } \\
=0.224)\end{array}$} & \multirow{8}{*}{723.52} & \multirow{8}{*}{749.33} \\
\hline & $\log (L)$ & 0.778 & 0.172 & $<0.001$ & & & \\
\hline & $\beta_{c m f}$ & 0.009 & 0.052 & 0.868 & & & \\
\hline & Constant & -9.634 & 1.707 & $<0.001$ & & & \\
\hline & \multicolumn{4}{|l|}{ Inflate } & & & \\
\hline & $\log (A A D T)$ & -3.059 & 2.352 & 0.194 & & & \\
\hline & $\log (L)$ & -1.192 & 2.111 & 0.572 & & & \\
\hline & Constant & 27.135 & 16.433 & 0.099 & & & \\
\hline \multirow{8}{*}{ ZINB5-5a } & $\log (A A D T)$ & 0.690 & 0.136 & $<0.001$ & \multirow{8}{*}{$\begin{array}{c}0.89 \\
(p- \\
\text { value } \\
=0.188)\end{array}$} & \multirow{8}{*}{720.06} & \multirow{8}{*}{745.86} \\
\hline & $\log (L)$ & 0.810 & 0.166 & $<0.001$ & & & \\
\hline & $\beta_{S D}$ & -0.069 & 0.037 & 0.060 & & & \\
\hline & Constant & -8.839 & 1.720 & $<0.001$ & & & \\
\hline & \multicolumn{4}{|l|}{ Inflate } & & & \\
\hline & $\log (A A D T)$ & -2.901 & 1.902 & 0.127 & & & \\
\hline & $\log (L)$ & -1.296 & 1.814 & 0.475 & & & \\
\hline & Constant & 26.737 & 14.233 & 0.060 & & & \\
\hline \multirow{8}{*}{ ZINB5-6a } & $\log (A A D T)$ & 0.730 & 0.133 & $<0.001$ & \multirow{8}{*}{$\begin{array}{c}0.95 \\
(p- \\
\text { value } \\
=0.171)\end{array}$} & \multirow{8}{*}{836.87} & \multirow{8}{*}{864.45} \\
\hline & $\log (L)$ & 0.834 & 0.169 & $<0.001$ & & & \\
\hline & $\beta_{\text {Roll }}$ & -0.168 & 0.087 & 0.055 & & & \\
\hline & Constant & -8.444 & 1.780 & $<0.001$ & & & \\
\hline & \multicolumn{4}{|l|}{ Inflate } & & & \\
\hline & $\log (A A D T)$ & -3.336 & 2.448 & 0.173 & & & \\
\hline & $\log (L)$ & -1.177 & 2.003 & 0.557 & & & \\
\hline & Constant & 29.104 & 17.169 & 0.090 & & & \\
\hline
\end{tabular}


Table G-13: ZINB Models to Predict Five-Year Collision Frequency- Cont.

\begin{tabular}{|c|c|c|c|c|c|c|c|}
\hline Model & Variable & Coef. & $S E E$ & $\begin{array}{c}p- \\
\text { value }\end{array}$ & $\begin{array}{c}\text { Vuong } \\
\text { test }\end{array}$ & AIC & $\mathrm{BIC}$ \\
\hline \multirow{9}{*}{ ZINB5-7a } & $\log (A A D T)$ & 0.134 & 4.760 & 0.377 & \multirow{9}{*}{$\begin{array}{c}0.91 \\
(p- \\
\text { value } \\
=0.180)\end{array}$} & \multirow{9}{*}{716.96} & \multirow{9}{*}{745.99} \\
\hline & $\log (L)$ & 0.900 & 0.170 & $<0.001$ & & & \\
\hline & $R$ & -0.001 & 0.000 & 0.025 & & & \\
\hline & $\beta_{V S}$ & -0.017 & 0.108 & 0.874 & & & \\
\hline & Constant & -8.816 & 1.709 & 0.000 & & & \\
\hline & \multicolumn{4}{|l|}{ Inflate } & & & \\
\hline & $\log (A A D T)$ & -3.121 & 2.160 & 0.148 & & & \\
\hline & $\log (L)$ & -1.084 & 1.813 & 0.550 & & & \\
\hline & Constant & 27.215 & 15.081 & 0.071 & & & \\
\hline \multirow{9}{*}{ ZINB5-8a } & $\log (A A D T)$ & 0.639 & 0.136 & $<0.001$ & \multirow{9}{*}{$\begin{array}{c}0.85 \\
(p- \\
\text { value } \\
=0.199)\end{array}$} & \multirow{9}{*}{716.13} & \multirow{9}{*}{745.17} \\
\hline & $\log (L)$ & 0.876 & 0.171 & $<0.001$ & & & \\
\hline & $R$ & -0.001 & $<0.001$ & 0.002 & & & \\
\hline & $\beta_{c m f}$ & 0.048 & 0.053 & 0.358 & & & \\
\hline & Constant & -8.779 & 1.683 & $<0.001$ & & & \\
\hline & \multicolumn{4}{|l|}{ Inflate } & & & \\
\hline & $\log (A A D T)$ & -3.296 & 2.355 & 0.162 & & & \\
\hline & $\log (L)$ & -0.981 & 1.950 & 0.615 & & & \\
\hline & Constant & 27.861 & 15.541 & 0.073 & & & \\
\hline \multirow{9}{*}{ ZINB5-9a } & $\log (A A D T)$ & 0.631 & 0.135 & $<0.001$ & \multirow{9}{*}{$\begin{array}{c}0.91 \\
(p- \\
\text { value } \\
=0.181)\end{array}$} & \multirow{9}{*}{716.61} & \multirow{9}{*}{745.64} \\
\hline & $\log (L)$ & 0.895 & 0.169 & $<0.001$ & & & \\
\hline & $R$ & -0.001 & $<0.001$ & 0.019 & & & \\
\hline & $\beta_{S D}$ & -0.025 & 0.041 & 0.538 & & & \\
\hline & Constant & -8.663 & 1.692 & $<0.001$ & & & \\
\hline & \multicolumn{4}{|l|}{ Inflate } & & & \\
\hline & $\log (A A D T)$ & -3.060 & 2.008 & 0.128 & & & \\
\hline & $\log (L)$ & -1.141 & 1.761 & 0.517 & & & \\
\hline & Constant & 27.109 & 14.345 & 0.059 & & & \\
\hline \multirow{9}{*}{ ZINB5-10a } & $\log (A A D T)$ & 0.646 & 0.135 & $<0.001$ & \multirow{9}{*}{$\begin{array}{c}0.88 \\
(p- \\
\text { value } \\
=0.190)\end{array}$} & \multirow{9}{*}{716.89} & \multirow{9}{*}{745.92} \\
\hline & $\log (L)$ & 0.900 & 0.169 & $<0.001$ & & & \\
\hline & $R$ & -0.001 & $<0.001$ & 0.025 & & & \\
\hline & $\beta_{\text {Roll }}$ & -0.032 & 0.104 & 0.758 & & & \\
\hline & Constant & -8.703 & 1.756 & $<0.001$ & & & \\
\hline & \multicolumn{4}{|l|}{ Inflate } & & & \\
\hline & $\log (A A D T)$ & -3.160 & 2.178 & 0.147 & & & \\
\hline & $\log (L)$ & -1.092 & 1.828 & 0.550 & & & \\
\hline & Constant & 27.524 & 15.272 & 0.072 & & & \\
\hline
\end{tabular}

